\title{
Proceedings of the 26th European Paediatric Rheumatology Congress: part 2
}

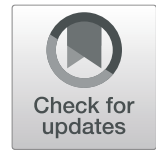

Virtual. 23 - 26 September 2020

Published: 28 October 2020

P122

Metabolic disorders in children with juvenile idiopathic arthritis on biological therapy

I. Chyzheuskaya ${ }^{1,2}$, A. Vishnevskaya², H. Voitova', M. Kastsianevich ${ }^{1}$

${ }^{1}$ Pediatrics, Belarusian Medical Academy of Postgraduate Education;

${ }^{2}$ Pediatrics, 4th City Children's Clinical Hospital, Minsk, Belarus

Correspondence: Yingli Fu

Pediatric Rheumatology 2020, 18(Suppl 2):P122

Introduction: Juvenile idiopathic arthritis (JIA) is a chronic immunoinflammatory joint disease with a high degree of disability and an unfavorable prognosis. In recent decades, drugs aimed at proinflammatory cytokines, such as tumor necrosis factor (TNF), have been used very often for the treatment of JIA. The effect of these drugs on metabolic processes is not well understood.

Objectives: The aim of the study was to study metabolic disorders in children with JIA receiving biological therapy.

Methods: 36 children with polyarticular JIA and 20 healthy children were examined in the rheumatology department of the 4th city children's clinical hospital in Minsk. All children with JIA have long received methotrexate, non-steroidal anti-inflammatory drugs and, if necessary, glucocorticoids. In connection with the preservation of a high degree of disease activity during therapy, patients were prescribed adalimumab.

All children were determined by the main indicators of the lipid spectrum of the blood. Proteins that make up lipoproteins (apoproteins $A p o A, A p o B, A p o E)$ were determined by the immunoturbidimetric method in the research laboratory of the Belarusian Medical Academy of Postgraduate Education. Statistical data processing was carried out by traditional methods of variation statistics on a personal computer using the program Statsoft Statistica 6.0.

Results: In children with JIA, the use of adalimumab showed a significant $(p<0.05)$ decrease in the concentration of ApoA (92.3 [69.7; 99.1] $\mathrm{mg} / \mathrm{dl}$ ) compared with the control group (127.2 [122.1; 132.3] $\mathrm{mg} / \mathrm{dl})$ and an increase in ApoB (60.9 [48.9; 73.4] mg / dl) compared with the control group $(32.1[19.9 ; 50.8] \mathrm{mg} / \mathrm{dl})$.

The determination of $A p o A$ and $A p o B$ is used to calculate the $A p o B /$ ApoA coefficient, which is a more reliable tool for assessing cardiovascular risk. With the $A p \circ B / A p o A$ index $<1$, atherogenicity is regarded as low, with the $A p \circ B / A p o A>1$, atherogenicity increases. ApoB / ApoA> 1 was established in 10 (27.8\%) children with JIA. Apolipoprotein $E$ (ApoE) plays an important role in the regulation of lipid metabolism, has a strong antiatherosclerotic effect. There is an assumption that apoE has allele-specific antioxidant abilities. The study found a reduced level of $A p o E$ in the blood serum of children with JIA compared with the control group.

During adalimumab treatment, a remission of the disease was achieved. According to the results of a second study of the indicators ApoA, ApoB and ApoE, 6 months after the start of biological therapy, an improvement in these indicators was found. Thus, the content of ApoA increased to $118.9[113.2 ; 129.4] \mathrm{mg} / \mathrm{dL}$, and the ApoB content decreased to $33.6[20.8 ; 49.4] \mathrm{mg} / \mathrm{dl}$. An increase in ApoE to reference values was also noted.

Conclusion: The results of the study indicate the likelihood of a reduction in cardiovascular risk in children with JIA in the treatment of adalimumab.

\section{Disclosure of Interest \\ None declared}

\section{P123}

Vaccination coverage in a cohort of patients with juvenile idiopathic arthritis: a single-centre experience

R. Alfani, R. Naddei, A. Vincenzi, F. Viscogliosi, A. Paonessa, A. Catzola, M. Alessio

Pediatric Rheumatology Unit, Mother and Child Department, University of Naples Federico II, Naples, Italy

Correspondence: R. Alfani

Pediatric Rheumatology 2020, 18(Suppl 2):P123

Introduction: Juvenile idiopathic arthritis (JIA) represents the most common pediatric chronic rheumatic disease. Children with JIA present an increased risk of infections, due to the immune-regulatory effects of disease modifying antirheumatic drugs (DMARDs); many of these infections are vaccine-preventable. Nevertheless, suboptimal vaccinations rates are reported in children with JIA.

Objectives: To evaluate vaccination coverage in a population of children with JIA and to describe the prevalence of the adverse events following immunization (AEFIs) in our cohort.

Methods: A single-centre retrospective study was conducted by reviewing medical records of all JIA patients, diagnosed according to ILAR criteria, admitted to the Pediatric Rheumatology Unit of University of Naples Federico II from January to December 2019. Parents were asked to provide the vaccinations records in form of the vaccination booklet. The occurrence of AEFIs was explored by telephone interviews.

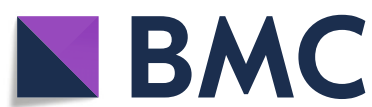

(c) The Author(s). 2020 Open Access This article is licensed under a Creative Commons Attribution 4.0 International License, which permits use, sharing, adaptation, distribution and reproduction in any medium or format, as long as you give appropriate credit to the original author(s) and the source, provide a link to the Creative Commons licence, and indicate if changes were made. The images or other third party material in this article are included in the article's Creative Commons licence, unless indicated otherwise in a credit line to the material. If material is not included in the article's Creative Commons licence and your intended use is not permitted by statutory regulation or exceeds the permitted use, you will need to obtain permission directly from the copyright holder. To view a copy of this licence, visit http://creativecommons.org/licenses/by/4.0/. The Creative Commons Public Domain Dedication waiver (http://creativecommons.org/publicdomain/zero/1.0/) applies to the data made available in this article, unless otherwise stated in a credit line to the data. 
Results: Data were obtained by 121 out of the 212 (57.1\%) invited patients (90 females; median age: 12.2 years, interquartile range 9.25 15). The most frequent diagnosis was oligoarticular JIA (69.4\%), followed by polyarticular (21.5\%), systemic ( $8.3 \%)$, and psoriatic (0.8\%) subtypes. Vaccination status was complete in 65 out of 121 of patients (53.7\%): anti-diphtheria-tetanus-pertussis (DTP) and antipoliomyelitis was complete in $76 \%$ and $72 \%$ of eligible cases, respectively; anti-hepatitis B virus in $99.2 \%$; anti-haemophilus influenzae b in $97.5 \%$; anti-measles-mumps-rubella (MMR) $69.4 \%$. The most frequently omitted vaccine is MMR. There was no association between vaccination coverage and age of onset of JIA ( $p=0.524)$, gen$\operatorname{der}(p=0.885)$ or JIA subtype $(p=0.298)$.

The vaccination status differed in a statistically significant manner with respect to JIA treatment: vaccination coverage was complete in $75 \%(21 / 28)$ of patients who underwent solely nonsteroidal antiinflammatory drugs and/or intra-articular injections of steroids compared to in $68.4 \%(26 / 38)$ of patients treated with methotrexate (MTX) and in $32.7 \%(18 / 55)$ of patients who underwent a biological DMARDs (bDMARDs) treatment $(p<0.001)$. In particular, in the group of patients treated with bDMARDs, coverages for DTP and MMR were $67.3 \%$ and $49.1 \%$, respectively.

In regard to non-mandatory vaccinations, 67 patients $(55.4 \%)$ received the pneumococcal vaccine, 56 meningococcal C $(46.3 \%)$, 14 meningococcal conjugate (ACW135Y) (11.6\%), 6 meningococcal B vaccine $(5 \%)$. The $15.7 \%$ of our population received at least one dose of influenza vaccine. $47.2 \%$ of eligible female patients did not receive any human papillomavirus (HPV) vaccine dose, 3.8\% 1 dose, 32.1\% 2 doses, and $17 \% 3$ doses.

37 AEFls were reported (30.6\%): 11 were local reactions (9.1\%), 11 fever episodes (9.1\%), 2 sleepiness (1.7\%). In 3 cases $(2.5 \%)$, the onset of JIA occurred approximately one month after the vaccine administration and was classified as a "coincidental event", according to WHO AEFI classification.

Conclusion: In our cohort, $46.3 \%$ of patients presented an incomplete vaccination status and a low coverage for non-mandatory, though recommended, vaccinations was observed. No serious AEFI was reported. Patients treated with bDMARDs showed the lowest vaccination coverage for live-attenuated vaccines, as expected, but also for non-live vaccines, resulting in a major risk for serious diseases preventable by vaccines. Further communication strategies are therefore needed.

\section{Disclosure of Interest}

None declared

\section{P124}

Minimal sedation/anxiolysis in children with juvenile idiopathic arthritis undergoing intrarticular injection of corticosteroids S. M. Orsi', M. Gattinara ${ }^{2}$, F. Baldo ${ }^{1,3}$, S. Carbogno ${ }^{1,3}$, A. Petaccia' ${ }^{1}$, C. Agostoni $^{1,3}$, G. Filocamo ${ }^{1}$, R. Cimaz ${ }^{2,4}$, S. Lanni ${ }^{1}$ on behalf of on behalf of the Pediatric Rheumatology Group of the Milan Area (PRAGMA) ${ }^{1}$ Fondazione IRCCS Ca' Granda Ospedale Maggiore Policlinico; ${ }^{2}$ ASST G. Pini-CTO; ${ }^{3}$ University of Milan; ${ }^{4}$ Department of Clinical Sciences and Community Health, and RECAP-RD, University of Milan, Milano, Italy Correspondence: S. M. Orsi

Pediatric Rheumatology 2020, 18(Suppl 2):P124

Introduction: Intrarticular corticosteroid injections (IACI) are widely used in the management of patients with juvenile idiopathic arthritis (JIA). General anesthesia can be avoided in case of a small number of joints to inject or in older children. However, pain and anxiety may reduce the patient compliance to $\mathrm{IACl}$, and may compromise the accuracy of the procedure. In order to overcame such problems, the use of appropriate methods of pain and anxiety control is advisable. Objectives: To assess the effectiveness and satisfaction of patients undergoing $\mathrm{IACl}$ with the use of topical numbing agent or under minimal sedation.

Methods: Patients with JIA who underwent an IACl of up to 3 joints were recruited. Depending on age and number of joints to treat, a group of patients (group A) were injected with the application $30 \mathrm{mi}-$ nutes prior the procedure of a topical numbing agent (prilocaine+lidocaine) to the skin over the injection site. Another group of patients (group B) were treated under minimal sedation (Ketorolac/Tramadol or Morphine + Midazolam). The physician was asked to record the degree of motion and pain of the patient during the procedure and the patient (or parents for patients aged less than 4 years) was asked to report the degree of pain and satisfaction on a Visual Analogue Scale (VAS) from 0 to 10.

Results: Twenty-seven patients were enrolled for a total of 30 procedures, 17 and 13 of them in group $A$ and $B$, respectively. The median age at the procedure was 10 years for group $A$ and 11 years for group B. For group A median pain scores for patients, parents and physicians were 2, 2 and 1.5, respectively. In patients of group $B$ who underwent the $\mathrm{IACl}$ under Ketorolac/Tramadol the median pain scores for patients, parents and physicians were 3, 5.25 and 2.5, whereas in patients treated with Morphine median pain scores were 6,6 and 2, respectively. Overall, we found that pain as reported by the patient/parent were higher with increase in the number of sites injected (and, consequently, duration of procedure) and age of patient. Amount of motion during procedures was overall negligible. The majority of patients/parents was satisfied for the procedures. Only 2 patients treated with Midazolam had psychomotor agitation during the $\mathrm{IACl}$.

Conclusion: $\mathrm{IACl}$ in a small number of sites without the use of general anesthesia is well tolerated by patients. The level of pain perceived from patients is irrespective of the power of the painkiller used, but seems to correlate with the duration of the procedures. It is possible that, in the paediatric age, the psychoemotional component seems to be decisive, with a progressive loss of tolerance with the increase in the number of injected joints.

Disclosure of Interest

None declared

\section{P125}

The effect of anti-TNF on adrenal steroid metabolism in juvenile idiopathic arthritis; a steroid metabolomics approach

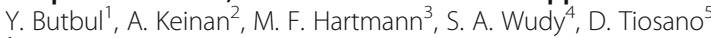

${ }^{1}$ Pediatric Rheumatology Service, Ruth Rappaport Children's Hospital, Rambam Medical Center, Haifa; ${ }^{2}$ Child and adolescent psychiatry unit, Ha'emek medical center., Afula, Israel; ${ }^{3}$ Pediatric Endocrinology \& Diabetology, Laboratory for Translational Hormone Analytics in Pediatric Endocrinology, Steroid Research and Mass Spectrometry Unit; ${ }^{4}$ Pediatric Endocrinology \& Diabetology, Laboratory for Translational Hormone Analytics in Pediatric Endocrinology, Steroid Research and Mass Spectrometry Unit, Center of Child and Adolescent Medicine, Justus Liebig University, Giessen, Germany; ${ }^{5}$ Pediatric endocrinology unit, Ruth Rappaport Children's Hospital, Rambam Medical Center, Haifa, Israel Correspondence: $Y$. Butbul

Pediatric Rheumatology 2020, 18(Suppl 2):P125

Introduction: Juvenile idiopathic arthritis (JIA) is the most common rheumatic disease in children and is a major cause of functional disability. The chronic inflammatory synovitis and systemic features of JIA are mediated by cytokine products of an activated immune system. Decreased production of adrenal androgens and cortisol production has been observed in RA. Recent studies showed that urinary free cortisol levels in active JIA patients are significantly lower than in remission periods and control groups.

Among the cytokines that are involved in the acute phase of the disease, TNFa has a major role.

Objectives: The aim of our study was to evaluate the impact of anti TNFa on adrenal activity by evaluating urinary adrenal steroid hormone metabolites by using GC-MS analysis before and after Etanercept treatment.

Methods: Eleven JIA patients were enrolled into the study, eight female and three male, average age was $12 \pm 6.2$ years (range 3 to 21 years). The average disease duration was $6.3 \pm 5.2$ years (range1.6-18 years) and the duration of Etanercept treatment was $3 \pm 2.8$ years (range 6 months to 10 years). The patients were treated once weekly with Etanercept injection $(0.8 \mathrm{mg} / \mathrm{kg})$. Urine was collected 3 times (just before and $+1,+3$ days following Etanercept injection) 
for GC-MS analysis of the steroid hormone metabolites age and sexmatched healthy controls were matched to each patient. Patients were excluded if they were treated with corticosteroids in the preceding 3 months.

Results: Of the 35 metabolites measured, 23 were significantly lower in JIA patients before the Etanercept treatment compared to the healthy control group. One day after the injection only 5 metabolites were still significantly lower in the JIA patients and all the other 30 metabolites normalized and were similar to the control group. Urine metabolite ratios reflecting CYP21 and $11 \beta$-HSD2 enzymatic activity indicate that these two enzyme activities were lower in JIA patients. The slowest recoveries noted were for metabolites of DHEAS and 17 $\mathrm{OH}$ pregnenolone.

Conclusion: Prior to Etanercept treatment almost all urine adrenal metabolites were significantly lower mainly due to the active inflammatory process. Immediately after the treatment many metabolites raised to normal values as in the control group. The two adrenal enzymes that were found to be affected in JIR are CYP21 and $11 \beta$ HSD2. Blocking TN alpha immediately restore adrenal function in JIA.

\section{Disclosure of Interest}

None declared

\section{P126}

Translation and validation of the MTX intolerance severity score questionnaire for Portuguese version in BRAZIL in juvenile idiopathic artrithis

A. C. Londe', R. Marini', S. Appenzeller ${ }^{3}$

${ }^{1}$ Child and Adolescent Health Graduate Program; ${ }^{2}$ Pediatrics; ${ }^{3}$ Medicine, UNICAMP, Campinas, Brazil

Correspondence: S. Appenzeller

Pediatric Rheumatology 2020, 18(Suppl 2):P126

Introduction: Methotrexate Intolerance Severity Score Questionnaire, MISS, is the tool in patients with juvenile idiophatic arthritis (JIA). During therapy, there are frequent reports of discontinuation of MTX, a drug classified as an the first choice for the treatment of JIA, either by physicians or patient's own conduct.

Objectives: Translate and validate the MTX Intolerance Severity Score questionnaire (MISS) to Portuguese of Brazil in patients with JIA.

Methods: The MISS was translated into Portuguese following the "Guidelines for the process of cross-cultural adaptation of self-report measures". The MISS consists of 4 domains: stomachache, nausea, vomiting, and behavioral complaints. The new version was tested on patients with JIA and relatives, both on the same day. The psychometric properties were analyzed according to the Consensus based Standards for the Selection of Health Measurement Instruments (COSMIN), analyzing acceptability for each item; internal consistency using Cronbach's alpha coefficient and reproducibility assessed by Kappa. We plot the ROC curve to evaluate the discriminant validity of the MISS compared to gold standard (clinical interview).

Results: We included 246 subjects to answer the MISS questionnaire, of these, 138 with JIA in use for least 3 months of MTX (oral/parenteral) and 108 relatives of children. All the subjects answered the MISS with less than 5 minutes and with no difficult of understanding it. The internal consistency of MISS had a Cronbach's considered good, with coefficient 0.88 (patients) and 0.79 (relatives). The reproducibility between the test (40 patients) and the retest done after 15 days (36 patients - less than $1 \%$ of missing data) was almost perfect (kappa $>0.8)$, except item restless (kappa $=0.54$ ). We didn't use test and retest in relative group. Reliability between patients and families was almost perfect (kappa>0.8), except stomachache by anticipatory (weak kappa $=0.30)$, stomachache by association $($ kappa $=0.54)$ and restless (kappa $=0.45$ ) when taking MTX; both values considered with moderate kappa. We plot the ROC curve and result was 0.90 $(95 \% \mathrm{Cl} 0.85-0.94)$ for the JIA patients. We found a cutoff score of 6 denoted the best sensitivity (84\% ICC $72.9-89.9)$ and specificity $(80 \%$ ICC 70.6-91.4) for the MISS. If we considered total sample (JIA and relative), the result was $0.89(95 \% \mathrm{Cl} 0.80-0.95)$ with score of 6 points denoted sensitivity $82 \%$ and specificity $79 \%$. The original MISS version found cutoff MISS score 5 and 6 during the validation, we found a good concordance between both points, with a kappa 0.73 and 0.79 , respectively; but the score 5 obtained a sensitivity $77 \%$ and specificity with $79 \%$ in the ROC curve. Finally, we considered MISS with a score 6 points through of these statistic data and comparing with other studies that used the MISS to measure the MTX intolerance in the literature. We found 96 intolerant patients (69.6\%) and 42 tolerant patients $(30.4 \%)$ of MTX. In intolerant, the median score MISS was 12.82 points SD \pm 5.47 , whereas tolerant were 2.42 points $\mathrm{SD} \pm 1.75$. The median score MISS for these both groups were 6.0 points $S D \pm 6.5$ in 246 subjects.

Conclusion: MISS is a viable and practical tool for the routine clinical, showing a good detection between intolerant and tolerant in MTX treatment in JIA. Children or relatives can complete the questionnaire with no discrepancy.

\section{Disclosure of Interest}

None declared

\section{P127}

Anti-adalimumab antibodies in patients with juvenile idiopathic arthritis: prevalence rate and clinical relevance

T. Marushko', Y. Holubovska', O. Onufreiv', Y. Marushko

${ }^{1}$ Pediatrics-2, Shupyk National Medical Academy of Postgraduate Education; ${ }^{2}$ Pediatrics of postgraduate education, Bogomolets National

Medical University, Kyiv, Ukraine

Correspondence: T. Marushko

Pediatric Rheumatology 2020, 18(Suppl 2):P127

Introduction: Patients with juvenile idiopathic arthritis (JIA) receive adalimumab treatment. Adalimumab is a monoclonal antibody that blocks TNF- $a$ and is structurally and functionally similar to human $\operatorname{lgG}_{1}$. Nevertheless, there are reports of the development of anti-drug antibodies. The production of these antibodies may be associated with treatment failures (a decrease in the effectiveness of therapy or drug inefficiency that developed over time) and hypersensitivity reactions. To our knowledge, there is currently limited information on the availability of adalimumab antibodies (AAA) in patients with JIA.

Objectives: to evaluate the prevalence rate and the clinical significance of AAA in patients with JIA on adalimumab treatment.

Methods: 26 patients with JIA were examined, 17 of whom had the oligoarticular form of the disease, 7 of them with uveitis, and 9 patients had the polyarticular form of the disease, 3 of them with uveitis. Among them, there were $13(50 \%)$ girls and $13(50 \%)$ boys. The mean age was $11.0 \pm 3.4$ years; the mean disease duration was $4.1 \pm$ 2.2 years. Patients received adalimumab (at least 1 year before the study) with concomitant administration of methotrexate (MTX) or adalimumab only - 13 children who did not receive MTX for at least 3 months prior to the study as a result of either adverse events of MTX administration (5 patients) or permanent drug remission (8 patients). Before starting adalimumab therapy, all participants were treated with MTX. The mean duration of adalimumab treatment for these patients was $1.8 \pm 1.0$ years. The serum AAA level of antibodies was determined using the enzyme immunoassay (EIA) method. This method determines both free and bound antibodies to adalimumab at reference values less than $10 \mathrm{AU} / \mathrm{ml}$. a and was used every 2 weeks for 3 months. The values were presented as mean \pm standard deviation. Data processing and analysis were carried out using Pearson's chi-squared test and Spearman's correlation test.

Results: 8 (31\%) of the 26 patients enrolled in the study had AAApositive results. The mean AAA level in positive patients was $40.8 \pm$ $20.1 \mathrm{AU} / \mathrm{ml}$. Further disease relapses tended to occur significantly more often in AAA-positive patients than in AAA-negative ones $(\mathrm{X} 2=$ $5.46, p=0.019)$. Thus, 5 of $8(62.5 \%)$ AAA-positive children had at least 1 exacerbation of the disease within 3 months, compared with 3 of $18(16.7 \%)$ in AAA-negative ones. 7 out of 8 (87.5\%) AAApositives did not take MTX for at least 3 months compared to 6 out of $18(33.3 \%)$ in AAA-negative ones. Thus, AAAs are found to be significantly more frequent without concomitant administration of MTX in the treatment of JIA $(x 2=6.5, p=0.01)$. There were no observed adverse events or side effects during adalimumab therapy. No significant correlation was found between the presence of AAA and sex, 
JIA form, uveitis presence, antinuclear antibodies high titer, rheumatoid factor, or disease duration.

Conclusion: We assume it would be appropriate to evaluate the AAA level in patients with JIA on adalimumab therapy, especially in the case of MTX withdrawal and the disease exacerbation. This gives a valuable advantage for further therapy enhancement.

\section{Disclosure of Interest}

None declared

\section{P128}

Establishment of a registry for juvenile idiopathic arthritis patients in South Australia: focus on patient-reported outcome and experience measures (PROMS/PREMS)

M. Min ${ }^{1,2}$, T. Crotti $^{2}$, C. Boros ${ }^{1,3}$

'University of Adelaide Discipline of Paediatrics, Women's and Children's Hospital; ${ }^{2}$ Faculty of Health and Medical Sciences, University of Adelaide; ${ }^{3}$ Women's and Children's Hospital Department of Rheumatology,

Adelaide, Australia

Correspondence: M. Min

Pediatric Rheumatology 2020, 18(Suppl 2):P128

Introduction: Patient outcomes and experiences are key components in the measurement of overall health outcomes in Juvenile Idiopathic Arthritis (JIA) and can be measured by validated patient-reported outcome and experience measures (PROMs and PREMs) questionnaires. There is little information in the published literature regarding the relationship between PROMs and PREMs and clinical disease activity in JIA.

Objectives: The objective was to establish a registry for JIA patients in South Australia (SA) and to understand the relationship between PROMs and PREMs and clinical disease activity.

Methods: JIA patients attending the Paediatric Rheumatology outpatient clinic at Women's and Children's Hospital were invited to participate. After obtaining written informed consent, data regarding demographics, JIA subtype, disease onset, investigation results, medications used and the clinical JIA Disease Activity Score (cJADAS) were documented. Patients/carers completed questionnaires at each clinic visit, namely the Childhood Health Assessment Questionnaire (CHAQ), the Quality of My Life (QoML) and the British Society for Paediatric and Adolescent Rheumatology (BSPAR) PROMs and PREMs Questionnaire. Disease activity states were defined based on cJADAS. Descriptive statistics, Spearman correlations and Kruskal-Wallis tests were used to analyse the data as appropriate.

Results: $A$ hundred and twelve patients were recruited (mean age $11.7 \pm$ SD 4.4 years) in this registry, including $n=11$ newly diagnosed JIA (9.8\%) and $n=75$ female (67\%). The median disease duration was 3.6 years [interquartile range (IQR) $1.2-7.7$ ]. $N=40,35.7 \%$ patients had oligoarticular onset (oligo) and $n=72,64.3 \%$ had polyarticular onset (poly) disease.

At the time of recruitment, the median CJADAS for oligo JIA was 0.5 (IQR 0.0-4.7) and for poly JIA was 1.9 (IQR 0.1-5.0). The median quality of life (QoL) was 7.9 (IQR 6.9-9.3) for oligo and 7.6 (IQR 6.4-8.9) for poly JIA. Functional ability was excellent, with a median CHAQ score of 0.1 (IQR 0-0.6) for oligo and 0.1 (IQR 0-0.7) for poly JIA. Among those patients with established disease ( $n=101 / 112,90.2 \%), n=20 / 33$, $60.6 \%$ of oligo JIA had clinically inactive disease (cJADAS $\leq 1)$, whilst only $n=28 / 68,41.2 \%$ of poly JIA had CJADAS $\leq 1$.

Patients with higher cJADAS reported lower QoL $(p<0.001)$ and lower health-related QoL (HRQoL, QoL affected by health or illness) ( $p<$ $0.001)$. In subgroup analysis, these associations remained significant for oligo and poly JIA. There was a statistically significant positive correlation between CHAQ score and cJADAS $(p=0.018$ for oligo, $p<$ 0.001 for poly), visual analogue pain score and cJADAS $(p<0.001$ for oligo, $p<0.001$ for poly).

Preliminary analysis of PROMs suggested a positive correlation $(p<$ 0.001 for all) between disease activity and fatigue $(R=0.39)$, pain $(R=$ $0.58)$, poor sleep $(R=0.40)$ and medication side effects $(R=0.33)$. Also, there was a negative correlation between disease activity and social wellbeing $(R=0.59, p<0.001)$, disease activity and emotional wellbeing $(R=0.47, p<0.001)$.
Most participants were satisfied with the clinic environment during their hospital visits (Fully 89.9\%, Mostly 9.2\%), whereas relatively fewer participants felt well supported in between hospital visits (Fully $75.2 \%$, Mostly 15.6\%). Twenty-nine out of 111 (26.1\%) participants experienced a delay in being seen during a clinic visit, and $n=5 / 29$, $17.2 \%$ of these patients felt the waiting time was unacceptable.

Conclusion: We have successfully commenced the development of a JIA registry in SA and have shown a direct relationship between clinical disease activity and PROMs, such as QoL, HRQoL, CHAQ score and PROM items for our JIA patients.

\section{Disclosure of Interest}

None declared

\section{P129}

Possible role of early-life exposures and environmental risk factors in juvenile idiopathic arthritis

O. Koker Turan', A. Aliyeva², M. Yildiz², A. Adrovic², S. Sahin², F. Haslak², K. Barut $^{2}$, R. Omeroglu' ${ }^{1}$, O. Kasapcopur ${ }^{2}$

${ }^{1}$ Pediatric Rheumatology, Istanbul Faculty of Medicine, Istanbul

University, ${ }^{2}$ Pediatric Rheumatology, Cerrahpasa Medical School, Istanbul University, Istanbul, Turkey

Correspondence: $\mathrm{O}$. Koker Turan

Pediatric Rheumatology 2020, 18(Suppl 2):P129

Introduction: Advances on molecular medicine, illumination of the cytokine network and the immune pathways shed light on the etiopathogenesis for a better understanding of Juvenile idiopathic arthritis (JIA). However, the fact that the course of the disease differs individually strongly suggests the effect of external factors.

Objectives: The current study was undertaken to evaluate sociodemographic and sociocultural features, parent behavior, the gestation and breastfeeding period, nutritional status of early childhood in our patients with JIA, and to determine their relationship with disease activity, damage index, remission time, and relapse rate.

Methods: The study was conducted with a face-to-face questionnaire method with the parents of 171 patients with JIA and 183 healthy children. The medical patient records were reviewed. Juvenile Arthritis Disease Activity Score (JADAS) 27, Wallace clinical inactive disease criteria, Juvenile Arthritis Damage Index (JADI), and relapse rates were used to assess the general medical condition of each patient.

Results: The median age of JIA patients $(n=171)$ was $13(3-20)$, with a female ratio of $59,1 \%$. Age at disease onset was $7(1-16)$ years. The first remission time was 5(1-17) months. The patients were evaluated according to disease subtypes and treatment modalities. There was no difference in the duration of breastfeeding according to the distribution of the subtypes $(p=0,97)$. When the breastfed and formula-fed patients were compared, there was a marginally significant difference in terms of first remission time $(p=0,05)$, whereas there was a significant difference in relapse rate in patients who introduced to cow milk early $(<12$ months) $(p=0,019)$. The early risk factors and their relationship with the disease are presented in Table 1. Both breastfeeding durations and maternal literacy levels showed a significant difference in terms of relapse rates ( $p=0,01 ; p=0,03$, respectively). There was no significant difference in breastfeeding durations and gestational risks between the patients and the healthy group $(p=0,1$; $p=0,65$ ), respectively. However, the smoking rate among family members was significantly higher in the patient group $(p=0,03)$. Conclusion: In patients with juvenile idiopathic arthritis, breastfeeding rate and duration did not differ when compared to healthy controls. However, breastfeeding duration, cow's milk commence age, and maternal literacy appeared to be relevant to the relapse rates. Going to preschool both influence the remission time and relapse rate. These findings suggest a role for parental attitude and nutritional status during early childhood in the course of JIA.

Disclosure of Interest

None declared 
Table 1 (abstract P129). Early Risk Factors and The Course Of Juvenile Idiopathic Arthritis

\begin{tabular}{|c|c|c|c|c|c|}
\hline Closed-ended Questions & $\begin{array}{l}\text { Positive } \\
\text { answers, } \\
\text { n (\%) }\end{array}$ & $\begin{array}{l}\text { JADAS, } \\
\text { (p) }\end{array}$ & $\begin{array}{l}\text { JADI, } \\
\text { (p) }\end{array}$ & $\begin{array}{l}\text { Remission, } \\
\text { (p) }\end{array}$ & $\begin{array}{l}\text { Relapse } \\
\text { Rate, }(p)\end{array}$ \\
\hline $\begin{array}{l}\text { Have you ever used a } \\
\text { cigarette during } \\
\text { pregnancy? }\end{array}$ & $41(24)$ & 0,20 & 0,72 & 0,13 & 0,63 \\
\hline $\begin{array}{l}\text { Have you had a major } \\
\text { illness during pregnancy? }\end{array}$ & $14(8,2)$ & 0,2 & 0,4 & 0,97 & 0,92 \\
\hline $\begin{array}{l}\text { Did you take any } \\
\text { medication during } \\
\text { pregnancy? }\end{array}$ & $8(4,7)$ & 0,14 & 0,41 & 0,95 & 0,54 \\
\hline $\begin{array}{l}\text { Has the child ever } \\
\text { breastfed? }\end{array}$ & $165(96,5)$ & 0,76 & 0,43 & 0,05 & 0,94 \\
\hline $\begin{array}{l}\text { Has the child fed with } \\
\text { cow's milk before } 12 \\
\text { months of age? }\end{array}$ & $21(12,3)$ & 0,50 & 0,36 & 0,54 & 0,01 \\
\hline $\begin{array}{l}\text { Has the child ever been } \\
\text { fed with formula? }\end{array}$ & $85(49,7)$ & 0,11 & 0,22 & 0,59 & 0,86 \\
\hline $\begin{array}{l}\text { Is the child's } \\
\text { immunization in line with } \\
\text { the vaccination schedule? }\end{array}$ & $155(90,6)$ & 0,63 & 0,92 & 0,10 & 0,24 \\
\hline $\begin{array}{l}\text { Has the child gone to } \\
\text { preschool? }\end{array}$ & $84(49,1)$ & 0,77 & 0,42 & 0,008 & 0,005 \\
\hline $\begin{array}{l}\text { Is there any smoking } \\
\text { indoors near the child? }\end{array}$ & $107(62,6)$ & 0,11 & 0,71 & 0,80 & 0,93 \\
\hline
\end{tabular}

\section{P130}

Articular and extra articular manifestations in JIA patients during transition

\author{
D. P. de Souza', R. Marini ${ }^{1}$, S. Appenzeller ${ }^{2}$ \\ ${ }^{1}$ UNICAMP, Campinas, Brazil; ${ }^{2}$ Medicine, UNICAMP, Campinas, Brazil \\ Correspondence: S. Appenzeller \\ Pediatric Rheumatology 2020, 18(Suppl 2):P130
}

Introduction: Juvenile idiopathic arthritis (JIA) constitutes the most common rheumatic disease in childhood. Few studies have evaluated the prevalence of activity in patients with JIA when they reach adulthood and how the presence of activity influences a transition.

Objectives: To evaluate the frequency of patients with JIA who reach 18 years of age with joint and extra articular activity.

Methods: A cross-sectional, descriptive study was conducted, including patients with JIA, aged 18 years or older. The variables studied were current age, sex, subtype of the disease and presence of articular and extra articular activity at 18 years old.Disease activity was evaluated by JDAS 78 and remission by preliminary criteria by Wallace.

Results: One hundered and thirty JIA patients were included, 77 (59.24\%) of the female sex. Of these, $19(14.62 \%)$ had the systemic form, 25 (19.3\%) Oligoarticular form, 19 (14.62\%) RF + polyarthritis, 42 (32.3\%) FR-Polyarthritis, 5 (3.85\%) Psoriatic arthritis, 17 (13.15\%) Enthesitis-Associated Arthritis and 3 (2.35\%) Undifferentiated arthritis. Of the 130 patients, $89(68.4 \%)$ were inactive at the age of 18.41 (31.6\%) had joint activity and $26(20 \%)$ active uveitis at age 18 . No association between subtype of the disease, age of disease-onset, treatment and immunological features were associated with remission at age of 18 .
Conclusion: Juvenile rheumatologic diseases frequently continue in adulthood requiring complex regimens of drug treatment, psychological support and complementary therapies. Our data showed that $32 \%$ of patients with JIA reached adulthood with disease activity and $20 \%$ of the patients had active uveitis, which warns of the vulnerability of young adults with JIA and the importance of a Coordinated Interdisciplinary Transition Program, focusing on orientation and education, patient satisfaction, quality of life and outcomes of the disease.

Disclosure of Interest

None declared

\section{P131}

Adalimumab trough concentrations are associated with treatment response in patients with juvenile idiopathic arthritis

M. Doeleman', S. De Roock', M. El Amrani', E. van Maarseveen², N.

Wulffraat $^{1}$, J. Swart ${ }^{1}$

${ }^{1}$ Paediatric Immunology and Rheumatology; ${ }^{2}$ Clinical Pharmacy,

University Medical Centre Utrecht (UMCU), Utrecht, Netherlands

Correspondence: $\mathrm{M}$. Doeleman

Pediatric Rheumatology 2020, 18(Suppl 2):P131

Introduction: Immunogenicity and low trough concentrations have been associated with adalimumab treatment failure in several studies of paediatric inflammatory diseases, indicating the possible value of therapeutic drug monitoring (TDM). Adalimumab efficacy may be improved by changing dose or treatment intervals based on drug concentrations. However, lack of standardization, assay heterogeneity, and paucity of research hinder the implementation of TDM in clinical practice.

Objectives: To assess the relationship of trough concentrations, immunogenicity and adalimumab response in paediatric patients with JIA.

Methods: Monocentric cohort study of patients $\leq 18$ years with JIA treated with adalimumab due to active arthritis. Clinical data and plasma samples were collected during routine follow-up. Adalimumab trough concentrations were measured by liquid chromatographytandem mass spectrometry (LC-MS/MS). Anti-adalimumab antibodies were measured in samples with trough concentrations $<5 \mathrm{mg} / \mathrm{l}$. Disease activity was evaluated using the clinical Juvenile Arthritis Disease Activity Score with 71 joint count (cJADAS71). Response to adalimumab was defined as at least $50 \%$ reduction of disease activity within 3 months of therapy followed by clinical inactive disease or minimal disease activity after 6 months. The latter was defined as CJADAS71 $\leq 1.5$ and $\leq 2.5$, for oligoarthritis and polyarthritis, respectively, or an active joint count equal to zero when CJADAS71 was unavailable.

Results: 36 adalimumab trough samples were available from 35 JIA patients. Although there was no significant difference in median adalimumab dose, trough concentrations were significantly lower in patients with secondary failure compared to primary failure or an adequate adalimumab response ( $p$-values $<0.01$ ). In addition, there were 11 samples with trough concentrations $<5 \mathrm{mg} / \mathrm{l}, 9$ in the group with secondary failure and 2 in the group with adequate adalimumab response (Table 1).

Conclusion: Adalimumab trough concentrations were significantly lower in JIA patients with secondary failure compared to primary failure or an adequate response to adalimumab. Anti-adalimumab antibodies were present in 8 out of 11 samples with trough concentrations $<5 \mathrm{mg} / \mathrm{l}$. Adalimumab trough concentration measurements may identify JIA patients that would benefit from increased doses or shorter treatment intervals. In addition, JIA patients with primary failure and adequate adalimumab trough concentrations may respond better to biologic agents with other therapeutic targets. Although biologic agents have improved disease outcome of patients with JIA, 
concentration measurements using reliable and cost-effective methods, such as LC-MS/MS, could further improve efficacy of biologic agents and guide treat-to-target strategies.

Disclosure of Interest

None declared

Table 1 (abstract P131). Characteristics of included JIA patients

\begin{tabular}{|c|c|c|c|}
\hline & Responders & Primary failure & $\begin{array}{l}\text { Secondary } \\
\text { failure }\end{array}$ \\
\hline Patients, $n$ & 16 & 8 & 12 \\
\hline MTX, n (\%) & $12(75)$ & $7(88)$ & $5(42)$ \\
\hline $\begin{array}{l}\text { MTX dosage }\left(\mathrm{mg} / \mathrm{m}^{2} /\right. \\
\text { week) }\end{array}$ & $6.7(5.3-12.3)$ & $12.1(7.7-12.8)$ & $7.3(5.3-8.4)$ \\
\hline ADA duration (days)* & $218(98-398)$ & $160(132-264)$ & $728(387-1021)$ \\
\hline $\begin{array}{l}\text { ADA dosage }(\mathrm{mg} / \mathrm{kg} / \\
\text { week) }\end{array}$ & $0.34(0.31-0.45)$ & $0.35(0.31-0.4)$ & $0.38(0.27-0.53)$ \\
\hline $\begin{array}{l}\text { ADA concentration }(\mathrm{mg} / \\
\mathrm{l})^{*}\end{array}$ & $\begin{array}{l}14.94(10.31- \\
16.19)\end{array}$ & $\begin{array}{l}13.37(10.85- \\
15.99)\end{array}$ & $1(1-5.3)$ \\
\hline ADAb-positive & $1 / 2$ & $0 / 0$ & $7 / 9$ \\
\hline$A J C^{*}$ & $0(0-0)$ & $2(2-5)$ & $1(1-3)$ \\
\hline CJADAS71 ${ }^{*^{\dagger}}$ & $0.2(0-0.4)$ & $5.5(3.9-9.2)$ & $6.5(4-9.2)$ \\
\hline
\end{tabular}

*Significant difference, $p$-values $<0.01$. + cJADAS71 was available for 10/16 patients with adequate response, $7 / 8$ with primary failure, and $11 / 12$ with secondary failure; Continuous data are presented as median (interquartile range); ADA: adalimumab; ADAb: anti-drug antibody; AJC: active joint count

\section{P132}

Comorbidities in juvenile idiopathic arthritis - important to evaluate patient mental health

E. Berthold ${ }^{1,2}$, A. Dahlberg ${ }^{2,3}$, B. Månsson 1', A. Jöud ${ }^{4}$, R. Kahn 2,3

'Department of clinical science Lund, Division of Rheumatology, Lund University; ${ }^{2}$ Wallenberg Centre for Molecular Medicine; ${ }^{3}$ Department of clinical science Lund, Division of Pediatrics, Lund University;

${ }^{4}$ Department of Laboratory Medicine, Lund University, Division of

Occupational and Environmental medicine, Lund, Sweden

Correspondence: $\mathrm{E}$. Berthold

Pediatric Rheumatology 2020, 18(Suppl 2):P132

Introduction: The role of comorbid disease among children with juvenile idiopathic arthritis (JIA) differ, and while the evidence for malignancy is inconclusive (1-5), there seems to be an increased prevalence of comorbid depressive and/or anxiety disorders (6) as well as an increased risk for other autoimmune diseases i.e. type 1 diabetes mellitus (DM1), hypothyroidism and coeliac disease (CD) (7-9).

Objectives: To study the long-term occurrence of comorbid malignancy, psychiatric and autoimmune disease in JIA in a populationbased cohort of children with a validated diagnosis of JIA collected over nine years.

Methods: The study cohort comprised of 252 validated cases of JIA diagnosed 2002-2010 in Skåne (10), the southernmost region of Sweden (population 1.24 million; 17.6\% aged < 16 years, year 2010). ICD-codes registered 1998-2019 for malignant tumours including hematologic malignancies (C00-C97), depression and anxiety disorders (F32, F33, F34.1, F41) and the autoimmune conditions DM1 (E10), hypothyroidism (E03) and CD (K90.0) were collected from the Skåne Health-care Register. Data is descriptively presented as percentage of comorbid disease in the cohort.

Results: No cases of malignant disease were found. Depression or anxiety occurred in $19.8 \%$ of the cohort. DM1 was present in $2.8 \%$ of the cohort. $5.6 \%$ of the patients had comorbid hypothyroidism; no case was due to exogenous substances or complication after infection. CD occurred in $5.2 \%$ of the cohort. (Table 1).
Conclusion: In all, 1/5 of the individuals with JIA in this validated population-based cohort were diagnosed with either depression and/or anxiety disorder over a period of 22 years. The presence of a comorbid autoimmune disease in this cohort was, except for a lower proportion of hypothyroidism, in line with previously published results. However, the results need to be compared with the agematched general population.Knowledge of high-risk comorbidities will contribute to better care for JIA patients, with potential development of new screening routines and our results highlight that it is important to continuously evaluate the patient's mental health to early detect symptoms of mental illness.

\section{References}

1. Zahedi N O, et al. RMD open. 2016;2(1):e000212.

2. Bernatsky S, et al. J Rheumatol. 2011;38(4):760-3.

3. Simard JF, et al. J Rheumatol. Arthritis Rheum. 2010;62(12):3776-82.

4. Nordstrom BL, et al. Arthritis Care Res (Hoboken). 2012;64(9):1357-64.

5. Beukelman T, et al. Arthritis Rheum. 2012;64(4):1263-71.

6. Fair DC, et al. Open Access Rheumatol. 2019;11:237-252.

7. Pohjankoski H, et al. Scand J Rheumatol. 2010;39(5):435-6.

8. Schenck S, et al. J Pediatr. 2018;192:196-203.

9. Stagi S, et al. Rheumatology (Oxford). 2005:44(4):517-20

10. Berthold E, et al. Arthritis Res Ther. 2019;21(1):218.

\section{Disclosure of Interest}

None declared

Table 1 (abstract P132). Comorbidities in JIA $(n=252)$

\begin{tabular}{ll}
\hline & Total (\%) \\
\hline Malignancy & 0 \\
Depression/Anxiety & 19.8 \\
Diabetes mellitus, type 1 & 2.8 \\
Hypothyroidism & 5.6 \\
Coeliac disease & 5.2 \\
\hline
\end{tabular}

\section{P133}

Echocardiographic findings of children with juvenile spondyloarthropathies

M. Yıldı' , R. Dedeoglu², B. Akdeniz', A. Adrovic ', F. Haslak', F. Karagozlu², O. Koker', S. Sahin' ${ }^{1}$, K. Barut ', F. Oztunc ${ }^{2}$, O. Kasapcopur

${ }^{1}$ Department of Pediatric Rheumatology; ${ }^{2}$ Department of Pediatric

Cardiology, Istanbul University-Cerrahpasa, Istanbul, Turkey

Correspondence: M. YIIdIZ

Pediatric Rheumatology 2020, 18(Suppl 2):P133

Introduction: Juvenile spondyloarthropathies (jSpA) is an umbrella definition of a group of inflammatory diseases characterized by asymmetric peripheral arthritis (especially in lower extremities), axial skeleton involvement and enthesitis. Although, cardiovascular findings of inflammatory diseases such as juvenile systemic lupus erythematosus, juvenile scleroderma, juvenile dermatomyositis welldocumented, it has not been extensively studied in children with $J \mathrm{SpA}$, and there are only few studies in the literature.

Objectives: This cross-sectional study had conducted for evaluating the cardiac functions of the children and adolescents with JSpA.

Methods: Forty patients with JSpA and twenty healthy control were included into the study. Healthy children or adolescents who attended the outpatient clinic for routine control were enrolled in the control group after the informed consent was taken. Patients with history/evidence of congenital heart disease, any rhythm abnormalities, other chronic systemic diseases (including renal, pulmonary diseases, diabetes, etc.), and those taking medications other than JSpA therapy were excluded. Cardiac functions of the patients and healthy controls were evaluated by conventional echocardiography and pulsed-wave tissue Doppler.

Results: Female/male ratio in patient and control groups were 0.6 and 0.81 respectively. The mean ages were $15 \pm 3$ years in patients with JSpA and $11.9 \pm 3.6$ years in control group. The mean age at 
diagnosis was $12.6 \pm 2.9$ years and the median follow-up duration was 23 (1-129) months. Tricuspid lateral annulus TDI-PW velocities E'/ $A^{\prime}$ ratio was significantly lower in patients with JSpA than in healthy controls [1.5 (0.72-4) versus $1.7(1.2-2.2), \mathrm{p}<0.05]$. Ejection fraction, right ventricle FAC and TAPSE were similar in both groups. Cardiac function parameters of patients were compared according to presence of enthesis, HLA-B27 positivity, morning stiffness, hypertension, medications they were on and erosion/sclerosis on X-ray of pelvis. There were no significant differences between the patients with and without enthesis, HLA-B27 positivity, morning stiffness and erosion/sclerosis on X-ray of pelvis. When cardiac function parameters were compared for magnetic resonance imaging (MRI) findings like bone marrow edema, enthesis, erosion, sclerosis, synovitis; there were no significant differences between groups except for enthesis. Patients with enthesis that is detected on MRI had lower ejection fraction $(p<0.05)$. The correlation analysis that was made for comparing the effect of the disease activity on the cardiac functions; showed significant, moderate correlation between BASDAI score and PWtrans mitral A velocity $(r=-0.352, p=0.03)$ and moderate negative correlation between BASDAI score and TAPSE ( $r=-0.407, p=0.03)$. There was significant, moderate negative correlation between follow-up duration and shortening fraction $(r=-0.41, p=0.009)$.

Conclusion: In this cross-sectional study, we are reporting disturbed $\mathrm{RV}$ diastolic function with preserved RV systolic function and possible association between MRI confirmed enthesis and lower LV systolic functions. Early identification of cardiac dysfunctions in these patients can help to prevent long-term cardiovascular complications in JSpA before irreversible changes take place.

\section{Disclosure of Interest}

None declared

\section{P134}

Gender differences in physical fitness related to selection in clubsport activities in JIA

M. Nørgaard', T. Herlin²

${ }^{1}$ Department of Physiotherapy; ${ }^{2}$ Department of Pediatrics, Aarhus

University Hospital, 8200 Aarhus N, Denmark

Correspondence: $\mathrm{M}$. Nørgaard

Pediatric Rheumatology 2020, 18(Suppl 2):P134

Introduction: Most studies of physical fitness (PF) in juvenile idiopathic arthritis (JIA) have shown decreased levels of maximal oxygen consumption $\left(\mathrm{VO}_{2} \mathrm{max}\right)$ compared to healthy peers. In JIA, boys have higher PF-levels than girls and younger children have higher levels than adolescents, congruently with data of healthy peers. Previously, we have shown that more than half of JIA-patients had below normative average of $\mathrm{VO}_{2}$ max. However, monitoring physical activity (PA) using accelerometry, $68 \%$ of boys and $39 \%$ of girls with JIA fulfilled the recommendations of WHO of $\geq 1$ hour of PA per day, which was comparable to normative values (61\%/39\%). Moreover, patients reporting engagement more than 7 hours per week in club-sports exceeded accelerometry values of healthy peers. Objectives: To explore the association between PF and specific sport habits in 10 to 16-year-old JIA-patients, related to gender, BMI, disease activity, and pain, and comparing the most fit quartile (Q4) of patients (respectively boys and girls) to the least fit quartile (Q1).

Methods: Sixty patients with JIA performed an indirect ergometertest of $\mathrm{VO}_{2} \max$ (Watt max test) and answered the Physical Activity and Sport Questionnaire (PASQ). Objective PA-monitoring for one week was conducted using the GT1M accelerometer. Cut-offs for moderate-high and high intensity PA were set to $>1000$ and $>2500$ counts per minute, respectively. Disease activity was assessed with the JADAS-27, current pain and worst pain last week were measured on visual analogue scales (VAS) and in a one-week pain diary using the Faces Pain Scale-Revised (FPS-R).

Results: Girls with JIA $(n=36)$ had lower mean PF than the boys ( $n=$ 24) $(36.5 \pm 8.2 / 43.4 \pm 6.73 \mathrm{ml} / \mathrm{kg} / \mathrm{min})$, being below normative values, respectively. In both genders the most fit boys and girls (Q4; 49.3-57/ 40.9-54) had average to well-above normative average PF. The least fit boys (Q1; 33.5-37.4) all had PF-levels well-below normative average. In girls Q1-levels (18.7-30.9) were well-below to below normative average.

We found significant differences between most fit (Q4) and least fit (Q1) patients regarding patient's global wellbeing $(p=0.040)$ and pain diary $(p=0.026)$. These differences were not significant when separating genders, though differences were more pronounced in girls. The least fit girls (Q1) had significantly higher disease activity (JADAS-27) than the most fit girls $(\mathrm{Q} 4)(\mathrm{p}=0.019)$.

The most fit boys and girls (Q4) engaged equally in high intensity sports (soccer: $3 / 24 ; 2 / 36$, handball: $0 / 24 ; 2 / 36$, gymnastics: $2 / 24 ; 4 /$ 36 , rowing: $1 / 24 ; 0 / 36)$. However, more boys than girls played soccer $(11 / 24 ; 3 / 36)$, whereas more girls preferred sports of lower intensity (riding: 8/36; 0/24). Eight of 11 boys in soccer and 2 boys in gymnastics or rowing had below average to well-above normative average of PF (Q3+Q4: 41.6-57). Three girls in gymnastics, 2 girls in soccer, and 2 girls in handball were in Q4 (40.9-54) with levels of average to well-above average PF. The third girl in soccer was in Q2 (31-36.3) with levels of well-below to below normative average. None of the riding girls were in Q4 and only 1 was in Q3 (36.3-40.8) (Below to average normative PF). Comparing accelerometer-monitored values of PA-intensity in girls with low (Q1) and high (Q4) PF, PAvalues of Q1 were significantly lower than in Q4. The same tendency was observed in boys, but not to significance.

Conclusion: Our results are in accordance with most other studies of $\mathrm{PF}$ in JIA, adding to the knowledge of gender-specific differences in PF and type and behavior in sport activities. It emphasizes the need of regular PF-testing and guidance in high intensity PA and sport in order to improve PF and avoid the risks of inactivity and lifestyle diseases in JIA.

Disclosure of Interest

None declared

\section{P135}

The ratio of some cytokines like a marker options and course of juvenile arthritis

O. A. Oshlianska', N. A. Melanchuk ${ }^{2}$

${ }^{1}$ Department of Pediatrics № 1, 2. Department of connective tissue disorders in children, 1.Shupyk National Medical Academy of

postgraduate education, 2.State Institute of Pediatrics, Obstetrics and Gynecology, Academy of Medical Sciences of Ukraine; ${ }^{2} 1$.Department of Pediatrics № 1, 2. Department of connective tissue disorders in children, 1.Shupyk National Medical Academy of postgraduate education, Kyiv, Ukraine

Correspondence: $\mathrm{O}$. A. Oshlianska

Pediatric Rheumatology 2020, 18(Suppl 2):P135

Introduction: The leading role in the implementation of inflammation in the human body belongs to cytokines. The study of the role of each of them and their cooperation continues with autoimmune diseases. New data on the importance of chronic inflammation of interleukin 17 (IL-17) have found. The effect of their ratio on the activity of inflammation is not well understood.

Objectives: To study the ratio of some initiating and blocking cytokines of IL-17 in children with juvenile idiopathic arthritis (JIA). Methods: 40 children with JIA (12 oJAA, 17 pJIA, 1 with positive RF, 6 eJIA, $5 \mathrm{sJIA}$ were examined during the period of exacerbation of the disease with an assessment of the activity and course of the disease. All cases were additionally studied IL-1 $\beta$, receptor (IL-17R), IFN- $\gamma$ in blood serum using linked immunosorbent assay sandwich option.

Results: The data obtained showed IL-1 $\beta$ was significantly increased only in sJIA $(30.15 \pm 17.09 \mathrm{pg} / \mathrm{ml})$, significantly $(p<0.05)$ higher than in other JIA $(6.71 \pm 0.91 \mathrm{pg} / \mathrm{ml}$ in oJIA, $8.61 \pm 1.11 \mathrm{pg} / \mathrm{ml}$ in $\mathrm{s} J \mathrm{~A})$. This was detected in $2 / 3$ of the patients, higher $(p<0.05)$ in adolescents $(16.69 \pm 8.32 \mathrm{pg} / \mathrm{ml})$ compared with children $4-12$ y.old $(6.69 \pm 0.86 \mathrm{pg} /$ $\mathrm{ml}$ ). IFN- $\gamma$ was increased above normal values (up to $50 \mathrm{pg} / \mathrm{ml}$ in healthy children) in all cases of JIA, it could be possible its role in the initiation of outbreak. Maximum values were noted in ollA, especially in cases with uveitis $(7029.2 \pm 4750 \mathrm{pg} / \mathrm{ml})$ and were higher $(p<0.1)$ in children 1-3 years old versus adolescents. IL-17R was maximum in pJlA patients $(2058936 \pm 35.21 \mathrm{pg} / \mathrm{ml})$ and eJlA $(22723.65 \pm 16022.54$ 
$\mathrm{pg} / \mathrm{ml}$ ), which also showed the highest the frequency of detection of its increase. It was absent in sJIA $(7.52 \pm 4.74 \mathrm{pg} / \mathrm{ml})$. The highest values of IL-17R $(1849836.4 \pm 176751 \mathrm{pg} / \mathrm{ml})$ were in the middle age group. The data obtained suggest the compensatory value of increasing IL-17R and the simultaneous initiation of inflammatory and anti-inflammatory processes during exacerbation of JIA. Assessment of the ratios of stimulating and inhibiting cytokines showed in patients with uveitis, the ratio of IFN- $/ / \mathrm{IL}-1 \beta(4379.29 \pm 476.83)$ was higher than with other JIA (from $60.84 \pm 14.92$ in oJlA to $105.20 \pm$ 66.01 in $\mathrm{pJIA}$ ) and IFN- $\gamma / \mathrm{IL}-17 \mathrm{R}(4474.01 \pm 3899.19$ versus from 20.14 \pm 11.48 in oJlA to $934.55 \pm 931.37$ in sJlA). An increase of IL-1B/IL-17R ratio was characteristic only for SJIA (34.12 \pm 26.17$)$. All of these ratios increased with disease activity $(r=0.22-0.37) \&$ they did not reflect the unpleasant course of the disease.

Conclusion: The balance of cytokines determines the direction of the inflammatory process in JIA. The most unfavorable presence of an increase IL-1 $\beta /$ IL-17R. The ratios IFN- $\gamma / \mathrm{IL}-1 \beta$ and IFN- $\gamma / \mathrm{IL}-17 \mathrm{R}$ could be considered as potential markers of uveitis \& IL-1 $\beta / \mathrm{IL}-17 \mathrm{R}$ - sJIA.

\section{Disclosure of Interest}

None declared

\section{P136}

Treatment with methotrexate and etanercept does not alter the fecal microbiota composition in children with juvenile idiopathic arthritis

A. I. Öman', J. Dicksved², L. Engstrand ${ }^{3}$, L. Berntson'

${ }^{1}$ Departement of womens and childrens health, Uppsala University;

${ }^{2}$ Department of Animal Nutrition and Management, Swedish University of Agricultural Sciences, Uppsala; ${ }^{3}$ Department of Microbiology, Tumour and Cell Biology (MTC), Karolinska Institutet, Solna, Sweden

Correspondence: A. I. Öman

Pediatric Rheumatology 2020, 18(Suppl 2):P136

Introduction: The first-line treatment for juvenile idiopathic arthritis (JIA) is usually methotrexate (MTX), followed by treatment with an anti-tumour necrosis factor alpha (anti-TNF-a) drug, such as etanercept (ETN). The effects of MTX and anti-TNF-a drugs on the fecal microbiota in children with JIA have not been studied previously.

Objectives: To study the effects of MTX and ETN treatments on the fecal microbiota composition in children with JIA.

Methods: In this multicenter, case-control study, 113 fecal samples were collected from 91 children with JIA, with 72 of these samples collected from untreated children (67 of whom were treatment-naïve children). Samples from 28 children with JIA were collected during treatment with MTX as single treatment and samples from 13 children during treatment with ETN. Of those 13 children, four were treated with ETN as single treatment and nine had a combination of ETN and MTX.

We compared 28 children on single treatment with MTX with 57 untreated children (52 treatment-naïve), and 13 children on treatment with ETN (nine in combination with MTX) with 62 untreated children (57 treatment-naïve). We also did pairwise comparisons of samples taken before any medication was given $(n=22)$ and samples taken during ongoing treatment with MTX $(n=14)$ or ETN $(n=$ 8 , four in combination with MTX).

The microbiota was characterized by sequencing amplicons from the V3 and V4 regions of the 16S rRNA gene.

Alpha diversity of the fecal samples was measured using the Chao-1 index and the Shannon diversity index. To compare these indices between treated children and untreated children, we used a logistic regression model with age at sampling as a covariate. For pairwise analyses, we used the Wilcoxon signed-rank test.

To analyze the community composition of the microbiota, principal coordinate analysis ( $\mathrm{PCOA}$ ) plots based on Bray-Curtis distances were generated for visual comparisons, and analysis of similarity (ANOSIM) was used to test for differences.
Analyses for relative abundances of taxa were performed at three taxonomic levels (phyla, families, and genera), and logistic regression with age as a covariate was used for calculations of differences between treated and untreated children, while the Wilcoxon signedrank test was used for pairwise comparisons. Significance was set to $p<0.05$ and corrections for multiple comparisons were made using the Benjamini-Hochberg method.

Results: Analyses showed no significant differences in a-diversity between children treated with MTX or ETN and untreated children, and pairwise comparisons of samples before and during treatment with MTX or ETN also showed no differences.

PCoA plots for children treated with MTX or ETN, in comparison with untreated children, did not show any clustering. ANOSIM showed no significant differences between treated and untreated children.

PCoA plots were also made for the pairwise comparisons of children sampled before and during treatment, and according to that analysis the community compositions of microbiota did not change in any uniform way during treatment with either MTX or ETN.

Furthermor, analyses of relative abundances of specific taxa did not reveal any significant results in any of the comparisons, after adjustment for multiple analyses.

Conclusion: Treatment with MTX or ETN did not alter the composition of fecal microbiota in children with JIA.

Disclosure of Interest

None declared

P137

Synovial fluid neutrophils from patients with juvenile idiopathic arthritis display a hyperactivated phenotype

M. Metzemaekers ${ }^{1}$, B. Malengier-Devlies ${ }^{1}$, K. Y $^{1}{ }^{1}$, S. Vandendriessche ${ }^{1}$, P. Matthys' ${ }^{1}$, L. De Somer ${ }^{1,2}$, C. Wouters' ${ }^{2}$, P. Proost ${ }^{1}$

${ }^{1} \mathrm{KU}$ Leuven; ${ }^{2}$ UZ Leuven, Leuven, Belgium

Correspondence: $\mathrm{M}$. Metzemaekers

Pediatric Rheumatology 2020, 18(Suppl 2):P137

Introduction: Juvenile idiopathic arthritis (JIA) is the most common rheumatic disease in childhood and an important cause of shortterm and long-term disability if patients are not treated appropriately. By definition, JIA clinically presents with peripheral joint inflammation of unknown origin, persisting for at least six consecutive weeks and starting before the age of 16 years. The predominant subtypes, i.e. oligoarticular (oligo) and polyarticular (poly) JIA, have long been assumed autoimmune diseases caused by dysregulation of the adaptive immune system, with a central role for autoreactive $T$ cells belonging to the Th1 and Th17 lineages and autoantigens that may include aggrecan, fibrillin, matrix metalloproteinase (MMP)-3 and heat shock proteins. Nevertheless, the original T cell-centered hypothesis has been challenged since it does not cover nor completely explain the full spectrum of immune-pathological phenomena observed in patients.

lien.desomer@uzleuven.b

Objectives: Emerging evidence suggests a potentially important role for neutrophils in JIA pathogenesis. Here, we investigated extensively the phenotypical features of neutrophils present in the peripheral blood and inflamed joints of JIA patients.

Methods: Synovial fluids and parallel blood samples from patients with oligo- or polyJIA and blood samples from healthy children were collected. Multicolor flow cytometry panels allowed for in-depth phenotypical analysis of neutrophils, focusing on the surface expression of adhesion molecules, activation and maturation markers, chemoattractant- and Toll-like receptors. Multiplex technology was exploited to quantify pro- and anti-inflammatory cytokines in plasma and synovial fluids.

Results: The vast majority of synovial fluid neutrophils displayed a strongly activated, hypersegmented phenotype with decreased Lselectin (CD62L) expression and increased numbers of nuclear lobes, 
upregulation of adhesion molecules CD66b, CD11b and CD15 and downregulation of chemokine receptors CXCR1/2. An elevated percentage of CXCR4-expressing aged neutrophils was detected in synovial fluids from patients. Strikingly, significant percentages of synovial fluid neutrophils showed a profound upregulation of atypical neutrophil markers, including CXCR3, ICAM-1 and HLA-DR.

Conclusion: Our data indicate that neutrophils present in inflamed joints of JIA patients are strongly activated cells with elevated proinflammatory and antigen presenting potential. This detailed molecular analysis supports the notion that a complex intertwining between these innate immune cells and adaptive immune events drives JIA.

\section{Disclosure of Interest}

None declared

\section{P138}

The main factors, associated with incomplete vaccination againts measels, parotitis, rubella and diphtheria in 170 juvenile idiopathic arthritis patients

N. Lyubimova', I. Fridman², O. Goleva², S. Kharit², M. Kostik ${ }^{3}$

${ }^{1}$ Almazov National Medical Research Centre; ${ }^{2}$ Pediatric Research and

Clinical Center for Infection Diseases, Saint Petersburg; ${ }^{3}$ Saint-Petersburg State Pediatric Medical University, Saint-Petersburg, Russian Federation

Correspondence: M. Kostik

Pediatric Rheumatology 2020, 18(Suppl 2):P138

Introduction: Patients with juvenile idiopathic arthritis (JIA)may have incomplete vaccination againts different vaccines leads to lower protective levels of anti-vaccine antibodies.

Objectives: The aim of our study was to evaluate the rate and the main factors of incomplete vaccination against measels, parotitis, rubella (MMR) and diphtheria in JIA patients.

Methods: In the present study were included data $170 \mathrm{JIA}(55$ boys and 115 girls)aged from 2 to 17 years, who received scheduled vaccination before the age of 2 years and before JIA onset against measles, parotitis,diphtheria and rubella.Incomplete vaccination means the reduced number of vaccine to age.In all patients the $\lg G$ anti-vaccine antibodies levels were detected with ELISA.JIA categories were:oligoarthritis - 73,polyarthritis - 61,systemic-16 and enthesitisrelated arthritis-20.Data presented with median and 25\%>75\%

Results: Incomplete vaccination against MMR was in 50 (42\%)diphtheria in $85(50 \%)$ of the JIA patients. The main differences in the studied groups are in the table.There were no differences in sex,JIA categories and treatment, except biologics compare to complete and complete vaccination in all vaccines.No differences in antimeasels $(p=0.18)$, antiparotitis $(p=0.1)$ and anti-rubella $(p=0.17)$ vaccination between complete and incomplete vaccination group.Number of patients with protective level of anti-vaccine antibodies was similar, except parotitis( $70 \%$ vs $84.2 \%, p=0.035)$.The anti-diphtheria antibodies lgG level was lower in the patients with incomplete vaccination group $(0.07 \mathrm{IU} / \mathrm{ml}[95 \% \mathrm{Cl}: 0.03 ; 0.22]$ vs 0.2 [95\%Cl:0.06; 0.4$]$, $\mathrm{p}=0.001)$ as well as number of patients with protective level $(34 \%$ vs $54 \%, p=0.002)$. In the multiple regression model only JIA onset age $(p=0.00001)$ and methotrexate treatment duration $(p=0.003)$ were predictors of incomplete vaccination against MMR and methotrexate treatment duration $(\mathrm{p}=0.005)$ and biologic treatment $(\mathrm{p}=0.05)$ for diphtheria incomplete vaccination. Incomplete MMR vaccination influence on the maintenance of the protective anti-parotitis level $(p=0.036)$ in regression model.In correlation analysis the number of vaccination influences on anti-diphtheria antibodies level $(\mathrm{p}=0.017)$ and number of patients with protective level of anti-diphtheria antibodies $(p=0.017)$. The main predictors in logistic regression for incomplete vaccination for MMR were:onset age $<6$ years $(\mathrm{OR}=7.8$ [95\%Cl:3.2;18.7], $\mathrm{Se}=0.6$, $\mathrm{Sp}=0.86, \mathrm{p}=0.000001)$, JIA duration $>3.1$ years $(\mathrm{OR}=4.4$ [95\%Cl:2.0;9.9], $\mathrm{Se}=0.5, \mathrm{Sp}=0.81, \mathrm{p}=0.0002)$, methotrexate duration $>3.1$ years $(\mathrm{OR}=5.7$ [95\% Cl:2.7;12.0], $\mathrm{Se}=0.74, \mathrm{Sp}=0.67, \mathrm{p}=0.0000012$ ); biologic treatmen$\mathrm{t}(\mathrm{OR}=2.5[95 \% \mathrm{Cl}: 1.3 ; 4.9], \mathrm{Se}=0.64, \mathrm{Sp}=0.58)$ and treatment $>1$ biologic $(\mathrm{OR}=3.3$ [95\%Cl:1.1;10.4], Se=0.63, Sp=0.67); for diphtheria were:JIA duration $>3.1$ years $\quad(\mathrm{OR}=3.4 \quad[95 \% \mathrm{Cl}: 1.8 ; 6.5], \mathrm{Se}=0.55, \mathrm{Sp}=0.73, \mathrm{p}=$ $0.0002)$, methotrexate duration $>2.8$ years $(\mathrm{OR}=4.1[95 \% \mathrm{Cl}: 2.1 ; 8.1], \mathrm{Se}=$ $0.73, \mathrm{Sp}=0.61, \mathrm{p}=0.00004)$,biologic treatment $(\mathrm{OR}=2.4$ [95\%Cl:1.3;4.4], $\mathrm{Se}=0.59, \mathrm{Sp}=0.62$ ).

\begin{tabular}{|c|c|c|c|c|c|c|}
\hline \multirow[t]{2}{*}{ Parameter } & \multicolumn{2}{|c|}{$\begin{array}{l}\text { MMR, } \\
\text { incomplete }\end{array}$} & \multirow[t]{2}{*}{$p$} & \multicolumn{2}{|c|}{$\begin{array}{l}\text { Diphtheria, } \\
\text { incomplete }\end{array}$} & \multirow[t]{2}{*}{$p$} \\
\hline & yes & no & & yes & no & \\
\hline Onset age, y & $\begin{array}{l}4.0(3.0 ; \\
5.9)\end{array}$ & $\begin{array}{l}7.0(4.9 ; \\
11.0)\end{array}$ & 0.000001 & $\begin{array}{l}5.0(3.0 ; \\
8.0)\end{array}$ & $\begin{array}{l}6.6(4.4 ; \\
10.1)\end{array}$ & 0.016 \\
\hline JIA duration, y & $\begin{array}{l}5.5(3.2 ; \\
8.6)\end{array}$ & $\begin{array}{l}3.1(1.5 ; \\
5.4)\end{array}$ & 0.0005 & $\begin{array}{l}4.5(2.8 ; \\
8.7)\end{array}$ & $\begin{array}{l}2.9(1.3 ; \\
5.5)\end{array}$ & 0.0006 \\
\hline Biologics, n (\%) & $32(64)$ & $50(42)$ & 0.008 & $50(59)$ & $32(38)$ & 0.006 \\
\hline $\begin{array}{l}\text { More than one } \\
\text { biologics, n (\%) }\end{array}$ & $10(20)$ & $6(5)$ & 0.002 & $10(12)$ & $6(7)$ & 0.022 \\
\hline $\begin{array}{l}\text { Methotrexate } \\
\text { duration, y }\end{array}$ & $\begin{array}{l}5.0(2.1 ; \\
7.5)\end{array}$ & $\begin{array}{l}1.8(0.9 ; \\
3.2)\end{array}$ & 0.000006 & $\begin{array}{l}3.3(1.7 ; \\
7.3)\end{array}$ & $\begin{array}{l}1.7(0.9 ; \\
2.9)\end{array}$ & 0.00002 \\
\hline
\end{tabular}

Conclusion: Younger onset of JIA age, longer duration of JIA and methotrexate treatment, biologics and more than 1 biologics are the main predictors of incomplete vaccination againt MMR and diphtheria.

\section{Disclosure of Interest}

None declared

\section{P139}

Biomarkers predicting the further disease course in children with juvenile idiopathic arthritis (JIA): results from the inception cohort of newly diagnosed patients with JIA (ICON-JIA)

M. Ganeva', S. Fuehner', C. Kessel2 , C. Hinze', J. Klotsche ${ }^{3}$, M. Niewerth ${ }^{3}$, K. Minden ${ }^{3}$, D. Foell ${ }^{2}$

'Department of Paediatric Rheumatology, Medical University Sofia, Sofia, Bulgaria; ${ }^{2}$ Department of Paediatric Rheumatology and Immunology, University of Münster, Münster; ${ }^{3}$ Department of Rheumatology and Clinical Immunology Charité, University Medicine Berlin, Berlin German Rheumatism Research Center, Leibniz Institute, Berlin, Germany

Correspondence: M. Ganeva

Pediatric Rheumatology 2020, 18(Suppl 2):P139

Introduction: Biomarkers have shown potential as diagnostic and prognostic tools in juvenile idiopathic arthritis (JIA).

Objectives: To describe the association of baseline serum biomarkers in patients with newly diagnosed JIA and their 1-year outcomes.

Methods: Serum samples of JIA patients enrolled in the German Inception Cohort of Newly diagnosed patients with JIA (ICON-JIA) at $\leq 1$ year of JIA diagnosis were collected at study enrolment and after 3 months. Standard laboratory markers of inflammation (CRP, ESR), as well as novel biomarkers - CXCL-9, CXCL-10, CXCL-11, G-CSF, IL-6, IL-17A, IL-18, MCP-1, MIP-1a, MMP-3, S100A8/A9, S100A12, TNFa, and 
TWEAK were analyzed for their potential to predict the 1-year outcome. Demographic and clinical parameters were also recorded. Disease activity was assessed with the clinical Juvenile Arthritis Disease Activity Score (cJADAS) 10.

Results: Two-hundred-sixty-six JIA patients had active disease at baseline, with oligoarthritis and rheumatoid factor-negative polyarthritis representing the largest proportion (72.9\%). CRP, ESR, IL-18, S100A8/A9 and S100A12 levels were higher in patients with systemic JIA compared to other JIA categories. Baseline levels of G-CSF, IL-18 and TWEAK were lower in oligoarthritic JIA patients with disease extension within one year. Higher baseline ESR, G-CSF, IL-6, and TNF levels indicated the risk of increased disease activity at 12 months. Additionally, higher levels of ESR, CRP, S100A8/A9, and S100A12 at baseline were associated with the necessity to escalate therapy during the first 12 month of follow-up and subsequent addition of biologic disease-modifying antirheumatic drugs.

Conclusion: Our data demonstrate that increased disease activity at baseline, defined through both clinical parameters and biomarker levels is associated with the risk of continued disease activity after 12 months.

\section{Disclosure of Interest}

None declared

\section{P140}

Screening for antithyroid antibodies in children with juvenile idiopathic arthritis: a single-centre experience from Southern Italy R. Naddei ${ }^{1}$, C. Esposito ${ }^{1}$, F. Orlando ${ }^{2}$, R. Alfani ${ }^{1}$, V. Discepolo ${ }^{1}$, M. Alessio 'Pediatric Rheumatology Unit, Mother and Child Department, University of Naples Federico II; ${ }^{2}$ Unit of Pediatrics 2, Santobono Pausilipon Children's Hospital, Naples, Italy

Correspondence: R. Naddei

Pediatric Rheumatology 2020, 18(Suppl 2):P140

Introduction: The prevalence of autoimmune thyroid disorders (AITD) has been reported to be higher in patients with juvenile idiopathic arthritis (JIA) in comparison to the general population. Nevertheless, there is a lack of studies investigating risk factors for AITD development in children with JIA.

Objectives: To investigate the co-occurrence of JIA and autoimmune thyroiditis in southern Italy and to identify potential predisposing factors to anti-thyroid antibodies (ATA) positivity in a JIA population.

Methods: A single-centre retrospective study was conducted. All JIA patients admitted to the Pediatric Rheumatology Unit of the University of Naples Federico II, from January 2001 to December 2019, tested for ATA at least once and with a minimum of 6-months follow-up, were included. For each patient, demographic, clinical and laboratory data were extracted from clinical charts. Differences between patients affected by JIA with or without ATA were analyzed.

Results: Three hundred thirty JIA patients (247 females; median age 12.5 years, IQR 9.1-16.1) were included in study. Median age at JIA onset was 4 years (IQR: 2.2-7.8). Twenty-three patients [7\% $(95 \% \mathrm{Cl}$ 4.5-10.3)] presented ATA positivity. Twenty-one of them (91.3\%) were females. Anti-thyroperoxidase was positive in $18 / 23$ patients (78.2\%) while 12 patients presented anti-thyroglobulin positivity (52.1\%). Both antibodies were present in 8/23 (34.8\%). 19 patients showed the typical ultrasound findings of autoimmune thyroiditis, resulting in a prevalence of Hashimoto's thyroiditis of $5.7 \%$ (95\% Cl $3.5-8.8)$ in our cohort. Three female patients developed subclinical hypothyroidism, whereas one male patient presented subclinical hyperthyroidism. The remaining 19 patients were euthyroid. No statistically significant difference was observed in regard to age of JIA onset, follow-up duration and JIA subtype between the patients with or without ATA. The proportion of females was marginally significantly higher $(p=0.059)$ in the group with ATA positivity compared to children without thyroid antibodies $(91.3 \%$ vs $73.6 \%$, respectively). $56.5 \%$ of patients with ATA showed ANA positivity compared to $37.5 \%$ of patients without ATA $(p=0.07)$. Family history for AITD was significantly higher in children with thyroid antibodies positivity ( $p=$ 0.01 ). Anti-TNF-alpha inhibitors were administered in only 3 children (13\%) with thyroid antibodies before their detection compared to
$35.5 \%$ of patients without thyroid antibodies $(p=0.028)$. Multivariate regression analysis showed that patients with a family history for AITD were about four times more likely to develop ATA (OR 3.75, $95 \% \mathrm{Cl} 1.401-10.017, \mathrm{p}=0.008)$ and confirmed that ATA positivity is less likely to occur in patients undergone anti-TNF-alpha therapy (OR $0.127,95 \% \mathrm{Cl} 0.031-0.518, \mathrm{p}=0.004$ ).

Conclusion: A high prevalence of ATA positivity and Hashimoto's thyroiditis in patients with JIA was found in our wide southern Italian cohort. As expected, a positive family history of AITD was found to be associated with a higher risk to ATA development during the follow-up. This finding supports the usefulness of an active screening for AITD in JIA children, in particular in patients with relatives affected by thyroid disorders. Notably, patients treated with TNF-alpha inhibitors resulted less likely to develop thyroid antibodies. Further studies are needed to investigate the effect of anti-TNF-alpha therapy on thyroid autoimmunity in JIA.

Disclosure of Interest

None declared

\section{P141}

Which musculoskeletal sites involved can we expect at juvenile idiopathic arthritis (JIA) onset?

G. Tarantino ${ }^{1}$, A. Uva ${ }^{2}$, D. Pires Marafon ${ }^{3}, H_{\text {. Jadoun }}^{3}$, A. Aquilani $^{3}$, R

Nicolai $^{3}$, F. De Benedetti ${ }^{3}$, S. Magni Manzoni ${ }^{3}$

${ }^{1}$ Catholic University of Sacred Heart; ${ }^{2}$ Sapienza-University; ${ }^{3}$ Division of

Rheumatology, IRCCS Ospedale Pediatrico Bambino Gesù, Rome, Italy

Correspondence: $\mathrm{G}$. Tarantino

Pediatric Rheumatology 2020, 18(Suppl 2):P141

Introduction: The knee is considered by far the joint most frequently affected at JIA onset. Nonetheless, JIA onset may present with unusual musculoskeletal involvement, eventually leading to a delay in the diagnosis and treatment.

Objectives: To identify the type and number of musculoskeletal sites affected at JIA onset in consecutive patients seen at the study center in an 8 years period.

Methods: Records of patients with new diagnosis of JIA from June 2012 to May 2020 available information in the medical history and standardized joint assessment at diagnosis, were retrospectively reviewed. Systemic JIA subtype according to ILAR classification criteria were excluded. Demographic and clinical features, including the type and number of joints at disease onset and diagnosis, were registered. Data were analyzed through descriptive statistics.

Results: Of a total of 333 Caucasian patients included in the study (75.7\% females), 241 patients $(72.4 \%)$ had oligoarthritis, $79(23.7 \%)$ RF-negative polyarthritis, 7 (2.1\%) RF-positive polyarthritis, 1 (0.3\%) psoriatic arthritis, $5(1.5 \%)$ enthesitis-related arthritis (ERA). Antinuclear antibody (ANA) were positive in 188 patients (56.5\%). The median age at onset was 4.8 years (IQR 2.3-9.3). At diagnosis 103 (30.9\%) patients had only 1 active joint, $143(43.0 \%)$ had 2-4 active joints, $87(26.1 \%)$ had $\geq 5$. As expected the knee, the tibiotalar and the wrist were the most frequently affected joints $(77.2 \%, 41.1 \%$, $21.0 \%$, respectively); cervical spine was involved only in patients with polyarthritis $(n=13)$. Notably, of 103 patients with monoarthritis at diagnosis 98 presented with large joints involvement, among which $\mathrm{n}=2$ isolated elbow and $\mathrm{n}=2$ isolated wrist, and 5 with small joints involvement (Table 1). No sufficient data were available regarding the involvement of tendons and bursae, since the standard joint assessment form did not include them. Nonetheless, additional 4 patients, not included in the sample analysis, had isolated tenosynovitis involvement at diagnosis ( $n=1$ both-sided ulnar extensor tendons; $n=2$ isolated tenosynovitis of the flexor digiti proprius; $n=1$ tenosynovitis of 2 flexors digiti proprii).

Conclusion: Our study confirms the knee, the tibiotalar and the wrist as the most frequently affected joints at JIA diagnosis. On the other hand, musculoskeletal sites, such as small joints of hands and feet, the hip and the shoulder, usually involved in polyarticular JIA, can be the site of disease presentation in oligo- and also mono-articular JIA. Further, JIA may present with isolated tendon involvement. Our results foster not to delay JIA diagnosis in persistent synovitis occurring 
in infrequent joints and to include musculoskeletal sites, other than joints, in the standard articular evaluation. This could be realized by merging clinical and imaging (i.e. ultrasound) musculoskeletal examinations in the same assessment.

\section{Disclosure of Interest}

None declared

Table 1 (abstract P141). Number of patients with mono- or oligoarthritis at disease diagnosis according to specific joint involvement

\begin{tabular}{lll}
\hline & $\mathbf{N A J = 1} \mathbf{( N = 1 0 3 )}$ & $\mathbf{N A J = 2 - 4}(\mathbf{N}=\mathbf{1 4 3})$ \\
\hline Temporomandibular joint & 0 & 2 \\
Shoulder & 0 & 1 \\
Hip & 1 & 1 \\
Metacarpophalangeal joint & 0 & 20 \\
Proximal interphalangeal joint & 2 & 31 \\
Distal interphalangeal joint & 2 & 5 \\
Metatarsophalangeal joint & 0 & 6 \\
Toe & 1 & 22 \\
\hline
\end{tabular}

P142

Combining age at JIA onset, female gender, ANA positivity and family history of autoimmune disease to predict autoimmune thyroid disease in patients with juvenile idiopathic arthritis

L. Baas', J. van Straalen ', P. Quartier ${ }^{2}$, V. Panaviene ${ }^{2}$, J. Anton Lopez ${ }^{2}$, I. Koné-Paut ${ }^{2}$, S. Hashad², V. Keltsev², J. Brunner', F. Gok ${ }^{2}$, G. Vega Cornejo $^{2}$, V. Chasnyk ${ }^{2}$, S. de Roock ${ }^{1}$, N. Wulffraat ${ }^{1}$, J. Swart ${ }^{1}$, N. Ruperto

${ }^{1}$ Paediatric Rheumatology, Wilhelmina Children's Hospital, Utrecht, Netherlands; ${ }^{2}$ Paediatric Rheumatology INternational Trials Organisation, CC at IRCCS Istituto Giannina Gaslini, Genoa, Italy

Correspondence: $L$. Baas

Pediatric Rheumatology 2020, 18(Suppl 2):P142

Introduction: Comorbidities occur more often in patients with juvenile idiopathic arthritis (JIA) than in the general population. In adults, the association between rheumatoid arthritis (RA) and other autoimmune diseases, such as autoimmune thyroid disease (AITD) is reported. Little is known about the association between JIA and other autoimmune diseases, like AITD.

Objectives: The purpose of this study is to evaluate the prevalence of symptomatic AITD in JIA patients and to investigate whether there are any factors associated with a higher risk of developing AITD.

Methods: Data of 8,971 patients, classified by the International League of Associations for Rheumatology (ILAR) criteria, were analyzed in a dataset from the worldwide PharmaChild registry. Patients with diagnosed Hashimoto's thyroiditis, Graves' disease and non-specified autoimmune thyroiditis were labeled as suffering from AITD. Logistic regression analyses were used and a prediction model was developed.

Results: In this study, the prevalence of symptomatic AITD was $1.1 \%$ of all JIA patients. In multivariate analyses, being older at JIA onset $(\mathrm{OR}=1.12 ; 95 \% \mathrm{Cl}=1.05-1.19)$, female gender $(\mathrm{OR}=2.33 ; 95 \% \mathrm{Cl}=$ 1.32-4.11), ANA positivity $(\mathrm{OR}=3.27 ; 95 \% \mathrm{Cl}=1.78-6.00)$ and family history of autoimmune disease $(\mathrm{OR}=3.07 ; 95 \% \mathrm{Cl}=1.76-5.37)$ were significantly associated with developing AITD ever. The final prediction model of developing AITD ever (AUC $=0.702 ; 95 \% \mathrm{Cl}=$
$0.64-0.77)$ included the predictors age at JIA onset $(p<0.001)$, gender $(p=0.058)$, ANA positivity $(p=0.001)$ and family history $(p<0.001)$.

Conclusion: The best predictors for the development of AITD in JIA patients ever were shown to be age at JIA onset, female gender, ANA positivity and family history of autoimmune diseases.

Disclosure of Interest

None declared

\section{P143}

Methotrexate response subgroups identified in two UK juvenile idiopathic arthritis cohorts

S. J. W. Shoop-Worrall ${ }^{1,2}$, K. L. Hyrich ${ }^{2,3}$, L. R. Wedderburn ${ }^{4,5,6}$, W.

Thomson $^{3,7}$, N. Geifman ${ }^{1}$ on behalf of BSPAR-ETN Study, BCRD Study, CLUSTER

${ }^{1}$ Centre for Health Informatics; ${ }^{2}$ Centre for Epidemiology Versus Arthritis, The University of Manchester; ${ }^{3}$ NIHR Manchester BRC, Manchester University NHS Foundation Trust, Manchester Academic Health Science Centre, Manchester; ${ }^{4}$ Centre for Adolescent Rheumatology Versus Arthritis, UCL, UCLH and GOSH; ${ }^{5}$ Infection Inflammation and

Rheumatology, UCL GOS Institute of Child Health; ${ }^{6}$ NIHR Great Ormond Street Hospital Biomedical Research Centre, London, ${ }^{7}$ Centre for Genetics and Genomics Versus Arthritis, The University of Manchester, Manchester, United Kingdom

Correspondence: S. J. W. Shoop-Worrall

Pediatric Rheumatology 2020, 18(Suppl 2):P143

Introduction: Treatment response in JIA is currently viewed as a binary outcome: response or non-response. However, JIA is a heterogeneous disease and it is likely that different, identifiable subgroups of children and young people (CYP) may demonstrate different patterns of disease following treatment. Identifying these response subgroups can assist the tailoring of stratified treatment approaches in JIA.

Objectives: To identify subgroups of CYP defined by different trajectories of juvenile arthritis disease activity score (JADAS) components following methotrexate (MTX) initiation for JIA.

Methods: MTX-naïve CYP with JIA were selected if enrolled prior to January 2018 in the BSPAR Etanercept Cohort Register or the Biologics for Children with Rheumatic Diseases Study at point of starting MTX. JADAS components (active joint count, physician's global assessment $(P G A, 0-10 \mathrm{~cm})$, parental global evaluation (PGE, 0-10 cm) and standardised ESR (0-10) were calculated based on data collected in the year following MTX initiation.

Multivariate group-based trajectory models were used to explore MTX response clusters across the different JADAS components, which were $\log 1 p$ transformed for analysis. Optimal models were selected based on a combination of model fit (BIC, relative entropy, average posterior probabilities), parsimony and clinical plausibility. Clinical and demographic characteristics and achievement of ACR Pedi 30/90 by six months were compared across identified groups.

Results: Of 658 CYP selected, the majority were female (68\%) and of white ethnicity $(86 \%)$, with RF-negative JIA the most common disease category (35\%).

Six subgroups of CYP were identified with differing patterns of disease activity following MTX initiation. Two groups improved across all JADAS components: Fast improvers (11\%), and Slow improvers (16\%). Persistent PGA (8\%), and Persistent PGE (13\%) groups maintained one persistent disease feature but otherwise improved. One group relapsed (7\%) and a final group had persistent disease overall $(44 \%)$.

There were no differences in active joint counts at MTX initiation between subgroups and all ILAR categories were represented across 
each subgroup. However, CYP in persistent disease and slow improver groups had higher CHAQ, PGA and PGE scores at MTX initiation. Those with persistent disease were also older at MTX initiation.

The majority of CYP fulfilled ACR Pedi 30 response ( $>60 \%$ across every group). ACR Pedi 90 achievement was low at 6 months for slow improvers (30\%) and high in the relapse group (68\%). Between $41 \%$ and $73 \%$ achieved ACR Pedi 90 response in groups with persistent disease in one JADAS component.

Conclusion: We identify different patterns of disease activity within CYP initiating MTX, suggesting a simple responder/non-responder analysis at a set point may be over-simplistic. Commonly used response measures did not adequately describe these heterogeneous response patterns. Understanding both clinical factors associated with, and biological mechanisms underpinning, these subgroups would aid stratified medicine in JIA.

\section{Disclosure of Interest}

S. Shoop-Worrall: None declared, K. Hyrich Speaker Bureau of: Abbvie, L. Wedderburn Speaker Bureau of: Pfizer, W. Thomson: None declared, N. Geifman: None declared

\section{P144}

Pressure pain thresholds in young adults with juvenile idiopathic E. D. Arnstad ${ }^{1,2}$, J. M. Iversen ${ }^{3}$, M. Uglem ${ }^{4,5}$, M. Glerup ${ }^{6}$, P. R. Romundstad 7 , T. Sand ${ }^{4,5}$, M. Rygg ${ }^{2,8}$

'Department of Pediatrics, Levanger Hospital, Nord-Trøndelag Hospital Trust, Levanger; ${ }^{2}$ Department of Clinical and Molecular Medicine, NTNU Norwegian University of Science and Technology, Trondheim; ${ }^{3}$ Bodø Municipality, Bodø; ${ }^{4}$ Department of Neuromedicine and Movement Science, NTNU - Norwegian University of Science and Technology; ${ }^{5}$ Department of Neurology and Clinical Neurophysiology, St. Olavs Hospital, Trondheim, Norway; ${ }^{6}$ Department of Pediatrics, Aarhus University Hospital, Aarhus, Denmark; ${ }^{7}$ Department of Public Health and Nursing, NTNU - Norwegian University of Science and Technology, ${ }^{8}$ Department of Pediatrics, St. Olavs Hospital, Trondheim, Norway Correspondence: E. D. Arnstad

Pediatric Rheumatology 2020, 18(Suppl 2):P144

Introduction: Despite modern treatment and improved disease control, pain is the most common complaint in juvenile idiopathic arthritis (JIA). Knowledge about pain thresholds and pain sensitivity among young adults with JIA is sparse.

Objectives: To study pressure pain thresholds (PPTs) in young adults with JIA, 16 years after disease onset compared with controls.

Methods: Consecutive newly diagnosed children with JIA and a disease onset between 1997-2004 from Central Norway, were included in this prospective population-based long-term follow-up study. Children with onset 1997-2000 were part of the Nordic JIA cohort $^{1,2}$. Age- and sex-matched controls were drawn from the National Population Register of Norway. Inactive disease and remission were defined according to Wallace ${ }^{3,4}$. At the follow-up between 2015-17, data from a clinical examination and blood tests were included in addition to an investigator-blinded quantification of PPTs. A digital algometer was used to manually apply pressure three times with an even rate at the upper and lower limb. PPTs from JIA and controls, and from subgroups of JIA defined by disease status, were compared with multilevel models in STATA.

Results: Among the 96 participants with $\mathrm{JIA}, 71 \%$ were female median age was 22.7 (IQR 18.7-26.2) years, median disease duration was 16.1 (IQR 14.2-17.1) years, $47 \%$ had an oligoarticular disease (persistent or extended), and $45 \%$ were in remission off medication. In the control group, $71 \%$ were female and median age was 23.5 (IQR 20.2-26.7) years. Results from the multilevel regression model showed significantly lower PPTs among participants with JIA compared to controls (Table 1). In the JIA group, participants with inactive disease had lower PPTs than both JIA in remission off medication and JIA with active disease (Table 1). The results were consistent for both upper and lower limb.

Conclusion: In this long-term follow-up study of young adults with JIA, we found significantly lower PPTs compared to controls. This may indicate that young adults with JIA have altered pain sensitivity possibly due to accumulated earlier pain experiences.

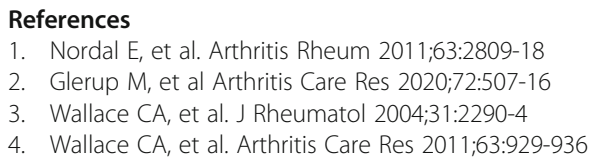

Disclosure of Interest

None declared

Table 1 (abstract P144). Pressure pain thresholds in young adults with JIA compared to controls

\begin{tabular}{llll}
\hline & & \multicolumn{2}{l}{ Pressure pain threshold } \\
\cline { 3 - 4 } & $\mathbf{n}$ & $\begin{array}{l}\text { Upper Limb kPa }(95 \% \\
\mathbf{C l})\end{array}$ & $\begin{array}{l}\text { Lower Limb kPa (95\% } \\
\mathbf{C l})\end{array}$ \\
\hline Control & 109 & $1029(999,1059)$ & $760(726,794)$ \\
IIA (total group) & 96 & $888(846,930)$ & $702(670,734)$ \\
$\begin{array}{l}\text { Remission off } \\
\text { medication }^{\mathrm{a}}\end{array}$ & 43 & $893(824,963)$ & $732(675,789)$ \\
Inactive disease $^{\mathrm{a}}$ & 20 & $836(762,911)$ & $626(575,678)$ \\
Active disease $^{\mathrm{a}}$ & 33 & $910(842,979)$ & $707(662,752)$ \\
\hline
\end{tabular}

$\mathrm{n}=$ number; $\mathrm{kPa}=$ kilopascal; $\mathrm{Cl}=$ confidence interval

${ }^{a}$ According to Wallace ${ }^{3,4}$

P145

Renal outcomes of a cohort of paediatric patients with juvenile idiopathic arthritis

M. F. Gicchino, A. Di Sessa, S. Guarino, E. Miraglia del Giudice, A. N.

Olivieri, P. Marzuillo

University of the Study of Campania Luigi Vanvitelli, Naples, Italy

Correspondence: M. F. Gicchino

Pediatric Rheumatology 2020, 18(Suppl 2):P145

Introduction: Juvenile Idiopathic Arthritis (JIA) represents the most common chronic rheumatic disease in childhood. Non-steroidal antiinflammatory drugs (NSAIDs) and intra-articular steroids are the first line treatment for JIA. Systemic steroids, disease modifying antirheumatic drugs (DMARDs) and biologic drugs are used in children with severe disease. It is not possible at onset of disease to predict when a child can suspend pharmacological treatment, so children affected from JIA have to continue pharmacological treatment for several months or years. Anecdotal reports showed that rarely JIA could present renal involvement due to uncontrolled inflammation or to long exposure to drugs.

Objectives: Because no cohort studies investigating renal injury in children with JIA are available, we designed this kind of study in our population.

Methods: We retrospectively evaluated 110 patients suffering from JIA. JIA diagnosis was made according to ILAR criteria, treatment was assigned with ACR recommendations. For each patient we recorded the type and the duration of pharmacological treatment and the presence of renal injury. Renal injury was defined by the presence of hypertension (systolic and/or diastolic blood pressure $>95^{\text {th }}$ percentile for age, sex and height), proteinuria (persistent confirmation within 3 months- urinary protein/creatinine ratio $>0.5$ $\mathrm{mg} / \mathrm{mg}$ for children $<2$ years old and $>0.2 \mathrm{mg} / \mathrm{mg}$ for patients $>2$ years old $)$ or reduced estimated glomerular filtration rate $(<90 \mathrm{~mL} /$ $\mathrm{min} / 1.73 \mathrm{~m} 2$ ). Development of renal injury was determined by survival analysis according to Kaplan-Meier method.

Results: All the patients underwent NSAIDs administration for a mean time of $44.3 \pm 42.6$ months. 63 patients $(57.3 \%)$ underwent also MTX administration for a mean time of $47.1 \pm 46.2$ months. 34 patients $(30.9 \%)$ underwent biologic agents with a mean duration of the treatment of $37.8 \pm 30.1$ months. Among these 34 patients, 30 patients underwent biological agents after administration of NSAIDs and MTX, while only 4 underwent biological agent administration 
without ever undergoing MTX administration. Mean age at the last follow-up was $13.3 \pm 5.6$ years. The mean duration of JIA was of $84.0 \pm$ 65.4 months. 9 of 110 patients (8.1\%) showed renal injury (8 with hypertension and 1 with proteinuria). Patients with renal injury presented longer duration of the disease $(152.8 \pm 58.2$ Vs $77.9 \pm 62.7$ months; $p=0.001)$, shorter intervals free from JIA relapses $(0(0 / 1) \mathrm{Vs}$ $12(6 / 40) ; p<0.001)$, longer duration NSAIDs treatment $(80.2 \pm 40.9$ Vs $41.13 \pm 40.5$ months; $p=0.008$ ) but with similar cumulative NSAIDs dose (270 Vs 252 grams; $p=0.83$ ) and higher rate of MTX prescription (100\% Vs 53\%; $p=0.007)$, longer time of MTX administration (86.0 \pm 50.5 Vs $40.5 \pm 38.86$ months; $\mathrm{p}=0.005)$ and higher cumulative MTX dose ( $4.8 \mathrm{Vs} 1.72$ grams $p=0.005$ ) compared with the patients without renal injury.

Conclusion: $8 \%$ of the children with JIA develop renal injury. The principal risk factor was longer exposure to NSAIDs and MTX for a more severe disease. Probably, the renal damage could be "time dependent" for NSAIDs exposure and "both time and dose dependent" for MTX exposure. Rheumatologists taking care of children with JIA should pay attention also to kidney health, avoiding long-time treatments with NSAIDs and/or MTX possibly preferring biological treatments in case of poor control of the disease. Moreover, in these patients, periodic evaluation of renal function, blood pressure and proteinuria should be warranted.

\section{Disclosure of Interest}

None declared

\section{P146}

Synovial neutrophils have an altered phenotype and impaired effector functions compared to circulating neutrophils in oligoarticular juvenile idiopathic arthritis

S. Arve-Butler ${ }^{1,2}$, T. Schmidt ${ }^{2,3}$, A. Mossberg ${ }^{2,3}$, E. Berthold ${ }^{1,2}$, B.

Gullstrand', R. Kahn $n^{2,3}$

${ }^{1}$ Clinical Sciences Lund, Rheumatology; ${ }^{2}$ Wallenberg Center for

Molecular Medicine; ${ }^{3}$ Clinical Sciences Lund, Pediatrics, Lund University, Lund, Sweden

Correspondence: $\mathrm{S}$. Arve-Butler

Pediatric Rheumatology 2020, 18(Suppl 2):P146

Introduction: Juvenile idiopathic arthritis (JIA) is a pediatric rheumatic disease with partially unknown etiology and pathogenesis. Neutrophils are the most common immune cell present in synovial fluid from inflamed joints in oligoarticular JIA, but the role of neutrophils in the immunopathogenesis of oligoarticular JIA has not been investigated.

Objectives: To characterize neutrophil phenotypes and effector functions in the circulation and in the inflamed joint during active arthritis in children with oligoarticular JIA.

Methods: Paired samples of blood and synovial fluid from 17 children with oligoarticular JIA were investigated regarding surface markers, phagocytic ability and oxidative burst. Healthy blood neutrophils exposed to cell-free JIA synovial fluid and healthy oral cavity neutrophils were studied as controls for synovial fluid exposure and transmigration respectively.

Results: Synovial neutrophils had a shifted phenotype, characterized by high surface levels of neutrophil activation markers CD11b and CD66b, and mannose receptor CD206 and decreased levels of adhesion molecule CD62L compared to circulating neutrophils. In comparison to oral cavity neutrophils, synovial neutrophils had higher levels of CD11b and a different overall phenotype, suggesting that the phenotype shift in synovial compared to circulating neutrophils is not dependent on transmigration alone. JIA synovial fluid in itself induced activation of healthy blood neutrophils, measured as increased CD11b levels. Synovial fluid neutrophils had impaired ability to phagocytose opsonized E. coli and to produce oxygen radicals upon neutrophil activation with phorbol-myristateacetate (PMA) compared to circulating neutrophils. The impaired effector functions in synovial neutrophils was not dependent on the synovial fluid alone, as addition of cell-free synovial fluid to blood neutrophils did not alter phagocytosis and oxidative burst.

Conclusion: JIA synovial fluid neutrophils are activated, have a "polarized" phenotype and impaired effector functions compared to neutrophils in the circulation. This study will help bridge the current knowledge-gap regarding the role of neutrophils in the immunopathogenesis in oligoarticular JIA.

Disclosure of Interest

None declared

P147

Withdrawn

P148

JIA-ACR50 response as a predictor of minimal disease activity in patients aged 2-17 years with polyarticular-course JIA treated with SC abatacept

N. Ruperto' ', H. I. Brunner', A. Berman', F. Ávila-Zapata', G. Horneff', M. Alessio ${ }^{1}$, M. Becker ${ }^{2}$, A. Belot ${ }^{1}$, R. Burgos-Vargas ${ }^{1}$, A. Boteanu', C. Goldenstein-Schainberg', I. Scheibel', M. T. Terreri', L. Zemel' ${ }^{2}$, R. Wong ${ }^{3}$, M. Nys ${ }^{4}$, M. Askelson ${ }^{3}$, A. Martini ${ }^{1}$, D. J. Lovell ${ }^{2}$ on behalf of Pediatric Rheumatology Collaborative Study Group (PRCSG) and Paediatric

Rheumatology International Trial Organization (PRINTO)

${ }^{1}$ PRINTO, Genova, Italy; ${ }^{2}$ PRCSG, Cincinnati; ${ }^{3}$ Bristol-Myers Squibb

Company, Princeton, United States; ${ }^{4}$ Bristol-Myers Squibb Company, Braine-L'Alleud, Belgium

Correspondence: N. Ruperto

Pediatric Rheumatology 2020, 18(Suppl 2):P148

Introduction: Effectiveness of SC abatacept (ABA) in patients (pts) with polyarticular-course JIA (pJIA) was shown in a 2-year (yr), openlabel Phase III international study (NCT01844518).

Objectives: To assess potential predictors of Juvenile Arthritis Disease Activity Score 27-CRP (JADAS27-CRP) minimal disease activity (MDA), inactive disease (ID) and remission.

Methods: Pts with pJIA aged 2-17 yrs received weight-tiered SC ABA $(10-<25 \mathrm{~kg}: 50 \mathrm{mg} ; 25-<50 \mathrm{~kg}: 87.5 \mathrm{mg} ; \geq 50 \mathrm{~kg}: 125 \mathrm{mg})$ weekly for 4 months (mos). ${ }^{1}$ JIA-ACR30 responders at Mo 4 could receive SC ABA for another 20 mos. ${ }^{1}$ Potential predictors of response over 11 time points to Mo 21 were determined with a multivariate logistic regression (MVR) analysis; Mo 4, 13 and 21 data are presented. MVR variables were baseline (BL) age, sex, race, weight, geographic region, CRP, MTX use, prior biologic use, number of active joints and joints with limitation of motion, physician's global assessment of disease activity, Childhood HAQ-DI (CHAQ-DI), parental assessment of well-being (PaGA) and JIA-ACR50 or JIA-ACR70 responses at Mo 3. Variables were deemed significant if corresponding $p$ values were $<0.05$ at $\geq 6$ time points. Missing values were imputed as nonresponders. BL continuous variable cut-offs (high/low) were determined with receiver-operator curve analysis. Outcomes analysed included JADAS27-CRP MDA ( $\leq 3.8)$, ID $(\leq 1)$ and remission (JADAS27-CRP ID for $\geq 6$ months) rates. Odds ratios and $95 \% \mathrm{Cls}$ were computed.

Results: In all treated pts ( $\mathrm{N}=219)$, median (range) $\mathrm{BL}$ characteristics were: age 11.0 (2.0-17.0) years, CRP $0.2(0.1-21.1) \mathrm{mg} / \mathrm{dL}$, CHAQ-DI $1.0(0.0-2.9)$ and PaGA $47.2(0.0-95.8)$. Variables with the highest number of significant $p$ values $(\leq 0.05)$ were BL CRP, CHAQ-DI, PaGA and Mo 3 JIA-ACR50 and JIA-ACR70. BL CRP, PaGA and CHAQ-DI were predictive of JADAS27-CRP MDA, ID and/or remission at multiple time points (Mo 13; Table 1). JIA-ACR50 response at Mo 3 significantly predicted achievement of JADAS27-CRP MDA at Mo 13 (Table 1) and Mo 21.

Conclusion: Clinically important JIA-ACR50 response at Mo 3 was predictive of the attainment of JADAS27-CRP MDA status at Mo 13 and Mo 21 in pts aged 2-17 yrs with pJIA treated with SC abatacept. 


\section{Reference}

1. Brunner HI, et al. Arthritis Rheumatol 2018:70:1144-1154.

Trial registration identifying number: NCT01844518

\section{Disclosure of Interest}

N. Ruperto Consultant for: Ablynx, AbbVie, AstraZeneca-Medimmune, Biogen, Boehringer Ingelheim, Bristol-Myers Squibb Company, Eli Lilly, EMD Serono, GlaxoSmithKline, F Hoffmann-La Roche, Janssen, Merck, Novartis, Pfizer, Pharma, Sanofi, Servier, Sinergie, Sobi, Takeda, Employee of: The IRCCS Istituto Giannina Gaslini (IGG), where NR works as a full-time public employee, has received contributions from Bristol-Myers Squibb Company, Eli Lilly, GlaxoSmithKline, F Hoffmann-La Roche, Janssen, Novartis, Pfizer, Sobi. This funding has been reinvested for the research activities of the hospital in a fully independent manner, without any commitment with third parties. The registry is funded by Bristol-Myers Squibb Company, Speaker Bureau of: Ablynx, AbbVie, AstraZeneca-Medimmune, Biogen, Boehringer Ingelheim, Bristol-Myers Squibb Company, Eli Lilly, EMD Serono, GlaxoSmithKline, F Hoffmann-La Roche, Janssen, Merck, Novartis, Pfizer, Pharma, Sanofi, Servier, Sinergie, Sobi, Takeda, H. Brunner Consultant for: Abbott, Amgen, AstraZeneca, Boehringer Ingelheim, Celgene, GlaxoSmithKline, F Hoffman-La Roche, Novartis, Pfizer, Takeda, UCB, Wyeth (funds paid to employer), Speaker Bureau of: F Hoffman-La Roche, GlaxoSmithKline, Novartis, A. Berman: None declared, F. Ávila-Zapata: None declared, G. Horneff Consultant for: AbbVie, Novartis, Pfizer, Sanofi, Speaker Bureau of: AbbVie, Novartis, Pfizer, Sanofi, M. Alessio: None declared, M. Becker: None declared, A. Belot: None declared, R. Burgos-Vargas: None declared, A. Boteanu: None declared, C. GoldensteinSchainberg: None declared, I. Scheibel: None declared, M. T. Terreri: None declared, L. Zemel: None declared, R. Wong Shareholder of: Bristol-Myers Squibb Company, Employee of: Bristol-Myers Squibb Company, M. Nys Shareholder of: Bristol-Myers Squibb Company, Employee of: Bristol-Myers Squibb Company, M. Askelson Employee of: Bristol-Myers Squibb Company, A. Martini Consultant for: Aurinia, Bristol-Myers Squibb Company, Eli Lilly, EMD Serono, Janssen, Pfizer, D. Lovell Consultant for: consultant: AstraZeneca, Boehringer Ingleheim, F Hoffman-La Roche, GlaxoSmithKline, Novartis, UCB; principal/co-principal investigator: Bristol-Myers Squibb Company, F Hoffman-La Roche, Janssen, Pfizer, UCB; board member: Forest Research

Table 1 (abstract P148). ORs (95\% Cl) for variables potentially predictive of JADAS27-CRP MDA, ID or remission at Mo 13

\begin{tabular}{|c|c|c|c|c|c|c|}
\hline & \multicolumn{2}{|l|}{ MDA } & \multicolumn{2}{|l|}{ ID } & \multicolumn{2}{|l|}{ Remission } \\
\hline & Cut-off & $\begin{array}{l}\text { OR }(95 \% \\
\text { Cl) }\end{array}$ & Cut-off & $\begin{array}{l}\text { OR }(95 \% \\
\mathrm{Cl})\end{array}$ & Cut-off & $\begin{array}{l}\text { OR }(95 \% \\
C I)\end{array}$ \\
\hline$\overline{C R P}$ & $>1$ vs $\leq 1$ & $\begin{array}{l}0.58(0.23 \\
1.42)\end{array}$ & $\begin{array}{l}>0.6 \text { vs } \\
\leq 0.6\end{array}$ & $\begin{array}{l}0.33 \\
(0.13 \\
0.83)^{a}\end{array}$ & $\begin{array}{l}>0.7 \text { vs } \\
\leq 0.7\end{array}$ & $\begin{array}{l}0.08 \\
(0.01 \\
0.78)^{a}\end{array}$ \\
\hline CHAQ-DI & $\begin{array}{l}>0.75 \text { vs } \\
\leq 0.75\end{array}$ & $\begin{array}{l}0.38(0.15 \\
0.94)^{\mathrm{a}}\end{array}$ & $\begin{array}{l}>0.75 \text { vs } \\
\leq 0.75\end{array}$ & $\begin{array}{l}0.53 \\
(0.23 \\
1.18)\end{array}$ & $\begin{array}{l}>0.5 \text { vs } \\
\leq 0.5\end{array}$ & $\begin{array}{l}0.39 \\
(0.13 \\
1.24)\end{array}$ \\
\hline PaGA & $\begin{array}{l}>43.16 \text { vs } \\
\leq 43.16\end{array}$ & $\begin{array}{l}0.48(0.21 \\
1.10)^{b}\end{array}$ & $\begin{array}{l}>39 \text { VS } \\
\leq 39\end{array}$ & $\begin{array}{l}0.41 \\
(0.18 \\
0.91)^{\mathrm{a}}\end{array}$ & $\begin{array}{l}>27.37 \text { vs } \\
\leq 27.37\end{array}$ & $\begin{array}{l}0.24 \\
(0.08 \\
0.75)^{a}\end{array}$ \\
\hline $\begin{array}{l}\text { JIA-ACR50 } \\
\text { at Mo } 3\end{array}$ & Yes vs no & $\begin{array}{l}6.93(2.20 \\
21.89)^{\mathrm{a}}\end{array}$ & $\begin{array}{l}\text { Yes vs } \\
\text { No }\end{array}$ & $\begin{array}{l}1.65 \\
(0.47 \\
5.73)\end{array}$ & Yes vs No & $\begin{array}{l}1.15 \\
(0.12 \\
11.08)\end{array}$ \\
\hline $\begin{array}{l}\text { JIA-ACR70 } \\
\text { at Mo } 3\end{array}$ & Yes vs no & $\begin{array}{l}1.53(0.58 \\
4.00)\end{array}$ & $\begin{array}{l}\text { Yes vs } \\
\text { No }\end{array}$ & $\begin{array}{l}3.01 \\
(1.09 \\
8.29)^{a}\end{array}$ & Yes vs No & $\begin{array}{l}4.14 \\
(0.72 \\
23.82)\end{array}$ \\
\hline
\end{tabular}

Statistically significant $(\mathrm{p}<0.05)$.

${ }^{\mathrm{b}}$ Showing trend $(\mathrm{p}=0.05-0.1)$.

$\mathrm{ID}=$ inactive disease; $\mathrm{MDA}=$ minimal disease activity; $\mathrm{OR}=$ odds ratio.

\section{P149}

Juvenile dermatomyositis (JDM) working party update

M. G. Wilkinson 1, L. J. Mccann ${ }^{2}$, on behalf of JDM Working Group

${ }^{1}$ UCL Great Ormond Street Institute of Child Health, London; ${ }^{2}$ Alder Hey Children's NHS Foundation Trust, Liverpool, United Kingdom

Correspondence: L. J. Mccann

Pediatric Rheumatology 2020, 18(Suppl 2):P149
Introduction: The JDM working party has 140 active members. Since June 2019, they have been represented by a core group of 7 elected members (Chair: Liza McCann, UK; Secretary: Meredyth Wilkinson, UK; Past Chair: Helga Sanner, Norway; Representative for basic science: Judith Wienke, Netherlands; Representative for clinical care / clinical science: Charris Papadopoulou, UK; Representative for education/ training (\& EMERGE): Raquel Campanilho-Marques, Portugal) and 3 co-opted members (Allied Health Professional Representatives: Sara Röstlund, Sweden and Mette Nørgaard, Denmark; Parent representative, Joanne Swan, Scotland).

Objectives: Our aim is to frame our work in line with the PReS pillars - clinical / research, basic science and education / training. We think that part of our role within the PReS Working Group is to help standardise care, facilitate multi-centre research projects and enhance educational opportunities.

Methods: The core group meet via teleconference every 2 months with a set agenda incorporating each of the PReS pillars. An update was distributed to all working party members in February 2020, encouraging submission of ideas and/or collaboration on proposed projects.

Results: The core group to date has:

- Established and completed a survey to define opinion / use in practice of the SHARE JDM consensus guidelines (Enders FB et al, Ann Rheum Dis 2017). Results of this $(n=46)$ submitted in abstract to PReS 2020; manuscript in preparation.

- Clarified the role of databases / data sharing in JDM across Europe; PReS JDM Working Party and Euromyositis Position Statement available on PReS website.

- Informed members of the EMERGE group of training opportunities / fellowships relating to JDM.

- Produced a summary of research projects and educational resources, available on PReS website.

- Collaborated with the International Myositis Assessment \& Clinical Studies Group (IMACS), promoting shared working.

Work currently in progress includes:

- Collaborating in the extension of the JDM SHARE consensus for management of JDM in North America (IMACS project).

- Research proposal on sleep in JDM - in development.

- Training package / educational resource on myositis specific antibodies near completion; will be available in PReS website.

- Proposal for a Policy Statement on management of JDM during Covid-19 with a particular focus on transition to adult services.

- Survey of practice in JDM proposed; in early stages of development.

Conclusion: The JDM PReS working party provides a platform for collaboration in JDM, incorporating clinical and research issues, basic science and education / training. Ideas and collaborations are welcomed and encouraged within an open membership structure. To join the group, e-mail: meredyth.wilkinson.14@ucl.ac.uk.

Disclosure of Interest

None declared

P150

Vitamin D supplementation of children with rheumatic diseases

M. Tikhaya, N. Podchernyaeva, E. Zholobova

I.M. Sechenov First Moscow State Medical University, Moscow, Russian

Federation

Correspondence: M. Tikhaya

Pediatric Rheumatology 2020, 18(Suppl 2):P150

Introduction: VITAMIN D SUPPLEMENTATION OF CHILDREN WITH RHEUMATIC DISEASES

Objectives: To determine supplementation level of vitamin $D$ in children with Juvenile idiopathic arthritis (JIA) and Juvenile dermatomyositis (JDM) 
Methods: 52 children ( 35 girls and 17 boys), aged from 5 to 17 years were observed. 29 children had JDM, JIA was in 23 children.The level of $25(\mathrm{OH}) \mathrm{D}$ in plasma was measured by chemiluminescent immunoassay method. All patients with JDM and 16 (69.5\%) JIA patients were taking vitamin D drugs (cholecalciferol $200 \mathrm{IU} /$ day and/ or alfacalcidol $0,25 \mu \mathrm{g}$ ). Concentration level $21-30 \mathrm{ng} / \mathrm{ml}$ was determined as insufficiency, less than $20 \mathrm{ng} / \mathrm{ml}$ as deficiency.

Results: Vitamin D insufficiency was in $50(94.3 \%)$ children of our cohort, including 100\% cases in patients with JIA (21 (91.3\%) children had a deficit, 4 of them (17.4\%) with extra deficit). $25(\mathrm{OH}) \mathrm{D}<30 \mathrm{ng} / \mathrm{ml}$ was observed in $90 \%$ cases in JDM patients, $70 \%$ of them with deficit. The average $25(\mathrm{OH}) \mathrm{D}$ level in JDM group was significantly higher than in JIA group $(21,51 \pm 11,87$ vs $14,98 \pm 0,91 \mathrm{ng} / \mathrm{ml})$

Conclusion: The overwhelming majority of children with JIA and JDM have an insufficiency or deficiency of vitamin $D$ in comparison with Russian population. Traditional therapy with complex calcium salts drugs and cholecalciferol in low doses is insufficient to achieve the optimal level of vitamin D.

At the same time, vitamin D supplementation in children with JDM is higher than in JIA patients. Perhaps this is partly due to more intensive vitamin $\mathrm{D}$ therapy.

\section{Disclosure of Interest}

None declared

\section{P151}

Favorable clinical response of refractory juvenile dermatomyositis to ruxolitinib

A. Heinen' ${ }^{1}$, A. Schnabel', N. Brück', M. Smitka', C. Wolf', N. Lucas' ${ }^{1}$, S. Dollinger ${ }^{2}$, G. Hahn ${ }^{3}$, C. Guenther ${ }^{4}$, R. Berner', M. A. Lee-Kirsch', C. Schuetz ${ }^{1}$

${ }^{1}$ Pediatrics, Technische Universität Dresden, Dresden; ${ }^{2}$ Pediatric Rheumatology, Deutsches Zentrum für Kinder- und

Jugendrheumatologie, Garmisch-Partenkirchen; ${ }^{3}$ Department of

Radiology; ${ }^{4}$ Department of Dermatology, TU Dresden, Dresden, Germany Correspondence: $\mathrm{C}$. Schuetz

Pediatric Rheumatology 2020, 18(Suppl 2):P151

Introduction: Juvenile dermatomyositis (JDM) is a rare systemic inflammatory disorder of childhood associated with vasculopathies and a constitutive type I interferon (IFN) activation. Standard immunosuppression does not always control disease activity and may have serious side effects.

Objectives: JAK1/2 inhibitors were recently shown to be therapeutically effective in patients with monogenic type I interferonopathies. We applied this rationale in a JDM patient with therapy-refractory course and elevated IFN signature.

Methods: A 14-year-old boy with NXP2-positive JDM presented with a relapse almost 10 years into remission. He failed to improve on first-line treatment and was transferred to our center with dysphagia due to severe myositis. His initial Childhood Myositis Assessment Scale (CMAS) score was 6/52. Besides elevation of CK and von-Willebrand antigen, he had a strong IFN signature consistent with the diagnosis of relapsed JDM.

Results: In view of insufficient disease control and the persistent high IFN signature, cyclophosphamide, rituximab and steroid pulses were stopped, and the patient was started on ruxolitinib. During the first three months of ruxolitinib therapy $(30 \mathrm{mg} / \mathrm{d})$, the patient experienced increasing muscle strength and significant clinical improvement. He was able to walk short distances and to climb one flight of stairs. At seven months, he is able to ride his bicycle for 10 minutes. His CMAS score improved from $18 / 52$ to $40 / 52$. This was accompanied by a sustained reduction the IFN signature.

Conclusion: The observation of a sustained reduction of the IFN signature under ruxolitinib supports a primary role of constitutive type I IFN activation in JDM pathogenesis. Thus, ruxolitinib should be considered early in the treatment of refractory JDM, not necessarily as second-line therapy.

\section{Disclosure of Interest}

None declared
P152

Overlap syndrome of idiopathic inflammatory myopathy with juvenile systemic lupus erythematosus presenting with macrophage activation syndrome

R. L. D. Bragança', R. P. Silva², J. F. Mendes², C. Rocha², J. L. Barreira', F. Aguiar $^{3}$, M. Rodrigues ${ }^{3}$, I. Brito ${ }^{3}$

'Serviço de Pediatria, Centro Hospitalar Universitário de São João, Porto;

${ }^{2}$ Serviço de Pediatria, Centro Hospitalar Entre Douro e Vouga, Feira;

${ }^{3}$ Unidade de Reumatologia Pediátrica e Jovem Adulto , Centro

Hospitalar Universitário de São João, Porto, Portugal

Correspondence: R. L. D. Bragança

Pediatric Rheumatology 2020, 18(Suppl 2):P152

Introduction: Some patients with connective tissue diseases cannot be assigned to a single disease category, presenting characteristics from two or more immune mediated conditions, the so-called overlap syndromes (OS). OS are infrequent in children and their description in literature is limited to some case series.

Objectives: Our aim is to present a rare case of an OS of idiopathic inflammatory myopathy (IIM) with juvenile systemic lupus erythematosus (jSLE) in a child that initially presented with macrophage activation syndrome (MAS).

Methods: A case report is described. Data was extracted from the medical chart of the patient and a literature review was undertaken. Results: A 7-year-old girl was transferred to our tertiary center after being admitted for prolonged intermittent fevers, abdominal pain, fatigue and polyarthralgias. On examination, there was symmetrical proximal muscle weakness, a vasculitic lower limb rash, facial erythema with eyelid edema (Fig. 1) and oral mucositis. Initial laboratory exams revealed pancytopenia, high muscle enzymes, increased erythrocyte sedimentation rate with moderately elevated reactive C-protein, and hypocomplementemia. She also had non-nephrotic proteinuria, without hematuria.Further investigations showed a positive direct antiglobulin test, antinuclear antibodies, antidouble-stranded DNA, anti-Mi 2 and anti-Ku. Serositis (pericardial and pleural effusions, ascitis) and hepatosplenomegaly were present. Lower limb MRI documented diffuse muscle edema. The diagnosis of an overlap syndrome of jSLE and IIM was established. While being treated for concomitant bacteremia, the patient became ill-appearing, with persistent fevers, worsened cytopenias, low fibrinogen and high ferritin and triglycerides, and a Macrophage Activation Syndrome (MAS) diagnosis was assumed. The patient received antibiotics and intravenous immunoglobulin, followed by methylprednisolone pulses, IV cyclosporine (CYC), hydroxychloroquine and supportive therapy with progressive improvement.

Due to hypertension (possibly related to CYC) and persistent proteinuria a renal biopsy was performed showing class IV lupus nephritis. After achieving clinical stability, CYC was switched to mycophenolate mofetil as an induction treatment, which is ongoing.

Conclusion: IMM with SLE OS is uncommon, and has seldom been described in children. In addition to fulfilling SLE criteria, our patient had clinical, laboratory and imagiologic evidence of IMM. The presence of myositis specific antibodies (especially anti-Mi 2) further supports the diagnosis of an OS rather than an atypical presentation of a lupus myopathy. Juvenile dermatomyositis appears to be the IMM subtype - it is associated with anti-Mi 2, and mild heliotrope and eyelid edema are compatible. Facial rash sparing the nasolabial folds is more suggestive of SLE.

MAS is a rare but life-threatening condition that should be suspected in rheumatologic conditions and might be triggered by infections or disease flares. Its identification may be particularly challenging at presentation, especially in SLE where cytopenias are common. The reported prevalence in adult SLE ranges from $0.9 \%$ to $4.6 \%$; disease-specific criteria have been proposed. MAS has occasionally been described in IIM. In a patient with a predisposing condition, persistent fevers and illappearance must always prompt a MAS workup, since early diagnosis and treatment are paramount.

Due to an early referral to a pediatric rheumatology center, the patient received a prompt diagnosis and treatment, which probably improved her prognosis.

Disclosure of Interest

None declared 
P153

Bilateral knee avascular necrosis in juvenile dermatomyositis: the pitfalls of diagnosis

I. Dabbak, F. Aeschlimann, S. Breton, P. Quartier, B. Bader Meunier

Hôpital Necker, Paris, France

Correspondence: $\mathrm{F}$. Aeschlimann

Pediatric Rheumatology 2020, 18(Suppl 2):P153

Introduction: Skeletal complications seem to be particularly frequent in JDM: bone mineral density loss before treatment initiation seems more frequent than in other inflammatory diseases and vertebral fractures may occur during the first months of CS therapy. Avascular osteonecrosis (AVN) is a rare complication of corticosteroid (CS) use.Rare cases of multifocal AVN in sometimes unusual locations have previously been described in JDM patients ${ }^{1,2}$, but information about associated myositisspecific autoantibodies was not available in these cases.

Objectives: To report the occurrence and characteristics of knee AVN in juvenile dermatomyositis (JDM) patients

Methods: Retrospective single-center study of JDM patients with knee AVN followed at the national paediatric rheumatology reference center of Necker hospital between January 2013 and December 2019 Results: Three female patients out of the 55 patients of our JDM cohort (5.5\%) developed knee AVN over a period of 6 years. Patient A, a 11-year old girl, was treated with the successive association of CS and mycophenolate mofetil (MMF), methotrexate (MTX) and eventually ruxolitinib due to persistent severe cutaneous ulcerations. Six months after diagnosis, she presented with bilateral knee swelling and pain of both inflammatory and mechanical character. An articular relapse was suspected, but MRI of the knees, revealed multiple bilateral epiphyseal, metaphyseal and diaphyseal bone infarction sites of the femur and tibia (fig. 1A). Patient B, a 14-year old adolescent, was treated with CS, MTX, and the subsequent addition of MMF and RTX. She presented 6 months after diagnosis with bilateral knee and ankle pain of mechanical character, with minimal knee effusion and normal range of motion. Whole-body MRI showed giant femorotibial (fig. 1B) and ankle bone infarction sites. Patient $\mathrm{C}$, a 6-year old girl, was treated with CS, MTX and RTX. Nine months after diagnosis she started complaining of mechanical left-knee pain. There was no swelling and range of motion was normal. MRI showed a focal epiphyseal lesion of the tibia compatible with bone infarction. The three patients were positive for antiMDA5 antibody (3/11 MDA5 positive JDM patients (27\%)), while none of the 44 MDA-negative patients had a diagnosis of AVN.

Conclusion: Knee AVN occurred in $5.5 \%$ (3/55) patients of our cohort. All of them had severe anti-MDA5-positive JDM, and AVN was diagnosed within the year following the diaagnosis.AVN should be considered as a differential diagnosis in JDM patients presenting with joint pain. Larger studies are warranted to assess the possible link between its occurrence and the presence of anti-MDA5 autoantibodies

\section{Disclosure of Interest}

None declared

\section{P154}

Successful use of cyclophosphamide in calcinosis in juvenile dermatomyositis

K. C. Warrier', S. Deepak', S. Rangaraj ${ }^{1}$

'Paediatric Rheumatology, Nottingham University Hospitals, Nottingham, United Kingdom

Correspondence: K. C. Warrier

Pediatric Rheumatology 2020, 18(Suppl 2):P154

Introduction: Juvenile Dermatomyositis (JDM) is the commonest inflammatory myopathy of childhood. One of the important causes of morbidity in JDM is calcinosis - dystrophic calcification affecting skin, soft tissue and muscles - seen in approximately one-third of patients. The longer duration of active disease, cardiac involvement and presence of certain myosotis specific antibodies have been suggested to increase the risk. The complications include secondary infection, panniculitis, and ulceration, in addition to pain and tenderness. The treatment strategies include anti-inflammatory medications, medications affecting calcium and phosphorus metabolism and surgical options, although the results are mixed with limited evidence. Intravenous Immunoglobulin (IVIG), anti-Tumour Necrosis Factor (TNF) agents, Abatacept and Rituximab have all been tried with varying success, in addition to corticosteroids.

Objectives: To describe the clinical and laboratory characteristics of five patients with JDM with calcinosis, who were treated successfully with Cyclophosphamide.

Methods: Retrospective analysis of digital health records.

Results: Four of the five patients were female (80\%) and all aged between 6 and 10 years. Four of them had calcinosis at the time of diagnosis, although they may have had symptoms for 12 to 18 months prior to diagnosis. Skin involvement was severe requiring multiple systemic and topical therapeutic agents in four out of the five patients - significantly more affected than the muscles. One patient had amyopathic subtype with normal Childhood Myositis Assessment Score (CMAS) throughout. None of them had cardiac involvement. All had weakly positive AntiNuclear Antibodies (ANA); but were negative for myositis antibodies except the patient with most severe skin involvement and calcinosis (patient 2), who was positive for Anti-TIF1gamma antibodies.

Two of the three patients with calcinosis at onset had Cyclophosphamide as the second line agent (chosen due to calcinosis) following systemic corticosteroids with complete resolution of the lesions after six cycles at $500 \mathrm{mg} / \mathrm{m} 2$. One patient responded to Infliximab, which failed to work after 20 months, following which Cyclophosphamide was tried with good response. The other two patients were given Cyclophosphamide after they failed to respond to Rituximab, which did work for muscle disease. One patient had recurrent episodes of calcinosis needing surgical curettage despite initial response to Cyclophosphamide and later IVIG.

\begin{tabular}{|c|c|c|c|c|c|}
\hline Gender & Female & Female & Female & Female & Male \\
\hline Age & $6 y 9 \mathrm{~m}$ & $7 y 4 m$ & $8 y 3 m$ & $\begin{array}{l}8 y \text { (rash - } \\
6 \mathrm{~m})\end{array}$ & $\begin{array}{l}9 y 2 \mathrm{~m}(12- \\
18 \mathrm{~m} \text { history) }\end{array}$ \\
\hline $\begin{array}{l}\text { Age at } \\
\text { calcification }\end{array}$ & $\begin{array}{l}\text { At } \\
\text { presentation }\end{array}$ & $13 y \mathrm{rm}$ & $\begin{array}{l}\text { At } \\
\text { presentation }\end{array}$ & $\begin{array}{l}\text { At } \\
\text { presentation }\end{array}$ & $\begin{array}{l}\text { At } \\
\text { presentation }\end{array}$ \\
\hline Site & $\begin{array}{l}\text { Upper arms, } \\
\text { elbows }\end{array}$ & $\begin{array}{l}\text { Upper arms, } \\
\text { elbows, } \\
\text { thighs, } \\
\text { knees, } \\
\text { occipital } \\
\text { area }\end{array}$ & Elbows & $\begin{array}{l}\text { Forearm, } \\
\text { thigh }\end{array}$ & $\begin{array}{l}\text { Elbows, } \\
\text { knees }\end{array}$ \\
\hline Skin disease & Severe & Severe & Severe & Moderate & Mild \\
\hline Muscle & $\begin{array}{l}\text { Affected - } \\
\text { CMAS 28/52 }\end{array}$ & $\begin{array}{l}\text { Affected - } \\
\text { CMAS 23/52 }\end{array}$ & Amyopathic & $\begin{array}{l}\text { Mild - } \\
\text { CMAS 44/ } \\
52\end{array}$ & $\begin{array}{l}\text { Affected - } \\
\text { CMAS 28/52 }\end{array}$ \\
\hline $\begin{array}{l}\text { Other } \\
\text { interventions }\end{array}$ & & $\begin{array}{l}\text { Surgical } \\
\text { curettage }\end{array}$ & $\begin{array}{l}\text { Surgical } \\
\text { excision }\end{array}$ & & \\
\hline Response & $\begin{array}{l}\text { Complete } \\
\text { resolution }\end{array}$ & $\begin{array}{l}\text { Stopped new } \\
\text { lesions - later } \\
\text { flared }\end{array}$ & $\begin{array}{l}\text { Stopped } \\
\text { new } \\
\text { lesions }\end{array}$ & $\begin{array}{l}\text { Complete } \\
\text { resolution }\end{array}$ & $\begin{array}{l}\text { Complete } \\
\text { resolution }\end{array}$ \\
\hline $\begin{array}{l}\text { Other } \\
\text { agents tried }\end{array}$ & $\begin{array}{l}\text { MTX } \\
\text { Rituximab } \\
\text { (muscles } \\
\text { responded) } \\
\text { HCQ } \\
\text { Dapsone }\end{array}$ & $\begin{array}{l}\text { MG - worked } \\
\text { for skin \& } \\
\text { calcinosis } \\
\text { Rituximab } \\
\text { (muscles } \\
\text { responded) } \\
\text { MTX }\end{array}$ & $\begin{array}{l}\text { Infliximab } \\
\text { (worked } \\
\text { for } 20 \\
\text { months) } \\
\text { MMF } \\
\text { MTX } \\
\text { Dapsone }\end{array}$ & $\begin{array}{l}\text { MTX } \\
\text { MMF }\end{array}$ & MTX \\
\hline
\end{tabular}

\section{Abbreviations}

MTX-Methotrexate

MMF-Mycophenolate Mofetil

IVIG-Intravenous Immunoglobulin

HCQ-Hydroxychloroquine

Conclusion: Although Cyclophosphamide does not feature prominently in literature in the management of calcinosis associated with JDM, it has proven to be useful in this cohort of patients, especially when tried early on for calcinosis. Based on our experience, although the numbers are small, Cyclophosphamide is worth trying in patients with calcinosis, after careful discussion with the patient and family about the risks against benefits.

Disclosure of Interest

None declared 


\section{P155}

Use of mycophenolate mofetil in inflammatory myopathies of childhood

G. C. Varnier ${ }^{1,2}$, A. Consolaro ${ }^{2,3}$, I. L. Cheng ${ }^{4}$, A. Silva Riveiro ${ }^{3}$, C

Pilkington ${ }^{4}, A$. Ravelli ${ }^{2,3,5}$ on behalf of Juvenile Dermatomyositis Research Group (JDRG)

'Paediatric Rheumatology, Royal Manchester Children's Hospital, Manchester, United Kingdom; ${ }^{2}$ University of Genoa; ${ }^{3}$ Paediatric Rheumatology, IRCCS G. Gaslini, Genoa, Italy; ${ }^{4}$ Paediatric Rheumatology, Great Ormond Street Hospital, London, United Kingdom; ${ }^{5}$ Sechenov First Moscow State Medical University, Moscow, Russian Federation

Correspondence: $\mathrm{G}$. C. Varnier

Pediatric Rheumatology 2020, 18(Suppl 2):P155

Introduction: The juvenile idiopathic inflammatory myopathies (JIIM) consist of heterogeneous inflammatory diseases that primarily affect the skeletal muscles, but can potentially also affect the skin and visceral organs. To date, there is still limited evidence regarding the treatment of these rare disorders.

Objectives: To evaluate the efficacy of mycophenolate mofetil (MMF) in the JIIM.

Methods: Patients diagnosed with JIIM and treated with MMF enrolled in the Juvenile Dermatomyositis Research Group (JDRG) in the United Kingdom and under the care of the G. Gaslini Institute (IGG) in Genoa, Italy were included in this study. Data collected included: sex, onset year, onset age, onset type, clinical manifestations, disease duration, disease course and activity, laboratory data and treatment received. Outcomes were muscle strength/endurance, cutaneous and global disease activity, cumulative damage and physical function. Data were retrospectively analysed at time of starting MMF, after 3, 6, 12 months and last clinical follow up.

Results: 29 children were included in this study, 25 were from the UK cohort and 4 were followed at IGG in Italy. Of these 29 patients, $79.3 \%$ were diagnosed with juvenile dermatomyositis and the remaining $20.7 \%$ were overlap myositis. We observed a significant improvement in the muscle-related outcome measures (Manual Muscle 8=80 from 50\% to 83.3\%; Childhood Myositis Activity Score= 52 from $53.5 \%$ to $88.9 \%$; Disease Activity Score-Muscle from $55.2 \%$ to $84.2 \%$ ) and overall disease activity (Global Visual analogue scale $(\mathrm{VAS})=0$ from $7.1 \%$ to $42.1 \%$ ), and also an improvement of the skinrelated outcome measures (Disease activity score-Skin $=0$ from $31 \%$ to $42.1 \%$; skin visual analogue scale $=0$ from $25 \%$ to $47.4 \%$ ). The number of patients with inactive disease significantly improved from $10.3 \%$ at baseline, to $68.5 \%$ at last study visit.

The corticosteroid dose was significantly reduced from 0.3 to $0.1 \mathrm{mg} /$ $\mathrm{Kg} /$ day. No significant side effects were reported.

Conclusion: The use of MMF has shown to be efficacious and safe in children with IIM, especially in patients with refractory muscle disease.

\section{Disclosure of Interest}

None declared

\section{P156}

Evaluation of disease activity in children with juvenile dermatomyositis: a comparison between electromyography and whole body-magnetic resonance imaging

S. Carbogno ${ }^{1,2}$, M. Brusamolino ${ }^{1}$, S. Lanni ${ }^{2}$, A. Petaccia 2 , C. Agostoni ${ }^{1,2}$, R. Cimaz $^{3,4}$, G. Filocamo², F. Minoia', A. Cappellari ${ }^{2}$ on behalf of on behalf of Pediatric Rheumatology Group of the Milan Area (PRAGMA)

${ }^{1}$ University of Milan; ${ }^{2}$ Fondazione IRCCS Ca' Granda Ospedale Maggiore Policlinico; ${ }^{3}$ ASST G. Pini-CTO; ${ }^{4}$ Department of Clinical Sciences and Community Health, and RECAP-RD, University of Milan, Milan, Italy Correspondence: $\mathrm{S}$. Carbogno

Pediatric Rheumatology 2020, 18(Suppl 2):P156

Introduction: Juvenile Dermatomyositis (JDM) is the most common pediatric inflammatory myopathy. The Juvenile Dermatomyositis Activity Index (JDMAl) is composed of four clinical items and has been proposed for use in both clinical and research settings. Needle electromyography (EMG) examination is the most informative part of the electro diagnostic study in myopathic disorders. Whole body-magnetic resonance imaging (WB-MRI) allows to reliably visualize the extent of the inflammatory process and to estimate the total disease burden. To date, the role of EMG and WB-MRI in assessing disease activity in JDM is still not fully defined.

Objectives: To perform a comparison between EMG testing and WBMRI with disease activity score in a group of JDM patients.

Methods: All patients diagnosed with JDM and referred to our Centre between January 2018 and January 2019 were enrolled. Clinical, laboratory and radiological data were collected. A standardized clinical evaluation through manual muscle test (MMT)-8, hybrid MMT/CMAS (hMC) and JDMAI was performed at each visit; laboratory test included muscle enzymes levels; WBMRI and EMG were performed within one month of the clinic visit. WB-MRI signal intensity was scored using a 0-2 point scale in 42 muscular groups; myofascial and subcutaneous tissue inflammation were assessed on the upper and lower extremities using a 0-1 point scale. The EMG evaluated the presence of fibrillation potentials on four muscles (deltoid and extensor digitorum communism for the upper limb, and vests medals and tibias anterior for the lower limb). The degree of fibrillation potentials in every muscle was scored using a $0-2$ point scale $(0=$ no fibrillation potentials; $1=$ presence of fibrillation potentials in $<50 \%$ of the sites analyzed; 2 = presence of fibrillation potentials in $>$ $50 \%$ of the sites analyzed).

Based on JDMAl, visits were grouped as follows: visits of patients in clinically active disease vs visits of patients in clinically inactive disease.

Mann-Whitney U test, Chi-square/Fisher test and Spearman's rank correlation coefficient were used for statistical analysis.

Results: Thirteen patients were included in the study for a total of 18 visits. WB-MRI score resulted significantly higher for visits of patients with active disease then in those of patients with clinically inactive disease ( $p 0.011$ and 0.007 respectively). No difference was found in EMG scores for both visits of patients with active and inactive disease ( $p 0.274$ and 0.310 , respectively). WBMRI score had a moderate to high correlation with all clinical evaluation tools of muscle strength or disease activity (Spearman's rank coefficient $=0.61,0.58$, and 0.86 with MMT-8, hMC and JDMAI respectively), while EMG score had a moderate correlation $(0.48,0.42$, and 0.62 with MMT-8, hMC and JDMAl respectively).

Conclusion: In this pilot study, WB-MRI seems to better discriminate between active and inactive disease compared to EMG in patients with JDM. Further studies on larger populations of children with JDM could contribute to define the role of EMG and WB-MRI in the assessment of disease activity.

Disclosure of Interest

None declared

P157

Withdrawn

P158

Pulmonary findings on high resolution computed tomography in patients with juvenile dermatomyositis: retrospective study

C. H. Capello ${ }^{1}$, E. Bronzatto ${ }^{2}$, R. Marini ${ }^{3}$, S. Dertkigil ${ }^{2}$, S. Appenzeller ${ }^{4}$

${ }^{1}$ Child and Adolescente Graduate Program, UNIVERSITY OF CAMPINAS;

${ }^{2}$ Radiology, UNICAMP; ${ }^{3}$ Pediatrics, UNIVERSITY OF CAMPINAS; ${ }^{4}$ Medicine,

UNICAMP, Campinas, Brazil

Correspondence: S. Appenzeller

Pediatric Rheumatology 2020, 18(Suppl 2):P158

Introduction: Juvenile Dermatomyositis (JDM), are complex, heterogeneous, autoimmune diseases that affect skeletal muscles and the skin. The interstitial lung disease (ILD) is a complication that has been observed in $20-78 \%$ of patients and can be a determining prognosis, causing greater morbidity and mortality. 
Objectives: To evaluate the finds pulmonary by high-resolution Computerized Tomography (HRCT) in accompanying patients in outpatient pediatric rheumatology unit.

Methods: Retrospective study of 62 patients diagnosed with JDM, which are submitted to the examination of image for clinical monitoring of the disease. TCAR tests were observed in approximately 10 years, including repeated CT scans assessed: interstitial pneumopatia; pneumomediastium; the presence of nodules; opacity in ground glass; fibros elastic stretch marks; septal thickening; bronquica wall thickening. Two patients were excluded because they had overlap with systemic lupus erythematosus.

Results: HRCT was retrieved for analysis in 62 of patients. We observed interstitial pneumopatia in 9 (14.51\%), showed; nodules/ micronodules in $20(32.25 \%)$, opacity in ground glass in 21 (33.87\%), fibroatelectasias in 13 (20.96\%), septal thickening in 7 (11.29\%), bronchial wall thickening in $10(16.12 \%)$ and pneumomediastium in $1(1.61 \%)$. Findings were observed during the first 2 years of disease In 62 patients.

Conclusion: We conclude that pulmonary involvement in the diseases devolps more frequently during the first two years of the disease. Carefull follow-up with HRCT is essential for early diagnosis and adequate treatment.

\section{Disclosure of Interest}

None declared

\section{P159}

Survey of the use of share JDM recommendations in clinical practise: report of the PRES JDM working group

C. Papadopoulou', M. G. Wilkinson' ${ }^{1}$, R. Campanilho-Marques², J. Wienke ${ }^{3}$ H. Sanner ${ }^{4}$, F. Bellutti Enders ${ }^{5}$, A. van Royen-Kerkhof ${ }^{6}$, L. J. McCann? ${ }^{7}$, on behalf of JDM Working Group

${ }^{1}$ UCL Great Ormond Street Institute of Child Health, London, United Kingdom; ${ }^{2}$ Hospital de Santa Maria, CHULN, Centro Académico de Medicina de Lisboa, Lisbon, Portugal; ${ }^{3}$ University Medical Center, Utrecht, Netherlands; ${ }^{4}$ Oslo University Hospital, Oslo, Norway; ${ }^{5}$ University Children's Hospital Basel, UKBB, Bassel, Switzerland; ${ }^{6}$ Wilhelmina Children's Hospital, University Medical Center, Utrecht, Netherlands; ${ }^{7}$ Alder Hey Children's NHS Foundation Trust, Liverpool, United Kingdom Correspondence: $L$. J. McCann

Pediatric Rheumatology 2020, 18(Suppl 2):P159

Introduction: The Single Hub and Access point for paediatric Rheumatology in Europe (SHARE) guideline for Juvenile Dermatomyositis (JDM)[Enders FB et al, Ann Rheum Dis 2017], directed towards healthcare professionals, aims to provide uniform, optimal access to care via evidence-informed consensus on diagnosis/treatment.

Objectives: Audit impact of SHARE guidance, ascertain opinion of Working Group regarding usefulness and if recommendations need to be revised/updated.

Methods: An online survey was written $(\mathrm{CH}, \mathrm{LM})$ using Survey Monkey ${ }^{\oplus}$, ratified/piloted by the Working Party core group, then distributed to membership of the JDM working party $(n=140)$ and EMERGE (PReS trainee/young scientist) group $(n=150)$ November 2019-February 2020. Reminders were sent to encourage participation. Results were collated and descriptive analysis performed. Responses were anonymous, but questions were asked regarding clinical role, time of practice and number of JDM cases treated/year.

Results: 46(16\%) responses were received from 39 paediatric rheumatologists, 3 adult rheumatologists, 2 physicians with rheumatology interest, 1 internist, 1 parent representative. 31 (67.4\%) had $\geq 10$ years' experience. Number of cases treated/year varied from $<5(17.4 \%)$ to $>30(23.9 \%)$. The majority of respondents reported SHARE guidance as important to their practice; $43.5 \%$ stated important/incorporated into hospital/national guidelines and $6.5 \%$ stated very important/critical for decision-making. Only 5(10.9\%) reported not using SHARE guidance. Overall opinion/experience was positive (Table). 33(73.3\%) thought the SHARE guidance was relevant to practice as it is, although $21(46.7 \%)$ suggested revision/update. Suggestions for revision included biomarkers, myositis antibodies, novel treatments (biologics/JAK inhibitors), managing resistant disease/calcinosis and addressing mental health. For effect of guidance on clinical practise, $60 \%$ reported no change, with only $20 \%$ giving a positive answer.

\begin{tabular}{|c|c|c|c|c|c|c|}
\hline $\begin{array}{l}\text { SHAR } \\
\text { E SURVEY }\end{array}$ & Question & $\begin{array}{l}\text { Yes } \\
\text { N (\%) }\end{array}$ & $\begin{array}{l}\text { No } \\
\text { N (\%) }\end{array}$ & $\begin{array}{l}\text { Unsure } \\
\mathrm{N}(\%)\end{array}$ & $\begin{array}{l}\text { Total } \\
\mathrm{N} \\
\text { (\%) }\end{array}$ & $\begin{array}{l}\text { Comments } \\
\text { (abbreviated) }\end{array}$ \\
\hline \multirow[t]{4}{*}{ Opinion } & $\begin{array}{l}\text { Clear \& easy } \\
\text { to use }\end{array}$ & $\begin{array}{l}41 \\
(89.1)\end{array}$ & $1(2.2)$ & $4(8.7)$ & $\begin{array}{l}46 \\
(100)\end{array}$ & \multirow{4}{*}{$\begin{array}{l}\text { - 'More on } \\
\text { treatment resistant } \\
\text { disease' } \\
\text { - 'NHSE restricts } \\
\text { treatment options } \\
\text { for biologics' } \\
\text { - 'Guidelines for } \\
\text { treatment should } \\
\text { be written more } \\
\text { accurately' } \\
\text { - - 'No mention for } \\
\text { professionals to } \\
\text { check mental } \\
\text { health in the flow } \\
\text { charts; (very } \\
\text { important in a } \\
\text { chronic illness)' }\end{array}$} \\
\hline & $\begin{array}{l}\text { Useful for } \\
\text { education \& } \\
\text { training }\end{array}$ & $\begin{array}{l}41 \\
(89.1)\end{array}$ & $1(2.2)$ & $4(8.7)$ & $\begin{array}{l}46 \\
(100)\end{array}$ & \\
\hline & $\begin{array}{l}\text { Helps get } \\
\text { investigations }\end{array}$ & $\begin{array}{l}28 \\
(60.9)\end{array}$ & $\begin{array}{l}10 \\
(21.7)\end{array}$ & $9(19.6)$ & $\begin{array}{l}46 \\
(100)\end{array}$ & \\
\hline & $\begin{array}{l}\text { Helps get } \\
\text { treatment }\end{array}$ & $\begin{array}{l}31 \\
(67.4)\end{array}$ & $\begin{array}{l}10 \\
(21.7)\end{array}$ & $5(10.9)$ & $\begin{array}{l}46 \\
(100)\end{array}$ & \\
\hline \multirow[t]{4}{*}{ Experience } & $\begin{array}{l}\text { Contains } \\
\text { investigations } \\
\text { that cannot } \\
\text { access in my } \\
\text { hospital }\end{array}$ & $\begin{array}{l}8 \\
(17.4)\end{array}$ & $\begin{array}{l}35 \\
(76.1)\end{array}$ & $3(6.5)$ & $\begin{array}{l}46 \\
(100)\end{array}$ & \multirow{4}{*}{$\begin{array}{l}\text { - 'Cannot access } \\
\text { TNF blockers' } \\
\text { - 'Do not go far } \\
\text { enough to help } \\
\text { specialists' } \\
\text { - - 'Revision should } \\
\text { be done on a } \\
\text { regular basis (2-5 } \\
\text { yearly) as for all } \\
\text { other } \\
\text { recommendations/ } \\
\text { guidelines. JDM } \\
\text { does not need it } \\
\text { yet.' }\end{array}$} \\
\hline & $\begin{array}{l}\text { Contains } \\
\text { treatments } \\
\text { that cannot } \\
\text { get in my } \\
\text { hospital }\end{array}$ & $\begin{array}{l}4 \\
(8.9 \%)\end{array}$ & $\begin{array}{l}36 \\
(80)\end{array}$ & $5(11.1)$ & $\begin{array}{l}45 \\
(97.8)\end{array}$ & \\
\hline & $\begin{array}{l}\text { Needs to be } \\
\text { revised \& } \\
\text { updated }\end{array}$ & $\begin{array}{l}21 \\
(46.7)\end{array}$ & $\begin{array}{l}16 \\
(35.56)\end{array}$ & $8(17.8)$ & $\begin{array}{l}45 \\
(97.8)\end{array}$ & \\
\hline & $\begin{array}{l}\text { Relevant to } \\
\text { my practice as } \\
\text { it is }\end{array}$ & $\begin{array}{l}33 \\
(73.3)\end{array}$ & $\begin{array}{l}5 \\
(11.11)\end{array}$ & $7(5.6)$ & $\begin{array}{l}45 \\
(97.8)\end{array}$ & \\
\hline
\end{tabular}

Conclusion: According to this survey, SHARE JDM guideline has been implemented widely. Despite not necessarily changing practice, the majority of respondents rate it as important, clear/easy to use and helpful for investigations/treatment. A small number of respondents cannot access investigations/treatments within the guidance, determined by local/national factors. Almost half of all respondents thought the guideline should be updated to include biomarkers, myositis antibodies, new treatments, resistant disease and mental health.

Disclosure of Interest

None declared 
P160

Clinical features, muscle biopsy scores, myositis specific antibody profiles and outcome of JDM patients

E. Sag ${ }^{1}$, S. Demir', Y. Bilginer', B. Talim², S. Ozen

${ }^{1}$ Pediatric Rheumatology; ${ }^{2}$ Pediatric Pathology Unit, Hacettepe

University, Ankara, Turkey

Correspondence: E. Sag

Pediatric Rheumatology 2020, 18(Suppl 2):P160

Introduction: Juvenile dermatomyositis (JDM) is the most common inflammatory myopathy of childhood.

Objectives: We aimed to analyse the clinical features, clinical implications of muscle biopsy scores and myositis specific antibodies (MSA), treatment responses and long-term outcomes of our JDM patients.

Methods: JDM patients followed at Hacettepe University, Department of Pediatric Rheumatology between 2000-2020 were included. Patients data were collected retrospectively from patient files.

Results: Fifty-eight $(60.3 \%$ F) JDM patients were included with a mean age of onset $8.1 \pm 4.3$ years. The mean follow-up period was $5.66 \pm 3.59$ years. The classical rash $(91 \%$; heliotrope rash and/or Gottron's papules) and muscle weakness (76\%) were the most common presenting features. Electromyography was positive in $23 / 25$ patients and muscle MRI revealed myositis in 12/15 patients. 35 patients had muscle biopsy and 16 of them were scored according to the score tool $^{1,2}$ with a mean total biopsy score of $18.5 \pm 5.7$ ( $\max 27$ ). Overexpression of MHC-I (94\%) was the most prominent feature followed by inflammatory cell infiltration $(78 \%)$ and perifascicular atrophy $(72 \%)$. Elevated creatine kinase levels were seen in $86 \%$ of the patients, ANA and ENA were positive in $77 \%$ and $13 \%$ of them respectively. 76\% (34/46) patients had MSA/MAA. The most common MSA was NXP2 (21.7\%) followed by TIF1-g (17.4\%), MDA-5 (8.7\%) and Mi-2 (8.7\%). Muscle involvement was less prominent in MDA-5 positive patients. TIF-1g and NXP2 positive patients had a severe course similar to previous cohorts; MDA- 5 positive patients had a milder disease course with only $25 \%$ of them having pulmonary involvement and Ku positive patients had a remarkably more severe course in contrast to previous studies. ${ }^{3-5}$ Remission rates did not differ but $43.9 \%$ of NXP2 positive and $33.3 \%$ of TIF-1g positive patients had a relapse. Corticosteroids (100\%) combined with methotrexate $(93 \%)$ was the initial treatment, hydroxychloroquine $(47 \%)$, cyclosporin-A (40\%), IVIG (34\%), azathioprine (14\%), cyclophosphamide (14\%) and pamidronate (10\%) were also used. Biological DMARDs (anti-TNFs, rituximab and abatacept) were used in $22 \%$ of the patients. Remission was achieved in $65.5 \%$ of the patients in a median 24 (IQR 11.8-42.5) months however $26.3 \%$ had a relapse. Overall disease course was monophasic in $31 \%$, polyphasic in $17.2 \%$ and chronic in $51.8 \%$ of the patients.

Calcinosis (36\%) was the most common long-term complication. The factors associated with the development of calcinosis were disease onset $\leq 6$ years, higher muscle biopsy scores, MDA-5, TIF- $1 \mathrm{~g}$ and NXP2 positivity. In a multivariate analysis, the disease onset $\leq 6$ years of age [5.3 (1.16-24.33) $\mathrm{p}=0.031]$ and MDA-5 positivity [42.4 (1.511190) $p=0.028$ ] were the predictive factors for the development of calcinosis during the disease course.

Conclusion: Recent advances on muscle biopsy scores, muscle imaging with MRI and myositis specific antibodies may provide us valuable informations for the diagnosis, disease course and prognosis of JDM.

\footnotetext{
References

1. Varsani H, et al. Ann Rheum Dis, 2015. 74(1): p. 204-10

2. Wedderburn LR, et al. Arthritis Rheum. 2007;57(7):1192-1201.

3. Tansley SL, et al. Arthritis Res Ther. 2014;16(4):R138.

4. Tansley SL, et al. Rheumatology (Oxford). 2014:53(12): p. 2204-2208.

5. Tansley SL,et al. J Autoimmun. 2017;84:55-64.
}

Disclosure of Interest

None declared

P161

Correlation of serum neopterin levels with disease activity and treatment in juvenile dermatomyositis (JDM).

R. Nicolai' ', I. Caiello', D. Pires Marafon', S. Rosina², L. Bracci Laudiero', F. Licciardi $^{3}$, A. Ravelli ${ }^{4}$, F. De Benedetti ${ }^{1}$, G. M. Moneta ${ }^{1}$

'Division of Rheumatology, IRCCS, Ospedale Pediatrico Bambino Gesù, Roma; ${ }^{2}$ University of Genova, Istituto Giannina Gaslini, IRCCS, Genoa, Italy, Genova; ${ }^{3}$ Division of Pediatric Immunology and Rheumatology, Regina Margherita Children Hospital, Torino; ${ }^{4}$ University of Genova,

Istituto Giannina Gaslini, IRCCS, Genova, Italy

Correspondence: R. Nicolai

Pediatric Rheumatology 2020, 18(Suppl 2):P161

Introduction: Activation of the type 1 and type 2 interferon (IFN) pathways seems to play a prominent role in the pathogenesis of dermatomyositis. Neopterin is a marker of immune activation induced by IFNY stimulation. Previous studies showed that serum neopterin levels are elevated in JDM at diagnosis and correlate strongly with muscle strength impairment.

Objectives: In this study we aimed to investigate neopterin levels in peripheral blood of JDM patients and assess their correlation with clinical and laboratory findings during follow-up.

Methods: We collected 276 blood samples from 55 JDM patients at different time points during follow-up. In 16 patients the first blood sample was obtained at diagnosis before starting treatment. Serum neopterin levels were analyzed by ELISA. We used sera from 19 healthy subjects as controls (HC). At each visit, the following data were recorded: physician's global assessment (PGA) of disease activity VAS (Visual Analogue Scale); cutaneous VAS, Cutaneous Assessment Tool (CAT) activity score; Childhood Myositis Assessment Score (CMAS); serum levels of creatine kinase (CK), AST, ALT and LDH; presence of myositis specific or myositis associated antibodies (MSA/MAA); prednisone (or equivalent) dose $(\mathrm{mg} / \mathrm{kg} /$ daily); ongoing immunosuppressive medications. Disease remission was defined according to modified Paediatric Rheumatology International Trials Organisation (PRINTO) criteria for clinically inactive disease. According to their disease status and treatment, patients were divided into 4 groups: patients with active disease not receiving medication $(A)$, patients with active disease receiving medication (AM), patients in remission receiving medication ( $\mathrm{RM})$ and patients in remission not receiving medication (R).

Results: Serum neopterin levels are significantly higher in JDM patients with active disease (both $A$ and AM group) when compared to $H C(P<0.0001, P<0.0001$, respectively), with active patients not receiving medication showing the highest levels $(P<$ 0.0001 when compared to the AM group). Patients of the RM group have neopterin levels that are comparable to $\mathrm{HC}$, whereas patients in in the R group tend to have higher levels than $H C(P<$ 0.01). Neopterin levels are highest in patients at time of diagnosis and decrease under treatment. Serum neopterin levels are significantly correlated with PGA, cutaneous VAS and CAT activity score (Spearman's rank coefficient ( $(r): 0.5,0.36$ and 0.39 respectively, $\mathrm{P}<0.0001, \mathrm{P}<0.0001$ and $\mathrm{P}<0.0001$ respectively) and inversely correlated with CMAS ( $\mathrm{r}:-0.35, \mathrm{P}<0.0001)$.

Conclusion: Neopterin levels are significantly correlated with global, cutaneous and muscular disease activity in JDM patients and are influenced by treatment. Our data support the role of neopterin as a biomarker for disease activity in JDM. 


\section{Disclosure of Interest}

R. Nicolai: None declared, I. Caiello: None declared, D. Pires Marafon: None declared, S. Rosina: None declared, L. Bracci Laudiero: None declared, F. Licciardi: None declared, A. Ravelli: None declared, F. De Benedetti Consultant for: Dr. De Benedetti's Institution received unrestricted research grants from BMS, Pfizer, Abbvie, Novartis, Novimmune, Roche, SOBI, Sanofi, UBC and travel support from Roche, Novartis, Novimmune, SOBI., G. M. Moneta: None declared

\section{P162}

Investigating novel mechanisms of T cells in the pathogenesis of juvenile dermatomyositis

L. R. Marshall ${ }^{1}$, C. T. Deakin 1,2,3 E. C. Rosser ${ }^{1,2}$, D. Eleftheriou', L. R.

Wedderburn ${ }^{1,2,3}$, on behalf of the UK Juvenile Dermatomyositis Research Group

${ }^{1}$ III, UCL Great Ormond Street Institute of Child Health; ${ }^{2}$ Centre for Adolescent Rheumatology Versus Arthritis at UCL, UCLH and GOSH; ${ }^{3} \mathrm{NIHR}$ Biomedical Research Centre at GOSH, London, United Kingdom Correspondence: $L$. R. Marshall

Pediatric Rheumatology 2020, 18(Suppl 2):P162

Introduction: Juvenile Dermatomyositis (JDM) is a rare autoimmune disease causing skin and muscle inflammation with an average onset of 7 years old. At present, JDM aetiology is poorly understood and current treatment options are not evidence based. This highlights the need for research investigating underlying disease pathogenesis. A skewed T helper (Th)17 phenotype in CD4+ T cells resulting in a Th1/17 imbalance has been observed in both child and adult-onset immune-mediated diseases including rheumatoid arthritis, and multiple sclerosis.

Objectives: The aim of this project is to investigate whether a Th1/17 imbalance can be observed in patients with JDM compared to age/ sex-matched child healthy controls $(\mathrm{CHC})$.

Methods: Expression of IL-17 and IFN- $\gamma$ in CD4+ T cells within peripheral blood mononuclear cells (PBMC) from JDM pre-treatment $(n=7)$, JDM on-treatment $(n=28)$ and CHC $(n=22)$ was assessed by flow cytometry after stimulation with PMA/lonomycin/Brefeldin A (P///B) for 4 hours. For secreted cytokine production, isolated $C D 4+T$ cells were isolated by magnetic separation and stimulated with anti-CD3 or antiCD3/anti-CD28 for 36 hours in the presence of IL-2. Supernatants were analysed for secreted IL-17 and IFN- $\gamma$ and measured by cytokine bead array. In parallel, extracellular Th1 (CD3+CD4+CXCR3+CCR6-), Th17 (CD3+CD4+CXCR3-CCR6+) and Treg (CD3+CD4+CD127-CD25hi) subsetting was carried out using flow cytometry and proliferative capacity of $T$ cells was assessed following stimulation by intra-nuclear staining for Ki67 protein. Finally, fluorescence co-staining of JDM muscle biopsies $(n=4)$ to identify Th1 cells $(C D 3+C D 4+I F N+)$ was performed to assess possible Th1 migration to the primary disease site.

Results: Both intracellular cytokine staining and stimulation experiments to assess secreted cytokine revealed a decreased trend of IFN-pproduction in JDM compared to CHC within CD4+ T cells, regardless of treatment status. The JDM CD4+ T cell phenotype was significantly skewed towards a Th17 phenotype $(p=<0.0016)$ compared to controls after ratio analysis of CD4+IFN- $\gamma+$ to CD4+IL-17+ cells within peripheral blood after PMA/lonomycin stimulation. A Th17 skew was confirmed when analysing surface markers for Th1 (CXCR3) and Th17 (CCR6) on JDM pre-treatment-treatment CD4+ T cells compared to controls $(p=0.0001)$. Central and Effector Memory compartments within CD4+ $T$ cells were reduced in JDM pretreatment patients compared to controls $(p=0.02, p=<0.001$ respectively). Co-staining of CD3+CD4+IFN- $\gamma+$ cells in JDM muscle were observed however a higher proportion of CD3-CD4+IFN- $\gamma+$ in JDM pretreatment patients were also identified.

Conclusion: These novel findings show a Th1/17 imbalance in JDM CD4+ T cells compared to CHC. Whilst results show promising avenues for further investigation there are no definitive explanations for this low Th1 response at present. Future work aims to investigate memory and naive compartments within JDM pre-treatment CD4+ T cells in addition to further testing of cell markers within the muscle.
Additionally, other immune and metabolic pathways that may explain this Th17 skew could be targeted to restore IFN- $\gamma$ loss in JDM patients.

\section{Disclosure of Interest}

None declared

\section{P163}

An unusual case of macrophage activation syndrome, complicated by thrombotic microangiopathy, with clinical response to IL-1 blockade

K. Mclellan', I. Shaheen², P. French³, V. Harkins'², N. Martin

${ }^{1}$ Rheumatology; ${ }^{2}$ Renal; ${ }^{3}$ Pathology, Royal Hospital for Children, Glasgow, United Kingdom

Correspondence: $\mathrm{K}$. Mclellan

Pediatric Rheumatology 2020, 18(Suppl 2):P163

Introduction: Macrophage activation syndrome (MAS) with associated thrombotic microangiopathy(TMA) is rare, but has been reported ${ }^{1}$. We present an adolescent with features of MAS and renal impairment with TMA on renal biopsy. Despite partial steroid response, he remained steroid-dependent until addition of an IL-1 blocker with rapid clinical and biochemical improvement.

Objectives: To report this case of an adolescent with MAS and TMA and describe use of IL-1 blockade in his management.

Methods: Retrospective case review

Results: This previously well 14 year old boy presented with 5 weeks malaise, lethargy, poor appetite and 2 weeks dyspnoea and productive cough. There was no history of fever or rash. Similar to other family members, he had a vomiting illness 5 weeks earlier.

$\mathrm{He}$ was tachycardic and tachypnoeic, with pleural effusions and hepatosplenomegaly. He had significant renal impairment (urea $18 \mathrm{mmol} / \mathrm{L}$, creatinine $124 \mu \mathrm{mol} / \mathrm{L})$, anaemia $(\mathrm{Hb} 10.7 \mathrm{~g} / \mathrm{dL})$, thrombocytopenia (platelets $75 \times 10^{9} / \mathrm{L}$ ), raised inflammatory markers (CRP 99mg/L, ESR $80 \mathrm{~mm} / \mathrm{hr}$, ferritin $1653 \mathrm{ng} / \mathrm{mL}$ ), albumin 19g/L. He received IV co-amoxiclav and albumin/frusemide infusions.

He continued to deteriorate; remaining febrile, hypertensive and oliguric, with worsening peripheral oedema, ascites and pleural effusions, requiring oxygen. Echocardiogram and MRI showed pericardial effusion, with thickened pericardium. Non-specific dural thickening identified on MRI brain. Extensive bacterial and viral investigations were negative. Bone marrow identified increased myeloid series with no haemophagocytosis. ANA, ANCA, ADAMTS13 and complement levels (CH100/AP100/terminal complement complex) were normal. Perforin and SAP(XLP1) expression were normal. XIAP(XLP2) expression was reduced, but with no pathogenic variant on sequence analysis XLP type 2 was excluded. No autoinflammatory diagnosis found on genetic testing. VIP3 panel ${ }^{2}$ identified class 3 variants of unknown significance in IFIH1, LACC1, NLRP6, HTR1A, SCN9A, TGFBR2 and NOTCH3.

On day 9 he was given $600 \mathrm{mg} / \mathrm{m}^{2}$ methylprednisolone followed by oral steroids. High dose steroids resulted in transient biochemical improvements, but this was not sustained, requiring further IV doses. Chest drains removed high volume inflammatory exudate. Renal biopsy on day 20 showed TMA; abnormal glomeruli, increased mesangial and endocapillary cellularity, fragmented red blood cells within the mesangium, with mesangiolysis and fibrin thrombus. Full body MRI and PET excluded malignancy. MMF, introduced on day 48 , was stopped due to worsening cytopenia. IVIG had no apparent effect. Anakinra, started on day 60 , had dramatic benefit clinically and biochemically. He reported rapid subjective improvement, renal function and ferritin levels improved and diuretics were stopped. He was subsequently managed as an outpatient; anti-hypertensives and oral steroids slowly weaned and stopped. 18 months later he had a mild recurrence with an intercurrent infection (ferritin 780ng/ $\mathrm{mL}$ ), which responded to IV methylprednisolone and twice daily anakinra. He responded clinically and biochemically and has remained well since. 
Conclusion: The association between MAS and TMA has recently been recognised ${ }^{1}$. Our case showed clear response to high-dose steroids and achieved sustained remission with IL-1 blockade. This could be a useful therapeutic option for future patients presenting with features of both MAS and TMA.

\section{References}

1. Minoia $F$ et al A multinational study of thrombotic microangiopathy in macrophage syndrome. Ann Rheum Dis 2019;978

2. Omoyinmi $E$ et al Clinical impact of a targeted next-generation sequencing gene panel for autoinflammation and vasculitis. PLUS ONE doi.org/ 10.1371/journal.pone.0181874

Disclosure of Interest

None declared

\section{P164}

Macrophage activation syndrome is a preventable life-threatening end point syndrome

D. B. Pandya, on behalf of Dr Jay Virani, Haresh Dobariya, Ambrish Panara, Nirav Karmta, Sudhir Rughani, Hitesh Bhambhani and Vishal Kalaria

Paediatric Rheumatology \& Immunology, Dev Children's Hospital, Rajkot, India

Correspondence: D. B. Pandya

Pediatric Rheumatology 2020, 18(Suppl 2):P164

\section{Introduction:}

Macrophage activation syndrome(MAS) is still underrecognized perplexed syndrome and usually results in delayed in diagnosis, which leads to high morbidity and mortality. ${ }^{1}$

\section{Objectives:}

To highlight frequent clinical and laboratory clues in MAS irrespective of its etiology for an early diagnosis

\section{Methods:}

I gathered a data of nine patients who were presented to Dev Children's Hospital as MAS or impending MAS between August 2018 and March 2020. I compared all available clinical and laboratory data of these nine patients in context of HLH-2004 protocol $^{2}$, Modified $2009 \mathrm{HLH}$ criteria $^{3}, 2014 \mathrm{H}$ score for reactive $\mathrm{HLH}^{4}, 2016$ sJIA-MAS diagnostic criteria ${ }^{5}$ and 2019 Ferritin:ESR Ratio $^{6}$.

Results: Table 1

\section{Conclusion:}

Most frequent early clinical findings in MAS are high grade fever , rash, hepatosplenomegaly and subtle CNS features irrespective of its etiology. More common changes in laboratory results are decreasing trend in $\mathrm{CBC}$, increasing trend in ferritin:ESR ratio, triglycerides, $\mathrm{LDH}$ and SGPT rather than an actual value. We can use these findings in a very large cohort to progress towards a new universal weightage based specific classification criteria for MAS irrespective of its etiology. It is very important to note that just because of an early diagnosis, all these patients responded to oral or IV steroids dramatically without any further requirement for more toxic immunosuppressive agents.

Trial registration identifying number:

1. Open access rheumatol 2018 aug 31;10:117-128. Doi: 10.2147/ oarrr.s151013. Ecollection 2018.

Macrophage activation syndrome: early diagnosis is key

butsabong lerkvaleekul ${ }^{1}$, soamarat vilaiyuk ${ }^{1}$

2. Pediatr blood cancer. 2007 feb;48(2):124-31. Doi: 10.1002/ pbc.21039.

HIh-2004: diagnostic and therapeutic guidelines for hemophagocytic lymphohistiocytosis

jan-inge henter 1, annacarin horne, maurizio aricó, $r$ maarten egeler, alexandra $\mathrm{h}$ filipovich, shinsaku imashuku, stephan ladisch, ken mcclain, david webb, jacek winiarski, gritta janka
3. Hematology am soc hematol educ program (2009) 2009 (1): 127-

131. Https://doi.org/10.1182/asheducation-2009.1.127

Hemophagocytic lymphohistiocytosis (hlh) and related disorders alexandra h. Filipovich

4. Arthritis rheumatol 2014 sep;66(9):2613-20. Doi: 10.1002/art.38690.

Development and validation of the hscore, a score for the diagnosis of reactive hemophagocytic syndrome

laurence fardet 1, lionel galicier, olivier lambotte, christophe marzac, cedric aumont, doumit chahwan, paul coppo, gilles hejblum

5. Arthritis \& rheumatology doi 10.1002 /art.39332 vc 2016, american college of rheumatology

Special article 2016 classification criteria for macrophage activation syndrome complicating systemic juvenile idiopathic arthritis a european league against rheumatism/american college of rheumatology/ paediatric rheumatology international trials organisation collaborative initiative angelo ravelli,1 francesca minoia,2 sergio davı,2 annacarin horne, 3 francesca bovis, 2 angela pistorio, 2 maurizio arico, 4 tadej avcin, 5 edward $\mathrm{m}$. Behrens, 6 fabrizio de benedetti,7 lisa filipovic,8 alexei a. Grom, 8 janinge henter,3 norman t. llowite, 9 michael b. Jordan, 8 raju khubchandani,10 paediatric rheumatology international trials organisation, the childhood arthritis and rheumatology research alliance, the pediatric rheumatology collaborative study group, and the histiocyte society 6. ACR brief report open access first published:13 july 2019 https:// doi.org/10.1002/acr2.11048

Ferritin to erythrocyte sedimentation rate ratio: simple measure to identify macrophage activation syndrome in systemic juvenile idiopathic arthritis. Esraa m. A. Eloseily md, mrcpch ,francesca minoia $\mathrm{md}$, courtney b. Crayne $\mathrm{md}$, $\mathrm{msph}$ timothy beukelman $\mathrm{md}$, msce, angelo ravelli $\mathrm{md}$ randy q. Cron $\mathrm{md}$, phd

Disclosure of Interest

None declared

Table 1 (abstract P164). showed detailed analysis of clinical and laboratory data of our cohort in context of parameters taken from above mentioned criterias

\begin{tabular}{ll}
\hline Parameter & $\begin{array}{l}\text { N (\%) - Number of } \\
\text { Patients }\end{array}$ \\
\hline Fever & $9(100 \%)$ \\
Rash & $6(66.6) \%)$ \\
Bleeding manifestations & $2(22.2 \%)$ \\
Hepatomegaly & $6(66.6 \%)$ \\
Splenomegaly & $2(22.2 \%)$ \\
Lymphadenopathy & $3(33.3 \%)$ \\
CNS dysfunction & $4(44.4 \%)$ \\
HB $(<9$ gm\%) & $6(66.6 \%)$ \\
Neutrophils $<1 * 10^{9} / L$ & $2(22.2 \%)$ \\
Platelets $</=181^{*} 10^{9} / L$ & $6(66.6 \%)$ \\
Platelets $</=100^{*} 10^{9} / L$ & $1(11.1 \%)$ \\
Number of Cytopenias & \\
1 lineage & $6(66.6 \%)$ \\
2 lineage & $2(22.2 \%)$ \\
3 lineage & $1(11.1 \%)$ \\
SGPT > 48 U/L & $6(66.6 \%)$ \\
SGPT >30 & $7(77.7 \%)$ \\
Triglycerides (mg/dl) & \\
$>156 m g / d l$ & $6(66.6 \%)$
\end{tabular}


Table 1 (abstract P164). showed detailed analysis of clinical and laboratory data of our cohort in context of parameters taken from above mentioned criterias (Continued)

\begin{tabular}{|c|c|}
\hline Parameter & $\begin{array}{l}\mathrm{N}(\%) \text { - Number of } \\
\text { Patients }\end{array}$ \\
\hline$>132 \mathrm{mg} / \mathrm{dl}$ & $7(77.7 \%)$ \\
\hline$>265 \mathrm{mg} / \mathrm{dl}-$ & $2(22.2 \%)$ \\
\hline \multicolumn{2}{|l|}{ Fibrinogen (mg/dl) } \\
\hline$</=360$ & $7(77.7 \%)$ \\
\hline$</=250$ & $5(55.5 \%)$ \\
\hline$</=150$ & $2(22.2 \%)$ \\
\hline \multicolumn{2}{|l|}{ Ferritin level (ng/ml) } \\
\hline$>684-$ & $9(100 \%)$ \\
\hline$>500$ & $9(100 \%)$ \\
\hline$<2000$ & $2(22.2 \%)$ \\
\hline $2000-6000$ & $2(22.2 \%)$ \\
\hline$<6000$ & $5(55.5 \%)$ \\
\hline Increased Ferritin : ESR Ratio & $9(100 \%)$ \\
\hline Bone Marrow & $1(11.1 \%)$ \\
\hline \multicolumn{2}{|l|}{ Hemophagocytosis ${ }^{\lessgtr}$} \\
\hline LDH (>500IU/L) & 7 (77.7\%) \\
\hline Low Sodium level & $3(33.3 \%)$ \\
\hline Abnormal PT,aPTT & $3(33.3 \%)$ \\
\hline Elevated bilirubin & $1(11.1 \%) 3$ \\
\hline Decreased albumin & $3(33.3 \%)$ \\
\hline CRP $>40 \mathrm{mg} / \mathrm{L}$ & $6(66.6 \%)$ \\
\hline $\begin{array}{l}\text { Subtle progressive changes in laboratory parameters } \\
\text { rather than actual value }\end{array}$ & $9(100 \%)$ \\
\hline Responded to Oral /IV steroids & $9(100 \%)$ \\
\hline
\end{tabular}

$\$=$ Bone marrow examination was performed only in five patients NK cell function test and CD25 level was not done for any of these patients

\section{P165}

MAS/sJIA working party activities

F. Minoia', S. Vastert ${ }^{2}$, C. Kessel $^{3}$, C. Bracaglia ${ }^{4}$, on behalf of on behalf of the MAS/sJIA Working Party

'Pediatric Rheumatology, Fondazione IRCCS Ospedale Maggiore

Policlinico, Milan, Italy; ${ }^{2}$ Pediatric Rheumatology and Immunology, University Medical Center Utrecht, Utrecht, Netherlands; ${ }^{3}$ Department of Pediatric Rheumatology and Immunology, University Hospital Muenster, Muenster, Germany; ${ }^{4}$ Division of Rheumatology, IRCCS Ospedale

Pediatrico Bambino Gesù, Rome, Italy

Correspondence: C. Bracaglia

Pediatric Rheumatology 2020, 18(Suppl 2):P165

Introduction: Systemic Juvenile Idiopathic Arthritis (sJIA) is a unique form of childhood arthritis. According to current understanding $\mathrm{SJIA}$ is primarily driven by innate immune mechanisms at disease onset, but can progress towards chronic destructive arthritis, which can involve $\mathrm{T}$ cellular immunity. For yet incompletely understood reasons, SJIA can be complicated by Macrophage Activation Syndrome (MAS), a severe hyperinflammatory condition characterized by a catastrophic cytokine storm resulting in multiple organ failure and high mortality.

Objectives: The sJIA/MAS Working Party (WP) aims to promote knowledge and international multidisciplinary collaboration among experts in the field of MAS and SJIA and to foster translational research in order to improve the care and outcome of these patients Methods: Currently 60 PReS members participate to the MAS/sJIA WP. The WP arranges an annual meeting during the PReS Congress, open to all members activities. The MAS/sJIA WP core team frequently report about ongoing activities by email.
Results: Several studies are currently ongoing. A project aimed to establish and validate a risk score for MAS in SJIA patients using routine laboratory parameters of disease activity and severity has already completed the construction phase. Recently, building of a validation cohort comprising data form 182 patients from 10 paediatric rheumatologic centers has been accomplished and is awaiting analysis (Claudia Bracaglia). A second project focused on MAS patients with systemic thrombotic microangiopathy (TMA) has just completed the collection of 27 patients with MAS and TMA from 18 centers in 9 countries and results will soon be published (Francesca Minoia). Furthermore, the MAS/sJIA WP participated in the data collection phase of a project on the development of new criteria for primary HLH (Jan-Inge Henter and AnnaCarin Horne).

A main goal of the WP is to improve clinical care. Within the project Current treatment in sJIA and MAS/secondary hemophagocytic lymphohistiocytosis (sHLH): a PReS/PRINTO survey (Francesca Minoia and Sebastiaan Vastert) the initiators aim to better understand the real-life experience in $\mathrm{sJIA}$ and MAS/sHLH treatment in pediatric rheumatology centers. To foster the achievement of a uniform approach, pediatric hemato-oncologists and Intensive Care Unit physicians will be involved, to capture all the different settings in which MAS/sHLH patients may be treated. The project was proposed for the first PReS/PRINTO grant and was ranked second.

Among translational science activities, MAS/sJIA WP members (Sebastiaan Vastert and Claudia Bracaglia) aim to collect biosamples and clinical data from patients with treatment refractory/resistant sJIA, connecting existing large national sJIA cohorts and open for new patients from paediatric rheumatology centers throughout Europe/world (ReSyst study).

A second translational project focuses on the role of viral triggers of MAS and proposes to analyze type I IFN score and IL-18 expression in MAS and MAS risk patients to deepen understanding on type 1 interferons as critical regulators of IL-18 expression (Christoph Kessel).

Finally, the MAS/sJIA WP aims to organize a focused PReS academic course on SJIA and MAS in 2021.

Conclusion: At present, the MAS/sJIA WP is coordinating several clinical and translational research activities. Any proposal for new collaborative projects is highly appreciated.

Claudia Bracaglia, Chair

Francesca Minoia, Secretary and Lead of Clinical Care Pillar

Sebastiaan Vastert, Lead of Training\&Education Pillar

Christoph Kessel, Lead of Science\&Research Pillar

Disclosure of Interest

None declared

\section{P166}

Hemophagocytic lymphohistiocytosis in infancy: a case series

H. Bhaskaran, S. Balan

Department of Clinical Immunology and Rheumatology, Amrita Institute of Medical Sciences and Research Centre, Kochi, Ernakulam, India

Correspondence: $\mathrm{H}$. Bhaskaran

Pediatric Rheumatology 2020, 18(Suppl 2):P166

Introduction: Hemophagocytic lymphohistiocytosis (HLH) is an immunological disorder characterized by clinical signs and symptoms of severe uncontrolled inflammation, due to massive release of inflammatory cytokines. A delay in diagnosis is common, and is one of the factors that determine the poor outcome. $\mathrm{HLH}$ is classified into primary $(\mathrm{pH} L \mathrm{H})$ and secondary $(\mathrm{sH} L \mathrm{H})$. It is important to differentiate between the two as management differs.

Objectives: To describe the clinical and laboratory profile of HLH in infancy.

Methods: The electronic case files of children (age $<1$ year) diagnosed with HLH at the AIMS, Kochi, Kerala, between January 2012 and December 2019, was retrospectively reviewed and described.

Results: Eight infants, with age range 1.5 months to 7 months, were clinically diagnosed with HLH. All were immunised and had normal 
development for age. None had a family history suggestive of HLH. Third degree consanguinity was present in parents of patient no.5 and second degree for patient no.7. Duration of symptoms before presentation ranged from 2 days to 68 days. Duration of follow up with us ranged from 12 days to 192 days, for those who expired.

All, eight of them, had fever, anemia, thrombocytopenia, hyperferritinemia, transaminitis, raised LDH and CRP. Lymphadenopathy was present only in patient no.4. Before starting specific treatment patient no. 7 had Pseudomonas sepsis, patient no. 5 had Roseomonas gilardii infection; patient no. 3 and 4 were IgM CMV positive but their PCR was negative. Both of them had received prior blood transfusion.

Before making a definitive diagnosis of $\mathrm{HLH}$ patients were treated for PUO, sepsis ? cause and acute liver failure. There was a delay in diagnosis for all patients except patient no.7.

All of them were treated with HLH 2004 protocol with modification according to clinical status of the patient. Later, broad spectrum antibiotics, antifungals and antivirals were used for all. Anakinra was tried for patient no.5.

Five patients (pHLH) succumbed to sepsis and MODS and three (one pHLH and two SHLH) are continuing follow up. HSCT was not done in any of them. Other clinical features are shown in Table 1.

Conclusion: Making a timely diagnosis of $\mathrm{HLH}$ is difficult. Differentiating $\mathrm{pHLH}$ from $\mathrm{sHLH}$ is very important as the management differs. Genetic testing should be done for all infants with HLH. Negative genetic study doesn't rule out $\mathrm{pHLH}$. The only curative treatment for pHLH is HSCT. sHLH infants, once their primary condition is treated, can have normal survival. Hyperbilirubinemia, splenomegaly, neutropenia, hepatomegaly, tissue hemophagocytes and hypertriglyceridemia were more common in $\mathrm{pHLH}$.

Disclosure of Interest

None declared

Table 1 (abstract P166). Clinical and lab characteristics in infantile HLH

\begin{tabular}{|c|c|c|c|c|c|c|c|c|c|}
\hline$\overline{\text { No }}$ & rash & jaundice & $\begin{array}{l}\text { Spleno- } \\
\text { megaly }\end{array}$ & $\begin{array}{l}\begin{array}{l}\text { Hepato- } \\
\text { megaly }\end{array} \\
\text { - }\end{array}$ & $\begin{array}{l}\text { Neutropenia } \\
<1000 / \mathrm{mm}^{3}\end{array}$ & $\begin{array}{l}\text { Triglyceride } \\
>265 \mathrm{mg} / \mathrm{dl}\end{array}$ & $\begin{array}{l}\text { Fibrinogen } \\
<150 \text { g/dl }\end{array}$ & $\begin{array}{l}\text { Hemo- } \\
\text { phagocyte }\end{array}$ & $\begin{array}{l}\text { Mutated } \\
\text { gene }\end{array}$ \\
\hline$\overline{i^{* \#}}$ & 7 & 1 & 1 & 1 & 0 & 1 & 1 & 1 & Neg \\
\hline $2^{*}$ & 1 & 1 & 0 & 1 & 1 & 1 & 1 & 0 & $\mathrm{Neg}$ \\
\hline $3^{* \#}$ & 0 & 1 & 0 & 1 & 0 & 1 & 1 & 1 & $\mathrm{Nd}$ \\
\hline $4^{*}$ & 0 & 0 & 1 & 1 & 1 & 0 & 0 & 1 & PRF1 \\
\hline $5^{* \#}$ & 1 & 1 & 1 & 1 & 1 & 1 & 0 & 0 & PRF1 \\
\hline 6 & 1 & 0 & 1 & 1 & 1 & 1 & 0 & 1 & UNC13D \\
\hline 7\# & 1 & 0 & 0 & 1 & 0 & 0 & 0 & 0 & $\mathrm{Nd}$ \\
\hline 8 & 1 & 0 & 0 & 0 & 0 & 1 & 1 & 0 & $\mathrm{Neg}$ \\
\hline
\end{tabular}

P167

Macrophage activation syndrome: 12 years data from an indian center

P. Pal, J. Bathia, P. P. Giri

Pediatric Rheumatology, Institute of Child Health Kolkata, Kolkata

700017, India

Correspondence: P. Pal

Pediatric Rheumatology 2020, 18(Suppl 2):P167

Introduction: Macrophage Activation Syndrome (MAS) is a dreaded complication of systemic inflammatory diseases and is most commonly seen in systemic onset juvenile idiopathic arthritis (sJA). Data of patients admitted in the Department of Pediatric Rheumatology, Institute of Child
Health, Kolkata diagnosed as having MAS, admitted between July 2008 and April 2020 was tabulated and retrospectively analyzed .

Objectives: To evaluate the clinical features, laboratory findings and outcomes in pediatric MAS, assess the response to different pharmacological therapies, and finally to identify possible factors associated with an unfavourable outcome.

Methods: The data of patients diagnosed with MAS over the study period was analyzed for the clinical and laboratory features, treatment details, response to therapy and outcome.

Results: 35 patients were diagnosed as having MAS. Primary illness was SJIA in $29(82 \%)$, SLE in $5(14 \%)$ and Kawasaki Disease (KD) in $1(4 \%)$. All had fever with varying degrees of multi systemic involvement. Hyperferritinemia was universally present. In the absence of Anakinra in India, pulse methylprednisolone with Cyclosporine was used for treating the majority.10 patients (28.5\%) expired. Patients on biologics and steroids can present with a silent MAS which may be difficult to diagnose.

Conclusion: MAS is a near fatal complication with protean manifestations and multi organ dysfunction. Hyperferritinemia is characteristic, higher values being associated with increased mortality. Patients resistant to steroids and cyclosporine had a poor prognosis. Early recognition with aggresive management forms the backbone of a successful outcome as reflected by improved prognosis over successive years. Late presentations with multiorgan dysfunction are associated with the poorest outcomes.

Disclosure of Interest

None declared

P168

Life-threatening macrophage activation syndrome with fulminant myocarditis successfully rescued by high dose intravenous

\section{Anakinra}

A. Meneghel', G. Martini' ${ }^{1}$, A. Amigoni², A. Pettenazzo², M. Padalino³ , F.

Zulian ${ }^{1}$

'Department for Women and Child Health-University Hospital of Padua,

Pediatric Rheumatology Unit; ${ }^{2}$ Department for Women and Child

Health-University Hospital of Padua, Pediatric Intensive Care Unit;

${ }^{3}$ Department of Cardiac, Thoracic and Vascular Sciences, Pediatric and

Congenital Cardiac Surgery, Padua, Italy

Correspondence: A. Meneghel

Pediatric Rheumatology 2020, 18(Suppl 2):P168

Introduction: Macrophage activation syndrome (MAS) is a rare, potentially life-threatening complication of some rheumatologic diseases ${ }^{1}$.

Objectives: We report the case of a child with systemic onset Juvenile Idiopathic Arthritis (sJA) complicated by severe MAS and acute myocarditis, needing veno-arterial Extracorporeal Membrane Oxygenation (VA-ECMO), successfully rescued by high dose intravenous Anakinra (HDIV-ANA).

Methods: Case report's description

Results: A two-year-old boy presented with one month history of fever associated with limping gait, cervical lymphadenopathy and skin rash. Laboratory tests showed elevation of inflammatory markers and ferritin. By exclusion criteria, sJlA was diagnosed and steroid therapy started. After a soft tissue bacterial infection, fever relapsed and laboratory tests were consistent with MAS (day 1): $\mathrm{Hb} 8.5 \mathrm{~g} / \mathrm{dL}$, PLT 44000/mm3; FDP 1522 ug/L, CRP 100 mg/L, ferritin 2200 ug/L. High doses intravenous metilprednisolone and oral Cyclosporin $A$ (CSA) were started. On day 2 he presented a Systemic Capillary Leak Syndrome and acute myocarditis. He was admitted into the pediatric intensive care unit (PICU) where intravenous immunoglobulin and subcutaneous Anakinra (ANA) were added. On day 4, due to an 
episode of cardiac arrest, VA-ECMO was started and we tried high dose intravenous ANA (HDIV-ANA, $8 \mathrm{mg} / \mathrm{Kg} /$ day q6h). This treatment brought immediate benefit: echocardiography showed progressive resolution of myocarditis so that VA-ECMO was definitely weaned off in six days. Laboratory test showed isolated neutropenia (PMNs 0$100 / \mathrm{mm}^{3}$ ). Suspecting a iatrogenic cause, HDIV-ANA was gradually reduced to the maintenance dose without benefit. On day 22, ANA was stopped and neutropenia resolved. Analysis of PRF1 gene revealed a mutation (c.[272C $>$ T] p.[Ala91Val]) in heterozygosis. 49 days after admission he was discharged on oral prednisone and CSA. Neither neurological nor other organ consequences related to MAS were reported. A few months later, on tapering down of therapy, he relapsed. ANA was restarted with rapid improvement and no side effects, including neutropenia. Currently, after 12 months, the disease is in clinical remission on medication.

Conclusion: MAS is a rare life-threatening complication of sJA, triggered by infections in up to one-third of the patients ${ }^{2}$. It is the result of a cytokine storm that lead to a dysregulated inflammatory activation of the immune system, with rapid progression to multiorgan failure. Treatment usually includes high dose corticosteroids and immunosuppressive agents. Recently, the use of selective cytokine inhibitors has been suggested. No standardized guidelines are available to date, but the use of ANA has been already reported, pointing out the need for a higher doses regimen in refractory cases. MAS in our patient appeared after a soft tissue infection which could have act as triggering factor in a patient with sJIA and genetic predisposing pattern. The choice of intravenous administration of ANA was partly due to the generalized edema and partly to the severe discoaugulopathy. Considering the higher doses needed for rapidly suppressing the cytokine storm and ANA pharmacokinetics, we split the daily dose into four administrations. No major adverse events were reported, except for a transient neutropenia, already reported ${ }^{6}$.

Based on our experience, HDIV-ANA is a safe and effective treatment for refractory life-threatening SJIA-related MAS. This therapeutic approach may be also considered in the current pandemic COVID-19 emergency where recent evidence showed IL1-driven MAS-like complication triggered by SARS-COV-2 virus as predictor of bad outcome ${ }^{7}$.

Disclosure of Interest

None declared

\section{P169}

Pulmonary involvement as initial manifestation of pediatric

Sjogren

C. S. Santos ${ }^{1}$, C. Moriano Morales ${ }^{2}$, E. Diez Alvarez ${ }^{3}$, E. F. Bollo de Miguel ${ }^{2}$

${ }^{1}$ complejo asistencial universitario de leon, LEON; ${ }^{2}$ Complejo Asistencial Universitario de León, León; ${ }^{3}$ Complejo Asistencial Universitario tatio de León, LEON, Spain

Correspondence: $C$. S. Santos

Pediatric Rheumatology 2020, 18(Suppl 2):P169

Introduction: Sjögren's syndrome is a systemic autoimmune disease characterized by dry syndrome and lymphocytic infiltration of the exocrine and extraglandular glands. Pulmonary involvement in primary Sjögren's syndrome occurs in $9-20 \%$ of patients, with very heterogeneous manifestations, and occasionally as an initial manifestation'. Diffuse interstitial lung involvement is one of the most characteristic pulmonary manifestations and the most frequent subtypes in lung biopsy are interstitial lymphocytic pneumonia and nonspecific interstitial pneumonia 2 .

Objectives: 14-year-old girl presented to our hospital because of bilateral interstitial involvement with ground glass areas in lower lobes of both lungs on thorax and abdominal CT scan after for kidney stones follow-up. The patient had grade 1 mMRC dyspnoea and dry cough but denied having symptoms of arthralgia or arthritis, photosensitivity, oral and genital ulcers, Raynaud's phenomenon or episodes of dry mucosa. She had no history of autoimmune disease nor family antecedents of any autoimmune disease. A physical examination disclosed no finger clubbing or swollen superficial lymph nodes but indicated crackles on pulmonary auscultation. Laboratory work showed elevated acute phase reactants, positive rheumatoid factor, positive antinuclear antibodies (1/ 40), positive cytoplasmic antineutrophil antibodies (1/320) and lgG and IgA hypergammaglobulinemia. An examination for autoantibodies were negative for anti-SS-A, anti-SS-B, anti Jo-1, anticentromere and anti-scl-70 antibodies. lontophoresis with pilocarpine and 6-minute walk test was also normal. Pulmonary function tests demonstrated a mild restrictive impairment and a reduced percent diffusion capacity for carbon monoxide of $55 \%$. Fibreoptic bronchoscopy showed acute inflammation in bronchial mucosa. Flow cytometry of bronchoalveolar lavage and cytology showed lymphocytosis with a 15\% of CD4 and $85 \%$ of CD8 lymphocytes in bronchoalveolar lavage fluid. Finally, a transbronchial lung biopsy lead to a definitive diagnosis, showing mixed interstitial inflammation and lymphocytic follicular hyperplasia with formation of germinal centers, suggestive of a lymphoid interstitial pneumonia of unreleased autoimmune etiology. Throughout time, the patient reported progression of her symptoms with increasing dyspnoea, persistent dry cough, xerostomia and arthralgia. Schirmer and Rose Bengal dye test were negative, and a salivary gland biopsy showed interstitial plasmacytosis and no lgG4 plasma cells expression which suggested Sjogren's disease. A high resolution computerized axial tomography was requested, suggesting organizing pneumonia in the context of Sjogren's disease.

Methods: Several studies indicate that lung involvement in Sjögren is more frequent in advanced stages of the disease and rarely as an initial manifestation. Sjögren's syndrome in paediatric age is rare and the subtype of secondary Sjogren's is the most common. The course is longer, and the symptoms are more heterogeneous than in adulthood $^{5}$. The diagnosis in children is delayed, because children less frequently report dryness and frequently present with extraglandular clinical features suggestive of other autoimmune diseases. A systematic review on primary Sjögren's syndrome in male and paediatric population reported a $2.4 \%$ of pulmonary involvement in paediatric patients. ${ }^{6}$ Pulmonary involvement is associated with an increase in the mortality of patients with Sjögren's, therefore, it is essential to periodically monitor patients with respiratory symptoms, making an early diagnosis and treatment of the disease.

Results: -

Conclusion: We present a case of a patient with childhood Sjögren's disease with atypical onset of disease with lung involvement.

Disclosure of Interest

None declared

P170

A frontal bone osseous lesion: an unusual presentation of childhood sarcoidosis

S. Blackstock, S. Compeyrot-Lacassagne

Paediatric Rheumatology, Great Ormond Street Hospital, London, United Kingdom

Correspondence: S. Blackstock

Pediatric Rheumatology 2020, 18(Suppl 2):P170

Introduction: Sarcoidosis is a multi-system disorder. Little is known about its pathogenesis. In children, the early onset sarcoidosis phenotype including Blau syndrome is more often seen. ${ }^{1,2}$ The diagnosis of sarcoidosis is confirmed by demonstrating a typical non-caseating granuloma on a biopsy specimen. Other granulomatous diseases should be excluded, in particular mycobacterial infections, Crohn's disease and immunodeficiencies. The clinical presentation may vary depending on the organs involved and the age of the patient. ${ }^{3,4}$

Objectives: We are reporting the case of a boy with a presentation of bone sarcoidosis at a young age. This is a rare phenotype in children.

Methods: Clinical details were retrospectively collated using routine clinical records. Confirmation of diagnosis was confirmed with bone biopsy.

Results: A 5 year old non-identical twin boy of Ghanaian descent born in the UK had a slowly growing, painless frontal bone mass which started to develop from 7 months of age. He was developmentally normal, with no history of fever, rashes or joint pains.

Examination findings revealed frontal bossing while the remainder of the musculoskeletal examination was normal. There was no evidence of rashes, hepatosplenomegaly and ocular examination was normal. 
The patient was initially referred for neurosurgical review with suspected fibrous dysplasia, after an initial MRI scan of the head revealed abnormal marrow signal and expansion of the frontal bone, with no soft tissue swelling. However, the CT scan of the calvarium was not suggestive of fibrous dysplasia. Consequently, bone biopsy was performed demonstrating inflammation with granuloma formation. He was referred to Infectious Diseases and Rheumatology.

There was no travel history and no TB contact. QuantiFERON TB was negative. Infectious work-up was negative especially for mycobacterial infections.

Rheumatology work-up identified on skeletal survey another bone location: a well-defined lytic lesion in the right distal fibula that was biopsied. Infection cultures and PCR were negative. Histopathology identified fibrous tissue and poorly formed granulomas. Laboratory investigations revealed a mild microcytic anaemia with iron deficiency and eosinophilia. He had normal serum calcium and vitamin D and his ESR was $25 \mathrm{~mm} / \mathrm{hr}$. ANA, ANCA and rheumatoid factor were negative, and complement $\mathrm{C} 3$ and $\mathrm{C} 4$ were normal. His serum Angiotensin Converting Enzyme (ACE) level was raised at $125 \mathrm{nmol} /$ $\mathrm{ml} / \mathrm{min}$ (normal $<40 \mathrm{nmol} / \mathrm{ml} / \mathrm{min}$ ). Investigations revealed mild renal impairment with normal urinary tests including normal calcium, protein and tubular proteins. Ultrasound of the kidneys was normal. Chest X-ray was normal. Lung function was performed and was normal. DLCO couldn't be performed due to low lung volume.

Vascular and inflammation genetic panel identified a variant in the NEMO gene. Functional studies excluded NEMO deficiency and patient did not display any of the clinical features.

However, a pattern of dysregulated T cells response was identified.

He was treated with oral steroids and methotrexate. The oral steroids were successfully weaned off. He has been successfully treated with Methotrexate $10 \mathrm{mg} \mathrm{s} / \mathrm{c}$ to initially stabilise disease with no bone growth, and had no significant side effects.

Repeat MRI 2 years later showed increased burden of disease with other newly affected sites however, including the right femoral diaphysis and signal changes in the left tibial metaphysis.

Based on the MRI and increasing musculoskeletal pain, decision was made to escalate to anti-TNF (adalimumab) with good clinical response.

Conclusion: Bone sarcoidoisis is rare in children but this should be considered in the differential diagnoses when granulomatous inflammation is identified on histopathology. Response to steroids and methotrexate is usually good but some patients will need escalation to anti-TNF.

Disclosure of Interest

None declared

\section{P171}

Night pains in children can become a nightmare for rheumatologist

D. B. Pandya, on behalf of Dr Haresh Dobariya, Pritesh Rathod, Nishant

Dharsandiya, Sandip G. Mori, Vishal Kalaria, Vinod Bhadukiya

Pediatric Rheumatology and Immunology, Dev children's hospital,

Rajkot, India

Correspondence: D. B. Pandya

Pediatric Rheumatology 2020, 18(Suppl 2):P171

\section{Introduction}

The most worrisome non-rheumatic condition causing persistent night pain in children which closely mimics arthritis is malignancy ${ }^{1,4}$. It is vital to pick up subtle clues at an early stage especially in absence of hematological manifestations, organomegaly and lymphadenopathy.

Objectives

To reveal early clinical clues in pediatric patients with predominant musculoskeletal (MSK) night pains who were initially diagnosed as suffering from some form of chronic arthritis but ultimately turned out to be affected by malignancy.

Methods

I gathered a data of five pediatric patients fulfilling above mentioned criteria who were seen at Dev Children's Hospital between January 2019 and March 2020. It included demographics, clinical presentation and laboratory results.

\section{Results}

Conclusion

All above cases reemphasize the need for an extremely detailed history pertaining to characteristics of pain \& pattern recognition in pediatric rheumatology. Prolonged fever, persistent MSK night pain, persistent limp, upper limb and hip joint involvement which is unlikely for JIA at onset are proven to be the earliest subtle clues which should not be missed.1, 2, 3, 4 LDH has served as a reliable screening tool here. Most of these patients have been diagnosed without organomegaly, lymphadenopathy and major changes in $C B C$ at this stage.

Trial registration identifying number

1. CMAJ. 2001 Jul 24; 165(2):183-8.

Rheumatology: 16. Diagnosing musculoskeletal pain in children.

Malleson PN ${ }^{1}$, Beauchamp RD. PMID: 11501459 PMCID: PMC81287

2. Am Fam Physician. 2013 Aug 1; 88(3):185-192.

Chronic musculoskeletal pain in children

Ioanna Fragkandrea, Md, Phd, The Royal Marsden Hospital, Sutton, London, United Kingdom. John Alexander Nixon, Md, Epsom And St. Helier Nhs University Hospital, Sutton, London, United KingdomParaskevi Panagopoulou, Md, Mph, Phd, Panagiotis And Aglaia Kyriakou Children's Hospital, Athens, Greece

3. Am fam physician. 2006 jul 1; 74(1):115-122.

Chronic musculoskeletal pain in children: part i. Initial evaluation

Jennifer I. Junnila, m.d., m.p.h., army medical department center and school, san antonio, texas

Victoria w. Cartwright, m.d., m.s., madigan army medical center, tacoma, washington

4.Am fam physician. 2006 jul 15;74(2):293-300.

Chronic musculoskeletal pain in children: part ii. Rheumatic causes Jennifer I. Junnila, m.d., m.p.h., army medical department center and school, san antonio, texas

Victoria w. Cartwright, m.d., m.s., madigan army medical center, tacoma, washington

Disclosure of Interest

None declared

Table 1 (abstract P171). showed characteristics of five children with persistent MSK night pains

\begin{tabular}{|c|c|c|c|c|c|}
\hline $\begin{array}{l}\text { Age (years)/ } \\
\text { Sex }\end{array}$ & $7 / \mathrm{M}$ & $4 / F$ & $5 / F$ & $2.5 / \mathrm{M}$ & $2.5 / \mathrm{F}$ \\
\hline $\begin{array}{l}\text { Joint } \\
\text { involvement }\end{array}$ & Hip & $\begin{array}{l}\text { Hip } \\
\text { Elbow }\end{array}$ & $\begin{array}{l}\text { Elbow } \\
\text { Knee } \\
\text { Ankle }\end{array}$ & $\begin{array}{l}\text { Hip } \\
\text { Elbow }\end{array}$ & - \\
\hline MSK pain & $\begin{array}{l}\text { Thigh } \\
\text { Back }\end{array}$ & $\begin{array}{l}\text { Thigh } \\
\text { Calf }\end{array}$ & - & - & $\begin{array}{l}\text { ?Calf } \\
\text { ?Thigh }\end{array}$ \\
\hline Fever & + & + & + & - & + \\
\hline $\begin{array}{l}\text { Persistent } \\
\text { Limp }\end{array}$ & + & + & + & + & - \\
\hline Organomegaly & + & - & - & - & + \\
\hline Lymphadenopathy & + & - & - & - & + \\
\hline $\mathrm{HB}(\mathrm{gm} \%)$ & 9 & 6.7 & 8 & 8 & 6 \\
\hline TLC(/cumm) & 7560 & 9300 & 9800 & 6500 & 30,300 \\
\hline Platelet (/ul) & $1,52,000$ & $1,90,000$ & $3,21,000$ & $1,65,000$ & $7,22,000$ \\
\hline $\operatorname{ESR}(\mathrm{mm} / \mathrm{hr})$ & 42 & 46 & 81 & 45 & 53 \\
\hline $\mathrm{CRP}(\mathrm{mg} / \mathrm{L})$ & 56 & 35 & 65 & 34 & 125 \\
\hline $\mathrm{LDH}(\mathrm{IU} / \mathrm{L})$ & 708 & 300 & 321 & 456 & 461 \\
\hline $\begin{array}{l}\text { Final } \\
\text { Diagnosis }\end{array}$ & $\mathrm{NHL}$ & ALL & ALL & ALL & $\begin{array}{l}\text { Multisystem } \\
\mathrm{LCH}\end{array}$ \\
\hline
\end{tabular}

Abbreviations: MSK=musculoskeletal, $\mathrm{NHL}=$ non-hodgkin lymphoma , $\mathrm{ALL}=$ acute lymphoblastic leukemia, $\mathrm{LCH}=$ langerhans cell histiocytosis, $\mathrm{LDH}=$ lactate dehydrogenase 
P172

Complete remission of auricular relapsing polychondritis by treatment with adalimumab and methotrexate

M. Tsinti ${ }^{1}$, K. Stefanaki ${ }^{2}$, V. Dermentzoglou ${ }^{1}$, E. Tsitsami ${ }^{1}$

'Pediatric Rheumatology Unit, First Department of Pediatrics, School Of

Medicine, University Of Athens, Children's Hospital "Aghia Sofia";

2Department of Pathology, Children's Hospital "Aghia Sofia", Athens,

Greece

Correspondence: M. Tsinti

Pediatric Rheumatology 2020, 18(Suppl 2):P172

Introduction: Relapsing polychondritis (RP) is a rare disease with wide spectrum, ranging from isolated auricular chondritis, to life threatening cardiopulmonary manifestations. Even isolated auricular inflammation may result in significant morbidity due to collapse of the external ear cannal leading to conductive deafness and to significant deformity. On the other hand auricular chondritis may represent the inaugural symptom of a more aggressive, systemic disease. In children RP is rarer than in adults and often has a more severe course and more frequent involvement of the respiratory tract. Heart valve damage may develop silently with significant morbidity and mortality. Isolated auricular involvement may be complicated with sensorineural or conductive deafness and significant structural damage. First line treatment is corticosteroids. There are conflicting reports on the efficacy of DMARDs and TNFa inhibitors

Objectives: To report the clinical picture of isolated auricular relapsing polychondritis and the response to anti-TNFa treatment with adalimumab

Methods: Case presentation

Results: A 9-year-old girl was reported to the pediatric rheumatology unit by the ENT department due to 4 recurrent episodes of bilateral ear (more severe left) inflammation, with painful violaceous edema confined to the cartilaginous part of the ear, sparing the lobe during the past year. Nonspecific back pain was the only other symptom. Other constitutional symptoms, respiratory, cardiovascular, ophthalmologic or osteoarticular involvement were absent. Growth was unaffected. Auditory tests were normal. Systemic antibiotic treatment and local steroids were ineffective. Laboratory findings were unremarkable, with only mild elevation of ESR $\left(28 \mathrm{~mm} / 1^{\text {st }} \mathrm{hr}\right)$. ANA and ANCA were absent in repeat meausrements (3 months intervals). Cardiovascular disease was excluded. Abdominal US was normal. On the basis of relapsing bilateral auricular chondritis and confirmatory histological findings revealing inflamed cartilage from the pinna of the ear with chondrocyte degeneration, perichondrial infiltrates of lymphocytes, plasma and polymorphonuclear cells and replacement of cartilage with fibrous tissue perivascular infiltrates of polymorphonuclear cells and lymphocytes, relapsing polychondritis was diagnosed. One month NSAIDS trial, pending histology results was ineffective. Methotrexate SC and steroids $1 \mathrm{mg} / \mathrm{kg} / \mathrm{d}$ gradually tapered over a 3-month period were given with significant improvement of auricular inflammation and normalization of markers of inflammation. Auricular chondritis worsened after steroid withdrawal and adalimumab was added to treatment with significant improvement of auricular inflammation in 2 months. In the following 8 months auricular chondritis relapsed during URIs with mild elevation of ESR $(25 \mathrm{~mm} \mathrm{1st} \mathrm{hr)} \mathrm{and} \mathrm{CRP}(13 \mathrm{mg} / \mathrm{l})$. After 15 months of treatment, in an effort to prolong the intervals of adalimumab administration, bilateral auricular chondritis relapsed. After 24 months of MTX and 21 months of adalimumab administration inflammation was put in complete remission. The following year no flares or involvement of other systems were observed, under methotrexate and adalimumab treatment.

Conclusion: In this patient isolated auricular relapsing polychondritis was unresponsive to NSAIDs. Steroids and methotrexate greatly improved inflammation but did not induce complete remission. Complete remission was achieved by addition of adalimumab to methotrexate treatment, which also allowed for steroids discontinuation.

\section{Disclosure of Interest}

None declared
P173

First ever single center study revealing spectrum of rheumatic diseases in $\mathbf{1 1 4}$ children from an Indian State of Gujarat

D. B. Pandya, on behalf of Dr Mehul Mitra, Pankaj Buch, Sonal Shah, Haresh Dobariya, Jay Virani, Jay Dhirwani, Nishant Dharsandiya, Nayan Kalawadia, Hitesh Bhambhani, Rakesh Patel

Pediatric Rheumatology \& Immunology, Dev Children's Hospital, Rajkot, India

Correspondence: D. B. Pandya

Pediatric Rheumatology 2020, 18(Suppl 2):P173

\section{Introduction}

There is very limited information and awareness about pediatric rheumatic and immunodeficiency diseases amongst primary physicians $s^{1,2,3}$ in Gujarat and to make this matter even worse, we are not having a single exclusive pediatric rheumatology and immunology centre for a population of around 60 million.

Objectives

To guesstimate a status of children with rheumatic and immunodeficiency diseases in Gujarat and spectrum of these diseases at Dev Children's Hospital.

Methods

I gathered a retrospective data of 174 patients who attended Dev Children's Hospital between January 2019 and January 2020. Out of these, 114 children with confirmed diagnosis of inflammatory rheumatic diseases and suspected primary immunodeficiencies were included. Patients with non-inflammatory musculoskeletal(MSK) pains and non-rheumatic diseases causing MSK pains were excluded. My collected data included referral details, demographics, clinical presentation, laboratory results and diagnosis.

Results

Majority of the cases were referred by pediatricians, orthopedicians, hemato-oncologist and general physicians. Main reasons for referral were joint involvement , undiagnosed fever , multisystem disease and elevated inflammatory markers. Many physicians had put a diagnosis like rheumatoid/rheumatic arthritis, autoimmune disease or connective tissue disease. Almost $80 \%$ of patients had been evaluated with RF, ASO titer, ANA and joint imaging irrespective of clinical pattern by their primary physicians before referral. Fever, MSK involvement, extreme fatigue, constitutional symptoms, skin and mucosal involvement were prominent complaints noted by me. Family history of rheumatic, primary immunodeficiency (PID) or consanguinity was found in $1 / 3$ of patients. Anemia of chronic disease, elevated ESR and thrombocytosis were almost universal laboratory findings in our cohort.

Conclusion

Rheumatic diseases in children are not anymore rare but due to lack of expertise and awareness, these children are not getting diagnosed. Many cases were advised unnecessary rheumatological investigations even before referral. Prolonged fever, MSK involvement, skin manifestations, fatigue, constitutional symptoms and unexplained multisystem features with systemic inflammation mainly elevated ESR are found to be very sensitive features to suspect rheumatological problems. The frequency of various rheumatic conditions can be depicted as Infections $>$ Vasculitis $=\mathrm{JIA}$ $>$ CTDs > PID in our cohort.

Trial registration identifying number

1. Review.Indian J Pediatr2010 Sep;77(9):993-6.doi: 10.1007/s12098010-0134-x. Epub 2010 Sep 3.

The Place of Pediatric Rheumatology in India

Sujata Sawhney ${ }^{1}$, Prudence Manners

2. Journal of Natural science, Biology \& Medicine-2018

Clinico-epidemiological profile of pediatric rheumatology disorders in Eastern India PratapKumarPatra, ManishKumar

Department of Pediatrics, All Institute of Medical Sciences, Patna, Bihar, India

3. International Journal of Advanced Medical Health \& Research (JIPMER)

Pediatric rheumatology: An under-recognized subspecialty in India

Year : 2017| Volume : 4 | Issue : 2 | Page : 47-53

AkhilaKavirayani $^{1}$, SumaBalan ${ }^{2}$ 
1 Department of Paediatric Rheumatology, Oxford University Hospitals NHS Foundation Trust, Oxford, United Kingdom

2 Department of Paediatric Rheumatology, Amrita Institute of Medical Sciences, Kochi, Kerala, India

4. Research Article: Pediatric Rheumatology: Toward New Classification Criteria for Juvenile Idiopathic Arthritis: First Steps, Pediatric Rheumatology International Trials Organization International Consensus

Alberto Martini, Angelo Ravelli, Tadej Avcin, Michael W. Beresford, Ruben Burgos-Vargas, Ruben Cuttica, Norman T. Ilowite, Raju Khubchandani, Ronald M. Laxer, Daniel J. Lovell, Ross E. Petty, Carol A. Wallace, Nico M. Wulffraat, Angela Pistorio, Nicolino Ruperto and for the Pediatric Rheumatology International Trials Organization (PRINTO)

\section{Disclosure of Interest}

None declared

Table 1 (abstract P173). Showed demographics and spectrum of pediatric rheumatic diseases in our cohort

\begin{tabular}{|c|c|c|c|c|}
\hline $\begin{array}{l}\text { Demographics } \\
\mathrm{N}(\%)\end{array}$ & $\begin{array}{l}\text { Age <5 } \\
\text { years } \\
48(42 \%)\end{array}$ & $\begin{array}{l}\text { Age 5-10 } \\
\text { years } \\
39(34 \%)\end{array}$ & $\begin{array}{l}\text { Age }>10 \\
\text { years } \\
27(24 \%)\end{array}$ & $\begin{array}{l}\text { Sex } \\
F: 61(53.5 \%) \\
\text { M:53(46.5\%) }\end{array}$ \\
\hline $\begin{array}{l}\text { Group of } \\
\text { Conditions } \\
N(\%)\end{array}$ & $\begin{array}{l}\frac{\text { Vasculitis }}{27(24 \%)} \\
\text { Kawasaki } \\
\text { Disease > } \\
\text { lgA- } \\
\text { Vasculitis } \\
\frac{\text { Infections }}{29(25 \%)} \\
\text { Brucella > } \\
\text { PSRA > } \\
\text { MRSA } \\
>\text { ARF > TB } \\
\text { arthritis } \\
=\text { ReA }\end{array}$ & $\begin{array}{l}\frac{\text { Juvenile }}{\text { Idiopathic }} \\
\frac{\text { Arthritis }}{(\text { JIA) 27 }} \\
\frac{(\mathbf{2 4 \% )}}{\text { RF +Ve JIA > }} \\
\text { SJIA > Early } \\
\text { onset } \\
\text { ANA+ve JIA } \\
>\text { ESRA } \\
\text { JIA>Other > } \\
\text { Unclassified } \\
\text { (as per } \\
\text { preliminary } \\
\text { PRINTO JIA } \\
\text { Criteria) }\end{array}$ & $\begin{array}{l}\frac{\text { Connective- }}{\text { tissue }} \\
\frac{\text { diseases }}{\text { (CTDs) }} \\
\frac{\mathbf{2 0 ( 1 7 . 5 \% )}}{\text { SLE }>} \\
\text { Overlap } \\
\text { syndromes > } \\
\text { MCTD > JDM } \\
>\text { systemic } \\
\text { sclerosis } \\
\text { >Inherited } \\
\text { CTDs }\end{array}$ & $\begin{array}{l}\frac{\text { Suspected Primary }}{\text { Immunodeficencies }} \\
\frac{\text { (PID) \& Auto }}{\text { inflammatory }} \\
\frac{\text { syndromes }}{11(9.5 \%)} \\
\frac{\text { Immune }}{\text { dysregulation }} \\
\text { (IPEX,APECED,HLH) > } \\
\text { Cryopyrinopathy > } \\
\text { CRMO > Bone } \\
\text { marrow failure > } \\
\text { cyclic neutropenia }\end{array}$ \\
\hline
\end{tabular}

Macrophage activation syndrome was seen to be associated with SJIA in 4 patients, SLE in 2 patients, PID in 1 patient and brucellosis in 2 patients (Abbreviations: PSRA=Post Streptococcal Reactive Arthritis,ARF=Acure Rheumatic Fever, ReA=Reactive Arthritis , ESRA= enthesitis-spondylitis related arthritis,APECED=Autoimmune-polyendocrinopathy-candidiasis-ectodermal dystrophy, IPEX=Immunodysregulation-polyendocrinopathy-enteropathy- $x$ linked)

\section{P174}

Parasites and arthritis: an unheard duo among children

S. Pellizzari ${ }^{1}$, E. Giacomelli ${ }^{1}$, A. Maguolo ${ }^{1}$, E. Tadiotto ${ }^{1}$, G. Melotti ${ }^{1}$, G.

Aiello', M. Maschio', F. Caldonazzi², S. Pieropan ${ }^{1}$

${ }^{1}$ Pediatrics, Azienda Ospedaliera Universitaria Integrata, Verona;

${ }^{2}$ Pediatrics, Ospedale di Rovereto, Rovereto (TN), Italy

Correspondence: S. Pellizzari

Pediatric Rheumatology 2020, 18(Suppl 2):P174

Introduction: Based on literature reports, arthritis is a rare manifestation of parasitic infestation and it occurs more frequently in adults with Giardia lamblia, Strongyloides stercoralis, Schistosoma mansoni and Toxocaridae infestations. Reports of this symptom in children are extremely rare. Enterobius vermicularis is the most common helmintic parasite in the Italian general population. It is usually asymptomatic or associated with mild and heterogeneous manifestations, including diffuse or perianal itching, restless sleeping, discomfort, gastrointestinal symptoms and arthralgia, with frequent evidence of peripheral eosinophilia.

Objectives: A cohort of pediatric patients evaluated in the Pediatric Rheumatology Medical Clinic of Verona from 2010 to 2020, affected by mono- and polyarthritis during parasitic infestation was described in this study with the aim of reporting clinical, instrumental and laboratory data, including research of parasites in order to raise awareness of this clinical entity in children and promote its systematic description.

Methods: Patients who presented joint pain or clinical signs of arthritis and evidence of parasitic infestation by scotch tape test or stool examination were selected among Pediatric Rheumatology patients of Verona Day Hospital from 2010 to 2020. The following indicators were considered: articular manifestations at onset, presence of eventual systemic symptoms associated, laboratory test abnormalities, elapsed time between onset and diagnosis, response to treatment and eventual relapse. Patients presenting signs of joint inflammation underwent an ultrasound examination.

Results: We selected 14 patients of prevalent age at onset of 7,71 years (2-14 years), male-to-female ratio 8:6. The majority of patients $(86 \%)$ presented a symmetric polyarthritis, exclusively involving the lower extremities in $54 \%$ of the cases. At onset of the articular manifestation, $78 \%$ of the patients presented one or more associated symptoms, such as diffuse or anal itching (30\%), gastrointestinal symptoms (abdominal pain 43\%) and a less specific symptomatic including headache (21\%), fatigue (14\%) and dermatitis or skin rash (21\%). The laboratory tests, performed by nearly all patients (93\%), showed eosinophilia in $92 \%$ of the cases, presence of antinuclear antibodies (ANA) (38\%) and elevated inflammation markers $(0,08 \%)$. Ultrasound was performed in $64 \%$ of the patients and showed synovial thickening and synovitis in all cases and joint effusion in $44 \%$ of the cases. Stool examination and scotch tape test repeated on 3 consecutive samples resulted positive for $E$. vermicularis in $93 \%$ of the cases and for Dientamoeba fragilis in one patient. After eradication therapy with repeated doses of mebendazole and pyrantel pamoate every 2-4 week for all cohabitants and pets, all the patients showed a significant reduction of symptoms and subsequent normalisation of test results. In 2 cases re-infestation occurred with recurrence of articular symptoms and treatment was restarted with benefit, as long as needed until complete eradication.

Conclusion: The study suggests that intestinal parasitic infestations are an under-reported cause of joint pain and arthritis in children. Indeed, parasites should be considered in the differential diagnosis in patients that present joint pain or signs of arthritis with clinical and laboratory findings suggestive of parasitic infestation. A re-infestation should also be excluded in case of recurrence of articular symptoms. A concomitant parasitic infestation should furthermore be excluded in chronic polyarthritis requiring immunosuppressant therapy, due to risk of disseminate infestation.

\section{Disclosure of Interest}

None declared

\section{P175}

Outcomes of mothers with rheumatic diseases and follow-up of with a focus on autoimmunity and neurodevelopment

K. V. Jirón Mendiola', S. Jiménez Hernández', F. García Rodriguez ${ }^{1}$, A. V. Villarreal Treviño ${ }^{1}$, M. E. Romero López ${ }^{1}$, E. S. Barriga Maldonado², L. Barbosa $^{2}$, C. Skinner ${ }^{2}$, F. Hernández ${ }^{3}$, Y. Medina ${ }^{4}$, M. de la O ${ }^{4}$, D. Galarza Delgado ${ }^{2}$, N. Rubio Pérez ${ }^{4}$

${ }^{1}$ Reumatología Pediátrica; ${ }^{2}$ Reumatología; ${ }^{3} \mathrm{GYO} ;{ }^{4}$ Pediatría, Hospital Universitario "Dr. José E. González", Monterrey, Nuevo León, Mexico Correspondence: K. V. Jirón Mendiola

Pediatric Rheumatology 2020, 18(Suppl 2):P175

Introduction: Autoimmune rheumatic diseases (ARD) predominantly affect women of childbearing age, including systemic lupus erythematosus (SLE) and rheumatoid arthritis (RA) being the most prevalent. Maternal ARD have been associated with early and late abortions, premature births, and low birth weight babies, which will subsequently have repercussions on their neurological development. Objectives: The aim of this study is to describe the birth outcomes of newborns born to woman with rheumatic disease, and the impact of their neurological development.

Methods: A prospective study from July 2017 to April 2020, from children born to women with ARD treated at our center. Sociodemographic and clinical characteristics of newborns and their 
mothers were evaluated; medication used during pregnancy, perinatal complications and prospective neurological evaluations were performed.

Results: 42 children born to 39 women were included,one twin and one triplet pregnancy.RA in 35\%.Median gestational age were 36 weeks (26-41), 38\% children were preterm,4.7\% extremely premature. Median weight 2509 grs (700-3920), 23\% low birth weight. The mortality was $9.5 \%$. 2.3\%was prenatally diagnosed with neonatal Lupus and complete AVB, treated with parenteral steroids prenatally and a pacemaker at birth. Neurological evaluations were abnormal in $4.7 \%$ in the first month and in $19 \%$ at 3 months, $7.1 \%$ patients had axial hypotonia,4.7\% a higher head circumference, $2.3 \%$ presented fists and no cephalic support.

Conclusion: Data collected during follow-up of this cohort of children born to women with rheumatic diseases show alterations in gestational age, antropometrical measures, neurological development and ARD features, at this moment to follow-up only $2.3 \%$ of children had an autoinmune disease. A more extensive follow-up is required to obtain noteworthy data on the impact of this deviations during children development.

Disclosure of Interest

None declared

Table 1 (abstract P175). RA=Rheumatoid Arthritis, APS= Antiphospholipid Syndrome, SLE=Systemic Lupus Erythematosus, AIJ= Juvenile Idiopathic Arthritis, AV=Atrioventricular

\begin{tabular}{llll}
\hline ARD & $\mathbf{n = 4 2}$ & Abnormal & Newborn \\
\hline RA & 14 & $1(2.3 \%)$ & Polycythemia \\
SLE & 5 & 0 & Normal \\
SJOGREN & 3 & $1(2.3 \%)$ & Patent ductus arteriosus \\
APS & 9 & $1(2.3 \%)$ & Cyanogenic heart disease \\
Asymptomatic & 2 & $1(2.3 \%)$ & Neonatal lupus AV block \\
SLE + APS & 1 & 0 & Normal \\
Dermatomyositis & 1 & 0 & Normal \\
Others & 7 & $7(16.6 \%)$ & Normal \\
\hline
\end{tabular}

\section{P176}

Ankle tenosynovitis in Poncet's disease

L. R. Campos' , K. L. Baptista', J. Caldas², V. Orenbuch ${ }^{2}$, I. Gomes ${ }^{3}$, C. A. A. Cardoso $^{3}$, C. Canella ${ }^{4}$

${ }^{1}$ Pediatric Rheumatology, Department of Pediatrics; ${ }^{2}$ Hospital

Universitário Antônio Pedro, Universidade Federal Fluminense, Niterói -

Rio de Janeiro, Brazil; ${ }^{3}$ Department of Maternal and Child; ${ }^{4}$ Department

of Radiology, Hospital Universitário Antônio Pedro, Universidade Federal

Fluminense, Niterói - Rio de Janeiro, Brazil

Correspondence: $L$. R. Campos

Pediatric Rheumatology 2020, 18(Suppl 2):P176

Introduction: Musculoskeletal manifestations of TB account for 10$19 \%$ of the cases of extrapulmonary form $(1,2)$. Poncet's disease is a reactive arthritis associated with active $T B$, with no evidence of $T B$ in the joints, bones or tendons(3). The typical manifestation is symmetric polyarthritis(4).

Objectives: Report a rare case of ankle tenosynovitis in Poncet's disease.

Methods: Case report and literature review.

Results: A 10-year-old female patient was referred to the rheumatology clinic at our hospital with a previous history of fever of $39^{\circ} \mathrm{C}$ $\left(102.2^{\circ} \mathrm{C}\right)$, loss of appetite, and acute polyarthritis of wrist, knees, and ankles. At that time, laboratory exams revealed a hemoglobin of 11.1 $\mathrm{g} / \mathrm{dL}$, C reactive protein $78.6 \mathrm{mg} / \mathrm{L}$, and antistreptolysin $O$ titers of
$400 \mathrm{UI} / \mathrm{mL}$ (normal range $<200 \mathrm{Ul} / \mathrm{ml}$. Clinical symptoms were relieved only after using NSAIDs. After 6 months, the patient returned to our hospital with a 7-month history of weight loss and claudication related to pain and daily morning stiffness (15 minutes) on her right ankle. New laboratory findings demonstrated positive antinuclear antibodies 1:320, negative rheumatoid factor, and alpha-1-acid glycoprotein of $171 \mathrm{mg} / \mathrm{dL}$ (normal range: $44-113 \mathrm{mg} / \mathrm{dL}$ ). Clinical signs suggestive of chronic arthritis with exuberant swelling of the ankles were observed on physical examination (figure A). She was screened for tuberculosis (TB) and had a positive $(18 \mathrm{~mm})$ tuberculin skin test (figure B). Chest CT revealed infiltrative soft tissue mass in the posterior mediastinum, with homogeneous contrast enhancement (figure C). Magnetic resonance imaging of both ankles was performed and demonstrated bilateral and symmetrical tibiotalar arthritis and prominent tenosynovitis of extensors, flexors, and fibularis tendons (figure D). Right ankle synovial biopsy revealed no granulomas and joint fluid culture was negative for Mycobacterium tuberculosis, confirming reactive arthritis (Poncet's) and tenosynovitis, that may follow mycobacterial infection with no infective agent in the joints.

Conclusion: To our knowledge, there is no report of Poncet's disease associated with inflammatory tenosynovitis, showing the particularity of this case. The patient's symptoms resolved after two months of anti-TB therapy.

Disclosure of Interest

None declared

\section{P177}

Camptodactyly-arthropathy-coxa vara-pericarditis syndrome: a rare and misdiagnosed condition

L. R. Campos' ${ }^{1}$, K. L. Baptista' ' L. Ferreira², L. Lima ${ }^{3}$, C. Canella ${ }^{3}$, A. Serfaty ${ }^{4}$

${ }^{1}$ Pediatric Rheumatology, Department of Pediatrics; ${ }^{2}$ Pediatrics;

${ }^{3}$ Department of Radiology, Hospital Universitário Antônio Pedro,

Universidade Federal Fluminense, Niterói - Rio de Janeiro; ${ }^{4}$ Department

of Radiology, Universidade Federal do Rio de Janeiro, Rio de Janeiro,

Brazil

Correspondence: L. R. Campos

Pediatric Rheumatology 2020, 18(Suppl 2):P177

Introduction: CACP is characterized by congenital or early-onset camptodactyly (usually bilateral); non-inflammatory arthropathy (more frequently in the wrists, knees, ankles, elbows, and hips); coxa vara (reduction of the angle between the neck and shaft of the femur); and non-inflammatory pericardial effusion (a late manifestation, less frequently reported). Recognizing the radiological aspects of this syndrome and differentiating it from JIA is crucial since CACP has no effective treatment and JIA is usually treated with NSAIDs and methotrexate $(2,3)$.

Objectives: To report a rare case of CACP syndrome mimicking JIA.

Methods: Case report and literature review.

Results: A 5-year-old male patient presented with arthropathy characterized by painless progressive swelling and restricted movement of the hands, hips, knees, and ankles since the first year of life. He had a family history of camptodactyly from his paternal grandfather. On physical examination, symmetric camptodactyly of the hands and feet was observed (A). He had no history of rash or weight loss and inflammatory markers were unremarkable. The echocardiogram was normal. The pelvic radiograph showed a widening of the joint space and bilateral coxa vara. Magnetic resonance imaging (MRI) of the hips (B) and knees (C) was performed and depicted large joint effusions (arrows, B and C) with normal synovial thickness and mild synovial enhancement in all joints, without bone marrow edema-like signal. A synovial biopsy of the knee was performed and revealed mild synovial hyperplasia without inflammatory cells. The patient was diagnosed with camptodactyly-arthropathy-coxa vara-pericarditis syndrome (CACP - OMIM 208250), a recently described genetic disorder with no gender predominance identified to date (1).

Conclusion: An important differential diagnosis of CACP is juvenile idiopathic arthritis (JIA), a painful inflammatory chronic arthritis that can cause not only joint effusions due to synovial inflammation, but 
also contractures resembling camptodactyly when not effectively treated.

Disclosure of Interest: None declared

\section{P178}

Features of childhood Sjogren's syndrome: a literature review based cohort

A. Marino ${ }^{1}$, M. Romano ${ }^{2}$, T. Giani ${ }^{3}$, C. Gaggiano ${ }^{4}$, S. Costi ${ }^{5}$, R. Singh ${ }^{6}$, J.

Mehta $^{7}$, S. Lieberman ${ }^{8}$, R. Cimaz ${ }^{2}$

${ }^{1}$ Department of Pediatrics, Desio Hospital, Desio, ${ }^{2}$ ASST G.Pini-CTO,

Milano, ${ }^{3}$ Pediatric Rheumatology, Meyer Children's Hospital, Florence,

${ }^{4}$ Department of Pediatrics, University of Siena, Siena, ${ }^{5}$ University of Milan,

Milano, Italy, ${ }^{6}$ Northwestern University, Evanston, ${ }^{7}$ Division of

Rheumatology, Children's Hospital of Philadelphia, Philadelphia,

${ }^{8}$ Department of Pediatrics, University of lowa, lowa City, United States

Correspondence: A. Marino

Pediatric Rheumatology 2020, 18(Suppl 2):P178

Introduction: Sjogren's syndrome (SS) is an autoimmune chronic disease characterized by inflammation of exocrine glands, but it can affect other organs as well.

Objectives: To describe childhood SS (CSS) features by reviewing pediatric published cases with individual data.

Methods: We conducted a literature review of CSS (age $<18$ years). Eligible papers were identified through a Medline search of English language articles published in the PubMed database until February 2020. Statistical analysis was performed in order to detect associations between clinical/laboratory features.

Results: Two-hundred-forty patients were identified (191 female); the median age at disease onset was 10 years (range 3 months17 years). Main clinical features are shown in Table 1. The most frequently reported clinical SS-specific feature was parotitis (134/198 patients; bilateral:unilateral=2.5:1); fewer patients had sicca symptoms (89/159 with dry eyes; $85 / 144$ with dry mouth). Arthritis was the most frequent extraglandular manifestation. Renal tubular acidosis represented the typical expression of renal involvement (19 cases). Neuromyelitis optica and aseptic meningoencephalitis ( 6 and 9 cases, respectively) were the most typical neurologic manifestations. Two cases of interstitial lung disease and one of pulmonary hypertension were reported. Almost all patients had autoantibodies, mostly ANA (200/224 patients) and anti-SSA/Ro (170/208 patients). The Schirmer test was performed in less than half of the patients, of whom $62 \%$ tested positive. A positive result of minor salivary biopsy was reported in 129/140 cases with available data. Juvenile idiopathic arthritis was the most frequently associated disease, followed by systemic lupus erythematosus ( 16 and 8 cases, respectively). No significant differences between patients with or without parotitis were found except that patients with parotitis showed increased levels of CRP more frequently than those without it $(p=0.00)$. Patients with anti-SSA/Ro had more frequently a positive Schirmer test $(p=0.04)$. The presence of RF was significantly associated with dry mouth $(p=0.00)$, arthritis $(p=0.00)$, and rash $(p=0.04)$. A positive minor salivary biopsy was more common in children with dry eyes than in those without this clinical feature $(p=0.02)$. Arthritis was more frequent in patients with other diseases than in those with primary SS $(p=0.00)$. We further investigated SS features according to the age groups ( $\leq 6$ years, 7-11 years, $\geq 12$ years). Parotid involvement was inversely proportional to the age and occurred more frequently in younger patients $(79 \%$ of those $\leq$ 6 years; $p=0.03$ ). Interestingly, the rate of anti-SSA/Ro positivity increased with age ( $97 \%$ of those $\geq 12$ years; $p=0.00$ ).

Conclusion: Even though parotitis was the most frequently reported feature, a wide range of clinical manifestations in children with SS has been reported so far. A better knowledge of cSS features will help to pave the way for the development of CSS specific diagnostic criteria.
Disclosure of Interest

None declared

Table 1 (abstract P178). Clinical features of cSS cohort

\begin{tabular}{lll}
\hline SS features & Positive pts & $\begin{array}{l}\text { Percentage } \\
\text { (\% of available data) }\end{array}$ \\
\hline Parotitis & 134 & 67.7 \\
Dry eyes & 89 & 56.0 \\
Dry mouth & 85 & 59.0 \\
Arthritis & 59 & 72.8 \\
Fever & 52 & 70.3 \\
Rash & 42 & 64.6 \\
CNS involvement & 40 & 85 \\
Renal involvement & 31 & 59 \\
Raynaud phenomenon & 14 & 58.3 \\
\hline
\end{tabular}

\section{P179}

Pachydermodactyly: a mimicker in the paediatric rheumatology outdoor clinical practice

E. Giacomelli' ${ }^{1}$ S. Pellizzari ${ }^{1}$, E. Tadiotto', G. Melotti ${ }^{1}$, G. Aiello ${ }^{1}$, M.

Maschio', F. Caldonazzi², S. Pieropan

${ }^{1}$ Pediatrics, Azienda Ospedaliera Universitaria Integrata, Verona;

${ }^{2}$ Pediatrics, Ospedale di Rovereto, Rovereto (TN), Italy

Correspondence: S. Pellizzari

Pediatric Rheumatology 2020, 18(Suppl 2):P179

Introduction: Pachydermodactyly (PDD) is a rare benign fibromatosis, characterized by progressive painless swelling of soft tissue of proximal interphalangeal (PIP) joints without inflammation signs. Generally PDD affects PIP joints of the fingers, rarely of the thumb. The involvement is typically symmetrical, in few cases unilateral. It usually occurs more frequently in young males. Etiology is unknown, but it arises from mechanical stimulation of periarticular skin (i.e repetitive rubbing, interlacing, and cracking of fingers). PDD has to be considered in the differential diagnosis of arthritis (i.e. juvenile idiopathic arthritis, JIA) and many syndromes (i.e. progressive pseudorheumatoid dysplasia). Prognosis is good with cessation of mechanical stimulation?.

Objectives: The aim of this study, is to improve our knowledge about this problem leading to a correct diagnosis. We describe a cohort of outdoor pediatric patients attending the Rheumathology Pediatric Medical Clinic in Verona.

Methods: A retrospective analysis was conducted in the Pediatric Rheumatology Day Hospital of Verona from January 2014 to April 2020. We selected six patients with painless swelling of soft tissue of PIP joints. Male-to female ratio was 1:1. Prevalent age at diagnosis was 11 years. Among all cases, only one practiced sport (16\%), in particular canoeing. All cases had painless swelling of soft tissue of PIP joints of fingers, bilateral in $66 \%$ of the cases. Associated symptoms were paresthesia and functional limitation (33\%), tic (16\%), anxiety disorders $(16 \%)$ and repetitive mechanical movements (i.e rubbing and interlacing of fingers) (33\%). None of our patients had findings of articular inflammation. Laboratory tests were completely normal objective and strumental for all reported cases,in particular all inflammatory markers were normal.

Results: Half of the evaluted patients had previously consulted a medical specialist (16\% orthopaedist, $33 \%$ rhumatologist). Ultrasonography was performed in $33 \%$ of patients and it was negative for joint damage in all cases. The hand MRI, performed in $66 \%$ of cases, excluded the presence of arthropathic or osteopathic lesions with exclusively swelling of the periarticular soft tissue. For $50 \%$ of the patients a genetic test for Progressive pseudorheumatoid dysplasia (gene WISP3) was conducted and it resulted negative in all cases. In one patient the exclusion of the diagnosis of JIA was complicated by concomitant knee pain and suprapatellar effusion on 
ultrasound; both findings successively regressed and were probably due to previous trauma.

Conclusion: Pachydermodactyly is a rare pathology that should be suspected in a patient who presents with swelling of PIP hand joints without clinical and laboratory findings of inflammation and related to the exclusive involvement of the soft tissue component on imaging. PDD resolves spontaneusly after behavioural therapy. PDD shares with JIA clinical appearance of joint swelling and its time of onset (months). However, a complete anamnesis, which should pay attention in particular to the habit behaviours and involuntary movements of the patient, and an accurate physical examination can help distinguish PDD from JIA, therefore prevent unnecessary immunosuppressant therapy; Refer child tot he neuropsychiatrist is indicated for the proper follow up.

\section{Reference}

1. Dallos T, Oppl B, Kovács L, Zwerina J. Pachydermodactyly: a review. Curr Rheumatol Rep. 2014;16(9):442.

\section{Disclosure of Interest}

None declared

\section{P180}

Treating two fibrodysplasia ossificans progressiva patients with interleukin-1 inhibitors

R. Haviv' ${ }^{1}$, V. Moshe ${ }^{2}$, F. De Benedetti ${ }^{3}$, G. Prencipe ${ }^{3}$, N. Rabinowicz ${ }^{2}$, Y. Uziel ${ }^{1}$ Pediatric Rheumatology Unit, Department of Pediatrics, Meir Medical Center, Sackler School of Medicine, Tel Aviv University; ${ }^{2}$ Pediatric Rheumatology Unit, Department of Pediatrics, Meir Medical Center, Kfar Saba, Israel; ${ }^{3}$ Division of Rheumatology, Bambino Gesù Children's Hospital IRCCS, Rome, Italy

Correspondence: R. Haviv

Pediatric Rheumatology 2020, 18(Suppl 2):P180

Introduction: Fibrodysplasia ossificans progressiva (FOP) is the most catastrophic form of heterotopic ossification ( $\mathrm{HO}$ ), due to activating mutations in the ACVR1/ALK2 gene, and ongoing intracellular signaling through the bone morphogenic protein (BMP) pathway. Painful soft tissue swellings usually appear by the age of 3-4 years, but the typical bilateral greater toe deformity can be noted at birth. Average life expectancy is 45 years. Currently, there is no proven effective treatment.

The recurrent paroxysmal appearance of inflammatory lumps (local erythematous tender swellings, which partially respond to antiinflammatory agents), accompanied by elevated inflammatory markers during flares, suggest that FOP may be an autoinflammatory disease. The episodic formation of bone, often following a trivial injury, suggests that innate immune-related triggers induce tissue transformation through the BMP pathway. Moreover interleukin-1 $\beta$ (IL-1 $\beta)$, a well-known mediator of the innate immune system, has been linked to $\mathrm{HO}$ and mineralization in mesenchymal stem cell cultures derived from human bone marrow. We hypothesized that treating FOP patients with anti-IL-1 agents could help ameliorate the progression of this devastating disease. We report our experience treating two FOP patients with anakinra and canakinumab.

Objectives: To decrease the frequency of FOP paroxysms, and/or limit the symptoms and extent of residual lesions, by using anti-IL-1 agents.

Methods: Patients' data and blood IL-1 levels were analyzed to characterize the efficacy of anti-IL-1 treatments in ameliorating the natural progression of FOP.

Results: A 13.5 year old boy and a 5 year old girl were diagnosed with FOP, both clinically and genetically (the typical R206H mutation was found). Various treatments, including high-dose corticosteroids, pamidronate infusions, celecoxib, monteleukast and sirolimus, did not change the course of the disease.
Both patients are receiving canakinumab (the male patient was initially treated with anakinra). The male patient has been treated for over 2 years. Flare rate was markedly reduced from one new lump every 8 days to approximately one every 25 days (Figure 1). The lumps involved in almost all of these flares are the same: at the left scapular base and within the sternocleidomastoid muscle. The female patient has been treated for a year, and has not experienced any $\mathrm{HO}$ flares during canakinumab treatment.

Temporarily withholding canakinumab in both patients, led to serious flares 8 weeks after the last dose. Notably, while undetectable levels of IL-1 $\beta(<0.125 \mathrm{pg} / \mathrm{ml})$ were found in the three plasma samples obtained from the male patient during treatment with anakinra or canakinumab, high levels (up to $21.52 \mathrm{pg} / \mathrm{ml}$, about 90-fold higher compared to average levels measured in healthy controls) were found in his plasma samples collected during the flare (Figure 2). In contrast, IL-18 and IL-6 plasma levels, measured before, during and after withholding treatment, were comparable or slightly higher than those observed in healthy controls (Figure 3A, B).

Conclusion: We report here, for the first time, that anti-IL-1 agents were found efficacious in treating two FOP patients. We also found markedly increased IL-1 $\beta$ levels during flares, which normalized following the treatment. We suggest a role for IL-1 $\beta$ in the pathogenesis of this disease. Although it is too soon to conclude whether FOP may be included under the umbrella of auto-inflammatory syndromes, anti-IL-1 agents can be effective in ameliorating the natural progression of FOP.

\section{Disclosure of Interest}

None declared

P181

Genetic syndromes mimicking rheumatologic diseases

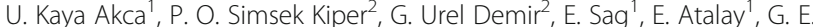

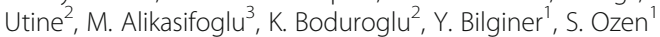

'Department of Pediatric Rheumatology; ${ }^{2}$ Department of Pediatric

Genetics, ${ }^{3}$ Department of Medical Genetics, Hacettepe University Faculty of Medicine, Ankara, Turkey

Correspondence: $U$. Kaya Akca

Pediatric Rheumatology 2020, 18(Suppl 2):P181

Introduction: Musculoskeletal symptoms are one of the common reasons for applying to rheumatology departments in general practice ${ }^{1}$. Although inflammatory causes are generally considered in the foreground, it is known that non-inflammatory causes including genetic diseases may also be responsible. The absence of signs of inflammation (morning stiffness, redness, tenderness) and normal inflammatory markers in laboratory findings may support nonrheumatologic diseases ${ }^{2}$.

Objectives: To present genetic disorders that can mimic rheumatologic symptoms and to answer when genetic diseases should be considered in the differential diagnosis in patients presenting with rheumatological complaints.

Methods: We retrospectively evaluated 60 patients who applied to Hacettepe University pediatric rheumatology department with musculoskeletal compliants between January 2015 and December 2019 and had been consulted to genetics departmant. The rate and degree of consanguinity, clinical diagnosis, indication for consultation, accompanying musculoskeletal and other findings had been recorded. The diagnosis of genetic diseases were based on physical examination, radiological evaluations and genetic analysis.

Results: A total of 60 patients, 19 boys (31.6\%), with a mean age $12.46 \pm 1.41$ years were included in the study. The rate of consanguinity was $25.0 \%$. The most frequent referral to the genetic department was the presence of skeletal anomalies (n:12) such as camptodactyly, clinodactyly, and bone shortness accompanying joint findings. Other causes include short stature $(n: 4)$, joint deformity $(n: 5)$, joint hyperlaxicity $(n: 10)$, dysmorphic findings such as atypic facial appearance $(n: 9)$, accompanying diseases that may be part of a syndrome $(n: 11)$, genetic diagnosis suspicion according to the results 
of radiological examination ( $n: 4)$ and joint findings without clinical and laboratory signs of inflammation (n:5). Distribution of joint involvement in 20 patients with genetic disease were hands, knees, and hips respectively. In the laboratory evaluation of patients presenting with joint swelling and arthralgia, acute phase reactants (erythrocyte sedimentation rate and C-reactive protein concentrations) were within normal reference values. One third of the patients (33.3\%) had a final diagnosis of a genetic disease. The diagnoses of these patients were as follows; CACP (camptodactyly, arthropathy, coxa vara deformity and pericarditis) syndrome $(n: 3)$, trichorhinophalangeal syndrome $(n: 1)$, progressive pseudoromatoid dysplasia $(n: 2)$, LIG4 syndrome (n:1), 3M syndrome (n:1), H syndrome ( $n: 1)$, SPENCD (spondyloenchondrodysplasia, $\mathrm{n}: 3$ ), and nonspecific connective tissue disease $(n: 8)$.

Conclusion: Genetic syndromes with musculoskeletal findings are often unrecognized and misdiagnosed as rheumatologic diseases leading to unnecessary procedures and treatments. Summarizing the genetic diagnosis spectrum that can be detected in these patients will increase the awareness of physicians.

\section{References}

1. Sills J. Non-inflammatory musculoskeletal disorders in childhood. Archives of disease in childhood. 1997;77(1):71-5.

2. Al-Mayouf SM. Noninflammatory disorders mimic juvenile idiopathic arthritis. International Journal of Pediatrics and Adolescent Medicine. 2018:5(1):1-4

Disclosure of Interest: None declared

\section{P182}

Infectious triggers of erythema nodosum in children

A. Akhenbekova, S. Akilbekov, G. Tashenova

Faculty of Medicine and Health Care, Al-Farabi Kazakh National

University, Almaty, Kazakhstan

Correspondence: A. Akhenbekova

Pediatric Rheumatology 2020, 18(Suppl 2):P182

Introduction: The aim of the study was to study the role of etiological triggers on the clinical course and severity of erythema nodosum in children.

Objectives: 65 children with nodular erythema aged from 3 years to 15 years

Methods: 65 children with nodular erythema aged from 3 years to 15 years were monitored. The examination included General clinical research, C-reactive protein (CPR) , antistreptolysin, rheumatoid factor, antinuclear factor (ANF) with method of immunofluorescence. To determine the etiological factor, bacteriological studies of nasopharyngeal smears, Epstein-Barr virus, fecal studies for intestinal diseases, diagnoses for Listeriosis, Yersiniosis, Chlamydia were conducted.

Results: According to the results of observation, the disease was more common in the age group of 7-11 years $(65 \%)$, to a lesser extent among children in the group of $12-15$ years $(35 \%)$, less often in the group of 3-7 years (5\%). When examining infectious agents, zoonotic infection was detected in $41 \%$ (Listeria monozytogenes, Yersinia enterocolitica). Clinical course of nodular erythema in this group was characterized by an expressed activity of the inflammatory process with multiple elements in the lower and upper extremities, joint syndrome, increased ESR to $45 \pm 3.8 \mathrm{~mm}$ per hour, CRP $28 \pm 2.5$ $\mathrm{mgl}$. The disease was preceded by an episode of acute infection with an increase in body temperature, intoxication, in some cases with short-term intestinal syndrome, pharyngitis. The rashes were persistent and recurrent, with a slow regression of laboratory activity. Streptococcal etiology of nodular erythema was detected in 37\% of cases. There was an increase in ESR to $25 \pm 3.8 \mathrm{~mm}$ per hour, CRP $15 \pm$ $2.7 \mathrm{mg} / \mathrm{l}$, a significant increase in antistreptolysin on average $480 \pm$ $34 \% \mathrm{IU} / \mathrm{ml}$. with an increase in individual cases to $870 \mathrm{IU} / \mathrm{ml}$. In $13 \%$ of cases, erythema nodosum developed after an intestinal infection. Among the pathogens were identified Sh. disenteria, E. coli, Yersinia enterocolitica, enterovirus. The disease was characterized by moderate activity, a good response to etiological therapy and a short course of NSAIDs. An interesting fact was the development of nodular erythema in $4 \%$ of cases caused by the Epstein-Barr virus in groups of children from 3 to 7 years and 7-9 years. They had clinic picture with normothermia, no symptoms of intoxication, periodically occurring elements of nodular erythema on the shins, no blood changes. Therapy aimed at eliminating the virus gave a positive result and did not require specific anti-rheumatic therapy. In $5 \%$ of cases, the etiology of nodular erythema was not defined.

Conclusion: The clinical course of nodular erythema in children depends on the infectious agent that was the trigger of the pathological process. The higher activity and duration of the disease is caused by zoonotic infection, which requires more active antiinflammatory therapy with corticosteroids, which may be associated with the activation of autoimmunity. This group of children was taken for further observation as a group at risk of developing systemic connective tissue disease. Changes in the etiological structure of nodular erythema and treatment tactics require further study.

Disclosure of Interest

None declared

\section{P183}

Pediatric Sjögren syndrome: an Italian case series

M. Romano ${ }^{1}$, T. Giani ${ }^{2}$, A. Marino ${ }^{3}$, C. Gaggiano ${ }^{4}$, G. Filocamo ${ }^{5}$, I. Pontikaki $^{6}$, R. Cimaz on behalf of Pediatric Rheumatology Group of the Milan Area

${ }^{1}$ Pediatric Rheumatology, ASST-G.Pini-CTO, Milan, Italy, Milan; ${ }^{2} \mathrm{AOU}$ Meyer, Florence; ${ }^{3}$ Department of Pediatrics, Desio Hospital, ASST Monza Desio, Monza; ${ }^{4}$ Clinical Pediatrics, Department of Molecular Medicine and Development, University of Siena, Siena; ${ }^{5}$ Pediatric Rheumatology, Fondazione IRCCS Cà Granda ospedale Maggiore Policlinico, Milan, Italy; ${ }^{6}$ Pediatric Rheumatology, ASST-PINI-CTO, Milan, Italy; ${ }^{7}$ Department of Clinical Sciences and Community Health, University of Milan, Milan, Italy

Correspondence: $\mathrm{M}$. Romano

Pediatric Rheumatology 2020, 18(Suppl 2):P183

Introduction: Sjögren syndrome (SS) is a chronic autoimmune disorder characterized by inflammation of the lacrimal and salivary glands leading to oral and ocular dryness. Childhood SS is rare and poorly defined and underdiagnosed owing to the lack of childspecific diagnostic or classification criteria.

Objectives: The purpose of this study is to describe 12 cases with pediatric SS in order to better clarify the characteristics of the disease in the pediatric age.

Methods: We retrospectively reviewed medical records of patients (pts) with pediatric SS referring to three Italian pediatric rheumatology centers. Due to lack of childhood validated SS-specific criteria, physician diagnosis was the only inclusion criteria.

Results: We collected data on 12 pts ( 9 females). The mean age of disease onset is $10.0 \mathrm{yrs}$ (median 10.2, range 4-17). The mean age of diagnosis is 11.83 (median 11.45, range 6-18). The follow up period varied from 0.1 to 9.3 yrs (mean 3.95, median 5.0). The most common manifestations were articular involvement (mainly with arthralgia) (9/12 pts) and parotid/salivary glands swelling (8/12 pts). Xerostomia and xerophthalmia were found in $6 / 12$ pts and in $4 / 12$ respectively. Vaginal dryness was reported only by one pt. Fever and fatigue occurred in 3/12 and 7/12 pts respectively. We also recorded 3 cases of circulating immune complexes manifestations in 3 pts, purpura $(n=2)$ and glomerulonephritis $(n=1)$. We observed an endocrine involvement in 3 pts (1 metabolic syndrome, 2 autoimmune thyroiditis). Abdominal pain was found in $4 / 12$ pts. All pts were positive for autoantibodies (positivity for ANA or anti-SSA or anti-SSB or $\mathrm{FR}$ ) at presentation. RF test results were available in $8 \mathrm{pts}$, all positive. Positive ANA (titer $>1 / 320$ ) and anti-SSA were present in 10/12 pts and in 9/12 respectively. Hypergammaglobulinemia (range 1,6-8.04 $\mathrm{g} / \mathrm{dl}$ ) was found in $8 / 11$ pts (1 NA).

Abnormal Schirmer test was observed in the half of cases (6/12). Minor salivary gland biopsy was performed in 10 pts resulting in histological evidence of focal lymphocytic sialadenitis in $9 / 10$. Sonographic evaluation of salivary glands was abnormal in all of the patients (10/10). 
With regard to treatment, $6 / 12$ pts received corticosteroids and eight were also treated with one or more DMARDs such a hydroxychloroquine $(n=8)$, methotrexate $(n=3)$, azathioprine $(n=1)$, leflunomide $(n=1)$. Biological therapy was used in 3 patients for systemic involvement: 1 received belimumab and then rituximab, while the other patients received rituximab.

Conclusion: Xerostomia and keratoconjunctivitis sicca were not common in our series while recurrent parotid swellings were more frequent than what reported in adults. Pediatric recurrent parotitis should increase the suspicion for Sjögren syndrome. Current diagnostic criteria for SS do not include parotitis and therefore, the incidence of SS may be under-recognized in childhood. The disease is not always benign and patients with severe course may need second line treatment including immunosuppressant and biologics.

Disclosure of Interest

None declared

\section{P184}

Withdrawn

\section{P185}

Rheumatological disorders presenting in the first year of life

T. L. Marques', A. Marinhas ${ }^{2}$, J. Nascimento ${ }^{3}$, P. Estanqueiro ${ }^{3}$, M. Salgado ${ }^{3}$ ${ }^{1}$ Pediatrics Department, Centro Hospitalar do Baixo Vouga, Aveiro;

${ }^{2}$ Hospital Pediatrico, Centro Hospitalar e Universitário de Coimbra;

${ }^{3}$ Pediatric Rheumatology Unit, Hospital Pediátrico, Centro Hospitalar e

Universitário de Coimbra, Coimbra, Portugal

Correspondence: A. Marinhas

Pediatric Rheumatology 2020, 18(Suppl 2):P185

Introduction: Improving our understanding of pediatric rheumatological (PR) patient population is crucial for pediatric rheumatologists to know rheumatic disease epidemiology and to raise awareness leading to early detection. We didn't find studies of PR disorders presenting in the first year of life.

Objectives: The aim of this study is to assess the prevalence of PR disorders with onset in the first year of life.

Methods: We retrospectively studied patients observed in our Pediatric Rheumatology Unit between January $1^{\text {st }}$ of 1987 and December $31^{\text {st }}$ of 2019 . We defined acute $(<2$ weeks), subacute $(\geq 2$ and $<6$ weeks) and chronic ( $\geq 6$ weeks).

Results: A total of 3751 patients were observed in 32 years. Diseases' onset occurred in the first decade of life in 2290 patients $(61 \%)$ and in the first year of life in $158(4,2 \%)$. Among the latest group, chronic inflammation was the most frequent group of diagnosis (30\%), followed by recurrent inflammation (23\%), acute inflammation (11\%), infection (9\%), infiltrative/ degenerative disorders (8\%) and subacute inflammation (3\%). The remaining patients $(16 \%)$ were diagnosed with other disorders classified as miscellaneous. Among chronic inflammation group, 14 patients were diagnosed with juvenile idiopathic arthritis (4 systemic); 14 had neonatal lupus and one patient had polyarteritis nodosa. Among recurrent inflammation group, 13 patients were later diagnosed with PFAPA (periodic fever, aphthous stomatitis, pharyngitis and adenitis), 8 were diagnosed with Behçet disease and 6 had an autoinflammatory disorder. Acute vasculitis was diagnosed in 13 patients (9 Kawasaki disease and 4 acute hemorrhagic edema of infancy). Among infectious diseases group, there were two cases of congenital syphilis with arthritis and two cases of osteomyelitis secondary to BCG vaccination.

Conclusion: Rheumatological diseases presenting in the first year of life are not exceptional. Although many patients didn't have a definitive diagnosis at the beginning of the symptoms, many of them were later diagnosed with rheumatic disorders, mostly chronic inflammation (30\%), which requires early diagnosis, specific treatment and long-term follow-up. Rheumatic diseases must be considered as differential diagnosis in the first year of life in order to avoid delayed intervention and long term disabilities and sequelae.
P186

Systemic JIA, Kawasaki syndrome and macrophage activation, what else? Not only COVID-19

R. Dagher ${ }^{1}$, E. Sfeir $^{1}$, V. Modica ${ }^{2}$

${ }^{1}$ Pediatric department, Notre Dame de Secours University Hospital,

Byblos; ${ }^{2}$ Holy Family University, Batroun, Lebanon

Correspondence: $\mathrm{R}$. Dagher

Pediatric Rheumatology 2020, 18(Suppl 2):P186

Introduction: Macrophage Activation Syndrome (MAS) is a wellknown complication of Systemic Juvenile Idiopathic Arthritis (sJiA) and a rare consequence of Kawasaki Disease (KD). The diagnosis of $M A S$, in patients with $K D$, could be very challenging especially when Measles infection is the primary trigger. If from one side, Measles and KD have very similar clinical features and can easily mask each other (1), on the other side measles-induced MAS has rarely been reported (2).

Objectives: We present the case of a child known to have SJIA in remission, who presented a Measles primary infection and a secondary KD complicated by MAS.

Methods: A 5 years old girl, not fully vaccinated and known to have SJIA in remission under Methotrexate, presented for frequent high grade fever of 3 days duration associated with flat flash red spots on the face and trunk as well as the palms and soles. A Koplik's spot was identified. Conjunctivitis and coryza were also present. Initial viral serology, including measles, returned negative. Fever persisted and on day 7 , edema of both hands and feet appeared with bilateral cervical adenopathy, erythematous tonsils, gingivitis, cracked lips and hepatomegaly was noted. All cultures were negative and chest X-ray was normal. Inflammatory markers rose up. Viral serology was repeated and measles $\operatorname{lgM}$ came back positive. Cardiac ultrasound ruled out coronary aneurism and the ophthalmic exam showed no uveitis. KD criteria were met and $2 \mathrm{~g} / \mathrm{kg}$ of intravenous immunoglobulins (IVIG) were administered. After 48 hours of clinical improvement, fever reappeared and the patient returned to be ill looking although the rash regressed. We noted high ferritine(2016 $\mathrm{ng} / \mathrm{ml})$ together with low $\mathrm{C} 3$, decrease in platelets $\left(170 \times 10^{3} / \mathrm{ml}\right)$ and elevation of hepatic enzymes, LDH and CPK, without increase in the inflammatory biomarkers. MAS was suspected and a bone marrow aspirate showed the presence of mild macrophage hemophagocytosis. Antibodies for Lupus and auto-immune myositis were all negative. Steroids were given, fever disappeared, and spectacular clinical and biological improvements were objected. 2 weeks later, desquamation of all extremities was noted. SARS-COV-2 was not investigated because historically this case presented 1 year earlier than the pandemic.

Results: We hereby report, for the first time, KD and MAS triggered by Measles infection in a child with SIIA in remission. The exact mechanism involved in KD-induced MAS and Measles-induced MAS has not yet been defined but a defective immune response is suspected (3).

Conclusion: Significant similarities and overlap between measles, KD, SJIA and MAS make an early diagnosis very challenging (1)(3). The recent COVID19 pandemic emphasizes how a viral illness can be responsible of KD and sometimes degenerating in MAS. We report this clinical case as an example of a Systemic Inflammatory Syndrome (SIS) taking place after a viral infection to Measles. In the era of COVID19 pandemic and secondary SIS in children, an additional challenge is present in regions lacking Measles vaccine coverage.

\section{References}

1. Buonsenso D, Macchiarulo G, Supino MC, et al. Laboratory biomarkers to facilitate differential diagnosis between measles and kawasaki disease in a pediatric emergency room: A retrospective study. Mediterranean journal of hematology and infectious diseases. 2018;10(1):e2018033.

2. Lagousi $T$, Korovessi $P$, Panagouli $E$, Tsagris $V$, Kostaridou S. A rare case of measles-associated hemophagocytic lymphohistiocytosis in an infant. Cureus. 2020. 
3. Wang W, MD, Gong, Fangqi, MD, PhD, Zhu W, MD, Fu S, MD, Zhang Q, MD. Macrophage activation syndrome in kawasaki disease: More common than we thought? Seminars in Arthritis and Rheumatism. 2014;44(4):405-410.

\section{Disclosure of Interest}

None declared

\section{P187}

The musculoskeletal manifestations of scurvy: a diagnostic challenge for the rheumatologist

T. Lastella', R. Naddei', F. Orlando', C. Porfito', F. Mozzillo', M. Amico', M. Alessio ${ }^{1}$

${ }^{1}$ Pediatric Rheumatology Unit, Mother and Child Department, University of Naples Federico II; ${ }^{2}$ Rheumatology Unit, Department of Pediatrics, Santobono-Pausilipon Children's Hospital, Naples, Italy

Correspondence: $T$. Lastella

Pediatric Rheumatology 2020, 18(Suppl 2):P187

Introduction: An increasing trend in the incidence of scurvy has been reported in the last years, especially in children with food selectivity associated to autism. Still, a diagnostic delay in the identification of scurvy is common, due to non-specific clinical features mimicking rheumatological, hematologic or infectious conditions.

Objectives: To describe clinical features of 5 patients with scurvy, referred to two pediatric Rheumatology Units in Southern Italy, from July 2018 to December 2019.

Methods: Case reports

Results: P1 was a 4-year-old boy presenting limp and bilateral hyperemic tibial swellings for a week. He suffered from autism spectrum disorder and, since the third year of life, he developed marked food selectivity. Joint assessment was normal. Laboratory tests showed elevated erythrocyte sedimentation rate (ESR) level (70 $\mathrm{mm} / \mathrm{h}$ ). Main causes of erythema nodosum were excluded. Vitamin C level resulted greatly reduced: $1.5 \mu \mathrm{mol} / /(26.1-84.69)$. Supplemental therapy was started with clinical resolution.

P2 was a 5-year-old boy, with autism spectrum disorder, malnutrition and severe food selectivity, admitted to our Unit for refusal to bear weight and bruises in lower limbs. The auxological evaluation showed a strongly dystrophic aspect. Coagulation profile and main organ function markers were normal. At nutritional biochemical parameters evaluation, iron and Vitamin $\mathrm{C}$ deficiencies were detected (vitamin C: $2 \mu \mathrm{mol} / \mathrm{l})$. Oral vitamin C therapy was started, with prompt clinical response.

P3 was a 7-year-old boy with autism spectrum disorder, admitted to our Unit for lameness and difficulty in walking for a month. At clinical examination, a mottled skin at lower limbs was noted. Joint examination was normal. Auxological parameters and main blood tests were adequate for age. Given the presence of food selectivity, he underwent serum vitamin $C$ dosage $(11 \mu \mathrm{mol} / \mathrm{L})$; hence he started oral vita$\min C$ therapy, with rapid clinical improvement.

P4 was a 2 years old boy who was referred for coxalgia and fever. At clinical examination, pale skin, gingival hyperemia, and pain in mobilization of the left hip were present. Microcytic anemia was detected, but main organ and inflammatory markers were normal. No evidence of infection was present. X-ray of femur and knee showed morpho-structural alteration of the distal metaphysis bilaterally. A low intake of fruit and vegetables was reported; hence, dosage of vitamin $C$ was performed, resulting reduced $(2.5 \mu \mathrm{mol} / \mathrm{L})$. He started Vitamin C oral therapy with clinical response.

P5 was a 13-year-old girl with behavioral disorder and intellectual disability, admitted for fever and right knee swelling which appeared two days after a right leg burning. C-reactive protein and ESR were elevated and ultrasound exam confirmed intra-articular knee effusion. Suspecting a septic arthritis, antibiotic therapy was started with laboratory normalization and partial clinical improvement. Considering the persistence of knee swelling after nine days of intravenous antibiotic therapy, the presence of gingival hyperemia and history of food selectivity, vitamin $C$ dosage was practiced $(12 \mu \mathrm{mol} / \mathrm{l})$. Oral vitamin $\mathrm{C}$ was administered with complete clinical resolution.
Conclusion: Although scurvy is considered a disease of the past, it still occurs nowadays. Food selectivity associated to autism is a major risk factor for vitamin C deficiency in childhood. Rheumatologists should take into account the diagnosis of scurvy in the diagnostic approach of musculoskeletal disorders in children, especially when development disorders are present.

Disclosure of Interest

None declared

\section{P188}

Interstitial lung disease in patients with rheumatic diseases

E. Atalay ${ }^{1}$, B. Ozsezen², D. D. Ersoz', E. Sag ${ }^{1}$, U. K. Akca', S. Demir ${ }^{1}$, M. K. Cuceoglu', H. U. Ozcelik², N. Kiper², E. Yalcın², N. Emiralioglu², D. A.

Tural', B. Sunman', E. D. Batu', Y. Bilginer', S. Ozen'

${ }^{1}$ Pediatric Rheumatology, ${ }^{2}$ Pediatric Pulmonology, Hacettepe University, Ankara, Turkey

Correspondence: E. Atalay

Pediatric Rheumatology 2020, 18(Suppl 2):P188

Introduction: Pulmonary involvement of systemic autoimmune and autoinflammatory diseases mostly occurs as pleural involvement and rarely as interstitial lung disease.

Objectives: The aim of this study is to retrospectively examine the demographic, clinical, laboratory findings, and clinical follow-up of patients diagnosed with interstitial lung disease during the course of rheumatic diseases.

Methods: Patients diagnosed with interstitial lung disease based on clinical and radiological findings or lung biopsy at Departments of Pediatric Rheumatology and Pulmonology in Hacettepe University, between 2005-2020 were included in the study. Data were recorded retrospectively from the patient files.

Results: There were 13 patients with systemic rheumatic disease who were diagnosed with interstitial lung disease during the course of the disease. Seven $(53.8 \%)$ of these patients were female and 6 $(46.2 \%)$ were male. The median age at the diagnosis of the rheumatic disease was 8 years. Primary rheumatic diseases were as follows; systemic juvenile idiopathic arthritis (JIA) ( $n=4 ; 30.8 \%)$, systemic sclerosis $(n=2 ; 15.4 \%)$, mixed connective tissue disease $(n=2$; $15.4 \%)$, juvenile dermatomyositis $(n=1)$, sarcoidosis $(n=1)$, granulomatous polyangiitis (GPA) $(n=1)$, Sting-associated vasculopathy with onset in infancy (SAVI) $(n=1)$, and oligoarticular JIA $(n=1)$. Respiratory symptoms were present in $6(46.2 \%)$ patients at the time of primary diagnosis. In other patients, the time period between the diagnosis of the rheumatic disease and the onset of the respiratory symptoms ranged from 1 to 12 years. Cough, the most common symptom, was present in $10(76.9 \%)$ patients. Six patients manifested with cough and sputum. Six (46.2\%) patients had shortness of breath and one patient had hemoptysis. On the physical examination of one patient, rales and clubbing were detected. High resonance computerized tomography (HRCT) was performed in all patients. HRCT findings were as follows; lymphadenopathy in 8 patients $(61.5 \%)$, ground glass appearance in 10 patients (76.9\%), consolidation in one patient, pleural effusion in one patient, pulmonary nodule in 4 patients (30.8\%), fibrosis in one patient, cystic lesions in 3 patients (23.1\%), septal thickening in 5 patients (38.5\%), bronchiectasis in one patient, and reverse halo sign in one patient. In echocardiographic examination, only one patient had pulmonary hypertension.

Three patients underwent open lung biopsy, and diagnosis was made with pathological examination of the lung tissue. Of these three patients, two (15.4\%) had lymphocytic interstitial pneumonia (LIP), and one patient had chronic inflammation and focal fibrosis. Infectious lung disease was not detected in any patient. Ten patients (76.9\%) had interstitial lung disease associated with rheumatic disease, one patient had pulmonary hemorrhage, one patient had pulmonary involvement of GPA, one patient had pulmonary involvement of sarcoidosis. There was no statistically significant difference between the first and last spirometry and DLCO values during the follow-up period. Mortality was 7.5\% (1/ 13) in this cohort. 
Conclusion: Although pulmonary complications of rheumatic diseases are rare in childhood, they can cause significant morbidity and mortality.

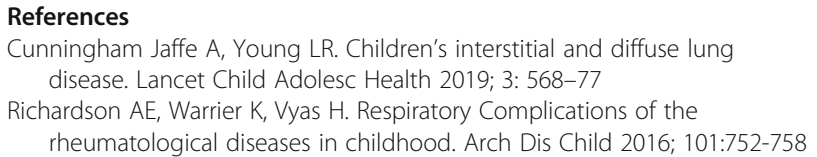

Disclosure of Interest

None declared

\section{P189}

Assessment of lipid profile in children with paediatric rheumatic diseases

S. I. Nasef', S. I. M. Abouzied'2, S. M. Elfiky ${ }^{3}$, A. Zeiton ${ }^{3}$

${ }^{1}$ Rheumatology and Rehabilitation, Faculty of Medicine, Suez Canal University, Ismailia; ${ }^{2}$ Paediatrics, Portfoad General Hospital, Portsaid; ${ }^{3}$ Paediatrics, Faculty of Medicine, Suez Canal University, Ismailia, Egypt Correspondence: S. I. Nasef

Pediatric Rheumatology 2020, 18(Suppl 2):P189

Introduction: Dyslipidemias are well-recognized risk factors for cardiovascular diseases (CVD) and are very common in patients with autoimmune rheumatic diseases. Lipid profile disorders are one of the most studied problems in adult patients with rheumatic diseases. However, few studies addressed this problem in paediatric rheumatic diseases patients.

Objectives: To assess the lipid profile in children with paediatric rheumatic diseases

Methods: Ninety patients diagnosed with pediatric rheumatic diseases were included. Exclusion criteria were patients previously treated with lipid lowering drugs, history of diabetes mellitus, thyroid disease and familial hyperlipidemia. After fulfilling the inclusion criteria, detailed history was taken. Data included age, sex, weight, body mass index (BMI), and duration of disease. Fasting lipid profiles were measured after overnight fasting and consumption of normal diet for previous 2 days (without fat restriction). Fasting lipid profiles included total cholesterol (TC), triglycerides (TG), low density lipoprotein cholesterol (LDL) and high density lipoprotein cholesterol (HDL). Normal values were considered according to reference values. Disease activity was classified according to clinical assessment and laboratory tests like ESR, CRP, complement levels and anti-ds DNA titres.

Results: The study included 90 children, suffering from rheumatic diseases. Out of them, 13 were males and 77 were females. Sixstyseven patients had juvenile idiopathic arthritis (JIA) of them, 48 patients Polyarticular type, 16 patients systemic onset type and 3 patients oligoarticular type. Twenty children were diagnosed with Juvenile systemic lupus erythematosus (jSLE), 2 children with Vasculitis (henoch schonlein purpura) and 1 child with juvenile scleroderma. Mean age was $11.58 \pm 3.6$ years. Mean age at diagnosis was $7.2 \pm 1.3$ years. Mean disease duration was $3.60 \pm 1.2$ years. Mean BMl was $20.55 \pm$ $8.42 \mathrm{Kg}$. Twenty-nine patients had active disease while sixty-one patients had inactive disease. Mean TC was $170.65 \pm 51.38 \mathrm{mg} / \mathrm{dl}$, mean $\mathrm{HDL}$ was $45.2 \pm 11.3 \mathrm{mg} / \mathrm{dl}$, mean LDL was $88.45 \pm 15.1$ and mean TG was $88.15 \pm 26.1 \mathrm{mg} / \mathrm{dl}$. The most common lipid abnormality was disturbed HDL, it was found in 29 patients followed by disturbed TC in 22 patients. Abnormal triglycerides was found in 22 patients and abnormal LDL in 16 patients. $14.9 \%$ of JIA patients had abnormal TC, $40.3 \%$ had abnormal LDL, $11.9 \%$ had abnormal LDL and $20.9 \%$ had abnormal TG. Sixty percent of jSLE patients had abnormal TC levels, $10 \%$ had abnormal HDL level, $40 \%$ had abnormal LDL and $40 \%$ had abnormal TG levels. Presence of jSLE and JIA was significantly associated with abnormal TC $\left(p<0.001^{*}\right)$, abnormal HDL $\left(p=0.005^{*}\right)$, abnormal LDL $\left(p=0.01^{*}\right)$ and abnormal TG $(p=0.07)$

Active disease was significantly associated with abnormal TC, HDL, and TG levels $\left(p=0.04^{*}\right),\left(p=0.03^{*}\right)$ and $\left(p=0.04^{*}\right)$ respectively. Multivariate analysis of the factors affecting abnormal cholesterol level revealed that
SLE is a significant predictor of abnormal cholesterol level . Presence of jSLE increase risk of abnormal cholesterol 9 times more than cases without jSLE. The overall percent predicted was $80 \%$. Active disease is a significant risk factor for abnormal TG with increased risk of abnormal TG by 3.2 among cases with active disease than cases with inactive disease. The overall percent predicted was $75.6 \%$.

Conclusion: children with rheumatic diseases showed significant lipid profile abnormalities. Abnormal TC, HDL and TG are significantly associated with active disease. Presence of jSLE increase risk of abnormal cholesterol. Active disease is a significant risk factor for abnormal TG. Therefore, lipid levels should be monitored regularly and managed in patients with paediatric rheumatic diseases to minimize the longterm risk of CVD.

\section{Disclosure of Interest}

None declared

\section{P190}

Definitions and concepts of muscular skeletal pain - what do physicians know?

V. L. Martins' ${ }^{1}$ M. Fernandes ${ }^{1}$, F. Costa ${ }^{2}$, A. Vaz ${ }^{1}$, M. Salgado

${ }^{1}$ Hospital Pediátrico de Coimbra; ${ }^{2}$ Centro Hospitalar e Universitario de Coimbra, Centro Hospitalar Universitário de Coimbra, Coimbra, Portugal

Correspondence: V. L. Martins

Pediatric Rheumatology 2020, 18(Suppl 2):P190

Introduction: Musculoskeletal pain (MSP) is the most frequent reason for seeking health care in adult and paediatric rheumatology. Semiological accuracy and the use of a standardized language will allow correct communication, avoiding ambiguities in definition and interpretation that may culminate in diagnostic errors. At least 82 concepts / definitions and clinical situations associated with musculoskeletal pain are known.

Objectives: Evaluate physicians' knowledge of the most used definitions of MSP in order to improve patient attendance.

Methods: Non-experimental, cross-sectional and descriptive study. A confidential survey was conducted online, aimed at residents and attendings who deal with musculoskeletal pain. Were addressed with the definitions of arthralgia, arthritis, myalgia, allodynia and hyperesthesia (between five to seven options) with only one correct answer.

Correct definitions: arthralgia (pain localized to the joint or periarticular structures, as a only manifestation); arthritis (Criterion one or criterion two: 1 - Joint swelling or intra-articular effusion / 2 Limitation of joint mobilization associated with at least one of the following: a) Pain b) Tenderness c) Swelling d) Heat); myialgia (pain with muscular origin or referred to muscle, regardless of its etiology); allodynia (pain resulting from usually non-painful stimulus); hyperesthesia (coexistence of allodynia plus hyperalgesia - exaggerated responses to tactile and thermal nociceptive and nonnociceptive stimuli). The study took place between September and October 2018.

Results: A total of 193 physicians participated, $71.5 \%$ (138) attendings and $28.5 \%$ (55) residents. Distribution by specialty: Paediatrics $51.3 \%$ (99), Rheumatology $14 \%$ (27), General and Family Medicine (GFM) $14 \%$ (27), Orthopedics $8.8 \% \quad$ (17), Paediatric Rheumatology $4.2 \%$ (8), Physical and Rehabilitation Medicine (PRM) $2 \%(4)$, other specialties $5.7 \%$ (11). Overall, the correct answers to each assessed item have the following distribution: isolated arthralgia $41.5 \%$ (80), arthritis $17.6 \%$ (34), myalgia $54.9 \%$ (106), allodynia $61.1 \%$ (118), hyperesthesia $64.8 \%$ (125). By specialty, the percentage of correct answers is shown in table 1.

Conclusion: The low percentage of correct answers, allows us to conclude that many physicians are unaware of the correct concepts / definitions related to MSP and do not evaluate the musculoskeletal system routinely, or in an appropriate way. This tendency must be counteracted through continuous medical training.

Disclosure of Interest: None declared 
Table 1 (abstract P190) Concept and percentage of correct answers by specialty

\begin{tabular}{|c|c|c|c|c|c|}
\hline & $\begin{array}{l}\text { Isolated } \\
\text { arthralgia }\end{array}$ & Arthritis & Myalgia & Allodynia & Hyperesthesia \\
\hline Paediatrics & $51.5 \%(51)$ & $\begin{array}{l}18.2 \% \\
(18)\end{array}$ & $\begin{array}{l}46.5 \% \\
(46)\end{array}$ & $\begin{array}{l}50.5 \% \\
(50)\end{array}$ & $59.6 \%(59)$ \\
\hline Rheumatology & $77.8 \%(21)$ & $\begin{array}{l}25.9 \% \\
(7)\end{array}$ & $\begin{array}{l}55.6 \% \\
(15)\end{array}$ & $\begin{array}{l}81.5 \% \\
(22)\end{array}$ & $77.8 \%(21)$ \\
\hline GFM & $25.9 \%(7)$ & $7.4 \%(2)$ & $\begin{array}{l}77.8 \% \\
(21)\end{array}$ & $\begin{array}{l}66.7 \% \\
(18)\end{array}$ & $70.4 \%$ (19) \\
\hline $\begin{array}{l}\text { Paediatric } \\
\text { Rheumatology }\end{array}$ & $75.0 \%(6)$ & $\begin{array}{l}50.0 \% \\
(4)\end{array}$ & $\begin{array}{l}50.0 \% \\
(4)\end{array}$ & $75.0 \%(6)$ & $50.0 \%(4)$ \\
\hline Orthopedics & $29.4 \%(5)$ & $5.9 \%(1)$ & $\begin{array}{l}58.8 \% \\
(10)\end{array}$ & $\begin{array}{l}70.6 \% \\
(12)\end{array}$ & $58.8 \%(10)$ \\
\hline PRM & $25.0 \%(1)$ & $0.0 \%(0)$ & $\begin{array}{l}75.0 \% \\
(3)\end{array}$ & $75.0 \%(3)$ & $75.0 \%(3)$ \\
\hline Overall & $41.5 \%(80)$ & $\begin{array}{l}17.6 \% \\
(34)\end{array}$ & $\begin{array}{l}54.9 \% \\
(106)\end{array}$ & $\begin{array}{l}61.1 \% \\
(118)\end{array}$ & $64.8 \%(125)$ \\
\hline
\end{tabular}

\section{P191}

Pure red cell aplasia, type 1 diabetes and poly arthritis in a 2.5 years old girl with a syndromic face - a new disease

D. B. Pandya on behalf of Dr Nishant Dharsandiya and Dr J.P. Keshrani Paediatric Rheumatology \& Immunology, Dev Children's Hospital, Rajkot, India

Correspondence: D. B. Pandya

Pediatric Rheumatology 2020, 18(Suppl 2):P191

\section{Introduction}

The association of pure red cell aplasia (PRCA) with thymoma led to the discovery of the autoimmune mechanisms involved in the pathogenesis of this rare disease. Till date many adult case reports have revealed a strong link between PRCA and autoimmune diseases, endocrine disorders, rheumatic diseases, autoinflammation and immune dysregulation. ${ }^{1-5}$

\section{Objectives}

To stimulate a search for the genetic and immunological roots for a 2.5 years old girl with syndromic face, pure red cell aplasia, type 1 diabetes and polyarthritis.

Methods

This is a story of a 2.5 years old girl with pure red cell aplasia, type 1 diabetes and polyarthritis. She was normal till 7 months of age. At the age of 8 months, she was diagnosed with type 1 diabetes. She was evaluated by her paediatrician in view of generalized hypotonia, deformed pinna, low set ears, midfacial hypoplasia, blue sclera, umbilical hernia and retracted eyelids. She had multiple episodes of seizures during next few months. To me, she was presented with one year history of polyarthritis with severe pallor requiring frequent blood transfusions. Family history was inconclusive. Musculoskeletal examination showed polyarthritis involving right knee, bilateral ankles, fingers and toes. Further examination revealed haemolytic facies and hepatosplenomegaly. I was not able to make out any facial dysmorphism mentioned earlier by her paediatrician.

Results: Table 1

\section{Conclusion}

Early age of onset, pure red cell aplasia, autoimmune and endocrine manifestations with some doubtful facial dysmorphism inspired me to suspect some known or unknown immune dysregulation syndrome in this child. Genetic analysis would be the best possible option in this scenario if financial condition permits.
Trial registration identifying number

1. Mol Immunol 2020 May;121:28-37. doi: 10.1016/ j.molimm.2020.02.014. Epub 2020 Mar 6. Autoinflammation in Addition to Combined Immunodeficiency: SLC29A3 Gene Defect Deniz Çağdaş 1, Naz Sürücü 2, Çağman Tan 3, Başak Kayaoğlu 2, Rıza Köksal Özgül 4

2. J Clin Pathol. 2007 Jun; 60(6): 717-720. Published online 2007 Jan 12. doi: $10.1136 / j c p .2006 .042671$ Pure red cell aplasia associated with type I autoimmune polyglandular syndrome-successful response to treatment with mycophenolate mofetil: case report and review of literature Milena Bakrac, Vladimir Jurisic, Tanja Kostic

3. J Pediatr Hematol Oncol. 2019 Nov;41(8):e542-e545. doi: 10.1097/ MPH.0000000000001292. Acquired Pure Red Cell Aplasia and Acquired Amegakaryocytic Thrombocytopenia Associated With Clonal Expansion of T-Cell Large Granular Lymphocytes in a Patient With Lipopolysaccharide-responsive Beige-like Anchor (LRBA) Protein Deficiency. Rajpurkar M, Buck S, Lafferty J, Wakeling E, Ravindranath Y, Savaşan $S$.

4. Indian J Pediatr 2015 Dec;82(12):1172-4. doi: 10.1007/s12098-0151779-2. Epub 2015 May 15.

Autoimmune Lymphoproliferative Syndrome With Red Cell Aplasia

K R Meena 1, Supriya Bisht 2, K C Tamaria 1

5. Eur J Pediatr 2011 Dec;170(12):1611-5. doi: 10.1007/s00431-0111588-1. Epub 2011 Oct 7.

A Novel Mutation and Unusual Clinical Features in a Patient With Immune Dysregulation, Polyendocrinopathy, Enteropathy, X-linked (IPEX) Syndrome. Keun Wook Bae 1, Bo Eun Kim, Jin-Ho Choi, Joo Hoon Lee, Young Seo Park, Gu-Hwan Kim, Han Wook Yoo, Jong Jin Seo

Disclosure of Interest: None declared

Table 1 (abstract P191) showed available investigation reports

\begin{tabular}{ll}
\hline Haemoglobin (11.5-14.5 gm/dl) & 3.6 \\
RBC Count (4-5.3 mill/cumm) & 1.16 \\
PCV (33-43\%) & 10.4 \\
WBC count(4000-12000 cells/ & 9100 \\
cumm) & \\
ANC (1800-6800 cells/cumm) & 5551 \\
ALC (1100-4700cells/cumm) & 3185 \\
Platelet count (1,50,000- & $5,62,000$ \\
4,50,000/ul) & \\
MCV (76-90 Fl) & 89.8 \\
Peripheral smear & Normocytic Normochromic RBCs \\
Reticulocyte count (0.8-2\%) & $0.5 \%$ \\
Absolute reticulocyte count & 0.0005 \\
(0.02-0.1 * 106 cells/ul) & \\
ESR (0-20 mm/hr) & 135 \\
CRP (0-5 mg/L) & 1.1 \\
HBA1c (<5.6\%) & 12.6 \\
TSH (0.45-4.5 microlU/ml) & 4.04 \\
Free T4 (0.91-1.44 ng/dl) & 1.24 \\
Bone Marrow Examination & Normocellular marrow with markedly reduced \\
ANA by IIF & erythropoiesis and occasional lymphoid \\
RF & Negative \\
GAD antibody & Negative \\
MRI Brain & \\
\hline
\end{tabular}


P192

Inflammatory arthritis complicating galactosialidosis: a case report and review of the literature

F. Verkuil ${ }^{1,2}$, A. M. Bosch ${ }^{3}$, P. A. Struijs ${ }^{4}$, R. Hemke², J. M. van den Berg

${ }^{1}$ Pediatric Immunology, Rheumatology and Infectious Diseases;

${ }^{2}$ Radiology and Nuclear Medicine; ${ }^{3}$ Pediatric Metabolic Diseases;

${ }^{4}$ Orthopedic Surgery, Amsterdam UMC, location AMC, Amsterdam,

Netherlands

Correspondence: F. Verkuil

Pediatric Rheumatology 2020, 18(Suppl 2):P192

Introduction: Galactosialidosis (GS) is a rare inherited lysosomal storage disorder (LSD) which is characterized by a defect in the lysosomal glycoprotein catabolism. Here we report, for the first time, a case of a child affected by GS who presented with recurrent episodes of extensive joint inflammation in both knees. Knowledge on GS related inflammatory joint pathology is lacking, which hampers evaluation of possible mechanisms that could give an explanation for the significant arthritic joint abnormalities as observed in our patient.

Objectives: The aim of this study is to describe the clinical presentation as well as the laboratory, radiologic and microscopic features of this extremely rare presentation of GS. Furthermore, we conduct a literature review on LSD's complicated by arthritis in order to evaluate potential mechanisms that could explain the extensive inflammatory joint swelling observed in our patient.

Methods: In this study we present a 12-year-old Turkish boy who was diagnosed with GS (late infantile form) at 17 months of age. From the age of 8 years, the boy presented with episodes of inflammatory joint pathology of the knee. Informed consent was obtained.

Alongside the case report, a literature review using Medline was conducted. An extensive list of known LSD's was combined with the terms: "arthritis", "joint inflammation", "synovitis" and "synovial inflammation". Cases in which joint inflammation was based on a probable cause other than the underlying LSD were excluded.

Results: In the present case, owing to comprehensive examinations (i.e. laboratory tests, imaging and microscopic examination) multiple possible causes for the recurrent inflammatory joint pathology could be rejected (i.e. no signs of infectious arthritis, reactive arthritis, osteoarthritis, arthritis secondary to a malignancy or crystal induced arthritis). A diagnosis which could explain the clinical picture is the JIA subtype: ANA negative oligo-articular JIA. However, microscopic examination showed numerous foamy macrophages with extensive vacuolization in the synovial tissue of the inflamed joint, which is not associated with JIA. Given the evidence of storage products within the macrophages of the inflamed synovial tissue and no conclusive diagnosis, GS itself should be considered as the primary cause for the recurrent arthritis.

An in-depth literature review using Medline for data on inflammatory joint pathology in LSD's showed that 7 LSD subtypes (i.e. Fabry disease, Farber lipogranulomatosis, Gaucher disease type 1, Mucopolysaccharidosis IX, a-Mannosidosis, Fucosidosis and Cystinosis) could present with disease related arthritis. Multiple potential arthritic mechanisms secondary to storage product accumulation in LSD's have been described, such as: dysregulation of innate immunity and increased upregulation of numerous pro-inflammatory proteins.

Conclusion: Given the evidence of storage products within macrophages of the inflamed synovial tissue and the absence of other etiological clues, our hypothesis is that GS itself is the primary cause for the inflammatory joint pathology in our patient. Although, GS cannot be linked directly to joint inflammation, lysosomal defects have been associated to pro-inflammatory effects that possibly could result in arthritic disease. Future identification of other patients with
GS is required to support the hypothesis of an arthritic clinical phenotype of GS and to assess underlying pathophysiology.

Disclosure of Interest: None declared

P193

Recurrent oral ulcers-a novel mutation

S. Guha

Pediatric, VIMS, Saltlake, Kolkata, India

Pediatric Rheumatology 2020, 18(Suppl 2):P193

Introduction: A 1 year old male infant presented with h/o recurrent oral ulcers and fever since 6 months of age. Provisional diagnosis were 1. Cyclic neutropenia. 2.Infantile behcet.3.A20 Haploinsuffiency

Objectives: To establish cause of recurrent fever and oral ulcers, to rule out immune deficiency

Methods: Blood sent for-Complete blood count, primary immune deficiency work up which included immunoglobulin levels, flow cytometry for lymphocytes subsets and phagocytic defects

Results: Thinking of cyclic neutropenia, a complete blood count was done twice a week for 6 weeks.There was no evidence of neutropenia.Primary immune deficiency work up was negative.Genetic analysis was sent to rule out A20 haploinsuffiency. However clinical exome sequencing showed homozygous deletion of MAG TI gene.

Conclusion: So this was a case of MAGTi deficiency.

The infant showed homozygous deletion and later on may be complicated with recurrent EBV infection and lymphomas.MAGT1deficiecy is an $\mathrm{x}$ linked immunodeficiency characterized by CD4 lymphopenia, and defective Tcell activation. These individuals have recurrent viral infections especially with Ebstein Barr Virus. They also have associated lymphoprolifeerative diseases especially lymphoma. Sometimes magnesium salts may prove useful in treatment. The index case is on magnesium and doing well. Though the usual age of presentation I 3to 45 years, our patient presented very early.

Later on they may have viral skin infections like molluscum,sinopulmonary infections and viral pneumonia.

The main aim of presentation is to

1.recognize immune deficiency early in life and not to ignore them 2.treat them accordingly

Disclosure of Interest: None declared

P194

Withdrawn

P195

Joint pain in children and adolescents: occurrence, causes and age profile

A. Kozhevnikov ${ }^{1,2}$, N. Pozdeeva', M. Nikitin', A. Moskalenko', Y.

Proshchenko', G.'Novik ${ }^{2}$

${ }^{1}$ H.Turner National Medical Research Center for Children's Orthopedics and Trauma Surgery; ${ }^{2}$ St. Petersburg State Pediatric Medical University, Saint-Petersburg, Russian Federation

Correspondence: A. Kozhevnikov

Pediatric Rheumatology 2020, 18(Suppl 2):P195

Introduction: Joint pain (JP) is a relatively common complaint among children and adolescents. A painful joint in children for many years continues to maintain the status of the most common symptom of juvenile arthritis. However this symptom should not always be interpreted as a manifestation of rheumatic diseases.

Objectives: The aim of current review is to debate of the structure in children with the chief complaint of JP. 
Methods: We retrospectively analysed our series of 600 children which attending outpatient department with complaint about pain lasting longer than two months in one or more joints. The clinical, instrumental and laboratory pictures were collected. Special attention was paid to certain aspect of medical complaints, a complete and accurate history and physical examination. Different categories as possible etiologies of JP in children were systematize and detailed.

Results: All children were divided into several groups based on their anatomical and physiological characteristics of osteoarticular system: the first group consisted of 240 children under 6-7 years old, the second group - 220 children 7-12 years old, the third group - 140 children over 12 years old.

Research suggests that more preschool children were experience bilateral lower extremity pain by "post-walk genesis" due to natural hypermobility, immaturity of sensory innervation of the joints and imbalance of the leg muscles (e.g. growing pains). The second most common cause of JP was associated with intra - or postinfectious factor (viral, streptococcal and chronic focal of infection). The frequency of juvenile arthritis and other rheumatic diseases in children of this age group did not exceed $10 \%$. Special attention was paid to fever, chills, malaise, nightpain and constitutional symptoms with changes in blood lab tests to exclude osteomyelitis (inc specific cause), malignancies manifestation and other bone tumors (less 5\%). The most common causes of joint pain of school-age children were hypermobility syndrome and enthesopathy (primary, secondary). Secondary enthesopathy were result of changes in nutrition, rapid growth and excessive exercise. Also enthesopathy were manifestation of endocrine, gastrointestinal or infectious diseases. The proportion of children with the onset of chronic inflammatory arthropathy also did not exceed $10 \%$. Hypermobility child's syndrome was characterized by harmless pain (inc low back pain), linked to physical activity (less morning stiffness).

Over the past decade, we've seen a gradual increase in the number of children (95\% were girls) with knee pain by diagnosed patellofemoral and mediopatellar plica syndromes, patellar tendinitis or idiopathic cause. In most cases children was complicated by syndrome of increased anxiety. The share of true chronic inflammatory arthropathies, including spondylitis, in children of this age group did not exceed $10 \%$. Fibromyalgia were diagnosed less $5 \%$.

Conclusion: Despite continuous improvements in examination technique and image quality there is no universal test to diagnose cause of chronic childhood arthralgia. Age features, individual nature of pain perception, the high frequency of incomplete and transient forms of arthropathy, cases atypical joint diseases have been intriguing problems for diagnostic pathology. Integrated assessment modelling framework of the clinical and instrumental pictures with understanding of the anatomical and physiological characteristics of childhood will help identify the true cause of chronic musculoskeletal pain.

Disclosure of Interest: None declared

\section{P196}

The main differences between children with inflammatory and non-inflammatory pain genesis

M. Dubko', F. Rokhlina ${ }^{2}$, E. Shebedya ${ }^{3}$

${ }^{1}$ Saint Petersburg State Pediatric Medical University; ${ }^{2}$ H.Turner National Medical Research Center for Children's Orthopedics and Trauma Surgery, Saint Petersburg; ${ }^{3}$ H.Turner National Medical Research Center for Children's Orthopedics and Trauma Surgery, Saint-Petersburg, Russian Federation

Correspondence: M. Dubko

Pediatric Rheumatology 2020, 18(Suppl 2):P196

Introduction: A significant part of patients in rheumatologist's practice is children and teenagers with complaints of pain. The further volume of examination and the choice of treatment course depends on the capability of the rheumatologist to define the inflammatory and non-inflammatory genesis of pain. That makes the problem of differential diagnosis very important.
Objectives: To conduct a comparative analysis of patients with a principal pain complaint to determine if there are significant differences in the groups with the inflammatory and noninflammatory pain genesis.

Methods: The retrospective study included children who consulted a rheumatologist in the outpatient clinic in the period 2018-2020 without preliminary selection $(n=176)$. Of them there were selected children with principal pain complaint $(n=120)$. According to the diagnosis, the children were divided into 2 groups: those who have inflammatory genesis of pain $(A, n=59)$ and those with noninflammatory genesis of pain $(B, n=61)$. The group $A$ included children with such diagnoses as: reactive, poststreptococcal and juvenile idiopathic arthritides. The group B included children with arthralgia, chronic pain syndrome, orthopedic pathology, fibromyalgia.

Results: 1. Groups A and B differ in the average age of the first complaints onset (t-criterion for equality of means) with a high degree of statistical significance (Group $A=7,4$ years; Group $B=9,3$ years; $p=$ 0.019). Which means that in Group A more often than in Group B first complaints appear in the age between 1 to 10 while in Group B more often than in Group A it happens in the age between 11 to 16 .

2. There was a statistically significant difference in the means between Groups A and B in time between the onset of first complaints and the first visit to a rheumatologist $(p=0.03)$

Also in favor of this conclusion speaks the fact that in Group A the number of visits to a rheumatologist in the same year when the first complaints appear is almost 2 times higher than in Group B.

$56 \%$ of cases in Group A consulted the rheumatologist the same year when the first complaints appeared in comparison to Group B where only $31 \%$ of patients did the same.

Below is the table with distribution of cases by the number of years between the first complaints onset and the first visit to a rheumatologist in both groups:

\begin{tabular}{lll}
\hline Number of years & Group A & Group B \\
\hline 0 & $56 \%$ & $31 \%$ \\
1 & $24 \%$ & $30 \%$ \\
2 & $10 \%$ & $16 \%$ \\
3 & $7 \%$ & $7 \%$ \\
4 & $2 \%$ & $5 \%$ \\
5 & $2 \%$ & $7 \%$ \\
6 & $0 \%$ & $0 \%$ \\
7 & $0 \%$ & $3 \%$ \\
8 & $0 \%$ & $0 \%$ \\
\hline
\end{tabular}

Conclusion: In children with arthritides, the first pain complaints appear at an earlier age (an average of 7.4), and in Group B (an average of 9.3). Patients with arthritis more often visit a rheumatologist earlier (within 1 year after the first complaints) than those with non-inflammatory genesis of pain complaints.

Disclosure of Interest: None declared

\section{P197}

Can artifical intelligence help with diagnosing growing pain?

E. D. Batu ${ }^{1,2}$, F. Akal ${ }^{3}$, S. G. Karadağ ${ }^{4}$, F. Demir ${ }^{5}$, N. Aktay Ayaz ${ }^{4}$, B. Sözeri ${ }^{5}$

${ }^{1}$ Department of Pediatrics, Division of Rheumatology, Hacettepe

University Faculty of Medicine; ${ }^{2}$ Department of Pediatrics, Division of

Rheumatology, Ankara Training and Research Hospital, University of

Health Sciences; ${ }^{3}$ Department of Computer Engineering, Hacettepe

University, Ankara; ${ }^{4}$ Department of Pediatrics, Division of Rheumatology,

Kanuni Sultan Suleyman Training and Research Hospital, University of

Health Sciences; ${ }^{5}$ Department of Pediatrics, Division of Rheumatology,

Umraniye Training and Research Hospital, University of Health Sciences,

Istanbul, Turkey

Correspondence: E. D. Batu

Pediatric Rheumatology 2020, 18(Suppl 2):P197 
Introduction: The most common cause of recurrent musculoskeletal pain is growing pain (GP) in children. Differential from rheumatic diseases could be challenging in some cases since there are no diagnostic criteria for GP.

Objectives: To analyze GP characteristics in a large cohort of patients in comparison with other non-inflammatory and inflammatory diseases causing limb pain, and to simplify the GP's diagnosis process by using machine learning $(\mathrm{ML})$ techniques.

Methods: This is a multicenter cross-sectional study. From February 2019 through August 2019, patients with GP and diseased controls were enrolled at the pediatric rheumatology units of three centers from Turkey. The gold standard for diagnosis of GP was the expert opinion.

A total of 398 patients with growing pain were enrolled (157 from Ankara Training and Research Hospital, Ankara; 128 from Umraniye Research and Training Hospital, Istanbul; and 113 from Kanuni Sultan Suleyman Training and Research Hospital, Istanbul, Turkey). The control group consisted of 254 patients with diseases causing limb pain other than GP; 212 of these had inflammatory diseases (e.g. juvenile idiopathic arthritis), while the etiology of limb pain was noninflammatory in 42 patients (e.g., trauma).

Once the data obtained from the participating hospitals as an Excel table, we performed exploratory data analysis. Consequently, columns with the missing value rate of more than $20 \%$ were removed. Iterative imputation methods were used to complete the rest of the missing values. Afterward, correlations among columns were investigated, and collinearity was removed. Finally, the data set used in this study consisted of 652 rows and 29 columns. We refer to columns as features as in ML vocabulary. Next, we developed several ML models by using a 10 -fold cross-validation method with algorithms frequently used in similar problems in literature.

Results: The female-to-male ratio was 1.3 , and the median age was $102(22-213)$ months in the GP group $(n=398)$. The pain was bilateral (86.2\%), localized at lower extremities (89.7\%), nocturnal (74\%), and led awakening at night $(60.8 \%)$ in the majority of GP patients. The pain was not daily $(58.4 \%)$ and was exacerbated by increased physical activity during the day (57.3\%) in more than half of the patients. History of arthritis, trauma, morning stiffness, limping, limitation of activities, and school abstinence were more prevalent among diseased controls then GP patients ( $p=0.016$ for trauma and $p<0.001$ for others). Hypermobility and pes planus were more frequent in the GP group than controls $(p<0.001$ and $p=0.02$, respectively). Anemia, leukocytosis, thrombocytosis, and elevated acute phase reactants were more prevalent among diseased controls than GP patients ( $p=$ 0.013 for thrombocytosis and $p<0.001$ for the rest).

Our experiments with different $\mathrm{ML}$ models revealed that the Random Forest (RF) algorithm provided with 0.98 accuracy, 1.0 sensitivity, and 0.97 specificity in our test set.

Conclusion: In our cohort, GP was bilateral, localized at lower extremities, nocturnal, and led awakening at night, which were consistent with the previous reports. Our cohort is the largest cohort of children with GP. We also developed an ML model to identify GP patients based on clinical features. The results show that our RF model can be used to facilitate diagnosing GP disease. To the best of our knowledge, this is the first study that attempts to diagnose GP in children by using $M L$ techniques.

Disclosure of Interest: None declared

\section{P198}

Structural and functional status of the bone tissue in children during growth spurt

N. Osman, T. Frolova, N. Stenkova, I. Siniaieva

Kharkiv National Medical University, Kharkiv, Ukraine

Correspondence: N. Osman

Pediatric Rheumatology 2020, 18(Suppl 2):P198

Introduction: It is a well-known fact that the period of intensive growth in children is associated with the processes of active bone mass accumulation and coincides with them in time. One of the most distinctive indicators of an increase in the disease incidence among children for the recent decade $(+105,3 \%)$ can be found in the skeletal disorders resulting from disrupted calcium metabolism and vitamin $\mathrm{D}$ deficit. The latter is widespread in Ukraine as it is observed in $92 \%$ of schoolers.

Objectives: Establish the specifics of the structural and functional status of the bone tissue in children during the growth spurt, taking account of the degree of vitamin D3 sufficiency.

Methods: The examination covered 147 children aged 9-17 who were divided into three groups depending on the presence of the growth spurt (GS) and its intensity: group $1-35$ children who had become 8-12 cm taller for the year in question; group $2-32$ children who had become taller by $12 \mathrm{~cm}$ or more, group $3-80$ children who had experienced no growth spurt. Inclusion criteria were the following: no chronic somatic or endocrine pathologies, no musculoskeletal disorders or mineral homeostasis disruptions; physical exertion corresponding to their age; the children had not been taking any complexes of vitamins and minerals, including vitamin $D_{3}$ for 6 months before the examination.

The examination included analysis of the medical history, general clinical examination and assessment of physical development (WHO "Child Growth Standards", 2007). Additional tests included ELISA aimed at determining the $25(\mathrm{OH}) \mathrm{D}_{3}$ level as well as Ultrasound densitometry (Sonost -2000, Korea) and X-ray densitometry(DXA) (HOLOGIC QDR W Explorer, USA). Z-score $\leq-2$ was used as a criterion determining reduced bone mineral density (BMD) (The International Society For Clinical Densitometry, 2013).

Results: Reduced BMD was found in $51,42 \%$ of the children in $1^{\text {st }}$ group, $62,5 \%$ of the children in the $2^{\text {nd }}$ group and $35,0 \%$ of the children in the control group. All the children under examination experienced a deficiency of $25(\mathrm{OH}) \mathrm{D}_{3}$ (M.F. Holicke at all., 2011). In the children of the $1^{\text {st }}$ group, the average $25(\mathrm{OH}) \mathrm{D}_{3}$ level reached $38,87 \pm$ $9,96 \mathrm{nmol} / \mathrm{L}$. In the children of the $2^{\text {nd }}$ group, the average $25(\mathrm{OH}) \mathrm{D}_{3}$ level was $42,58 \pm 8,99 \mathrm{nmol} / \mathrm{L}$. In the 3 rd group, the $25(\mathrm{OH}) \mathrm{D}_{3}$ level reached $40,68 \pm 9,29 \mathrm{nmol} / \mathrm{L}$ on average.

Spearman's correlation showed that in groups 1 and 2 there was no interrelation between the levels of $25(\mathrm{OH}) \mathrm{D}_{3}$ and $\mathrm{BMD}$; in group 3 , it revealed a positive relation between the $25(\mathrm{OH}) \mathrm{D}_{3}$ level and $\mathrm{BMD}(\mathrm{r}=0,45)$. Conclusion: Children aged 9-17 showed deficiency of vitamin $D_{3}$ reaching 100\% which had no correlation with the presence or intensity of the growth spurt. In children who experienced growth spurt, a reduced BMD proved more frequent and correlated with the spurt intensity, however, it did not depend on sufficiency of vitamin $\mathrm{D}_{3}$. Therefore, during the growth spurt, disrupted mineralization of the bone tissue was influenced not only by the vitamin $D$ deficit but also by the correlation between the bone tissue mineralization rate and intensity of growth in the children.

Disclosure of Interest: None declared

\section{P199}

Patient perspectives on living with sJIA or Still's disease: impact on quality of life

R. Sinha' ${ }^{1}$ K. Durrant ${ }^{2}$, M. C. Marques ${ }^{3}$, N. Tennermann ${ }^{3}$, P. Reardon' ${ }^{1}$ J. Tousseau', S. Angevare ${ }^{4,5}$, S. Lapidus', G. S. Schulert', F. Dedeoglu ${ }^{3}$

${ }^{1} \mathrm{~s} J$ IA Foundation, National Organization; ${ }^{2}$ Autoinflammatory Alliance, San

Francisco; ${ }^{3}$ Boston Children's Hospital, Boston, ${ }^{4}$ Autoinflammatory

Alliance, National Organization, United States; ${ }^{5}$ KAISZNAISZ, ENCA,

National Organization, Netherlands; ${ }^{6}$ Hackensack Meridian, Hackensack;

${ }^{7}$ Cincinnati Children's Hospital, Cincinnati, United States

Correspondence: R. Sinha

Pediatric Rheumatology 2020, 18(Suppl 2):P199

Introduction: Systemic juvenile idiopathic arthritis (sJIA) and Adultonset Still's disease (AOSD) are related arthropathies with features of autoinflammation and onset in children and adults, respectively. Due to their chronic course, we hypothesized that sJIA and AOSD have negative impact on quality of life (QOL). We also hypothesized that children with sJIA, adults diagnosed with sJIA as children, and adults with AOSD have differing effects on QOL.

Objectives: To investigate patient-reported factors underlying the negative impact of $s J A$ and AOSD on QOL. 
Methods: A self-reported 25 question online survey on $\mathrm{QOL}$ of patients with $s J A$ and AOSD was developed by the non-profit organizations, the Autoinflammatory Alliance, KAISZ/VAISZ, ENCA and sJIA Foundation in English and translated to Dutch. Respondents were recruited by convenience sampling through online social media posts. Data on flares, triggers, family history, and correlation of symptoms with labs were collected in addition to detailed information on QOL during and in-between flares.

Results: Between 2017 and 2019, there were 109 responses; 54 were from parents of children with SJIA, 18 from adults with $\mathrm{SJIA}$, and 37 from adults with AOSD. Interestingly, adults (whether diagnosed with sJIA or AOSD) were more likely to report pain, fatigue, joint swelling or arthritis, nausea \& vomiting, and diarrhea during flares than children. Adults were also more likely to describe flares >one month. $80 \%$ of patients reported being "greatly" or "severely" limited during flares. Between flares, $20 \%$ reported being "greatly" or "severely" limited while 59\% were "somewhat" limited. $80 \%$ felt their condition affected their studies, job, and career, including $66 \%$ of children with sJIA, $100 \%$ of adults with sJIA, and $92 \%$ with AOSD.

Respondents were asked open-ended questions regarding their experience with disease flares and impact on their lives, and specifically how SJIA and AOSD affected work, career and schooling. Responses regarding the disease experience were classified into 7 theme areas: 1) experience with disease onset and process of diagnosis; 2) health care access, quality, and drug safety concerns; 3 ) physical impact of the disease including pain and chronic fatigue; 4) social impact of the disease; 5) mental health and emotional impact of the disease; 6) impact on work, career, and employment; and 7) broad impact on life and lifestyle. Responses regarding effect on work, career, and schooling were categorized into 3 theme areas: 1) physical impact negatively influencing school/work productivity; 2) lost work and wages, including unemployment and needing disability benefits, and parents missing work to care for the child; and 3) the socialemotional impact as well as negative effects on mental health. About half of patients regardless of age reported the name sJIA did not represent well the disease, specifically that it did not emphasize the systemic symptoms, and that the disease gets confused with other types of arthritis. Adult patients with sJIA did not like to have juvenile in the name.

Conclusion: Children and adults with sJIA and AOSD report high levels of QOL limitation and effect on school, work, and career, both during and between flares. Our qualitative data emphasizes the importance of multidimensional evaluation of disease with ongoing input from the patients, which will provide a foundation for asking more relevant research questions to foster better care and improve QOL.

Disclosure of Interest: R. Sinha: None declared, K. Durrant: None declared, M. Marques: None declared, N. Tennermann: None declared, P. Reardon: None declared, J. Tousseau: None declared, S. Angevare : None declared, S. Lapidus: None declared, G. Schulert Consultant for: Novartis and SOBI, F. Dedeoglu Consultant for: Novartis

\section{P200}

Reducing fatigue in paediatric rheumatic conditions: a systematic review

K. Kant-Smits, M. van Brussel, J. Van Der Net

Center for Child Development, Exercise and Physical literacy, University

Medical Center Utrecht; Wilhelmina Children`s Hospital, Utrecht, Netherlands

Correspondence: J. Van Der Net

Pediatric Rheumatology 2020, 18(Suppl 2):P200

Introduction: Although fatigue is a generic and profound symptom among children and adolescents with Paediatric Rheumatic Conditions (PRCs), intervention studies designed for reducing fatigue in PCRs are limited.

Objectives: To systematically review contemporary evidence regarding the efficacy of interventions intended to reduce fatigue in patients with PRCs.

Methods: Comprehensive electronic searches were performed in PubMed/ MEDLINE, Embase, Web of Science and Cinahl. Risk of bias was assessed using the 'Revised Cochrane risk-of-bias tool for randomized trials' and 'Quality Assessment Tool for Before-After Studies With No Control Group' for respectively studies with and without a control group. Level of evidence was assessed according to the rating system of Proper et al.

Results: Eight out of 385 studies were included for review with a total of 213 participants (age range 5-23 years). Applied interventions included exercise therapy, prednisolone therapy, vitamin-D supplementation, psychological therapy and a transition programme. Fatigue was assessed with self-reported questionnaires in all included studies. Four studies displayed a small but significant positive effect, one study a small non-significant positive effect and three studies found no effects in reducing subjective fatigue. Five studies showed a high risk of bias and three studies showed a moderate risk of bias. Conclusion: Insufficient evidence is provided to substantiate the efficacy of current interventions to reduce fatigue in PCRs. The low number of studies, non-comparable interventions, risk of bias and inclusive outcomes of the included studies denote future research should focus more on understanding the underlying mechanisms of fatigue. Identification and development of and need to effective multifactorial interventions to reduce complaints of fatigue in children and adolescents with PCRs is warranted.

Trial registration identifying number: NA

Disclosure of Interest: None declared

P201

Quality of life in juvenile idiopathic arthritis patients in Latvia

A. Baršuka ${ }^{1}$ I. Saulitete ${ }^{1,2}$, K. Pudāne ${ }^{1}$, S. Feierābende ${ }^{2}$, I. Rumba-

Rozenfelde ${ }^{1,2}$

${ }^{1}$ University of Latvia; ${ }^{2}$ Children's Clinical University Hospital, Riga, Latvia

Correspondence: A. Baršuka

Pediatric Rheumatology 2020, 18(Suppl 2):P201

Introduction: Dimensions of health releated quality of life (HRQoL) includes both physial and psychosocial well-being and reveals how health status influence on the patient's well-being. Juvenile idiopathic arthritis can be a reason for children's low indicators of quality of life.

Objectives: The objective of the study was to analyse health releated quality of life in Juvenile idiopathic arthritis patient comparing to their parents opinion's and healthy children's answers about their health releated quality of life.

Methods: In this study, juvenile idiopathic arthritis patients aged 8 to 17 years old and their parents were surveyed at the Children's Clinical University Hospital outpatient department. The participants voluntarily filled Kidscreen - 52 questionnaire ${ }^{1}$. Survey includes 10 HRQoL dimensions such as physical, psychological and social life well-being. Control group consisted of 100 healthy children. Statistical analysis was performed using Mann-Whitney U-test (SPSS Statistics 22). The level of statistical significance was set at $p<0.05$.

${ }^{1}$ Ravens-Sieberer U. et al., 2004, The ....KIDSCREEN Group" Europe, 2006

Results: In total 111 participants were included in the study : 62 Juvenile idiopathic arthritis (JIA) patients, and 49 their parents. The mean age of the patients was $13.7 \pm 2.3$ years. Significant differences were found between patients and healthy children in such HRQoL survey categories like "Autonomy" and "Financial resources" $(p<$ 0.05). Although quality of life in children's with Juvenile idiopathic arthritis was lower than in healthy children in HRQoL survey category "Self perception" $(p<0.05)$. After analyzing data no significantly differences were found between patients and parents' assessment scores in HRQoL survey categories $(p>0.05)$.

Conclusion: Juvenile idiopathic arthritis has a moderate negative influence on HRQoL survey categories "Self perception", "Autonomy" and "Financial resources" $(p<0.05)$ according KIDSCREEN-52 queationnaire. The evaluation of children and their parents' opinions in their children's quality of life did not differ significantly. We need to continue study about children's with Juvenile idiopathic arthritis quality of life.

Disclosure of Interest: None declared 
P202

"Tomato face" - the visible / invisible implications of JDM from a child and young person's perspective

P. Livermore ${ }^{1,2,3}$, C. T. Deakin ${ }^{3,4,5}$, L. R. Wedderburn ${ }^{3,6,7}$

${ }^{1} \mathrm{NIHR}$ BRC; ${ }^{2} \mathrm{ORCHID}, \mathrm{GOSH} ;{ }^{3} \mathrm{ICH} / \mathrm{UCL}, \mathrm{ICH} / \mathrm{UCL} ;{ }^{4} \mathrm{NIHR}$ BRC GOSH, Great

Ormond Street Children's Hospital; ${ }^{5} \mathrm{Centre}$ for Adolescent

Rheumatology Versus Arthritis at UCL UCLH and GOSH, , UCL ICH GOS;

${ }^{6} \mathrm{NIHR}$ BRC GOSH, GOSH; ${ }^{7}$ Centre for Adolescent Rheumatology Versus

Arthritis at UCL UCLH and GOSH, UCL, ICH, GOS, London, United

Kingdom

Correspondence: P. Livermore

Pediatric Rheumatology 2020, 18(Suppl 2):P202

Introduction: Juvenile Dermatomyositis (JDM) is often first identified by parents and carers as the red facial rash develops. The rash can progress and lead to young people being misdiagnosed with eczema, scarlet fever or psoriasis. However, over time the obvious signs of JDM can become "invisible" as treatment calms the rashes and masks the outward signs of JDM, until a flare occurs, when the rashes can be a marker for disease activity or progression. As part of a larger study, children around the United Kingdom were asked to discuss their views on whether they wanted people to be able to see their JDM.

Objectives: To understand the implications for children and young people from having a disease that has visible and invisible phases and whether they want others to see their JDM, or not.

Methods: Children and young people around the United Kingdom who were already consented and enrolled into the UK Juvenile Dermatomyositis Cohort and Biomarker Study were asked to complete a bespoke questionnaire. There was a mix of open and closed questions, and it was administered in paper format to all children and young people between the ages of 8 and 19 years of age for self-completion, either on the paper forms, or via a secure web-based software system. The questionnaires were administered at the end of 2018. Numeric data were described and qualitative data were analysed using standard content analysis.

Results: 246 questionnaire packs were sent out, with 127 (52\%) being returned. Of these 4 could not be used due to practical reasons, such as only demographic data being completed, which left a sample of $123.98(80 \%)$ of the 122 who responded said other people could not see their JDM, with only 11 (9\%) saying it was visible and $13(11 \%)$ saying they did not know if others can see it. 1 did not respond to the question as said their JDM has gone away. They were then asked whether it was a good or bad thing for others to be able to see their JDM or for others not to be able to see it. 41 young people left comments as to why it was a good thing, 36 left comments as to why it was a bad thing and 14 left comments to why they said don't know, table 1 presents the top ranking response for the three multichoice answers.

Conclusion: This study has highlighted the disparity between young people wanting others to see their JDM so that they gain more understanding and empathy from those around them, but equally, wanting their JDM to be invisible, so that they feel the same as their peers. Whilst many paediatric rheumatic conditions are in fact invisible, our data illustrate that JDM often gives children and young people a taste of both visible and invisible phases of disease activity. As one young person said "It's not good, nor bad - it's good that it's invisible sometimes so I can blend in without the disabled stereotype. However, sometimes it needs to be seen so I can be understood and not challenged".

Disclosure of Interest: None declared
Table 1 (abstract P202) Presenting the top coded theme for each ranked answer

\begin{tabular}{lll}
\hline Answer Option & Theme & Number \\
\hline Good & So others can understand & 23 \\
& So I can be the same as others & 10 \\
& Both/neither & 4 \\
\multirow{3}{*}{ Bad } & Makes me embarrassed & 14 \\
& I get treated negatively & 11 \\
& It means I don't get any leeway or understanding & 5 \\
No answer & Don't mind & 6 \\
& Neither & 4 \\
& Don't know & 4 \\
\hline
\end{tabular}

\section{P203}

A novel multidimensional assessment tool for clinical care of patients with juvenile fibromyalgia syndrome

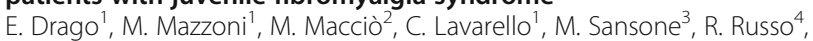
E. Pescio ${ }^{4}$, A. Ronchetti ${ }^{5}$, A. Ravelli ${ }^{1}$, C. Malattia ${ }^{1}$

${ }^{1}$ Clinica Pediatrica e Reumatologia, IRCSS Giannina Gaslini; ${ }^{2}$ Università degli studi di Genova; ${ }^{3}$ Child Neuropsychiatry Unit, IRCSS Giannina Gaslini; ${ }^{4}$ Clinical Psicology Unit, AMRI Onlus; ${ }^{5}$ UOC Medicina Fisica e Riabilitazione, IRCSS Giannina Gaslini, Genova, Italy

Correspondence: E. Drago

Pediatric Rheumatology 2020, 18(Suppl 2):P203

Introduction: Juvenile fibromyalgia syndrome (JFS) is a chronic disabling condition characterized by widespread musculoskeletal pain in combination with several somatic symptoms including fatigue, non restorative sleep, headaches. Although 2-6\% of school age children are estimated to suffer from JFS, patients often go undiagnosed for years; in addition, recommendations for the treatment and validated outcome measures for JFS are currently lacking.

Objectives: 1) To describe clinical features of JFS patients followed at our Centre

2) To develop a new multidimensional outcome measure for the assessment of patients with JFS in standard clinical care.

Methods: We included 43 patients diagnosed with JFS according to the 2010 criteria of the American College of Rheumatology. All patients were administered the Juvenile Fibromyalgia Multidimensional Assessment Report (J-FiMAR) which includes comprehensive patient self-report questionnaire and numerical rating scales to measure pain, fatigue, headache, sleep quality, physical function, psychological state, health-related quality of life, satisfaction with illness course. The J-FiMAR has been devised according to the Outcome measure in Rheumatology (OMERACT) guidelines. Discriminant ability of the multidimensional tool was evaluated by testing it in a control group including healthy controls and patients affected by active juvenile idiopathic arthritis (JIA). The psychosocial consequences of chronic pain were evaluated by using the Children Depression Index (CDI) and the Multidimensional Anxiety Scale for Children (MASC). The objective sleep quality was measured by overnight polysomnography.

Results: Table 1 shows characteristics and the most represented somatic symptoms in our cohort of JFS patients at the study enter. Polysomnography was performed in 21 patients with sleep disturbance; $8 / 21 \quad(38.1 \%)$ showed an electroencephalographic pattern of alpha wave intrusion in slow wave sleep (SWS). The presence of objective sleep disorders was significantly correlated to CDI score rs $-0,775(p \leq 0,0001)$ and MASC $0,61 \quad(p=0,005)$. From November 2016 to April 2020 J-FiMAR was completed by 43 JFS patients ( $\mathrm{F} 35$ (81.4\%), median age 14.7 years [7.1-17.6], median disease duration 1.9 years [0.1-7.8]) in 125 visits. All patients filled out the questionnaire in a short time ( $<15$ minutes) and considered it simple 
and easy to understand. JFS patients showed significantly higher score for pain, fatigue, poor physical function and measure of psychological distress than healthy controls and JIA patients $(p<0.05$ for each item).

Conclusion: JFS patients presented significantly higher pain experience, functional disability, and impaired quality of life than patients with active JIA. A relevant percentage of JFS patients experience sleep disturbances, which were correlated with mood and anxiety disorders. Our multidimensional tool was feasible and able to quantify global JFS severity. This multidimensional tool, by measuring the main domains affected by the disease, could be promising to individualize treatment strategy and to test its efficacy.

Disclosure of Interest: None declared

Table 1 (abstract p203). See text for description

\begin{tabular}{ll}
\hline Widespread musculoskeletal pain & $38(88.4 \%)$ \\
Fatigue & $37(86 \%)$ \\
Headache & $27(62.8 \%)$ \\
Concentration or Memory Problems & $22(55 \%)$ \\
Sleep disturbance & $22(51.7 \%)$ \\
Anxiety and/or depression & $17(39.5 \%)$ \\
Irregular School attendance & $15(34.9 \%)$ \\
IBS and abdominal pain & $11(25.6 \%)$ \\
Body mass index $>25$ & $12(27.9 \%)$ \\
Family history of fibromyalgia & $11(25.6 \%)$ \\
\hline
\end{tabular}

\section{P204}

Fatigue in children and teens with autoinflammatory disease

S. Angevare ${ }^{1,2,3}$, K. Durrant $^{3}$, R. Beesley ${ }^{1,4}$

${ }^{1}$ ENCA, Zurich, Switzerland; ${ }^{2}$ KAISZ, Amersfoort, Netherlands;

${ }^{3}$ Autoinflammatory Alliance, San Fransico, United States; ${ }^{4}$ Juvenile

Arthritis Research, Tonbridge, United Kingdom

Correspondence: $\mathrm{S}$. Angevare

Pediatric Rheumatology 2020, 18(Suppl 2):P204

Introduction: Fatigue is a subjective state of overwhelming, sustained exhaustion and decreased physical and mental capacity, which is not relieved by rest. Fatigue is the most common complaint in children and teens with an autoinflammatory disease, besides the disease related flares. The purpose for this study was to show that fatigue is a serious issue for children and young people with autoinflammatory diseases. We hypothesized that age, gender and/ or the type of autoinflammatory disease could have differing effects on the fatigue experience.

Objectives: We aimed to investigate fatigue in children and young people (CYP) with autoinflammatory disease, including how this affected them on a daily basis.

Methods: CYP with autoinflammatory diseases were invited to complete an online survey, providing details about their fatigue and how it affected them. The survey was developed by the non-profit organizations Autoinflammatory Alliance and KAISZ/VAISZ, in English. Respondents were recruited by convenience sampling through online social media posts. Data on age, gender and disease were collected in addition to information on their experience of fatigue on school and social interaction.

A total of 114 CYP (age range 7-18 years) with an autoinflammatory disease responded to the survey ( $52 \%$ female).

Results: The majority of respondents (81\%) reported experiencing both mental and physical fatigue. Respondents were asked how much their fatigue affected them, on a scale of 0 to 10; overall, the mean fatigue score was 6.6. However, young people aged 15 or over reported a significantly higher impact than those aged 11-14 years (mean 7.5, $\mathrm{p}=0.012$ ). Different autoinflammatory diseases were surveyed: CRMO $25 \%$, CAPS 20\%, PFAPA $12 \%$ also Unclassified SAID (USAID) with $23 \%$.
In the open-response portion of the survey, $81 \%$ of respondents reported that fatigue was physical, as well as mental, in their experience.

Most $(89 \%)$ reported that someone had doubted their fatigue in the past; $29 \%$ had found their teachers had doubted them, $26 \%$ had friends who doubted them, and $24 \%$ reported that they felt their doctors had doubted them. Children and young people also felt a number of activities made their fatigue worse (table 1)."

Conclusion: CYP with autoinflammatory diseases experience physical and mental fatigue. Health professionals and teachers should listen to patients reporting fatigue, validate their experience, and help find ways to support them. Identifying resources to help the patients with fatigue, and referrals to therapy and mental health resources as needed may help the patients to better cope and manage their chronic disease. Further studies will include patient engagement in designing questionnaires about all aspects of life and autoinflammatory disease will help our understanding of these complex conditions and how they affect patients.

Disclosure of Interest: None declared

Table 1 (Abstract P204). Proportion of respondents reporting what they felt makes their fatigue worse.

\begin{tabular}{lll}
\hline & Total & $\%$ \\
\hline School & 77 & $20 \%$ \\
Extracurricular activities & 76 & $20 \%$ \\
Sports & 72 & $19 \%$ \\
Social interaction & 62 & $16 \%$ \\
Family activities & 50 & $13 \%$ \\
Medication & 16 & $4 \%$ \\
Don't know & 16 & $4 \%$ \\
Nothing & 8 & $2 \%$ \\
Total & 377 & \\
\hline
\end{tabular}

P205

Psychological involvement in young people with chronic rheumatological disease: what input do patients need?

O. Ogundiran 1,2, A. Leahy 1,2, E. Lee', A. Griffin ${ }^{3}$, S. Hartfree², S. Small², B. Davidson ${ }^{2}$, A. De Schutter ${ }^{3}$, H. De Graaf ${ }^{2}$

${ }^{1}$ Faculty of Medicine, University of Southampton; ${ }^{2}$ Paediatric

Rheumatology; ${ }^{3}$ University Hospital Southampton, Southampton, United Kingdom

Correspondence: $\mathrm{O}$. Ogundiran

Pediatric Rheumatology 2020, 18(Suppl 2):P205

Introduction: Paediatric rheumatological diseases increase the risk of co-morbid mental health disorders and symptoms, yet the optimum psychological intervention to address mental health symptoms in this patient group has not been established.

Objectives: This study set out to establish patient and parent views on the suitability of different interventions that seek to support the mental health of patients with paediatric rheumatological diseases.

Methods: Patients with inflammatory diseases and their parents attending the Paediatric Rheumatology Department or Young Adult Clinic (YAC) at the University Hospital Southampton were invited to take part in the study. Questionnaires and semi-structured interviews, developed with paediatric psychologists, and a medical anthropologist, were used to examine the experience of emotional difficulties amongst patients and views on suitability of different intervention formats. Patients and their parents were invited to complete the questionnaires however only patients were invited to take part in the interviews. Interviews were audio recorded and transcribed verbatim by the interviewer. Codes were generated inductively from the interview transcripts and manually grouped into themes. 
Quantitative data from completed forms were analysed descriptively using SPSS and Excel. Qualitative data from extended answer responses and patient interviews were thematically analysed.

Results: 72 patients, (IQR of age in years 12.25 - 17.00) and 47 parents (IQR of age in years 12.00 - 15.25) completed questionnaires. $80 \%$ of patients reported experiencing at least one emotional difficulty, related to their rheumatological condition. Sleeping problems $(49.3 \%)$ and anxiety $(46.5 \%)$ were the most commonly reported symptoms in patient participants. $91.4 \%$ of patient participants agree or strongly agree that intervention deliverers should understand their condition. $50 \%$ of patients and $64.4 \%$ of parents reported psychologists as suitable interventionists for emotional difficulties followed by paediatric rheumatologists (29.4\% and $57.8 \%$, respectively).

Five patients were interviewed. Key themes from the interviews include experience with emotional difficulties (e.g. anxiety around taking medication and the effect of disease on future life); variety of interventions (e.g. educational and psychological support to overcome emotional challenges related to disease), and awareness of available support.

Conclusion: This study highlighted there are high levels of emotional difficulties in paediatric rheumatology patients. Psychologists and paediatric rheumatologists are deemed the most suitable interventionists by participants. Patients demonstrate a need for emotional and educational support to overcome emotional difficulties associated with their rheumatological disease.

Disclosure of Interest: None declared

\section{P206}

Scleroderma-polymyositis overlap syndrome in pediatric age: a case report

M. Amico, R. Naddei, L. Pierri, R. Alfani, T. Lastella, C. Porfito, M. Alessio Pediatric Rheumatology Unit, Mother and Child Department, University of Naples Federico II, Naples, Italy

Correspondence: $\mathrm{M}$. Amico

Pediatric Rheumatology 2020, 18(Suppl 2):P206

Introduction: Scleromyositis is the most common overlap syndrome but is rarely observed in childhood. This disorder involves two different autoimmune diseases: systemic scleroderma (SSc) and polymyositis (PM).

Objectives: To describe the clinical course of a SSc/PM syndrome in a young girl.

Methods: Case report

Results: An 11-year-old female was admitted to the Neurological Unit of our hospital for creatine phosphokinase (CPK) increase and hypertransaminasemia associated to sporadic episodes of right calf pain. Familiarity for muscular dystrophy was reported in the maternal branch. Muscle tone and trophism were preserved at initial neurological evaluation. Laboratory investigation confirmed increased muscle enzyme levels, including CPK (x70) (CK-MM 94.5\%, CK-MB $5.5 \%)$, aldolase $(x 7)$, cardiac troponin $(x 10)$ and myoglobin $(x 10)$. Suspecting a primary muscle disease, she underwent a total body STIRMRI which showed a diffuse edema of gluteus medius bilaterally and a muscle biopsy revealing a marked muscle damage with dystrophic aspects and normality of the tested proteins. A genetic extended panel for congenital myopathies resulted negative.

After 4 months, a new clinical examination showed the occurrence of general skin induration, sclerodactyly and tightening of the face skin. Appearance of dysphagia was also reported, and muscle enzyme increase persisted. In suspicion of an SSc/PM overlap syndrome, she was referred to our Unit. Nailfold capillaroscopy showed capillary dilatation and branching, megacapillaries and diffuse microhemorrhages. Reduction of esophageal contractions amplitude and hypotensive lower esophageal sphincter pressure were observed at esophageal manometry test. High-resolution CT of lungs and pulmonary function testing were normal. Skin biopsy showed sclerodermiform findings. Immunological studies revealed a positivity of antinuclear antibody (1:320) and anti-Ku. Anti-PM-Scl resulted negative. An oral corticosteroid therapy (prednisone, $1.5 \mathrm{mg} / \mathrm{kg} /$ day) was started in association with subcutaneous Methotrexate $\left(15 \mathrm{mg} / \mathrm{m}^{2} /\right.$ week) and intravenous immunoglobulins (IVIG) (2gr/kg every two weeks). Improvement of skin manifestation, joint mobility, as well as normalization of serum CPK levels were observed. Over 3 months, prednisone and IVIG were slowly discontinued up to the ongoing dosage of $0.9 / \mathrm{mg} /$ day and $2 \mathrm{gr} / \mathrm{kg}$ every 4 weeks, respectively. MTX is still ongoing at the same dosage.

Conclusion: The diagnosis of overlap connective tissue disease syndromes may be challenging in pediatric age. Different symptoms may be prevalent at different stages throughout the course of the disease. In our patient, a localized myositis preceded the SS onset by about four months. Even though the use of high dose of corticosteroids is associated to a higher incidence of renal crisis in patients with CSS, a combined therapy with high doses of oral steroids, IVIG and MTX was safe and effective in skin, muscle and joint symptoms in our patient.

\section{Disclosure of Interest: None declared}

\section{P207}

Tocilizuab as a treatment option for a Libyan female child with juvenle systemic sclerosis

A. A. Abushhaiwia, M. A Zletni ${ }^{1}$

Pediatric Rheumatology, Tripoli faculty of medicine, Tripoli , Libya

Correspondence: A. A. Abushhaiwia

Pediatric Rheumatology 2020, 18(Suppl 2):P207

Introduction: Juvenile systemic sclerosis is a rare autoimmune disease characterized by skin stiffness and fibrosis of internal organs (e.g.lung,heart,and gastrointestinal system).The efficacy and safety of tocilizumab (TCZ), an interleukin 6 receptor- inhibitor,and reports on its efficacy among children have been the subject of a few recent studies.This is a first report on the treatment of a JSS patient with TCZ in Libya.

Objectives: We aimed to present a case with Juvenile systemic sclerosis to evaluate the efficacy of tocilizumab in the treatment of systemic sclerosis in children.

Methods: DESIGN AND methods: Case report study

Results: A 16-year-old female who was diagnosed with juvenile scleroderma was referred to our rheumatology clinic in September 2018 due to progressive dysphagia specifically for solid food associated with coffee ground vomiting and sometimes, shortness of breath during exertion.The patient had been diagnosed with juvenile scleroderma 3 years ago based on skin tightness, Raynaud phenomena,sclerodactly with digital ulcers,an upper GIT endoscopy which revealed lower esophagus ulceration,decreased pulmonary function test,a high resolution CT scan chest which showed interstitial pneumonitis,holter ECG showed ventricular arrhythmia with normal conduction, and an EMG revealed myopathy.She had been treated with a B.bloker (Nebiodol 5mg) $1 \backslash 4$ tab once per day and flecaine tab 50mg,in addition to a methotrexate tab and an omeprazole capsule by a 
rheumatologist.She showed generalized fatigability,increased tightness of skin on her face as well as her upper and lower extremities,limitation of range of motions of most joints,and an abnormal handgrip.Laboratory tests revealed the following: low CBC at HGB 9.6,ANA 1:1280 positive and antids DNA was negative,ENA was negative,SCL-70 was positive,holter ECG showed ventricular arrhythmia,EMG revealed myopathy,high resolution chest showed fibrosing alveolitis worse than the first one,PFT was decreased.A diagnosis of diffuse systemic sclerosis (JSS) was made,and cyclophosphamide IV was administrated for 6 months.Methotrexate $15 \mathrm{mg} \backslash \mathrm{m} /$ week SC was initiated,well as a,low dose of steroids for 3 months,along with B blocker tab and an omeprazole capsule; however,there was no improvement.In April 2019, she started using Tocilizumab infusion once per month accompanied with methotrexate SC and MMF tab,and the effects of therapy have been very good.

Conclusion: JSS is a rare condition characterized with internal organ involvement.Tocilizumab represents an efficient treatment option for patients unresponsive to standard treatment.

Disclosure of Interest: None declared

\section{P208}

How the adult criss works in pediatric jssc patients - results from the juvenile scleroderma inception cohort

J. Klotsche', I. Foeldvari ${ }^{2}$, O. Kasapcopur ${ }^{3}$, A. Adrovic ${ }^{3}$, K. Torok ${ }^{3}$, V. Stanevicha ${ }^{3}$, J. Anton ${ }^{3}$, E. Marrani ${ }^{3}$, W.-A. Sifuentes-Giraldo ${ }^{3}$, M. T. Terreri ${ }^{3}$ F. Sztajnbok ${ }^{3}$, C. Battagliotti ${ }^{3}$, L. Berntson ${ }^{3}$, D. Eleftheriou ${ }^{3}$, G. Horneff $^{3}$, F. Nuruzzaman ${ }^{3}$, N. Helmus ${ }^{2}$

${ }^{1}$ German Rheumatism Research Center, Berlin; ${ }^{2}$ Hamburg Center for Pediatric and Adolescent Rheumatology, Am Schoen Klinik Eilbek; ${ }^{3}$ SSSC Collaborative Group, Hamburg, Germany

Correspondence: I. Foeldvari

Pediatric Rheumatology 2020, 18(Suppl 2):P208

Introduction: The Composite Response Index in Systemic Sclerosis (CRISS) was developed by Dinesh Khanna as a response measure in patients with adult systemic sclerosis. CRISS aims to capture the complexity of systemic sclerosis and to provide a sensitive measure for change in disease activity. The CRISS score is based on a two-step approach. First, significant disease worsening or new-onset organ damage is defined as non-responsiveness. In patients who did not fulfill the criteria of part one, a probability of improvement is calculated for each patient based the Rodnan Skin Score (mRSS), percent predicted forced vital capacity (FVC\%), patient and physician global assessments (PGA), and the Health Assessment Questionnaire Disability Index (HAQ-DI). A probability of 0.6 or higher indicates improvement.

Objectives: to validate the CRISS in a prospectively followed cohort of patients with juvenile systemic sclerosis (jSSc)

Methods: Data from the prospective international inception cohort for jSSC was used to validate the CRISS. Patients with an available 12months follow-up were included in the analyses. Clinically improvement was defined by the anchor question about improvement (much better or little better versus almost the same, little worse or much worse) in patients overall health due to scleroderma since the last visit provided by the treating physician.

Results: Forty seven jSSc patients were included in the analysis. $74.2 \%$ had diffuse subtype. The physician rated the disease as improved in 34 patients (72.3\%) since the last visit. No patient had a renal crisis or new onset of left ventricular failure during the 12months follow-up. Three patients (3.4\%) each had a new onset or worsening of lung fibrosis and new onset of pulmonary arterial hypertension. In total, 6 patients resulted in a rating of not improved based on the CRISS in part I. The mRSSS, FVC\%, CHAQ and PGA significantly improved during the 12-months follow-up in patients who were rated as improved. The predicted probability based on the CRIS $\mathrm{S}$ algorithm resulted in an area under curve of 0.77 predicting the anchor question of improvement. In summary, 33 (70.0\%) patients were correctly classified by the adult CRISS score resulting in an overall area under curve of 0.7 .
Conclusion: The CRISS score was evaluated in a pediatric jSSc cohort for the first time. It showed a good performance. However, it seems that the formula of part II of the CRISS score needs a calibration to pediatric jSSc patients.

\section{Disclosure of Interest: None declared}

\section{P209}

Description of the characteristics of the nailfold capillary structure in healthy children: a pilot study

H. Adiguzel Dundar', A. Adrovic ${ }^{2}$, S. Demir ${ }^{3}$, F. Demir ${ }^{4}$, F. Cakmak ${ }^{5}$, N.

Aktay Ayaz ${ }^{5}$, B. Sozeri ${ }^{4}$, Y. Bilginer ${ }^{3}$, O. Kasapcopur², E. Unsal ${ }^{1}$

'Department of Pediatrics, Pediatric Rheumatology Unit, DOKUZ EYLUL

UNIVERSITY FACULTY OF MEDICINE, izmir; ${ }^{2}$ Department of Pediatrics,

Pediatric Rheumatology Unit, Istanbul University-Cerrahpaşa, Cerrahpaşa

Medical Faculty, Istanbul; ${ }^{3}$ Department of Pediatrics, Pediatric

Rheumatology Unit, Hacettepe University Faculty of Medicine, Ankara;

${ }^{4}$ Department of Pediatrics, Pediatric Rheumatology Unit, Health Sciences

University, Ümraniye Training and Research Hospital; ${ }^{5}$ Department of

Pediatrics, Pediatric Rheumatology Unit, Istanbul University Çapa Medical

Faculty, Istanbul, Turkey

Correspondence: $\mathrm{H}$. Adiguzel Dundar

Pediatric Rheumatology 2020, 18(Suppl 2):P209

Introduction: Nailfold capillaroscopy is the best method for the early diagnosis of connective tissue diseases, especially systemic sclerosis, and evaluation of microcirculation in children and adolescents. Although there are many studies to identify normal capillaroscopic findings in healthy adults, there are limited number of studies for normal reference ranges by age and gender in the children and adolescents.

Objectives: The aim is to define and standardize the nail bed capillary properties in healthy Turkish children and adolescents.

Methods: This multicenter cross-sectional pilot study included; 118 healthy children and adolescents from 5 pediatric rheumatology centers. Using the Dino-Lite CapillaryScope 200 Pro / MEDL4N Pro capillaroscopy device, two images of $1 \mathrm{~mm}$ radial and ulnar edge were obtained from the $4^{\text {th }}$ fingernail bed of the non-dominant hand at 200x magnification. Capillary density, capillary morphology (i.e., capillary tortuosity, capillary crossing, giant capillary, capillary meandering and branched capillary), microhemorrhage and avascular area were the parameters. Also 3 consecutive capillaries from each image; capillary length, capillary width, apical loop, arterial and venous width, and distance between capillaries were measured. The children included in the study were classified according to their age; Group 1: 5-7 years, Group 2: 8-10 years, Group 3: 11-14 years, and Group 4: $15-18$ years old.

Results: A total of 336 images were obtained from 118 healthy children included in the study and 708 capillary measurements were made. Capillary density was significantly higher in Group 4 than in Groups 1 and 2. Arterial width was significantly lower in Group 1 as compared to Group 3 and 4, and in Group 2 as compared to Group 4. Apical loop width and capillary distance were significantly lower in Group 1 compared to Group 2 and 3 and 4. There was no significant difference between the age groups in terms of capillary length and venous width. There was no difference between the groups in terms of capillary morphology. In total 336 image evaluations, capillary tortuosity was $<50 \%$ in $67.8 \%$, and $>50 \%$ in $4.2 \%$, and capillary crossing were $<50 \%$ in $52.5 \%$ and $>50 \%$ in $3.4 \%$. While the enlarged capillary was $4.7 \%$ and the avascular area was $4.2 \%$, capillary branching, capillary meandering, microhemorrhage, and giant capillary were not detected in any case. There was a good level of agreement between the researchers, as 20 cases with 120 capillaries were evaluated with a good level of agreement (Table 1).

Conclusion: This is the first study to evaluate capillary morphology in healthy Turkish children. This study also adds that some special forms such as enlarged capillary and avascular area, which is always named as pathological in adult age, can be seen in healthy children. These data will be guiding in capillaroscopic studies in various patient groups, particularly in children with collagen vascular diseases. 


\section{Disclosure of Interest: None declared}

Table 1 (Abstract P209). Evaluation of compatibility between the researchers for capillary density / $\mathrm{mm}$, capillary length, capillary width, arterial width, venous width, apical width and intercapillary distance measurements.

\begin{tabular}{llll}
\hline & ICC (\%) & Confidence Interval (\%95) & p \\
\hline Capillary density & 96.1 & $0.944-0.975$ & $<0.001$ \\
Capillary length & 94.3 & $0.895-0.974$ & $<0.001$ \\
Capillary width & 90.6 & $0.866-0.937$ & $<0.001$ \\
Arterial width & 90.7 & $0.868-0.938$ & $<0.001$ \\
Venous width & 89.6 & $0.853-0.930$ & $<0.001$ \\
Apical loop width & 91.1 & $0.874-0.941$ & $<0.001$ \\
Capillary distance & 94.3 & $0.918-0.962$ & $<0.001$ \\
\hline
\end{tabular}

ICC: Interclass correlation coefficient

\section{P210}

Is there a different presentation of juvenile systemic diffuse and limited subset? data from the juvenile scleroderma inception cohort. www.juvenile-scleorderma.com

I. Foeldvari ${ }^{1}$, J. Klotsche ${ }^{2}$, O. Kasapcopur ${ }^{3}$, A. Adrovic ${ }^{3}$, K. Torok ${ }^{3}$, M. T. Terreri $^{3}$, A. P. Sakamoto ${ }^{3}$, V. Stanevicha ${ }^{3}$, F. Sztajnbok ${ }^{3}$, J. Anton ${ }^{3}$, B. Feldman ${ }^{3}$, E. Alexeeva ${ }^{3}$, M. Katsikas ${ }^{3}$, V. Smith ${ }^{3}$, T. Avcin ${ }^{3}$, E. Marrani ${ }^{3}$, M. Kostik $^{3}$, T. Lehman ${ }^{3}$, W.-A. Sifuentes-Giraldo ${ }^{3}$, N. Vasquez-Canizares ${ }^{3}$, A. S. Appenzeller, Simone ${ }^{3}$, M. Janarthanan ${ }^{3}$, M. Moll ${ }^{3}$, D. Nemcova ${ }^{3}$, A. Patwardhan $^{3}$, M. J. Santos 3 , S. Sawhney ${ }^{3}$, D. Schonenberg-Meinema ${ }^{3}$, C. Battagliotti ${ }^{3}$, L. Berntson ${ }^{3}$, B. Bica ${ }^{3}$, J. Brunner ${ }^{3}$, P. Costa Reis ${ }^{3}$, D.

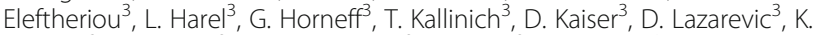
Minden ${ }^{3}$, S. Nielsen ${ }^{3}$, F. Nuruzzaman ${ }^{3}$, Y. Uziel ${ }^{3}$, N. Helmus ${ }^{1}$

${ }^{1}$ Hamburg Center for Pediatric and Adolescent Rheumatology, Am Schoen Klinik Eilbek, Hamburg; ${ }^{2}$ German Rheumatism Research Center, Berlin; ${ }^{3}$ jSSc Collaborative Group, Hamburg, Germany

Correspondence: I. Foeldvari

Pediatric Rheumatology 2020, 18(Suppl 2):P210

Introduction: Juvenile systemic scleroderma (jSSc) has a prevalence of 3 per 1000000 children. There are limited data regarding the clinical presentation of jSSc. The Juvenile Systemic Scleroderma Inception Cohort (jSScC) is the largest multinational registry that prospectively collects information about jSSc patients.

Objectives: To assess of there is a difference in the clinical characteristics in diffuse and limited subtype jSSc patients at the time of inclusion in the jSScC

Methods: Patients were included, if they fulfilled the adult ACR/ EULAR classification criteria for systemic scleroderma, if they presented the first non-Raynaud symptom before 16 years of age and were younger than 18 years of age at time of inclusion. Patients' characteristics at time of inclusion were evaluated.

Results: Until December 2019 hundred fifty patients were included, $83 \%$ of them being Caucasian and $80 \%$ female. The majority had the diffuse subtype $(72 \%)$ and $17 \%$ of all jSSc had overlap features. The mean age of first presentation of Raynaud's phenomenon was 9.8 years in the diffuse subtype (djSSc) and 10.7 years in the limited subtype (ljSSc) $(p=.197)$. The mean age at first non-Raynaud's symptoms was 10.0 years in the djSSc and 11.2 years in the ljSSc ( $p=$ 0.247). Mean disease duration at time of inclusion was 3.4 years in the djSSc and 2.4 years in the ljSSc group.

Significant differences were found between the groups regarding mean modified Rodnan skin score, 18.2 in the djSSc vs 6.2 in the ljSSc $(p=0.02)$; presence of Gottron's papulae (djSSc $30 \%$ vs ljSSc 13\%, $\mathrm{p}=0.43$ ); presence of teleangiectasia ( djSSc $42 \%$ vs $18 \%$ ljSS, $p=0.01$ ); history of ulceration (djSSc $42 \%$ vs $18 \%$ ljSSc, $p=0.008$ ); 6 Minute walk test below the $10^{\text {th }}$ percentile ( djSSc $85 \%$ vs ljSSc $54 \%$, $\mathrm{p}=0.044$ ), total pulmonary involvement ( djSSc 49\% vs ljSSc $31 \%, p=$
0.045), cardiac involvement (ljSSc 17\% vs djSSc 3\%, p=0.002). djSSc patients had significantly worse scores for Physician Global Assessment of disease activity compared to ljSSc patients (VAS 0$100)$ (40 vs 15) $(p=0.001)$ and for Physician Global Assessment of disease damage (VAS 0-100) (36 vs 17) $(p=0.001)$.

There were no statistically significant differences in the other presentations. Pulmonary hypertension occurred in approximately $6 \%$ in both groups. No systemic hypertension or renal crisis was reported. ANA positivity was $90 \%$ in both groups. Anti-Scl70 was positive in $35 \%$ in djSSc and $36 \%$ in the ljSSc group. Anticentromere positivity occurred in 3\% in the djSSc and 7\% in the ljSSc group.

Conclusion: In this unique large cohort of jSSc patients there were significant differences between djSSc and ljSSc patients at time of inclusion into the cohort regarding skin, vascular, pulmonary and cardiac involvement. According to the physician global scores the djSSc patients had a significantly more severe disease. Supported by the "Joachim Herz Stiftung"

Disclosure of Interest: None declared

P211

The comparison of the HLA-B27-positive and HLA-B27-negative patients with juvenile idiopathic arthritis in the single center R. Raupov1, L. Sorokina2, M. Gharabaghtsyan³, N. Garipova", I. Avrusin', L. Snegireva', M. Dubko', A. Santimov', E. Gaidar', V. Masalova', E. Isupova', I. Chikova', T. Likhacheva', F. Vinokurova ${ }^{5}$, M. Kostik'

${ }^{1}$ Saint Petersburg State Pediatric Medical University; ${ }^{2}$ Leningrad's Regional Children's Clinical Hospital, Saint Petersburg, Russian Federation; ${ }^{3}$ Institute of Child and Adolescent Health with the Arabkir Medical Center, Yerevan, Armenia; ${ }^{4}$ Almazov National Medical Research Centre, Saint Petersburg, ${ }^{5}$ Yakut Science Centre of complex medical problems, Yakutsk, Russian Federation

Correspondence: R. Raupov

Pediatric Rheumatology 2020, 18(Suppl 2):P211

Introduction: Juvenile spondyloarthropathies (JSA) are the group of diseases with axial involvement in childhood. EULAR and ASAS classifications are known for diagnosis of JSA, the last one is less sensitive. The $70 \%$ of patients are HLA-B27 positive and have sacroiliitis or cervical involvement in the onset or developing of the disease. There's limited information about seronegative JSA in childhood.

Objectives: to compare the patients with JIA depending on HLA-B27 positivity. To identify the rate of axial involvement depending HLAB27 positivity.

Methods: 308 patients with JIA were tested for HLA-B27. They were divided into 2 groups: 1) HLA-B27 positive and 2) HLA-B27 negative.

Results: 100 patients (32,5\%) were HLA- B27 positive and all of them are fulfilled the EULAR criteria of entesitis-related arthritis (ERA). The group 2 consists of 208 patients (67.5\%). There's no statistical difference between both groups in active joint count, ANA-positivity and uveitis frequency, the rate of use methotrexate and time before biologics. No difference in axial cervical spine $12(12.0 \%)$ vs $21(10.1 \%)$ $(p=0.613)$ and sacroiliac joints $18(18.0 \%)$ vs 23/207 $(11.1 \%)(p=0.097)$ involvement was observed. HLA B27(+) patients often received pulse therapy with methylprednisolone due to increased inflammatory activity and severe arthritis ( $22 \%$ vs $11.1 \%, p=0.011)$. Other parameters are listed in Table1.

Conclusion: patients with HLA-B27 positivity were characterized by male predominance, more often hip involvement, higher laboratory activity and the need for more frequent use biologics. The rate of axial involvement wasn't different in HLA-B27 positive and negative patients, that needs further study and creating more accurate classification criteria for JSA.

Disclosure of Interest: None declared 
Table 1 (abstract P211). The difference between HLA-B27 positive and negative arthritis

\begin{tabular}{llll}
\hline Parameters & HLAB27 (+) $(\mathbf{n}=\mathbf{1 0 0})$ & HLAB27 (-) (n=208) & $\mathbf{p}$ \\
\hline Onset age, years & $9.3(6.4 ; 11.8)$ & $6.1(3.5 ; 10.3)$ & 0,00001 \\
ESR, mm/h & $16.0(4.0 ; 30.0)$ & $5.0(3.0 ; 14.0)$ & 0,00002 \\
CRP, g/l & $2.2(0.2 ; 15.7)$ & $0.8(0.0 ; 5.2)$ & 0,016 \\
PLT, $\times 10^{9} / /$ & $336.0(257.0 ; 430.0)$ & $303.5(255.0 ; 366.5)$ & 0,034 \\
Male, $\mathrm{n}(\%)$ & $66(66.0)$ & $93(44.7)$ & 0,001 \\
Hip arthritis & $34(34.0)$ & $41(19.7)$ & 0.007 \\
Knee arthritis & $56(56.0)$ & $155(74.5)$ & 0.002 \\
Sulfasalazine & $42 / 64(65.6)$ & $34 / 156(21.8)$ & 0,001 \\
Biologics & $61(61.0)$ & $82(39.4)$ & 0,001 \\
\hline
\end{tabular}

\section{P212}

Cross-sectional study of fecal calprotectin in children with various forms of arthritis and non-inflammatory musculoskeletal disorders: a single centre experience

L. Lamot ${ }^{1,2}$, A. Kovacevic ${ }^{1}$, M. Miler ${ }^{3}$, M. Lamot ${ }^{1}$, N. Nikolac Gabaj ${ }^{3}$, M. Vidović $^{1}$, M. Harjaček ${ }^{1,2}$

${ }^{1}$ Pediatrics, Sestre milosrdnice University Hospital Center; ${ }^{2}$ Pediatrics, University of Zagreb School of Medicine; ${ }^{3}$ Clinical Chemistry, Sestre milosrdnice University Hospital Center, Zagreb, Croatia

Correspondence: L. Lamot

Pediatric Rheumatology 2020, 18(Suppl 2):P212

Introduction: Although gut is increasingly recognized as origin and/or target of inflammation in adult onset spondyloarthritis $(\mathrm{SpA})$, the incidence of gut involvement in juvenile $\mathrm{SpA}$ (jSpA) patients is still largely unknown, mostly due to the lack of reliable non-invasive tests.

Objectives: We performed a cross-sectional study of fecal calprotectin ( $f C A L)$, a surrogate marker of gut inflammation, in patients with jSpA, other forms of juvenile idiopathic arthritis (JIA) and noninflammatory (NI) conditions.

Methods: fCAL was measured by commercially available assay in stool samples of enthesitis related (ErA), psoriatic (PsA) and patients with other JIA subtypes (oligo- and poly- articular) who fulfilled ILAR criteria, as well as in children with $\mathrm{NI}$ causes of musculoskeletal pain (NI-MSD), regardless of the gastrointestinal (GI) symptoms (Table 1). fCAL was compared among different groups of patients and correlated with demographic data, clinical characteristics, treatment modalities and disease activity measured by jSpADA. The values were also dichotomized to $<50$ $\mathrm{mg} / \mathrm{kg}, 50-200 \mathrm{mg} / \mathrm{kg}$, and $>200 \mathrm{mg} / \mathrm{kg}$, which was regarded as normal, slightly increased and increased, respectively. Ileocolonoscopy was performed in one patient.

Results: The median fCAL levels were highest in ErA patients ( $p=$ 0.04). Moreover, in ErA patients moderate correlation between $\mathrm{fCAL}$ levels and MRI signs of $\mathrm{SI}$ inflammation $(r=0.4, p=$ 0.39 ) was found, while the patients with inflammation had higher fCAL concentrations than those without ( 22.6 vs $54.3, p=0.048$ ). There was no significant difference in $\mathrm{fCAL}$ concentration between ErA patients with inactive (jSpADA $\leq 1$ ) or active (jSpADA $\geq 1$ ) disease (39.7 vs 30.9, $\mathrm{p}=0.66$ ). In all patients, NSAID use was not associated with increased fCAL ( 20 vs $23, p=0.18$ ), although weak correlation was found with the duration of the use $(r=0.25, p=0.03)$. No correlation was observed between fCAL level and age at the time of sampling, duration of the disease, CRP or ESR, number of active joints and/or enthesis, physician global assesment, morning stiffnes, uveitis, back mobility, abdominal pain, diarrhea, B27 presence in a patinet or a faimly and disease activity in ErA and other JIA patients measured by jSpADA and JADAS, respectively. Microscopic gut inflammation was observed in one ErA patient with fCAL concentration of 839 $\mathrm{mg} / \mathrm{kg}$.

\begin{tabular}{|c|c|c|c|c|c|}
\hline & & ErA & PsA & JIA & NI-MSD \\
\hline \multicolumn{2}{|l|}{$\mathbf{N}$ (\% female) } & $26(65 \%)$ & $4(100 \%)$ & $29(57 \%)$ & $12(67 \%)$ \\
\hline \multicolumn{2}{|l|}{ Age $^{*}(y r s)$} & $12(7.7-14.5)$ & $\begin{array}{l}10.8(8- \\
13.9)\end{array}$ & $11(7-14)$ & $\begin{array}{l}13(6.1- \\
14)\end{array}$ \\
\hline \multicolumn{2}{|l|}{ fCAL* $(\mathrm{mg} / \mathrm{kg})$} & $\begin{array}{l}33.20(20- \\
84.8)\end{array}$ & $20(20-30.7)$ & $\begin{array}{l}20(20- \\
31.5)\end{array}$ & 20 \\
\hline \multirow{3}{*}{$\begin{array}{l}\text { fCAL (mg/ } \\
\mathrm{kg})\end{array}$} & $<50$ & 18 & 4 & 24 & 10 \\
\hline & $50-200$ & 5 & / & 4 & 2 \\
\hline & $>200$ & 3 & / & 1 & / \\
\hline \multirow[t]{3}{*}{ TREATMENT } & NSAIDS & 14 & 2 & 23 & 0 \\
\hline & $\begin{array}{l}\text { DMAR } \\
\text { DS }\end{array}$ & 2 & 0 & 9 & 0 \\
\hline & GC & 1 & 0 & 1 & 0 \\
\hline
\end{tabular}

Conclusion: Our study has shown that fCAL levels are significantly higher in ErA patients compared to other JIA $(p=0.03)$ and/or NI-MSD $(p=0.03)$ patients. Moreover, almost a third of patients with ErA had levels of fCAL above the range regarded as normal, which adds to the number of evidences for a gut inflammation in this particular type of JIA. Besides, the fCAL levels were higher in those with axial involvement, which further suppots the association of gut and axial inflammation in children with ErA. Although endoscopy remains a gold standard for the diagnosis of gut inflammation, fCAL can help to select children with ErA who might benefit from this invasive procedure, regardless of the Gl symptoms, as shown in one patient with the highest fCAL concentration in our study. Moreover, fCAL levels seems not to be influenced by disease characteristic and/or concomitant therapy intake. Therefore, fCAL should be a part of diagnostic workup in children with any type of JIA, but most importantly in those with ErA.

Disclosure of Interest: None declared

\section{P213}

The features of the juvenile idiopathic arthritis in Sakha Republic (Yakutia)

F. Vinokurova', V. Argunova², P. Sleptsova², T. Burtseva 1,3, M. Kostik ${ }^{4}$ ${ }^{1}$ Yakut science center of complex medical problems; ${ }^{2}$ Republic hospital № 1 - National center of Medicine, Pediatric center; ${ }^{3}$ North-Eastern Federal University, Yakutsk; ${ }^{4}$ SAINT-PETERSBURG STATE PEDIATRIC MEDI CAL UNIVERSITY, Saint-Petersburg, Russian Federation

Correspondence: M. Kostik

Pediatric Rheumatology 2020, 18(Suppl 2):P213

Introduction: Juvenile idiopathic arthritis (JIA) is not uncommon disease among aboriginals in Sakha Republic (Yakutia) - SR(Y), which can be related to high spreading of HLA B27 antigen. Adult aboriginals of $\mathrm{SR}(\mathrm{Y})$ have ankylosing spondylitis several times higher than Caucasians. According the epidemiological studies HLA B27 antigen distributes in 33\% of healthy aboriginals.

Objectives: The aim of our study was to describe features of JIA in aboriginals of $\mathrm{RS}(\mathrm{Y})$.

Methods: In the retrospective study were included 184 JIA patients before age 18 consisted of 155 SR(Y) aboriginals, 26 Caucasians and 3 mixed (Yakutia and Caucasians) whom have been living in SR(Y). We compare data of clinical course and compare aboriginals with Caucasians.

Results: The JIA prevalence in aboriginals is approximately $137 / 100$ 000 children and $30 / 100000$ in Caucasians. Clinical features describes in the table. HLA B27 distributed in aboriginals with JIA two times higher than in Caucasians and in healthy aboriginals. Positive family history in HLA B27 related diseases in 78 (53.1\%) of aboriginals compare to $8(36.4 \%)$ in Caucasians. ANA positivity was only in $4 / 53(7.6 \%)$ that is less than in other populations. Uveitis is in $16(10.5 \%)$ of aboriginals, usually associated with HLA B27 rather than ANA-positivity. Biologics received $44.5 \%$ of JIA aboriginals, 91.3\% received TNF-a inhibitors. HLA B27 presented in all JIA 
categories, $48.7 \%$ in oligoarthritis, in $42.9 \%$ of $\mathrm{RF}(-)$ polyarthritis, in $50 \%$ of $\mathrm{RF}(+)$ polyarthritis, $66.7 \%$ of systemic JIA, $71.3 \%$ of enthesitisrelated arthritis and in $40 \%$ of psoriatic arthritis patients. $70.3 \%$ of biologic users had HLA B27 antigen ( $p=0.028)$.

\begin{tabular}{llll}
\hline Parameter & Aboriginals $(\mathbf{n}=155)$ & Caucasians $(\mathbf{n}=\mathbf{2 6})$ & $\mathbf{p}$ \\
\hline Males, $\mathrm{n}(\%)$ & $92(85.6)$ & $10(38.6)$ & 0.047 \\
HLA B27, n (\%) & $87 / 145(60.0)$ & $6 / 20(30.0)$ & 0.012 \\
JIA categories & & & \\
Oligoarthritis & $39(25.1)$ & $6(23.1)$ & 0.13 \\
Polyarthritis, RF (-) & $25(16.1)$ & $4(15.4)$ & 7 \\
Polyarthritis, RF (+) & $2(1.3)$ & $0(0.0)$ & \\
Systemic & $4(2.6)$ & $4(15.4)$ & \\
Enthesitis-related arthritis & $79(51.0)$ & $10(38.5)$ & \\
Psoriatic arthritis & $6(13.9)$ & $2(7.7)$ & \\
\hline
\end{tabular}

Conclusion: Aboriginals in $\mathrm{SR}(\mathrm{Y})$ have higher prevalence of JIA, HLA B27 antigene, enthesitis-related category of JIA and juvenile ankylosing spondylitis, increased family history of rheumatic diseases, especially arthritis and have higher requirement in biologic medicine (anti-cytokine antibodies) for arthritis control compare to Caucasians. Trial registration identifying number: This work was supported by the Budget Projects of YNC CMP "Monitoring the state of children's health of the Republic of Sakha (Yakutia)" (\#0120-128-07-98), by the Project of the Ministry of Science and Higher Education of the Russian Federation (basic part of funding to M.K. Ammosov NorthEastern Federal University \#FSRG-2020-0016) and by the RFBR grant \#18-05-600035_Arctika.

\section{Disclosure of Interest: None declared}

P214

Achieving inactive disease in era with secukinumab following TNF inhibitors failure; a real-life, dual-center experience

S. Papailiou', M. Onoufriou², F. Dasoula ${ }^{1}$, M. N. Tsolia', D. N. Maritsi ${ }^{1,3}$

'Second Department of Paediatrics, 'P. \& A. Kyriakou' Children's Hospital, National and Kapodistrian University of Athens, Athens, Greece;

${ }^{2}$ Rheumatology Unit, Department of Paediatrics, 'Makarios III' Children's

Hospital, Nicosia; ${ }^{3}$ Rheumatology Unit, Department of Paediatrics,

'Makarios III' Children's Hospital, Nicosia, Cyprus

Correspondence: S. Papailiou

Pediatric Rheumatology 2020, 18(Suppl 2):P214

Introduction: TNF-inhibitors (TNFi) have greatly improved the clinical outcome of patients with Enthesitis-Related Arhtritis (ERA), there is however a minority of patients who fail to respond to standard treatment.

Objectives: We describe the efficacy and safety results of a secukinumab (monoclonal antibody neutralizing interleukin-17A) compassionate use in patients with active ERA following failure of disease remission by TNFi.

Methods: In this case-series, 4 patients diagnosed with ERA (based on ILAR criteria) with a mean age of 16.2 years (range 15-17) received secukinumab $150 \mathrm{mg}$ subcutaneously at weekly intervals and each 4 weeks thereafter for a total period of 18 months. All patients showed mild/no improvement to treatment with adalimumab (TNFi) received for at least six months. Clinical response was assessed at weeks 24, 52, 76(jSpADA). Safety and tolerability were also assessed at the same key time points during the course of the study

Results: Clinical and demographic data were collected. The jSpADA response rate was $70 \%$ at week 24 , which was sustained and further improved until week 76. Secukinumab was effective in multiple clinical outcomes including physician's global assessment of disease activity, CHAQ score and CRP level (table 1). Secukinumab was welltolerated with a safety profile consistent with reports in adult studies.
Disease duration, prior use of NSAIDS, age and gender did not affect the clinical outcome. There have been no reports of uveitis, psoriasis, IBD or any new-onset autoimmune disease. No major adverse events were reported.

Conclusion: This was a retrospective study regarding secukinumab effectiveness in patients with ERA who failed TNFi treatment, showing optimistic results. Secukinumab is usually effective in inducing and maintaining remission in Ankylosing Spondylitis and psoriatic patients and thus long-term therapy is recommended. Overall, biologic-naïve patients demonstrate a swift and sustained response to biologics (TNFi); however not all patients who receive TNFi will reach a state of inactive disease. Further studies are required to address the effectiveness of secukinumab treatment in patients with ERA, with the aid of appropriate disease-associated risk-assessment markers. Our study demonstrated that secukinumab is safe and effective in ERA patients, provided it is initiated promptly following TNFi failure. In addition, prolonging the duration of treatment in clinical remission prior to attempting discontinuation may show favorable results in contrast to other studies endeavoring earlier discontinuation. Minimizing exposure to TNFi especially within the case of partial response or no response may lead to decreased adverse events and costs. In addition, secukinumab provided sustained improvement in the signs and symptoms of ERA patients through 18 months, with no new or unexpected safety signals.

Disclosure of Interest: None declared

Table 1 (abstract P214). Patient and disease characteristics at inception and at one year follow-up First line indicates initial assessment and second line (in bold) follow up assessment

\begin{tabular}{|c|c|c|c|c|c|c|c|c|}
\hline $\begin{array}{l}\text { Patient } \\
\text { Characteristics }\end{array}$ & Gender & $\begin{array}{l}\begin{array}{l}\text { Age } \\
\text { (years) }\end{array}\end{array}$ & $\begin{array}{l}\text { CRP } \\
\text { (mg/L) }\end{array}$ & $\begin{array}{l}\text { SI } \\
\text { involvement }\end{array}$ & $\begin{array}{l}\text { Physician } \\
\text { global } \\
\text { assessment }\end{array}$ & $\begin{array}{l}\text { Spinal } \\
\text { Pain VAS } \\
\text { (0-10 scale) }\end{array}$ & CHAQ & jSpADA \\
\hline Patient 1 & male & 16 & $\begin{array}{l}32 \\
5\end{array}$ & bilateral & $\begin{array}{l}4.5 \\
1\end{array}$ & $\begin{array}{l}6 \\
0.3\end{array}$ & $\begin{array}{l}3 \\
0\end{array}$ & $\begin{array}{l}7 \\
0.5\end{array}$ \\
\hline Patient 2 & male & 17 & $\begin{array}{l}45 \\
4\end{array}$ & bilateral & $\begin{array}{l}8 \\
2\end{array}$ & $\begin{array}{l}7 \\
0\end{array}$ & $\begin{array}{l}2 \\
0.5\end{array}$ & $\begin{array}{l}5 \\
1\end{array}$ \\
\hline Patient 3 & female & 15 & $\begin{array}{l}76 \\
5\end{array}$ & bilateral & $\begin{array}{l}7.2 \\
1.5\end{array}$ & $\begin{array}{l}6 \\
1\end{array}$ & $\begin{array}{l}6 \\
1\end{array}$ & $\begin{array}{l}6 \\
1\end{array}$ \\
\hline Patient 4 & male & 17 & $\begin{array}{l}19 \\
\mathbf{3}\end{array}$ & unilateral & $\begin{array}{l}5 \\
1\end{array}$ & $\begin{array}{l}4 \\
0\end{array}$ & $\begin{array}{l}2 \\
0\end{array}$ & $\begin{array}{l}5 \\
0\end{array}$ \\
\hline
\end{tabular}

P215

Impact of anti-tumor necrosis factor therapy on spondyloarthritis research consortium of Canada sacroiliac joint inflammation score in children with enthesitis-related arthritis

M. Katsicas' ${ }^{1}$, C. Anoni ${ }^{2}$, G. Villarreal ${ }^{1}$, M. Galeano ${ }^{2}$

${ }^{1}$ Immnunology \& Rheumatology; ${ }^{2}$ Radiology, HOSPITAL DE PEDIATRÍA

GARRAHAN, Buenos Aires, Argentina

Correspondence: M. Katsicas

Pediatric Rheumatology 2020, 18(Suppl 2):P215

Introduction: Enthesitis-related arthritis (ERA) is a category of Juvenile idiopathic arthritis considered to be a form of Juvenile Spondyloarthropathy (jSpA). Tumor necrosis factor (TNF)-blocking strategies have proven to be effective in the treatment of jSpA. The Spondyloarthritis Research Consortium of Canada sacroiliac joint score (SPAR CC- SIS), measures inflammation in sacroiliac joints (SIJ). It has been used in adults as a tool to assess response to anti-TNF agents, however, limited experience exists in JSpA cohorts

Objectives: To evaluate effectiveness of anti-TNF treatment in SIJ inflammation in a cohort of patients with ERA using SPARCC-SIS. To quantify the effect size of TNF-blockers on SIJ inflammation. To 
evaluate potential predictor variables of Magnetic Resonance Imaging (MRI) SIJ remission

Methods: Retrospective review of prospectively collected data. We included patients with ERA (according to ILAR criteria) continuously treated with anti-TNF agents for $\geq 12$ months who had at least two MRIs of the sacroiliac joints performed before starting anti-TNF therapy (baseline) and during the follow up ( $>12$ months after anti-TNF treatment). SI joints were examined using T1-weighted images, T2 fastsuppressed and short-tau inversión recovery. The SPARCC-SIS was scored by two pediatric radiologists. SPARCC-SIS assessed the presence, depth and intensity of bone marrow edema (BME) on consecutive six slices in the iliac and sacrum bones. Scoring is composed by: BME (048), BM intensity (0-12), BM depth (0-12).Maximum:72. MRI SIJ remission was defined as SPARCC-SIS score < 2. Magnitude of change on MRI SIJ score was assessed. Treatment with TNF-blockers and exposure time were recorded. Outcome measures were collected: pain score (0-10), wellbeing according to the patient using a visual analogue scale (VASp, $0-10$ ), disease activity according to the physician (VASphy, 0-10), JADAS-10, JSpADA. Functional capacity was assessed by CHAQ. Statistical analysis included: Intraclass Correlation Coefficient (ICC) for readers, magnitude of change using Cohen's $d$ test, comparisons by Wilkoxon Signed Rank test and Mc Nemar test.

\section{Results:}

Twelve (75\% male) patients fulfilled inclusion criteria. Median (range) age at start of TNF-blocker therapy was 10.5 years (6-15) and disease duration 8 years(4-14). Presence of HLA-B27 positive 33\%. Anti-TNF therapy consisted of: etanercept (7, two of them were later switched to adalimumab) and adalimumab (5). Median (range) exposure to anti -TNF was 30.5 months (12-60). All patients had evidence of SIJ inflammation at baseline on MRI. SPARCC-SIS at baseline was (me$\operatorname{dian} \pm$ SD) $=36 \pm 16.87$. SPARCC-SIS over time (post treatment) was $12 \pm 7.44$. Magnitude of change on score (Cohen`s:1.84, r: 0.68). MRI SIJ remission was achieved on $33 \%$. ICC for concordance between readers $=0.73$. Outcome measures are shown in table1.

There was no significant association between exposure time to antiTNF treatment and MRI SIJ remission. Neither predictor variables on MRI SIJ remission were found.

Conclusion: Anti-TNF blockers showed to be effective in reducing MRI SIJ inflammation. Magnitude of change in SIJ inflammation had a large effect size. One third of patients achieved MRI SIJ remission on TNF-agents

Disclosure of Interest: None declared

Table 1 (abstract P215). See text for description

\begin{tabular}{llll}
\hline & Before TNF- blockers & After TNF -blockers & p value \\
\hline Pain Score $(0-10)^{*}$ & $3.5(0-7.5)$ & $0.25(0-5)$ & 0.019 \\
VASp $(0-10)^{*}$ & $4(0-9.5)$ & $0(0-6)$ & 0.03 \\
VASphy $(0-10)^{*}$ & $3.5(0-7.5)$ & $0.5(0-6)$ & 0.009 \\
CHAQ $\geq 0.5^{* *}$ & $6(50 \%)$ & $1(8 \%)$ & $\mathrm{ns}$ \\
JADAS-10* & $14.8(1-28)$ & $1(0-18)$ & 0.003 \\
JSpADA $^{*}$ & $2.5(0.5-6)$ & $0.75(0-3.5)$ & 0.013 \\
\hline
\end{tabular}

${ }^{*}$ median (range) ${ }^{* *} \mathrm{n}(\%)$

\section{P216}

Distinct disease profile in Hong Kong Juvenile Idiopathic Arthritis (JIA) patients

A. C. H. Ho, O. M. Chan, C. Y. Leung

Paediatrics, PRINCE OF WALES HOSPITAL, CHINESE UNIVERSITY OF HONG KONG, Hong Kong, Hong Kong

Correspondence: A. C. H. Ho

Pediatric Rheumatology 2020, 18(Suppl 2):P216

Introduction: The prevalence and disease pattern of JIA vary across different ethnicities. Up till now, data on Chinese children is lacking. Different disease profile may potentially impact management strategies and resource allocation.
Objectives: To study the characteristics of JIA in Hong Kong.

Methods: A retrospective review of patients being seen at the rheumatology clinic of a tertiary referral centre from 1990 to 2020 was performed. Eligible patients were: 1) diagnosed JIA according to the ILAR criteria; 2) those having more than 1 clinic visits; 3) those data retrievable from hospital record. Results were presented in descriptive manner.

Results: 94 of 100 patients were eligible for analysis. The mean duration of follow up was $7.5+5.5$ years. The mean age at diagnosis was 11.6+4.5 years. There were more boys than girls ( 55 vs $39,1: 1.28$ ). Among them Enthesitis Related Arthritis (ERA) was the most prevalent subtype (39.4\%), followed by Oligoarticular JIA (20.2\%). Undifferentiated JIA accounted for $10.6 \%$ of the total cases.

The data also reveal a large proportion of HLA B27+ individuals develops axial disease early. It is our practice to perform imaging to look for SI joint involvement if there is any suggestive signs and symptoms. Among ERA (all were HLA B27+), 78\% had sacroiliitis and $57 \%$ low back pain. 4 of the 10 undifferentiated JIA were HLA B27+, 2 had sacroiliitis. For the 13 patients who are still being managed by paediatricians, sacroiliitis are already established in 10 (77\%). Taking together, an overwhelming proportion of paediatric patients had established axial involvement.

$70.2 \%$ patients required treatment other than NSAIDs. DMARDs was used as $57.4 \%$. Biologics was used in $20 \%$. Despite the fact that many ERA had axial disease, biologic was prescribed in only $22 \%$ of them. This proportion is lower than other ERA cohorts ${ }^{1}$.

72 of $94(77 \%)$ patients still have rheumatology clinic FU at the end of the review period.

\begin{tabular}{ll}
\hline Category & Number (\%) \\
\hline Oligoarticular JIA & $19(20.2 \%)$ \\
Polyarticular RF negative JIA & $10(10.6 \%)$ \\
Polyarticular RF positive JIA & $6(6.4 \%)$ \\
Psoriatic arthritis & $3(3.2 \%)$ \\
Systemic JIA & $9(9.6 \%)$ \\
Undifferentiated JIA & $10(10.6 \%)$ \\
Enthesitis related arthritis & $37(39.4 \%)$ \\
Sacroiliitis & $29(78.4 \%)$ \\
Low Back Pain & $21(56.8 \%)$ \\
Treatment other than NSAIDs & $66(70.2 \%)$ \\
DMARDs & $54(57.4 \%)$ \\
Prednisolone & $4(4.3 \%)$ \\
Intra-articular steroid & $8(8.5 \%)$ \\
Biologics & $18(19.1 \%)$ \\
\hline
\end{tabular}

Discussion: In Hong Kong ERA is the most prevalent JIA subtype. A striking $78 \%$ develop sacroiliitis early in the course. This is somewhat different from what being suggested by a recent Taiwanese cohort, in which only $16 \%$ has sacroiliitis ${ }^{2}$. It was not mentioned in their study how SIJ was assessed. The possible difference in SIJ assessment may explain the discrepancy.

Biologics, instead of DMARDs, is indicated when the sacroiliitis fails to response to NSAIDs ${ }^{3}$. The finding of such a sizable number of sacroiliitis in ERA may have an impact on treatment algorithm and resource justification in the future.

Conclusion: ERA is the predominant JIA subtype in Hong Kong. Sacroiliitis development are common and probably early.

References

1 Glerup M, Rypdal V, Arnstad ED et al. Arthritis Care Res (Hoboken).2020;72(4):507-516

2 Shih YJ, Yang YH, Lin CY et al. Pediatr Rheumatol Online J.2019;17:58

3 Ringold S, Angeles-Han ST, Beukelman T et al. Arthritis Care Res (Hoboken).2019;71(6):717-734

Disclosure of Interest: None declared 
P217

Enthesitis-related arthritis: is there a relationship between structural hip damage and ultrasound synovitis?

K. Zouaoui, H. ferjani, K. maatallah, L. Ben ammar, D. Kaffel, W. Hamdi

Rheumatology, Kassab Institute of orthopedics, Ksar Said, Tunis, Tunisia

Correspondence: $\mathrm{K}$. Zouaoui

Pediatric Rheumatology 2020, 18(Suppl 2):P217

Introduction: Juvenile idiopathic arthritis (JIA) includes all inflammatory joint damage starting before the age of 16 , lasting more than or equal to 6 weeks and with no identifiable cause. Enthesitis-related arthritis (ERA) accounts for $15-20 \%$ of all JIAs. Hip involvement is particularly important in this disease as it represents a major functional prognostic factor.

Objectives: The objective of our study is to clarify the correlation between the presence of a structural hip damage on X-Ray and the presence of synovitis on hip ultrasound.

Methods: This is a monocentric retrospective study, 35 patients with ERA were enrolled (ILAR Criteria). We have identified the epidemiological, clinical and radiological characteristics. The damage of hip was assessed by the Bath Ankylosing Spondylitis Radiology Index hip (BASRI) score.

Results: Our study included 35 patients, 91,4\% of whom were male. The average age at diagnosis was 13.08 years [6-16]. At the time of inclusion, $88,5 \%$ of patients had coxitis, $77,1 \%$ of which had bilateral coxitis. The median time to onset of coxitis from the onset of ERA was 0,2 years [0-3,4]. Nearly $60 \%$ of patients had destructive coxitis. The average BASRI was 2.57 [0.4]. The ultrasound performed in 17 patients had demonstrated synovitis in 6 cases $(n=6)$ and effusion in 3 cases. There was no statistically significant correlation between the BASRI Hip Score and hip synovitis $(p=0.053)$.

Conclusion: According to our study, there was no correlation between structural hip damage assessed by BASRI and ultrasound synovitis in ERA. These two examinations must be complementary and if necessary supplemented by an MRI in order to better document the involvement of the hip.

Disclosure of Interest: None declared

\section{P218}

Spider bite mimicking pyoderma gangrenosum in sJlA: a case report

M. C. Maggio ${ }^{1}$, S. Giordano ${ }^{2}$, F. Cardella ${ }^{3}$, G. Corsello ${ }^{4}$

${ }^{1}$ PROMISE "G. D'Alessandro", University of Palermo; ${ }^{2}$ U.O.C. of Paediatric

Infectious Diseases, Paediatric COVID Center, Children Hospital "G. Di

Cristina"; ${ }^{3}$ Paediatric Clinic, Children Hospital "G. Di Cristina", ARNAS,

Palermo; "Department PROMISE "G. D'Alessandro", University of Palermo, Palermo, Italy

Correspondence: M. C. Maggio

Pediatric Rheumatology 2020, 18(Suppl 2):P218

Introduction: Several paediatric patients manifest conditions commonly misdiagnosed as spider bites, which however, can include other arthropods bites; bacterial, viral, and mycotic infections; vasculitis; dermatological diseases; miscellaneous conditions as drug reactions, chemical injuries.

Objectives: In Italy, spiders which are likely to be associated with severe toxin mediated tissue damage are uncommon, especially in urban zones. However, a minor trauma may be a precipitating factor for pyoderma gangrenosum particularly over the legs, in association with inflammatory bowel disease, haematologic diseases and Juvenile Idiopathic Arthritis (JIA). Methods: We describe a 11-years old boy with pyoderma gangrenosum complicated spider bite in association with systemic JIA (sJIA). The patient was in clinical remission after the start of the sJIA, occurred two months before, still treated with tapering doses of steroids and canakinumab, with the normalization of inflammatory parameters (CRP, ESR, SAA, ferritin) and clinical manifestations. Only a mild arthritis of the knee persisted and for this reason he was still treated with steroids. Furthermore, he developed hyperglycemia, requiring insulin treatment. The first dermatological manifestation which he referred was a red dot of the leg skin. In a few days, the erythema enlarged, involving an area of $7 \times 7 \mathrm{~cm}$, with oedema, pain, and blisters, evolving in a necrotic lesion, with purulent exudate, surrounded by a haemorrhagic zone.

Results: Haematological controls revealed neutrophilic leucocytosis, increased CRP and procalcitonin. He started treatment with intra venous administration of teicoplanin plus ceftriaxone, with no resolution of the clinical manifestations and the reduction of leukocytosis, CRP, procalcitonin.

A culture swab was performed and was positive for Pseudomonas Aeruginosa, confirmed by PCR on the culture. He started ciprofloxacin and surgical curettage of the lesion, with the resolution of the lesion and the normalization of biochemical parameters.

Conclusion: The aspect of the lesion and its evolution were evocative of a spider bite suggested by anamnestic records, complicated by a pyoderma gangrenosum secondary to Pseudomonas Aeruginosa. The underlying disease, the immune suppressive treatment, with steroids and biological drugs, the hyperglycaemic pattern of the patient allowed the severe evolution of the spider bite.

Children in treatment with immune suppressive and/or biologic drugs are at high risk of infections. Skin lesions, as arthropods bites, can be a facility for superinfection, with possible haematological and systemic diffusion.

Disclosure of Interest: None declared

\section{P219}

Application of preliminary printo classification criteria for sJIA in an Indian cohort

D. B. Pandya, on behalf of Dr Jay Virani, Dr Haresh Dobariya, Dr Harsh Bala, Dr Hitesh Bhambhani, Dr Ambrish Panara and Dr Vinod Bhadukiya.

Pediatric Rheumatology \& Immunology, Dev Children's Hospital, Rajkot, India

Correspondence: D. B. Pandya

Pediatric Rheumatology 2020, 18(Suppl 2):P219

\section{Introduction}

The strict application of the ILAR ${ }^{1}$ requirement for the presence of documented arthritis for the diagnosis of SJIA, early in the disease course, may result in unnecessary delays in initiating appropriate treatment. In preliminary PRINTO $^{2}$ classification criteria for SJIA, this mandatory requirement of documented arthritis has been modified. Objectives

To measure performance of preliminary PRINTO classification criteria for sJlA in our Indian cohort.

Methods

I gathered a data of seven SJIA patients who attended dev children's hospital between Jan 2019 and Jan 2020. My data included demographics,clinical presentation, laboratory parameters and outcome of these patients. All these patients were diagnosed at an early stage by clinical judgement irrespective of fulfilment of ILAR criteria. I applied preliminary PRINTO classification criteria for all. Results

Average age of selected children (4 girls and 3 boys) was 5.1 years. Conclusion

A preliminary PRINTO classification criteria for SJIA has been validated in our cohort. There are many raised inflammatory markers in most of these patients other than WBC count. These markers should be considered to be added in supportive laboratory criteria to be more specific towards the diagnosis. It is important to add PID in exclusion list especially in a case of sJA with MAS at onset. ${ }^{3}$

Trial registration identifying number

1.R.E. Petty, T.R. Southwood, P. Manners, et al., International League of Associations for Rheumatology classification of juvenile idiopathic arthritis: second revision, Edmonton, 2001, J. Rheumatol. 31 (2) (2004) 390-392. 2. Toward New Classification Criteria for Juvenile Idiopathic Arthritis: First Steps, Pediatric Rheumatology International Trials Organization International Consensus Alberto Martini, Angelo Ravelli, Tadej Avcin, Michael W. Beresford, Ruben Burgos-Vargas, Ruben Cuttica, Norman T. llowite, Raju Khubchandani, Ronald M. Laxer, Daniel J. Lovell, Ross E. Petty, Carol A. Wallace, Nico M. Wulffraat, Angela Pistorio, Nicolino Ruperto and for the Pediatric Rheumatology International Trials Organization (PRINTO) 
The Journal of Rheumatology February 2019, 46 (2) 190-197; DOI: https://doi.org/10.3899/jrheum.180168

3. The syndrome of hemophagocytic lymphohistiocytosis in primary immunodeficiencies: implications for differential diagnosis and pathogenesis. Sebastian FN Bode,1,2 Sandra Ammann,1,3 Waleed AlHerz,4 Mihaela Bataneant,5 Christopher C Dvorak. Haematologica. 2015 Jul; 100(7): 978-988. doi: 10.3324/haematol.2014.121608

Disclosure of Interest: None declared

Table 1 (abstract P219). Showed performance of preliminary PRINTO classification criteria for sJIA in our cohort

\begin{tabular}{|c|c|c|}
\hline SJIA & Preliminary PRINTO Classification Criteria & $\begin{array}{l}\text { No of patients } \\
\text { fulfilled respective } \\
\text { criteria } \\
N \text { (total=7) (\%) }\end{array}$ \\
\hline $\begin{array}{l}\text { Mandatory } \\
\text { criterion }\end{array}$ & $\begin{array}{l}\text { Fever of unknown origin that is } \\
\text { documented to be daily, quotidian; } \\
\text { fever that rises }>/=39 \text { C once a day } \\
\text { and returns to }\end{array}$ & $\begin{array}{l}7(100 \%) \text { at } \\
\text { diagnosis }\end{array}$ \\
\hline \multirow[t]{3}{*}{ Major criteria } & $\begin{array}{l}\text { 1.Evanescent (nonfixed) erythematous } \\
\text { rash }\end{array}$ & $6(\mathbf{8 6 \%})$ at diagnosis \\
\hline & 2.Arthritis & $2(\mathbf{2 8} \%)$ at diagnosis \\
\hline & & $\begin{array}{l}4 \text { (57\%) within } 4 \\
\text { months of diagnosis }\end{array}$ \\
\hline \multirow[t]{4}{*}{ Minor Criteria } & $\begin{array}{l}\text { 1.Generalized lymph node enlargement } \\
\text { and/or hepatomegaly and/or } \\
\text { splenomegaly }\end{array}$ & $3(43 \%)$ at diagnosis \\
\hline & 2.Serositis & $3(43 \%)$ at diagnosis \\
\hline & $\begin{array}{l}\text { 3.Arthralgia lasting } 2 \text { weeks or longer } \\
\text { (in absence of arthritis) }\end{array}$ & $3(43 \%)$ at diagnosis \\
\hline & $\begin{array}{l}\text { 4.Leukocytosis (>/= 15,000/mm3) with } \\
\text { neutrophillia }\end{array}$ & $\begin{array}{l}7(100 \%) \text { at } \\
\text { diagnosis }\end{array}$ \\
\hline \multirow{5}{*}{$\begin{array}{l}\text { Exclusions( ruled } \\
\text { out in our cohort) }\end{array}$} & EBV & $3(43 \%)$ \\
\hline & Neoplastic conditions & $2(28 \%)$ \\
\hline & Autoimmune conditions & $2(28 \%)$ \\
\hline & $\begin{array}{l}\text { Monogenic auto-inflammatory condi- } \\
\text { tion- CAPS }\end{array}$ & $3(43 \%)$ \\
\hline & PID & $1(14 \%)$ \\
\hline \multirow{5}{*}{$\begin{array}{l}\text { Common } \\
\text { laboratory } \\
\text { parameters }\end{array}$} & $\begin{array}{l}\text { Anaemia of chronic disease (age } \\
\text { matched) }\end{array}$ & $7(100 \%)$ \\
\hline & Platelet count (> 5 lakhs/ul) & $5(71 \%)$ \\
\hline & $E S R(>80 \mathrm{~mm} / \mathrm{hr})$ & $5(71 \%)$ \\
\hline & $C R P(>60 \mathrm{mg} / \mathrm{L})$ & $6(86 \%)$ \\
\hline & Ferritin $(>500 \mathrm{ug} / \mathrm{dl})$ & $2(28 \%)$ \\
\hline \multirow{5}{*}{$\begin{array}{l}\text { Medicines used in } \\
\text { our cohort }\end{array}$} & Oral Naproxen with oral steroids & $7(100 \%)$ \\
\hline & SC Methotrexate & $5(71 \%)$ \\
\hline & IV Methylprednisolone & $1(14 \%)$ \\
\hline & IV Tocilizumab & $2(14 \%)$ \\
\hline & $\begin{array}{l}\text { IV Ig (in one case of suspected } \\
\text { incomplete KD) }\end{array}$ & $1(14 \%)$ \\
\hline \multirow[t]{3}{*}{ Final Outcome } & Death (due to MAS) & $1(14 \%)$ \\
\hline & Monocylic course & $2(28 \%)$ \\
\hline & Chronic persistent course & $4(57 \%)$ \\
\hline
\end{tabular}

Abbreviations: PRINTO: Paediatric Rheumatology INterrnational Trail Organization, sJIA: systemic juvenile idiopathic arthritis, ILAR: International League of Associations for Rheumatology, EBV: Epstein-Barr-virus, CAPS: Cryopyrin Associated Periodic Syndromes, KD: Kawasaki Disease, MAS: Macrophage Activation Syndrome, PID: Primary Immunodeficiency Disease
P220

Allogenic hematopoietic stem cell transplantation for refractory childhood rheumatic diseases

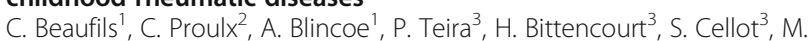
Duval $^{3}$, M.P. Morin', J. J. De Bruycker', J. Couture ${ }^{1}$, K. Samaan', H.

Decaluwe' ${ }^{1}$, F. Touzot' ${ }^{1}$, E. Haddad' ${ }^{1}$, J. Barsalou'

${ }^{1}$ Pediatric Rheumatology and Immunology; ${ }^{2}$ Pediatrics; ${ }^{3}$ Pediatric

Hemato-Oncology, CHU Sainte-Justine, Université de Montréal, Montreal, Canada

Correspondence: $C$. Beaufils

Pediatric Rheumatology 2020, 18(Suppl 2):P220

Introduction: Patients with refractory rheumatic diseases face poor quality of life, long-term sequelae and life-threatening complications. With advances in allogenic hematopoietic stem cell transplantation (allo-HSCT), this procedure becomes an interesting therapeutic option, allowing patients to achieve complete remission (CR) and withdrawal of most medications.

Objectives: Report two cases of allo-HSCT performed in children with refractory rheumatic diseases at a tertiary care paediatric hospital since 2017.

Methods: Patients' demographics, disease course, allo-HSCT course and outcome are described. A review of the literature was performed, and clinical outcomes were compared to our local experience. Patients/parental consent was obtained from both subjects.

Results: Two patients with systemic-onset juvenile idiopathic arthritis (sJIA) underwent allo-HSCT. Patients' characteristics are shown in Table 1. Both had failed multiple lines of immunosuppressive treatments and experienced severe disease and treatment-related complications. Both experienced early complications following HSCT but recovered without major sequelae. Patient 1 is in CR off all immunosuppressive medications at 2.6 years post-HSCT. Patient 2 experienced a disease flare at 11 months post-HSCT. He received one pulse of methylprednisolone and was started on tofacitinib. At 1.3 years post-HSCT, he is now in $C R$ on tofacitinib monotherapy, which represents a significant improvement from his pre-HSCT status. Engraftment was excellent with most recent chimerism at 100\% (2.6 years post-HSCT) and 95\% (1.1 years post HSCT), for Patient 1 and 2, respectively. Allo-HSCT is reported as a treatment option in very few paediatric JIA patients. In a recently described cohort of 16 JIA patients who underwent allo-HSCT (11/16 had SJIA), CR was achieved in $90 \%$ and $9 \%$ had transplantation-related mortality (TRM) (median follow-up 2.4 [range 0.2-8.0] years) [1].

Conclusion: Allo-HSCT should be considered as an alternative therapeutic option early in the course of severe paediatric rheumatic diseases refractory to conventional treatments as it is currently the only curative treatment. The decision to proceed to allo-HSCT should not be delayed since it increases TRM, and allo-HSCT does not correct disease and treatment's sequelae. With current TRM rates $<10 \%$, allo-HSCT should be considered sooner after failure of conventional lines of treatment, and not only in patients with no other therapeutic options. Our data may suggest that full donor chimerism is necessary in some patients to maintain $\mathrm{CR}$ off immunosuppression.

\section{Reference}

1. J MFS, Ladomenou F, Carpenter B, et al. Allogeneic hematopoietic stem cell transplantation for severe, refractory juvenile idiopathic arthritis. Blood advances 2018;2(7):777-86.

Disclosure of Interest: None declared 
Table 1 (abstract P220). sJlA patients characteristics and evolution post-HSCT

\begin{tabular}{|c|c|c|}
\hline & Patient 1 & Patient 2 \\
\hline Sex & $F$ & $M$ \\
\hline $\begin{array}{l}\text { Refractory } \\
\text { disease } \\
\text { manifestations }\end{array}$ & Fever, polyarthritis, MAS & Fever, polyarthritis \\
\hline $\begin{array}{l}\text { Previous } \\
\text { medications }\end{array}$ & $\begin{array}{l}\text { NSAID, Corticosteroids, } \\
\text { Methotrexate, Tocilizumab, } \\
\text { Anakinra, Canakinumab, } \\
\text { Cyclosporine, Tofacitinib. }\end{array}$ & $\begin{array}{l}\text { NSAID, Corticosteroids, } \\
\text { Methotrexate, Tocilizumab, } \\
\text { Canakinumab, Infliximab, } \\
\text { Tofacitinib, Siltuximab }\end{array}$ \\
\hline $\begin{array}{l}\text { Age at HSCT, } \\
\text { years }\end{array}$ & 5 & 8 \\
\hline $\begin{array}{l}\text { Disease } \\
\text { duration at } \\
\text { HSCT, years }\end{array}$ & 2 & 3 \\
\hline Donor type & MUD & MRD \\
\hline $\begin{array}{l}\text { Time to stop IS } \\
\text { post-HSCT, } \\
\text { months }\end{array}$ & 14 & Ongoing \\
\hline $\begin{array}{l}\text { Disease } \\
\text { outcome } \\
\text { (years post- } \\
\text { HSCT) }\end{array}$ & $\begin{array}{l}\text { CR off IS } \\
(2.6)\end{array}$ & $\begin{array}{l}\text { Relapsed at } 11 \text { months; CR } \\
\text { on Tofacitinib } \\
\text { (1.3) }\end{array}$ \\
\hline
\end{tabular}

F: Female; IS: Immunosuppression; M: Male; MAS: Macrophage Activation Syndrome; MRD: Matched-Related Donor; MUD: Matched Unrelated Donor; NSAID: Non Steroidal Anti-Inflammatory Drugs

\section{P221}

The role of Intra-Articular Glucocorticoid Injections (IAGI) in Systemic Juvenile Idiopathic Arthritis (sJA): a single center experience

A. Uva ${ }^{1}$, G. Tarantino ${ }^{2}$, D. Pires-Marafon ${ }^{3}$, A. Aquilani ${ }^{3}$, H. Jadoun ${ }^{3}$, R. Nicolai $^{3}$, F. De Benedetti ${ }^{3}$, S. Magni-Manzoni ${ }^{3}$

${ }^{1}$ Sapienza-University; ${ }^{2}$ Catholic University of Sacred Heart; ${ }^{3}$ IRCCS Bambino Gesù Children's Hospital, Rome, Italy

Correspondence: A. Uva

Pediatric Rheumatology 2020, 18(Suppl 2):P221

Introduction: $|A G|$ are a safe and widespread treatment option in patients with JIA. Little is known about the benefits of IAGI in sJIA Objectives: The aim of the study is to explore whether IAGI can spare the amount of systemic glucocorticoids (GC) in achieving remission of arthritis in SJIA

Methods: Records of patients with sJIA at the study center were retrospectively reviewed. All sJIA patients at their first assessment of arthritis (baseline) and treated with systemic GC (with or without c/ bDMARDs) were included and divided into two groups: group $A$ comprised children who were also treated with IAGI within the first five months of arthritis occurrence; group B the others. Patients with disease onset before 2015 or treated for more than 3 months before accessing to the study center were excluded. Demographic and clinical features, administration of intravenous (IV) GC pulses from baseline to six months from arthritis occurrence (T6), total amount of systemic GC $(\mathrm{mg} / \mathrm{kg})$, remission of arthritis and achievement of clinical inactive disease (CID, according to Wallace criteria) at T6 were registered and compared in the two groups

Results: From a total of $23 \mathrm{~s}$ اA patients at first assessment of arthritis at the study center, 11 were excluded because of treatment before $2015(n=2)$, treatment for more than 3 months before coming at the study center $(n=6)$, no systemic $G C(n=2)$ or follow-up less than six months from IAGI $(n=1)$. Twelve patients were included in the study. In group A ( $n=6, F$ 83.3\%) IAGI was performed in a median of 3.8 months (range 1.6-4.9) from baseline, with a median of 12 injected sites/IAGI procedure (range 2-26), including 30 large joints, 19 small joints and 24 tendon sheaths. Triamcinolone hexacetonide and methylprednisolone acetate were injected in large joints and small joints+tendon sheaths, respectively. Children in group $B$ ( $n=6, F$ $33.3 \%$ ) presented arthritis of large joints ( 2 wrists, 2 knees, 1 ankle, 1 elbow). In both groups $4 / 6$ (66.7\%) patients received IV GC pulses from baseline to T6. Table 1 shows demographic features at baseline and clinical assessment at T6 of the two groups

Conclusion: Our study represents the first attempt to define the role of IAGI in children with sJIA. Despite a clear trend in sparing systemic GC in achieving remission in children with $\mathrm{SJ}$ IA treated also with IAGI, our results highlight the complexity of $\mathrm{s} J \mathrm{IA}$ features presentation and treatment. This limits the possibility of comparing the therapeutic strategies in our cohort. Nonetheless, IAGI appear to represent an efficacious option in SJIA, particularly in those patients with widespread articular involvement. Prospective randomized studies may further explore this benefit

Disclosure of Interest: None declared

Table 1 (abstract P222). Demographic features at baseline and clinical assessment at T6 in sJIA patients with arthritis.

\begin{tabular}{|c|c|c|}
\hline & $\begin{array}{l}\text { Group A (IAGI; } \\
\mathrm{N}=6)\end{array}$ & $\begin{array}{l}\text { Group B (No } \\
\text { IAGI; N=6) }\end{array}$ \\
\hline Age at baseline, years, median (range) & $3.5(1.0-7.9)$ & $7.4(0.8-15.1)$ \\
\hline $\begin{array}{l}\text { Concomitant systemic treatment at } \\
\text { baseline, } n(\%)\end{array}$ & $3(50.0)$ & $5(83.3)$ \\
\hline $\begin{array}{l}\text { Systemic treatment (apart from GC) } \\
\text { baseline-T6, } n(\%)\end{array}$ & $6(100)$ & $6(100)$ \\
\hline Systemic GC baseline-T6, $n$ (\%) & $5(83.3)$ & $6(100)$ \\
\hline $\begin{array}{l}\text { Cumulative systemic GC baseline-T6 (mg/ } \\
\text { Kg), median (range) }\end{array}$ & $54(21-147)$ & $102(54-200)$ \\
\hline $\begin{array}{l}\text { Triamcinolone hexacetonide (mg/kg), } \\
\text { median (range) }\end{array}$ & $2.7(1.1-6.7)$ & - \\
\hline $\begin{array}{l}\text { Methylprednisolone acetate }(\mathrm{mg} / \mathrm{kg}) \\
\text { median (range) }\end{array}$ & $4.8(1.1-8.0)$ & - \\
\hline Remission of arthritis at T6, $n$ (\%) & $5(83.3)$ & $6(100)$ \\
\hline CID at T6, $n(\%)$ & $4(66.7)$ & $5(83.3)$ \\
\hline
\end{tabular}

P222

Coxarthrosis risk factors in systemic and Non-Systemic Juvenile Idiopathic Arthritis

L. Sorokina', I. Avrusin², R. Raupov², N. Garipova ${ }^{3}$, M. Gharabaghtsyan ${ }^{4}$, S. Khrypov $^{5}$, E. Isupova ${ }^{2}$, E. Gaidar ${ }^{2}$, A. Santimov', I. Chikova², M. Dubko², V. Masalova ${ }^{2}$, T. Likhacheva ${ }^{2}$, L. Snegireva ${ }^{2}$, M. Kostik ${ }^{2}$

'Leningrad's Regional Children's Clinical Hospital, Saint-Petersburg, Russia; ${ }^{2}$ Saint-Petersburg State Pediatric Medical University; ${ }^{3}$ Almazov National Medical Research Centre, Saint Petersburg, Russian Federation; ${ }^{4}$ Institute of Child and Adolescent Health with the Arabkir Medical

Center, Yerevan, Armenia; ${ }^{5}$ Saint-Petersburg clinical research-practical center for specialized healthcare (oncology), Saint Petersburg, Russian Federation

Correspondence: $L$. Sorokina

Pediatric Rheumatology 2020, 18(Suppl 2):P222

Introduction: Hip involvement in juvenile idiopathic arthritis (JIA) is an alarming sign for patients and physicians. It can lead to coxarthrosis (CA), severe loss of function and decreased quality of life, and may require total hip arthroplasty (THA).

Objectives: To compare the frequency of hip involvement, progression to CA and a requirement for THA in patients with systemic and non-systemic JIA categories.

Methods: $753 \mathrm{JIA}$ patients aged 2 to 17 years included in the retrospective study. JIA was diagnosed by ILAR criteria. Patients divided into two groups: systemic-onset JIA ( $n=58)$ and non-systemic, included other JIA categories $(n=695)$. We compared demographic and clinical data, frequency and character of hip damage (coxitis, CA and THA), and treatment regimens too, especially, corticosteroid (CS) administration.

Results: the data presented in table 1. Patients with sollA developed CA earlier than non-systemic $13.7(9.5 ; 15.4)$ vs $15.2(13.5 ; 16.4)$ years 
$(p=0.045)$. There were no differences in time to $C A$ ( 4.5 vs 5.1 years, $p=$ 0.956 ), time to THA ( 7.4 vs 9.5 years, $p=0.571$ ) and time since CA to THA ( 2.1 vs 1.1 years, $p=1.0$ ). Patients with sollA had more markers of inflammation (ESR, CRP, PLT, WBC) and lower Ca (2.35 vs $2.4 \mathrm{mmol} / \mathrm{l}$, $\mathrm{p}=0.006$ ) and $25 \mathrm{OHD}(14.0 \mathrm{vs} 19.0 \mathrm{ng} / \mathrm{ml}, \mathrm{p}=0.039)$. Systemic JIA increased the cumulative probability of CA development in Coxregression model: $R R=2.7(1.4 ; 5.4), p=0.009$. For whole studied population CS per os (PO) $(p=0.008)$, pulse-therapy with $C S(p=0.012)$, cumulative doses of CS $(p=0.023)$, WBC $(p=0.044)$, soJIA $(p=0.007)$ and delayed hip involvement $(p=0.00002)$ were predictors of $C A$ in univariate regression analysis. In multiple regression analyses only delayed hip involvement $(p=0.023)$ and cumulative doses of $C S>2700 \mathrm{mg}$ ( $p=$ 0.02 ) were independent risk factors of CA development. In logistic regression delayed hip involvement ( $\mathrm{OR}=4.9$ [95\%Cl: 1.2; 20.4], $\mathrm{p}=0.027$ ) and cumulative doses of $\mathrm{CS}>2700 \mathrm{mg}(\mathrm{OR}=5.7$ [95\%Cl: 1.2; 27.9], $\mathrm{p}=$ 0.025 ) increase the risk of CA development. Patients with systemic and non-systemic JIA had different risk factors of coxarthrosis: onset age $(p=0.049), C S$ PO $(p=0.033)$, CS pulse-therapy $(p=0.023), C S>2700 \mathrm{mg}$ $(p=0.025)$ and WBC $(p=0.013)$ were risk factors in systemic JIA; alkaline phosphatase (AP) $(p=0.013), C S$ PO $(p=0.047), C S$ pulse-therapy $(p=$ $0.026), C S>2700 \mathrm{mg}(p=0.01)$ were risk factors in non-systemic JIA. In discriminant analysis only CS $>2700 \mathrm{mg}(\mathrm{p}=0.008)$ and WBC $(p=0.024)$ were CA predictors in systemic JIA and calcium $(p=0.026), A P(p=$ $0.019)$, CS PO ( $p=0.041), C S$ pulse-therapy $(p=0.031), C S>2700 \mathrm{mg}(p=$ 0.012 ) were risk factors in non-systemic JIA.

\begin{tabular}{llll}
\hline Investigated parameters & soJIA, $\mathbf{n = 5 8}$ & $\begin{array}{l}\text { non-systemic JIA, } \mathbf{n =} \\
\mathbf{6 9 5}\end{array}$ & p-value \\
\hline JIA onset age, years & $4.3(2.6-7.3)$ & $6.15(3.0-10.5)$ & 0.022 \\
Any hip involvement, n (\%): & $19(32.8)$ & $134(19.3)$ & 0.015 \\
Coxitis & $8(13.8)$ & $97(14.0)$ & $<0.001$ \\
CA & $11(19.0)$ & $37(5.3)$ & \\
THA, n (\%) & $5(8.6)$ & $16(2.3)$ & 0.005 \\
Delayed hip involvement, n & $11 / 19(57,9)$ & $41 / 134(30,6)$ & 0,019 \\
(\%) & & & \\
CS, PO, n (\%) & $47(81)$ & $105 / 694(15,1)$ & 0,000001 \\
Pulse-therapy of CS, n (\%) & $46 / 57(80,7)$ & $89 / 693(12,8)$ & 0.000001 \\
Total CS, mg & $3085(1500-$ & $2000(750-4500)$ & 0,005 \\
& $7000)$ & & \\
\hline
\end{tabular}

Conclusion: to avoid coxarthrosis development required excluded corticosteroids as well as possible or applied steroid-sparing agents, e.g., biologics, especially in soJIA.

Trial registration identifying number: This work supported by the Russian Foundation for Basic Research (grant № 18-515-57001).

Disclosure of Interest: None declared

\section{P223}

Drug survival for IL-1 inhibitor canakinumab: data from a singlecenter observation

E. Krekhova ${ }^{1,2}$, E. Alexeeva ${ }^{1,2}$, T. Dvoryakovskaya ${ }^{1,2}$, A. Mamutova ${ }^{1}$, K. Isayeva', R. Denisova', A. Chomahidze', A. Fetisova', O. Lomakina', K. Chibisova', M. Gautier', D. Vankova', I. Kriulin $^{1,2}$, M. Shingarova ${ }^{1,2}$ ${ }^{1}$ Rheumatology, National Medical Research Center for Children's Health; ${ }^{2}$ Sechenov First Moscow State Medical University, Moscow, Russian Federation

Correspondence: E. Alexeeva

Pediatric Rheumatology 2020, 18(Suppl 2):P223

Introduction: The efficacy of canakinumab for treatment patients with systemic juvenile idiopathic arthirtis (sJlA) was demonstrated before. Our aims was to describe canakinumab drug survival based on data from a single-center observation.

Objectives: To analyze the drug survival of canakinumab in patients with SJIA treated at the National Medical Research Center of Children`s health, Moscow, Russia.
Methods: Medical records from sJlA patients treated with canakinumab (CAN) were analyzed retrospectively from the National Medical Research Center of Children`s health, Moscow, Russia.

Results: Seventy-four patients presenting with SJIA were included in this observation, with a median age at treatment initiation of 8,2 (interquartile range, IQR 3,9-12.7) years and a median disease duration of 5,3 (IQR 2,9-10,7) years. Most patients (66/74) had been treated previously with one or more biologic agents for SJIA, 44/74 patients have received CDMARDs. As of 30 March of 2020, the median time of follow up was 97,3 $(37,4-153,8)$ months, with all patients being followed for at least 6 months. The most patients $(66 / 74)$ were previously treated with other biologic drugs. Eleven (11/74) patients stopped treatment with canakinumab, three of them - because of achievement of remission. Two patient stopped treatment within 6 months from therapy start: due to primary inefficiency (1) and allergic reaction (1). Five $(5 / 11)$ patients were-co-administered with CDMARDs, other 5 with oral GC, and 6 subjects had been previously exposed to other biologic drugs. Whole 5 patients stopped therapy due to secondary inefficiency: 2 patients were switched on TOC, other children were switched on ETA (1), RIT (1), ADA (1). There were 1 death recorded because of MAS, not associated with receiving CAN.

Conclusion: Our results have shown an excellent survival of the IL-1 inhibitor CAN. Survival was not affected by the concomitant use of cDMARDs. These data underline the effectiveness of CAN in sJIA patients as monotherapy.

Disclosure of Interest: E. Krekhova: None declared, E. Alexeeva Speaker Bureau of: Novartis, Pfizer, Sanofi, MSD and Roche, T. Dvoryakovskaya Speaker Bureau of: Novartis, Pfizer, MSD and Roche, A. Mamutova Speaker Bureau of: Novartis, K. Isayeva: None declared, R. Denisova Speaker Bureau of: Novartis, MSD and Roche, A. Chomahidze: None declared, A. Fetisova: None declared, O. Lomakina: None declared, K. Chibisova: None declared, M. Gautier: None declared, D. Vankova: None declared, I. Kriulin: None declared, M. Shingarova: None declared

P224

The share recommendations on diagnosis and treatment of systemic JIA

A. Leek' , J. Anton' 2 , T. Avcin ${ }^{3}$, F. De Benedetti ${ }^{4}$, V. Boom ${ }^{5}$, C. Bracaglia ${ }^{6}$, P.

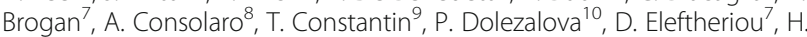
Foster $^{11}$, C. Hinze ${ }^{12}$, I. Kone-Paut ${ }^{13}$, K. Minden ${ }^{14}$, F. Minoa ${ }^{15}$, P. Quartier ${ }^{16}$ A. Ravelli ${ }^{17}$, N. Ruperto ${ }^{18}$, J. Swart ${ }^{19}$, Y. Uziel ${ }^{20}$, H. Wittkowski ${ }^{21}$, C. Wouters $^{22}$, M. Zajc Avramovitz ${ }^{23}$, N. Wulffraat', S. Vastert ${ }^{24}$

${ }^{1}$ Pediatric Rheumatology and Immunology, UMC Utrecht, Utrecht, Netherlands; ${ }^{2}$ Reumatologia Pediàtrica, Hospital Sant Joan de Déu, Barcelona, Spain; ${ }^{3}$ epartment of Allergology, Rheumatology and Clinical Immunology, University Children's Hospital University Medical Center Ljubljana, Ljubljana, Slovenia; ${ }^{4} \cup O$ Rheumatologia, IRCCS Ospedale Pediatrico Bambino Gesù, Rome, Italy; Internal Medicine, University Medical Center Utrecht, Utrecht, Netherlands; ${ }^{6}$ UO Reumatologia, IRCCS Ospedale Pediatrico Bambino Gesù, Roma, Italy; ${ }^{7}$ Infection,Inflammation and Rheumatology, UCL GOS Institute of Child Health, London, United Kingdom; ${ }^{8}$ U.O.C. Pediatria II - Reumatologia, IRCCS Istituto Giannina Gaslini, Genova, Italy; ${ }^{9}$ Pediatric Rheumatology-Immunology,

Semmelweiss University, Budapest, Hungary; ${ }^{10}$ Centre for paediatric rheumatic and autoinflammatory diseases, General University Hospital Prague, Prague, Czech Republic; ${ }^{11}$ Pediatric Rheumatology, Newcastle University, Newcastle upon Tyne, United Kingdom; ${ }^{12}$, University Children's Hospital, University Children's Hospital, Muenster, Germany; ${ }^{13}$ Department of rheumatoogy and immunology, Necker Hospital, Paris, France; ${ }^{14}$ Pediatric Rheumatology, DRFZ, Berlin, Germany;

${ }^{15}$ Reumatologia Pediatrica, UOC Pediatria a Media Intensità di Cure, Milano, Italy; ${ }^{16}$ Pediatric Immunology-Hematology and Rheumatology Unit, Necker Hospital, Paris, France; ${ }^{17}$ Rheumatologia pediatrico, istituto G. Gaslin; ${ }^{18}$ Clinica Pediatria e Reumatologia, Istituto G. Gaslini, Genova, Italy; ${ }^{19}$ Pediaric Rheumatology and Immunology, University Medical Center Utrecht, Utrecht, Netherlands; ${ }^{20}$ Pediatric Rheumatology Unit, Department of Pediatrics, Meir Medical Center, Kfar Saba, Israel; 
${ }^{21}$ Department of Pediatric Rheumatology and Immunology , , University Children's Hospital, Muenster, Germany; ${ }^{22}$ Pediatric rheumatology and immunology, Universitye Hospital Leuven, Leuven, Belgium; ${ }^{23}$ Pediatric Rheumatology, University Hospital, Ljubljana, Slovenia; ${ }^{24}$ Pediatric Rheumatology \& Immunology, University Medical Center Utrecht, Utrecht, Netherlands

Correspondence: S. Vastert

Pediatric Rheumatology 2020, 18(Suppl 2):P224

Introduction: Systemic juvenile idiopathic arthritis (sJIA) is a rare, complex auto-inflammatory disease with significant morbidity including fever, rash, serositis and articular problems. With the availability of interleukin-1 (IL-1) and IL-6 inhibitor treatment, morbidity has significantly reduced and the outcome for SJIA patients has improved. However, differences in access to care and differences in treatment strategies between countries in and outside of Europe remain a concern.

Objectives: The Single Hub and Access point for paediatric Rheumatology in Europe (SHARE) consortium aimed to develop best practices for paediatric rheumatic diseases in order to decrease differences in care between European countries. Here, we present the final results of the literature review and a series of consensus meetings on defining overarching, diagnostic and therapeutic recommendations for diagnosis and treatment of sJIA.

Methods: The SHARE methodology has been previously published, including the use of the EULAR standardized operating procedure for developing best practice recommendations. As per these guidelines, a methodologist provided supervision during the process and consensus meetings.

A systematic literature search of Medline, Embase and Cochrane databases was performed in 2013 and again in November 2019. Results were used to develop and support recommendations on diagnosis, treatment and complications of systemic JIA. These recommendations were presented in an online survey to a taskforce of expert paediatric rheumatologists to assess potential agreement and enable rewording. The participating experts convened in 3 consensus meetings (Genoa 2014, Barcelona 2015 and Utrecht 2020) to develop the recommendations. Making use of the Nominal Group Technique, recommendations were proposed, discussed and voted on. Recommendations that reached $\geq 80 \%$ were accepted.

Results: The 2-step systematic literature review included 98 papers on SJIA. Quality and grade of evidence was assessed and a categorized overview was used as backbone during the consensus meeting. In total, 15 recommendations were developed and accepted: 4 overarching principles, 3 diagnostic recommendations and 8 recommendations on management of sJIA and its complications. Recommendations were presented with accompanying level of evidence (LoE), strength of recommendation (SoR) and percentage of agreement (PoA). In addition, adherence to the 2018 EULAR Treat-to-Target principles on JIA was confirmed by the expert panel.

Conclusion: These SHARE best practice recommendations for sJIA are based on the best available evidence and expert opinion, and provide guidance for the diagnosis and management of $s J \mathrm{~A}$, aiming to improve the outcome for all sJIA patients in Europe and beyond.

Disclosure of Interest: A. Leek: None declared, J. Anton Consultant for: SOBI, Roche, Novartis, Speaker Bureau of: SOBI, Roche, Novartis, T. Avcin Consultant for: AbbVie, Octapharma, Takeda, Alexion, F. De Benedetti Consultant for: Institutional grants from Abbvie, SOBI, Novimmune, Novartis, Roche, Pfizer, Abbvie, SOBI, Novimmune, Novartis, Roche, Pfizer, Employee of: Family member SOBI employee, V. Boom: None declared, C. Bracaglia Consultant for: SOBI and Novartis , P. Brogan Consultant for: SOBI, Roche, Novartis; UCB, institutional grants from $\mathrm{SOBI}$, Roche, Novartis, A. Consolaro Speaker
Bureau of: AbbVie, T. Constantin: None declared, P. Dolezalova Consultant for: SOBI, Novartis, Sanofi Genzyme, D. Eleftheriou: None declared, H. Foster Consultant for: Unrestricted educational grants from Pfizer, SOBI, Sanofi-Genzyme, C. Hinze Consultant for: Novartis, I. Kone-Paut: None declared, K. Minden Consultant for: Abbvie, Sanofi, gsk, Roche, F. Minoa Consultant for: SOBI, P. Quartier Consultant for: consultancy or speaking fees from AbbVie, Bristol-Myers Squibb, Chugai-Roche, Lilly, Novartis, Novimmune, and Swedish Orphan Biovitrum, A. Ravelli Consultant for: SOBI, Novartis and Roche, N. Ruperto Consultant for: consultancy or speakers fee from: Ablynx, AstrazenecaMedimmune, Aurinia, Biogen, Boehringer, Bristol Myers and Squibb, Centrical Global, Domain Therapeutics, Eli-Lilly, EMD Serono, Glaxo Smith and Kline, Hoffmann-La Roche, Idorsia, Janssen, Merck, Novartis, Pfizer, R-Pharma, Sanofi, Servier, Sinergie, and Sobi, J. Swart Paid Instructor for: SOBI, Y. Uziel Speaker Bureau of: Pfizer, Abbvie, H. Wittkowski Speaker Bureau of: Novartis, Shire/Takeda and CSL-Behring, C. Wouters Consultant for: institutional grants from Roche, Novartis, Sobi, GSK, M. Zajc Avramovitz: None declared, N. Wulffraat Consultant for: SOBI, Novartis, Roche, institutional grants from SOBI, S. Vastert Consultant for: SOBI, Novartis, unrestricted institutional grant $\mathrm{SOBI}$

\section{P225}

Effect of dose and duration of glucocorticoid treatment on prognosis of Systemic Juvenile Idiopathic Arthritis

A. Paç Kısaarslan', S. Ozdemir Cicek', N. Şahin', M. Basol ${ }^{2}$, S. Doğantan'

S. N. Taşkın', M. H. Poyrazoğlu

${ }^{1}$ Pediatric Rheumatology, Erciyes University Faculty of Medicine, Kayseri;

${ }^{2}$ Biostatistics, Abant Izzet Baysal University Faculty of Medicine, Bolu, Turkey

Correspondence: S. Ozdemir Cicek

Pediatric Rheumatology 2020, 18(Suppl 2):P225

Introduction: Systemic Juvenile idiopathic arthritis(sJIA) is characterised with high level of inflammation, high disease activity, and risk of development of macrophage activation syndrome that is high life threatening condution. Due to all of these reasons,the rate of glucocorticoid usage is very higher in sJIA than other JIA subtypes. Although the cessation of glucocorticoid was recommended as soon as possible, there is no consensus on the duration and dosage of glucocorticoid treatment.

Objectives: We aimed to investigate to effect of dose and duration of glucocorticoid treatment on SJIA disease prognosis.

Methods: Forty two patients diagnosed with sJIA and had duration of disease upper than 2 years were involved in this study. Demographic, clinic, laboratory data, and treatments were collected from patients records. Affecting factors which were patients clinical, labaratory findings, treatment options, dose and duration of steroid treatments were evaluated on the duration of achieving remission period (period of active disease) and duration of remission period with cox regression analysis.

Results: Half of patients had monophasic course. Age at diagnosis; HR(95\% Cl):1,095 (1,006-1,192),p: 0,036, platelet values; HR $(95 \% \mathrm{Cl}): 0,997(0,995-0,999), p: 0,008$, duration of steroid treatment; $\mathrm{HR} \quad(95 \% \quad \mathrm{Cl}): \quad 0,837 \quad(0,754-0,929), \mathrm{p}: \quad 0,001$ were determined as risk factors on duration of achieving remission period (period of active disease) with multivariate cox regression analysis (table). There was no determined risk factors on duration of remission period.

Conclusion: In our preliminary study showed that duration and dose of glucocorticoid treatment did not change prognosis and disease course. The best dose and duration of the treatment should be evaluated further studies.

Disclosure of Interest: None declared 
Table 1 (abstract P225). Factors Affecting on the Duration of Achieving Remission Period

\begin{tabular}{|c|c|c|c|c|c|}
\hline \multirow[t]{2}{*}{ Variables } & \multicolumn{3}{|c|}{$\begin{array}{l}\text { Univariate Cox } \\
\text { Regression }\end{array}$} & \multicolumn{2}{|l|}{$\begin{array}{l}\text { Multivariate Cox } \\
\text { Regression* }\end{array}$} \\
\hline & $\mathrm{n}$ & $\begin{array}{l}\text { HR } \\
(95 \% \text { Cl) }\end{array}$ & $p$ & $\begin{array}{l}\mathrm{HR} \\
(95 \% \mathrm{Cl})\end{array}$ & $p$ \\
\hline Age at diagnosis & 42 & $1,098(1,015-1,188)$ & 0,019 & $1,095(1,006-1,192)$ & 0,036 \\
\hline Gender & 42 & $0,986(0,530-1,835)$ & 0,965 & & \\
\hline \multicolumn{6}{|l|}{ Features at first flare } \\
\hline Fever & 42 & & & & \\
\hline Serositis & 5 & 1,460 $(0,556-3,830)$ & 0,442 & & \\
\hline Rash & 23 & $1,594(0,846-3,001)$ & 0,149 & & \\
\hline Joint involvement & 38 & $0,644(0,223-1,864)$ & 0,417 & & \\
\hline Morning stiffness & 9 & $0,440(0,189-1,026)$ & 0,057 & & \\
\hline Lymphadenopathy & 6 & 1,603 $(0,653-3,936)$ & 0,303 & & \\
\hline Hepatosplenomegaly & 10 & 2,099 $(0,963-4,574)$ & 0,062 & & \\
\hline Macrophage activating syndrome & 14 & $1,108(0,578-2,125)$ & 0,757 & & \\
\hline Patient/parent VAS & 42 & $0,846(0,648-1,105)$ & 0,219 & & \\
\hline PGA & 42 & $1,002(0,777-1,293)$ & 0,987 & & \\
\hline \multicolumn{6}{|l|}{ Laboratory parameters at first flare } \\
\hline $\mathrm{HGB}(\mathrm{g} / \mathrm{dL})$ & 42 & $0,885(0,677-1,158)$ & 0,374 & & \\
\hline $\mathrm{WBC}(/ \mu \mathrm{L})$ & 42 & $1,000(1,000-1,000)$ & 0,407 & & \\
\hline PLT $(/ \mu \mathrm{L})$ & 42 & $0,998(0,997-1,000)$ & 0,028 & $0,997(0,995-0,999)$ & 0,008 \\
\hline ESR $(\mathrm{mm} / \mathrm{h})$ & 42 & $1,000(0,991-1,008)$ & 0,925 & & \\
\hline CRP (mg/L) & 42 & $1,000(0,995-1,006)$ & 0,892 & & \\
\hline AST (U/L) & 42 & $1,001(0,998-1,005)$ & 0,471 & & \\
\hline ALT (U/L) & 42 & $1,002(0,999-1,005)$ & 0,134 & & \\
\hline BUN (mg/dL) & 42 & $1,014(0,945-1,089)$ & 0,694 & & \\
\hline Creatinine (mg/dL) & 42 & $1,133(0,429-2,992)$ & 0,802 & & \\
\hline Ferritin (ng/mL) & 24 & $1,000(1,000-1,000)$ & 0,662 & & \\
\hline Fibrinojen (mg/dL) & 17 & $1,001(0,997-1,004)$ & 0,711 & & \\
\hline LDH (U/L) & 27 & $1,000(0,999-1,001)$ & 0,924 & & \\
\hline Trigliserid (mg/dL) & 17 & $1,000(0,998-1,002)$ & 0,999 & & \\
\hline \multicolumn{6}{|l|}{ Treatments at the first flare } \\
\hline PMP treatment & 15 & $0,561(0,282-1,115)$ & 0,099 & & \\
\hline Oral steroid & 40 & 1,295 (0,308-5,444) & 0,724 & & \\
\hline DMARDs & 31 & $0,761(0,367-1,579)$ & 0,464 & & \\
\hline Biologic drugs & 7 & $0,552(0,243-1,261)$ & 0,159 & & \\
\hline Dosage of corticosteroid treatment $\left(\mathrm{g} / \mathrm{m}^{2}\right)$ & 40 & $0,890(0,808-0,980)$ & 0,018 & - & - \\
\hline Duration of corticosteroid treatment (months) & 40 & $0,894(0,824-0,970)$ & 0,007 & $0,837(0,754-0,929)$ & 0,001 \\
\hline
\end{tabular}

P226

Performance of the "ms-score" and "hscore" in the diagnosis of MAS in systemic JIA patients

E. Sag, A. Keskin, S. Demir, M. K. Cuceoglu, E. Atalay, U. K. Akca, E. D. Batu, Y. Bilginer, S. Ozen

Pediatric Rheumatology, Hacettepe University, Ankara, Turkey

Correspondence: E. Sag

Pediatric Rheumatology 2020, 18(Suppl 2):P226

Introduction: Macrophage activation syndrome (MAS) is a very devistating complication of Systemic JIA (sJA), seen in approximately $15-25 \%$ of the sJIA patients. There are many tools to differentiate activation of SJIA and MAS including HScore and the recently proposed MS-score. This is the first study comparing MS-score and HScore in sJA. Objectives: We aimed to compare the performances of MSscore ${ }^{1}$ and $\mathrm{HScore}^{2}$ for the diagnosis of MAS in SJA patients

Methods: Systemic JIA patients followed at Hacettepe University Pediatric Rheumatology Department were included in the study. Clinical features and laboratory findings at the time when patients were most active or diagnosed as MAS were recorded retrospectively. HScore and MS-score were calculated respectively and the diagnostic capacity of MAS was compared by means of receiver operating characteristic (ROC) curve analysis.

Results: Seventy-one sJIA patients were included (23 MAS, 48 activation). There was no difference in the age of onset (median 4.7 vs 5.0 ) and gender ( $73.9 \%$ vs $54.2 \%$ ) between patients who had MAS and SJIA activation. There was no significant difference in the frequency of fever, rash and LAP between the two groups, but the frequency of fever $\geq 39.4^{\circ} \mathrm{C}$ was higher in the MAS group. Hepatomegaly, splenomegaly, central nervous system involvement, haemorrhagic manifestations, and hemophagocytosis in the bone marrow were also common in the MAS group, while the presence of active arthritis and the number of affected joints were higher in the sJA activation group. Hemoglobin, white blood cell count, platelet count, fibrinogen, erythrocyte sedimentation rates were lower; ALT, AST, LDH, triglyceride and ferritin were higher in the MAS group as expected. There was no significant difference in C-reactive protein levels between two groups. While $47.8 \%$ of MAS patients required intensive care hospitalization, this rate was $6.5 \%$ in patients who had disease activation. Although there was no significant difference between two groups in terms of mean intensive care stay $(11.7 \pm 12.3$ vs $8 \pm 4.2$ days; $p=0.23$ ), the total duration of hospital stay was longer in the MAS group $(25.9 \pm 17.9$ vs $10.1 \pm 8.6$ days; $p<0.0001)$. The most common disease course in both the MAS group and the activation group was monocyclic disease (43.5\% vs. $45.8 \%)$. Polycyclic course was observed more frequently in MAS group $(43.5 \%$ vs $10.4 \%)$, polyarticular course was more common in activation group (13\% vs $43.8 \%)$.

MS-score (median [range] $1.8[(-5.7)-(9.3)]$ vs (-4.0) [(-7.2)-(3.8)] $\mathrm{p}<$ $0.0001)$ and HScore (median [range] 241 [51-337] vs 51 [18-202] $\mathrm{p}<$ 0.0001 ) were higher in the MAS group. ROC curve analysis revealed that HScore performed slightly better in diagnosing MAS, compared with MS-score (AUC=0.965 and 0.901 for HScore and MS-score repectively, $\mathrm{P}<0.001)$. In our cohort, MS score $\geq-1.64$ yielded a sensitivity of $91.3 \%$ and a specificity of $83.8 \%$; HScore $\geq 162.5$ yielded a sensitivity of $91.3 \%$ and specificity of $90.2 \%$.

Conclusion: HScore seems to perform slightly better than MS-score for the diagnosis of MAS in our cohort.

\section{References}

1. Minoia F, Bovis F, Davi S, Horne A, Fischbach M, Frosch M, et al. Development and initial validation of the MS score for diagnosis of macrophage activation syndrome in systemic juvenile idiopathic arthritis. Ann Rheum Dis. 2019;78(10):1357-1362.

2. Fardet L, Galicier L, Lambotte O, Marzac C, Aumont C, Chahwan D, et al. Development and validation of the HScore, a score for the diagnosis of reactive hemophagocytic syndrome. Arthritis Rheumatol. 2014;66(9):2613-2620.

Disclosure of Interest: None declared

P227

A randomized, double-blind, placebo-controlled study of anakinra in pediatric and adult patients with Still's disease

L. Schanberg ${ }^{1}$, P. Nigrovic ${ }^{2}$, A. Cooper ${ }^{3}$, W. Chatham ${ }^{4}$, S. Akoghlanian ${ }^{5}$, N. Singh ${ }^{6}$, E. Rabinovich ${ }^{1}$, A. Thatayatikom7, A. Taxter ${ }^{8}$, J. Hausmann ${ }^{2,9}$, M.

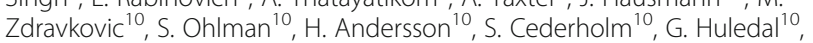
R. Schneider ${ }^{11}$, F. De Benedetti ${ }^{12}$

${ }^{1}$ Duke Children's Hospital \& Health Center, Durham; ${ }^{2}$ Boston Children's Hospital, Boston; ${ }^{3}$ Children's Mercy Kansas City, Kansas City; ${ }^{4}$ University of Alabama at Birmingham, Birmingham; ${ }^{5}$ Nationwide Children's

Hospital, Columbus; 6 University of lowa Hospitals and Clinics, lowa City; ${ }^{7}$ University of Florida, Gainesville; ${ }^{8}$ Wake Forest Baptist Medical Center, Winston-Salem; ${ }^{9}$ Beth Israel Deaconess Medical Center, Boston, United States; ${ }^{10}$ Sobi, Stockholm, Sweden; ${ }^{11}$ The University of Toronto, Hospital for Sick Children, Toronto, Canada; ${ }^{12}$ Ospedale Pediatrico Bambino Gesù, Rome, Italy

Correspondence: L. Schanberg

Pediatric Rheumatology 2020, 18(Suppl 2):P227 
Introduction: Still's disease, including both systemic juvenile idiopathic arthritis (sJIA) and adult-onset Still's disease (AOSD), is a rare systemic auto-inflammatory disorder associated with an activated IL-1 pathway.

Objectives: To build on earlier evidence and evaluate in a controlled setting the efficacy and safety of anakinra, an IL-1 receptor antagonist, in patients with active, newly diagnosed Still's disease across all age groups.

Methods: The anaSTILLs study (anakinra in Still's disease) was a randomized, double-blind, placebo-controlled, 12 -week study including patients with active and newly diagnosed Still's disease (adapted ILAR criteria if $<16$ years of age, Yamaguchi criteria if $\geq 16$ years of age at disease onset). Patients were randomized to anakinra $2 \mathrm{mg} / \mathrm{kg}$ ( $\max 100 \mathrm{mg} /$ day), $4 \mathrm{mg} / \mathrm{kg}$ ( $\max 200 \mathrm{mg} /$ day) or placebo. The primary objective was to demonstrate the efficacy of anakinra versus placebo as assessed by ACR30 response with an absence of fever at Week 2. Secondary objectives included: early onset of efficacy, sustained efficacy, time to study drug discontinuation, safety, pharmacokinetics (PK), clinical signs and biomarkers.

Results: Twelve patients were randomized and received study treatment: 6 to anakinra ( $2 \mathrm{mg} / \mathrm{kg} \mathrm{n}=2,4 \mathrm{mg} / \mathrm{kg} \mathrm{n}=4$ ) and 6 to placebo. Due to slow recruitment the study was terminated early. One patient was excluded from the efficacy analyses since he/she was later diagnosed with lymphoma, not Still's disease. 11 patients were analyzed for efficacy (anakinra $\mathrm{n}=6$, placebo $\mathrm{n}=5$ ), 8 children (median [range] age $=4.0$ [1-11] years) and 3 adults (median [range] age $=32.0$ [25-51] years). $55 \%$ were male and the mean (range) symptom duration was 74.2 (12-222) days. All patients on anakinra but none on placebo achieved ACR30 response with absence of fever at Week 2 ( $p$-value $=0.0022$ ). The efficacy of anakinra was supported by superiority to placebo in ACR50/70/90 responses with absence of fever at Week 2. All placebo patients discontinued the study within 6 weeks, 2 due to progressive disease, 2 due to lack of efficacy and 1 due to withdrawal by the patient. A numerically greater proportion of patients in the anakinra group had early onset of efficacy (Week 1) compared to placebo, as assessed by ACR30. ACR30/50/70/90 responses in anakinra-treated patients were sustained throughout the study period. No unexpected safety findings were observed. Based on serum anakinra concentrations Week 12, Cmax was $2920 \mathrm{ng} / \mathrm{mL}$ at 4.0 hours (dose: 4.1 $\mathrm{mg} / \mathrm{kg}$ ) in a 1-year-old patient, and Cmax was $1060 \mathrm{ng} / \mathrm{mL}$ at 2.1 hours (dose: $1.5 \mathrm{mg} / \mathrm{kg}$ ) in a 6-year-old patient.

\begin{tabular}{|c|c|c|c|c|c|}
\hline \multicolumn{6}{|c|}{ ACR response with absence of fever at Week 2} \\
\hline $\begin{array}{l}\text { Primary \& } \\
\text { secondary } \\
\text { endpoints }\end{array}$ & $\begin{array}{l}\text { Response } \\
\text { (Anakinra) } \\
n(\%) \\
N=6\end{array}$ & $\begin{array}{l}\text { Response } \\
\text { (Placebo) } \\
\text { n (\%) } \\
\mathrm{N}=5\end{array}$ & $\begin{array}{l}\text { Difference } \\
\text { (Anakinra- } \\
\text { Placebo) }\end{array}$ & $\begin{array}{l}95 \% \\
\text { exact } \\
\mathrm{Cl} \text { for } \\
\text { difference }\end{array}$ & $\begin{array}{l}\text { Fisher's } \\
\text { exact } \\
\text { testp-value }\end{array}$ \\
\hline ACR30* & $6(100.0)$ & $0(0.0)$ & 1.00 & $0.42,1.00$ & 0.0022 \\
\hline ACR50* & $6(100.0)$ & $0(0.0)$ & 1.00 & $0.42,1.00$ & 0.0022 \\
\hline ACR70* & $6(100.0)$ & $0(0.0)$ & 1.00 & $0.42,1.00$ & 0.0022 \\
\hline ACR90* & $5(83.3)$ & $0(0.0)$ & 0.83 & $0.24,1.00$ & 0.0152 \\
\hline
\end{tabular}

Conclusion: Anakinra is superior to placebo in the treatment of Still's disease. The safety and PK results are consistent with previous anakinra experience. These results confirm the benefits of anakinra treatment in patients with active, newly diagnosed Still's disease across ages.

Trial registration identifying number: NCT03265132

Disclosure of Interest: L. Schanberg Consultant for: Sobi, P. Nigrovic Consultant for: Novartis, BMS, Pfizer, Sobi, Miach Orthopedics, Simcere, XBiotech, Quench Bio, Siglion, Cerecor, A. Cooper: None declared, W. Chatham Consultant for: Sobi, S. Akoghlanian: None declared, N. Singh: None declared, E. Rabinovich: None declared, A. Thatayatikom: None declared, A. Taxter: None declared, J. Hausmann Consultant for: Novartis, M. Zdravkovic Shareholder of: Sobi, Employee of: Sobi, S. Ohlman Shareholder of: Sobi, Employee of: Former employee of Sobi, H. Andersson Employee of: Sobi, S. Cederholm Shareholder of: Sobi, Employee of: Sobi, G. Huledal Employee of: Sobi, R. Schneider Consultant for: Novartis, Novimmune, Sobi, F. De Benedetti Speaker Bureau of: AbbVie, Novartis, Roche and Sobi
P228

Canakinumab as first- or second-line DMARD in systemic-onset Juvenile Idiopathic Arthritis (sJIA) - data from the German biker registry

R. Heidebrecht ${ }^{1}$, A. Klein ${ }^{1}$, K. Minden², F. Dressler ${ }^{3}$, G. Horneff ${ }^{1}$

${ }^{1}$ Asklepios Klinik Sankt Augustin GmbH, Sankt Augustin; ${ }^{2}$ Charité

University Hospital Berlin, Berlin; ${ }^{3}$ Hannover Medical School, Hannover,

Germany

Correspondence: R. Heidebrecht

Pediatric Rheumatology 2020, 18(Suppl 2):P228

\section{Introduction}

Early inhibition of IL-1 is discussed to play an important role in the disease course of $\mathrm{sJA}^{1}$. Assuming that pretreatment with other DMARDs leads to a later start of therapy with canakinumab, this analysis evaluates the effectiveness of canakinumab as first-line vs. second-line DMARD.

Objectives

To evaluate the effectiveness of canakinumab as first used biological DMARD in sJIA compared to canakinumab in sJIA-patients pretreated with other DMARDs.

Methods

SJIA-patients documented in the German Biologic Registry for Pediatric Rheumatology (BiKeR), who were exposed to canakinumab, were identified. For the first-line $(\mathrm{FL})$ group DMARD naïve patients were selected, prior treatment with corticosteroids and/or NSAIDs was allowed. Patients receiving any DMARD prior to canakinumab entered the second-line (SL) group. Both groups were compared in a retrospective intention-to-treat-analysis. Effectiveness was determined by analyzing JIA American College of Rheumatology (ACR) Inactive Disease (defined by Wallace et al. ${ }^{2}$ ), 10-joint Juvenile Arthritis Disease Activity Score Remission (JADAS-10-Remission) and JADAS10-Minimal Disease Activity (-MDA). Safety was analyzed based on adverse event (AE) reports.

Results

Altogether 60 patients with sJA were included, 13 patients (21.7\%) in the FL-group, $47(78.3 \%)$ in the SL-group. Mean disease duration until baseline was $10.3 \pm 22.9$ months in the $\mathrm{FL}$ - and $35.7 \pm 46.7$ months in the SL-group $(p=0.0647)$. Patients in the $F L$-group had significantly higher scores for CRP (FL 76.7 $\pm 56.8 \mathrm{mg} / \mathrm{l}, \mathrm{SL} 27.2 \pm 46.5 \mathrm{mg} / \mathrm{l}, \mathrm{p}=$ 0.002 ) and JADAS-10-mean (FL 17.7 $\pm 7.0, \mathrm{SL} 10.5 \pm 8.5, \mathrm{p}=0.0073$ ) at baseline than patients in the SL-group. In both groups a significant improvement in the number of patients reaching JADAS-10-MDA, JADAS-10-Remission and ACR-Inactive-Disease was achieved from month 0 to month 3. Significantly more patients in the FL-group reached ACR-Inactive-Disease ( $\mathrm{FL} 81.8 \%, \mathrm{SL} 40.0 \%, \mathrm{p}=0.0351$ ) at month 6 than in the SL-group. 122 adverse events (AE) were reported, 22 of them were serious (SAEs). Most events were classified as infections and infestations (49 AEs, 5 SAEs).

\begin{tabular}{|c|c|c|c|c|}
\hline & & $\begin{array}{l}\text { First Line }(n= \\
13)\end{array}$ & $\begin{array}{l}\text { Second Line }(n= \\
47)\end{array}$ & $\begin{array}{l}\text { P- } \\
\text { Value }\end{array}$ \\
\hline \multirow{3}{*}{$\begin{array}{l}\text { ACR-Inactive Disease, } n \\
(\%)\end{array}$} & \multirow{3}{*}{$\begin{array}{l}\text { Mo } \\
0 \\
\text { Mo } \\
3 \\
\text { Mo } \\
6\end{array}$} & $0(0.0)$ & $7(14.9)$ & 0.3288 \\
\hline & & $6(60.0)$ & $18(64.3)$ & 1.000 \\
\hline & & $9(81.8)$ & $14(40.0)$ & 0.0351 \\
\hline \multirow{3}{*}{$\begin{array}{l}\text { JADAS-10-Remission, n } \\
\text { (\%) }\end{array}$} & \multirow{3}{*}{$\begin{array}{l}\text { Mo } \\
0 \\
\text { Mo } \\
3 \\
\text { Mo } \\
6\end{array}$} & $0(0.0)$ & $11(23.4)$ & 0.0998 \\
\hline & & $8(80.0)$ & $16(57.1)$ & 0.2685 \\
\hline & & $9(81.8)$ & 17 (48.6) & 0.0821 \\
\hline
\end{tabular}

\section{Conclusion}

Canakinumab treatment showed good effectiveness in SJIA both as first- and second-line DMARD. After 6 months the use of canakinumab as first-line DMARD is associated with higher response rates compared to second-line use. Our data support the hypothesis that early 
treatment with canakinumab is associated with good therapeutical response and a positive effect on the disease course of sJIA.

\section{References}

[1] Toplak N, Blazina Š, Avčin T. The role of IL-1 inhibition in systemic juvenile idiopathic arthritis: current status and future perspectives. Drug Des Devel Ther. 2018 Jun 8;12:1633-1643. doi: 10.2147/DDDT.S114532. PMID: 29922038; PMCID: PMC5996857.

[2] Wallace C.A. et al. Childhood Arthritis Rheumatology Research Alliance; Pediatric Rheumatology Collaborative Study Group; Paediatric Rheumatology International Trials Organisation. American College of Rheumatology provisional criteria for defining clinical inactive disease in select categories of juvenile idiopathic arthritis. Arthritis Care \& ResearchVol. 63, No. 7, July 2011, pp 929-936.

Disclosure of Interest: R. Heidebrecht: None declared, A. Klein: None declared, K. Minden Speaker Bureau of: Abbvie, GSK, Roche, Sanofi \& Biermann, F. Dressler Speaker Bureau of: Novartis, Pfizer \& Abbvie, G. Horneff Speaker Bureau of: Pfizer, Abbvie, Novartis, Chugai

\section{P229}

Childhood systemic lupus erythematosus: experience from sub- Himalayan region of North West India

Y. Sharma, A. Sharma

Pediatrics, Dr Rajendra Prasad Government Medical College, kangra, India

Correspondence: $Y$. Sharma

Pediatric Rheumatology 2020, 18(Suppl 2):P229

Introduction: Systemic lupus erythematosus (SLE) is a multisystem autoimmune disease. Clinical presentation can vary from mere cutaneous involvement to more severe multisystem involvement. SLE usually presents with rash, fatigue and fever but may sometimes present with unusual, non-specific manifestations in children.

Objectives: To describe a cohort of children with SLE from tertiary care centre in a resource limited setting in North west India

Methods: Retrospective case review of all children diagnosed as SLE from July 2017-December 2018 at a single tertiary care hospital in North India was done. Diagnosis of SLE was based on Systemic Lupus International Collaborating Clinics(SLICC) classification criteria.

Results: A total of 11 children (9 girls) with SLE were identified. Median age of symptom onset and diagnosis was 14 years(range 817 years) and 11 years respectively. The presenting manifestations were fever(5), oral ulcers(3), alopecia(3), malar rash(4), photosensitivity(5), renal involvement(5), seizures(1) and gastrointestinal complaints (1) apart from some unusual manifestations of isolated peripheral arthritis(1), isolated bilateral pleural effusion(1), macrophage activation syndrome(2).

Laboratory investigations: Hemogram revealed anemia in 8 children and thrombocytopenia in 5 . Urine examination showed nephrotic range proteinuria in 1 child and subnephrotic proteinuria in 2 . Microscopic hematuria was noted in 2 pateints. Renal function tests were deranged in 2 cases. ANA, Anti dsDNA positivity and hypocomplimentemia were present in all. Renal biopsy was done in 4 patients, 2 had class IV, one class III and one had class V lupus nephritis. All patients were initiated on hydroxychloroquine and photoprotection. Children with renal involvement were given pulse methylprednisolone followed by tapering doses of oral prednisolone and intravenous, monthly cyclophosphamide. Azathioprine was used as maintenance therapy in all. Subcutaneous weekly methotrexate was used in 2 patients. One child (MAS) died during disease course. Disease continues to be in remission in rest.

Conclusion: We found a significant female preponderance in our study group. Renal involvement was the commonest presentation. Some unusual presentations were also seen. Early recognition of SLE is critical for timely initiation of appropriate treatment. This is the first report of a cohort of Pediatric SLE from this part of India.
P230

A rare case of mixed type autoimmune hemolytic anemia in a 15-years old adolescent- don't always blame lupus

C. D. A. S. Faria', J. A. Guimarães', L. R. Campos², K. L. Baptista², F. P. L. F. Veiga $^{3}$, T. G. Noronha', D. P. Bulkool', R. D. C. Villalba', A. C. F. Bassani', C. Pinheiro ${ }^{4}$, T. R. D. Souza ${ }^{4}$, B. Cohen ${ }^{4}$

${ }^{1}$ Pediatrics; ${ }^{2}$ Pediatric Rheumatology, Department of Pediatrics; ${ }^{3}$ Pediatric Nephrology, Hospital Universitário Antônio Pedro, Universidade Federal Fluminense, Niterói - Rio de Janeiro; ${ }^{4}$ Hematologistas Associados, Rio de Janeiro, Brazil

Correspondence: C. D. A. S. Faria

Pediatric Rheumatology 2020, 18(Suppl 2):P230

Introduction: Autoantibodies in AHAI may be $\mathrm{lgG} / \mathrm{lgM} / \mathrm{lgA}$. AHAI can be divided into primary or secondary (e.g. SLE, lymphoproliferative diseases, infections, medications). It is also classified based on the temperature at which the antibody reacts to erythrocytes, and can be warm (IgG or $\lg A$ ) or cold (IgM or C3). In warm AHAl, the antibodies react at temperatures $\geq 37^{\circ} \mathrm{C}$, not activating the complement system and not undergoing agglutination in vitro. In cold $\mathrm{AHAl}$, antibodies react at temperatures below $37^{\circ} \mathrm{C}$, activating the complement system with in vitro agglutination.Mixed AlHA (warm and cold) is rare and occurs in $<10 \%$ of AlHA cases and can occur at any age, but is extremely rare in children. The prevalence of the mixed form is less than $1 / 1,000,000$ patients with AHAI.

Objectives: To report a rare case of mixed AHAI and idiopathic intracranial hypertension $(\mathrm{IIH})$ in a 15 -years old female patient with a previous diagnosis of SLE and APS.

Methods: Case report and literature review.

Results: A 15-years old female adolescent previously diagnosed with SLE/APS since 2017 was in remission on hydroxychloroquine(400mg);azathioprine(150mg); aspirin $(100 \mathrm{mg})$;vitaminD3(1.000IU);calcium(1g), and sunscreen. In April 2020' she had a relapse presenting with fatigue, myositis, headache, hypocomplementemia, and severe autoimmune hemolytic anemia ( $\mathrm{Hb}$ of $4 \mathrm{~g} / \mathrm{dL}$ ) (SLEDAI-2K=18 points). Mixed AHAI was diagnosed base on a Direct/Indirect Coombs test 4/4+;DirectAntiglobulinTesting showing anti-lgA(weak), anti-IgM(3+/4+), anti-lgG(3+/4+), anti-C3c(weak), antiC3d (3+/4+);lgG1/3subclasses with a reaction of 1:100(2+/4+);an eleven cell antibody panel positive revealing a cold and warm antibody, and adsorption technique revealing a cold and warm autoantibody. Chest CT showed bibasilar subsegmental atelectasis, head CT/MRI was normal and LP showed a high opening pressure of $45 \mathrm{cmH} 2 \mathrm{O}$ with a normal cell count. After the procedure, the patient reported improvement in the pain and was diagnosed with $\mathrm{IIH}$. The patient was screened for secondary causes for AHAI (table 1) due to the unusual mixed type pattern and serology was positive for Chlamydia trachomatis (IgM) and Mycoplasma pneumoniae (indeterminate-lgM/positive-lgG) suggesting a recent infectious trigger causing reactivation of the underlying disease with a probable cross-reactivity. The patient treated with 10-days of clarithromycin. Before the infectious screening came back negative, AHAI was treated with a single dose of $\mathrm{IVIG}(1 \mathrm{~g} / \mathrm{kg})$ and then, with 3 -days of methylprednisolone $(1 \mathrm{~g} /$ day). Azathioprine was replaced by mycophenolate mofetil. Due to headache recurrence, acetazolamide $(500 \mathrm{mg} /$ day) was started, and the patient referred no pain. The patient was discharged with a resolution of the symptoms.

Conclusion: The diagnosis of AHAl should alert pediatricians for the possibility of underlying causes other such as infectious, autoimmune diseases or neoplasms. In this case, SLE reactivation was a clear cause of AHAl but the mixed type is not what usually occurs in SLE. AHAI in SLE presents with a warm type(lgG). The presence of a cold antibody(lgM) is usually associated with infectious diseases such as Mycoplasma pneumoniae. This could contribute to the disease relapse. Atypical patterns of AHAI should be investigated for other causes even if a clear cause such as SLE is identified. 
P231

Bickerstaff encephalitis with overlapping Guillain-Barré syndrome as a first manifestation of juvenile systemic lupus erythematosus: a case report

A. Koryllou', M. Mejbri', S. Garcia-Tarodo², P. Parvex', E. Gonzalez Nguyen Tang ${ }^{3}$, C. M. Korff?, M. Hofer ${ }^{1}$

'Pediatric Immuno-Rheumatology Unit, Lausanne University Hospital (CHUV), Lausanne, ${ }^{2}$ Pediatric Neurology Unit, ${ }^{3}$ Pediatric Nephrology Unit, University Hospitals of Geneva, Geneva, Switzerland

Correspondence: A. Koryllou

Pediatric Rheumatology 2020, 18(Suppl 2):P231

Introduction: Pediatric systemic lupus erythematosus (pSLE) is an autoimmune disease with multisystemic involvement. More than $50 \%$ of the patients present neurological or psychiatric manifestations, with $43.9 \%$ of them presenting these symptoms at the time of diagnosis (REF). Rarely, Guillain-Barré syndrome (GBS) and its subtypes have been described in association with active SLE. Bickerstaff's brainstem encephalitis (BBE) is a rare immune-mediated disorder characterized by ophthalmoplegia, ataxia and disturbance of consciousness, which symptoms may overlap with GBS.

Objectives: To our knowledge, the association of GBS and BBE has been described in adults only.

Methods: We here describe a child presenting at SLE disease-onset with an overlap of peripheral (GBS) and central (BBE) nervous system manifestations, highlighting the possible association between these two entities in children.

Results: An 11-year-old healthy girl presented with acute ataxia, ophtalmoparesis and altered level of consciousness, rapidly followed by areflexia, facial paresis, swallowing difficulties, sensory deficits, paresis in all four limbs and respiratory insufficiency. These symptoms were accompanied by pleuro-pericardial serositis, proteinuria and hypertension. Immunological investigations revealed the presence of positive ANA and ds-DNA antibodies. The renal biopsy showed a stage III lupus nephritis. Hence, the clinical, laboratory findings and biopsy report led to the diagnosis of pSLE. Brain and spine MRI did not show any abnormalities; diffuse slowing compatible with nonspecific encephalopathy was seen on EEG. Nerve conduction studies (NCS) confirmed the clinical suspicion of acute polyradiculoneuropathy with proximal interruption of motor nerve conduction, compatible with Guillain-Barré-like syndrome. CSF analysis (performed twice) remained normal. The patient was treated with glucocorticoids, intravenous immunoglobulins, cyclophosphamide as well as plasmapheresis. The neurological and physical symptoms improved gradually with complete neurological recovery four months after onset.

Conclusion: Overlapping forms of BBE/GBS have never been described in association to SLE in children. Our patient's presentation and evolution fulfilled the criteria for such an overlap, occurring at pSLE onset. Although SLE and BBE/GBS are rare entities, our case suggests that there may be a common underlying immune background. This association should be recognized early for rapid and appropriate treatment initiation.

Disclosure of Interest: None declared

P232

Infantile antiphospholipid antibody syndrome: acquired and de novo APL appearance in four infants

T. Giani ${ }^{1}$, G. Ferrara ${ }^{2}$, A. Mauro ${ }^{3}$, R. Cimaz $^{4}$

${ }^{1}$ Department of Medical Biotechnology, University of Siena, Siena; ${ }^{2}$ Department of Pediatric, ASL 10, Florence; ${ }^{3}$ Pediatric Emergency Department, Santobono Children Hospital, Napoli; ${ }^{4}$ Department of Clinical Sciences and Community Health, University of Milan, Milan, Italy Correspondence: $\mathrm{T}$. Giani

Pediatric Rheumatology 2020, 18(Suppl 2):P232

Introduction: Antiphospholipid syndrome (APS) is a rare condition in the neonatal age. In most cases it is considered a passively acquired autoimmune disease, due to a transplacental passage of maternal antiphospholipid antibodies (aPL). Exceedingly unusual is the de novo production of aPL in newborns and infants.

Objectives: To describe four infants who developed an early brain stroke with increased and persistent levels of $\mathrm{aPL}$, even after six months of life.

Methods: We reviewed the clinical charts of four such infants, followed from diagnosis up to two years after the disappearance of aPL.

Results: Four babies ( $3 \mathrm{~F}$ and $1 \mathrm{M}$ ) came to our attention at 5, 7, 9 and 22 months of life, respectively, because of perinatal stroke and presence of aPL. Clinical and laboratory data of mother-infant pairs are described in the table. Three out of 4 children (cases 1-2-3) had only IgG antibodies. One mother had both aCL and anti-beta2GP1 IgM and IgG. In the first 3 cases antibodies disappeared within 2 years of life $(6$, 16 e 20 months respectively), in the last case at 26 months of life. Two cases ( 1 and 3 ) had genetic pro-thrombotic factors and received baby aspirin until the disappearance of aPL. Case 4 had a perinatal risk factor and was the only one to have positive aPL IgM.

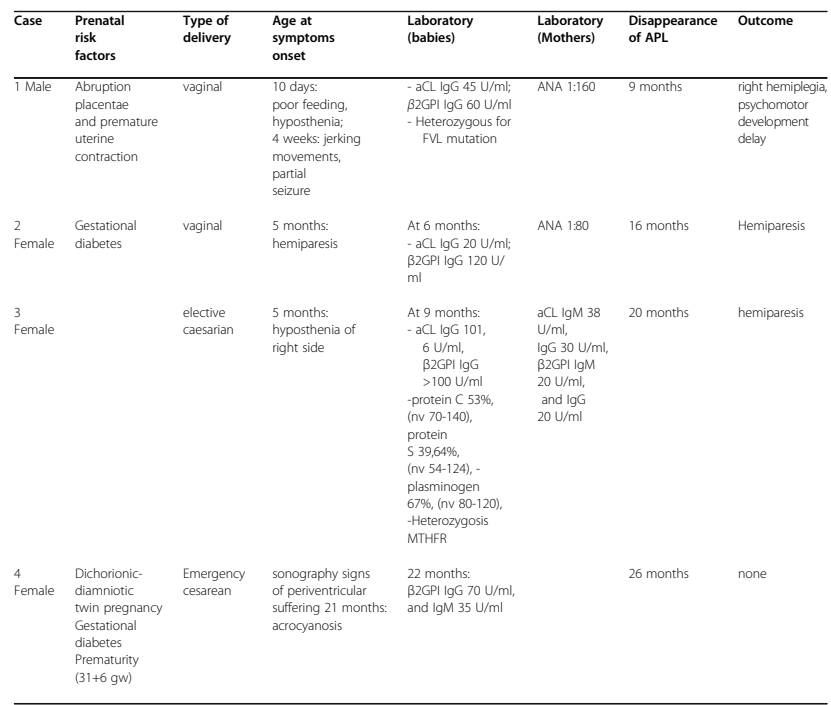

Conclusion: Common characteristics of these four children are the development of brain stroke and the increased and persistent aPL levels even after six months of life. This opens the window on a gray zone related to the origin of these antibodies (maternal or neonatal) and on their role in the pathogenesis of the infantile brain stroke.

Disclosure of Interest: None declared

P233

Spectrum of childhood-onset systemic lupus erythematosus and its clinical course

P. Morán Álvarez', A. Boteanu², M. Vázquez Díaz ${ }^{2}$

${ }^{1}$ Rheumatology: ${ }^{2}$ Hospital Ramón y Cajal, Madrid, Spain

Correspondence: P. Morán Álvarez

Pediatric Rheumatology 2020, 18(Suppl 2):P233

Introduction: Childhood systemic lupus erythematosus (CSLE) is an autoimmune systemic disease diagnosed in children under the age of 18 years old, leading to an important morbidity and mortality. Typically in the literature, CSLE is described in the literature with a more severe clinical course compared to adult-onset.

Objectives: To analyze clinical manifestations within the first year after the diagnosis of CSLE and its subsequent clinical course. 
Methods: A descriptive, observational, cross-sectional study was carried out. Inclusion criteria: all patients with diagnosis of CSLE based on 1997 updated American College of Rheumatology (ACR) criteria or 2012 Systemic International Collaborating Clinics (SLICC), in a tertiary hospital. Exclusion criteria: patients with a diagnosis of CSLE in another center, as data of the onset of the disease was not available. Demographic, clinical and analytical data were collected.

Results: 42 patients were included, 38 (90.5\%) girls and $4(9.5 \%)$ boys. 39 (92.9\%) were Caucasians. Mean age at diagnosis was 13.3 years (range: 7-18). Clinical manifestations within the first year of CSLE and complementary tests are shown in Table 1.

$31(73.8 \%)$ children developed a major organ involvement within the first year of the disease. Renal impairment was the most frequent manifestation (20 patients, 47.6\%); followed by neurological (8 patients, 19\%), lung (2 patients, $4.8 \%$ ), and cardiac (1 patient, $2.4 \%$ ) involvement.

Class IV lupus nephritis, based on 2003 International Society of Nephrology and the Renal Pathology Society (ISN/RPS) classification, was the most frequent (50\%). The most common neurological manifestations was the presence of seizures (4 patients, 9.5\%), followed by: 1 pseudotumor (2.4\%), 1 chorea (2.4\%), 1 aseptic meningitis $(2.4 \%)$, 1 peripheral nervous system (2.4\%) and 1 lupus psychosis (2.4\%). Regarding lung disorders, 2 (4.8\%) lupus pneumonitis were registered; and within cardiac involvement, 1 (2.4\%) tamponade.

Following the first year of the onset (maximum follow-up period: 46 years), just 6 (14.2\%) patients suffered from major organ involvement, with a mean time of 29.5 months (range 12 months - 17 years).

$20(47.6 \%)$ children required a strong immunosuppressant drug as mycophenolate, cyclophosphamide, azathioprine or biologic therapy within the first year. 1 death was registered.

Conclusion: In our patient cohort with CSLE, 88\% patients developed a major organ involvement. $73 \%$ children suffered from this kind of manifestations during the first year after diagnosis, being less frequent $(14.2 \%)$ as the disease progresses. Renal impairment was the most common, followed by neurological, lung and cardiac involvement.

Disclosure of Interest: None declared

Table 1 (abstract P234). Clinical manifestations and complementary tests within the first year of CSLE. ANA: antinuclear antibodies (>1:80); ESR: erythrocyte sedimentation rate; PCR: protein-C reactive

\begin{tabular}{|c|c|c|c|}
\hline Joint & $34(81 \%)$ & & Total $(n=42)$ \\
\hline Mucocutaneous & $31(73.8 \%)$ & ANA+ & $41(97.6 \%)$ \\
\hline Hematological & $29(6 \%)$ & $\begin{array}{l}\text { antiDNA+ } \\
(\mathrm{IU} / \mathrm{ml})\end{array}$ & $132(12-624)$ \\
\hline Renal & $20(47.6 \%)$ & C3 (mg/dL) & $38.7(9.8-75.8)$ \\
\hline Systemic & $19(45.2 \%)$ & $\mathrm{C} 4(\mathrm{mg} / \mathrm{dL})$ & $6(1.34-9.9)$ \\
\hline Neurological & $8(19 \%)$ & $\operatorname{ESR}\left(\mathrm{mm} / 1^{\mathrm{a} h}\right)$ & $44(4-120)$ \\
\hline Serositis & $6(14.3 \%)$ & PCR (mg/L) & $2.44(0.2-17.8)$ \\
\hline Pulmonary & $5(11.9 \%)$ & & \\
\hline Cardiac & $4(9.5 \%)$ & & \\
\hline Raynaud's phenomenon & $2(4.8 \%)$ & & \\
\hline
\end{tabular}

\section{P234}

The use of 'the lupus checklist' in consultations with paediatric patients with systemic lupus erythematosus in improving patient monitoring outcomes

K. A. Alexander ${ }^{1}$, C. Scott ${ }^{2}$, L. B. Lewandowski ${ }^{3}$

${ }^{1}$ Western General Hospital, Edinburgh, United Kingdom; ${ }^{2}$ Department of Paediatric Rheumatology, Red Cross War Memorial Childrens Hospital and University of Cape Town, Cape Town, South Africa; ${ }^{3}$ National Institute of Arthritis, Musculoskeletal and Skin Diseases, Bethesda, United States

Correspondence: K. A. Alexander

Pediatric Rheumatology 2020, 18(Suppl 2):P234
Introduction: The Lupus Checklist is designed for use in a Paediatric Rheumatology Clinic to help doctors keep track of the monitoring of patients with Juvenile Systemic Lupus Erythematosus, a complex, multi-organ condition which requires rigorous and regular monitoring. ${ }^{1}$ Juvenile Systemic Lupus Erythematosus (JSLE) patients in South Africa are at high risk of poor outcomes, so stringent monitoring of disease activity, vaccination, and medication safety are important. $^{2}$

Objectives: The two objectives of this audit were, firstly, to determine if the appropriate level of rigorous monitoring (as set out by the Lupus Checklist) for each patient was completed and secondly, to determine whether the Lupus Checklist was useful in assisting doctors in monitoring their patients' condition.

Methods: All patients treated in the Paediatric Rheumatology Clinic at the Red Cross War Memorial Children's Hospital, Cape Town with JSLE were included (20 patients). Patient notes and laboratory records were used to determine if the appropriate monitoring checks, as laid out by the Lupus Checklist, had been completed.

Results: Overall $37.7 \%$ of audited checks were completed. 19 patients had over $20 \%$ of their monitoring completed but only 2 had over $80 \%$. Aspects of monitoring that were more time intensive or were required less regularly were most frequently overlooked. There was a statistically significant increase in the percentage of completed monitoring in those patients for whom the Lupus Checklist was used compared to patients where a checklist was not used $(p=0.00)$.

Conclusion: There is significant room for improvement in the monitoring of these patients with JSLE in the rheumatology clinic. This audit illustrates that more diligent use of the Lupus Checklist and an overall improvement in sustained use of the checklist will help to improve monitoring of these patients. Evidence suggests that checklists are underutilised in medicine and wider implementation of this simple tool could improve patient outcomes. ${ }^{3,4,5}$ Interventions such as in person or electronic reminders, or audits with feedback to physicians could improve usage over time. The application of the Lupus Checklist or a similar document in other paediatric clinics is important for comprehensive monitoring of a condition as complex as JSLE and has the potential to prevent ongoing damage and medication toxicity in this high-risk population.

\section{References}

1. Morgan TA, Watson L, McCann LJ, Beresford MW. Children and adolescents with SLE: not just little adults. Lupus 2013; 22: 1309-1319.

2. Lewandowski LB, Schanberg LE, Thielman N, Phuti A, Kalla AA, Okpechi I, et al. Severe disease presentation and poor outcomes among pediatric systemic lupus erythematosus patients in South Africa. Lupus. 2017;26(2):186-94

3. Gawande, A. The Checklist Manifesto: How to Get Things Right. New York: Metropolitan Books, 2010

4. Winters BD, Gurses AP, Lehmann H, Sexton B, Rampersad CJ, Pronovost PJ. Clinical review: Checklists - translating evidence into practice. Crit Care 2009; 13: 210-219.

5. Alnaib $M$, Samaraee AA, Bhattacharya $V$. The WHO surgical safety checklist: a review. J Perioper Pract 2012; 22(9): 289-292.

Disclosure of Interest: None declared

P235

Disease course and treatment responses in juvenile systemic lupus erythematosus; a single center experience

B. Sözeri', F. Demir ${ }^{1}$, D. Kilit ${ }^{2}$, C. Pehlivanoğlu ${ }^{3}$

${ }^{1}$ Department of Pediatric Rheumatology; ${ }^{2}$ Department of Pediatrics:

${ }^{3}$ Department of Pediatric Nephrology, UMRANIYE TRAINING AND RESEAR CH HOSPITAL, Istanbul, Turkey

Correspondence: $B$. Sözeri

Pediatric Rheumatology 2020, 18(Suppl 2):P235

Introduction: Systemic lupus erythematosus (SLE) is a chronic autoimmune inflammatory disease that may cause morbidity and mortality by affecting multiple systems. The $10-20 \%$ of patients have 
juvenile onset and this cluster have may more severe kidney, neuropsychiatric or hematological involvement.

Objectives: The aim of this study was to assess the clinical and laboratory characteristics, disease activity, and treatment response of patients with juvenile SLE (jSLE).

Methods: This is a retrospective study involving patients between 1 July 2016 and 1 January 2020. The data of patients diagnosed with jSLE and followed up for a minimum of 6 months, were collected. The SLEDAI-2K scores at initiation and at the follow-up (1st, 3rd, 6th, and 12th months of treatment) were examined. The SLEDAI-2K score was considered to be $\leq 4$, for disease remission status.

Results: A total of 49 children were included in to the study. The female/male ratio was $4.4 / 1$ and the median age of the patients at the diagnosis was 13 (IQR: 11.1-15.2) years. The median follow-up of patients was 19 (IQR: 12-25) month. Four of the patients were diagnosed with monogenic SLE. Two siblings were diagnosed with c3 deficiency and two were diagnosed with familial chilblain lupus. The most common clinical findings were found musculoskeletal complaints $(69.4 \%)$, malar rash $(51 \%)$, oral ulcers $(38.8 \%)$, and fever $(30.6 \%)$, respectively in over all the group. The frequency of involvement of the system and organs was as follows; mucocutaneous $77.6 \%$, musculoskeletal $69.4 \%$, renal $44.9 \%$, hematological $34.7 \%$, serous membranes $16.3 \%$, neuropsychiatric $12.2 \%$, respectively. All patients had anti-nuclear antibody positivity, while $46.9 \%$ had anti-ds DNA, $14.3 \%$ had anti-Sm and $8.2 \%$ had antiphospholipid antibody positivity. While all patients received hydroxychloroquine treatment, $22.4 \%$ of the patients were received were mycophenolate mofetil, $22.4 \%$ were azathioprine, $14.3 \%$ cyclophosphamide, $12.2 \%$ methotrexate and $10.2 \%$ were rituximab. The median SLEDAI-2K score was 14 (IQR: 10-18.5) at admission, besides it was found to 6 (IQR: 4-12), 4 (IQR: 2-6), 2 (IQR: 0-6) in the 1st, 6th and 12th months of treatment, respectively. While $98 \%$ of the patients had active disease at admission, $67.3 \%$ at 1 months, $32.7 \%$ at 6 months and $22.4 \%$ at 12 months still had active disease (SLEDAI-2K >4). Patients with initially high SLEDAI-2K scores had significantly lower remission rates in the first month $(p=0.003)$. It was observed that patients with high SLEDAI-2K scores in admission were more resistant to conventional immunosuppressive treatments and the use of rituximab was more frequent in these patients. At least one major organ (renal, hematological, neurological) were affected in $57 \%$ of patients. The remission rate of these patients at 6 months was found significantly decreased compared to the others $(p<0.005)$. Renal biopsy was performed in 21 patients (42.9\%). 12 of them had type 4 lupus nephritis (LN), 5 had type 2, 2 had type 3, and 1 had type 5. It was observed that patients with renal involvement were the group that reached remission latest.

Conclusion: The presence of high initial SLEDAI-2K scores and the major organ involvement have poor predictive value to achieve inactive disease.

Trial registration identifying number: None

Disclosure of Interest: None declared

\section{P236}

Hypophosphatemia secondary to FGF23 increase associated with systemic autoimmune disease: a report of 2 cases

A. Schvartz, G. Dorval, O. Boyer, F. Bienaimé, D. Prié, B. Bader Meunier

'Hôpital Necker, Paris, France

Correspondence: B. Bader Meunier

Pediatric Rheumatology 2020, 18(Suppl 2):P236

Introduction: Fibroblast Growth Factor 23 (FGF23) is a hypophosphatemic hormone playing a key role in phosphatemia homeostasis. Antibodies blocking FGF23 signaling can cause hyperphosphatemic tumoral calcinosis. To our knowledge, there is no description of systemic autoimmune disease associated with FGF23 signaling excess.
Objectives: To report two patients with systemic autoimmune diseases and severe hypophosphatemia secondary to FGF23 level increase.

Methods

Results: The first patient was diagnosed with an early-onset Sjogren syndrome at 4 years old with anti-SSa and SSb antibody positivity and focal lymphocytic sialadenitis with a focus score of $\geq 1$ foci/4 $\mathrm{mm}^{2}$, as well as joint, parotid, hepatic, hematological and neurological involvement. During a disease flare, hypophosphatemia occurred $(0.51 \mathrm{mmol} / \mathrm{L}$ (Normal values:1.3-1.85)) with intact FGF23 level at $215 \mathrm{ng} / \mathrm{mL}(\mathrm{N}=23.2-95.3)$ associated with hyperphosphaturia with a normal renal function. FGF23 level normalized at $62.4 \mathrm{ng} / \mathrm{mL}$ and phosphatemia went back up at $1.19 \mathrm{mmol} / \mathrm{L}$ after intensification of immunosuppressive drugs. The second patient suffers from systemic erythematous lupus diagnosed at age of 10 year, with cutaneous, joint, and hematological involvement, positive anti-Sm, anti-SSA, anti-RNP, , anti-DNA antibodies. She developed hypophosphatemia at $0.35 \mathrm{mmol} / \mathrm{L}$ with a $70 \%$ phosphate reabsorption rate, resulting from a renal phosphate leak. An elevation of intact FGF23 to $135.7 \mathrm{ng} / \mathrm{L}$ without renal dysfunction was demonstrated. The level of intact FGF23 then normalized with the intensification of immunosuppression.

Conclusion: Conclusion: We report for the first time the occurrence of severe hypophosphatemia secondary to an increase of intact FGF23 level in two patients with systemic autoimmune disease. Correlation between disease activity and the level of FGF23 suggests that this secretion may be secondary to an activating autoimmune mechanism.

Disclosure of Interest: None declared

\section{P237}

Longitudinal evaluation of executive functions in patients with childhood-onset systemic lupus erythematosus

J. C. de Amorim', C. L. Elorza', B. Bellini', P. T. Fernandes², R. Marini ${ }^{3}$

S. Appenzeller ${ }^{4}$

${ }^{1}$ Child and Adolescente Graduate Program; ${ }^{2}$ Sports Psychology;

${ }^{3}$ Pediatrics; ${ }^{4}$ Medicine, UNICAMP, Campinas, Brazil

Correspondence: S. Appenzeller

Pediatric Rheumatology 2020, 18(Suppl 2):P237

Introduction: Among the neuropsychiatric manifestations of Childhood-onset Systemic Lupus Erythematosus , cognitive losses are frequent.

Objectives: To analyze the fluctuation of executive functions these patients in treatment at a tertiary hospital rheumatology outpatient clinic.

Methods: This is a longitudinal study with application of validated instruments: Coding subtest, Picture Arrangement, Block Design, Vocabulary and Picture Completion in patients with confirmed diagnosis of Childhood-onset SLE . In this study 24 patients were included, with a mean of 16.83 years, of whom 23 were women and 1 were man. The subjects were evaluated in two different periods with mean separation time of 9.5 months.

Results: The Coding subtest showed the largest difference between the scoring means, with the first being $58.83 \pm 15.18$ (mean \pm SD) and the second $55.20 \pm 20.63$, followed by the arrangement of figures, with $22.87 \pm 12.69$ and $20.5 \pm 8.88$, and Block Design with $42.37 \pm 13.73$ and $41.62 \pm 13.66$. On the other hand, when analyzing the substandard Vocabulary and Picture Completion, there is an increase in the mean between evaluations, the first one being from $26 \pm 3.21$ to $26.41 \pm 6.48$, and the second from $22.79 \pm 3.28$ to 23.95 \pm 9.03 . When performed the T-test for paired samples, with a $95 \%$ confidence interval, it was not possible to prove the statistical significance when comparing the data obtained in the two evaluations. The Block Design subtest was closest to significance, with a result of 0.328 , followed by Coding with 0.524 .

Conclusion: Although the data analyzed do not have statistical significance it's possible to realize a tendency in the decrease of the ability to perform tasks. In this way, the study provides an indication of impairment in spatial visualization capabilities, selective and focused 
attention, and mental flexibility and perceptual and visual organization. Further studies are needed to better evaluate the long-term impairment of executive functions on patients with Childhood-onset SLE

Disclosure of Interest: None declared

\section{P238}

Neurological manifestations of pediatric systemic lupus erythemtosus

Y. Gazar', S. Y. Mostafa'2, H. M. Emam², S. S. Rashed²

${ }^{1}$ Rheumatology; ${ }^{2}$ Neurology, Al-Azhar University, Cairo, Egypt

Correspondence: $Y$. Gazar

Pediatric Rheumatology 2020, 18(Suppl 2):P238

Introduction: SLE is a complex autoimmune disorder, characterized by multisystem involvement including the nervous system, juvenile onset SLE has more aggressive clinical course in comparison with adult-onset SLE. Neuro Psychatric (NP) symptoms may be the initial presentation of SLE in children. The mortality rate is relatively low, but morbidity may be significant and permanent damage can occur.Early recognition of symptoms is crucial in prevented of permanent neurological sequalae and patients' quality of life.

Objectives: The aim of the work is to study the neurological manifestations of pediatric SLE in a sample of Egyptian children.

Methods: We studied 54 children and adolescents $<18$ years old who were undergoing treatment or follow-up at the paediatric neurology unit, neurology department at Al-Azhar University Hospitals (Al-Hussein and Bab-Al-Shaaria), and children referred from rheumatology department from June 2018 to November 2018. All patients fulfilled the new EULAR/ACR SLE classification criteria 2017.Patients were excluded from the study when their NP manifestations were secondary to other causes, such as hypertensive encephalopathy uremia infection or congenital or acquired CNS disease not related to SLE.

Results: out of 54 children with SLE, 30 (55.6\%) had neuropsychiatric (NP) manifestations, the mean age at onset of the disease was 13.6 years. The mean period between onset of SLE and NP manifestations 15.5 months. NP manifestations was the presenting feature in 3 patients. Headache was the initial symptom of central nervous system (CNS) involvement in 35\% of patients seizures was the most frequent CNS finding seen in $7(23.3 \%)$ patients, $6(20 \%)$ patients had congnifive impairment, $6(20 \%)$ patient had cognitive impairment, 6(20\%) patients had CVA, 2(6.7\%) had chorea, 2(6.7\%) had psychosis, $2(6.7 \%)$ had depression, $1(3.3)$ had cerebritis, 1(3.3\%) had peripheral neuropathy. Lupus anticoagulant was high in patients with chorea, seizures or cerebrovascular accidents (CVA). Electroencephalogram (EEG) was abnormal in $30 \%$ of patients presented by seizures and rarely helpful in patients with diffuse NP symptoms. Magnetic resonance imaging (MRI) was abnormal in 13 cases, long term outcome was good, 3 patients had significant persistent CNS deficits, the majority of patients (90\%) had excellent recovery from neuropsychiatric SLE.

Conclusion: NPSLE is one of the most common serious complications of pediatric SLE, so early recognition and management are of paramount importance. CNS involvement was observed in 55\% of our pediatric patients with SLE, $76 \%$ of whom developed symptoms during the first year of onset of the disease Headache and seizures were the most common neurological manifestations of pediatric SLE, followed by Cerebro Vascular Accident CVA and intellectual disability. Psychosis, depression and chorea were less frequent in our study group, while peripheral neuropathy and cerebritis were rare. Neuroimaging were generally unhelpful in patients with diffuse CNS disease, in contrast, it was more helpful in patients with focal neurological findings such as CVA. Patients with CVA or chorea, usually had positive lupus anticoagulant (LAC) antibody, and all of these patients should be investigated thoroughly for the presence of antiphospholipid antibodies. Thus, the clinicians should make a reasonable prediction of individual patients to reduce morbidity and mortality of SLE in children and improve patients' outcome
Disclosure of Interest: None declared

P239

Lupus manifestations in children with primary immunodeficiency diseases: phenotypic and genetic features and outcome

S. M. Al-Mayouf ${ }^{1,2}$, H. Alreefi ${ }^{3}$, T. Alsinan ${ }^{3}$, G. AlSalmi ${ }^{4}$, A. AlRowais ${ }^{5}$, W. Al$\mathrm{Herz}^{6}, \mathrm{~A}$. Alsonbull ${ }^{4}, \mathrm{H}$. Al-Mousa ${ }^{4}$

${ }^{1}$ Pediatric Rheumarology, King Faisal Specialist Hospital and Research

Center; ${ }^{2}$ Pediatric Rheumarology; ${ }^{3}$ Alfaisal University; ${ }^{4}$ King Faisal

Specialist Hospital; ${ }^{5}$ Prince Sultan Military Medical City, Riyadh, Saudi

Arabia; ${ }^{6}$ Al-Sabah Hospital, Kuwait, Kuwait

Correspondence: $\mathrm{H}$. Alreefi

Pediatric Rheumatology 2020, 18(Suppl 2):P239

Introduction: Systemic lupus erythematosus (SLE) is a complex systemic inflammatory disease with a wide spectrum of clinical and laboratory features. Increasing evidence has shown a strong association between autoimmune diseases and primary immunodeficiency diseases (PIDs).

Objectives: To report the phenotypic features, underlying genetic defects and outcome of patients with SLE and SLE-like associated with PIDs.

Methods: Data retrospectively collected on patients with lupus manifestations and clinically/ or genetically proven PIDs seen between 1998 to 2019. The collected data comprised the clinical findings and diagnostic evaluation including genetic testing, the response to therapeutic intervention and the accrual damage related to SLE.

Results: A total of 40 patients ( 22 female) with a median age of 13 (range of 2-24) years were reviewed. Thirty-four patients had SLE and 6 with SLE-like. Genetic analysis was performed in 23 patients, all had positive results for monogenic PIDs. Complement deficiency was the most frequent PIDs; 26 patients were C1q deficient, 3 patients had C3 deficiency, 2 patients had C4 deficiency and one patient with heterozygous C $8 b$ variant. The other 8 patients had different PIDs genetic defects that include severe combined immunodeficiency caused by PNP deficiency, chronic granulomatous disease, common variable immunodeficiency (PIK3CD), IL-2RB mutation, DNase II deficiency, STAT1 mutation and Griscelli syndrome type 3, in addition to a possible novel genetic defect in FGL2. Mucocutaneous lesions, arthritis and lung involvement were the main clinical features. Most of the patients (84.2\%) experienced recurrent infections. Complement-deficient patients were younger and more likely to have a familial disease with severe mucocutaneous lesions. All patients treated with corticosteroid and immunosuppressive medications. Seven patients received biologic agents, either rituximab or belimumab. The mean accrual damage was $2.7 \pm$ 2.2. There were 5 deaths because of infection.

Conclusion: This study showed a variable pectrum of SLE associated with heterogeneous group of PIDs. Our data suggest that SLE patients with early onset disease, family history of SLE or recurrent infections should undergo immunological work-up and genetic testing to rule out PIDs.

Disclosure of Interest: None declared

P240

Ofatumumab use in juvenile systemic lupus erythematosus: a single centre experience

O. Kul Cinar' ${ }^{1}$, M. Marlais', M. Al-Obaidi', F. I. Cheng ${ }^{3}$, K. Tullus' ${ }^{2}$,

P. Brogan ${ }^{1,4}$, E. Moraitis ${ }^{1}$

${ }^{1}$ Paediatric Rheumatology; ${ }^{2}$ Paediatric Nephrology; ${ }^{3}$ Pharmacy, Great Ormond Street Hospital for Children NHS Trust; ${ }^{4}$ Infection, Immunity and Rheumatology Section, UCL Great Ormond Street Institute of Child Health, London, United Kingdom

Correspondence: $\mathrm{O}$. Kul Cinar

Pediatric Rheumatology 2020, 18(Suppl 2):P240

Introduction: Ofatumumab is a fully humanized anti-CD20 monoclonal antibody ( $\mathrm{mAb}$ ) which has been licensed for use in haematological malignancies, rheumatoid arthritis and paediatric nephrotic syndrome. There are limited data on the off-label use of Ofatumumab as an alternative B-cell depletion agent for patients with 
systemic lupus erythematosus (SLE) allergic to Rituximab, particularly juvenile SLE (jSLE).

Objectives: To describe a single-centre experience of use of ofatumumab for jSLE.

Methods: A single-centre retrospective case series of patients treated with off-label ofatumumab for jSLE between June 2018-April 2020 at Great Ormond Street Hospital, UK. Demographics, clinical and laboratory characteristics and treatment were collected.

Results: Three patients were identified: Laboratory and clinical data including active organs/systems involved prior to ofatumumab are summarized in Table 1 . All patients received Rituximab, Mycophenolate Mofetil (MMF) and steroids prior to Ofatumumab. 2/3 patients had cyclophosphamide (cases 1 and 2). Post-Ofatumumab, all patients remained on MMF maintenance therapy, and weaning course of steroids. The indication for ofatumumab in 3 patients was active SLE, with severe prior reaction to Rituximab. The median number of Rituximab infusions received was 2 (range 1-4).

Cases 1 and 3 received one course of Ofatumumab (700 mg/dose, 2 doses, administered on day 0 and day 14); case 2 received 2 courses, same dose, 9 months apart. The median follow up post-Ofatumumab was 14 months (range 8-23 months).

Ofatumumab was well tolerated without any infusion reactions or other adverse events for all 3 patients. B cell depletion was achieved in $3 / 3$ patients within three months (range 1-3 months). Significant clinical improvement was observed in all cases (Table 1), mirrored by improved laboratory markers of disease activity including anti-dsDNA antibody, complement levels, and proteinuria. At 6 months follow up, BILAG-2004 index had improvement for all patients (Table 1) At 6 months followup, the disease remained well-controlled for $2 / 3$ patients, whereas $1 / 3$ patient had a disease flare 9 months after the Ofatumumab course and received a second course with good response. Lymphocyte subsets were only available for $2 / 3$ patients at 6 months post Ofatumumab. Two of the patients had repopulated B cells at this time point.

\begin{tabular}{|c|c|c|c|c|c|c|}
\hline & \multicolumn{2}{|c|}{ Case 1} & \multicolumn{2}{|c|}{ Case 2} & \multicolumn{2}{|c|}{ Case 3} \\
\hline Age, gender & \multicolumn{2}{|c|}{14 years, $F$} & \multicolumn{2}{|c|}{16 years, $F$} & \multicolumn{2}{|c|}{$\begin{array}{l}12 \text { years } 8 \\
\text { months, } F\end{array}$} \\
\hline Ethnicity & \multicolumn{2}{|l|}{ Asian } & \multicolumn{2}{|c|}{ Afro-Caribbean } & \multicolumn{2}{|c|}{ Asian } \\
\hline $\begin{array}{l}\text { Duration jSLE } \\
\text { (months) }\end{array}$ & \multicolumn{2}{|c|}{31 months } & \multicolumn{2}{|c|}{71 months } & \multicolumn{2}{|c|}{16 months } \\
\hline $\begin{array}{l}\text { Disease manifestations } \\
\text { Pre Ofatumumab }\end{array}$ & \multicolumn{2}{|c|}{$\begin{array}{l}\text { Neurological } \\
\text { (headaches, } \\
\text { memory loss, } \\
\text { non-specific } \\
\text { white matter } \\
\text { changes on } \\
\text { MRI Brain) } \\
\text { Renal } \\
\text { (Lupus } \\
\text { nephritis, ISN/ } \\
\text { RPS Class III) } \\
\text { Haematological }\end{array}$} & \multicolumn{2}{|c|}{$\begin{array}{l}\text { Lupus } \\
\text { nephritis, ISN/ } \\
\text { RPS Class III } \\
\text { Serositis } \\
\text { (pleural and } \\
\text { pericardial } \\
\text { effusions) } \\
\text { Haematological } \\
\text { Arthritis } \\
\text { Chronic } \\
\text { cutaneous } \\
\text { lupus } \\
\text { Non-scarring } \\
\text { alopecia }\end{array}$} & \multicolumn{2}{|c|}{$\begin{array}{l}\text { Haematologica } \\
\text { (ITP, anaemia) } \\
\text { Arthritis }\end{array}$} \\
\hline Ofatumumab & Pre & Post & Pre & Post & Pre & Post \\
\hline BILAG-2004 & A & D & B & D & A & D \\
\hline dsDNA & 318.0 & 104.0 & 412 & 156 & 3.5 & 1.8 \\
\hline $\begin{array}{l}\text { Urine Alb/Cr ratio } \\
\text { (RR: } 0.7-7.4 \mathrm{mg} / \mathrm{mmol} \text { ) }\end{array}$ & 561.9 & 172.6 & 1.4 & 3.7 & 2.0 & 1.1 \\
\hline $\begin{array}{l}\text { B cell repopulation } \\
\text { (months/CD19) }\end{array}$ & N/A & & $\begin{array}{l}5 \mathrm{mo} \\
\mathrm{CD} 1\end{array}$ & $\begin{array}{l}\text { hs } \\
4.1 \%\end{array}$ & $\begin{array}{l}6 \mathrm{mc} \\
\mathrm{CD} 1\end{array}$ & $\begin{array}{l}\text { ths } \\
13.5 \%\end{array}$ \\
\hline
\end{tabular}

Conclusion: In this small series, treatment with Ofatumumab for patients with jSLE allergic to Rituximab was a safe and well-tolerated alternative to Rituximab therapy for B cell depletion. The clinical and serological outcomes were favourable. Clinical trials of Ofatumumab in jSLE are needed to prove efficacy and to determine the optimal treatment regimen.
P241

A novel mutation in the $\mathrm{C} 1 \mathrm{qA}$ gene in a toddler with $\mathrm{C} 1 \mathrm{q}$ deficiency associated with monogenic lupus and recurrent infection

S. Darwish', S. Bout tabaku², B. Al Adba ${ }^{2}$

${ }^{1}$ paediatric; ${ }^{2}$ Paediatric Rheumatology, Sidra Medicine, Doha, Qatar Correspondence: S. Darwish

Pediatric Rheumatology 2020, 18(Suppl 2):P241

Introduction: C1q deficiency is a rare hereditary disorder, which is strongly associated with Systemic Lupus Erythematosus (SLE).To date more than 60 cases of C1q deficiency have been published with various mutations

Objectives: We are reporting a novel homozygous mutation in the C1qA gene in a two year old girl who presented with SLE

Methods: Chart review of clinical data, laboratory data and molecular analysis

Results: A two year old girl of consanguineous parents presented to hospital at 13 months of age with fever and erythematous macular rash on her cheeks which spread to her nose, chin, and ears. The rash started a month prior, and progressed over her entire body. A skin swab grew staphylococcus aureus but the rash didn't respond to topical antibiotics. Review of systems was unremarkable except for longstanding oral thrush and diaper rash. Birth and family history were unremarkable. On exam she had a diffuse, erythematous, morbilliform eruption over her face and body. She had facial swelling, orbital edema and vasculitic oral ulcers. She had leukopenia mainly neutropenia, low hemoglobin, with normal platelets. Her liver enzymes and erythrocyte sedimentation rate (ESR) were high while C-reactive protein, immunoglobulins, C3 and C4 were normal. Cultures were negative, however she was positive for adenovirus, mycoplasma and EBV (EBV load was $6000 \mathrm{IU} / \mathrm{ml}$ ). Autoimmune hepatitis work up was negative. The direct coombs test, antinuclear antibodies (1:640), Ro, RNP and SmD were positive. CH50 came low as well as C1q level of $4 \mathrm{mg} / \mathrm{dL}$ (normal range 12-22 mg/dL). Lymphocyte subsets showed reduced CD4 and NK cells. Bone marrow aspiration showed active marrow. Skin biopsy showed chronic non-specific inflammation (immunofluorescence and electron microscopy were not available). Echocardiogram showed dilatation of the left coronaries. She was treated with intravenous immunoglobulin (IVIG) for Kawasaki disease with no improvement. Therefore pulse steroid $30 \mathrm{mg} / \mathrm{kg}$ followed by $2 \mathrm{mg} / \mathrm{kg}$ was initiated. Her rash, facial swelling and abnormal blood counts improved dramatically. Whole Exome sequence showed homozygous variant C.469G>T p.G157C at the C1qA gene. While tapering steroids she flared so subcutaneous methotrexate was started. Unfortunately, she continued to have rash, leukopenia and high liver enzymes, so treatment was switched to mycophenolate mofetil and hydroxychloroquine. However she did not improve and started to have recurrent bacterial and viral infections that included cellulitis, gastroenteritis and upper respiratory tract infection. We started her on regular IVIG, which helped with infections and allowed for weaning of steroids. However she developed alopecia and lower limb spasticity with delayed walking. MRI brain and spine was normal. Upon reanalysis of the WES, two other homozygous mutations at KIF1C and APG7 were identified and associated with spastic paraplegia, but reported as variants of unknown significant. Fresh frozen plasma (FFP) transfusions were started, initially weekly, then every two weeks and subsequently every four weeks. The rash disappeared, leukopenia and ESR improved and we were able to discontinue steroids

Conclusion: Early-onset SLE with a severe course of disease raises the possibility of a genetic etiology. We are reporting, for the first time, a rare missense mutation $\mathrm{G}>\mathrm{T}$ in exon 3 of the C1qA gene that resulted in an amino acid substitution that is pathogenic. Interestingly, she had other mutations associated with neurological manifestation that never reported together before and altered her phenotype. She has responded well to FFP as has been reported in a few case reports

Disclosure of Interest: None declared 


\section{P242}

How do not to miss the systemic lupus erythematosus in patients with juvenile idiopathic arthritis: preliminary results of a retrospective study

A. Kupreeva', E. Kuchinskaya², L. Sorokina ${ }^{3}$, M. Dubko ', A. Mazing ${ }^{4}, 0$. Tkachenko ${ }^{4}$, S. Lapin ${ }^{4}$, M. Kostik ${ }^{1,2}$

${ }^{1}$ Saint-Petersburg State Pediatric Medical University; ${ }^{2}$ Almazov National Medical Research Centre; ${ }^{3}$ Leningrad's Regional Children's Clinical Hospital; ${ }^{4}$ Pavlov First Saint Petersburg State Medical University, Saint Petersburg, Russian Federation

Correspondence: A. Kupreeva

Pediatric Rheumatology 2020, 18(Suppl 2):P242

Introduction: Systemic lupus erythematosus (SLE) - is an autoimmune disease with a multisystem lesion. In some cases, mild SLE may resemble juvenile idiopathic arthritis (JIA), leading to misdiagnosis. Both diseases have common features, such as arthritis and antinuclear antibodies (ANA) seropositivity.

Objectives: To conduct a comparative study analysis of patients with SLE and JIA, to develop discriminating criteria for both conditions.

Methods: In a retrospective study, ANA-positive children $(\geq 1 / 160)$ from laboratory reports $(n=281)$ selected. We chose patients meeting the criteria for SLE $(n=55)$ aged $4-18$ years and selected RF-negative ANA-positive polyarticular JIA patients $(n=52)$ of the same age. We exclude patients with other reasons for ANA-positivity. Clinical and laboratory characteristics compared using descriptive statistics, Mann-Whitney test, $X^{2}$ test, AUC-ROC analysis, calculation of odds ratio (OR), analysis of sensitivity (Se) and specificity (Sp). Univariate, multivariate, and logistic regression analysis applied.

Results: The results of comparison of studied groups in the table. The cut-offs of continued variables and their OR associated with SLE are: onset age $>9,5$ years (OR=9,0 (95\% Cl: 3,6; 22,6), $\mathrm{Se}=89,3, \mathrm{Sp}=$ $63,3, p=0,000001), A N A>1280(\mathrm{OR}=4,2(95 \% \mathrm{Cl}: 1,5 ; 11,7), \mathrm{Se}=48,2$ $\mathrm{Sp}=81,8, \mathrm{p}=0,005)$; platelets $\leq 267 \times 109 / \mathrm{I}(\mathrm{OR}=7,3$ (95\% Cl: 2,6; 20,4), $\mathrm{Se}=89,3, \mathrm{Sp}=46,8, \mathrm{p}=0,00004)$ and active joints $<7 \quad(\mathrm{OR}=3,7(95 \% \mathrm{Cl}$ : $1,6 ; 8,5), \mathrm{Se}=61,8, \mathrm{Sp}=69,6, \mathrm{p}=0,002)$. In univariate, multivariate regression analysis, only onset age $>9.5$ years $(p=0.00002)$ and active joints $<7 \quad(p=0.009)$ were independent discriminators between SLE and JIA. In logistic regression onset age $>9.5$ years $(\mathrm{OR}=9.1[95 \% \mathrm{Cl}$ : 2.8; 29.8], $\mathrm{p}=0.0002)$ and active joints $<7$ (OR=5.4 [95\% Cl: 1.4; 20.1], $\mathrm{p}=0.012$ ) increase the probability of SLE in JIA-like cohort. The presence of 2 mentioned above criteria has $\mathrm{Se}=75.0, \mathrm{Sp}=87.5, \mathrm{OR}=18.0$ (95\% Cl: $6.6 ; 49.1)$ for discrimination of SLE from JIA.

Conclusion: In ANA-positive patients with arthritis older than 9.5 years with active joints $<7$, it should be included in the differential diagnosis with the obligatory determination of all immunological tests specific for SLE.

Trial registration identifying number: This work supported by the Russian Foundation for Basic Research (grant № 18-515-57001).

Disclosure of Interest: None declared

Table 1 (abstract P242). Comparison SLE and JIA patients

\begin{tabular}{|c|c|c|c|}
\hline Parameter & SLE $(n=49)$ & JIA $(n=56)$ & $p$ \\
\hline Onset age, year & $12.3(10.1 ; 14)$ & $8.0(6.2 ; 11.0)$ & 0.00004 \\
\hline ANA (titer) & $\begin{array}{l}1 / 1280(1 / 640 ; 1 / \\
5120)\end{array}$ & $\begin{array}{l}1 / 1280(1 / 640 ; 1 / \\
1280)\end{array}$ & 0.007 \\
\hline Active joints, $n$ & $0.5(0 ; 3.5)$ & $5.0(1.0 ; 10.0)$ & 0.00009 \\
\hline CRP, $\mathrm{mg} / \mathrm{l}$ & $1.0(0.3 ; 3.9)$ & $1.4(0.5 ; 4.5)$ & 0.345 \\
\hline $\mathrm{ESR}, \mathrm{mm} / \mathrm{h}$ & $10.5(5 ; 20.5)$ & $8.0(4.0 ; 18.0)$ & 0.282 \\
\hline WBC $\times 10^{9} / I$ & $6.0(4.4 ; 8.2)$ & $6.7(5.7 ; 8.1)$ & 0.164 \\
\hline Platelets $\times 10^{9} / 1$ & $245.5(217.5 ; 333.0)$ & $298.0(251.0 ; 363.0)$ & 0.015 \\
\hline Hemoglobin, g/l & $121.5(113.0 ; 131.5)$ & $124.0(118.0 ; 130.0)$ & 0.256 \\
\hline $\begin{array}{l}\text { Absolute lymphocyte count, } \\
\times 10^{3} / 1\end{array}$ & $\begin{array}{l}1845.8(1320.5 ; \\
2459.0)\end{array}$ & $\begin{array}{l}2046.0(1711.0 ; \\
2557.8)\end{array}$ & 0.137 \\
\hline
\end{tabular}

P243

Hypocomplementemic urticarial vasculitis in pediatric systemic lupus erythematosus

R. Abdwani

Child Health, SQUH, Muscat, Oman

Pediatric Rheumatology 2020, 18(Suppl 2):P243

Introduction: Urticarial vasculitis (UV) is a clinico-pathologic entity typified by recurrent episodes of urticaria that have the histopathologic features of leukocytoclastic vasculitis. In additions, patients with hypocomplementemic urticarial vasculitis (HUV) exhibit systemic manifestations, including constitutional symptoms, arthralgia or arthritis, serositis, glomerulonephritis, conjunctivitis and recurrent abdominal pain.

An association between HUV and SLE is hypothesized as both share similar clinical anf pathological features. Low serum complement measurements ( $\mathrm{C} 3, \mathrm{C} 4$ and $\mathrm{C} 1 \mathrm{q}$ ) as well as anti C1q autoantibodies are also observed in both conditions. Some authors suggest the possible progression of HUV to SLE and others have defined HUV as an SLE subtype. HUV is present in 7-8\% of SLE patients and $54 \%$ of HUV patients are diagnosed with SLE upon followup.

Objectives: To determine the prevalence HUV in pediatric systemic lupus erythematosus (pSLE) cohort of Arab ethnicity from Oman and to describe their demographic, clinical and laboratory features.

Methods: We conducted a retrospective multicenter study among pediatric rheumatology centers in Oman over a 10-year period from 2008-2018. Analyses were performed using univariate statistics.

Results: A total of 148 pSLE under the age of 13 years were included, $30 \%(n=44)$ were males. The overall mean age at diagnosis was $7.6 \pm 3.5$ years and median disease duration was 9.5 (5-13) years. HUV was diagnosed in 34.5\% $(n=51)$ of pSLE cohort. pSLE with UV were more likely to be males (57\% vs $15 \% ; P<0.001$ ), diagnosed at a younger age (5.9 vs 8.5 years; $P<0.001)$, have a family history of SLE (53\% vs 36\%; $P=0.044$ ) and have conjunctivitis more frequently $(32 \%$ vs $5.3 \% ; P<0.001)$ than pSLE without UV. pSLE with UV were also less likely to have CNS involvement ( $7.6 \%$ vs $20 \% ; P=0.045)$ and hematological manifestations such as leukopenia (9.4\% vs 24\%; $P=0.028$ ) and thrombocytopenia (5.7\% vs $18 \% ; P=0.045)$. In addition, pSLE with UV were more likely to be associated with low C3 complement count (94\% vs 66\%; $P<0.001$ ) and positive cytoplasmic ANCA (11\% vs $0 \% ; P=0.022)$. However, the pSLE with UV cohort were less likely to be associated with ANA (65\% vs $83 \% ; P=0.016)$, DsDNA (56\% vs $72 \% ; P=0.042)$ and perinuclear anti-neutrophil cytoplasmic antibodies (33\% vs 55\%; $P=$ 0.047).

Conclusion: We report a high occurrence of HUV in pSLE cohort (34.5\%) associated with unique demographic, clinical features and laboratory features. The debate regarding whether HUV is a rare subset or unusual type of SLE, or is a separate entity altogether, continues. However, the overlap in clinical, laboratory and genetic mutation supports the notion that HUV and SLE fall into the same spectrum of autoimmune disease with similar disease pathogenesis. However, further studies are needed to reach clear conclusions regarding the relationship between HUV and SLE.

Disclosure of Interest: None declared

P244

Clinical features and long-term outcome in juvenile-onset systemic lupus erythematosus

I. Pontikaki ${ }^{1}$, A. Petaccia ${ }^{2}$, A. F. Luppino ${ }^{3}$, M. Gerosa ${ }^{3,4}$, L. M. Argolini ${ }^{3}$, F. Corona $^{2}$, M. Torcoletti $^{2}$, R. Cimaz ${ }^{1,4}$ on behalf of on behalf of Pediatric

Rheumatology Group of the Milan Area

Pediatric Rheumatology, ASST-Pini-CTO; ${ }^{2}$ Pediatric Rheumatology,

Fondazione IRCSS Cà Granda-Ospedale Maggiore Policlinico;

${ }^{3}$ Rheumatology, ASST-Pini-CTO; ${ }^{4}$ Department of Clinical Science,

University of Milan, Milan, Italy

Correspondence: I. Pontikaki

Pediatric Rheumatology 2020, 18(Suppl 2):P244 
Introduction: Juvenile-onset Systemic Lupus Erythematosus (jSLE) is a rare multisystem, autoimmune/inflammatory disease with onset before the age of 18 , that accounts for $15-20 \%$ of all SLE patients. Clinical manifestations are extremely heterogeneous and disease course is unpredictable, varying from mild to life-threating disease. jSLE has been associated with a worse prognosis than adult-onset disease and can be a life-long disease. However, long term outcomes are poorly described.

Objectives: The aim of this study was to describe clinical features and long-term outcome of a cohort of adult patients diagnosed with jSLE and to evaluate their long-term outcome.

Methods: Charts of adult patients diagnosed with jSLE in two tertiary centres in Milan were analyzed. Data regarding clinical features, laboratory findings, treatments, long-term complications and longterm outcome were recorded retrospectively.

Results: In total, 183 patients with jSLE were included, 88\% were female. Mean age at diagnosis was 12.5 years, while median disease duration at last clinical evaluation was 26 years.

At the time of diagnosis 102 patients (55.7\%) presented cutaneous involvement, 115 patients (62.8\%) showed heamatologic manifestations, while renal disease was described in 59 patients (32.2\%); neurologic involvement was less frequently observed (14.2\%).

We reported deaths in nine patients: two patients died due to infectious complications, one of kidney failure; two of myocardial infarctions and two of malignancies.

Corticosteroids were used in 74 patients (40.4\%); $62.8 \%$ of patients received hydroxycloroquine, $35.5 \%$ azathioprine, $9.2 \%$ cyclosporine $\mathrm{A}$ and $30.6 \%$ cyclophosphamide. Four patients required the use of biologic agents (2 Rituximab and 2 Belimumab).

Major long term complications were hypercorticism due to long use of corticosteroids and infections.

Conclusion: In our cohort of patients, heamatologic, cutaneous and renal involvement were the most common clinical features, while neurologic manifestations were rarer. Our patients showed active disease also during adulthood confirming that jSLE is a life-long multisystem disease with an important impact on health-related quality of life. Moreover treatment strategies contribute to the development of irreversible damage, determining hypercorticism and higher risk of infections.

\section{References}

Amaral B, Murphy G, loannou Y, Isenberg DA. A comparison of the outcome of adolescent and adult-onset systemic lupus erythematosus. Rheumatology (Oxford). 2014;53(6):1130-1135. doi:10.1093/rheumatology/ ket488

Groot N, Shaikhani D, Teng YKO, et al. Long-Term Clinical Outcomes in a Cohort of Adults With Childhood-Onset Systemic Lupus Erythematosus. Arthritis Rheumatol. 2019:71(2):290-301. doi:10.1002/art.40697

Smith EMD, Lythgoe H, Midgley A, Beresford MW, Hedrich CM. Juvenile-onset systemic lupus erythematosus: Update on clinical presentation, pathophysiology and treatment options. Clin Immunol. 2019;209:108274. doi:10.1016/j.clim.2019.108274

Disclosure of Interest: None declared

\section{P245}

Genetic ancestry and its contribution to complex traits in childhood-onset systemic lupus erythematosus

F. B. Barbosa', L. de Oliveira², N. A. Sinicato ${ }^{2}$, R. Marini ${ }^{3}$, V. L. Gil-da-SilvaLopes $^{4}$, S. Appenzeller ${ }^{2}$

'Laboratory of Recognition Biology, Center of Biosciences and Biotechnology, State University of North Fluminense, Rio de janeiro;

${ }^{2}$ Medicine; ${ }^{3}$ Pediatrics; ${ }^{4}$ Genetics, UNICAMP, Campinas, Brazil

Correspondence: S. Appenzeller

Pediatric Rheumatology 2020, 18(Suppl 2):P245

Introduction: Several studies have shown that ancestry influences the susceptibility and development of systemic lupus erythematosus (SLE). However, determining ancestry in an admixture population like Brazilians is difficult.
Objectives: To evaluated the role of admixture degree in clinical manifestations of childhood-onset systemic lupus erythematosus (CSLE) in a tri-hybrid population.

Methods: The genome-wide human Cytoscan HD array was applied to genotype $2.6 \mathrm{~K}$ markers in 107 CSLE Brazilian patients and 110 healthy controls. Quality control of the chip was carried out using Chromosome Analysis Suite software. A panel of 345 ancestry informative markers (AIMs) based on SNP data from Cytoscan HD array was used to infer the proportion of European, African and Amerindian ancestries of each CSLE and control subjects. The individual ancestral composition based on the SNP-AIMs set was estimated using Admixture. Statistical analyses to associate the genetic ancestry with different clinical traits were performed using the computing environment R.

Results: Ancestral composition analysis revealed that the main component of patients and control group was European $(66.9 \%$ and $80.2 \%)$, followed by African (21.6\% and $11.5 \%$ ) and Amerindian (11.5\% and $8.4 \%)$. Comparisons using the proportions of each ancestral component showed significant differences in European $(p$ $=4.7 \times 10-11,95 \% \mathrm{Cl}-0.18--0.10)$, African $(\mathrm{p}=4.1 \times 10-9,95 \% \mathrm{Cl}$ $0.05-0.12)$ and Amerindian ( $p=1.9 \times 10-6,95 \% \mathrm{Cl}-0.03-0.06)$ ancestries between CSLE and control groups.A higher proportion of Amerindian ancestry in CSLE was associated to development of photosensitivity $(p=0.035)$ and hematologic alterations $(p=0.025)$. Otherwise, a higher African component was protective against hypocomplementemia $(p=0.021)$.

Conclusion: Results described here show novel associations to clinical manifestations in cSLE according to the ancestry profile. The deeper investigation of its relation to the pathogenesis may contribute to the understanding of the genetic basis of the disease.

Disclosure of Interest: None declared

\section{P246}

Reliable detection of subtypes of nailfold capillary haemorrhages in childhood-onset systemic lupus erythematosus

S. C. Bergkamp 1 , D. Schonenberg-Meinema' on behalf of EULAR study group on Microcirculation in Rheumatic Diseases, A. Nassar-Sheikh Rashid ${ }^{1}, \mathrm{~K}$. Melsens ${ }^{2,3}$ on behalf of EULAR study group on Microcirculation in Rheumatic Diseases, A. Vanhaecke ${ }^{2,3}$ on behalf of EULAR study group on Microcirculation in Rheumatic Diseases, M. J. Boumans', P. C. Hissink Muller', T. W. Kuijpers', J. M. van den Berg ${ }^{1}$, V. Smith ${ }^{2,3,6}$ on behalf of EULAR Study Group on

Microcirculation in Rheumatic Diseases

'Department of Pediatric Immunology, Rheumatology and Infectious diseases, Amsterdam University Medical Centers, Amsterdam, Netherlands; ${ }^{2}$ Department of Rheumatology, Ghent University Hospital; ${ }^{3}$ Faculty of Internal Medicine, Ghent University, Ghent, Belgium; ${ }^{4}$ Department of Rheumatology, Maxima Medical Centre, Eindhoven; ${ }^{5}$ Department of Pediatric Rheumatology, Leiden University Medical Center , Leiden, Netherlands; ${ }^{6}$ Unit for Molecular Immunology and Inflammation, VB Inflammation Research Center (IRC), Ghent, Belgium

Correspondence: S. C. Bergkamp

Pediatric Rheumatology 2020, 18(Suppl 2):P246

Introduction: Systemic Lupus Erythematosus (SLE) is a severe chronic disease for which it is necessary to obtain more indicators of disease severity that predict disease damage. Nailfold capillary abnormalities could be such an indicator or biomarker in SLE. Nailfold capillary haemorrhages have been observed in adults and children with SLE as an abnormality that was significantly correlated with disease activity. Recently, different subtypes of capillary haemorrhages have been described in a cross-sectional casecontrol study of childhood-onset (c)SLE.

Objectives: The aim of this current study was to evaluate the inter- and intra-observer reliability for detection of different subtypes of capillary haemorrhages, as identified by nailfold videocapillaroscopy (NVC) in CSLE patients.

Methods: Five raters from three different centres blindly evaluated 140 NVC images from 35 CSLE-patients, diagnosed according to the Systemic Lupus International Collaborating Clinics (SLICC) criteria. The raters assessed the anonymized NVC images qualitatively (present or absent) and quantitatively (total number) on four different subtypes of haemorrhages: 
1) punctate extravasations; point-shaped, localized around, or in the apical centre of capillary loop

2) perivascular haemorrhage; as 1) but in grouped/confluent aspect

3) large confluent haemorrhage; migrating in line with the capillary to distal towards the cuticle

4) non-definable; not matching criteria 1-3

As punctate extravasations and perivascular haemorrhages were interpreted as a continuous spectrum, an analysis with groups 1-2 merged (mean) kappa/ICC was also calculated as one sub-group. For qualitative data, Fleiss' and Cohen's kappa analyses were calculated. For quantitative data, the intraclass correlation coefficient (ICC) was calculated.

Results:

\begin{tabular}{|c|c|c|c|c|}
\hline Inter-rater scores & $\begin{array}{l}\text { Fleiss' kappa } \\
\text { (qualitative counts) }\end{array}$ & $\begin{array}{l}95 \% \\
\mathrm{Cl}\end{array}$ & $\begin{array}{l}\text { ICC } \\
\text { (quantitative counts) }\end{array}$ & $\begin{array}{l}95 \% \\
\mathrm{Cl}\end{array}$ \\
\hline $\begin{array}{l}\text { Punctate\&perivascular } \\
\text { haemorrhages }\end{array}$ & 0.62 & $\begin{array}{l}0.57- \\
0.67\end{array}$ & 0.82 & $\begin{array}{l}0.76- \\
0.87\end{array}$ \\
\hline $\begin{array}{l}\text { Large confluent } \\
\text { haemorrhages }\end{array}$ & 0.78 & $\begin{array}{l}0.72- \\
0.83\end{array}$ & 0.93 & $\begin{array}{l}0.91- \\
0.95\end{array}$ \\
\hline Intra-rater scores & $\begin{array}{l}\text { Mean Kappa } \\
\text { (qualitative counts) }\end{array}$ & & $\begin{array}{l}\text { Mean ICC } \\
\text { (quantitative counts) }\end{array}$ & \\
\hline $\begin{array}{l}\text { Punctate\&perivascular } \\
\text { haemorrhages }\end{array}$ & 0.70 & & 0.84 & \\
\hline $\begin{array}{l}\text { Large confluent } \\
\text { haemorrhages }\end{array}$ & 0.86 & & 0.96 & \\
\hline \multirow{4}{*}{$\begin{array}{l}\mathrm{Cl}=\text { Confidence } \\
\text { Interval }\end{array}$} & Kappa interpretation: & & ICC interpretation: & \\
\hline & \multirow{2}{*}{$\begin{array}{l}0.61-0.80 \text { substantial } \\
\text { agreement }\end{array}$} & & \multirow{3}{*}{$>0.90$ excellent } & \\
\hline & & & & \\
\hline & $\begin{array}{l}>0.81 \text { almost perfect } \\
\text { agreement }\end{array}$ & & & \\
\hline
\end{tabular}

Conclusion: This is the first study on intra- and inter-rater reliability for different subtypes of nailfold capillary haemorrhages in (c)SLE. Our findings show that different subtypes of capillary haemorrhages in CSLE-patients can be reproduced with a good inter- and intrarater reliability. This confirms our recent observation of two haemorrhagesubtypes in a cross-sectional case-control study. Thus, it also indicates the potential diagnostical value for NVC in CSLE. Future observational studies should elucidate whether the different subtypes of capillary haemorrhages are specific for (c)SLE compared to (juvenile) dermatomyositis and (juvenile) systemic sclerosis. Longitudinal studies are needed to investigate the role of capillary haemorrhages as a prognostic biomarker for disease damage in (c)SLE.

Disclosure of Interest: None declared

\section{P247}

The performances of different classification criteria in pediatric systemic lupus erythematosus

E. D. Batu', U. Kaya Akca' ${ }^{1}$, A. Pac Kisaarslann', E. Sag ${ }^{1}$, F. Demir ${ }^{3}$, S. Demir ${ }^{1}$, S. Ozdemir Cicek', H. Poyrazoglu², B. Sozeri ${ }^{3}$, Y. Bilginer' ${ }^{1}$ S. Ozen ${ }^{1}$ ${ }^{1}$ Department of Pediatrics, Division of Rheumatology, Hacettepe University Faculty of Medicine, Ankara; ${ }^{2}$ Department of Pediatrics, Division of Rheumatology, Erciyes University Faculty of Medicine, Kayseri; ${ }^{3}$ Department of Pediatrics, Division of Rheumatology, Umraniye Training and Research Center, University of Health Sciences, Istanbul, Turkey

Correspondence: E. D. Batu

Pediatric Rheumatology 2020, 18(Suppl 2):P247

Introduction: Systemic lupus erythematosus (SLE) is a chronic autoimmune disease. The three available classification criteria for SLE are ACR 1997, SLICC 2012, and EULAR/ACR 2019 all of which are formed based on data mainly derived from adult SLE cohorts. Objectives: We aimed to test the performances of ACR 1997, SLICC 2012, and EULAR/ACR 2019 SLE criteria among pediatric SLE patients. Methods: One hundred and eleven SLE patients from Hacettepe University, Ankara; 102 from Erciyes University, Kayseri; and 49 SLE patients from Umraniye Training and Research Hospital, Istanbul,
Turkiye were included. As controls, 172 children with different rheumatic diseases (including mixed connective tissue disease and juvenile dermatomyositis) who had ANA test were included. The disease onset was before 18 years of age in all patients. The sensitivity and specificity of the ACR 1997, SLICC 2012, and EULAR/ ACR 2019 criteria were evaluated based on the features of the patients at the time of diagnosis. The gold standard for the diagnosis of SLE was expert opinion at each center.

Results: In total, 262 SLE (80.9\% female) and 172 control patients were included. The sensitivities of ACR 1997, SLICC 2012, and EULAR/ACR 2019 criteria were 68.7\%, 95.4\%, and 92\%, respectively. The specificities of ACR 1997, SLICC 2012, and EULAR/ACR 2019 criteria were 94.8\%, 89.5\%, and 87.8\%, respectively. There were 17 SLE patients who met SLICC criteria but did not fulfill ACR/EULAR 2019 criteria. Among these patients, hematologic involvement was prominent $(12 / 17 ; 70.6 \%)$. On the other hand, there were 8 SLE patients fulfilling ACR/EULAR 2019 criteria but not SLICC 2012 criteria. In these patients, joint involvement was prominent $(6 / 8 ; 75 \%)$. The characteristics of these patients are presented in Table 1.

Conclusion: In our study, the sensitivity of SLICC 2012 criteria was the best, while the best specificity was that of ACR 1997 criteria. SLIC C 2012 criteria performed better than EULAR/ACR 2019 criteria with higher sensitivity and specificity. Separation of different hematological manifestations in SLICC 2012 criteria might have contributed to the higher performance of this criteria set.

Disclosure of Interest: None declared

Table 1 (abstract P247). SLE (systemic lupus erythematosus) patients who met either one of the SLICC 2012 or EULAR/ACR 2019 criteria but not the other

\begin{tabular}{|c|c|c|}
\hline $\begin{array}{l}\text { Characteristics, } \\
\text { n (\%) }\end{array}$ & $\begin{array}{l}\text { SLE patients who met SLICC } \\
2012 \text { but not EULAR/ACR } \\
2019(n=17)\end{array}$ & $\begin{array}{l}\text { SLE patients who met } \\
\text { EULAR/ACR } 2019 \text { but not } \\
\text { SLICC } 2012(n=8)\end{array}$ \\
\hline $\begin{array}{l}\text { Joint } \\
\text { involvement }\end{array}$ & $3(17.6)$ & $6(75)$ \\
\hline Oral ulcers & $8(42)$ & $0(0)$ \\
\hline $\begin{array}{l}\text { Hematologic } \\
\text { involvement }\end{array}$ & $12(70.6)$ & $1(12.5)$ \\
\hline $\begin{array}{l}\text { ANA positivity } \\
(\geq 1 / 80)\end{array}$ & $15(88.2)$ & $8(100)$ \\
\hline $\begin{array}{l}\text { Anti-cardiolipin } \\
\text { antibodies }\end{array}$ & $6(35.3)$ & $0(0)$ \\
\hline $\begin{array}{l}\text { Anti- } \beta 2 \\
\text { glycoprotein }\end{array}$ & $3(17.6)$ & $0(0)$ \\
\hline $\begin{array}{l}\text { Lupus } \\
\text { anticoagulant }\end{array}$ & $4(23.5)$ & $0(0)$ \\
\hline Renal biopsy & $\begin{array}{l}2 \text { (11.8) (class l; class III lupus } \\
\text { nephritis) }\end{array}$ & $0(0)$ \\
\hline $\begin{array}{l}\text { SLE according to } \\
\text { the ACR } 1997 \\
\text { criteria }\end{array}$ & $7(41.2)$ & $2(25)$ \\
\hline
\end{tabular}

ACR, American College of Rheumatology; ANA, anti-nuclear antibody; anti-dsDNA, anti-double stranded DNA; EULAR, European League Against Rheumatism; SLE, systemic lupus erythematosus; SLICC, Systemic Lupus International Collaborating Clinics

P248

Validation of the 2019 ACR/EULAR classification criteria of systemic lupus erythematosus in a Mexican pediatric population

R. E. Loor, E. Faugier

Reumatología Pediátrica, HOSPITAL INFANTIL DE MÉXICO FEDERICO

GÓMEZ, México, Mexico

Correspondence: R. E. Loor

Pediatric Rheumatology 2020, 18(Suppl 2):P248 
Introduction: Systemic Lupus Erythematosus (SLE) is the prototype autoimmune disease, characterized by the presence of multiple autoantibodies with the consequent formation and deposition of immunocomplexes, and other immune processes, causing a multisystemic disease. Over the years, various classification criteria have been published with variable performance. In 2019 the European League Against Rheumatism (EULAR) and the American College of Rheumatology (ACR) developed a set of criteria, which the validity has not been studied so far in the pediatric population with juvenile Systemic Lupus Erythematosus (SLEj).

Objectives: To determine the sensitivity, specificity, positive predictive value and negative predictive value of the 2019 EULAR / ACR criteria for SLE in children who attended in the pediatric rheumatology service of the Hospital Infantil de México Federico Gómez and compare them with the ACR criteria of 1997 and the International Collaborating Clinics of Systemic Lupus (SLICC) 2012. Methods: Retrospective collection of clinical and paraclinical data during the first month of illness in patients with SLEj and control group with rheumatologic disease other than SLE who have a positive determination of antinuclear antibodies. The ACR 1997, SLICC 2012 and 2019 EULAR / ACR classification criteria were applied to each patient to determine the sensitivity, specificity, positive predictive value and negative predictive value for each classification criteria.

Results: A total of 100 patients with LESj diagnosis and 100 patients in the control group were included. $88 \%$ of the cases and $76 \%$ of the controls were female. The average age at diagnosis was $10.5 \pm 3.78$ years (2 - 17 years). When comparing the criteria proposed by 2019 EULAR-ACR, ACR 1997 and SLICC 21012, greater sensitivity, specificity, positive predictive value and negative predictive value were obtained in the 2019 EULAR-ACR criteria $(98 \%, 100 \%, 100 \%$ and $98 \%$ respectively) as reflected in Table 1.

Conclusion: It is the first study in pediatric population where a comparison of the three groups of classification criteria is performed and reveals a sensitivity of $98 \%$ and specificity of $100 \%$ for the 2019 EULAR-ACR criteria, demonstrating greater sensitivity and specificity in comparison with the criteria of ACR 1997 and SLICC 2012. The report identifies the applicability of the 2019 EULAR / ACR criteria and sets a guideline for future studies.

\section{Disclosure of Interest: None declared}

Table 1 (abstract P248). Sensitivity, Specificity, Positive Predictive Value and Negative Predictive Value of the Classification Criteria of the European League Against Rheumatism and the American College of Rheumatology of 2019, American College of Rheumatology 1997 and the group of International Collaborating Clinics of Systemic Lupus from 2012

\begin{tabular}{|c|c|c|c|c|}
\hline Criteria & Sensitivity (\%) & Specificity (\%) & PPV (\%) & NPV (\%) \\
\hline 2019 EULAR-ACR & 98 & 100 & 100 & 98 \\
\hline ACR 1997 & 91 & 98 & 98 & 92 \\
\hline SLICC 2012 & 88 & 97 & 97 & 89 \\
\hline
\end{tabular}

\section{P249}

Molecular findings in patients with systemic lupus erythematosus from Sakha Republic (Yakutia)

E. Suspitsin ${ }^{1,2}$, M. Kostik ${ }^{1,3}$, V. Argunova ${ }^{4}$, A. Kosmin² ${ }^{2}$ E. Kuchinskaya ${ }^{3}$

${ }^{1}$ St.- Petersburg State Pediatric Medical University, ${ }^{2}$ N.N. Petrov Scientific Research Center of Oncology, ${ }^{3}$ Almazov National Medical Research Center, St.- Petersburg, ${ }^{4}$ Republican Hospital №1, National Center of Medicine, Yakutsk, Russian Federation

Correspondence: E. Suspitsin

Pediatric Rheumatology 2020, 18(Suppl 2):P249

Introduction: In the last few years, many monogenic defects leading to a lupus-like phenotype have been described. Sakha Republic (Yakutia) is a large north - eastern region of Russia where an increased incidence of systemic lupus erythematosus (SLE) is reported. In general, like the native Americans from Alyaska, the Yakutia aboriginals have a load of autoimmune diseases, especially HLA-B27associated conditions.

Objectives: Yakut population of 500000 has peculiar genetic background and high level of consanguinity; thus, we expected to reveal specific lupus - associated genes and founder variants characteristic for this ethnic group.

Methods: We performed clinical exome sequencing of 14 Yakut SLE patients ( $9-18$ years old) primarily focusing on analysis of the 51 genes currently associated with monogenic lupus and lupus - like conditions.

Results: Likely causative variants of SLE-associated genes were detected in 7 of 14 patients. Rare variants of RNASEH2B, TREX1, PTPN22, DNASE1L3, TMEM173 and SAMHD1 including some recurrent alleles have been revealed. It is also of interest that TNFRSF13C c.62C $>$ G (p.P21R) polymorphism having reported frequency of 1 $7 \%$ in different world populations was apparently overrepresented in the group of SLE patients.

Conclusion: Genetic defects may be detected in a substantial fraction of non-early onset Yakut SLE thus making this population worthy of further analysis. This pilot study extends existing data on spectrum of genetic variants associated with SLE.

This work was supported by the RSF grant № 20-45-01005

Disclosure of Interest: None declared

P250

Withdrawn

P251

Rates of tuberculosis infection in patients with Juvenile Idiopathic Arthritis preceding anti-tnf therapy for more than 2 years: a singlecenter observational study

N. Tsurikova', E. Ligostaeva', V. Avdeenko', N. Kobzeva' ', I. Tsiganok', O. Lagunova ${ }^{2}$

${ }^{1}$ Rostov Regional Children Hospital; ${ }^{2}$ Rostov regional phthisiological dispensary, Rostov-on-don, Russian Federation

Correspondence: $\mathrm{N}$. Tsurikova

Pediatric Rheumatology 2020, 18(Suppl 2):P251

Introduction: Currently, the assessment of the incidence of tuberculosis infection in patients with juvenile idiopathic arthritis (JIA) receiving therapy with TNF-alpha inhibitors remains relevant.

Objectives: To evaluate the incidence of tuberculosis infection in patients with JIA receiving anti-TNF therapy (Adalimumab (ADA), Etanercept (ETA)) for 2 years or more.

Methods: This was a retrospective study that included JIA patients treated with anti-TNF therapy for more than 2 years.

48 JIA patients with current age $9.2 \pm 4.0$ years, mean disease duration of $3.4 \pm 1.8$ years were included. All patients received a single anti-TNF therapy: 28(58\%) patients were treated with etanercept, 20(42\%) patients were treated with adalimumab. All patients were screened for latent tuberculosis infection(LTBI) prior to anti-TNF therapy using tuberculin skin test (TST), chest computer tomography (CT) and history of exposure to tuberculosis. Patients were regularly followed at 6month intervals. Before patients started receive anti-TNF therapy, LTBI screening (solely TST-positive) was positive in $8(16.6 \%)$ patients: 5 patients in group of ETA and 3 in group of ADA, no one had active tuberculosis (TB) or history of TB exposure.

Results: After 2 years of therapy with ADA latent tuberculosis infection (LTBI) was diagnosed in $6(12.5 \%)$ patients $(10.3 / 100$ patientyears); spontaneously cured primary tuberculosis was diagnosed in $1(2 \%)$ patient (1.7/100 patient-years); pulmonary tuberculosis (positive TST and changes on chest CT) was diagnosed in $1(2 \%)$ patient (1.7/100 patient-years).

After 2 years of ETA therapy LTBI was diagnosed in 4(8.3\%) patients (4.6/100 patient-years); spontaneously cured primary tuberculosis developed in $1(2 \%)$ patient (1.1/100 patient-years).

Conclusion: According to the obtained data it can be concluded that the risk of developing tuberculosis infection among patients receiving anti-TNF therapy remains. Our observation shows that the 
risk of developing tuberculosis infection is slightly higher in the group of children receiving ADA, compared with patients treated with ETA.

\section{Disclosure of Interest: None declared}

\section{P252}

\section{Hypersensitivity reactions with DMARDS}

M. Kasap Cuceoglu', E. Sag ${ }^{1}$, S. Demir ${ }^{1}$, U. Kaya Akca', E. Atalay ${ }^{1}$, Ö. Uysal Soyer $^{2}$, E. D. Batu', Y. Bilginer', S. Ozen

${ }^{1}$ Pediatric Rheumatology; ${ }^{2}$ Pediatric Allergy, Hacettepe University, Ankara, Turkey

Correspondence: M. Kasap Cuceoglu

Pediatric Rheumatology 2020, 18(Suppl 2):P252

Introduction: Disease-modifying antirheumatic drugs (DMARDs), are widely used for the treatment of rheumatologic diseases including juvenile idiopathic arthritis (JIA), psoriatic arthritis (PsA), entesitis related arthritis (ERA), systemic sclerosis (SSC), systemic lupus erythematosus (SLE), and Sjogren syndrome (SS), juvenile dermatomyositis, Behçet disease. We aimed to study the hypersentivity reaction findings associated with the commonly used conventional DMARDs include methotrexate, leflunomide, hydroxychloroquine and sulfasalazine.

Objectives: We aimed to study the hypersentivity reaction findings associated with the commonly used conventional DMARDs include methotrexate, leflunomide, hydroxychloroquine and sulfasalazine.

Methods: We evaluated the patients followed up between January 2019 and January 2020 in Department of Pediatric Rheumatology in Hacettepe University, Ankara, Turkey. 128 pediatric patients used DMARDs were accommodated in the study. The demographics, disease course, atopy history, familial history of atopy and drug allergies were analysed.

Results: 128 pediatric rheumatology patients including 46 juvenile idiopathic arthritis, 23 familial mediterranean fever patients, 19 polyarteritis nodosa, 17 systemic lupus erythematosus, 7 Behçet's disease, 5 juvenile dermatomyozitis, 5 enthesitis-related arthritis, 4 scleroderma, and 2 psoriatic arthritis were the subject of this study. Drug allergy was found in 14 patients: 7 against to antibiotics, 4 against to biologic agents and 3 to DMARDs. The use of DMARDs agents among the group were: 62 patients (48\%) using methotrexate, 38 patients $(29 \%)$ colchicine, 18 patients $(14 \%)$ hydroxychloroquine, 18 patients (14\%) mycophenolate mofetil,12 patients (9\%) cyclosporine A, 19 patients (14.8\%) AZA, 2 patients sulfasalazine. No allergic reactions to MTX, AZA, MMF were detected. On the other hand, 1 patient developed a hypersensitivity reaction against hydroxychloroquine similar to erythroderma, and another patient developed allergic reactions to sulfasalazine, one to infliximab, one to colchicine and one to rituximab.

Conclusion: DMARDs are commonly used agents in rheumatology departments, hypersensitivity reactions against them are seldom reported. It is usually known that the subcutaneous form results in more allergic reactions than the oral form, however it is discovered that oral forms of DMARDs is very likely to lead to allergenic reactions.

Key words: childhood, DMARDs, hypersensitivity reactions, rheumatologic disease, methotrexate

\section{Disclosure of Interest: None declared}

\section{P253}

Drug survival and safety of biological therapies in patients with Juvenile Idiopathic Arthritis

A. Boteanu', A. Briones Figueroa', L. Calvo Sanz' 'Á. Andreu Suarez², A. García Fernández

${ }^{1}$ Rheumatology, Ramon y Cajal University Hospital; ${ }^{2}$ Rheumatology, Ramón y Cajal University Hospital, Madrid, Spain

Correspondence: A. Boteanu

Pediatric Rheumatology 2020, 18(Suppl 2):P253
Introduction: Biological treatment (BT) has changed perspectives of JIA patients. Increasing data from real life experience have been reported.

Objectives: To compare drug survival, safety and efficacy of BT in patients with Juvenile Idiopathic Arthritis (JIA).

Methods: A retrospective observational study was conducted on JIA patients followed in a referal hospital and who had received at least one BT between 1999 and 2019.

Results: 218 BT in $130 \mathrm{JIA}$ patients were analyzed. $67.7 \%$ were women with a median age at diagnosis of 8 years old IQR (3-13) and a median age at the beginning of the BT of 15 years old IQR(7.8-21). $21.5 \%$ of the patients had uveitis during follow-up. BT were indicated due to: arthritis $(73.9 \%)$, uveitis (10.1\%), arthritis and uveitis (2.7\%), systemic activity (8.3\%) and macrophage activation syndrome $(1,8 \%)$. There were 130 BT started in $1^{\text {st }}$ line, 55 in $2^{\text {nd }}$ line, 20 in $3^{\text {rd }}$ line, 10 in $4^{\text {th }}$ line and 3 in $5^{\text {th }}$ line.

The $1^{\text {st }}$ line BT most frequently indicated was Etarnecept (ETN) up to $40 \%$, followed by $30 \%$ Adalimumab (ADA) and 16,2\% Infliximab (INF). The median duration of the $1^{\text {st }}$ line was 51 months IQR (14$109,3)$. However, $53.8 \%$ of the $1^{\text {st }}$ line BT were swiched: $28.3 \%$ due to adverse events, $25.7 \%$ due to $1^{\circ}$ failure and $25.7 \%$ due to $2^{\circ}$ failure. The BT that were discontinued were: INF $(76.2 \%)$ and Anakinra (ANAK) (75\%) due to adverse events and ETN (59.6\%) due to $1^{\circ}$ and $2^{\circ}$ failure. 55 patients started a $2^{\text {nd }} B T: 43.6 \%$ received ADA and $20 \%$ Tocilizumab (TCZ) with a median duration of 43 months IQR (12-90). 22 of 55 BT required a change: $75 \%$ of ETN and $59 \%$ of INF prescribed in $2^{\text {nd }}$ line were discotinued. The causes were: $40 \% 1^{\circ}$ failure, $28 \% 2^{\circ}$ failure and $12 \%$ remission. In $1^{\text {st }}$ line $87,6 \%$ of patients received TNF inhibitors, $74 \%$ mantained the target in $2^{\text {nd }}$ line. In $3^{\text {rd }}$ line TCZ was the most frequent BT. $71.5 \%$ of patients continue on BT. BT was withdrawn in 20 of 130 patients due to remission ( $40 \%)$, adverse events (30\%), and pregnancy (10\%). In the analysis by decades, 80 BT (36.7\%) were started from 1999 to 2008 and 138 BT (63.3\%) from 2009 to 2019. In the $1^{\text {st }}$ decade ETN and INF were the most frequently prescribed and in the $2^{\text {nd }}$ decade, ADA and TCZ $(p<0.0001)$. The $1^{\text {st }}$ BT in the $2^{\text {nd }}$ decade were indicated sooner compared to the $1^{\text {st }}$ decade $\left(1^{\text {st }}\right.$ decade: mean 119.5months SD(109.2); $2^{\text {nd }}$ decade: mean 53.9 months SD(99.7); $\mathrm{p}<$ $0.0001)$. In $1^{\text {st }}$ line BT, the BT prescribed in the $2^{\text {nd }}$ decade had a shorter duration than those in the $1^{\text {st }}$ decade $\left(1^{\text {st }}\right.$ decade: mean 84.1 months SD(71.8); $2^{\text {nd }}$ decade: mean 51.7 months SD(5); $\left.p<0.0001\right)$.

In the survival analysis, TCZ and ADA were the BT with the highest survival $(p=0.001)$. Of the 31 patients that startedTCZ, $61.3 \%$ continue on TCZ, with a median duration of 46 months IQR(25-99) and $36 / 68(52,9 \%)$ still on ADA with a median duration of 61,5 months IQR(30.5-98).

Conclusion: $42.3 \%$ of patients required more than one BT. Since the onset of the BT there has been a change in prescription, probably related to the emerge of new targets and the evidence provided by clinical trials and guidelines. TCZ and ADA were the BT with the highest survival rate. On the other hand, INF and ANAK were the ones with the lowest survival rate. The most common causes of BT change in $1^{\text {st }}$ line were adverse events in relation to INF and ANAK. In $2^{\text {nd }}$ line there was a high rate of change in those patients who maintained $\mathrm{TNFi}$, related to $1^{\circ}$ failure.

Disclosure of Interest: None declared

P254

Revising the who essential medicines list for paediatric rheumatology

C. Scott ${ }^{1}$, N. Smith², R. James ${ }^{3}$, B. Whitehead ${ }^{3}$, R. Green ${ }^{4}$, H. Foster ${ }^{2}$ on behalf of on behalf of the Paediatric Global MSK Task Force

'Department of Paediatrics, University of Cape Town, Cape Town, South Africa; ${ }^{2}$ Translational and Clinical Research Institute, Newcastle University, Newcastle Upon Tyne, United Kingdom; ${ }^{3}$ Paediatric Rheumatology,

${ }^{4}$ Pharmacy, Queensland Children's Hospital, Brisbane, Queensland, Australia

Correspondence: C. Scott

Pediatric Rheumatology 2020, 18(Suppl 2):P254 
Introduction: The World Health Organisation (WHO) Essential Medicines List (EML) informs countries about the minimum medicine items necessary to meet priority health needs of the population (both adults and children). The EMLs guide national and institutional medicine lists, especially in Low and Middle Resource Income Countries. The current EML for paediatric rheumatology does not reflect current best practice (Foster and Scott 2020 Nature Reviews Rheumatology). The Paediatric Global Musculoskeletal Health Task Force (TF) is working to revise the WHO EML.

Objectives: To explore which drugs are considered 'essential' and 'ideal' for listing in the EML under paediatric rheumatic diseases.

Methods: Healthcare professionals working in paediatric rheumatology and who are TF members were invited to take part in an anonymous online survey. We had 97 responses from 43 countries (across all continents), with 1-35 years of clinical practice including: consultant grade paediatric rheumatologists $(n=77)$, consultant general paediatricians with interest in rheumatology $(n=13)$, paediatric rheumatology trainees $(n=3)$, adult rheumatologists $(n=3)$ and a nurse working in paediatric rheumatology $(n=1)$. The survey data was analysed applying descriptive statistics and free-text comments using qualitative techniques.

Results: Most respondents ( $n=70 / 97,72 \%)$ reported that a revised EML would improve access to medicines in their country, improve drug provision and accessibility within their clinical practice, provide assistance when negotiating with healthcare agencies, funding bodies or insurance companies and inform paediatric trainees and adult rheumatologists about paediatric rheumatology issues. They deemed that the EML should list the following drugs (Table 1).

Conclusion: Respondents confirmed the need to update the WHO EML and anticipate considerable impact on clinical practice in many countries around the world. More than $80 \%$ of respondents identified 5 agents as 'essential' (oral, intra-articular and intravenous steroids, NSAIDS, Hydroxychloroquine and Methotrexate [oral and subcutaneous]) and indicated that the EML should include a wide range of synthetic and biologic DMARDS as well as other immunosuppressive agents used in the management of rheumatic diseases in children. This data will form the basis of the TF application to the WHO to revise the EML with submission planned for 2020.

Disclosure of Interest: None declared

Table 1 (abstract P254). Rheumatic disease drug inclusion within the WHO EML

\begin{tabular}{|c|c|c|c|c|c|c|c|c|}
\hline$\overline{\text { Drug }}$ & $\begin{array}{l}\text { Should } \\
\text { Include } \\
\text { (Ideal) }\end{array}$ & $\begin{array}{l}\text { Inclusion } \\
\text { 'Essential' }\end{array}$ & Drug & $\begin{array}{l}\text { Should } \\
\text { Include } \\
\text { (Ideal) }\end{array}$ & $\begin{array}{l}\text { Inclusion } \\
\text { 'Essential' }\end{array}$ & Drug & $\begin{array}{l}\text { Should } \\
\text { Include } \\
\text { (Ideal) }\end{array}$ & $\begin{array}{l}\text { Inclusion } \\
\text { 'Essential' }\end{array}$ \\
\hline $\begin{array}{l}\text { Oral } \\
\text { prednisolone }\end{array}$ & $100 \%$ & $92 \%$ & $\begin{array}{l}\text { Intravenous } \\
\text { cyclophosphamide }\end{array}$ & $91 \%$ & $77 \%$ & $\begin{array}{l}\text { Subcutaneous } \\
\text { Tocilizumab }\end{array}$ & $81 \%$ & $46 \%$ \\
\hline $\begin{array}{l}\text { Oral } \\
\text { NSAADs }\end{array}$ & $99 \%$ & 93\% & Adalimumab & $91 \%$ & $71 \%$ & Infiliximab & $80 \%$ & $52 \%$ \\
\hline $\begin{array}{l}\text { Hydro- } \\
\text { xychloroquine }\end{array}$ & $98 \%$ & $88 \%$ & Anakinra & $90 \%$ & $60 \%$ & $\begin{array}{l}\text { Intrravenous } \\
\text { bisphosphonate } \\
\text { (e.g. pamidronate) }\end{array}$ & $76 \%$ & $37 \%$ \\
\hline $\begin{array}{l}\text { Intravenous } \\
\text { Methyl- } \\
\text { prednisolone }\end{array}$ & $98 \%$ & $83 \%$ & Etanercept & $87 \%$ & $70 \%$ & $\begin{array}{l}\text { Intra-articular } \\
\text { corticosteroid } \\
\text { Triamcinolone } \\
\text { Acetonide }\end{array}$ & $72 \%$ & $28 \%$ \\
\hline $\begin{array}{l}\text { Methotrexate } \\
\text { oral }\end{array}$ & $96 \%$ & $81 \%$ & $\begin{array}{l}\text { Intra-articular } \\
\text { corticosteroid } \\
\text { Triamcinolone } \\
\text { Hexacetonide }\end{array}$ & $86 \%$ & $64 \%$ & $\begin{array}{l}\text { Intra-articular } \\
\text { corticosteroid } \\
\text { Methyl- } \\
\text { prednisolone }\end{array}$ & $45 \%$ & $25 \%$ \\
\hline Mycophenolate Mofetil & $95 \%$ & $77 \%$ & $\begin{array}{l}\text { Intravenous } \\
\text { Tocilizumab }\end{array}$ & $86 \%$ & $63 \%$ & $\begin{array}{l}\text { Oral } \\
\text { cyclo- } \\
\text { phosphamide }\end{array}$ & $41 \%$ & $16 \%$ \\
\hline Azathioprine & $94 \%$ & $71 \%$ & $\begin{array}{l}\text { Oral } \\
\text { prednisolone } \\
\text { (soluble) }\end{array}$ & $86 \%$ & $55 \%$ & $\begin{array}{l}\text { Inhaled } \\
\text { analgesia } \\
\text { (nitrous oxide) }\end{array}$ & $36 \%$ & $15 \%$ \\
\hline $\begin{array}{l}\text { Methotrexate } \\
\text { subcutaneous }\end{array}$ & $91 \%$ & $80 \%$ & Ciclosporin & $85 \%$ & $52 \%$ & Thalidomide & $34 \%$ & $8 \%$ \\
\hline
\end{tabular}

P255

The range of biological drugs used in patients with juvenile idiopathic arthritis, saratov region example

Y. M. Spivakovskiy, A. Y. Spivakovskaya, Y. V. Chernenkov

Depertment of Hospitality Pediatrics, SARATOV STATE MEDICAL

UNIVERSITY, Saratov, Russian Federation

Correspondence: A. Y. Spivakovskaya

Pediatric Rheumatology 2020, 18(Suppl 2):P255

Introduction: The last decade has brought a lot to the approaches to the diagnosis and treatment of juvenile arthritis. In Russia, the actualization of the problem of diagnosis and treatment of JIA required the development of federal standards, which provide the most detailed algorithms for medical care, both at the stage of inpatient and outpatient care. In the regions of the Russian Federation, the effective use of these documents required a whole range of additional educated activities, both with students of medical universities, as well as with the medical and nursing community, in addition, a set of work was carried out to create a regional regulatory framework. The first genetically engineering drug with children's indications in the Russian Federation was registered at the end of 2009. Over the past decade, the range of biological agents used in pediatrics has expanded significantly and requires constant replenishment of the level of knowledge of doctors.

Objectives: To analyze the structure of biological therapy in patients with juvenile idiopathic arthritis living in the Saratov region of the Russian Federation.

Methods: The study included generalized information on 253 patients aged 1-17 years with a diagnosis of JIA verified by the ILAR criteria and living in the Saratov Region on January 1, 2019.

Results: According to medical statistics in the region, a diagnosis of JIA has been made in 253 children and adolescents. A significant increase is noted annually in children receiving biological therapy, so in 2014 there were 30 patients, which accounted for 9,3\% of the total number of patients with $\mathrm{JIA}$, at the beginning of 2019 this figure was $19 \%(n=48)$, and by the end of $2019-22,1 \%(n=56)$. In the total biological therapy pool, $67 \%$ of patients receive TNF-alpha inhibitors, antibodies to IL- 6 receive $27 \%$ of patients, antibodies to IL$1-6,25 \%$. It is worth noting that when using biological agents in $60 \%$ of cases, the criterion of an inactive disease was achieved by $4-$ 5 months, which was characterized by the absence of acute inflammatory symptoms, normalization of ESR and CRP. Monitoring of patients with JIA receiving biological agents required the conduct of a number of educational activities for medical personnel, the creation of an additional methodological base. For further training of young specialists at the regional medical university, a program of an additional educational course in pediatric rheumatology was developed and introduced. A regional patient organization was established and also required a set of information activities by the medical community.

Conclusion: In the Saratov region of the Russian Federation, about $20 \%$ of patients with JIA receive biological therapy, which corresponds to the average indicators according to the literature. In the structure of the biological drugs used, the group of TNF-alpha inhibitors is preserved $-67 \%$. The introduction of modern methods of treatment using biological agents in JIA has significantly increased the effectiveness of treatment, but it required the organization of additional information support for medical personnel.

Disclosure of Interest: None declared 
P256

Withdrawn

P257

Biological therapies in Juvenile Idiopathic Arthritis: are there any differences between categories?

L. Calvo Sanz, A. García Fernández, A. Briones Figueroa, Á. Andreu

Suárez, A. Boteanu

Rheumatology, Ramón y Cajal University Hospital, Madrid, Spain

Correspondence: A. García Fernández

Pediatric Rheumatology 2020, 18(Suppl 2):P257

Introduction: Juvenile Idiopathic Arthritis (JIA) is a heterogeneous group of pediatric diseases. Different response to biological treatment (BT) has been reported according to disease subtype.

Objectives: to analyze the presctiption and withdrawal of BT in JIA patients with focus on JIA category.

Methods: A retrospective observational study was conducted on JIA patients followed in a referal hospital and who had received at least one BT between 1999 and 2019.

Results: $130 \mathrm{JIA}$ patients were analyzed: 29 (22,4\%) were Oligoarticular Persistent (OligP), 22 (16,9\%) Enthesitis related Arthritis (ERA), 20 (15,4\%) Systemic (sJIA), 19 (14,6\%) Polyarticular RF- (PolyRF-), 14 (10,8\%) Polyarticular RF+(PolyRF+), 13 (10\%) Oligoarticular-Extended (OligE), 11 (8,4\%) Psoriatic Arthritis (APso) and $2(1,5 \%)$ Undifferentiated (Und).

The main characteristics are summarized in table 1.

The first line BT most frequently indicated was Etanercept up to $40 \%$ in all the categories except for ERA, where the most frequent BT was Adalimumab and SJIA, where the most frequent BT was Anakinra. The time between diagnosis and start of BT was different among the categories $(p=0,007)$. In the Und category, the time until BT was the shortest (median: 1 month), since both patients had coxitis, followed by APso [median: 9 months IQR(1-57)] and sJIA [median: 17,5 months IQR(0,3-146,8)].

The survival of the first BT was different among the categories $(p=0,006): 94,7 \%$ of the ERA continue receiving the first BT, followed by $76,2 \%$ of OligP and $50 \%$ of PolyRF+ and APso. Only $42 \%$ of sJIA continue on the first BT prescribed [up to $53,3 \%$ were TNF inhibitors (TNFi)]. The categories with less retention of the first BT were: OligE (25\%) ; PolyRF- $(27,3 \%)$ and Und (0\%). The most frequent cause of discontinuation, among these categories, was secondary failure.

In the survival analysis between categories, there were differences on OligP $(p=0,004)$, OligE $(p=0,042)$ and PolyRF- $(p=$ 0,017 ). Tocilizumab and Adalimumab were the BT with highest survival with regards to Infliximab, Etanercept, Rituximab (OligE, PolyRF-), Abatacept (OligE, PolyRF-) and Certolizumab (OligP). The survival rate of IL1 inhibitiors and IL6 inhibitiors was higher regarding to TNFi in sJIA patients $(p=0,013)$.

Conclusion: Taking into account JIA category is mandatory to choose $\mathrm{BT}$ and to understand the response and discontinuation of BT. OligE and PolyRF - showed a high rate of change of the first BT related to secondary failure of Etanercept and Infliximab when compared to Adalimumab and Tocilizumab, as described in the survival analysis. The category with the highest retention of the first BT was ERA. UND patients started sooner BT due to the presence of coxitis. In SJIA, IL1 inhibitors and IL6 inhibitors were superior to TNFi in the survival analysis, as reported in existing literature.

Disclosure of Interest: None declared
Table 1 (abstract P257). See text for description

\begin{tabular}{|c|c|c|c|c|c|c|c|c|}
\hline & OligP & ERA & SJIA & PolyRF- & PolyRF+ & OligE & Apso & UND \\
\hline \multicolumn{9}{|l|}{ Sex,n\% } \\
\hline M & $4(13,8)$ & $17(77,3)$ & $11(55)$ & $2(10,5)$ & $2(14,3)$ & $1(7,7)$ & $4(36,4)$ & $1(50)$ \\
\hline$F$ & $25(86,2)$ & $5(22,7)$ & $9(45)$ & $17(89,5)$ & $12(85,7)$ & $12(92,3)$ & $7(63,6)$ & $1(50)$ \\
\hline \multicolumn{9}{|l|}{$\begin{array}{l}\text { Age at } \\
\text { diagnosis }\end{array}$} \\
\hline me, IQR & $\begin{array}{l}4(2,6- \\
5)\end{array}$ & $\begin{array}{l}12(9,8- \\
15)\end{array}$ & $\begin{array}{l}7(3- \\
13)\end{array}$ & $8(2-13)$ & $\begin{array}{l}12(8,5- \\
15)\end{array}$ & $\begin{array}{l}3,5(2- \\
8,3)\end{array}$ & $\begin{array}{l}12(3- \\
15)\end{array}$ & 12,5 \\
\hline Uveitis,n\% & $12(41,4)$ & $7(31,8)$ & $0(0)$ & $3(15,8)$ & $0(0)$ & $3(25)$ & $2(18,2)$ & $1(50)$ \\
\hline ANA, n (\%) & $22(75,9)$ & & & $8(42,1)$ & $12(85,7)$ & $9(75)$ & $545,5)$ & \\
\hline ACPA, n (\%) & & & & & $9(64,3)$ & & & \\
\hline B27, n (\%) & & $18(81,8)$ & & & & & $3(27,3)$ & \\
\hline \multirow[t]{5}{*}{ BT lines: $n$} & $1 \mathrm{a}: 29$ & 1a: 22 & 1a: 20 & 1a: 11 & 1a: 14 & 1a:13 & $1 \mathrm{a}: 11$ & $1 \mathrm{a}: 2$ \\
\hline & $2^{\mathrm{a}:}: 11$ & $2^{\mathrm{a}}: 2$ & $2^{\mathrm{a}}: 10$ & $2^{\mathrm{a}}: 9$ & $2^{\mathrm{a}:} 7$ & $2^{\mathrm{a}}: 9$ & $2^{\mathrm{a}}: 5$ & $2^{\mathrm{a}}: 2$ \\
\hline & $3^{a}: 1$ & & $3^{\mathrm{a}}: 5$ & $3^{\mathrm{a}}: 6$ & $3^{\mathrm{a}}: 2$ & $3^{\mathrm{a}}: 2$ & $3^{\mathrm{a}:} 4$ & \\
\hline & & & $4^{\mathrm{a}}: 1$ & $4^{\mathrm{a}}: 3$ & $4^{\mathrm{a}}: 2$ & $4^{\mathrm{a}}: 1$ & $4^{\mathrm{a}}: 3$ & \\
\hline & & & $5^{a}: 1$ & & & $5^{\mathrm{a}}: 1$ & 5a: 1 & \\
\hline
\end{tabular}

P258

Comparison of different pharmaceutical preparations of colchicine in children with FMF: is colchicine opocalcium a good alternative? S. Turkucar', G. Otar Yener ${ }^{2}$, H. Adiguzel Dundar ', C. Acari ${ }^{3}$, B. Makay', S. Yuksel $^{4}$, E. Unnsal ${ }^{1}$

'Departments of Pediatrics, Pediatric Rheumatology, Dokuz Eylul University Faculty of Medicine, Izmir; ${ }^{2}$ Departments of Pediatrics, Pediatric Rheumatology, Sanliurfa Research and Training Hospital, Sanliurfa; ${ }^{3}$ Departments of Pediatrics, Pediatric Rheumatology, Malatya Research and Training Hospital, Malatya; ${ }^{4}$ Departments of Pediatrics, Pediatric Rheumatology, Denizli Pamukkale University, Faculty of Medicine, Denizli, Turkey

Correspondence: $\mathrm{S}$. Turkucar

Pediatric Rheumatology 2020, 18(Suppl 2):P258

Introduction: Familial Mediterranean fever (FMF) is the most common autoinflammatory disease among Mediterranean populations that can cause serious complications such as amyloidosis, without proper treatment (1). Colchicine is an antiinflammatory agent used for preventing FMF attacks and amyloidosis. Remarkable numbers of patients are non-responsive or intolerant to domestic drug colchicum dispert (2).

Objectives: This study aimed to compare the efficacy and side effects of colchicum dispert (CD) and colchicine opocalcium (CO) in children with FMF.

Methods: Twenty-nine children with FMF, who used CD at least six months initially, and another consecutive 6 months of $\mathrm{CO}$ were included. Clinical features, visual analog scale (VAS) for pain scores, exercise induced leg pain (EILP), and FMF severity scores with laboratory parameters were evaluated for both treatment periods. Bristol stool chart and number of stools per 24 hours were recorded for comparing gastrointestinal side effects.

Results: There were 13 female (43.4\%) and 16 male (56.6\%) patients and the mean age was $14 \pm 3.8$ years. Non-responsiveness to $C D$ was the major indication for switching preparation in 18 patients (62\%), and also intolerance was noted in 11 patients (38\%) due to gastrointestinal symptoms, i.e. diarrhea. 19 patients had homozygous M694V mutation (62 \%) and 27 patients had exon-10 mutation in at 
least one allele of MEFV gene (93\%). The median duration for CD treatment was higher than that for $\mathrm{CO}$.

The mean dosage of $\mathrm{CO}$ was higher $(1.71 \pm 0.44 \mathrm{mg} /$ day $)$ than CD $(1.49 \pm 0.41 \mathrm{mg} /$ day $)$, and the difference was statistically significant $(p<0.001)$. Besides, usage of CO (significantly higher dosage than $(D)$ showed beneficial effects on number and duration of attacks, VAS for pain and EILP scores, also on FMF severity scores, statistically significantly $(p<0.05$ for each parameters) (Table 1).

Usage of colchicine opocalcium was successful also in terms of controlling gastro7intestinal symptoms. Bristol stool chart questionnaire scores decreased from $5.62 \pm 1.56$ to $4.15 \pm 1.73$ points, and scores of daily stool number decreased from $0.46 \pm 0.894$ points to $0.03 \pm 0.118$. Decrease in gastrointestinal complaints were statistically significant $(p<0.05)$ (Table 1$)$.

There were 12 patients in which colchicine dose remained the same. We further analyzed this group separately to see if there was a bias in terms of change of dosage. The results were similar to the whole group analysis. There was a significant decrease of the clinical findings such as number of attacks at the last six months, average duration of attacks, FMF severity score and VAS scores of EILP $(p<0.05)$. Also, the laboratory findings such as NLR, ESR and CRP values were decreased following switch.

Conclusion: The patients with FMF in pediatric age group who have active disease and/or gastrointestinal complaints during the use of colchicum dispert, may benefit from colchicine opocalcium. It might be a valuable treatment option before considering biological agents.

Disclosure of Interest

None declared

Table 1 (abstract P258). Comparison of clinical results for both preparations $(n=29)$.

\begin{tabular}{llll}
\hline & $\begin{array}{l}\text { Colchicum } \\
\text { Dispert }\end{array}$ & $\begin{array}{l}\text { Colchicine } \\
\text { Opocalcium }\end{array}$ & $\begin{array}{l}\boldsymbol{p} \\
\text { value }\end{array}$ \\
\hline Duration of treatment (months) & $59.54 \pm 36.43$ & $26.39 \pm 16.64$ & $<0.001$ \\
Dosage (mg/day) & $1.49 \pm 0.41$ & $1.71 \pm 0.44$ & $<0.001$ \\
$\begin{array}{l}\text { Number of attacks at the last 6 } \\
\text { months }\end{array}$ & $4.83 \pm 2.1$ & $1.89 \pm 1.50$ & $<0.001$ \\
$\begin{array}{l}\text { Average duration of FMF attacks } \\
\text { (hours) }\end{array}$ & $63.98 \pm 25.84$ & $44.41 \pm 21.81$ & $<0.001$ \\
$\begin{array}{l}\text { ElLP (attack-free period) } \\
\text { FMF severity score }\end{array}$ & $7.36 \pm 1.43$ & $2.84 \pm 1.77$ & $<0.001$ \\
Bristol stool scale & $8.88 \pm 2.08$ & $6.52 \pm 1.83$ & $<0.001$ \\
Number of stools/24 h & $5.62 \pm 1.56$ & $4.15 \pm 1.73$ & 0.044 \\
\hline
\end{tabular}

\section{P259}

Anti-adalimumab antibodies detection using a novel peptidebased assay in a cohort of pediatric patients with chronic rheumatic disorders: a pilot study

E. Marrani ${ }^{1}$, H. Rusche ${ }^{2}$, F. Terzani ${ }^{3}$, E. Peroni ${ }^{2}$, F. Real-Fernandez ${ }^{4}, \mathrm{O}$.

Monasson ${ }^{2}$, R. Ponti ${ }^{5}$, G. Simonini, A. M. Papini ${ }^{3}$, P. Rovero ${ }^{4}$

${ }^{1}$ Rheumatology Unit, Anna Meyer Children Hospital, Firenze, Italy;

2Peptlab@CY and Laboratory of Chemical Biology, Cergy-Paris University, Cergy-Pontoise, France; ${ }^{3}$ PeptLab, Dep. Chemistry, University of Florence, sesto fiorentino; ${ }^{4}$ PeptLab, Dep. NEUROFARBA, University of Florence, firenze; ${ }^{5}$ Pediatric Department, University of Udine, Udine;

${ }^{6}$ Rheumatology Unit, AOU Meyer, NEUROFARBA, University of Florence, firenze, Italy

Correspondence: E. Marrani

Pediatric Rheumatology 2020, 18(Suppl 2):P259
Introduction: Immunogenicity and development of anti-drug antibodies have been associated with treatment failure and adverse events during biologic treatment. Anti-drug antibodies (ADAs) have been reported in $21 \%$ of Juvenile Idiopathic Arthritis patients treated with Adalimumab. However, their role in reducing adalimumab efficacy is still debated due to conflicting results. No study has been directed toward identification of neutralizing ADAs in paediatric rheumatic disorders.

Objectives: Aim of our study was to detect ADAs, along with their clinical relevance, using a new theranostic peptide-base assay in a cohort of children with inflammatory chronic diseases on Adalimumab treatment.

Methods: Six candidate Adalimumab derived peptide antigens (HCCDR1, HC CDR2, HC CDR3, LC CDR1, LC CDR 2, LC CDR3) have been developed and optimized to be tested. Their performance has been compared with commercial ELISA kit and a SPR-based optical assay (Biacore ${ }^{\circledR}$ ). Assays have been performed in sera of a cohort of children receiving Adalimumab due to an inflammatory chronic disease. Mean age, disease duration, concomitant treatment with methotrexate (MTX), ANA positivity, disease activity parameters and scores at the time of ADA determination have been recorded. Chisquare, and Fisher exact test were used to compare data. Pearson's and Spearman's correlation tests were used to determine correlation coefficients for entered variables.

Results: Eighteen (14 F, median age 12.6, range 3.8-16, yrs) patients were enrolled: 16 affected by Juvenile Idiopathic Arthritis, 7 of whom complicated by JIA -associated chronic uveitis, and 2 patients affected by chronic idiopathic uveitis. Peptide assay revealed ADAs in 8 children, Biacore in 6, commercial Elisa in 5. Of note, we found total concordance among the 3 tests just in 2 patients. No significant correlation has been proven among the 3 ADA determinations. Biacore and ELISA determination showed significant concordance $\left(r_{s}\right.$ : $0.72, p<0.006)$. The presence of HC CDR3 and LC CDR 3 resulted significantly correlated with disease activity $\left(r_{\mathrm{s}}: 0.57, \mathrm{p}<0.05\right)$, and, inversely, with disease remission on treatment $\left(r_{s}=-0.523, p<0.05\right)$. No patient experienced severe adverse events and no correlation with ADAs has been revealed

Conclusion: In chronic rheumatic disorders, novel reliable methods are urgently required to guide clinical decision and support decisions about switching within or between drugs in refractory children. The 3 different methods, since based on different antigenic probes, detect different antibody populations. The present peptide-based assays might contribute to identify neutralizing ADAs in patients treated with Adalimumab. Further validation in larger cohort is required.

\section{Disclosure of Interest}

None declared

\section{P260}

TNF-inhibitors therapy in 13 patients with non-bacterial multifocal osteomyelitis: lessons from real clinical practice of the single center

I. Nikishina, S. V. Arsenyeva, M. Kaleda, D. Alekseev, T. Pachkoria, O. Kostareva, O. Borodacheva, S. Salugina

Paediatric, V.A. Nasonova Scientific Research Institute of Rheumatology, Moscow, Russian Federation

Correspondence: I. Nikishina

Pediatric Rheumatology 2020, 18(Suppl 2):P260

Introduction: Non-bacterial multifocal osteomyelitis (NBO) is a rare polygenic autoinflammatory disease, which is difficult to diagnose and treat. Because of combination of bone lesions with arthritis and/ or axial skeleton damage in most cases the diagnosis of juvenile idiopathic arthritis (JIA) or juvenile ankylosing spondylitis (JAS) may be establish as a concurrent diagnosis, so this allows to legal use of Biologics (BA) for the treatment. 
Objectives: To analyze the single center experience of clinical and laboratory features of multifocal NBO in patients (pts) who were treated by BA for the last 8 years.

Methods: The study involved a retrospective cohort of multifocal NBO pts treated by different BA in our clinic from 2013 to 2020. All of them underwent standard rheumatological examination. In order to examine all localizations of the bone damage, a scintigraphy and/ or "whole body" MRI scan was performed.

Results: Among the whole group of pts with NBO $(n=40)$ we identified 13 pts treated by BA (TNF-inhibitors only). The majority were girls ( $n=9$, $69 \%$ ). Age at disease onset was 10.2 years in average (Me 10.2 range 1.3-16.5). For legal reason of BA administration, we classified our patients according to rheumatological features as JIA or JAS. 7 pts had JIA (5 girls), 6 pts had JAS (4 girls). Among 13 pts 9 had oligoarthritis (69\%), 4 had polyarthritis of low limbs (hip, knee, ankle). Axial involvement was represented by active erosive sacroiliitis with deep bone marrow edema on MRI scan in 9 pts (69\%), active spondylitis of several bodies in thoracic spine - in 2; erosive arthritis with partial ankyloses of facet joints of neck in 3 pts, multiple syndesmophytes in 1 girl. We found that definite axial lesions in NBO developed in very young children (in 2 y.old at minimum), much earlier than in "idiopathic" JAS. HLA B27 was presented in 5 pts (39\%), 5 pts had ANA in high titer (all of those HLA B27-negative). The pts had bone lesions in different parts of skeleton: vertebral bodies - 5 pts, clavicle - 1, sternum, ribs -1 , extremities bones, metaphysic mostly (tibial, fibular -7 pts), sacroiliac region - 4 pts. Extraskeletal manifestations were observed in 3 pts, one in each condition - uveitis, psoriasis pustulosus, acnae conglobate. In a girl with very severe course of disease, not responded to any therapy NBO was combined with familial Mediterranean fever. High level of laboratory activity were detected before biologics in 10 pts (77\%): ESR acceleration up to $60 \mathrm{~mm} / \mathrm{h}$, increase of CRP - up to $80 \mathrm{mg} / \mathrm{l}$. Treatment included NSAIDS (all), methotrexate (7 pts), sulfasalazine (6 pts, but it was withdrawn in all pts), bisphosphonates (1 pt), prednisolone (3 pts). Because of high activity of NBO with appearance of new bone lesions and persistent arthritis TNF inhibitors were administrated: etanercept in 10 pts, adalimumab - 4 (2 as first line, 2 second line), golimumab - 1. At the start of BA the average age was 13.7 years (range 7.2-17.9); mean disease duration was 3,4 years (range 0.3-8.1). There were 2 cases of withdrawals. Due to inefficacy etanercept was switched to adalimumab. Disease activity decreasing was reached in the most of the patients ( 12 from 13). Among them 2 pts developed the whole remission with resolving of active arthritis and bone marrow edema spots. Skin lesions (psoriasis pustulosis and acnae conglobate) were significantly improved. There were no adverse events during the TNF therapy.

Conclusion: Our experience of the therapy with TNF inhibitors in patients with high NBO activity has shown that this is a good and safe therapeutic option that is expected to prevent progression and bone destruction.

Disclosure of Interest

None declared

\section{P261}

Safety on the use of bisphosphonates in paediatrics

S. Odorico ${ }^{1}$, E. Jeziorski ${ }^{2}$, F. Rivier ${ }^{3}$, T.-A. Tran ${ }^{1}$

${ }^{1}$ Pediatrics, Nîmes University Hospital, Nîmes; ${ }^{2}$ Pediatrics; ${ }^{3}$ Pediatric

Neurology, Montpellier University Hospital, Montpellier, France

Correspondence: T.A. Tran

Pediatric Rheumatology 2020, 18(Suppl 2):P261

Introduction: The use of bisphosphonates has increased in paediatrics in the last twenty years.

Objectives: The study describes safety of bisphosphonate therapy in children in Montpellier and Nimes University Hospitals, France.

Methods: In our retrospective study, all patients treated with bisphosphonates between January 2012 and August 2018 were included. The main endpoint was safety using adverse events (AE) and serious adverse events (SAE) reported in medical files.

Results: 120 children, median age [IQR $25 \%>75 \%$ ] of 13 years [1 month-18 years], with osteogenesis imperfecta (22), secondary osteoporosis (77: 63 immobility, 5 nutritional diseases, 7 corticosteroids, 1 sickle cell anaemia, 1 growth hormone deficiency) and nonfragility bone disorders (21: 10 fibrous dysplasia, 4 bone cysts and tumour, 7 inflammatory bone diseases), were included: 29 using zoledronate, 91 using pamidronate. The median duration of treatment [IQR 25\%>75\%] was 12 months [6-27 months]. AE were reported for $71.7 \%$ of patients, most within 24 to 48 hours after the first or second injection: flu-like symptoms (57.5\%), hypocalcaemia (37.5\%) and hypophosphatemia (20\%). Underweight patients (body mass index < $18.5 \mathrm{~kg} / \mathrm{m}^{2}$ ) accounted for $50 \%$ of hypocalcaemia. The frequency of all the AE not significantly decreased with the reduction of the first dose. Only one serious hyponatremia occurred corresponding to a patient with renal failure before treatment.

Conclusion: Our results were similar to those previously published: bisphosphonates are safe for osteoporosis in children. In the literature, SAE are very rare in children, being limited to anecdotal osteopetrosis in cases of higher doses and long-term treatment, and delayed bone healing. Anecdotal osteonecrosis of the jaw in adults has never been described in children. The use of bisphosphonates beforehand requires dietary measures (vitamin $\mathrm{D}$ and calcium supplementation). Furthers systematic collection on efficacy and safety parameters for each Bisphosphonates drug should confirm these data.

\section{Disclosure of Interest}

None declared

\section{P262}

A real-life study of effectiveness and tolerability of adalimumab biosimilar in JIA-A single centre experience

V. Choida ${ }^{1,2}$, S.-A. Yeoh ${ }^{2}$, C. Fisher ${ }^{1,2}$, M. Leandro ${ }^{1,2}$, D. Sen ${ }^{1,2}$, C. Ciurtin 1,2

${ }^{1}$ Centre for Adolescent Rheumatology Versus Arthritis, University College London; ${ }^{2}$ Rheumatology, University College London Hospitals, London,

United Kingdom

Correspondence: $\mathrm{V}$. Choida

Pediatric Rheumatology 2020, 18(Suppl 2):P262

Introduction: The use of biosimilars in rheumatology has increased significantly over the last 5 years and has resulted in considerable cost savings. Objectives: To assess the effectiveness and tolerability of the Adalimumab biosimilar ABP 501 in patients with JIA.

Methods: A database of patients prescribed Adalimumab in our service has been screened to identify patients with JIA, who switched from the originator to the biosimilar. Only patients who had a clinical review since they had started the biosimilar were included. A paired-samples t-test was conducted to compare the number of active joints at the clinic appointment before and after the initiation of the biosimilar treatment. The frequency and type of side effects, the clinical response and the number of patients who switched back to the originator have been collected.

Results: Sixty-one patients who switched to the biosimilar ABP 501 between February 2019 and February 2020 were included. They were comprised of 30 enthesitis-related arthritis (ERA), 13 polyarthritis, 9 oligoarthritis, 6 psoriatic and 3 systemic JIA patients. Their baseline characteristics and outcomes are summarised in Table. The mean duration of follow-up after the switch to biosimilar was 10 months (range 2-23). Eleven patients (18\%) reported side effects; the most common side effect $(n=7,63.6 \%)$ was injection site reactions and the remaining 4 consisted of anaphylaxis, druginduced lupus, dizziness and bone pain, respectively. Seven patients (11.5\%) reverted to the Adalimumab originator, 4 as a result of side effects, 3 because of ineffectiveness and one patient for both reasons. In addition, 3 patients were changed to a different biologic, one patient due to allergy to both the originator and biosimilar and the other two patients had active disease on the originator and biosimilar Adalimumab. Two patients stopped the biosimilar and remained off any biologic, in the first case this was due to a side effect and in the second case it was patient's choice. On the whole, $78.7 \%$ of patients had remained on ABP 501 at their last visit. There was no significant difference in the active joint count before the biosimilar was started (mean 0.55+/-1.11) and after the switch (mean 0.6+/-1.59), ( $p=$ 0.855). 


\begin{tabular}{ll}
\hline Age, mean (range) & $21(15-36)$ \\
Female, $\mathbf{n}(\%)$ & $40(65.6 \%)$ \\
Disease duration in years, mean (range) & $11.5(0-29)$ \\
Prior exposure to biologics other than the originator, $\mathbf{n}(\%)$ & $25(40.9 \%)$ \\
Concomitant Methotrexate, $\mathbf{n}(\%)$ & $31(50.8 \%)$ \\
Side effects, $\mathbf{n}$ (\%) & $11(18 \%)$ \\
Switched back to originator & $5(45.4 \%)$ \\
Remained on biosimilar & $4(36.4 \%)$ \\
Changed to different biologic & $1(9.1 \%)$ \\
Not on biologic & $1(9.1 \%)$ \\
Patient-reported reduced effectiveness, $\mathbf{n}(\%)$ & $6(9.8 \%)$ \\
Switched back to originator & $3(50 \%)$ \\
Remained on biosimilar & $3(50 \%)$ \\
\hline
\end{tabular}

Conclusion: During a mean follow-up period of 10 months, $78.7 \%$ of JIA patients who switched to the Adalimumab biosimilar have remained on treatment with no significant difference in their disease activity. Overall, the tolerability and effectiveness of the Adalimumab biosimilar is acceptable, but $11.5 \%$ of patients required to be switched back to the originator.

\section{Disclosure of Interest}

None declared

\section{P263}

Matched controlled surveillance of golimumab treatment for polyarticular Juvenile Idiopathic Arthritis-an interim analysis A. Klein ${ }^{1}$, A. Zimmer ${ }^{1}$, K. Minden ${ }^{2}$, A. Hospach³ , F. Weller-Heinemann ${ }^{4}$, J. Kuemmerle-Deschner $^{5}$, M. Fasshauer ${ }^{6}$ I. Foeldvari ${ }^{7}$, M. Hufnage ${ }^{8}, D$. Foell ${ }^{9}$, P. T. Oommen ${ }^{10}$, N. Hofmann ${ }^{11}$, F. Dressler ${ }^{12}$, A. Helling-Bakki ${ }^{13}, H$. E. Koessel ${ }^{14}$, S. Mrusek ${ }^{15}$, D. Windschall ${ }^{16}$, N. Brueck ${ }^{17}$, G. Horneff $^{1}$

${ }^{1}$ ASKLEPIOS, Sankt Augustin; ${ }^{2}$ DRFZ, Berlin; ${ }^{3}$ Olgahospital, Stuttgart; ${ }^{4}$ Prof. Hess Kinderklinik, Bremen; ${ }^{5}$ University Tuebingen, Tuebingen; ${ }^{6}$ St Georg, Leipzig; ${ }^{7}$ Centre of ped. Rheumatology, Hamburg; ${ }^{8}$ University, Freiburg; ${ }^{9}$ University, Muenster; ${ }^{10}$ University, Duesseldorf; ${ }^{11}$ klinikum Bamberg, Bamberg; ${ }^{12} \mathrm{MHH}$, Hannover; ${ }^{13}$ University, Heidelberg; ${ }^{14}$ Klinikum Brandenburg, Brandenburg; ${ }^{15}$ Private office, Badedn-Baden; ${ }^{16}$ St Josef Stift, Sendenhorst; ${ }^{17}$ University, Dresden, Germany

Correspondence: G. Horneff

Pediatric Rheumatology 2020, 18(Suppl 2):P263

Introduction: Golimumab (GOL) is approved for polyarticular juvenile idiopathic arthritis $(\mathrm{p} / \mathrm{A})$ in patients of $\geq 2$ years but long-term safety data are limited.

Objectives: Prospective monitoring of long-term safety and effectiveness of GOL in routine care using the BIKER-registry.

Methods: Baseline demographics, clinical characteristics, disease activity and safety parameters were compared to a contemporary 1:2 matched control cohort using alternative TNF inhibitors or methotrexate without exposure to a biologic. Efficacy outcomes were JADAS10, joint counts and functional status. Safety assessments were based on adverse events (AE) reports.

Results: In this ongoing study, 65 pts initiating GOL were matched to 130 with alternative TNFi and 65 biologic-naïve pts. Pts starting GOL had a longer disease duration $(p<0.0001)$ and use of $\mathrm{GOL}$ was significantly more often second line ( $84.6 \%$ vs $22.3 \%, p<0.0001)$ and thus disease activity was lower at baseline. Pts in the GOL cohort used less corticosteroids, otherwise patients were comparable with pts treated with other TNFi (Table 1).

In GOL treated ps a marked clinical response was noted at 6 months and beyond, indicating the effectiveness of $\mathrm{GOL}$ in the treatment of pJIA. A significant decrease of the mean JADAS 1011.3 to 5.3 ( $p=$ 0.0008 ) after 6 months of treatment was observed, as well as JIA ACR $30 / 50 / 70 / 90$ response rates of 61/59/42/29\%. JADAS remission and minimal disease activity was observed in $27 \%$ and $53.7 \%$ after 6 months and in 39\% and 54\% after 12 months of treatment.

Rates of $A E, S A E$ and infectious $A E$ were comparable in the $G O L$ cohort (87.5/100PY, 3.4/100PY and 11.1/100PY), the alternative TNFi cohort (92.3/100PY, 2.9/100PY and 9.7/100PY) and the MTX only cohort (121.2/100PY, 2.1/100PY and 18.5/100PY). SAE reported in the GOL cohort were flares of uveitis and of JIA (each 1) and fibromyalgia syndrome (1). SAE reported in the alternative TNF cohort was two serious infections (both influenza), one knee ligament injury, one flare of arthritis and one hyperventilation. No case of pregnancy, malignancy or death was reported.

Conclusion: Golimumab seems an effective in treatment of pJIA. Tolerability was acceptable and comparable to alternative TNFi or MTX. Recruitment to the project is ongoing.

Trial registration identifying number: EUPAS20781

Disclosure of Interest

None declared

Table 1 (abstract P263). Baseline parameters and adverse events. Comparison of GOL cohort with $1^{\text {st }}$ Other TNFi cohort and $2^{\text {nd }}$ MTX cohort. \# t-test or c2-test as appropriate. SD standard deviation, RF rheumatoid factor, bDMARD biologic disease modifying antirheumatic drug

\begin{tabular}{|c|c|c|c|c|}
\hline & $\begin{array}{l}\mathrm{GOL} \\
\mathrm{N}=65\end{array}$ & $\begin{array}{l}\text { Other TNFi } \\
\mathrm{N}=130\end{array}$ & $\begin{array}{l}\text { MTX } \\
\mathrm{N}=65\end{array}$ & $\begin{array}{l}\text { p\# } \\
\text { GOL vs } \\
\text { TNFi/GOL } \\
\text { vs. MTX }\end{array}$ \\
\hline $\begin{array}{l}\text { Disease duration, } \\
\text { mean (SD), years }\end{array}$ & $7.0(4.4)$ & $4.2(3.8)$ & $1.2(2.2)$ & $\begin{array}{l}<0.001 / \\
<0.001\end{array}$ \\
\hline $\begin{array}{l}\text { RF - Poly/RF+ } \\
\text { Poly/ext Oligo/ } \\
\text { PSA, n(\%) }\end{array}$ & $\begin{array}{l}32(49.2) / \\
6(9.2) / \\
24(36.9) / \\
3(4.6)\end{array}$ & $\begin{array}{l}63(48.5) / \\
20(15.4) / \\
44(33.8) / 3(2.3)\end{array}$ & $\begin{array}{l}43(66.2) / \\
12(18.5) / \\
9(13.8) / 1(1.5)\end{array}$ & ns \\
\hline $\begin{array}{l}\text { Pretreatment } \\
\text { bDMARD n(\%) }\end{array}$ & $56(86.2)$ & $29(22.3)$ & 0 & $\begin{array}{l}<0.001 / \\
<0.001\end{array}$ \\
\hline $\begin{array}{l}\text { Concomitant } \\
\text { steroids, n(\%) }\end{array}$ & $9(13.8)$ & $33(25.4)$ & $32(49.2)$ & $\mathrm{ns} /<0.001$ \\
\hline $\begin{array}{l}\text { Active joints, } \\
\text { mean (SD) }\end{array}$ & $4.3(3.8)$ & $5.2(5.8)$ & $10.1(6.7)$ & $\mathrm{ns} /<0.001$ \\
\hline $\begin{array}{l}\text { JADAS10, mean } \\
\text { (SD) }\end{array}$ & $11.3(5.6)$ & $12.2(6.0)$ & $17.1(5.4)$ & $\mathrm{ns} /<0.001$ \\
\hline $\mathrm{AE}, \mathrm{n}$ (rate/100PY) & $71(87.5)$ & $161(92.3)$ & $59(121.2)$ & ns \\
\hline $\begin{array}{l}\text { SAE, n (rate/ } \\
\text { 100PY; 95\%Cl) }\end{array}$ & $3(3.4 ; 1.2-8.3)$ & $5(2.9 ; 1.2-6.9)$ & $1(2.1 ; 0.3-14.6)$ & ns \\
\hline $\begin{array}{l}\text { Serious infections, } \\
\text { n (rate/100PY; } \\
95 \% \mathrm{Cl})\end{array}$ & 0 & $2(1.1 ; 0.3-4.6)$ & 0 & na \\
\hline
\end{tabular}




\section{P264}

Methotrexate intolerance: children vs adults

U. Kaya Akca', B. Farisogullari², G. K. Yardimci², S. Demir', E. Sag ${ }^{1}$,

E. Atalay ${ }^{1}$, M. Kasap Cuceoglu', L. Kilic ${ }^{2}$, S. Ozen' ${ }^{1}$, Y. Bilginer ${ }^{1}$

'Division of Pediatric Rheumatology, Department of Pediatrics; ${ }^{2}$ Division

of Rheumatology, Department of Internal Medicine, Hacettepe University Faculty of Medicine, Ankara, Turkey

Correspondence: $U$. Kaya Akca

Pediatric Rheumatology 2020, 18(Suppl 2):P264

Introduction: Methotrexate (MTX) is one of the most commonly used disease-modifying anti-rheumatic drug in rheumatology practice. It has some side effects that can impair quality of life. The most common of them is associated with the gastrointestinal tract.

Objectives: The aim of the study is to evaluate and compare the frequency of methotrexate intolerance in adult and pediatric patients.

Methods: Patients with rheumatologic diseases followed in Hacettepe University Pediatric Rheumatology and Rheumatology departments who used oral or parenteral methotrexate for at least 3 months were included in the study. Methotrexate intolerance was assessed using 'Methotrexate Intolerance Severity Score (MISS) questionnaire. The MISS questionnaire consisted of 5 parts: abdominal pain, nausea, vomiting, fatigue and behavioral symptoms. The patients scored the severity of each symptom separately; 0: no symptoms, 1: mild symptoms, 2: moderate symptoms, 3: severe symptoms. A total score of 6 or more was defined as MTX intolerance. Visual analogue scale (VAS) ranging from $0 \mathrm{~cm}$ to $10 \mathrm{~cm}$ was performed to each patient concurrently with the MISS questionnaire. In the pediatric patient group, MISS questionnaire and VAS assessment were applied to both patients and families.

Results: A total of 100 patients, 50 of whom were children, enrolled in the study. The mean age for children and adults were $11.78( \pm 3.4)$ and 52.9 ( \pm 11.8) respectively. The most frequent diagnosis of patients was juvenile idiopathic arthritis (78.0\%) in children and rheumatoid arthritis in adults (68.0\%). The mean MTX dose in adults and pediatric group was $12.5( \pm 3) \mathrm{mg}$ vs $14.5( \pm 3.6) \mathrm{mg}$ (p: 0.004).

The prevalence of MTX intolerance in children and adults were $66.0 \%$ ( $n: 33)$ and $14.0 \%(n: 7)$ respectively. The mean MISS score in the pediatric group was higher compared with the adults $(12.4 \pm 9.4$ vs $1.84 \pm 4.5, p<0.001)$. Similarly, the mean VAS scores were higher in pediatric group $(1.2 \pm 2.4$ vs $4.2 \pm 3.2(p<0.001))$. There was a strong correlation between MISS and VAS scores between family and child evaluations ( $p<0.01, r=0.95 / p<0.01, r=0.94)$.

Abdominal pain, nausea, vomiting and behavioral symptoms were observed more frequently in children compared to adults. The rate of subcutaneous use of MTX was $74.0 \%$ in pediatric patients and $4.0 \%$ in adult patients. Of 61 patients receiving oral MTX, $17(27.8 \%)$ experienced MTX intolerance, whereas $23(58.9 \%)$ of 39 patients receiving parenteral MTX experienced intolerance to the drug symptoms $(p=0.001)$. Complaints were started after the first dose in $2 / 7$ in adults and $8 / 33$ in children. MTX intolerance decreased with increasing folic acid dose $(r=-0.26, p=0.007)$.

MTX intolerance developed in 16 of 21 pediatric patients who were informed about side effects of drug by their families. In addition, 17 of 45 patients (\%37.7) who read the drug prospectus had MTX intolerance. Conclusion: Methotrexate intolerance was higher in childhood. Folic acid supplementation should be recommended for patients taking MTX treatment.

\section{Disclosure of Interest}

None declared

\section{P265}

Rates of psoriasis de-novo in patients with Juvenile Idiopathic Arthritis preceding anti-tnf therapy: a single-center observational study

N. Tsurikova, E. Ligostaeva, V. Avdeenko, N. Kobzeva, I. Tsiganok

Rostov Regional Children Hospital, Rostov-on-don, Russian Federation

Correspondence: $\mathrm{N}$. Tsurikova

Pediatric Rheumatology 2020, 18(Suppl 2):P265
Introduction: nowadays there are known cases of psoriasis de-novo in patients with juvenile idiopathic arthritis(JIA) receiving therapy with TNF-alpha inhibitors.

Objectives: to evaluate frequency of psoriasis de-novo in patients with JIA receiving TNF-alpha inhibitors.

Methods: this prospective study included 73 patients with different types of JIA (persistent or extended oligoarthritis, RF-negative polyarthritis, enthesitis-related arthritis and undifferentiated arthritis) who were treated with TNF-alpha inhibitors.

Children with psoriatic JIA were excluded from this study. All patients had no previous clinical manifestations of psoriasis. TNF-induced psoriasis had been identified as a case of psoriasis development after initiation of TNF-alpha inhibitors.

The average age of patients was $11.7 \pm 3.7$ years, the average duration of the disease was $4.1 \pm 2.1$.

24 (33\%) children received Adalimumab(ADA), 49(67\%) - Etanercept (ETA). The average duration of ADA therapy was $2.1 \pm 0.7$ years. The average duration of ETA therapy was $2.9 \pm 1.1$ years. All children received methotrexate (the average duration of methotrexate therapy was $3.4 \pm 0.7$ years.

Presence of HLA B27 antigen had been detected in $14(19 \%)$ patients: $9(64 \%)$ boys, $5(36 \%)$ girls.

Antinuclear factor (ANF) had been detected in 38 (52\%) patients: $31(81 \%)$ girls, $7(19 \%)$ boys.

Results: 3(4\%) out of 73 patients were diagnosed with psoriasis denovo. One patient was treated with ADA (a girl with undifferentiated arthritis who had positive HLA-B 27, ANF and family history of psoriasis - her grandmother had psoriasis), 2 patients were treated with ETA (both female, one patient had undifferentiated arthritis, the other had enthesitis-related arthritis; both patients had positive HLA - B 27 and ANF negative).

2 patients achieved significant improvement after changing TNFalpha inhibitor (1-ADA, 1-ETA), 1 patient (was treated with ETA) had significant improvement after discontinuation of biological therapy. Conclusion: This single-center observational study demonstrates the possibility of developing psoriasis de-novo in patients with JIA receiving TNF-alpha inhibitors.

Although more extensive research is needed, our data suggest that discontinuing the TNF-alpha inhibitor or switching to another TNFalpha inhibitor in patients with psoriasis de-novo should be considered as a treatment strategy in such cases.

Disclosure of Interest

None declared

P266

The possibility of increasing the intervals between injections of tnf-alfa inhibitors in children with Juvenile Idiopathic Arthritis: a single-center observational study

N. Tsurikova, E. Ligostaeva, V. Avdeenko, N. Kobzeva, I. Tsiganok

Rostov Regional Children Hospital, Rostov-on-don, Russian Federation

Correspondence: $\mathrm{N}$. Tsurikova

Pediatric Rheumatology 2020, 18(Suppl 2):P266

Introduction: Nowadays many patients who are suffering from juvenile idiopathic arthritis (JIA) are treated with TNF-alpha inhibitors. The question of the duration of therapy with TNF-alpha inhibitors in children receiving TNF-alpha inhibitors and achieving remission on it is relevant.

Objectives: To evaluate the efficacy of therapy with TNF-alpha inhibitors in children suffering from JIA on different therapy regimens after 2 years of treatment with TNF-alpha inhibitors and having a remission for at least one year, which was achieved after prescribing TNFalpha inhibitors.

Methods: This single-center observational study included 44 children suffering from JIA receiving anti-TNF therapy for 2 consecutive years in standard dose and standard scheme and reached remission on it

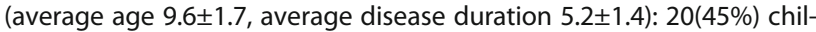
dren were treated with adalimumab(ADA), 24(55\%) - etanercep$\mathrm{t}(\mathrm{ETA})$; all children received methotrexate (the average duration of methotrexate therapy was $4.7 \pm 0.4$. The first group included children 
who after 2 years of therapy with TNF-alpha inhibitors, was continued this therapy at the standard dose and standard scheme (10 children treated with ADA and 10 children treated with ETA). The comparison group included children who after 2 years of therapy with TNF-alpha inhibitors was continued this therapy at the standard dose, but the intervals between injections of TNF-alpha inhibitors were doubled (this group included 10 children treated with ADA and 14 children treated with ETA). Effectiveness was determined by using the Pediatric American College of Rheumatology Criteria (PedACR) and the Juvenile Disease Activity Score 27 (JADAS-27) at the time of inclusion in the study and after 12, 24, 36 and 52 weeks of therapy. The criteria for exclusion from the study was absent $30 \%$ improvement according to PedACR.

Results: In both groups at the time of inclusion all patients had a $90 \%$ improvement according to PedACR criteria, JADAS 27 less than 1.

After 12 weeks in the first group and in the comparison group JADAS $27-0.75(0.1 ; 1.5)$ and $0.7(0.2 ; 1.5)$, respectively; $100 \%$ of children in the first group had $90 \%$ improvement according to PedACR criteria, in the comparison group - PedACR 70/90 improvement registered in $24(100 \%) / 22(90 \%)$ children.

After 24 weeks in the first group JADAS 27-0.75(0.1; 1.5), in the comparison group - 2(1.5;5). In the first group 100\% of children had $90 \%$ improvement according to PedACR criteria, in the comparison group - 2(8\%) children didn't achieved 30\% improvement according to the PedACR criteria (1 child was treated with ADA, 1-ETA), PedACR 70/90 improvement registered in 22(92\%)/17 (71\%) patients.

After 36 weeks in the first group JADAS $27-1.5(0.5 ; 2.5)$, in the comparison group- $2(1.5 ; 2.5)$. In the first group $18(90 \%)$ children maintained a $90 \%$ improvement PedACR criteria, $2(10 \%)$ children ( 1 were treated with ADA, 1 - ETA) didn't reached 30\% improvement according to the PedACR criteria; in the comparison group PedACR 70/90 improvement registered in 22(92\%)/19(79\%) patients.

After 52 weeks JADAS 27 in the first group - 0.75(0.1; 1.5), in the comparison group - $1(0.5 ; 2.0)$. In the first group $18(90 \%)$ children have a $90 \%$ improvement in PedACR criteria, in the comparison group - PedACR 70/90 improvement registered in 22(92\%) /20(83\%) patients.

Conclusion: According to the obtained data, it can be concluded that after 2 years of therapy with TNF-alpha inhibitors at the standard dose and standard scheme and if there is a remission on this therapy for at least a one year, it is possible to correct therapy with TNF-alpha inhibitors in the form of doubling the intervals between the injections of TNF-alpha inhibitors.

\section{Disclosure of Interest}

None declared

\section{P267}

Matched controlled surveillance of tocilizumab treatment for polyarticular Juvenile Idiopathic Arthritis-an interim analysis G. Horneff' ${ }^{1}$, A. Hospach², F. Weller-Heinemann ${ }^{3}$, J. KuemmerleDeschner ${ }^{4}$, M. Fasshauer ${ }^{5}$, K. Minden $^{6}$, I. Foeldvari ${ }^{7}$, C. Rietschel ${ }^{8}, T$. Schwarz ${ }^{9}$, R. Trauzeddel ${ }^{10}$, M. Hufnagel ${ }^{11}$, D. Foell ${ }^{12}$, R. Berendes ${ }^{13}$, P. T. Oommen $^{14}$, F. Dressler ${ }^{15}$, A. Helling-Bakki ${ }^{16}$, A. Klein ${ }^{1}$

${ }^{1}$ ASKLEPIOS, Sankt Augustin; ${ }^{2}$ Olgahospital, Stuttgart; ${ }^{3}$ Prof. Hess Kinderklinik, Bremen; ${ }^{4}$ University Tuebingen, Tuebingen; ${ }^{5}$ St Georg , Leipzig; ${ }^{6}$ DRFZ, Berlin; ${ }^{7}$ Centre of ped. Rheumatology, Hamburg;

${ }^{8}$ Clementinen Hospital, Frankfurt; ${ }^{9}$ University, Wuerzburg; ${ }^{10}$ Helios Kliniken, Berlin; ${ }^{11}$ University, Freiburg; ${ }^{12}$ University, Muenster; ${ }^{13}$ St Marien Hospital, Landshut; ${ }^{14}$ University, Duesseldorf; ${ }^{15} \mathrm{MHH}$, Hannover;

${ }^{16}$ University, Heidelberg, Germany

Correspondence: $\mathrm{G}$. Horneff

Pediatric Rheumatology 2020, 18(Suppl 2):P267

Introduction: Tocilizumab (TOC) is approved for treatment of polyarticular JIA. Data out of clinical practice are limited.

Objectives: Long-term surveillance of patients newly initiating TOC treatment for at least 5 years compared to a cohort of patients newly initiating a comparator biologic using the BIKER-registry.

Methods: Baseline demographics, clinical characteristics and disease activity, efficacy and safety parameters were compared. Efficacy outcomes were JADAS10, joint counts and functional status Safety was assessed by adverse events (AE) reports.

Results: 161 patients with 161 matched controls have been recruited. Patients starting on TOC were older at treatment start (12.1 vs. 10.1 years $(y) ; p<0.0001)$ and had a longer disease duration $(p<$ 0.0001 ). TOC was significantly more often a second line biologic $(p<$ $0.0001)$. Baseline JADAS10 (17+/-10 vs 15+/-6), CHAQ-DI (0.63+/-0.63 vs $0.65+/-0,64)$, ESR $18+/-15 \mathrm{~mm} / \mathrm{h}$ vs. $21+/-21 \mathrm{~mm} / \mathrm{h}$ and active joint counts $(7+/-7$ vs. $6+/-5)$ were comparable.

Upon TOC a substantial response with a significant reduction in JADAS 10 from 16.8 to 3.4 ( $p<0.0001)$ after 12 months of treatment was observed. There were no significant differences between patients from the TOC cohort and their matched controls in the JIA ACR 30/50/70/90 criteria, JADAS 10, JADAS remission and minimal disease activity was reached by comparable numbers (TOC $37 \%$ and $58 \%$; control cohort $37 \%$ and $60 \%$ ).

The total number of AE was comparable (TOC cohort $\mathrm{n}=201 \mathrm{AE}$; (77/) $100 \mathrm{PY})$; control cohort $\mathrm{n}=207$; (65/100PY; RR 1.2; $95 \% \mathrm{Cl} 0.99-1.4)$. More serious AE (SAE) were reported with TOC. Serious infections were documented at lower frequency with TOC. Uveitis events were documented at significantly higher frequency with TNF inhibitors most likely due to a selection bias(Table 1 ). SAE with TOC were depression $(n=3)$ in 2 with suicidal intent, exacerbation of JIA $(n=2)$, septic arthritis, gastrointestinal infection, abdominal pain, colitis, paronychia and fracture. SAE in the control cohort were depression, osteomyelitis, gastrointestinal infection and disease flare. No significant differences regarding cytopenias and elevated transaminases were observed. No gastrointestinal perforation, no vascular events and no deaths occurred.

\begin{tabular}{|c|c|c|c|}
\hline & $\begin{array}{l}\text { TCZ mono } \\
\mathrm{N}=161\end{array}$ & $\begin{array}{l}\text { Matched } \\
\text { controls } \\
\mathrm{N}=161\end{array}$ & $\mathrm{p}$ \\
\hline $\begin{array}{l}\text { RF neg. Poly/RF pos. Poly/ } \\
\text { ext.Oligo n (\%) }\end{array}$ & $\begin{array}{l}110(68)(15(9) / \\
36(22)\end{array}$ & $\begin{array}{l}97(60) / 19(12) / \\
45(28)\end{array}$ & n.s. \\
\hline $\begin{array}{l}\text { Pretreatment with biologics, } \\
\mathrm{n}(\%)\end{array}$ & $127(83.5)$ & $20(13.2)$ & $<0.0001$ \\
\hline Efficacy Month 12 & $N=87$ & $N=105$ & \\
\hline JADAS MDA/REM, n (\%) & $\begin{array}{l}50(57.5) / 32 \\
(36.8)\end{array}$ & $\begin{array}{l}63(60.0) / \\
39(37.1)\end{array}$ & n.s. \\
\hline JIA ACR 30/50/70/90, \% & $80 / 75 / 61 / 53$ & $86 / 84 / 70 / 56$ & $\begin{array}{l}0.34 / 0.15 / 0.17 / \\
0.66\end{array}$ \\
\hline $\mathrm{AE}, \mathrm{N}($ rate/100PY/95\%Cl) & $\begin{array}{l}\text { Observation: } \\
260 \text { PY }\end{array}$ & $\begin{array}{l}\text { Observation: } \\
312 \text { PY }\end{array}$ & $\mathrm{RR}(95 \% \mathrm{Cl}) ; \mathrm{p}$ \\
\hline Serious AE (SAE) & $13(5 ; 3-9)$ & $4(1.3 ; 1-3)$ & $\begin{array}{l}3.9(1.3-12.0) ; \\
0.02\end{array}$ \\
\hline SAE infection & $3(1.2 ; 0.4-3.6)$ & $6(1.9 ; 0.8-4.2)$ & $\begin{array}{l}0.6(0.2-2.4) ; \\
\text { n.s. }\end{array}$ \\
\hline Uveitis event & $2(0.8 ; 0.2-3.1)$ & $12(3.8 ; 2.2-6.8)$ & $\begin{array}{l}0.2(0.04-0.9) ; \\
0.03\end{array}$ \\
\hline
\end{tabular}

*Number and rate of patients are given \# t-test or c2-test as appropriate

Conclusion: TOC was effective and comparable to treatment with alternative biologics. Tolerability was acceptable. As TOC was given as a second-line biologic in the vast majority of patients comparisons between the 2 cohorts have to be interpreted carefully. Observation is ongoing.

Disclosure of Interest:

G. Horneff Speaker Bureau of: Pfizer, Abbvie, Novartis, Chugai, A. Hospach: None declared, F. Weller-Heinemann: None declared, J. Kuemmerle-Deschner: None declared, M. Fasshauer: None declared, K. Minden: None declared, I. Foeldvari: None declared, C. Rietschel: None declared, T. Schwarz: None declared, R. Trauzeddel: None declared, M. Hufnagel: None declared, D. Foell: None declared, R. Berendes: None declared, P. Oommen: None declared, F. Dressler: None declared, A. Helling-Bakki: None declared, A. Klein: None declared 


\section{P268}

Secukinumab efficacy and safety in Juvenile Idiopathic Arthritis

I. Kriulin ${ }^{1,2}$, E. Alexeeva ${ }^{1,2}$, T. Dvoryakovskaya ${ }^{1,2}$, R. Denisova', A. Fetisova', A. Mamutova', K. Isayeva', A. Chomahidze', O. Lomakina', K. Chibisova', M. Gautier', D. Vankova', E. Krekhova ${ }^{1,2}$, M. Shingarova ${ }^{1,2}$

${ }^{1}$ Rheumatology, National Medical Research Center for Children's Health; ${ }^{2}$ Sechenov First Moscow State Medical University, Moscow, Russian

Federation

Correspondence: $\mathrm{E}$. Alexeeva

Pediatric Rheumatology 2020, 18(Suppl 2):P268

Introduction: Juvenile idiopathic arthritis (JIA) is the most common rheumatic disease in children, characterized by chronic inflammation of synovium, cartilage and bones destruction and wide spectrum of extra-articular manifestations. Often JIA is associated with eye damage -uveitis. Rheumatoid uveitis is the most common extra-articular JIA manifestation. Currently there is a large number of biological monoclonal antibodies therapy for the treatment of JIA. Monoclonal antibodies now represent an important series of options in the treatment of rheumatoid arthritis. However, some patients do not response to standard biologic therapy. However, in some cases there is no response to standard therapy. Secukinumab is a human antiinterleukin-17A monoclonal antibody, which is used in JIA. Secukinumab is being used off-label in Russia for JIA, so it is important to evaluate efficacy and safety in children.

Objectives: To evaluate efficacy and safety of Secukinumab in children with JIA.

Methods: 25 children (13 boys (52\%) and 12 girls (48\%)) with JIA who received Secukinumab in the rheumatological department of the National Medical Research Center for Children's health of Ministry of health were included. Efficacy of therapy was evaluated by uveitis activity, joint inflammation activity according to pediatric criteria of the American College of rheumatologists (ACRpedi), juvenile arthritis disease activity score (JADAS).

Results: Secukinumab was used in children with following types of JIA: enthesitis-related arthritis - 9 (36\%), oligoarthritis - 4 (16\%), RF(-) polyarthritis - $6(24 \%)$, psoriatic arthritis - $4(16 \%)$, undifferentiated arthritis - 2 (8\%). Among these patients $4(16 \%)$ children had rheumatoid uveitis and $15(60 \%)$ children were HLA-B27 positive. We consider important to notice that $17(68 \%)$ patients were switched on Secukinumab from another biologic agents, the average number of switches was 1,3 [min 0; $\max 3$ ]. Secukinumab demonstrated high efficacy in treatment of JIA and rheumatoid uveitis. Average treatment duration was 17,6 months [min 0; max 28,5]. From 20 patients who received Secukinumab more than 6 months after six months 20 $(100 \%), 18(90 \%), 16(80 \%), 9(45 \%)$ patients achieved 30\%, 50\%, $70 \%, 90 \%$ improvements in ACRpedi and $8(40 \%)$ children reached inactive disease stage in JADAS (less than 1 point). 2 (50\%) patients out of 4 with rheumatoid uveitis achieved remission. 5 (20\%) patients developed adverse effects: in $3(12 \%)$ children high levels of hepatic transaminases was observed (in 1 child it required to stop therapy), in $1(4 \%)$ patient - leucopenia and neutropenia and in 1 (4\%) patient - an severe allergic reaction and secondary MAS.

Conclusion: High efficiency and safety of Secukinumab in patients with JIA was demonstrated and we consider Secukinumab can be used as first-line therapy for JIA, associated with HLA-B 27 gene and as an alternative therapy when standard biologic ani-TNF therapy is ineffective. Only one serious adverse effect was reported.

\section{Disclosure of Interest}

I. Kriulin: None declared, E. Alexeeva Speaker Bureau of: Novartis, Pfizer, Sanofi, MSD and Roche, T. Dvoryakovskaya Speaker Bureau of: Novartis, Pfizer, MSD and Roche, R. Denisova Speaker Bureau of: Novartis, MSD and Roche, A. Fetisova: None declared, A. Mamutova Speaker Bureau of: Novartis, K. Isayeva: None declared, A. Chomahidze: None declared, O. Lomakina: None declared, K. Chibisova: None declared, M. Gautier: None declared, D. Vankova: None declared, E. Krekhova: None declared, M. Shingarova: None declared
P269

Neutralizing anti-rituximab antibodies in children with immune mediated diseases

I. Oomen', A. Nassar-Sheikh Rashid' ${ }^{1}$, A. H. Bouts ${ }^{2}$, S. C. Gouw ${ }^{3}$, T. W. Kuijpers' ${ }^{1}$, T. Rispens ${ }^{4}$, A. de Vries ${ }^{4}$, G. Wolbinkk, J. M. van den Berg ${ }^{1}$, D. Schonenberg-Meinema'

'Department of Pediatric Immunology, Rheumatology and Infectious diseases; ${ }^{2}$ Department of Pediatric Nephrology; ${ }^{3}$ Department of Pediatric Hematology, Amsterdam University Medical Centers; ${ }^{4}$ Department of Immunopathology, Sanquin Research and Landsteiner Laboratory, Amsterdam, Netherlands

Correspondence: I. Oomen

Pediatric Rheumatology 2020, 18(Suppl 2):P269

Introduction: Rituximab is frequently used as a therapeutic drug in different B-cell mediated autoimmune diseases and leads to B cell depletion. Different treatment responses have been observed in patients with B-cell mediated diseases. It has been suggested that a lack of efficacy may be related to the formation of anti-drug antibodies (ADA). The presence of ADA has been correlated with the failure of $B$ cell depletion as well as the occurrence of infusion-related reactions. It is unknown if these ADA neutralize in-vivo rituximab levels.

Objectives: The primary objective of this study is to determine if ADA neutralize rituximab levels and if a specific patient-group has a higher susceptibility for developing ADA. The secondary objective is to correlate ADA with B-cel depletion and infusion-related reactions. Methods: Retrospectively, children with different B-cell mediated diseases, treated with rituximab between December 2006 and November 2019, were included. Plasma samples for B-cell measurments had been collected at standard clinical visits every three months until six months after rituximab treatment. Rituximab-specific ADA levels and rituximab serum concentrations were determined, from frozen rest material, by radio-immunoassay and enzyme-linked immunosorbent assay, respectively. ADA presence was defined as a titer above 12 $\mathrm{AU} / \mathrm{ml}$. Patient charts were screened for infusion-related reactions.

Results: ADA levels were measured in 29 patients $(n=9$ nephrotic syndrome, $n=8$ Systemic Lupus Erythematosus (SLE), $n=2$ systemic vasculitis, $\mathrm{n}=2$ pulmonary hemosiderosis, $\mathrm{n}=2$ juvenile systemic sclerosis, $n=2$ other renal disease, $n=1$ juvenile dermatomyositis, $n=3$ other).

Of these, 35.4\% ( $n=10 / 29)$ tested ADA-positive, after a median of 93 days after RTX infusion (IQR 127.5 - 108.5). Median ADA-titer was $345.0 \mathrm{AU} / \mathrm{ml}$

RTX concentrations were measured in 28 patients after a median of 92 days after RTX infusion (IQR 62-113). Rituximab concentrations in seven ADA-positive patients were measured and all $(n=7)$ showed undetectable low RTX-concentrations. These RTX-levels differed significantly when compared to ADA-negative patients $(p<0.005)$.

Three autoantibodies were significantly more present in ADA-positive patients (anti-ds-DNA (OR 12.5, p=0.045), anti-RNP (OR 14.7, p=0.038) and anti-Sm (only present in ADA-positive patients, $p=0.036)$ ).

Five ADA-positive patients $(n=5 / 8,62.5 \%)$ did not show B-cell depletion after RTX-treatment, compared to one of 19 (5.3\%) ADAnegative patients ( $p=0.002$; OR $26.7 ; 95 \% \mathrm{Cl}, 2.24-317.25$ ).

Severe anaphylaxis during rituximab infusion occurred only in the ADA-positive patients.

Conclusion: In this retrospective cohort study in pediatric patients on RTX-treatment, we found undetectable low drug levels in ADApositive patients, indicative for their neutralizing capacity. Consequently, the lack of B-cel depletion leads to reduced treatment efficacy. Patients with SLE seem more susceptible to develop ADA. If ADA are detected, continuation of treatment seems non-effective and changing medication is advised. Certainly when considering that, in this study, anaphylactic reactions only occurred in ADA-positive patients.

Disclosure of Interest

None declared 
P270

Switching from reference to biosimilars does not reduce efficacy and safety in Juvenile Idiopathic Arthritis

I. Maccora' , G. Simonini', A. Bettiol ${ }^{2}$, I. Pagnini ${ }^{3}$, N. Lombardi', V. Maniscalco ${ }^{1}$, G. Crescioli ${ }^{4}$, E. Marrani ${ }^{1}$, R. Bonaiuti ${ }^{4}$, M. V. Mastrolia', C. Ravaldi ${ }^{5}$, A. Vannacci ${ }^{4}$

${ }^{1}$ Rheumatology Unit, Anna Meyer Children's Hospital and Department of Neurosciences, Psychology, Drug Research and Child Health, Section of Paediatric, , University of Florence; ${ }^{2}$ Department of Neurosciences, Psychology, Drug Research and Child Health, Section of Pharmacology and Toxicology,University of Florence; ${ }^{3}$ Rheumatology Unit, Anna Meyer Children's Hospital and Department of Neurosciences, Psychology, Drug Research and Child Health, Section of Paediatric, University of Florence; ${ }^{4}$ Department of Neurosciences, Psychology, Drug Research and Child Health, Section of Pharmacology and Toxicology, University of Florence; ${ }^{5}$ Department of Health Sciences, University of Florence, Florence, Italy Correspondence: I. Maccora

Pediatric Rheumatology 2020, 18(Suppl 2):P270

Introduction: Limited data about the use of biosimilar are available in children with Juvenile Idiopathic Arthritis (JIA).

Objectives: To evaluate the long-term efficacy and safety of switching from the etanercept (ETA) and adalimumab (ADA) originators to their biosimilars, in children with JIA.

Methods: Medical charts of JIA children who switched from ETA or ADA originators to the biosimilars were retrospectively evaluated. Efficacy of anti-TNF therapy was evaluated at last follow-up during the originator therapy and at 3,6 and 12 months following the switch to biosimilar, assessing number of inflamed joints, CRP, ESR, Juvenile Arthritis Disease Activity Score (JADAS 10), Visual Analog Scale (VAS) and Childhood Health Assessment Questionnaire (CHAQ). Occurrence of adverse event ( $\mathrm{AE}$ ) during treatment was evaluated. Continuous variables were reported as median value and interquartile range (IQR) and compared using the Wilcoxon test for paired data, and csquare analysis.

Results: 43 children (31 Female, median age at onset 65 months (IQR $31-125)$ received originator ETA $(n=14)$ or ADA $(n=29)$, as first-line antiTNF treatment for refractory JIA. Due to healthcare politics, patients have been switched to the biosimilar: Benepali $(n=13)$, Erelzi ${ }^{\oplus}(n=1)$ for ETA; Imraldi $^{\circledR}(n=24)$, Amgevita ${ }^{\oplus}(n=5)$ for ADA, after 40.5 months (IQR 19.173.8) duration of originator treatment. At time of switch, 10/14 patients on ETA and 19/29 on ADA were on complete disease remission. No significance difference of entered parameters has been found at 3, 6 and 12 months thereafter the switch. Nine patients discontinued biosimilars, due to disease remission (5), to family willing (2), to occurrence of burning at injection site (2, on Benepali). The number of patients who experienced an $\mathrm{AE}$ was not different in different frame follow-up when comparing exposure to the originator and that to biosimilar, respectively: during 0-3 months, $15 / 42$ (35.7\%) vs $7 / 37$ (18.9\%), $c^{2}: 2.76$; during 3-6 months, 16/40 (40.0\%) vs 17/31 (54.8\%), $c^{2}: 1.54$; during 6-12 months, $15 / 39$ (38.5\%) vs $11 / 24(45.8 \%), c^{2}: 0.33$. Most frequent AEs were upper respiratory tract infections (31) and injection site reactions (7).

\begin{tabular}{|c|c|c|c|c|c|}
\hline & & $\begin{array}{l}0 \\
\text { Time of } \\
\text { switch }\end{array}$ & $\begin{array}{l}3 \\
\text { months }\end{array}$ & $\begin{array}{l}6 \\
\text { months }\end{array}$ & $\begin{array}{l}12 \\
\text { months }\end{array}$ \\
\hline \multirow{3}{*}{$\begin{array}{l}\text { Amgevita }(\mathrm{N}= \\
\text { 5) }\end{array}$} & $n$ & 5 & 5 & 3 & 2 \\
\hline & $\begin{array}{l}\text { Number of active } \\
\text { joints }\end{array}$ & $0(0-0)$ & $0(0-0)$ & $0(0-0)$ & $0(0-0)$ \\
\hline & JADAS10 & $0(0-0.1)$ & $0(0-1)$ & $0(0-0)$ & $\begin{array}{l}0.1(0- \\
0.2)\end{array}$ \\
\hline \multirow{3}{*}{$\begin{array}{l}\text { Benepali }(N= \\
\text { 13) }\end{array}$} & $\mathrm{n}$ & 13 & 11 & 9 & 10 \\
\hline & $\begin{array}{l}\text { Number of active } \\
\text { joints }\end{array}$ & $0(0-0)$ & $0(0-0)$ & $0(0-0)$ & $0(0-0)$ \\
\hline & JADAS10 & $0(0-6)$ & $0(0-0.8)$ & $0(0-0.4)$ & $0(0-0.8)$ \\
\hline \multirow{3}{*}{$\begin{array}{l}\text { Imraldi }(N= \\
24)\end{array}$} & $\mathrm{n}$ & 24 & 20 & 19 & 12 \\
\hline & $\begin{array}{l}\text { Number of active } \\
\text { joints }\end{array}$ & $0(0-0.5)$ & $0(0-0)$ & $0(0-0)$ & $0(0-0)$ \\
\hline & JADAS10 & $0(0-3.4)$ & $0(0-2.8)$ & $0(0-0)$ & $0(0-0)$ \\
\hline
\end{tabular}

Conclusion: Data from this small, retrospective inception cohort, showed similar efficacy and safety of the originator and a type of ETA and ADA biosimilars in JIA.

Disclosure of Interest

None declared

P271

Long term treatment with adalimumab in Juvenile Idiopathic

Arthritis associated uveitis - our experience

D. S. Lazarevic ${ }^{1,2}$, M. Jakovljevic ${ }^{2}$, J. Vojinovic ${ }^{2,3}$

${ }^{1}$ Faculty of Medicine, University of Nis; ${ }^{2}$ Pediatric Rheumatology, CLINIC OF PEDIATRICS, CLINICAL CENTER NIS; ${ }^{3}$ Faculty of Medicine, University of Niš, Nis, Serbia

Correspondence: D. S. Lazarevic

Pediatric Rheumatology 2020, 18(Suppl 2):P271

Introduction: Juvenile idiopathic arthritis (JIA) associated uveitis is one of the most severe extra-articular manifestations in JIA. Delayed diagnose time and inadequate treatment could lead to serious structural consequences. In the last years new treatment novelties and TNF inhibitors have shown excellent control of ocular inflammation.

Objectives: The aim of this study was to evaluate retrospectively the long-term efficacy and safety of adalimumab in patients with JIAassociated uveitis.

Methods: We have retrospectively analysed nineteen JIA patiens data with associated uveitis from our centre registry between 2010 and 2020, treated with adalimumab after failure of treatment with corticosteroids and metotrexate. Demografic data and blood samples were collected at different time points while uveitis activity was evaluated by slit-lamp biomicroscopy. Adverse events were recorded. Results: Registry records provided 10 years follow up of $19 \mathrm{JIA}$ patients data with associated uveitis. Eleven patients were females (57.90 \%) diagnosed as oligo/extended oligoarticular JIA while eight (42.10\%) were males diagnosed as enthesitis related arthritis (ERA). Before adalimumab was prescribed, all patients were previously treated with metotrexate during 3.5 years in avarage dose of $10 \mathrm{mg} /$ $\mathrm{m}^{2}$ weekly. The mean uveitis duration, before adalimumab administration was 9 months. Ten years long follow up period have showed that there were no new relapsis of uveitis while patients were receiving adalimumab and metotrexate. All of our patients were able to gradually tapper and stop treatment with topical steroids two months after adalimumab commencing. Seven patients were able to stop biological treatment after 4.3 years of adalimumab usage. Uveitis relapsed three monts after the adalimumab discontinuation only in one patient. Two patient were lost to follow up during the transitional period. No serious adverse events were recorded.

Conclusion: During the long term follow up period adalimumab have shown good efficacy and safety profile in JIA patients with active inflammatory ocular disease.

Disclosure of Interest

None declared

\section{P272}

Prolonged fever, arthritis and uveitis: a surprising diagnosis

T. L. Marques', A. Marinhas' ${ }^{2}$ P. Estanqueiro ${ }^{3}$, M. Salgado ${ }^{4}$

${ }^{1}$ Serviço de Pediatria, Centro Hospitalar do Baixo Vouga, Aveiro;

${ }^{2}$ Hospital Pediátrico - Centro Hospitalar e Universitário de Coimbra,

Coimbra, Portugal; ${ }^{3}$ Unidade de Reumatologia, Hospital Pediátrico -

Centro Hospitalar e Universitário de Coimbra; ${ }^{4}$ Unidade de

Reumatologia, Hospital Pediátrico, Centro Hospitalar e Universitário de

Coimbra, Coimbra, Portugal

Correspondence: A. Marinhas

Pediatric Rheumatology 2020, 18(Suppl 2):P272

Introduction: Post-streptococcal syndrome is a systemic immunemediated complication of beta-haemolytic streptococci infection, mostly seen as post-streptococcal arthritis, rheumatic fever or glomerulonephritis. Uveitis is an uncommon manifestation of this syndrome. 


\section{Objectives: Case report} Methods: Case report

Results: A previously healthy 7-year-old female was admitted at the emergency department with prolonged fever, arthritis and red eye. She had a 4-month history of febrile episodes every two weeks, with axillary temperature ranging from 37,8 to $39^{\circ} \mathrm{C}$. Migratory arthralgia affecting both knees and tibiotarsal joints showed up two months after the fever onset and worsened in the previous week, with refusal to walk. Non-painful bilateral red eye for several weeks was mentioned. Other symptoms were absent. Recent infections were denied and family history was irrelevant.

Physical examination revealed lower limb muscular atrophy, knees pain and impaired function and bilateral tibiotarsal arthritis with inability to walk. Ophthalmological observation showed a bilateral non-granulomatous anterior uveitis. Sequential laboratory work up revealed a maximum eritrocitary sedimentation rate of $135 \mathrm{~mm} / \mathrm{h}$, maximum c-reactive protein of $5,3 \mathrm{mg} / \mathrm{dL}$, microcytic hypochromic anemia, positive antistreptolysin O titer (ASOT) (initial result of 1250 that increased to 2500 in 4 weeks and later decreased to 500) and negative anti-nuclear antibodies. Cardiac involvement was excluded. The diagnosis of rheumatic fever with concomitant poststreptococcal uveitis was assumed and the patient was treated with oral and topical ophthalmic corticosteroids with prompt clinical resolution of fever, acute polyarthritis and uveitis. No relapse occurred in a 5-year follow-up.

Conclusion: Juvenile idiopathic arthritis (JIA) is the most common cause of uveitis in childhood. Although our patient clinical course could initially raise the possibility of systemic JIA (sJIA), the criteria that define this entity weren't all present and clinical and laboratory findings were more supportive of rheumatic fever. Besides, uveitis occurs exceptionally in sJIA, which turned this diagnosis even less reasonable. In our Rheumatology Unit, among 563 patients diagnosed with JIA in 32 years, 89 had uveitis. However, in the group of 51 patients with SJIA only one had ocular involvement, a boy with isolated vitritis. Post-streptococcal uveitis (PSU) typically presents as bilateral, non-granulomatous anterior uveitis, as described in this case. As streptococcal infection is very common among children and many patients may experience subclinical infection. PSU should be considered in all patients with uveitis along with positive ASOT and negative routine investigations for other causes.

Although PSU has been described in literature, to the best of our knowledge, this is the first reported case of concomitant rheumatic fever and PSU.

\section{Disclosure of Interest}

None declared

\section{P273}

To taper or not to taper in juvenile idiopathic arthritis: is there a risk of development of uveitis flares?

M. A. TERAN, A. BOTEANU, C. GUILLEN, C. PIJOAN

REUMATOLOGIA, HOSPITAL RAMON Y CAJAL, MADRID, Spain

Correspondence: M. A. TERAN

Pediatric Rheumatology 2020, 18(Suppl 2):P273

Introduction: Juvenile Idiopathic Arthritis (JIA) is the most common extra-ocular disease and is associated with chronic anterior uveitis during childhood. JIA-associated uveitis (JIAU) is a serious, sightthreatening disease with multiple complications and even blindness if untreated. Although most treatments used improve simultaneously both arthritis and uveitis, there is low correlation between the activity and damage for both conditions.

Objectives: To determine the association between the occurrence of uveitis flares in patients with Juvenile Idiopathic Arthritis (JIA) and the de-intensification of immunosuppressive treatment.
Methods: We conducted a retrospective longitudinal cohort study, including a single-centre consecutive cohort of patients diagnosed with oligoarticular JIA antinuclear antibody (ANA) positive, who had had at least one uveitis flare during their follow-up up to 19.5 years. Patiens with the same JIA category, ANA positive, with no history of uveitis flare were considered controls. Epidemiological data, age of first uveitis flare, number of previous episodes, treatments prescribed at the time of the flare and time since the last treatment modification were recorded. Treatment tapering was defined as a reduction in dose or increase in the inter-doses period, according to datasheet of the corresponding treatment. The relative risk (RR) for the development of uveitis flare and treatment tapering were determined by contingency tables.

Results: We included 68 patients of which 22 had had uveitis flares during their follow-up, and 46 controls. The mean age of patients at JIA diagnosis was $3.56 \pm 2.17$ years. A total of 107 uveitis flares were recorded with an average of $4.54 \pm 4.70$ episodes per patient. The first uveitis flare was registered at an average age of $6.57 \pm 5.79$ years. Four patients (18.1\%) had had only one episode. Among patients with more than one flare, the inter-flare period was $17.84 \pm$ 21.8 months. Thirty flares (27\%) were registered in patients without immunosuppressive treatment. Twenty patients (90\%) required the initiation of biological therapy specific for uveitis. Adalimumab (ADA) was chosen in 19 (86.3\%) patienst and avoided further uveitis flares in $15(68 \%)$ cases. Treatment with Tocilizumab (TCZ) was used in 6 (27.7\%) cases and avoided further uveitis flares in 5 (27.3\%). Thirtythree episodes (33.1\%) were registered in patients with Methotrexate (MTX) of which, $8(7.5 \%)$ were receiving doses below datasheet $(<$ $10 \mathrm{mg} / \mathrm{m} 2$ ). Forty-four uveitis flares $(41 \%)$ took place in patients on biological treatment, of which 27 were receiving ADA (25.3\%), 2 (1.9\%) TCZ and 15 (14\%) other therapies. Thirty-seven flares $(32.1 \%)$ took place in patients on tapered treatments and 11 (10.3\%) after non scheduled withdrawal. In terms of risk of developing a new uveitis flare, tapering had a RR of $2.79(\mathrm{Cl} 2.01-3.7 ; P<0.05)$ while therapy withdrawal had a RR of 5.91 (Cl 3.23-10.8; $p<0.05$ ). MTX tapering had a RR of 12.5 (Cl 6.4-24.5 $p<0.05)$. Patients with ADA had a RR of 0.88 (CI 0.4-1.6; $P=0.84$ ) of developing uveitis flares, with TCZ a RR of 4,65 (CI 1.2-17.8; $P<0.05$ ) and with other biological therapy (Etanercept, Infliximab, Abatacept) a RR of $3.56(\mathrm{Cl} 2.05-6.2 ; P<0.05)$.

Conclusion: Tapering immunosuppressive treatment in oligoarticular JIA ANA positive patients, increases the risk of developing uveitis flares.

Disclosure of Interest

None declared

P274

Practice patterns for tapering medications in the treatment of JIAassociated uveitis

I. Foeldvari ${ }^{1}$, J. Klotsche², S. Angeles-Han³ ${ }^{3}$ J. Anton ${ }^{4}$, G. Simonini ${ }^{5}$, N.

Groesch², J. Baer

${ }^{1}$ Hamburg Center for Pediatric and Adolescent Rheumatology, Am

Schoen Klinik Eilbek, Hamburg; ${ }^{2}$ German Rheumatism Research Center, Berlin, Germany; ${ }^{3}$ Cincinnati Children's, Cinicinnati, United States;

${ }^{4}$ Hospital Sant Joan de Déu, Barcelona, Spain; ${ }^{5}$ Anna Meyer Children's Hospital, Florence, Italy

Correspondence: I. Foeldvari

Pediatric Rheumatology 2020, 18(Suppl 2):P274

Introduction: Juvenile idiopathic arthritis associated uveitis (JIAU) is the most common extra-articular manifestation of JIA, and occurs in approximately $10 \%$ of affected children. Although there are effective medications to treat JIAU, guidelines and large studies that inform of tapering treatment after disease remission are lacking. 
Objectives: To understand the current medication tapering practice patterns of pediatric rheumatologists and pediatric ophthalmologists specialized in children with JIAU.

Methods: We surveyed vie email international pediatric rheumatologists: 1. Multinational Interdisciplinary Working Group for Uveitis in Childhood (MIWGUC), 2. Pediatric Rheumatologic Email-Listserve, 3. CARRA uveitis workgroup, and international

ophthalmologic specialized in children with JIAU. Survey questions focused on the definition of remission, duration of remission prior to initiation of medication tapering, and method of tapering. Specific medications included methotrexate (MTX), adalimumab (ADA), infliximab (IFX), abatacept (ABA), and tocilizumab (TOC).

Results: Of 45 responses, $88 \%$ were from pediatric rheumatologists with a mean work experience of 18 years. The regional distribution was 31 form Europa, 9 from North-America, 3 from South-America and 2 from Asia. The responding colleagues managed a mean number of 43 JIAU patients. Remission on medication was defined as no cells in the anterior chamber (78\%), followed by no need for eye drops (36\%), and no uveitis flares (32\%). Tapering practices were described for MTX monotherapy (100\%) ADA (100\%), IFX (80\%), TOC (56\% [25\% s.c.]) and ABA (46\% [30\% s.c.]).

Standardized protocol for tapering exists in $32 \%$ of centers for MTX, in $26 \%$ for ADA, and $20 \%$ for IFX. The timepoint for tapering was after 6 months of remission on medication by $14 \%$ of respondents, 12 months for $38 \%, 24$ months for $56 \%$ and 36 months for $12 \%$.

MTX was tapered by dose in $42 \%$, dose and interval in $40 \%$, and interval in $15 \%$. The lowest dose of MTX was $6 \mathrm{mg} / \mathrm{m} 2 /$ week at the time of tapering and the longest mean interval 2.5 weeks (1 to 4 weeks). ADA was first tapered to every 3 weeks by $76 \%$ of the responders and then to every 4 weeks by $49 \%$ before discontinuing. Fewer respondents used or tapered IFX, TOC or ABA. Around $65 \%$ tapered the interval and $20 \%$ tapered the dose and interval for $A B A$ $26 \%$ for TOC and $37 \%$ IFX

There were differences in the duration of tapering prior to discontinuation of specific medications. For ADA it was 6 months in $62 \%, 12$ months in $36 \%$, and 24 months in $10 \%$. For IFX it was 6 months in 27\%, 12 months in $45 \%$, and 24 months in 33\%. For TOC it was $40 \%$ after 4 weeks, $87 \%$ after 6 weeks and $53 \%$ after 24 weeks. For ABA i.v. it was $30 \%$ after 8 weeks, and $90 \%$ after 12 weeks.

If combination therapy was used, $36 \%$ tapered the bDMARD first, $62 \%$ csDMARD first, and $12 \%$ both simultaneously.

Conclusion: This is the first survey to describe "real world" medication tapering and discontinuation practices of pediatric rheumatologists and ophthalmologists globally. Most physicians start to taper medication after 24 months of remission on medication and discontinue after the 6 to 12 months of tapering.

Acknowledgement:

We would like to thank all the participating colleagues, who took time to fill out our surve

\section{Disclosure of Interest}

None declared

\section{P275}

The clinical features of uveitis de-novo in juvenile idiopathic arthritis patients developed on biologic treatment

M. Chakhalian, E. Gaidar, E. Isupova, I. Chikova, M. Dubko, V. Masalova, T. Likhacheva, A. Santimov, T. Nikitina, L. Snegireva, M. Kostik

Saint Petersburg State Pediatric Medical University, Saint-Petersburg,

Russian Federation

Correspondence: M. Chakhalian

Pediatric Rheumatology 2020, 18(Suppl 2):P275

Introduction: Uveitis de-novo means the new cases of uveitis, developed after the initiation of the biologic treatment. More often uveitis de-novo occurred in the juvenile idiopathic arthritis patients and after etanercept.

Objectives: The aim of our study was to evaluate clinical features of uveitis de novo and compare to other autoimmune uveitis.
Methods: in the retrospective study 225 pediatric autoimmune uveitis included. The onset age ranged from 1 to 16 years, 144 girls (64\%) and 81 boys (36\%), ANA positivity was in 106/180 (58.9\%), HLA B27 was in 23/107 (21.4\%). JIA-associated uveitis was in 90\% (59.2\% - oligoarthritis, $21.6 \%$ - polyarthritis, $9.2 \%$ enthsytis-related arthritis) and $10 \%$ of the patients had uveitis solely. The distribution of uveitis types: anterior $78 \%$, peripherial and posterior uveitis in $2.8 \%$ each, and panuveitis in $16.4 \%$. Unilateral uveitis at onset was in $34.7 \%$ and bilateral in $65.3 \%$.

Results: uveitis de-novo occurred in $12(5.3 \%)$ patients of all uveitis in 1-48 months (median=26 months, 25-75\%: 17-41 months) after start of biologic. Gender distribution: 4 boys (33.3\%) and 8 girls (66.7\%). Anterior uveitis was in 11/12 (91.7\%) patients and 1/12 (8.3\%) had panuveitis; unilateral involvement in $5(41.7 \%)$ and bilateral in 7 patients (58.3\%). HLA B27 antigen was in 3/10 (30\%) in uveitis de-novo and in $20 / 98(20.4 \%)$ in other uveitis $(p=0.366)$, ANA positivity in $4 / 12$ (33.3\%) in uveitis de-novo and in 102/171 (59.7\%) in other uveitis ( $p=$ 0.366 ). The main features in both studied groups are in the table 1. All cases of the uveitis de-novo developed under etanercept (100\%) and 4 of them (33.3\%) discontinued methotrexate before uveitis. Before uveitis 10/12 (83.3\%) had remission in arthritis. All patient discontinued etanercept and 10 patients switched etanercept on adalimumab: eight patients with methotrexate and 2 pattients - adalimumab monotherapy. In two remaining patients, one only discontinued etanercept and continue methotrexate with mild flares and using the topical steroids, and second patient discontinued etanercept and restarted methotrexate. Remission in uveitis achieved in 6/10 (60\%) who switched etanercept on adalimumab, 4/10 (40\%) experienced flares despite adalimumab treatment with methotrexate $(n=3)$.

\begin{tabular}{|c|c|c|c|}
\hline Parameter & $\begin{array}{l}\text { Uveitis de-novo } \\
(n=12)\end{array}$ & $\begin{array}{l}\begin{array}{l}\text { Other } \\
\text { uveitis } \\
(n=213)\end{array}\end{array}$ & $\mathrm{p}$ \\
\hline \multicolumn{4}{|l|}{ JIA category } \\
\hline $\mathrm{OA}$ & $2(16.7)$ & $127(69.5)$ & 0.002 \\
\hline PA & $6(50)$ & $40(21.9)$ & \\
\hline ERA & $4(33.3)$ & $16(8.6)$ & \\
\hline $\mathrm{ESR}, \mathrm{mm} / \mathrm{h}$ & $30(19 ; 57)$ & $20(7 ; 28)$ & 0.03 \\
\hline Arthritis before uveitis, n (\%) & $12(100)$ & $\begin{array}{l}123 / 202 \\
(60.9)\end{array}$ & 0.007 \\
\hline Biologics for uveitis treatment, $\mathrm{n}(\%)$ & $10(83.3)$ & $\begin{array}{l}113 / 206 \\
(54.9)\end{array}$ & 0.042 \\
\hline Time before uveitis, years & $3.5(2.4 ; 4.9)$ & $\begin{array}{l}0.2(0.0 ; \\
1.7)\end{array}$ & 0.000008 \\
\hline $\begin{array}{l}\text { Time before biologics treatment } \\
\text { (indication: uveitis), years }\end{array}$ & $0.2(0.1 ; 0.6)$ & $\begin{array}{l}2.1(0.8 ; \\
4.9)\end{array}$ & 0.002 \\
\hline
\end{tabular}

Conclusion: uveitis de-novo is a challenging problem, associated with biologic treatment. Further investigation required for finding the predictors of this condition.

Trial registration identifying number: This work supported by the Russian Foundation for Basic Research (grant № 18-515-57001)

Disclosure of Interest

None declared

P276

Changing evidence over time: updated meta-analysis regarding anti-tnf efficacy in childhood chronic uveitis

I. Maccora' ${ }^{1}$ E. Fusco ${ }^{2}$, E. Marrani ${ }^{2}$, A. V. Ramanan ${ }^{3}$, G. Simonini ${ }^{4}$

${ }^{1}$ Rheumatology Unit, Anna Meyer Children's Hospital, School of Human Health Science; ${ }^{2}$ Rheumatology Unit, Anna Meyer Children's Hospital,

School of Human Health Science, University of Florence, Florence, Italy;

${ }^{3}$ Bristol Royal Hospital for Children, UK \& University of Bristol, Bristol,

United Kingdom; ${ }^{4}$ Rheumatology Unit, Anna Meyer Children's Hospital,

NEUROFARBA Department, University of Florence, Florence, Italy

Correspondence: I. Maccora

Pediatric Rheumatology 2020, 18(Suppl 2):P276 
Introduction: Childhood uveitis is a sight-threatening condition and it may lead to ocular complications. In the last 15 years the biologic therapy, specifically anti-TNF, has revolutionised the management of uveitis refractory to conventional immunomodulatory approaches.

Objectives: To summarize evidence regarding efficacy of anti-tumour necrosis factor-a (anti-TNFa) in childhood autoimmune chronic uveitis (CACU), refractory to common disease modifying antirheumatic drugs (DMARDs).

Methods: An updated systematic search was conducted between November 2012 and January 2020. Studies investigating the efficacy of anti-TNFa therapy, in children ages $<16$ years, as the first biologic treatment for $C A C U$, refractory to topical and/or systemic steroid and at least one DMARD, were eligible for inclusion. The primary outcome measure was the improvement of intraocular inflammation according to Standardization of Uveitis Nomenclature Working Group criteria. A combined estimate of the proportion of children responding to etanercept (ETA), infliximab (INF), and adalimumab (ADA) was determined.

Results: We identified 1677 articles and 37 articles were eligible. Three were randomized clinical trials (RCTs), one on ETA and 2 on $A D A$, and were excluded from pooled analysis. From the observational studies, a total of 487 children were identified: 226 received ADA, 213 INF and 48 ETA. The proportion of responding children was $86 \%(95 \% \mathrm{Cl} 76-95 \%)$ for ADA, 68\% (95\% Cl 50-85\%) for INF, and $36 \%$ (95\% Cl 9-67\%) for ETA. Pooled analysis showed clear differences $(x 2=32.2, p<0.0001)$ : ADA and INF were both significantly superior to ETA $(X 2=26.8, p<0.0001$, and $X 2=7.41, p<$ 0.006 respectively), ADA significantly superior to INF $(x 2=13.4, p<$ 0.0002).

Conclusion: This metanalysis, consistent with recent RCT data, suggests the efficacy of ADA and INF in CACU treatment. However, ADA results superior to INF in this clinical setting.

\section{Disclosure of Interest}

None declared

\section{P277}

Drug survival of the infliximab biosimilar (CT-P13) in pediatric patients with non-infectious uveitis

B. Sozeri ${ }^{1}$, E. Kardes ${ }^{2}$, F. demir ${ }^{1}$, E. Sali ${ }^{3}$, D. cakir $^{3}$

${ }^{1}$ pediatric rheumatology: ${ }^{2}$ Ophtalmalogy: ${ }^{3}$ pediatric infectious disease,

Health Sciences University, Umraniye Traning and Research Hospital,

Istanbul, Turkey

Correspondence: B. Sozeri

Pediatric Rheumatology 2020, 18(Suppl 2):P277

Introduction: Uveitis is uncommon in the children, accounting for 2 to $14 \%$ of all uveitis cases Pediatric non-infectious uveitis (NIU) is an important cause of significant long term complications and blindness in children.Biosimilar drugs are reproductions of their originator counterparts, are usually less expensive

Objectives: In this report, we studied children with uveitis who had Infliximab Biosimilar (CT-P13) therapy focusing on demographics, anatomic distribution, etiologies, outcome, and complications.

Methods: This non-interventional, retrospective, single center analysis collected medical record data for pediatric patients with non-infectious uveitis who received CT-P13 treatment at the referral center for pediatric rheumatology between January 2016 and January 2020.

Results: Forty six eyes of twenty six patients were enrolled in this study. The gender distrubiton was equal The median age at presentation was 9.7 years. The median age (IQR) at diagnosis of uveitis was $9.41(5-12.3)$ years, the median age at primary diagnosis was 7.8 years and the median age at the beginning of the symptoms was 6.6 years. The median time between primary diagnosis and uveitis was 1 months.The mean number of uveitis episodes was $5.4 \pm 2.75$. Bilaterally was more commonly encountered in the older age group, $p=0.32$. Anterior uveitis was the most common site of inflammation followed by panuveitis then posterior and finally intermediate uveitis. Of the 26 patients evaluated, the primary diagnosis of 16 was JIA, 3 was Behçet disease, 6 were idiopathic and 1 was sarcoidosis. In the patients with
$\mathrm{BD}$, the mean age (IQR) at diagnosis was 17.7 (12.9-17.7) years and the mean age (IQR) at diagnosis was 8.2 (7.3-12.3) years in patients with idiopathic uveitis. Both the age at uveitis and primary diagnosis were detected younger in JIA groups than others $(p=0,04)$. While the rate of patients with controlled uveitis was $80.7 \%$, disease control was not achieved in 7 patients with INF treatment. At presentation, there was good VA (LogMAR VA $<0.3,>20 / 40$ ) in sixty percent of the eyes and significant improvement in VA during follow-up in most eyes (81\%).Overall, all patients were treated with CT-P13 (22 patients infliximab-naïve; 4 switched from adalimumab). Drug survival was similar in naïve and switched patients. Treatment-emergent adverse events (TEAEs) occurred in $26,9 \%$.

Conclusion: In our present study, we report the 4-year follow up results on the safety and efficacy of biosimilar infiliximab of pediatric non-infectious uveitis. This is first report about biosimilar infliximab (CT-P13) efficacy and safety in pediatric patients with uveitis. IFX biosimilar (CT-P13) is remarkably safe and effective for long-term treatment of non-infectious pediatric uveitis. Higher dosage and shorter intervals may be necessary to achieve successful control in a greater percentage of patients.

\section{Disclosure of Interest}

None declared

\section{P278}

Morbidity of JIA-associated uveitis: half of patients despite systemic treatment still show ocular damage during a long-term follow-up

G. B. Beretta', L. Marelli ${ }^{2}$, V. Casotto ${ }^{1}$, M. Romano ${ }^{3}$, E. Miserocchi ${ }^{3}$, C. Mapelli', A. Petaccia' ${ }^{1}$ S. Lanni ${ }^{1}$, I. Pontikaki ${ }^{3}$, F. Minoia', R. Cimaz ${ }^{3}$ on behalf of on behalf of the Pediatric Rheumatology Associated Group of Milan Area

${ }^{1}$ Fondazione IRCCS Ca' Granda Ospedale Maggiore Policlinico; ${ }^{2}$ San Giuseppe Hospital, IRCCS Multimedica; ${ }^{3}$ ASST Gaetano Pini-CTO Institute, Milan, Italy

Correspondence: G. B. Beretta

Pediatric Rheumatology 2020, 18(Suppl 2):P278

Introduction: Uveitis is the most common extra-articular complication of juvenile Idiopathic arthritis (JIA). Due to its typical indolent and chronic course, children with this condition are at risk for ocular morbidity with a significant impact on their quality of life.

Objectives: To describe demographic and clinical features, treatment approaches and outcome of a population of patients with JIAassociated uveitis.

Methods: Charts of patients with JIA-associated uveitis, followed in two tertiary Pediatric Rheumatology Centres were retrospectively reviewed with regard to clinical features, therapeutic choices and outcome.

Results: Data from $162 \mathrm{JIA}$ patients with uveitis were analysed (81.5\% female), with a mean follow up of 8.9 years (SD \pm 2.56 ). Mean age at JIA onset was 3.6 years $(\mathrm{SD} \pm 3.1)$ and the mean JIA duration at uveitis onset was 2.5 years $(S D \pm 4.3)$. Uveitis was diagnosed at JIA onset in $9.9 \%$ of patients. The most frequent JIA category was oligoarthritis (88.9\%), which was persistent in $72.8 \%$ of cases, followed by RF- polyarthritis (9.3\%). No systemic JIA was reported. Uveitis was predominantly anterior (96.9\%) and reported bilateral in $65.4 \%$ of cases. In almost all patients $(87.6 \%)$ antinuclear antibodies (ANA) were positive. Systemic medications were required in 134 (82.7 \%) patients. Methotrexate and cyclosporine were used in $66.0 \%$ and $7.4 \%$ of cases, respectively, while 86 patients $(53.1 \%)$ required biologic therapy, mainly adalimumab (34.6\%), followed by infliximab (10.5\%) and tocilizumab (3.7\%). In $28.4 \%$ of cases more than 1 biologic was needed. Mean recurrence rate in our cohort was 1.3 per year $(\mathrm{SD} \pm 1.1)$. In 79 patients $(49.8 \%)$ uveitis was complicated by ocular damage, which is summarized in Table 1. A best-corrected visual acuity $(B C V A) \leq 0.4$ and $\leq 0.1$ were observed in $14.2 \%$ and $10.5 \%$ of patients, respectively. Clinical remission at last follow-up was reached in $26(70.3 \%) / 37$ patients with available data. 
Table 1 (abstract P278). See text for description.

\begin{tabular}{ll}
\hline Ocular damage, $\mathbf{n}(\%)$ & JIA patients with uveitis \\
& N 162 \\
\hline Synechiae & $56(34.6 \%)$ \\
Glaucoma & $9(5.6 \%)$ \\
Cataract & $29(17.9 \%)$ \\
Band keratopathy & $32(19.8 \%)$ \\
Cystoid macular edema & $7(4.3 \%)$ \\
Any surgery & $28(17.3 \%)$ \\
Cataract & $24(14.8 \%)$ \\
Synechiotomy & $2(1.2 \%)$ \\
Other (band keratopathy, glaucoma) & $2(1.2 \%)$ \\
\hline
\end{tabular}

Conclusion: Despite continue improvement in therapeutic options, uveitis remains a high morbidity complication of JIA. Clinical predictors and biomarkers are needed to identify patients at higher risk of unfavourable outcome. Careful monitoring and follow-up are crucial for timely detection of ocular inflammation and prevention of damage.

\section{Disclosure of Interest}

None declared

\section{P279}

Assessing s100 proteins and cytokines in tears as potential biomarkers for uveitis diagnosis and activity in JIA patients J. Rodriguez-Smith ${ }^{1}$, V. Miraldi Utz ${ }^{2}$, S. Thornton ${ }^{1}$, G. Schulert ${ }^{1}$, A. Sproles $^{1}$, N. Mwase', T. Hennard ${ }^{1}$, A. A. Grom', M. Altaye ${ }^{3}$, S. T. Angeles$\mathrm{Han}^{\top}$

${ }^{1}$ Rheumatology; ${ }^{2}$ Ophthalmology, Cincinnati Children's Hospital Medical Center; ${ }^{3}$ Biostatistics and Epidemiology, University of Cincinnati,

Cincinnati, United States

Correspondence: $J$. Rodriguez-Smith

Pediatric Rheumatology 2020, 18(Suppl 2):P279

Introduction: JIA-associated uveitis (JIA-U) occurs in $10-20 \%$ of children with Juvenile Idiopathic Arthritis (JIA) and typically asymptomatic, and sight-threatening complications occur in 50\% of children, (i.e. cataracts, vision loss). Frequent ophthalmic examinations are important for early diagnosis and monitoring of uveitis activity. Even after uveitis is controlled, risk of disease exacerbation still exists. Therefore, frequent ophthalmic screening and monitoring is important for detection and management of JIA-associated uveitis (JIA-U). S100 proteins, cytokines, and chemokines detected in aqueous humor of patients with uveitis are also detected in tears. Biomarker discovery using tears is promising since collection is noninvasive, feasible, well-tolerated, and close to the target organ.

Objectives: We aim to determine if $\mathrm{S} 100$ proteins, cytokines, and chemokines levels differ in tears of children with JIA and JIA-U and in children with JIA-U by uveitis activity.

Methods: Tears were collected using Schirmer strips from children $\geq 5$ years old with oligo- or polyarticular RF negative JIA with (JIA-U) and without uveitis (JIA-no-U), and in children with JIA-U at time of active and inactive eye disease. Activity was defined by Standardization of Uveitis Nomenclature (SUN) criteria. Active uveitis was anterior chamber inflammation grade $\geq 0.5+$ cells. S100A8, A9, and A12 were measured by ELISA, and IL-18, IL-8, IP-10, MCP-1, RANT $\mathrm{ES}$, and sICAM-1 by Luminex assays. Biomarker levels were compared in children with 1) JIA-no-U (n=8) to active JIA-U (n=8), and 2) JIA-U $(n=8)$ at time of active and inactive uveitis.

Results: Children with JIA-no-U and JIA-U were matched by JIA subtype and arthritis activity. They had primarily oligoarticular JIA (63\%), active arthritis $(25 \%)$, and were on systemic medication (75\%). At time of active uveitis, $75 \%$ had grade $0.5+$, and $25 \%$ had $1+$ and mean interval between time of active and inactive disease was 11 months. We found that levels of biomarkers in tears of children with JIA-no-U compared to active JIA-U were similar. Although not statistically significant, levels of S100A12 (mean difference $12,190 \mathrm{pg} / \mathrm{ML}$ [95\% Cl -4847 to 29,227 ], $\mathrm{P}=$ $0.14)$ and sICAM-1 (5329 pg/ML [95\% Cl -5372 to 16,031$], \mathrm{P}=0.28)$ were higher when uveitis was active compared to inactive.

Conclusion: Our results suggest that S100A12 and sICAM-1 are potential biomarkers of uveitis activity in JIA-U, but not uveitis diagnosis. Thus, neutrophils may play a role in the pathogenesis of anterior uveitis which has been reported in an animal model of acute anterior uveitis. Identifying biomarkers using tears provides a noninvasive and objective method of monitoring uveitis. Limitations are our heterogeneous cohort that varied by arthritis severity and immunosuppression, and minimally active uveitis. We were underpowered to detect statistically significant differences and continue to collect tears prospectively in children with JIA-U with goal of $n=28$. Despite low uveitis activity, we were still able to detect differences. Further studies in larger and diverse cohorts are necessary to assess the role of S100A12 and SICAM-1 in JIA-U.

\section{Disclosure of Interest}

J. Rodriguez-Smith: None declared, V. Miraldi Utz : None declared, S. Thornton: None declared, G. Schulert Consultant for: Novartis, SOBI, A. Sproles: None declared, N. Mwase: None declared, T. Hennard: None declared, A. Grom Consultant for: AB2Bio, Novartis, SOBI, M. Altaye: None declared, S. Angeles-Han: None declared

\section{P280}

Case report of acute digital ischaemia in a 12 year old boy with granulomatosis with polyangiitis

K. Gallagher, S. Deepak, S. Rangaraj, K. Warrier

Nottingham Children's Hospital, Nottingham, United Kingdom

Correspondence: K. Gallagher

Pediatric Rheumatology 2020, 18(Suppl 2):P280

Introduction: Granulomatosis with polyangiitis(GPA) is a rare vasculitis involving medium and small arteries, affecting predominantly upper and lower respiratory tracts, often with glomerulonephritis. Typically it is characterised by necrotising granulomatous inflammation and the presence of anti-neutrophil cytoplasmic antibodies(ANCA).

Objectives: To report an extremely rare presentation of GPA in a 12 year old with acute digital ischemia.

Methods: A 12 year old boy, with a background of poorly controlled type 1 diabetes and hypothyroidism, initially presented to hospital unwell with diabetic ketoacidosis. Treatment was initiated promptly with good response. Furthermore, he was found to have weight loss, productive cough and hearing loss over the past 3 months.

He was haemodynamically stable, but very pale and cachectic. He had reduced air entry and crackles on the right. There was hypertonia and clonus in his lower limbs.

Blood tests showed microcytic hypochromic anaemia ( $\mathrm{Hb} 82 \mathrm{~g} / \mathrm{L})$, normal white cell count, thrombocytosis and raised inflammatory markers (CRP $138 \mathrm{mg} / \mathrm{L}$ and ESR $68 \mathrm{~mm} / \mathrm{hr}$ ). His chest $\mathrm{x}$-ray showed enlargement of the right hilum with consolidation/ atelectasis extending into the middle and lower lobes. MRI scans of head and spine were normal apart from fluid opacification in the paranasal sinuses. He was screened for infections including Tuberculosis and started on intravenous antibiotics.

On day 13 , he developed painful bluish discolouration of his left hand, particularly his thumb, index and middle fingers. His left radial and brachial pulses weren't palpable. A heparin infusion was started. A Doppler scan showed occlusion of radial and ulnar arteries proximal to the wrist with no clear thrombus.

He had a CT thoracic aorta with contrast which showed proximal left radial artery occlusion and distal ulnar artery occlusion with no evidence of proximal embolic source or vasculitis. It showed multiple perihilar masses (lymph nodes) in the right lung and peripheral parenchymal masses in both lungs, suggestive of atypical infection or connective tissue disease.

Blood tests still showed raised inflammatory markers(CRP $107 \mathrm{mg} / \mathrm{L}$, ESR $86 \mathrm{~mm} / \mathrm{hr}$ and platelets $65810^{9} / \mathrm{L}$ ). An autoantibody screen showed positive ANCA with strongly positive anti PR3(>100 U/mL); 
other autoantibodies, including ANA, ds DNA and anti-phospholipid antibodies, were negative.

He developed further ischaemia with bluish, painful discoloration of his right foot, especially right great toe, with a weakly palpable dorsalis pedis pulse. Doppler scan revealed occlusion/narrowing of the posterior tibial artery $6 \mathrm{~cm}$ proximal to the ankle. Following vascular team advice, he was started on ilioprost infusion to aid reperfusion of the extremities involved, with good results.

Based on clinical and lab features of systemic inflammation, evidence of upper airway involvement(bilateral conductive hearing loss and sinusitis on MRI scan), parenchymal lesions on CT chest and strong PR3 positivity, a diagnosis of GPA was made.

Results: Our patient responded well to therapy including multiple pulses of high dose methylprednisolone and cyclophosphamide, with improvement of all organs involved and no further digital ischemia.

Conclusion: Although GPA is very rare in children, it is associated with high morbidity and mortality. Many studies show that the spectrum of paediatric GPA is not vastly different from adults, except for higher gender bias towards female, more constitutional and musculoskeletal symptoms and higher risk of subglottic stenosis. Although there are a handful of case reports of digital ischaemia in adults with GPA, to our knowledge this is the first case report of acute digital ischaemia in paediatric GPA. Early diagnosis and prompt treatment with a multidisciplinary team approach is paramount for good outcome.

\section{Disclosure of Interest}

None declared

\section{P281}

Childhood polyarteritis nodosa - diagnosis using selective visceral angiography

T. Hospach, F. Blankenburg, F. Glowatzki, C. Stirnkorb, A. Heinkele, T. von Kalle, U. Schweigmann, F. Uhlemann

Olgahospital, Stuttgart, Germany

Correspondence: $T$. Hospach

Pediatric Rheumatology 2020, 18(Suppl 2):P281

Introduction: To diagnose polyarteritis nodosa (PAN), either histopathological or imaging evidence of vasculitis is necessary. In our case, the diagnosis could not be confirmed either by histology or by angiography MRI. It was only when conventional selective visceral angiography was carried out that the decisive evidence about the diagnosis was made

Objectives: To report on the difficulties in diagnosing childhood PAN:

Methods: Case report: This 10 year old girl with parents of Turkish origin presented with fever up to $40{ }^{\circ} \mathrm{C}$ over 14 days, distinct myalgia and cutaneous efflorescences. The general condition was massively impaired, the girl could not turn around in bed without foreign help. The CRP was $24 \mathrm{mg} / \mathrm{dl}$, the ESR $100 \mathrm{~mm} / \mathrm{h}$. The girl had microscopic hematuria, the blood pressure was intermittently greater than 95th centile for height. The MRI showed distinct increased signal intensity in the muscles of the lower limbs. Under the suspected diagnosis of PAN, a deep muscle biopsy in the area of the signal intense muscles was performed. However, no arteries were detectable in the biopsy, so that a diagnosis could not be made. The angiography MRI performed afterwards was normal. It was only through conventional selective visceral angiography that the diagnosis could be confirmed by detecting multiple aneurysms.

Results: Discussion: To ensure the diagnosis of a PAN, one of the two mandatory criteria must be met. A definitive confirmation of the diagnosis is of crucial importance, since the disease can be complicated and result in intensive and aggressive therapy. In our case, the high degree of clinical suspicion could not be confirmed histopathologically or by angiography MRI. Conventional selective visceral angiography. This method offers the advantage of a good detailed representation of small aneurysms and is superior in resolution to MRI and - although less - to CT angiography. It should be noted that all intra-abdominal vessels (Celiac trunk, renal-, mesenteric-, hepatic- artery) are examined. However, at an early stage of the disease or under therapy, aneurysms may not be detectable by imaging. However, as an invasive procedure, conventional selective visceral angiography may have complications and should therefore only be carried out by experienced examiners. It is generally recommended that an MRI-based targeted deep biopsy should be performed. This should measure a size of at least $2.5 \times 0.5 \mathrm{~cm}$ to capture arterial vessels as possible. If the biopsy is negative, a CT or conventional selective visceral angiography can be performed afterwards.

Conclusion: To ensure the diagnosis of a PAN, all diagnostic options - including conventional selective visceral angiography - should be consistently exhausted. Therefore a close cooperation between the involved different disciplines is recommended.

Disclosure of Interest

None declared

\section{P282}

Different clinical presentation of adenosine deaminase-2 deficiency in two sisters

P. O. Avar-Aydin, F. Yalcinkaya, Z. B. Ozcakar, N. Cakar

Department of Pediatric Rheumatology, Ankara University School of

Medicine, Ankara, Turkey

Correspondence: P. O. Avar-Aydin

Pediatric Rheumatology 2020, 18(Suppl 2):P282

Introduction: Adenosine deaminase-2 deficiency (DADA2) is a monogenic vasculitis syndrome whose presentation ranges from recurrent fevers and livedo reticularis to systemic vasculitis, hematologic and immunologic abnormalities, and early-onset stroke. It is characterized by biallelic loss-of-function mutations in the encoding gene of ADA2 protein and low levels of ADA2 enzymatic activity in the peripheral blood. The genotype and phenotype features of DADA2 has a wide spectrum. Treatment with anti-TNF inhibitors is effective in controlling vascular inflammation and reducing strokes.

Objectives: To describe two sisters with different presentations of DADA2 and a deletion mutation on exon 7 of the ADA2 gene.

Methods: Medical data was used to describe the clinical manifestations of two siblings. Parental informed consent was obtained.

Results: Patient 1: A 10-year-old female had presented with fever, rash, arthralgia, hepatosplenomegaly, and coombs positive autoimmune hemolytic anemia (AIHA) at the age of 7 years. She had been followed with a suspected diagnosis of systemic lupus erythematosus (SLE) and steroids, azathioprine, mycophenolate mofetil had been used. Her ANA and complement levels were normal. Because of unmet classification criteria of SLE, genetic testing had been done, and no mutation found in the ADA2 gene. Cranial MR and MR angiography was normal.

She was referred to our clinic after 2.5 years of the first manifestation. Physical examination revealed Raynaud phenomenon on both hands and feet, livedo reticularis, arthritis, and splenomegaly. Laboratory tests indicated an increase in acute phase reactants, CD19, CD20, and switched memory B cell lymphopenia, and hypogammaglobulinemia. Because of prolonged fevers, a thorax CT was obtained and aneurisms of the renal artery were seen. Abdominal CT angiography indicated multiple aneurysms of both renal, intercostal, and hepatic arteries. Repeated genetic analysis of the ADA2 gene showed a homozygous deletion mutation on exon 7. She has been followed on anti-TNF and IV immunoglobulin without severe symptoms for a year.

Patient 2: The older sister had been followed with a diagnosis of familial Mediterranean fever with E148Q heterozygous mutation because of recurrent fever, abdominal pain, erysipelas-like erythema, elevated acute phase reactants, and splenomegaly. She did not have any other cutaneous or systemic findings. Because of parental consanguinity, the ADA2 gene was analyzed and a homozygous deletion mutation on exon 7 was found. She has been followed without any symptoms after anti-TNF treatment. 
Conclusion: We presented two siblings from a consanguineous marriage with different clinical presentations of DADA2. Further, we emphasize that genetic testing should be repeated in the presence of clinical suspicion.

Disclosure of Interest

None declared

\section{P283}

Gastrointestinal Henoch-Schönlein purpura treated with mycophenolate mofetil: description of two case reports

M. F. Gicchino, D. lafusco, M. M. Marrapodi, R. Melone, G. Cuomo, A.

Zanfardino, E. Miraglia del Giudice, A. N. Olivieri

University of the Study of Campania Luigi Vanvitelli, Naples, Italy

Correspondence: M. F. Gicchino

Pediatric Rheumatology 2020, 18(Suppl 2):P283

Introduction: Henoch Schönlein purpura (HSP) is the most common vasculitis in children. HSP prognosis is generally good, recurrence is common among children. Morbidity and mortality are associated with gastrointestinal erosions and nephritis.Treatment is supportive,with control of pain with analgesics.There are retrospective studies that suggest a benefits of steroids to treat abdominal,renal involvement and severe orchitis. Immunosuppressive treatment of HSP nephritis is used in patients with severe renal involvement. There are few data in literature about immunosuppressive treatment of recurrent HSP without kidney involvement

Objectives: We report the successful use of Mycophenolate Mofetil( MMF) in two patients affected from recurrent gastrointestinal HSP

Methods: Case 1: A 15 years old girl after a pharyngitis presented rash, so she was hospitalized, vasculitis was diagnosed, and steroids treatment was started. At steroids suspension patient presented hematemesis and purpuric lesions, so underwent both to an esophagogastroduodenoscopy that was negative and to a skin biopsy, suggestive of leukocytoclastic vasculitic with IgA deposition. HSP was confirmed and oral prednisone was prescribed. At steroids reduction purpuric lesions, hematemesis, abdominal pain came again, so she came to our observation. She presented purpuric lesions and abdominal pain. Blood tests were unremarkable for: complete blood count,ANA, ENA,ANCA, RF,immunoglobulins, complement C3 and C4. Factor XIII activity was reduced. Inflammatory parameters were slightly increased: ERS $25 \mathrm{~mm} / \mathrm{h}, \mathrm{CRP}$ $1 \mathrm{mg} / \mathrm{dL}$. Urinalysis was normal. Occult blood in the stool was present. Throat swab was negative. Abdomen ultrasound showed bowel wall thickening. Oral prednisone was prescribed, with initial improvement of symptoms. After few days she presented new purpuric lesions and abdominal pain. Due to the recurrent nature of her symptoms and the lack of a sustained response to steroids, $\operatorname{MMF}\left(600 \mathrm{mg} / \mathrm{m}^{2}\right.$ twice a day) was started and prednisone was gradually tapered in a month

Case 2: After a respiratory tract infection a 13 years old boy presented purpuric lesions on the limbs with spontaneous resolution in 3 days. After a week purpuric lesions and abdominal pain came again so he was evaluated to emergency department. Abdominal ultrasound was normal, urinalysis revealed proteinuria. HSP was diagnosed, but no treatment was prescribed. Due to the persistence of vasculitic lesions, abdominal pain and the onset of gastrointestinal bleeding patient was hospitalized to our Department. On examination purpuric lesions were detected. Patient referred both abdominal and testis pain. Blood tests were unremarkable for: complete blood count, ERS, CRP,ANA,ENA, ANCA, RF, immunoglobulins, complement C3 and C4. Factor XIII activity was reduced. Virological screening showed Immunoglobulin M versus Influenza Virus. Urinalysis revealed proteinuria. Throat swab was negative. Abdomen ultrasound showed bowel wall thickening, testis ultrasound was normal. HSP diagnosis was confirmed. Methylprednisolone iv was administered for three days, then oral prednisone was started. Purpuric lesions, abdominal pain persisted, so we decided to add MMF $(600 \mathrm{mg} /$ $\mathrm{m}^{2} /$ day) and prednisone was tapered in a month.
Results: Thanks to MMF vasculitis lesions and abdominal symptoms disappeared in few days. MMF was continued for a month, tapered in 6 months. There was no evidence of relapse in a 6 months follow up. Conclusion: These cases suggest that MMF may be useful to induce and maintain remission of recurrent HSP with gastrointestinal involvement. Multicenter clinical trials with long-term follow up to confirm the efficacy of MMF in the treatment of HSP with gastrointestinal involvement are needed.

Disclosure of Interest

None declared

\section{P284}

Henoch-Schönlein purpura complicated by calculus cholecystitis

A. A. Abushhaiwia', M. A Zletni ${ }^{2}$, A. Ateeg ${ }^{3}$, A. Alwaddan $^{4}$, A. Malek $^{1}$

${ }^{1}$ pediatric Rheumatology, Tripoli faculty of medicine; ${ }^{2}$ pediatric rheumatology, Tripoli university Faculty of Medicine; ${ }^{3}$ pediatric departement, Tripoli Faculty of Medicine; ${ }^{4}$ pediatric departement

Tripoli children's hospital , Tripoli , Libya

Correspondence: A. A. Abushhaiwia

Pediatric Rheumatology 2020, 18(Suppl 2):P284

Introduction: Henoch-Schönlein purpura (HSP), the most common childhood vasculitis. Cholecystitis is extremely rare in patients with HSP. This is the first case of a Libyan child presenting with HSP complicated by calculus cholecystitis HSP nephritis.

Objectives: our aim is to present an unusual case of gall bladder involvement in an 8-year-old Libyan female affected by HSP.

Methods: a case reports study

Results: : we report an unusual case of gall bladder involvement in an 8-year-old Libyan female with HSP. She was referred to a rheumatology clinic due to HSP with chronic calculus cholecystitis and distended small bowel with fluid-like fecal material with no evidence of intussusception on an abdominal ultrasound.

The patient had a one-month history of abdominal pain, purpuric lesion on lower limbs and swelling in both feet. She was admitted 3 times to another hospital before being referred to the rheumatology clinic. An abdominal sonography revealed a distended small bowel with fluid-like fecal material with no evidence of intussusception and chronic calculus cholecystitis; they treated her with Urosdoxycholic acid tab at $250 \mathrm{mg}$ per day and ibuprofen syrup. Then referred to our rheumatology clinic. After 40 days, she showed a purpuric rash over her lower extremities, mainly over her thighs and buttocks, microscopic hematuria, no arthritis, no fever, no abdominal pain; her blood pressure was normal at $90 \backslash 55 \mathrm{mmHg}$, and she had normal laboratory tests (CBC, WBC 7.7, HGB 10.8, Platelets 356 ESR 20ml hour, CRP $1 \mathrm{mg} \backslash \mathrm{dl}$ was negative, $\mathrm{C} 3$ was $150 \mathrm{mg} \backslash \mathrm{dl}$ within normal range $90-$ $180 \mathrm{mg} \backslash \mathrm{dl}$, C4 was $35.4 \mathrm{mgldl}$ within normal range 10-40, ANCA, $A N A$, as well AntidsDNA Ab yielded negative, Antistreptolysin-O (ASO) titer was 250 Todd, LFT included total bilirubin , direct , indirect GPT,GOT, UIE, creatinine ) except urine routine showed mild microscopic hematuria RBC $100 \mathrm{HPF}$, protein was Nil ) urine for pro-

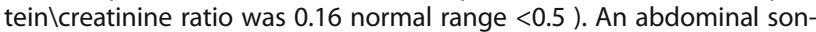
ography showed multiple stones, with faint acoustic shadow, the largest of which were $7 \mathrm{~mm}$, no signs of acute inflammation. We initially treated her with oral prednisolone tab at $1 \mathrm{mg} \backslash \mathrm{kg} \backslash$ day and enalopril tab $2.5 \mathrm{mg}$ once per day, All clinical manifestations resolved within one week, including normal gallbladder sonography finding, besides microscopic hematuria RBS was $80-85 \mathrm{HPF}$; she had nephrotic syndrome urine proteinlcreatinine ratio was 1.99 (normal range is < 0.5 ) in spite of taking prednisolone tab for 4 weeks. The treatment with MMF $30 \mathrm{mg} / \mathrm{kg} / \mathrm{day}$ commenced and the oral prednisolone (2 $\mathrm{mg} / \mathrm{kg} / \mathrm{every}$ other day for 2 months only) after treatment with MMF for two months, microscopic hematuria continued for 3 months and recovery from nephrotic syndrome within 2 weeks. After six months, MMF was discontinued and there was no clinical or laboratory abnormalities. The patient achieved complete remission at the end and after discontinuation of the therapy. There was no evidence of recurrence over one-year follow-up.

Conclusion: Physicians should be aware that HSP might present initially as calculus cholecystitis. Repeat abdominal ultrasonography 
is essential in order to detect complications in patients with HSP. MMF was found to be a useful immune- suppressant and effective for maintenance of remission in HSP patients.

Disclosure of Interest

None declared

\section{P285}

Inflammatory bowel disease and takayasu arteritis: a case report

R. L. Pybus ${ }^{1}$, J. Sims², M. Wood ${ }^{3}$, N. Tahir ${ }^{4}$, V. Zamvar ${ }^{1}$

${ }^{1}$ Paediatric Gastroenterology, Leeds Teaching Hospitals Trust, Leeds;

${ }^{2}$ Paediatrics, Bradford Teaching Hospitals Trust, Bradford; ${ }^{3}$ Paediatric

Rheumatology; ${ }^{4}$ Paediatric Radiology, Leeds Teaching Hospitals Trust,

Leeds, United Kingdom

Correspondence: R. L. Pybus

Pediatric Rheumatology 2020, 18(Suppl 2):P285

Introduction: Takayasu's arteritis (TA) is extremely rare, especially in childhood, and its occurrence with inflammatory bowel disease (IBD) is even rarer. There have been suspected associations between TA and IBD in adult literature, but only a few paediatric case reports.

Objectives: We describe a case of a girl $(\mathrm{X})$ with Pakistani origin, diagnosed with indeterminate pan colitis, age 4 following her presentation with abdominal pain, weight loss, blood and mucus PR. Rescoping 2 years later, as steroid dependant, confirmed ulcerative colitis and she subsequently went into remission with Sulfasalazine and Azathioprine. Age 12, X presented with 3 months history of right sided neck pain, fatigue, pain in her lower limbs, intermittent fevers, night sweats and weight loss but no changes in her bowel symptoms. On examination she was pale, tachycardic at $135 \mathrm{bpm}, \mathrm{BP}$ $101 / 61 \mathrm{mmHg}$ with no discrepancy between limbs. She had right sided torticollis despite full range of neck movements.

Methods: X was admitted for pain management and noted to have raised inflammatory markers, anaemia and thrombocytosis. She had a neck ultrasound which revealed evidence of large vessel arteritis. CT angiogram showed arteritis of the thoracic aorta with aneurysmal change with involvement of the proximal arch vessels and proximal superior mesenteric artery; consistent with a diagnosis of Takayasu arteritis. MRI confirmed the findings. X was started on IV pulsed Methylprednisolone $30 \mathrm{mg} / \mathrm{kg}$ for 3 days followed by weaning dose of oral Prednisolone, weekly SC Methotrexate and low dose Aspirin therapy. Treatment was switched to Mycophenolate Mofetil and later Tocilizumab, due to inadequate control of TA with SC Methotrexate, suggested by blood tests and serial US of large artery walls. Her disease remains stable and Prednisolone has been weaned to $5 \mathrm{mg}$ daily.

Results: The exact causative mechanism for both IBD and TA is unclear; however there may be genetic markers which link both diseases. A significantly higher proportion of patients with IBD and TA were HLA-B52 positive compared to TA alone. Other genetic markers including HLA-DR2 and IL-12B have also been shown to be present in both diseases. There are reports that Infliximab has been effective to treat combined IBD and TA; this could support the theory that TNF-alpha may have a role in both conditions leading to mucosal and vessel inflammation. There is currently no NHS England funding pathway for anti-TNF alpha medications for TA, however Tocilizumab can be used.

Conclusion: TA and IBD is extremely rare and requires a high degree of suspicion. Ongoing symptoms of pain, weight loss, fever and high inflammatory markers, in patients with otherwise well controlled IBD, should raise suspicion of alternative pathologies such as Takayasu arteritis. Neck and back pain in this child aided the journey to a diagnosis of TA due to direct pressure effects of enlarged arteries, but these symptoms are unusual. Early diagnosis and prompt treatment are likely to reduce morbidity and mortality in these patients who already suffer a high disease burden.

\section{Disclosure of Interest}

None declared

\section{P286}

Kawasaki disease in early infancy - case series

K. Rücklová ${ }^{1,2}$, Š. Fingerhutová2, P. Doležalová

${ }^{1}$ Faculty Hospital Královské Vinohrady; ${ }^{2}$ General University Hospital,

Prague, Czech Republic

Correspondence: K. Rücklová

Pediatric Rheumatology 2020, 18(Suppl 2):P286

Introduction: Kawasaki disease in infants typically presents as an incomplete or atypical form, which often results in delayed diagnosis and treatment thereby increasing the risk of coronary artery involvement.

Objectives: To draw attention to diagnostic and therapeutic challenges posed by patients with Kawasaki disease manifesting at very early age.

Methods: Medical reports and laboratory and echocardiographic findings of three infants treated at our centre for Kawasaki disease presenting below four months of age were analyzed retrospectively. Results: The first patient presented at the age of almost four months with fever and shock that required inotropes and artificial ventilation. Due to the atypical presentation the diagnosis was delayed until day seventeen of the illness when the patient developed giant coronary aneurysms and severe mitral valve insufficiency. Another patient manifested at three months of age and fulfilled most of the clinical criteria and received appropriate treatment already on the fifth day of the illness. He exhibited multiple risk factors for an adverse outcome including low age, very high CRP, thrombocytopenia, hyponatremia and hypalbuminemia with pronounced vascular leakage. After administration of IVIG and corticosteroids his clinical condition improved significantly. He was afebrile but his CRP remained mildly elevated and he experienced another flare of the disease within four weeks after the initial treatment with newly formed giant coronary aneurysms. He received adalimumab and pulsed methylprednisolone that finally terminated the inflammation. The last patient manifested at two months with a complete form of Kawasaki disease that prompted timely IVIG treatment. Despite repeated administration of IVIG only partial improvement was achieved and the patient developed giant coronary aneurysms during the second week of illness. Further therapeutic decision was complicated by the patient's concurrent primary CMV infection.

Conclusion: The first case demostrates a diagnostic challenge posed by patients presenting with Kawasaki disease shock syndrome. The second patient shows the need for very close follow-up of high risk patients. Even though our patient was afebrile after the initial treatment, his CRP remained elevated and the inflammatory process continued requiring biological therapy and escalation of antiaggregation to anticoagulation. The last patient emphasizes the importance of treating both Kawasaki and the concurrent illness that may potentiate the vasculitis as a trigger.

Supported by Ministry of Health of the Czech Republic, grant nr. NV18-02-00237.

\section{Disclosure of Interest}

None declared 


\section{P287}

Kawasaki disease complicated by macrophage activation syndrome and parvovirus b19 infection: which relationship? M. G. Magnolia', S. Martelossi $i^{2}$, C. Stefani $i^{3}$, L. Da Dalt ${ }^{4}$, G. Martini ${ }^{5}$

'Pediatric and Neonatologic Unit, "Santa Maria della Stella" Hospital, Orvieto, ${ }^{2}$ Pediatric Unit, "Ca' Foncello Hospital", ${ }^{3}$ Pediatric Unit, "Ca' Foncello" Hospital, Treviso, ${ }^{4}$ Pediatric Emergency Unit, ${ }^{5}$ Pediatric Rheumatology Unit, Department of Woman and Child Health, Padova, Italy

Correspondence: G. Martin

Pediatric Rheumatology 2020, 18(Suppl 2):P287

Introduction: Macrophage activation syndrome (MAS) is characterized by massive production of cytokines leading to macrophage activation and haemophagocytosis presenting with prolonged fever, rash, hepatosplenomegaly, pancytopenia, liver dysfunction, hypertriglyceridemia, hyperferritinemia and coagulopathy that can complicate rheumatic conditions such as Systemic Juvenile Idiopathic Arthritis (sJIA) and Systemic Lupus Erythematosus (SLE). Incidence of MAS in Kawasaki Disease (KD) has been estimated in about $1.1 \%$ patients but subclinical MAS may be detected in $30-40 \%$ of $\mathrm{KD}$.

Objectives: Case description

Methods: A previously healthy 10 years-old girl presented high grade fever for 4 days, pharyngitis and vomiting. After 24 hours, she developed diffuse maculo-papular rash and oedema on extremities. She presented progressive worsening of general conditions and bilateral bulbar conjunctivitis, mucositis with strawberry-like tongue and left cervical lymph nodes enlargement. On admission remarkable laboratory tests were increased $C$ reactive protein (CRP), neutrophilic leucocytosis, low sodium and albumin, increased gGT and gallbladder hydrops on abdominal ultrasound. Suspecting Kawasaki disease $2 \mathrm{gr} /$ $\mathrm{kg}$ IVIG were administered with salicylic acid ( $50 \mathrm{mg} / \mathrm{kg} /$ day). Nevertheless, she presented persistent remitting fever, low consciousness, diffuse vasculitic rash, worsening of mucositis and pericardial and pleural effusion. Lab tests showed low haemoglobin, platelets and fibrinogen $(9,3 \mathrm{~g} / \mathrm{L}, 65.000 / \mathrm{ml}$ and $1.05 \mathrm{~g} / \mathrm{L}$, respectively), ferritin $16.492 \mathrm{~g} / \mathrm{L}$, SGOT $487 \mathrm{U} / \mathrm{L}$, SGT $351 \mathrm{U} / \mathrm{L}$, triglycerides $345 \mathrm{mg} / \mathrm{dl}$, Ddimers $10.353 \mathrm{microgr} / \mathrm{L}$ and soluble interleukin-2 receptor (sIL-2R), $6464 \mathrm{kU} / \mathrm{L}$. Active haemophagocytosis was retrieved in bone marrow and cerebrospinal fluid (CSF) so MAS was diagnosed. Three consecutive iv methylprednisolone pulses $(30 \mathrm{mg} / \mathrm{kg})$ were administered followed by dexamethasone $10 \mathrm{mg} / \mathrm{m} 2 /$ day and cyclosporin A $2 \mathrm{mg} /$ $\mathrm{kg} /$ day as well as plasma infusions and oxygen supplementation ( $6 \mathrm{l} /$ min) for 48 hours. Parvovirus B19 (HPVB19) DNA was found in peripheral blood, bone marrow and CSF, while other microbiological analysis (EBV, CMV, HHV6, VZV, Influenza A-B, Measles, Adenovirus, HSV) were negative. The patient progressively improved with reduction of fever, oedema of extremities and skin rash and after 6 days presented extensive desquamation on hands, feet and limbs. Lab tests slowly improved and normal values were achieved on day 23 . Echocardiogram did not show any coronary aneurism or dilatation, cerebral MRI was normal and neurological impairment gradually disappeared. Primary HLH mutations for UNC13D, STXBP2, STX11, RAB27a, SH2D1A, XIAP were not found. Corticosteroids and Ciclosporin were gradually tapered and discontinued after 7 and 12 months respectively, whilst acetylsalicylic acid was stopped after 2 months.

Results: MAS is a relatively infrequent complication in KD and may be associated with severe course and poor outcome. Several potential infectious agents have been suggested as trigger factors of both MAS and KD, such as Epstein Barr virus, Influenza virus etc. and, more recently, the SARS-COV-2 epidemic has been associated with severe forms of systemic inflammatory syndrome resembling KD and MAS.
Conclusion: To the best of our knowledge, this is the first case in which demonstration of HPVB19 DNA in peripheral blood, bone marrow and CSF during acute phase strongly suggests a direct role of the virus in triggering both KD and MAS rather than an antibody or immune-complex mediated mechanism.

Disclosure of Interest

None declared

P288

Spectrum of vasculitis in $\mathbf{2 7}$ children from a single center in an Indian state of Gujarat

D. B. Pandya ${ }^{1}$, on behalf of Dr Haresh Dobariya, Dr Hitesh Bhambhani,

Dr Jay Dhirwani, Dr Rakesh Patel, Dr Nayan Kalawadia,Dr Sudhir

Rughani and Dr Savan Vamja.

Pediatric Rheumatology \& Immunology, Dev Children's Hospital, Rajkot,

India

Correspondence: D. B. Pandya

Pediatric Rheumatology 2020, 18(Suppl 2):P288

\section{Introduction}

There is a limited awareness about vasculitis amongst primary physicians in our region. There is not a single exclusive pediatric rheumatology center in our region catering around 20 million people. $^{1-3}$

Objectives

To unveil characteristics of vasculitis in children in our region.

Methods

I gathered a data of 27 children with confirmed diagnosis of some form of vasculitis who attended Dev Children's Hospital between January 2019 and January 2020. It included demographics, clinical presentations, laboratory results, treatment and follow up.

Results

\section{Conclusion}

The most common vasculitis in our cohort is Kawasaki disease. The number of incomplete KD patients was almost same as complete KD. Almost all our KD patients responded to IV Ig. None of the children with KD developed coronary artery abnormalities. Gastrointestinal complications were seen to be associated with four patients of $\lg A$ vasculitis at onset. Two children with IgA vasculitis developed renal complication within 3 months of disease onset.

Trial registration identifying number

1. Review.Indian J Pediatr2010 Sep;77(9):993-6.

doi: 10.1007/s12098-010-0134-x. Epub 2010 Sep 3.

The Place of Pediatric Rheumatology in India

Sujata Sawhney 1, Prudence Manners

2. Journal of Natural science,Biology \& Medicine-2018

Clinico-epidemiological profile of pediatric rheumatology disorders in Eastern India

PratapKumarPatra, ManishKumar

Department of Pediatrics, All Institute of Medical Sciences, Patna, Bihar, India

3. International Journal of Advanced Medical Health \& Research (JIPMER)

Pediatric rheumatology: An under-recognized subspecialty in India

Year : 2017 | Volume : 4 | Issue : 2 | Page : 47-53

AkhilaKavirayani1, SumaBalan2

1 Department of Paediatric Rheumatology, Oxford University Hospitals NHS Foundation Trust, Oxford, United Kingdom

2 Department of Paediatric Rheumatology, Amrita Institute of Medical Sciences, Kochi, Kerala, India

Disclosure of Interest

None declared 
Table 1 (abstract P288). showed detailed analysis of children with Vasculitis at Dev Children's Hospital

\begin{tabular}{|c|c|}
\hline Parameter & $\begin{array}{l}\text { No }(\%) \text { of children fulfilled a } \\
\text { respective parameter }\end{array}$ \\
\hline Age $<3$ months & $1(3.5 \%)$ \\
\hline 3 months- 2 years & $8(29.5 \%)$ \\
\hline 2 years -5 years & $9(33.5 \%)$ \\
\hline$>5$ years & $9(33.5 \%)$ \\
\hline Sex : Male & $18(66.6 \%)$ \\
\hline Female & $9(33.5 \%)$ \\
\hline Incomplete Kawasaki disease (KD) & $8(29.5 \%)$ \\
\hline Complete Kawasaki disease & $9(33.5 \%)$ \\
\hline IgA Vasculitis (IgA-V) & $10(37 \%)$ \\
\hline $\begin{array}{l}\text { Coronary artery involvement in KD } \\
\text { patients at onset }\end{array}$ & None \\
\hline $\begin{array}{l}\text { Renal involvement in IgA-V patients at } \\
\text { onset }\end{array}$ & $1(10 \%)$ \\
\hline $\begin{array}{l}\text { Gastrointestinal complications in lgA-V } \\
\text { patients }\end{array}$ & $4(40 \%)$ \\
\hline $\begin{array}{l}\text { Echocardiography findings at diagnosis } \\
\text { in } \mathrm{KD}\end{array}$ & $7(44 \%)$ \\
\hline \multicolumn{2}{|l|}{ Pericarditis } \\
\hline $\begin{array}{l}\text { Treatment (IV Ig within } 12 \text { days of fever } \\
\text { in all KD patients) }\end{array}$ & $15(88 \%)$ \\
\hline $\begin{array}{l}\text { Intravenous Immunoglobulin Infusion (IV } \\
\text { Ig) - single dose }\end{array}$ & $2(12 \%)$ \\
\hline IV Ig - two doses & 2 (KD and $\lg A-V$ patient each ) \\
\hline IV methyl prednisolone & 9 (lgA-V patients) \\
\hline \multicolumn{2}{|l|}{ Oral steroids } \\
\hline $\begin{array}{l}\text { Coronary artery involvement in } \mathrm{KD} \\
\text { patients at 2,4,6 weeks }\end{array}$ & 0 \\
\hline $\begin{array}{l}\text { Renal involvement in IgA-V patients at } \\
1,2,3 \text { months }\end{array}$ & $1(10 \%)$ \\
\hline
\end{tabular}

\section{P289}

Autoimmune diseases as non-cardiogenic complications of Kawasaki disease

V. Osterman', T. Avramoska', M. Bizjak², G. Markelj', M. Pokorn ${ }^{3,4}$, T. Avčin 2,4

${ }^{1}$ Department of Infectious diseases, University Medical Centre Ljubljana; ${ }^{2}$ Department of Allergology, Rheumatology and Clinical Immunology; ${ }^{3}$ University Children's Hospital; ${ }^{4}$ Faculty of Medicine, University of Ljubljana, Ljubljana, Slovenia

Correspondence: $\mathrm{V}$. Osterman

Pediatric Rheumatology 2020, 18(Suppl 2):P289

Introduction: Kawasaki disease (KD) is an acute, immune-mediated multisystem vasculitis of unknown etiology that most frequently affects infants and young children. Immunological abnormalities during the acute phase of KD have been described extensively and coronary artery aneurysms (CAA) identified as a main long term complication. The occurrence of non-cardiac, autoimmune complications in patients with history of KD was rarely reported.

Objectives: Our aim was to evaluate the presence of autoimmune diseases (AID) in patients after KD diagnosis and to compare clinical, demographic and laboratory characteristics between patients who developed AID (group 1) and patients who did not (group 2).

Methods: A single-center, retrospective cohort study with longitudinal follow-up of all children newly diagnosed with KD between June 2006 and December 2018 was performed. In total, 151 children
(91 males and 60 females, median age 3.4 years) with KD were enrolled. Range of follow up was from 1 to 13 years after KD (mean follow-up 16.8 months).

Results: Of 151 children with KD, 16 (10.6\%) developed AID after KD including: 6 juvenile idiopathic arthritis (JIA), 6 macrophage activation syndrome (MAS), 3 coeliac diseases, 1 ulcerative colitis, 1 psoriasis, 1 hypothyroidism and 1 demyelination. Three patients presented with more than one AID. The mean time interval between diagnosis of KD and diagnosis of AID was 30.5 months (range 0.2 149 months). We found no difference in age distribution, clinical characteristics and incidence of CAA between the two groups. Group 1 had significantly lower median serum sodium (135 vs $137 \mathrm{mmol} / \mathrm{L}$, $\mathrm{p}=0.002$ ), lower median platelet count ( 262 vs $363 \times 10^{9} / \mathrm{L}, \mathrm{p}=$ $0.026)$ and higher neutrophil to lymphocyte ratio (9.4 vs $4, p=$ 0.009). There was also significantly higher percentage of IVIG resistance in group $1(37.5 \%$ vs $12.6 \%, p=0.022)$. Moreover, we found that children with KD had twice as many AID compared to CAA on long term follow-up ( $10,6 \%$ and $5,3 \%$ respectively).

Conclusion: In our cohort, children with KD had twice as many AID when compared to CAA on long term follow up. Monitoring of children after KD for AID should be considered, especially in patients who were resistant to IVIG treatment. Additional studies are necessary to clarify the possible role of sodium, platelet count and neutrophil to lymphocyte ratio for risk stratification of AID after KD.

Disclosure of Interest

None declared

\section{P290}

Clinical features and therapy in children who have granulomatosis with polyangiitis (Wegener's) in one Russian center

E. Alexeeva ${ }^{1,2}$, R. Denisova', T. Dvoryakovskaya ${ }^{1,2}$, A. Mamutova' ${ }^{1}, K$. Isayeva', A. Chomahidze', A. Fetisova', O. Lomakina', K. Chibisova', M. Gautier $^{1}$, D. Vankova ${ }^{1}$, I. Kriulin ${ }^{1,2}$, E. Krekhova ${ }^{1,2}$, M. Shingarova ${ }^{1,2}$

${ }^{1}$ Rheumatology, National Medical Research Center for Children's Health;

${ }^{2}$ Sechenov First Moscow State Medical University, Moscow, Russian Federation

Correspondence: E. Alexeeva

Pediatric Rheumatology 2020, 18(Suppl 2):P290

Introduction: The clinical presentation of Granulomatosis With Polyangiitis (GPA) is often heterogeneous, with frequent involvement of the respiratory tract, the kidney, the skin and the joints. GPA vasculitis is rare in childhood but cohort studies performed during the last decade have clarified their phenotype, patterns of renal involvement and their prognostic implications, and outcome. We provided preliminary data on demographic characteristics and organ manifestations of Russian cohort in one center.

Objectives: To describe demographic characteristics, presenting clinical features, and initial treatments in patients with granulomatosis with polyangiitis (Wegener's) (GPA).

Methods: The European Medicines Agency (EMA) classification algorithm was applied. The EMA algorithm was used to uniquely distinguish children with GPA from children with other vasculitis, whose diagnoses had been classified according to both adult- and pediatric-specific criteria. Descriptive statistics were used for comparisons.

Results: In total, 15 patients ( $67 \%$ female) fulfilled the classification criteria for GPA ( $\mathrm{n}=15)$. The median time to diagnosis was 3.5 months (ranging to 24 and 46 months, respectively). The median age was 13 years. Pulmonary manifestations were in $53 \%(n=8)$ of patients (alveolar hemorrhage $(n=2)$, dyspnea $(n=5)$, chronic cough $(n=4)$, pleural effusion $(n=1)$, lung nodules or cavity $(n=3)$, lung infiltration $(n=6)$. Renal pathologic features were found in $53 \%(n=8)$. Eye involvement was in $33 \%(n=5)$, ENT involvement was in $67 \%$ $(n=10)$, cutaneous involvement was in $26 \%(n=4)$, CNS involvement was in $6 \%(n=1)$, cardiovascular involvement was in $6 \%(n=1)$, musculoskeletal involvement was in $60 \%(n=9)$, gastrointestinal involvement was in $13 \%(n=2)$, fever, fatigue were in $87 \%(n=13)$. All patients with GPA for induction remission received combination therapy with corticosteroids plus immunosuppressive agents 
(cyclophosphamide $(n=4)$, mycophenolate $(n=10)$, rituximab $(n=6)$, tocilizumab $(n=1))$, for maintenance therapy - combination therapy with corticosteroids plus immunosuppressive agents (azathioprine $(n=5)$, mycophenolate $(n=10)$, rituximab $(n=2))$.

Conclusion: GPA is rare disease in childhood. Their clinical presentation is often similar to that adult patients, although some differences exist. Renal involvement drives the prognosis of pediatric GPA. The treatment of pediatric GPA still relies on the studies performed in adults.

\section{Disclosure of Interest}

E. Alexeeva Speaker Bureau of: Novartis, Pfizer, Sanofi, MSD and Roche, R. Denisova Speaker Bureau of: Novartis, MSD and Roche, T. Dvoryakovskaya Speaker Bureau of: Novartis, Pfizer, MSD and Roche, A. Mamutova Speaker Bureau of: Novartis, K. Isayeva: None declared, A. Chomahidze: None declared, A. Fetisova: None declared, O. Lomakina: None declared, K. Chibisova: None declared, M. Gautier: None declared, D. Vankova: None declared, I. Kriulin: None declared, E. Krekhova: None declared, M. Shingarova: None declared

\section{P291}

Erythrocyte glutathione s-transferase activity in children with Henoch-Schönlein purpura

M. Frkovic', A. Mlinarić, S. Sršen ${ }^{3}$, A. Gagro ${ }^{4}$, S. Huljev Frković ${ }^{1}$, D. Rogić 2 , M. Jelušić

${ }^{1}$ Department of pediatrics; ${ }^{2}$ Department for laboratory diagnostics, UHC ZAGREB, Zagreb; ${ }^{3}$ Department of pediatrics, UHC Split, Split; ${ }^{4}$ Department of pediatrics, Clidren's Hospital Zagreb, Zagreb, Croatia Correspondence: M. Frkovic

Pediatric Rheumatology 2020, 18(Suppl 2):P291

Introduction: Henoch-Schönlein purpura (HSP) is the most common vasculitis of the childhood. Among all possible symptoms / complications, nephritis (HSPN) is the main and almost only cause of morbidity and mortality in HSP.

Objectives: The aim of this study was to investigate the value of erythrocyte glutathione S-transferase (e-GST) activity as an early predictor of nephritis development in HSP.

Methods: Ninety-seven children with HSP were enrolled into the study. The control group consisted of 52 children without clinical and laboratory signs of inflammation. In all patients e-GST activity was determined spectrometrically for three times during six-month period and corelated with clinical characteristics of the disease, routine blood and urine laboratory findings as well as with e-GST activity in healthy children.

Results: At the beginning of the disease the e-GST activity values were significantly higher in the group of HSP patients who developed proteinuria above $0.15 \mathrm{~g} / \mathrm{dU}$ and/or haematuria above $5 \mathrm{E} / \mathrm{mm}^{3}$ (i.e. HSPN) during six-month follow-up period, compared to the group of HSP patients without nephritis during same time span: median (interquartile range) $5,70 \mathrm{U} / \mathrm{g}_{\mathrm{Hb}}\left(4,38-7,50 \mathrm{U} / \mathrm{g}_{\mathrm{Hb}}\right)$ compared to $3,10 \mathrm{U} / \mathrm{g}_{\mathrm{Hb}}\left(2,20-4,20 \mathrm{U} / \mathrm{g}_{\mathrm{Hb}}\right) ; \mathrm{P}<0,001$. Similar results were obtained after the comparison of the patients with HSPN and control group: $5,70 \mathrm{U} / \mathrm{g}_{\mathrm{Hb}}\left(4,38-7,50 \mathrm{U} / \mathrm{g}_{\mathrm{Hb}}\right)$ vs. $3,13 \mathrm{U} / \mathrm{g}_{\mathrm{Hb}}\left(1,91-4,20 \mathrm{U} / \mathrm{g}_{\mathrm{Hb}}\right) ; \mathrm{P}<$ 0,001 . There were no statistically significant differences between the group of HSP patients without nephritis and a control group ( $P=$ 0,837 ). After the period of three and six months, a decrease of e-GST activity was observed in the HSPN patients, but it was still significantly higher compared to the group of HSP patients without nephritis $(\mathrm{P}<0,001 / \mathrm{P}<0,001)$.

In the ROC analysis of the e-GST activity determination value in the prediction of HSP nephritis, for the e-GST values $>4,1 \mathrm{U} / \mathrm{g}_{\mathrm{Hb}}$ a significant area under the curve (AUC) of $91.1 \%(P<0.001)$ and sensitivity of $90.5 \%$ and specificity of $72.7 \%$ was found at the beginning of the study. The sensitivity of the nephritis detection tests decreased, and the specificity increased during the follow-up period. No significant correlation between e-GST activity and severity of skin changes, abdominal pain and arthralgia/arthritis, or used therapy was found. Among the routine laboratory tests, a consistent, statistically significant, positive correlation was found only between e-GST activity and the number of erythrocytes per $\mathrm{mm}^{3}$ in urine samples.

Conclusion: e-GST activity is a reliable, independent marker of early nephritis risk assessment in children with HSP. As a sensitive and specific, feasible and inexpensive laboratory test, it has potential practical utility in the diagnostic algorithm and monitoring of the children with HSP.

Disclosure of Interest

None declared

P292

Gastrointestinal manifestations and their association with the risk for renal disease in patients with henoch-schönlein's purpura M. Sestan', N. Kifer', S. Srsen?2, A. Ovuka ${ }^{3}$, M. Batnozic Varga ${ }^{4}$, M. Sapina ${ }^{5}$, M. Held ${ }^{1}$, M. Ban ${ }^{1}$, A. Kozmar ${ }^{1}$, M. Coric ${ }^{1}$, S. Bulimbasic ${ }^{1}$, K. Crkvenac $^{1}$, D. Milosevic ${ }^{1}$, M. Frkovic', A. Gagro ${ }^{6}$, M. Jelusic ${ }^{1}$

${ }^{1}$ University Hospital Centre Zagreb, University of Zagreb School of Medicine, Zagreb; ${ }^{2}$ University Hospital Centre Split, University of Split School of Medicine, Split; ${ }^{3}$ University Hospital Centre Rijeka, University of Rijeka School of Medicine, Rijeka; ${ }^{4}$ University Hospital Centre Osijek, Josip Juraj Strossmayer University of Osijek, Medical Faculty Osijek; ${ }^{5}$ University Hospital Centre Osijek, Josip Juraj Strossmayer University of Osijek, Medical Faculty Osijek, Faculty of Dental Medicine and Health, Osijek; ${ }^{6}$ Children's Hospital Zagreb, Josip Juraj Strossmayer University of Osijek, Medical Faculty Osijek, Zagreb, Croatia

Correspondence: M. Sestan

Pediatric Rheumatology 2020, 18(Suppl 2):P292

Introduction: Henoch-Schönlein's purpura (HSP) is the most frequent systemic vasculitis in childhood. Although the disease is most often self-limiting, more than $50 \%$ of children may develop gastrointestinal (Gl) symptoms, most commonly manifested by nausea, vomiting, and blood in the stool, while in about $10-20 \%$ of patients serious complications such as intussusception, bowel perforation, and massive bleeding occur.

Objectives: The aim of this research was to analyze clinical and biochemical parameters in patients with HSP and GI manifestations.

Methods: This retrospective study included children with HSP reviewed in five Croatian University Centers for pediatric rheumatology in the period 2009 to 2019. Differences between categorical variables were examined using the $x^{2}$ and Fisher exact test, and among the numerical using the Mann Whitney $\mathrm{U}$ test.

Results: Out of 611 children with HSP, 320 were males and 291 were females. The overal Gl prevalence was $45.9 \%$ with a $95 \% \mathrm{Cl}$ of 41.9 to $50 \%$ and the median (range) age at diagnosis was 6.42 (4.5-8.83) years. Among patients with $\mathrm{Gl}$ symptoms there were 1.44 times more males $(\mathrm{N}=166)$ than females $(\mathrm{N}=115)$, which was statistically significant ( $p=0.003)$, higher proportion of patients came from the Mediterranean area (54\% vs. $42 \%, p=0.007)$. Patients with $\mathrm{Gl}$ simptoms had less prodromal infections before the appearance of purpura $(59.8 \%$ vs. $70.9 \%, p=0.005)$ and less respiratory infections $(35.6 \%$ vs. $45.2 \%, p<0.001)$, while regarding intestinal infections there was no difference. Patients with Gl symptoms were 1.68 times more likely to develop renal symptoms, and if Gl symptoms occured before other symptoms of HSP, then this probability was 3.55 times higher. There was no difference in involvement of the joints and central nervous system, whereas patients suffering from HSP with GI symptoms were found to be significantly more likely to have rash distributed on the trunk (61.9\% vs. $48.5 \%, p=0.001)$, and upper extremities $(35.2 \%$ vs. $24.7 \%, p=0.006)$, as well as generalized rash (38.8\% vs. $28.3 \%, p=0.008)$. These patients also had statistically significant higher values of C-reactive protein, leukocyte count, erythrocytes and platelets, hemoglobin, hematocrit and D-dimer concentrations and lower levels of IgG and IgM. In our cohort 42 out of 281 
children (14.9\%) had the most severe Gl manifestations (intussusception and/or massive Gl bleeding) with statistically significant higher values of 24-hour urine protein levels and D-dimer concentrations and lower total serum protein, albumin, IgG, IgM and C3 levels in comparison with children whose $\mathrm{Gl}$ manifestations were less severe (abdominal pain, vomiting, and blood in the stool).

Conclusion: We detected a group of patients with HSP and GI symptoms that differed in their demographic, clinical, and biochemical characteristics from patients without Gl symptoms. This group of patients was found to be significantly more likely to develop renal disease and thus cumulatively have a higher risk of acute and chronic complications of HSP.

SUPPORT: Croatian Science Foundation project IP-2019-04-8822.

\section{Disclosure of Interest}

None declared

\section{P293}

Does the patients with Kawasaki disease diagnosed before one year of age have distinct presentations?

N. Aktay Ayaz', F. Çakmak' , S. G. Karadağ², A. Tanatar', H. E. Sönmez², M. Cakan $^{2}$, F. Demir ${ }^{3}$, B. Sözeri ${ }^{3}$

Pediatric Rheumatology, Istanbul University Medical School; ${ }^{2}$ Pediatric Rheumatology, Kanuni Sultan Süleyman Research and Training Hospital, University of Health Sciences; ${ }^{3}$ Pediatric Rheumatology, Ümraniye Research and Training Hospital, University of Health Sciences, İstanbul, Turkey

\section{Correspondence: N. Aktay Ayaz}

Pediatric Rheumatology 2020, 18(Suppl 2):P293

Introduction: Kawasaki disease (KD) is one of the most common vasculitidis of childhood. It may present at an early age with atypical findings and even more severe course.

Objectives: In this study, we aimed to examine the clinical and laboratory features of patients diagnosed with KD below one year of age and compare the data with KD patients diagnosed over one year of age.

Methods: The demographic data, clinical features, laboratory findings and treatment modalaties of patients diagnosed with KD between January 2009 and January 2019 were reviewed retrospectively. Patients were goruped according to age of presentation as group 1 (diagnosed before 1 year of age) and group 2 (diagnosed after 1 year of age).

Results: The study included 68 patients ( 37 boys / 31 girls) below one year of age and 97 ( 60 boys / 37 girls) patients over one year of age. The mean age of diagnosis was 7 months (1-12) in patients under one year and 50 months (12-119) in patients over one year of age. Twenty-six of all patients $(\% 15,7)$ were atypical KD; 18 of these children were below one year and 8 were over one year of age $(p=$ $0,001)$. Fifty-eight of all patients $(\% 35,1)$ were diagnosed with incomplete KD; 34 of these children were in group 1 and 24 were in group $2(p<0,001)$. Unilateral cervical lymphadenopathy was obviously more common in group $2(p<0.001)$, while aseptic meningitis and BCG scar erythema was significantly more common in group 1 . Coronary artery involvement was present in 27 of 68 patients $(\% 39,7)$ in group 1 and in 15 of 97 patients $(\% 15,4)$ in group $2 ;(p<0,001)$. IVIG resistance was detected in $23 / 165(\% 13,9)$ of the patients, 12 of whom were in group $1(p=0,178)$. In addition to IVIG treatment, steroid therapy was commenced to 17 patients in group 1 and 12 patients in group 2 $(p=0,30)$ Aspirin was started to all patients $(40-80 \mathrm{mg} / \mathrm{kg} /$ day) and saliciliysm findings were observed in 3 patients in group 1 and 3 patients in group 2.

We applied Kobayashi, Harada, Egami and Formosa risk score systems for each of the two groups and analyzed the accuracy of each score in detecting high risk patients for coronary artery aneurysms and IVIG resistance. None of the scores could predict high risk patients in both of the groups. When we use Harada score to predict coronary artery involvement it was statistically high in both groups that had CALs $(p=0,001)$

Conclusion: In our cohort, incomplete KD, atypical KD and cardiac involvement were more common in children diagnosed before one year of age, So, while evaluating patients under 1 year of age, phyicians should be aware of the distinctive features of presentation, severe course of disease and follow these patients accordingly.

Disclosure of Interest

None declared

\section{P294}

Is Kawasaki disease or systemic juvenile idiopathic arthritis in children with fever without a source?

B. Sozeri ${ }^{1}$, F. Demir ${ }^{1}$, T. Merter ${ }^{2}$, M. karacan ${ }^{3}$

${ }^{1}$ Pediatric Rheumatology; ${ }^{2}$ Pediatrics; ${ }^{3}$ Pediatric cardiology, Health Sciences University, Umraniye Traning and Research Hospital, Istanbul,

Turkey

Correspondence: B. Sozeri

Pediatric Rheumatology 2020, 18(Suppl 2):P294

Introduction: Fever without a source (FWS) is caused by various diseases, making differential diagnosis difficult. Clinical similarities between Kawasaki disease (KD) and systemic Juvenile Idiopathic Arthritis (sJIA) are well known. Kawasaki disease (KD), a self-limiting systemic vasculitis, remains of unknown etiology and can cause irreversible coronary artery aneurysms (CAAs). SoJIA is sometimes confused with incomplete KD because both diseases have overlapping clinical features and can be accompanied with CAAs and/or sJIA with macrophage activation syndrome (MAS).

Objectives: In this study, the frequency of both KD and SJIA among the patients evaluated with FWS and the clinical features of patients diagnosed with Kawasaki disease.

Methods: Medical records of patients who first visited our department between January 2016 and December 2019 were reviewed

Results: A total of 107 patients were enrolled in this study, including 43 patients $(40.2 \%, 23$ males) who fulfilled the criteria of Kawasaki disease and 64 patients $(59.8 \%, 39$ males) who did not fulfill them. In patients who fulfilled the criteria of classical FWS, 36(33.6\%, 20 males) patients were diagnosed with systemic juvenile idiopathic arthritis. The mean age of the patients with Kawasaki disease was $30.0 \pm 20,4$ months (median 25 months), the mean age of other patients was $52,6 \pm 40$ months (median 39,5 months). The mean age of the patients with sJIA patients was $87,6 \pm 49,8$ (median 80 months). Kawasaki patients were younger than others $(p=0.01)$. There was no difference in gender between groups.

In Kawasaki patients, the most common clinical feature at diagnosis was fever $(100 \%)$ followed by conjunctival congestion and mucosal changes (69\%). The last two findings are more significant in kawasaki patients than others $(p<0,00)$. Twenty-six $(59 \%)$ patients had completed KD while $25 \%$ had incomplete KD. 7 (16\%) patients had atypical KD. The mean fever duration was longer in SJIA patients than KD and others (median 14,8 and 7 days, $p<0.00$ ). All patients with $\mathrm{KD}$ received IVIG ( $2 \mathrm{~g} / \mathrm{kg}$, infusion in $12 \mathrm{~h}$ ) and aspirin $(60 \mathrm{mg} / \mathrm{kg} /$ day). $13.6 \%$ of the patients also received oral corticosteroids because of IVIG resistance. Thirty-one patients $(72.1 \%)$ responded to IVIG treatment, whereas 12 ( 6 female, 6 male) were IVIG resistant. CAI was detected in echocardiography at diagnosis in $10(22.7 \%)$ (6 female ; 4 male) patients. We also detected 4 patients pericarditis with /without $\mathrm{CIA}$.

Conclusion: The clinical presentations of KD and sJA are quite similar with fever, rash, hepatomegaly, and lymphadenopathy. All 2 entities may provide clues to potentially shared immunopathology.

Disclosure of Interest

None declared 


\section{P295}

Japanese predictive scores in Slovenian population of children with Kawasaki disease

T. Avramoska', V. Osterman', M. Bizjak², G. Markelj', T. Avcin ${ }^{3}$, M. Pokorn² ${ }^{1}$ Department of Infectious diseases; ${ }^{2}$ Children's University Hospital;

${ }^{3}$ Department of Rheumatology, Children's University Hospital, University Medical Centre, Ljubljana, Slovenia

Correspondence: $\mathrm{T}$. Avramoska

Pediatric Rheumatology 2020, 18(Suppl 2):P295

Introduction: There are several scoring systems developed in Japan that are clinically used to stratify high risk KD patients and thus identify the ones that may benefit from early adjunctive therapy. There are increasing reports from all over the world on poor performance of these scores in other ethnic populations.

Objectives: The aim of our study was to evaluate the Kobayashi, Egami, Sano and Kawamura scores in our population which is homogenous Caucasian.

Methods: Hospital database was retrospectively searched for code M30.3 of the International Classification of Diseases, 10th Revision, Clinical Modification Code: Mucocutaneous lymph node syndrome [Kawasaki], over the period from January 2006 to December 2019. All patients who were seen in this period for the first time for complete or incomplete Kawasaki disease, as defined by the American Heart Association, were included.

We applied IVIG resistance prediction scores (Kobayashi, Sano, Egami and Kawamura scores) to our cohort. Only patients who received 2g/ $\mathrm{kg}$ IVIG within the first 10 days of the disease were included in this analysis. The scores of prediction models were calculated for each patient and patients were assigned to high- or low-risk group accordingly.

Results: During the study period a total of 169 children were diagnosed with KD (61.5\% males, median age 3.28 years). All of them were Caucasian except one child who was biracial (Caucasian and African American). Among them, 158 children were hospitalized in the acute phase of the disease and 11 children were seen in the subacute phase of the disease. 151 children were followed-up for at least one year to evaluate persistent coronary artery aneurysms (CAA), which were observed in 8 (5.3\%) patients. Among them, 2 were not treated with IVIG and 2 received IVIG after 10 days of illness. 125 patients were treated with IVIG within first 10 days of illness and were included in the calculation of IVIG resistance prediction scores. 24 (19.2 \%) were IVIG resistant.

Sensitivity of Kobayashi, Sano, Egami and Kawamura scores were $0.53,0.47,0.61$ and 0.58 , respectively. Specificity of those scores were $0.77,0.87,0.75$ and 0.58 , respectively. We found no difference in demographic or clinical characteristics between IVIG resistant and IVIG responsive patients. Patients with IVIG resistance had significantly higher $\operatorname{ALT}(p=0.025)$, neutrophil-to-lymphocyte ratio ( $p$ $=0.036)$ and lower serum sodium $(p=0.009)$.

Conclusion: By applying the Japanese scores to our population, we were able to identify most of the low-risk, but missed many of the high-risk patients. Our results are consistent with Caucasi $n$ based population studies available to date.

\section{Disclosure of Interest}

None declared

\section{P296}

Long term outcome of post-varicella arteriopathy in children S. Signa', M. Bertamino ${ }^{2}$, M. Veneruso ${ }^{3}$, G. Prato ${ }^{3}$, R. Caorsi ${ }^{1}$, M. Di Rocco ${ }^{4}$, G. Losurdo ${ }^{5}$, G. Amico ${ }^{6}$, A. Zanetti ${ }^{2}$, D. Tortora ${ }^{7}$, S. Volpi ${ }^{1}$, A. Rossi ${ }^{7}$, P. Moretti ${ }^{2}$, A. Ravelli ${ }^{8}$, M. Gattorno ${ }^{7}$, M. Severino ${ }^{7}$

${ }^{1}$ UOSD Centro Malattie Autoinfiammatorie e Immunodeficienze; ${ }^{2} \mathrm{UOC}$ Medicina Fisica e Riabilitazione; ${ }^{3}$ UOC Neuropsichiatria Infantile; ${ }^{4}$ UOSD Malattie Rare; ${ }^{5}$ OOC Malattie Infettive; ${ }^{6}$ OOC Genetica Medica; ${ }^{7}$ UOC Neuroradiologia; ${ }^{8}$ UOC Clinica Pediatrica e Reumatologia, IRCCS Istituto Giannina Gaslini, Genoa, Italy

Correspondence: S. Signa

Pediatric Rheumatology 2020, 18(Suppl 2):P296
Introduction: Varicella zoster virus (VZV) related arterial ischemic stroke (AIS) has been described in literature in pediatric age However, the long-term course of post-VZV vasculopathy need to be inquired: clear information about prevalence of recurrence and severity of clinical outcome are lacking, even if a favorable evolution was initially described, and therapeutic protocols are not currently standardized.

Objectives: We aimed to describe the clinical, laboratory and neuroradiologic features of children affected by AIS due to post-VZV referred to our Institute and to present our experience in their therapeutic management.

Methods: We selected 22 pediatric patients ( 6 females) with AIS and a CNS confirmed VZV reactivation and/or with a VZV history in the previous 12 months. Other causes of pediatric stroke (systemic disease, cardiac disease, trauma, major thrombophilia) were excluded. Clinical, neuroimaging, laboratory and treatment data were reviewed, focusing on pediatric score outcome measure (PSOM) and executive functions final outcome.

Results: Average age of AIS onset, VZV primary infection and interval between infection and AIS were: 4 years 10mo (range: 1 year and 8 mo-9 years and 11 months), 4 years and 5 months (range 8 months-9.4 years), and 7 months (range 10days-34 months), respectively. The AIS involved the nucleo-capsular region in 18 cases, the cerebral cortex in 9 cases, the thalamus in 4 cases, and the pons in 3 subjects. Seventeen patients had inflammatory focal cerebral arteriopathy (iFCA). Virological confirmation (VZVDNA or anti-VZV IgG in the cerebrospinal fluid) was obtained in 11 patients. Three patients were treated with trombectomy and one with rTPA. Thirteen patients were treated with antiviral agents associated with steroids in 8 cases, with different administration schedules. Only in one case steroid treatment was given without association with antiviral agents. One patient received a short course of steroid and antiviral treatment at the time of the stroke and then a more prolonged course after six months at the time of the virological diagnosis. Prophylactic antiaggregants were administered to all patients. Mean follow-up was 2 years and 5 months (range 6 mo -10 years); iFCA was persistent in 12 cases and transient in 5 subjects. Four patients presented a recurrence of post VZV arteriopathy, two of them presenting new stroke events. Twelve patients presented a variable motor deficit at last follow up. The mean PSOM score of the cohort at the last visit was 1 (range 0-2). Executive functions were evaluated at last follow up in twelve patients, showing no deficit in seven patients, a mild deficit in two patients and a severe deficit in the last three.

Conclusion: Albeit a favourable evolution was initially described, our experience suggests that VZV-related AIS may result in persistent FCA and significant neurological impairment in the majority of cases. Therapeutic approach, particularly involving steroid administration, still need to be validated.

\section{Disclosure of Interest}

None declared

\section{P297}

Can we predict coronary artery involvement in Kawasaki disease?

U. Kaya Akca', O. Serin², E. Arslanoglu Aydin², E. Sag', S. Demir', E.

Atalay', M. Kasap Cuceoglu', E. D. Batu', Y. Bilginer', S. Ozen'

'Division of Pediatric Rheumatology, Department of Pediatrics;

${ }^{2}$ Department of Pediatrics, Hacettepe University Faculty of Medicine,

Ankara, Turkey

Correspondence: U. Kaya Akca

Pediatric Rheumatology 2020, 18(Suppl 2):P297

Introduction: Coronary artery involvement is the most important complication of Kawasaki disease (KD). While $25 \%$ of the untreated patients develop coronary artery aneurysms, this rate decreases to 3$5 \%$ in patients who started treatment rapidly.

Objectives: We aimed to evaluate the clinical and laboratory features which could predict coronary artery involvement in these patients. 
Methods: This study included retrospective analysis of patients who diagnosed with Kawasaki disease at the Hacettepe University Children's Hospital between June 2007 and September 2019. Complete and incomplete KD patients were included in the study.

Results: A total of 129 patients, 79 boys (61.2\%), with a median age 36 (IQR 19.5-57.0) months were included in the study. The median duration of fever was 7.0 (IQR 5-10) days. Complet KD was diagnosed in 87 patients $(67.4 \%)$. The number of patients with IVIG resistance were $16(12.4 \%)$. Coronary artery involvement was detected in 44 of 129 patients (34.1\%). There were coronary artery dilatation (Z score 2 to $<2.5)$ in 14 patients, small aneurysm $\left(Z\right.$ score ${ }^{3} 2.5$ to $\left.<5\right)$ in 18 patients, medium aneurysm ( $Z$ score $\geq 5$ to $<10$, and absolute dimension $<8 \mathrm{~mm}$ ) in 7 patients, and giant aneurysm ( $Z$ score $\geq 10$, or absolute dimension $\geq 8 \mathrm{~mm}$ ) in 5 patients. Patients with extremity changes had more common coronary artery involvement $(p=0.04)$. We observed a significant association with young age, male gender, high levels of white blood cell count, high lymphocyte count and high lymphocyte percentage. In univariate analysis, male gender, age under 1 year, changes in extremities, and high lymphocyte counts were associated with the coronary involvement (OR: $0.393 ; 95 \% \mathrm{Cl}$ : 0.176-0.879; $\mathrm{p}=0.023$, OR: 3.873; $95 \% \mathrm{Cl}: 1.303-11.507 ; \mathrm{p}=0.015$, OR: $2.523 ; 95 \% \mathrm{Cl}: 1.008-6.313 ; \mathrm{p}=0.048$, and OR: $1.239 ; 95 \% \mathrm{Cl}: 1.046-$ 1.467; $p=0.013$, respectively) while duration of fever, IVIG resistance, incomplet form of disease, white blood cell count, erythrocyte sedimentation rate, and $\mathrm{C}$-reactive protein were not found as a risk factor. The multivariate analysis identified young age $(<1$ year of age) and high lymphocyte count as independent risk factors for coronary involvement (OR: 4.384; 95\%Cl: 1.192-16.128; $\mathrm{p}=0.026$ and OR: 1.215 ; 95\%Cl: $1.017-1.452 ; p=0.032$, respectively).

Conclusion: Children under 1 year of age and high lymphocyte counts were the risk factors of coronary involvement in KD. However, in order to accurately determine the risk of coronary artery involvement, there is a need to clarify the pathophysiology.

\section{Disclosure of Interest}

None declared

\section{P298}

Kawasaki disease in infants below 6 months: case series

P. R. Chickermane, S. Balan, A. Tiwari

Department of Clinical Immunology and Rheumatology, Amrita Institute of Medical Sciences, Kochi, India

Correspondence: P. R. Chickermane

Pediatric Rheumatology 2020, 18(Suppl 2):P298

Introduction: Kawasaki Disease (KD), a medium vessel vasculitis having predilection for the coronaries is the commonest cause of myocardial infarction in children. Although infants tend to have incomplete forms of KD, they are at a greater risk for the development of coronary artery aneurysms.

Objectives: To describe our experience with KD in infants below 6 months of age.

Methods: Retrospective review of 4 infants below 6 months of age with $\mathrm{KD}$ who received treatment at the Amrita Institute of Medical Sciences, Kochi, India during the period from January 2019 to May 2020.

Results: We describe four infants below 6 months of age diagnosed with KD with coronary artery aneurysms (CAA). Cases 1, 2 and 4 were referred to our centre after diagnosis and primary treatment for the management of refractory KD.

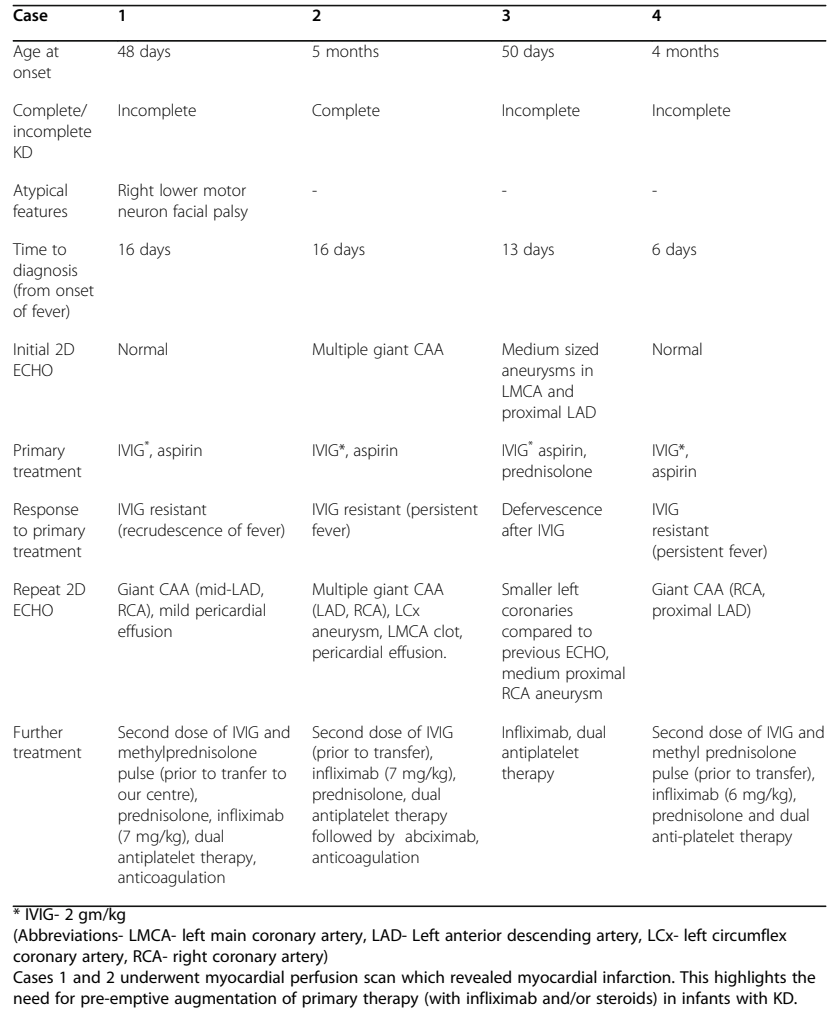

Conclusion: Infants with KD often present with incomplete forms of the disease, resulting in delayed diagnosis and treatment, with catastrophic consequences. The clinical possibility of KD needs to be considered in infants below 6 months of age presenting with unexplained fever for more than 5 days. Infants with KD are at a high risk for developing coronary artery aneurysms and should be considered for primary adjunctive therapy.

Disclosure of Interest

None declared

P299

The severity of skin symptoms in patients with childhood IgA vasculitis - five tertiary centres in Croatia experience

S. Srsen ${ }^{1,2}$, M. Sapina ${ }^{3}$, M. Batnozic Varga ${ }^{3}$, M. Sestan ${ }^{4,5}$, N. Kifer ${ }^{4,5}$, A.

Ovuka ${ }^{6,7}$, M. Held ${ }^{4,5}$, M. Ban ${ }^{4,5}$, A. Kozmar ${ }^{4,8}$, M. Coric ${ }^{4,9}$, S. Bulimbasic ${ }^{4,9}$, K. Crkvenac Gornik ${ }^{4,8}$, D. Milosevic ${ }^{4,5}$, M. Frkovic ${ }^{4,5}$, A. Gagro ${ }^{10,11}$, M. Jelusic ${ }^{4,5}$

${ }^{1}$ University of Split, School of Medicine Split; ${ }^{2}$ Department of Pediatrics, University Hospital Centre Split, Split; ${ }^{3}$ Department of Pediatrics,

University Hospital Centre Osijek, Osijek; ${ }^{4}$ University of Zagreb, School of Medicine Zagreb; ${ }^{5}$ Department of Pediatrics, University Hospital Centre Zagreb, Zagreb; ${ }^{6}$ University of Rijeka, School of Medicine Rijeka; ${ }^{7}$ Department of Pediatrics, University Hospital Centre Rijeka, Children's Hospital Kantrida, Rijeka; ${ }^{8}$ Department of Laboratory Diagnostics;

${ }^{9}$ Department of Pathology, University Hospital Centre Zagreb, Zagreb;

${ }^{10}$ University of Osijek, School of Medicine Osijek, Osijek, ${ }^{11}$ Department of Pediatrics, Children's Hospital Zagreb, Zagreb, Croatia

Correspondence: S. Srsen

Pediatric Rheumatology 2020, 18(Suppl 2):P299 
Introduction: Henoch Schonlein purpura or $\lg A$ vasculitis (IgAV) is the most common childhood vasculitis which is characterized by nonthrombocytopenic purpura of the skin, gastrointestinal, renal and joint involvement. Skin symptoms can be localized on legs, affecting the glutes, or rarely presented atypically, affecting the head and neck area. In the most severe cases, ulcerations and necroses can be seen. Objectives: To evaluate the prevalence and severity of skin involvement in patients with childhood IgAV, and its relationship with other clinical and biochemical characteristics.

Methods: In this retrospective study patients with IgAV referred to five tertiary teaching hospitals between 2009 and 2019 were included in the study. The severity and distribution of skin symptoms were classified into four categories: localized only to legs, extended to the gluteal region, generalized, and severe skin symptoms with ulcerations and necrosis.

Results: 611 patients, of which 320 boys and 291 girls with a median age of $6.42(4.42-8.92)$ years were included in the study. All patients had skin involvements. In 205 (33.72\%) patients, only legs were affected, in 207 (34.05\%) the gluteal region was affected besides the legs, in $181(29.77 \%)$ the rash was generalized, and 15 patients (2.47\%) had the most severe skin symptoms which included ulcerations and necroses.

Patients with generalized (27.68\%), and severe skin (28.57\%) involvement had almost twice as much relapses compared to patients with legs $(16.08 \%)$, legs and gluteal (16.1\%) affection ( $p=$ 0.011).

Patients with more severe skin symptoms had significantly more gastrointestinal symptoms (generalized: 53.59\%, necroses and ulcerations: $66.67 \%$, legs only: $36.1 \%$, legs and glutes: $47.83 \%$; $p=$ 0.002 ), joint involvements (generalized, necroses and ulcerations: $29.08 \%$, legs only: $16.59 \%$, legs and glutes: $21.27 \%, p=0.01$ ), renal involvement (generalized: $28.18 \%$, necroses and ulcerations: $46.67 \%$, legs only: $21.46 \%$, legs and glutes: $21.46 \% ; p<0.001$ )

Regarding the treatment, patients with more severe skin involvement needed more corticosteroids (generalized: 52.78\%, necroses and ulcerations: $93.33 \%$, legs only: $21.11 \%$, legs and glutes: $34.15 \% ; \mathrm{p}<0.001$ ), and immunesuppressants (generalized: $2.22 \%$, necroses and ulcerations: $20 \%$, legs only: $1.97 \%$, legs and glutes: $1.97 \% ; p=0.017$ ).

Conclusion: The prevalence of generalized skin symptoms, as well as the most severe forms in childhood IgAV is much less than the classical findings. However, the more severe the skin symptoms are, the more severe the course of the disease is, and aggressive treatment will be needed.

SUPPORT: Croatian Science Foundation project IP-2019-04-8822.

\section{Disclosure of Interest}

None declared

\section{P300}

Venous wall thickness in Behçet's disease: can it be a finding supporting our diagnosis?

E. Atalay', B. Oguz', E. Sag', S. Demir', U. K. Akca', M. K. Cuceoglu', E. D. Batu', Y. Bilginer', S. Ozen

${ }^{1}$ Pediatric Rheumatology; ${ }^{2}$ Radiology, Hacettepe University, Ankara,

Turkey

Correspondence: E. Atalay

Pediatric Rheumatology 2020, 18(Suppl 2):P300

Introduction: Behçet's Disease (BD) is a systemic inflammatory disease that can involve both the arteries and veins. The lower extremity venous wall thickness (VWT) of patients who were followed with the diagnosis of Behçet's disease increased significantly compared to healthy controls in two studies conducted in adults.

Objectives: The aim of this prospective study was to investigate the lower extremity VWT in childhood Behçet's patients and in incomplete $\mathrm{BD}$.

Methods: Pediatric patients classified according to the 2015 international pediatric BD criteria in Hacettepe University Department of Pediatric Rheumatology were included in the study. The control group was age and gender matched healthy children. VWT measurements of the lower extremity veins including common femoral vein (CFV), superficial femoral vein (FV), vena saphena magna (VSM), vena saphena parva (VSP) and popliteal vein (PV) were recorded.

Results: Venous wall thickness was measured in 20 patients $(70 \%$ male) and 5 healthy controls (60\% male). Eight of 20 patients fully met the criteria for the diagnosis of $\mathrm{BD}$. The remaining 12 had incomplete BD. The median age was 18 (range 9,5-24) years in the $\mathrm{BD}$ group and 16 (range 12-17) in the control group. The median VWT values of $B D$ patients were significantly higher than the control group in the right CFV, right superficial FV, right VSM, right PV, left CFV, left superficial FV and left PV (Table 1). Vascular involvement was present in $5(25 \%)$ patients. Of those; sagittal vein thrombus was detected in two patients, thrombus in deep lower extremity veins in two patients and superficial thrombophlebitis in one patient. Venous wall thickness of the right CFV, right FV, left CFV and left FV in patients with vascular involvement were higher than those without vascular involvement. However, the difference was not statistically significant.

Conclusion: The majority of pediatric patients do not meet the full criteria, and are followed with a diagnosis of incomplete Behçet's Disease. Increased venous wall thickness might serve as a biomarker supporting the diagnosis of Behçet's disease.

\section{References}

Koné-Paut I, Shahram F, Darce-Bello M, et al. Consensus classification criteria for paediatric Behçet's disease from a prospective observational cohort: PEDBD. Ann Rheum Dis Published Online First doi:10.1136/ annrheumdis2015-20849

Oner FA, Ergelen R, Mutis A et al. Venous vessel wall thickness in lower extremity is increased in male patients with Behcet's disease. Clinical Rheumatology-2019 https://doi.org/10.1007/s10067-019-04470-z

Seyahi E, Gjoni M, Durmaz ES et.al. Increased vein wall thickness in Behçet disease. Journal of Vascular Surgery: Venous and Lymphatic Disorders Volume 7, Issue 5, September 2019, Pages 677-684.e2

Disclosure of Interest

None declared

Table 1 (abstract P300). Behçet's patients ( $n=20)$ WWT values (median and IQR) vs healthy controls $(n=5)$ WWT values (median and IQR) respectively.

\begin{tabular}{|c|c|c|c|}
\hline Right Common Femoral Vein & 0.77 (IQR 0.7-0.88) & $\begin{array}{l}0.57(\text { IQR } 0.56- \\
0.59)\end{array}$ & $\begin{array}{l}P= \\
0.003\end{array}$ \\
\hline $\begin{array}{l}\text { Right Superficial Femoral } \\
\text { Vein }\end{array}$ & 0.67 (IQR 0.6-0.75) & $\begin{array}{l}0.54 \text { (IQR 0.53- } \\
0.58)\end{array}$ & $P=0.02$ \\
\hline Right Vena Saphena Magna & $\begin{array}{l}0.58(\text { IQR } 0.46- \\
0.62)\end{array}$ & $\begin{array}{l}0.42(\text { IQR } 0.40- \\
0.42)\end{array}$ & $\begin{array}{l}P= \\
0.038\end{array}$ \\
\hline Right Vena Saphena Parva & $\begin{array}{l}0.45(\text { IQR } 0.38- \\
0.57)\end{array}$ & $\begin{array}{l}0.40(\text { IQR } 0.35- \\
0.41)\end{array}$ & $P=0,31$ \\
\hline Right Popliteal Vein & $\begin{array}{l}0.65(\mathrm{IQR} 0.56- \\
0.74)\end{array}$ & $\begin{array}{l}0.53 \text { (IQR } 0.51- \\
0.52)\end{array}$ & $\begin{array}{l}\mathrm{P}= \\
0.025\end{array}$ \\
\hline Left Common Femoral Vein & 0.78 (IQR 0.6-0.86) & $\begin{array}{l}0.57 \text { (IQR 0.55- } \\
0.58)\end{array}$ & $\begin{array}{l}P= \\
0.025\end{array}$ \\
\hline Left Superficial Femoral Vein & $\begin{array}{l}0.65(\text { IQR } 0.55- \\
0.75)\end{array}$ & $\begin{array}{l}0.57 \text { (IQR 0.46- } \\
0.53)\end{array}$ & $\begin{array}{l}P= \\
0.035\end{array}$ \\
\hline Left Vena Saphena Magna & $\begin{array}{l}0.50(\mathrm{IQR} 0.42- \\
0.64)\end{array}$ & $\begin{array}{l}0.40 \text { (IQR 0.39- } \\
0.41)\end{array}$ & $P=0.057$ \\
\hline Left Vena Saphena Parva & $\begin{array}{l}0.40(\mathrm{IQR} 0.33- \\
0.58)\end{array}$ & $\begin{array}{l}0.35 \text { (IQR 0.31- } \\
0.37)\end{array}$ & $P=0.185$ \\
\hline Left Popliteal Vein & $\begin{array}{l}0.66(\text { IQR } 0.51- \\
0.81)\end{array}$ & $\begin{array}{l}0.47 \text { (IQR } 0.44- \\
0.49)\end{array}$ & $\begin{array}{l}\mathrm{P}= \\
0.012\end{array}$ \\
\hline
\end{tabular}




\section{P301}

The changing epidemiology of kawasaki disease in Eastern India

P. Pal, N. Ahmed

Pediatric Rheumatology, Institute of Child Health Kolkata, Kolkata

700017, India

Correspondence: $\mathrm{P}$. Pal

Pediatric Rheumatology 2020, 18(Suppl 2):P301

Introduction: Kawasaki disease(KD) is an emerging disease in India. Though there is an overall agreement that the disease is on the rise but there is no nation wide data. Publications from Northern India show an overall increase in disease incidence. Institute of Child Health Kolkata is a tertiary care pediatric hospital in eastern India with dedicated pediatric rheumatology services. In this study we have attempted to analyse data of KD patients from the Pediatric Rheumatology Unit of our hospital from April 2009 to April 2020. Objectives: To evaluate the epidemiology of KD in Eastern India. Methods: Medical records of 266 children admitted with KD during the study period was evaluated for annual incidence, complete and incomplete KD, incidence of coronary artery abnormalities and IVIG resistance.

Results: 1. A significant rise in the overall incidence of the disease was observed with number of cases doubling over the last 10 years; from 18 in 2009 to 35 in 2018 \& 40 in 2019. In 2020, 13 cases have been diagnosed till April.

2. Majority (80\%) of the children presented with complete form of KD.

3. Till 2017 the incidence of coronary artery aneurysms (CAA) was 12 to $15 \%$. A rise in the CAA was observed over the last 2 years with 6 cases in $2018(17 \%)$ \& 13(32.5\%) in 2019. It is interesting to note that majority of the 13 patients in 2019 was diagnosed and administered IVIG within 10 days of disease onset. In the current year, 3 of the 13 children had CAA at diagnosis.

4. There has been a significant increase in IVIG resistant KD. 12 patients with IVIG resistance were recorded from 2009 to 2017. However, in 2018 there were 7 cases (20\%) and 8 (20\%) in 2019. 5. 10 children over the last 4 years have developed giant aneurysms ( $z$ score $>10$ ) inspite of timely initiation of IVIG. 4 of them had persistent ballooning even after Infliximab administration post IVIG. Conclusion: 1. The number of patients diagnosed with KD has doubled over the last 10 years. Whether it is a true increase in incidence or because of increased detection remains speculative.

2. It seems that the disease is behaving more aggressively with higher incidence of coronary aneurysms and IVIG unresponsiveness inspite of timely IVIG administration.

Disclosure of Interest

None declared

\section{P302}

Predictive biomarkers of $i \lg A$ vasculitis with nephritis by metabolomic analysis

S. Demir ${ }^{1}$, M. CELEBIER ${ }^{2}$, O. KAPLAN ${ }^{3}$, E. SAG ${ }^{4}$, Y. BILGINER ${ }^{1}$, I. LAY ${ }^{5}$, S. OZEN ${ }^{\prime}$

${ }^{1}$ Department of Pediatric Rheumatology; ${ }^{2}$ Department of Analytical Chemistry; ${ }^{3}$ Department of Analytical Chemistry; ${ }^{4}$ Depatrment of Pediatric Rheumatology; ${ }^{5}$ Department of Biochemistry, Hacettepe University Medical Faculty, Ankara, Turkey

Correspondence: S. Demir

Pediatric Rheumatology 2020, 18(Suppl 2):P302

Introduction: IgA vasculitis/ Henoch Schönlein Purpura (IgAV/HSP) is the most common vasculitis of childhood and renal involvement is the most serious long-term complication. A better understanding of the pathophysiology of the progression to kidney disease is required for better treatment to be achieved and current biomarkers of $\lg A$ vasculitis with nephritis (IgAVN) lack the predictive value.

Objectives: In this study, an untargeted metabolomics approach was performed to reveal the underlying molecular mechanism of disease pathogenesis and to find potential biomarkers of plasma samples from patients with IgAV and IgAVN.
Methods: IgAV was diagnosed according to the Ankara criteria in 2008 (1). Forty-five patients, including 39 active IgAV patients (H), 6 $\operatorname{lgAVN}(\mathrm{N})$, and 6 age- and gender- matched healthy controls (C), were enrolled in the study. Plasma samples from subjects were collected on the same day of $\lg \mathrm{AV}(\mathrm{HSP})$ diagnosis and before steroid or other immunosuppressive treatment initiated. This study has utilized liquid chromatography-mass spectrometry (LC-MS/ Q-TOF) to investigate the alterations in plasma metabolomic profiles. Three separate pools, health controls, active IgAV, and IgAVN were created. Peak picking, grouping, and comparison parts were performed (metabolite profiling) via XCMS (https://xcmsonline.scripps.edu/) software.

Results: Totally 2618 peaks were detected for group H, N and C. Among them 355 peaks were found to be statistically significant and reliable $(p<$ 0.05 ) and 155 of these peaks were found to be changed (fold change $>1.5$ ) between the groups $\mathrm{C}$ and $\mathrm{H}$. On the other hand, 66 peaks were found to be changed (fold change $>1.5$ ) between the groups $\mathrm{H}$ and $\mathrm{N}$. The number of the peaks on the intersection of the peaks found to be changed between the groups $(\mathrm{C}$ and $\mathrm{H})$ and $(\mathrm{H}$ and $\mathrm{N})$ was 39. Based on putative identification results, 15 peaks were matched with 11 metabolites. We found an up-regulated level of $\operatorname{DHAP}(18: 0)$, prostaglandin $D 2 / 12$, 5methyltetrahydrofolic acid, porphobilinogen and N-Acetyl-4-O-acetylneuraminic acid/N-Acetyl-7-O-acetylneuraminic acid, 5-Aminopentanamide /5Aminopentanoic acid, Glycocholic acid, Saccharopine, N2-Succinyl-L-ornithine, gamma Tocopherol, and Galactosylsphingosine /Glucosylsphingosine in IgAV patients.

Conclusion: In conclusion, we have identified a number of metabolites that may be associated with the pathogenesis of IgAV. We also suggest that $\operatorname{DHAP}(18: 0)$, prostaglandin D2/I2, porphobilinogen, 5-methyltetrahydrofolic acid and N-Acetyl-4-Oacetylneuraminic acid/N-Acetyl-7-O-acetylneuraminic acid may serve as biomarkers for predicting kidney disease since they were increased only in the patients who developed renal involvement at follow-up.

Grant Support: This work was supported by Hacettepe University Scientific Research Coordination Unit

Disclosure of Interest

None declared

P303

Spatial analysis of childhood IgA-vasculitis in Croatia - a pilot study

M. Sapina', M. Frkovic ${ }^{2}$, M. Sestan'2 S. Srsen ${ }^{3}$, A. Ovuka ${ }^{4}$, M. Batnozic Varga ${ }^{5}$, N. Kifer ${ }^{2}$, K. Kramaric 6 , D. Brdaric ${ }^{7}$, K. Milas ${ }^{5}$, A. Gagro ${ }^{8}$, M. Jelusic ${ }^{2}$

1 University Hospital Centre Osijek, Josip Juraj Strossmayer University of Osijek, Medical Faculty Osijek, Faculty of Dental Medicine and Health, Osijek; ${ }^{2}$ University Hospital Centre Zagreb, University of Zagreb School of Medicine, Zagreb; ${ }^{3}$ University Hospital Centre Split, University of Split School of Medicine, Split; ${ }^{4}$ University Hospital Centre Rijeka, University of Rijeka School of Medicine, Rijeka; ${ }^{5}$ University Hospital Centre Osijek, Josip Juraj Strossmayer University of Osijek, Medical Faculty; ${ }^{6}$ University

Hospital Centre Osijek, Josip Juraj Strossmayer University of Osijek,

Faculty of Dental Medicine and Health Osijek; ${ }^{7}$ Josip Juraj Strossmayer

University of Osijek, Faculty of Dental Medicine and Health, Institute of Public Health for the Osijek-Baranja County, Osijek; ${ }^{8}$ Children's Hospital

Zagreb, Josip Juraj Strossmayer University of Osijek, Medical Faculty Osijek, Zagreb, Croatia

Correspondence: M. Sapina

Pediatric Rheumatology 2020, 18(Suppl 2):P303

Introduction: Henoch-Schönlein purpura or IgA vasculitis (IgAV) is the most common childhood vasculitis. Although the etiology of $\operatorname{IgAV}$ is still unclear, however, it seems that a combination of genetic and environmental factors may contribute. Spatial analyses have previously been used in the analysis of infectious diseases spreading, and several researches showed promising applications in noncommunicable diseases.

Objectives: To estimate the incidence and describe the spatial distribution of the incidence of IgAV in Croatia.

Methods: In this retrospective pilot study patient's data were collected over a six-year period between 2013 and 2019 in five 
tertiary hospitals in Croatia. The average annual incidence of IgAV was calculated with the population census data from 2011 as the denominator. To investigate the spatial distributions of IgAV, a choropleth map was created based on the raw and Bayesian adjusted incidence data.

Results: A total of 402 domestic patients were included in the study, of which $223(55.47 \%)$ were male, and $179(44.53 \%)$ were female, with a median age of 6.42 (4.5 to 9.08) years. The estimated average annual incidence was 8.4 with a $95 \%$ confidence interval between 6.51 to 10.66 per 100000 children. As expected, the raw data showed that the highest number of cases was detected in administrative areas with a higher population. A statistically significant higher percentage of patients came from the continental $(69.15 \%)$, when compared to the Mediterranean part $(30.85 \%), p<$ 0.001. Box maps and standard deviation maps showed with incidences higher (hot-spots) and lower (cold-spots) than the average annual incidence. However, when the standardized average annual incidence was plotted, the spatial distribution correlates with the location of higher incidence levels. Therefore, the spatial distribution pattern showed the existing of hot-spot clusters with higher incidences around large cities both in the continental, and Mediterranean part of Croatia.

Conclusion: This pilot study investigated the usefulness in expanding the epidemiological toolbox with applying spatial analyses. The results of this study suggested that the IgAV incidence might be clustered in space. However, for a more definitive conclusion, a geostatistical analytical approach is needed to evaluate the significance of observed clusters.

SUPPORT: Croatian Science Foundation project IP-2019-04-8822.

\section{Disclosure of Interest}

None declared

\section{P304}

Eleven children with primary angiitis of the central nervous system: the experience of a single center

T. Giani $i^{1,2}$, G. Abbati ${ }^{1}$, M. Mortilla ${ }^{3}$, G. Simonini ${ }^{1}$, A. Rosati ${ }^{4}$

${ }^{1}$ Rheumatology Unit, AOU Meyer, Florence; ${ }^{2}$ Department of Medical Biotechnology, University of Siena, Siena; ${ }^{3}$ Radiology Unit; ${ }^{4}$ Neurology Unit, AOU Meyer, Florence, Italy

Correspondence: $T$. Giani

Pediatric Rheumatology 2020, 18(Suppl 2):P304

Introduction: Childhood primary angiitis of the central nervous system (CPACNS) is a rare condition, whose clinical presentation may embrace a large, but non-specific range of manifestations.

Objectives: To describe clinical presentation, treatment and outcome in our childhood PACNS cohort.

Methods: We retrospectively reviewed all cases of cPACNS followed at Anna Meyer Children Hospital from January 1st, 2010 through March 31st, 2020 analysing demographic, anamnestic, clinical, radiological, and laboratory data, type of treatment, and outcome.

Results: We included 11 patients, 2 females, median age at disease onset 6.8 years (range $0.9-15.8$ ), median age at diagnosis 7.6 years (range 1.11-16.5). Motor deficits were the most frequent presenting symptoms (8/11), followed by consciousness impairment (5/11) and headache (5/11). All patients underwent magnetic resonance imaging (MRI) including magnetic resonance angiography (MRA) sequences in 10/11 cases. In 8/11 children digital subtraction angiography was also performed. Basal ganglia resulted the most frequently damaged area (6/11), and middle cerebral artery the most affected territory $(8 / 11)$.

Two out of eleven patients remained stable during the follow-up. Three out of eleven patients had new clinical manifestations, before starting mycophenolate mofetil (MMF): Pt \#1 experienced a second stroke in a different cerebral vascular territory 1 month later the first one, Pt \# 2 had a second stroke 8 months later, and Pt \#3 had two consecutives strokes, one month a part each other, associated with MRA documented progressive radiological worsening at 6 and 12 months from the disease onset.

Six out of eleven children showed a radiological worsening without new clinical manifestations: $4 / 6$ at the $3^{\text {th }}$ month [of these $3 / 4$ showed a new territory involvement and $1 / 4$ a territory extension] while $2 / 6$ had additional worsening with new territories involvement at the $6^{\text {th }}$ month and at $12^{\text {th }}$ month respectively.

All patients received MMF $\left(650-1100 \mathrm{mg} / \mathrm{m}^{2} /\right.$ day), after a median latency period of 6 months (range 2-32) from the disease onset.

MMF has been administered for a median period of 32 months (range 2-58). Five out of 11 patients were still receiving MMF at last follow-up. Five other children concluded the treatment due to the sustained clinical remission and stable/improved radiological findings. One child switched to Methotrexate plus Infliximab due to the progressive worsening despite MMF treatment. No MMF serious adverse events were registered.

All patients received a corticosteroid cycle during the acute phases. At last follow-up (42 months, range 11-108), 10/11 patients showed radiological abnormalities, 5/11 patients had associated neurological symptoms or signs as motor deficits (5/11), cognitive dysfunction (2/ $11)$, concentration impairment (2/11), and behaviour changes (2/11).

Conclusion: Children with cPACNS may present clinical and/or radiological worsening few months after the disease onset. $\mathrm{A}$ strict MRI/MRA follow-up is recommended.

MMF has proven to be effective and safe in our cohort of children.

Disclosure of Interest

None declared

P305

Infliximab in Kawasaki disease: sharing an experience over 4 years

P. Pal, M. Roy

Pediatric Rheumatology, Institute of Child Health Kolkata, Kolkata

700017, India

Correspondence: P. Pal

Pediatric Rheumatology 2020, 18(Suppl 2):P305

Introduction: Tumor Necrosis Factor(TNF-a) blocker Infliximab (IFX) is emerging as an important drug in management of Kawasaki Disease (KD). This study was undertaken to evaluate the effectiveness of IFX in severe KD on 33 children over a period of 4 years (2015 to 2019).

Objectives: 1.Determine effectiveness of IFX in IVIG resistant KD.

2.Evaluate response on developing/ increasing coronary artery aneurysms (CAAs).

Methods: The study was carried out in the pediatric Rheumatology Unit of the Institute of Child Health Kolkata, India. 33 children aged between 6 weeks to 7 years with KD were included who received IFX after 1-2 doses of IVIG due to persistent fever/ increasing CAA or developing new CAA. Patients were analyzed for response in terms of achievement of defervescence in hours, normalization of CRP and improvement in echocardiographical findings specially CAAs. Children with severe CAAs were followed up by echocardiography weekly upto 6 weeks, monthly upto 3 months, and then 6 monthly.

Results: IFX was used in 1) IVIG resistant fever(23/33) and 2) increasing CAAs post IVIG (15/33). Overlapping indications ie. IVIG resistance with increasing CAAs were also present (7/33).IFX was administered at $5 \mathrm{mg} / \mathrm{kg}$ and defervescence was achieved within 24 hours in all of the 23 resistant cases along with normalization/ drastic fall in CRP. 2 children whose fever persisted was later diagnosed as Systemic Arthritis and hence excluded from the study. Diminution in size of aneurysm was seen in $80 \%$ (12 out of 15) cases on follow up, giant aneurysms being converted to medium or small sized aneurysms over 6 to 18 months. Interestingly $50 \%$ reduction in the aneurysm size was noted in $60 \%(n=9)$ within first 6 months of administration. 
Conclusion: 1.IFX showed remarkable success in rapidly lowering the inflammation ( fever and CRP) in IVIG resistant patients. Superiority over 2nd dose IVIG cannot be commented though 2 patients were enrolled who had failed to respond to the 2 nd dose IVIG.

2.Majority of patients with developing or increasing aneurysms receiving IFX post IVIG showed diminution in size on follow up. Limitations:

1.The number of patients is relatively small and there was no control arm.

2.Coronary angiographic corroboration of the echocardiographic findings could be performed in only 2 of the patients.

\section{Disclosure of Interest}

None declared

\section{P306}

Systematic review of childhood-onset polyarteritis nodosa and

DADA2

M. Kasap Cuceoglu, S. Demir, E. Sag, U. Kaya Akca, E. Atalay, E. D. Batu, Y. Bilginer, S. Ozen

Pediatric Rheumatology, Hacettepe University, Ankara, Turkey

Correspondence: M. Kasap Cuceoglu

Pediatric Rheumatology 2020, 18(Suppl 2):P306

Introduction: Polyarteritis nodosa (PAN) is a vasculitic disease characterized primarily by necrotizing vasculitis that may present with fever, weight loss, severe muscle and joint pains, and abdominal pain. Effective treatment is now available for PAN. ${ }^{1}$

Objectives: We aimed at assesing the characteristics of childhoodonset PAN in our center in the last ten years along with a systematic review.

Methods: We reviewed the charts of all pediatric PAN patients from 2010 onwards, in the Department of Pediatric Rheumatology in Hacettepe University, Ankara, Turkey. 47 pediatric patients who had fulfilled the Ankara 2008 for PAN, were included in the study. ${ }^{2}$ The demographics, clinical findings and treatment were evaluated. A systematic literature review was conducted by using keywords 'Polyarteritis Nodosa' and 'childhood' in PubMed databases in the English literature.

Results: 12 children had cutaneous PAN, 18 patients had systemic PAN. After 2014, 17 patients who we had originally classified as PAN, were diagnosed as DADA2. Skin involvement $(86 \%)$ was the most common feature of PAN, followed by abdominal pain (73\%), arthralgia/arthritis, weight loss, renal and neurologic involvement. Cutaneous PAN patients were treated with corticosteroids. In systemic PAN both IV cyclophosphamide and mycophenolate mofetil were used for the induction phase. None of the patients died. All patients were ANCA negative. MEFV mutations were screened among 20 patients, 17 of them had mutations in at least one allele. Biopsy was performed in 21 patients and angiography was performed in 33 patients.

The literature review yielded 937 articles about PAN, 170 articles in childhood.

Conclusion: Early recognition and treatment accounts for a good prognosis in childhood PAN. A careful attention must be given to DADA2 in the differential diagnosis, since the DADA2 patients also meet both the Ankara 2008 and ACR criteria for PAN and have mimicking features.

\section{References}

1. De Virgilio A, Greco A, Magliulo G, et al. Polyarteritis nodosa: A contemporary overview. Autoimmun Rev. 2016;15(6):564-570:10.1016/ j.autrev.2016.02.015

2. Ozen S, Pistorio A, lusan SM, et al. EULAR / PRINTO / PRES criteria for Henoch - Schönlein purpura, childhood polyarteritis nodosa, childhood Wegener granulomatosis and childhood Takayasu arteritis : Ankara 2008. Part II : Final classifi cation criteria. 2010;(1):798-806. doi:10.1136/ ard.2009.116657

Disclosure of Interest

None declared
P307

PRES vasculitis working party annual report for 2020 year

M. Jelusic ${ }^{1}$, on behalf of the PRES Vasulitis Working Party,

D. Eleftheriou ${ }^{2}$, T. Giani ${ }^{3}$, E. D. Batu ${ }^{4}$, N. Martin ${ }^{5}$, M. Sestan

${ }^{1}$ University Hospital Centre Zagreb, University of Zagreb School of Medicine, Zagreb, Croatia; ${ }^{2}$ UCL Great Ormond St Institute of Child Health, London, United Kingdom; ${ }^{3}$ Anna Meyer Children's Hospital,

Florence, Italy; ${ }^{4}$ Ankara Training and Research Hospital, Ankara, Turkey;

${ }^{5}$ Royal Hospital for Children, Glasgow, United Kingdom

Correspondence: $M$. Jelusic

Pediatric Rheumatology 2020, 18(Suppl 2):P307

Introduction: The PRES Vasulitis Working Party is an international group of clinicians, scientists and trainees with about 50 members, and its main goal is to facilitate translational research in the field of vasculitis of the young. This encompasses basic science with potential to improve patient care, molecular genetics, clinical trials (investigator led and industry sponsored) and clinical guidelines.

Objectives: Current activities of this Working Party are divided into three areas: educational activities, activities for clinical care and activities in science and research.

The main educational activity includes endorsement of the First European Congress on Kawasaki Disease EURO - KiDs Congress from 14 to 16 January 2021 in Paris, France. A two-day main meeting aimed to update on clinical innovation, recommendations, basic science and sharing clinical experiences. We have applied for the PReS Educational Course "Vasculitis in paediatric age", which is planned to be held in Barcelona in March 2022.

Activities for clinical care include: implementation of existing diagnostic and treatment recommendations into clinical practice and development of new recommendations with translations to other languages; encouraging the application of clinical questionnaires in assessing the quality of life and disease activity; encouraging the issuance and use of brochures and educational materials for patients and their family members that will be available online; connection with organizations of patients to better understand their needs; developing web-based platform for healthcare professionals with virtual panels in order to discuss difficult cases in the field of vasculitis with the possibility of sharing medical imaging.

Currently there are three ongoing scientific projects.

The first project entitled "Comparison of pediatric criteria with adult classification criteria in granulomatosis with polyangiitis" is a multicentre study which will include at least 2 patients with granulomatosis with polyangiitis per center, and 2 controls (patients with other primary systemic vasculitis or mimicks such as sarcoidosis) per 1 granulomatosis with polyangiitis patient. For further information please contact principal investigator of the project, Prof. Seza Ozen (sezaozen@gmail.com). The second project entitled "Multi-centre, randomised, open-label, blinded endpoint assessed, trial of corticosteroids plus intravenous immunoglobulin (IVIG) and aspirin, versus IVIG and aspirin for prevention of coronary artery aneurysms in Kawasaki disease" is a multicentre European study (funding already approved) to establish the efficacy and safety of added corticosteroids to IVIG and aspirin for treatment of Kawasaki disease. It aims to recruit 262 children over 30 months from around 40 centres in 12 countries. Chief investigators of the project are Dr. Despina Eleftheriou (d.eleftheriou@ucl.ac.uk) and Prof. Paul Brogan (p.brogan@ucl.ac.uk). The third project entitled "Histological predictors of outcome in patients with Henoch-Schönlein purpura and nephritis" is a multicenter, multinational study which aims to compare the International Study of Kidney Disease in Children, Haas, Oxford and modified semiquantitative classification for their ability to predict the clinical outcomes in Henoch-Schönlein purpura and to establish which variables of each histological classification most significantly predict renal outcome/have the strongest association with unfavorable outcome. The principal investigator of this project is Prof. Marija Jelusic (marija.jelusic.drazic@gmail.com).

Disclosure of Interest

None declared 


\section{AB001}

A case of myositis in a patient with possible familial mediterranean fever,successfully treated with colchicine

S. Aristakesyan ${ }^{1}$, A. Ayvazyan²

${ }^{1}$ Rheumatology, Yerevan State Medical University; ${ }^{2}$ Internal Medicine, Muratsan clinic, Yerevan, Armenia

Correspondence: S. Aristakesyan

Pediatric Rheumatology 2020, 18(Suppl 2):AB001

Introduction: Although Myalgia in FMF is quite common,reported cases of Myositis in FMF are rare.

Objectives: To report an unusual clinical presentation of possible FMF.

Methods: A 18 year old,male patient was admitted to the clinic,complaining of severe bilateral calf muscle pain. No edema or erythema was visible. No chest or abdominal pain was mentioned. Peripheral joints were not swollen,range of motion was preserved. His parents mentioned,that this was his second episode of calf muscle pain,but no documented evidence was present. Body temperature was $37,5^{\circ} \mathrm{C}$. On laboratory tests-CRP-50mg/L,ESR-30mm/ h,transient mild proteinuria,Creatine phosphokinase 1995U/L. A number of viruses(AIDS,Hep.B,C, etc) was excluded. On EMG-definite pathological myopathy-like changes were visible.Muscle biopsy was not performed due to technical problems. Genetics-his uncle has definite diagnosis of FMF.MEFV gene mutation test revealed M694V/N genotype.

A possible diagnosis of FMF by Tel-hashomer criteria was made. Colchicine $1 \mathrm{mg} /$ day was prescribed.

Results: A total clinicolaboratory remission on Colchicine $1 \mathrm{mg} /$ daily was achieved.

Conclusion: Several types of Myalgia can occur in FMF patients. Exertional,spontaneous and protracted febrile myalgia syndrome are quite common. In all these cases CPK level and EMG are normal. So,we report an unusual case of Myositis in possible FMF,treated by Colchicine.

\section{Disclosure of Interest}

None declared

\section{AB002}

Abdominal syndrome in a monogenic autoinflammatory disease not only a familial mediterranean fever

S. Salugina, E. Fedorov

V. A. Nasonova Research Institute of Rheumatology, Moscow, Russian Federation

Correspondence: S. Salugina

Pediatric Rheumatology 2020, 18(Suppl 2):AB002

Introduction: Manifestations of the gastrointestinal tract (GIT) (abdominal pain, nausea, vomiting, diarrhea) are frequent symptoms of autoinflammatory diseases (AID). Acute abdominal pain occurs in $95 \%$ of patients with FMF and not rarely leads to unreasonable surgical interventions. This symptom is included in the diagnostic criteria for FMF. However, abdominal pain may be a serious symptom in patients with TRAPS, leading to occurrence of severe complications (peritonitis, peritoneal commissures, intestinal perforation) and emergency surgeries. The core targeted drug in the treatment of TRAPS is IL-1 inhibitor - Canakinumab, that prevents development of organ-lesions, including gastrointestinal disorders.

Objectives: to present a female patient with genetically confirmed TRAPS, suffering, in addition to standard manifestations (fever, rash, periorbital edema, arthritis, acute phase markers), from profound abdominalgia during seizures, formation of severe adhesive disease, which led to perforation of the intestine and an emergency surgical intervention.

Methods: Case report

Results: A female patient P., aged 13, for the first time was admitted to the Federal Rheumatology Center in March 2015 with complaints of intermittent episodes of fever, rash, periorbital edema, arthritis, and abdominal pain lasting for 10-20 days every 2-3 weeks. From the disease anamnesis: she has been sick almost from the birth, as episodes of a macular rash began to occur. From the age of 3 months, monthly fever up to $39.6-40.4^{\circ \mathrm{C}}$, lasting for 10 days (sometimes even up to 20 days), accompanied by a confluent macular and annular rash, myalgia, arthralgia, conjunctivitis, periorbital erythema, a significant increase in acute phase markers (ESR, CRP). ANA,RFnegative. During the hospitalization period, 2 disease recurrences were noted, with febrile fever up to $40.4^{\circ \mathrm{C}}$, erythematous rash, conjunctival hyperemia, periorbital erythema, myalgia, arthritis, profound abdominal pains. ESR: 43mm / h, leuk: 23.5, CRP: $211 \mathrm{mg} /$ I (normal $\leq$ 5). The use of pulse therapy of GS by a single intravenous administration interrupted the attack. The patient's condition between the attacks is relatively satisfactory. A molecular-genetic study was conducted: by the direct sequencing method a partial analysis of the TNFRSF1A gene was performed. In exon 3, a single nucleotide substitution c295T> C (pCys99Arg) in a heterozygous state was detected. The TRAPS diagnosis was confirmed. Canakinumab was prescribed in a dosage of $150 \mathrm{mg}$ once every 4 weeks, which led to relief of the disease main manifestations and improvement of lab tests. An increase in the interval up to 8 weeks and interruptions in treatment resulted in acute exacerbations with fever, rash, increase CRP, ESR, diarrhea, nausea, vomiting, abdominal pains. In November 2018: an acute exacerbation, acute abdominal pains, increase in ESR level up to $82 \mathrm{~mm} / \mathrm{h}, \mathrm{CRP}$ up to $31 \mathrm{mg} / \mathrm{dl}$ (normal $\leq 0.5$ ), $\mathrm{Hb}-8.7 \mathrm{~g} / \mathrm{dl}$. In December 2018, an emergency laparotomy was performed - perforation of the transverse colon, widespread serofibrinous peritonitis, massive adhesion process in the abdominal cavity were diagnosed. Treatment with Canakinumab re-initiation starting from January 2019 led to a complete relief of clinical manifestations, abdominal symptoms and normalization of ESR,CRP.

Conclusion: treating patients suffering from severe gastrointestinal manifestations during seizures, it is necessary to consider not only FMF, but also other autoinflammatory diseases, including TRAPS. Well-timed prescribed targeted therapy, strict adherence to the dosage and intervals between drug administrations, as well as a proper monitoring may help prevent severe gastrointestinal disorders and eliminate them in a prompt manner.

\section{Disclosure of Interest}

None declared

\section{AB003}

Severe glomerulonephritis complicating traps syndrome

P. Mesa-Del-Castillo B. ', M. J. Lorente Sánchez ${ }^{2}$, J. A. Piñero², C. Vicente Calderón ${ }^{2}, J$. M. de la Peña Moral ${ }^{3}$

${ }^{1}$ Pediatric Rheumatology; ${ }^{2}$ Pediatric Nephrology; ${ }^{3}$ Pathology, Hospital Universitario Virgen de la Arrixaca, Murcia, Spain

Correspondence: P. Mesa-Del-Castillo B

Pediatric Rheumatology 2020, 18(Suppl 2):AB003

Introduction: TRAPs is an autosommal dominant autoinflammatory disease. To date no severe inflammatory glomerular lesions leading to renal failure have been described associated to disease or its treatment. We present a case of severe glomerulonephritis in a TRAPs patient in treatment with IL-1 blocker canakinumab.

Objectives: To describe the clinical features, treatment and outcome of an acute renal failure due to glomerulonephritis in a 5 year old toddler who was under treatment with IL-a blocker canakinumab.

Methods: A retrospective chart review was conducted to extract clinical, laboratory, histology and evolutive data of the patient.

Results: A previously steady 5 year old male in chronic treatment with canakinumab $4 \mathrm{mg} / \mathrm{Kg}$ for a TRAPs due to an heterozygotic de novo mutation in TNFRSF1A p.(Cys43Tyr), presents in emergency room with a 12 hour macrohematuric oligoanuria and generalized oedema. He referred an auto limited febrile spike two weeks before, and a few days vomiting and nausea previous to consultation. Initial exams showed renal injury with urea $227 \mathrm{mg} / \mathrm{dl}$ and creatinin $4.24 \mathrm{mg} / \mathrm{dl}$, hematuria, leucocituria and nephrotic range proteinuria (3.7 $\mathrm{mg} / \mathrm{Cr} \mathrm{mg}$ ) with normal seric albumin, compatible with acute glomerulonephritis. Autoimmunity, lupus anticoagulant, and rheumatoid factor were negative, serology was normal apart from antistreptolysin $\mathrm{O}$ titres of $1663 \mathrm{UI} / \mathrm{L}$. Levels of C4 were normal and 
C3 was low (10.7 mg/dL) with an ESR of $67 \mathrm{~mm} / \mathrm{h}$ and a CRP of 0.95 $\mathrm{mg} / \mathrm{dL}$. First day after admission a drug refractory hypertension, metabolic acidosis and hyperpotasemia developed, requiring admission in intensive care unit for veno-venous continuous hemofiltration (HDFVVC). Metil prednisolone bolus $\left(1 \mathrm{~g} / 1.73 \mathrm{~m}^{2}\right)$ every 48 hours were initiated and a renal biopsy performed, showing generalized endocapilar proliferation with neutrophil infiltration and exclusive abundant mesangial pseudolineal deposits of $\mathrm{C} 3$ on immunofluorescence, no signs of amyloidosis. After five days of HDFVVC and two metilprednisolone bolus, oral prednisone $(2 \mathrm{mg} / \mathrm{Kg})$ was initiated and a 10 day course of amoxiciline administered. Evolution was satisfactory thereafter, at two months follow-up visit only minimal hematuria (5-10 erytrocites/field) persisted, with normal renal function, complement levels, prednisone $2.5 \mathrm{mg}$ every 48 hour and no hypertensive drugs required; canakinumab was reinitiated 8 weeks after renal failure, no further major events ocurred up to date. Conclusion: We present a case of acute severe postinfectious glomerulonephritis in a TRAPs patient, presumably poststreptococal. This is to our best knowledge the first case reported and questions arise on the possible association of this severe event to TRAPs syndrome, ongoing treatment or just an unfortunate coincidence. Differential diagnosis was mandatory to discard a rapidly progressive glomerulonephritis and renal amyloidosis in this context. Mild and generally transient proteinuria and creatinine clearance reduction have been reported in association with canakinumab treatment but to no acute glomerulonephritis has been previously described.

\section{Disclosure of Interest}

None declared

\section{AB004}

Clinical case of the hyperimmunoglobulinemia $\mathrm{D}$ with periodic fever

Y. Vyzhga, N. Sinchuk

Pediatric department, National Pirogov Memorial Medical University,

Vinnytsya, Vinnitsya, Ukraine

Correspondence: $Y$. Vyzhga

Pediatric Rheumatology 2020, 18(Suppl 2):AB004

Introduction: In abstract presented clinical case of the hyperimmunoglobulinemia D syndrome, that usually characterized by earl onset and favorable course.

Objectives: Patient O., 27 years old, came to the center of the orphans diseases a year ago. Patient became sick at 10 months with the first episode of a fever occurred, with diarrhea, macular skin rush. Methods: With a fever child was admitted to the infectious department, after the treatment with improved condition discharged home. In a 3 month, and than after 4 months the same episodes of a sudden fever and skin rush were observed, with nausea, vomiting, diarrhea. Child was excluded with common causes of prolonged infection disease. At the age of 1,6 together with fever and rush, episode of arthritis appeared. Child was admitted to the rheumatologist and confirmed with systemic JIA, treated with high dose of steroids. In a next 2 years involvement of both knee and ankle joints was observed, fever appeared at least once in 2-3 months and was treated with steroids. At the age of 5, child got long therapy with steroids, but frequency of fever episodes was high, followed by typical inflammatory signs in laboratory investigations, in periods between fever attacks - all laboratory and instrumental results were normal. At the age of $8-13$ years, episodes of fever became rare, 2-3 times a year, and less severe. At the age of 13 treatment was added with metotrexate, as joint syndrome was still ongoing, steroids were stopped. Patients condition remain stable, with minimal arthritis of knee joint, fever was occurred once in a year, and at the age of 18 patient was transferred to adult rheumatologist. At the background of an argument between the patient and a doctor, he didn't come to his office for regular check-ups and discontinued treatment by himself. At the age of 20 new episodes of a fever started to appear, with rush, arthritis. Episodes of vomiting and diarrhea appeared as well. Patient by himself started to take steroids, but took them just during fever attacks. Patient started to suffer from muscle weakness and mother admitted that son became depressed, refused even from small walks. At the age of 24, during fever attack acute cerebrovascular accident happened. After the stroke, patient developed neurological symptoms that accompanied periodic fever, he got poor memorizing, difficulties with speech. Rheumatologist initiated systemic steroids, metotrexate, recommended pulse therapy with steroids. Patient refused from pulse therapy and didn't continue systematically treatment. At the age of 25,5 patient developed second acute cerobravascular accident during fever episode. This time patient was admitted to the ICU for long time, after the treatment discharged home with severe neurological complications. After the second stroke he got inversion of the day/night sleep, slept 18-20 hours a day, didn't recognize and communicate with no one, except mother and still had periodic fever and arthritis. Patient totally refused from the treatment and was deeply depressed.

Results: Ptient came to the center of orphan disease and was examined by genetic and pediatric rheumatologist, prescribed with additional investigations and mutation V377I was detected, as well high level of mevalonic acid in urine and decreased activity of mevolanatkinase. Patient was recommended to initiate treatment with anakinra and canakinumab. In a 4 months after the treatment was initiated, neurological symptoms normalized, disappeared fever and arthritis.

Conclusion: Adequate and in a timely manner diagnostic measures of the periodic fever syndrome with deficiency of mevalonatkinase allows initiate appropriate treatment, improve quality of patients life and avoid dozen complications. Estimation of the correct diagnose in case of our patient was late for 25 years.

Disclosure of Interest

None declared

\section{AB005}

Hyper - Immunoglobulin D Syndrome (HIDS)

E. Rapushi', E. Rapushi' ${ }^{2}$ L. Nuhaj ${ }^{1}$, I. Xhemali ${ }^{3}$

${ }^{1}$ Internal Medicine, Service of Rheumatology, 1. U.H.C.. "Mother Theresa";

${ }^{2}$ Service of Pediatry, 2. Hygeia Hospital; ${ }^{3}$ Internal Medicine, 1. U.H.C..

"Mother Theresa", Tirana, Albania

Correspondence: E. Rapushi

Pediatric Rheumatology 2020, 18(Suppl 2):AB005

Introduction: HIDS is a rare genetic inflammatory disease (autosomal recessive), characterized by periodic episodes of fever accompanied by other symptoms such as joint pain, skin rash and abdominal pain. The fever episodes have a duration of 4-6 days, they begin from the first year of life and appear periodically throughout the whole life. The mutation in the case of HIDS leads to a decrease in the activity of mevalonate kinase, an enzyme important for cholesterol synthesis, and febrile episodes can further decrease this activity. Thus, during these episodes high levels of mevalonic acid can be found on blood serum and urine.

Objectives: Case presentation, a child with HIDS

Methods: A 12-year-old female patient, whose parents refer for recurrent episodes of fever $\left(38-39.5^{\circ} \mathrm{C}\right)$, that are accompanied with diffuse joint disease, skin rash, oral aphthous lesions and abdominal pain. The episodes had begun nearly at 18-months-old with a duration of 3-5 days up to 6-years-old. Then again at 11-years-old. The fever episodes did not improve with the use of antibiotics, NSAIDs, CS or antipyretics. The mother had a normal pregnancy, the child was born on term and the psycho-motor development was conform the age.

Results: In the examination performed during the febrile episodes the patient did have joint pain on pressure, non itchy skin rash in the abdomen and inferior limbs, appearing during the fever but that endure even outside the febrile episodes. Aphthous lesions can be seen on tongue and soft palate. Moreover, non painful, mobile nodules of $1-1.5 \mathrm{~cm}$ can be palpated in the cervical, axillary and inguinal area. Spleen at the level of iliac crest. In the laboratory evaluation: inflammatory anemia in the blood

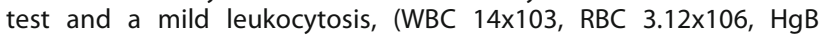


$9.7 \mathrm{mg} / \mathrm{dl}, \mathrm{MCV} 86 \mathrm{fl}$, ESR $40 \mathrm{~mm} / \mathrm{h}$ ). The biochemical profile within range. In the blood and urine culture no pathogenic increases were found. Febrile infectious antigens were negative. Bone marrow examination was normal. Immunological evaluation: FR neg, AAN neg, ENA neg, ANCAp/c neg, C3-C4 within the range, PCR $9.6(<5 \mathrm{UI} / \mathrm{ml})$. Protein electrophoresis revealed an increase in á1. Immunoelectrophoresis revealed an increase in IgD 350UI/ $\mathrm{ml}(100 \mathrm{Ul} / \mathrm{ml})$. Blood ferritin level was $60 \mathrm{mg} / \mathrm{dl}(20-50 \mathrm{mg} / \mathrm{dl})$. Head MRI was normal. In the abdominal CT with contrast infusion, cervical, axillary and inguinal nodules of $1-2 \mathrm{~cm}$ can be seen, liver of $16 \mathrm{~cm}$ without any visible lesions and homogenous spleen of $13.4 \mathrm{~cm}$. The data of lymphocyte immunophenotyping of peripheral and central blood within the range, no pathological lymphocytic populations were found. Nothing abnormal was found during nodule biopsy. HLA-B27 neg, HLA-B51neg. The level of mevalonic acid in urine resulted $130.8 \mathrm{mmol} / \mathrm{ml}(<69 \mathrm{mmol} / \mathrm{ml})$. Discussion: For the case the diagnostic differentiation between several diseases was made, including lymphoproliferative disorders (the data of bone marrow examination, biopsy and lymphocyte immunophenotyping did not support the diagnosis), infectious diseases (the data of febrile antigens were negative and febrile episodes were not affected by use of antibiotics), JRA- Still's disease (blood ferritin slightly increased and recurrent febrile episodes that resolve by themselves), inflammatory connective tissue disease ( $F R$, AAN, ENA, HLA-B27, HLA-B51 neg).

Conclusion: Since all other diseases were ruled out and both the level of $\lg D$ in blood and the level of mevalonic acid in urine were found to be elevated, besides the periodic febrile episodes and clinical symptoms, the patient was diagnosed with HIDS. In all patients with periodic febrile episodes the electrophoresis of immunoglobulins should be performed and also the evaluation of mevalonic acid both in blood and urine.

\section{Disclosure of Interest}

None declared

\section{AB006}

The evaluation of calcium metabolism and bone mineral density in patiens with CAPS

S. Cekic ${ }^{1}$, S. S. Kilic ${ }^{2}$

'Pediatric Immunology and Rheumatology, Uludag University Faculty of Medicine; ${ }^{2}$ Uludag University Faculty of Medicine, Pediatric Immunology and Rheumatology, Bursa, Turkey

Correspondence: S. S. Kilic

Pediatric Rheumatology 2020, 18(Suppl 2):AB006

Introduction: Cryopyrin-associated periodic syndrome (CAPS) includes a group of genetic conditions, caused by dominantly inherited mutations in the NLRP3 gene in Muckle-Wells syndrome (MWS) and familial cold autoinflammatory syndrome (FCAS), and de novo mutations in cases of chronic inflammatory neurologic cutaneous and articular syndrome (NOMID/CINCA).

Objectives: In this study, we aimed to investigate bone mineral density (BMD) and Ca metabolism in our patients with CAPS, mainly MWS.

Methods: Eight patients with MWS were included in the study. The clinical data were obtained from the file records of patients, retrospectively. Calcium (Ca), Phosphorus (P), Magnesium (Mg), Alkaline Phosphatase (ALP), $25 \mathrm{OH}$ vitamin $\mathrm{D}$, and bone mineral densitometry (BMD) tests were performed in all cases, but parathyroid hormone (PTH) levels were in 7 cases.

Results: The female to male ratio was $5 / 3$ and their ages ranged from 13.5 to 70 years. Most patients had had a history of coldinduced urticaria (88.8\%) and arthritis (100\%) since . their early childhood. Hearing loss was detected in 3 out of 8 (55.5\%). Alkaline Phosphatase levels ranged between 57-271 U / L, Ca 9-9.6 mg / dL, Mg 2$2.3 \mathrm{mg} / \mathrm{dL}, \mathrm{P} 2.6-4.4 \mathrm{mg} / \mathrm{dL}, 25-\mathrm{OH}$ vitamin D 3.7- $20.2 \mu \mathrm{g} / \mathrm{L}$ and PTH 28.8-162.7 ng / L. Vitamin D level was low in 7 out of 8 cases $(87,5 \%)$. The results of BMD tests showed that ; 2 cases were normal, 5 cases had osteopenia and 1 had osteoporosis. T scores of lumbar spine were between $-2.4-0.5$ in adult patients, the $z$ score was -0.5 of the pediatric case. Six patients were treated with canakinumab and 2 patients with anakinra.

Conclusion: It is known that excessive production of IL-1 $\beta$ can cause inflammatory bone loss and abnormality. Vitamin D deficiency and osteopenia/ osteoporosis may cause additional musculoskeletal problems besides arthritis and joint destruction in CAPS. We think that $\mathrm{Ca}$ metabolism and bone mineral density measurements should be a part of routine controls in patients with CAPS.

Disclosure of Interest

None declared

\section{AB007}

Clinical and genetic features of patients with periodic syndrome associated with mutation of the tumor necrosis factor receptor gene and juvenile arthritis having mutations in TNFRSF1A gene M. Shingarova ${ }^{1,2}$, E. Alexeeva ${ }^{1,2}$, T. Dvoryakovskaya ${ }^{1,2}$, A. Mamutova ${ }^{1}, K$. Isayeva', R. Denisova', A. Chomahidze', A. Fetisova', O. Lomakina', K. Chibisova', M. Gautier', D. Vankova', I. Kriulin ${ }^{1,2}$, E. Krekhova ${ }^{1,2}$, A. Pushkov ${ }^{3}$, K. Savostyanov ${ }^{3}$

'Rheumatology, National Medical Research Center for Children's Health; ${ }^{2}$ Sechenov First Moscow State Medical University; ${ }^{3}$ Genetic, National

Medical Research Center for Children's Health, Moscow, Russian

Federation

Correspondence: $\mathrm{E}$. Alexeeva

Pediatric Rheumatology 2020, 18(Suppl 2):AB007

Introduction: The use of a new generation of sequencing allows us to evaluate the genetic characteristics of patients with JIA in the Russian Federation, as well as to optimize the treatment strategy for JIA and TRAPS.

Objectives: To reveal the clinical and genetic features of JIA and TRAPS in patients with a genetically confirmed nucleotide variant in the TNFRSF1A gene.

Methods: Retrospective analysis of 21 case histories of patients with JIA-12 and TRAPS-9 observed, of which 14 girls, median age-14.1, median debut age-4.7. All children underwent molecular genetic research by Next Generation Sequencing and revealed nucleotide variants of the TNFRSF1A gene.

Results: An analysis of clinical symptoms and laboratory activity in children with TRAPS was performed: in the onset of the disease, systemic manifestations were observed in 8/9: fever in 8/9, maculopapular rash in 5/9, hepatosplenomegaly occurred in 5/9, and lymphadenopathy in $4 / 9$. High laboratory activity was recorded in 7/9. Active arthritis in $8 / 9$, it was poly in 2 , oligo in 6 . When assessing the clinical symptoms and laboratory activity of patients with JIA, it was revealed that in the onset of the disease, systemic manifestations were observed in 8/12: fever in $8 / 12$, rash in $4 / 12$, hepatosplenomegaly in $5 /$ 12 , pneumonitis in $2 / 12$, carditis in $1 / 12$ and lymphadenopathy in $5 / 12$. High laboratory activity was recorded in $11 / 12$. Active arthritis in $10 / 12$, it was polys in 4 , oligo in 6 . In all $100 \%$ of patients, the nucleotide variants of the TNFRSF1A gene were identified in the study. 9/21 of patients were diagnosed with TRAPS. The most frequent heterozygous variant of TNFRSF1A gene with nucleotide substitution of $c .362 G>A$ was found in $7 / 9$ of patients, in $1 / 9$ of patients it was found homozygous variant with nucleotide substitution of $c .362 G>A$, in $1 / 9$ of children it was found heterozygous variant with deletion of c.337_339del. All of these variants are pathogenic. 12/21 of patients were diagnosed with JA: juvenile arthritis with a systemic onset was in $7 / 12$, paucarticular arthritis was in $2 / 12$, in $1 / 12$ it was poly RF- and in $1 / 12$ it was psoriatic arthritis. It is worth noting to note that in $6 / 12$ a heterozygous version of the TNFRSF1A gene was detected with a nucleotide substitution c.362G>A, however, considering the absence of clinical manifestations of autoinflammatory disease and active articular syndrome in these patients, children were diagnosed with JA. In addition, in 2/12 a heterozygous variant with a nucleotide substitution $c .1110 C>T$ was detected, which is an undetermined value variant; in 2/12 a heterozygous variant with a nucleotide substitution $c .43 \mathrm{C}>\mathrm{T}$ was detected, in 1/12 heterozygous variant c.374G $>A$ was detected, and 1/ 12 heterozygous variant $c .369 C>T$ was detected. 
Conclusion: Systemic manifestations were more prevalent in patients with TRAPS - 91.6\%, and active articular syndrome was approximately the same in patients with JIA and TRAPS. In patients with TRAPS, the most common was the heterozygous version of the TNFRSF1A gene with the nucleotide substitution c.362G>A. Despite the fact that in $57.1 \%$ of JIA patients, variants of TNFRSF1A gene were identified, it is not possible to establish a diagnosis of auto-inflammatory syndrome in such patients. It is necessary to correctly interpret the results of molecular genetic research in accordance with the severity of the course of the disease, clinical manifestations.

\section{Disclosure of Interest}

M. Shingarova: None declared, E. Alexeeva Speaker Bureau of: Novartis, Pfizer, Sanofi, MSD and Roche, T. Dvoryakovskaya: None declared, A. Mamutova Speaker Bureau of: Novartis, K. Isayeva: None declared, R. Denisova Speaker Bureau of: Novartis, MSD and Roche, A. Chomahidze: None declared, A. Fetisova: None declared, O. Lomakina: None declared, K. Chibisova: None declared, M. Gautier: None declared, D. Vankova: None declared, I. Kriulin: None declared, E. Krekhova: None declared, A. Pushkov: None declared, K. Savostyanov: None declared

\section{AB008}

Does corona virus cause a specific inflammatory toe abnormality N. Hasson

THE PORTLAND HOSPITAL, London, United Kingdom Pediatric Rheumatology 2020, 18(Suppl 2):AB008

Introduction: During the recent coronavirus pandemic there was a description of inflammation of toes possibly related to Covid 19 infection. I have seen 4 patients present with inflamed toes.

Objectives: To ascertain if Covid-19 causes a specific toe inflammation or is this due to another problem

Methods: I reviewed the 3 patients who presented with toe inflammation doing a full rheumatological examination. They were all seen via Skype.

Results: None of the 4 patients had had any symptoms of Covid 19 and all had self isolated. Examination of the toes revealed chilblain type lesions and all 3 patients had evidence of Raynaud's syndrome with markedly delayed capillary refil times in the feet of around 10 seconds. 2 of the patients had had a previous diagnosis of Primary Raynaud's and the two were new patients both with Primary Raynauds and no underlying rheumatological disease. Hands were much less affected in the patients than feet with slight prolongation of CRT in hands compared to feet.

Conclusion: I think that although the weather was warm in the UK at the time of lockdown, indoor temperatures are significantly lower than outdoors. I think due to decreased exercise, lack of wearing socks and shoes indoors and cooler temperatures indoors these patients had suffered from acute Raynaud's syndrome with chilblains rather than immune mediated vasculitic type illness causing their toe problems. I question if 'Covid Toes' as it was described is actually a condition or a manifestation of lockdown and Raynaud's with chilblains. Once the patients wore socks and shoes and kept warm no further lesions occured.

\section{Disclosure of Interest}

None declared

\section{AB009}

COVID-19 and relapsing Kawasaki disease: a case report during the pandemia

M. C. Maggio', S. Giordano², M. C. Failla², A. Alaimo ${ }^{3}$, C. Gioè ${ }^{4}$, G. Sireci ${ }^{5}$ 'PROMISE "G. D'Alessandro", University of Palermo; ${ }^{2}$ U.O.C. of Paediatric Infectious Diseases, Paediatric COVID Center, Children Hospital "G. Di Cristina"; ${ }^{3}$ U.O.C. of Paediatric Cardiology, Children Hospital "G. Di Cristina"; ${ }^{4}$ B U.O.C. of Paediatric Infectious Diseases, Paediatric COVID Center, Children Hospital "G. Di Cristina", ARNAS, Palermo; ${ }^{5} C L A D I B I O R$, University of Palermo, Palermo, Italy

Correspondence: M. C. Maggio

Pediatric Rheumatology 2020, 18(Suppl 2):AB009
Introduction: The pandemia of COVID-19 remains a global health alarm with high incidence of lethality, especially in older age groups who suffer from underlying medical conditions. However, children are less likely to manifest severe conditions.

Objectives: COVID-19 was correlated to a higher incidence and a suspected increased risk of Kawasaki Disease (KD) in children.

Methods: We describe the case of a 2.2-year-old infant admitted for fever $\left(>5\right.$ days; $\left.>39^{\circ} \mathrm{C}\right)$, pharyngitis, cheilitis, arthralgia, feet oedema, rash, perineal and scrotal region erythema, bilateral lymphadenopathy of the neck, cough, rhinorrhea. He was extremely irritable. Heart rate: 140/min; capillary saturation $99 \%$ in air. Laboratory tests showed: leukocytes 13.4 $\times 10^{3} / \mu$ l (neutrophils: $7.4 \times 10^{3} / \mu$ ); platelets $502 \times 10^{3} / \mu$ l; haemoglobin $11.1 \mathrm{~g} / \mathrm{dl}$; increased inflammatory markers, with C-reactive protein (CRP) of $14.7 \mathrm{mg} / \mathrm{dl}$ (n.v.: < 0.5); hyponatremia $(133 \mathrm{mEq} / \mathrm{l})$. The nasal swab for respiratory viruses, IgM and IgG anti-EBV, CMV, Parvovirus, Mycoplasma, Chlamydia were negative. Anamnestic records revealed a previous KD, without coronary artery lesions (CAL), 1 year before.

Results: He was treated with antibiotics, intravenous infusion of Immunoglobulins (IVIG) (2 gr/Kg), acetylsalicylic acid (ASA) $(50 \mathrm{mg} / \mathrm{Kg}$ in 4 doses/day) and reached defervescence into 2 days. Echocardiography excluded CAL. The nasopharyngeal swab for SARS-COV-2 was doubt. The second throat swab done the day after IVIG infusion, was negative; however, the third nasopharyngeal swab for SARS-COV-2, done 4 days after IVIG infusion, was positive. Chest $x$-ray showed a significant lung interstitial thickening. IL-6 levels were $<6.25 \mathrm{pg} / \mathrm{ml}$ (n.v. $<6.25 \mathrm{pg} / \mathrm{ml}$ ).

He continued treatment with antibiotics, ASA $(5 \mathrm{mg} / \mathrm{Kg} /$ day), with the progressive resolution of the clinical symptoms and of the normalization of laboratory findings.

Conclusion: The peculiar outcome of the patient is the correlation of COVID-19 with KD, recently reported as associated. KD is considered as a multifactorial autoinflammatory disease, induced by a cytokine hypersecretion with a systemic vasculitis. COVID-19 is considered a cytokine storm syndrome, with a severe systemic vasculitis. SARS-COV-2 infection could be the trigger that could lead to hyperinflammation of KD.

The IVIG infusion could explain the transient negative swab for SARSCOV-2, with the successive positive relieve lasting 7 days, and the normal levels of IL-6, detected after IVIG infusion.

Relapsing KD is rare (1.7-3.5\%); in our patient this event could be triggered by the documented SARS-COV-2 infection.

\section{Disclosure of Interest}

None declared

\section{AB010}

Management of children with rheumatic diseases in covid-19 outbreak in the tertiary pediatric rheumatology center in Iran V. Javadi Parvaneh, K. Rahmani, R. Shiari, M. H. Yeganeh, M. Abdollahi Pediatric rheumatology, shahid beheshti university of medical sciences, tehran, Iran, Islamic Republic Of

Correspondence: $\mathrm{V}$. Javadi Parvaneh

Pediatric Rheumatology 2020, 18(Suppl 2):AB010

Introduction: The COVID-19 disease identified and reported from Wuhan, Hubei, China. At the time being, the disease is a pandemic and has involved the entire world.

Objectives: The aim of this study is reporting the planning and actions after the COVID-19 epidemic for prevention of spread of the disease, supporting patients, and managing the disease at our center's outpatient clinic as a referral center for children suffering rheumatic diseases.

Methods: Since the first report of disease, we tried to reply to the patients' questions in a virtual social network. We were following lab data, radiograms, sonograms, CT scans, and MRI results in this network and provided solutions for the management of the children's problems without need to encountering.

Results: In cases that were controlled and their diseases were in remission, we invited one of the patient's parents to come and receive the prescriptions. We put some instructive short films about COVID-19 in the virtual network and in LED screens of our outpatient hospital clinic to provide useful information. About the turn rating system (queuing system) we omitted manual turn-taking stands to prevent virus transmission. In the peak of the epidemic, we stopped outpatient 
clinics for three weeks. After that, we started clinics with the least number of patients and the most standard protection measures for physicians, patients, and other staff. All people were triaged in the hospital yard before entering the waiting hall of the clinic by taking their temperature and screening questions. If they didn't have a fever and symptoms compatible with the disease, we let the patient and only one compeer enter the clinic hall with giving a free three-layer surgical mask to each of them. We requested the physicians to increase the time of their clinics to distance the appointments of the patients and to prevent overcrowding in the clinic. Each clinic was held with the presence of the attending physician and only one fellow physician. Gowns, protective glasses and face shields, surgical masks, and latex gloves were provided for secretaries and the other staff in the clinic. In the first eight weeks, daily disinfection was performed for all the surfaces of the clinic and after that, a disinfecting tunnel at the door of the clinic was added. With the screening of about 100 staff in the clinic, we found only one IgM positive person for COVID-19 during the first eight weeks who was one of the secretaries without any signs and symptoms and were quarantined at home. Among the physicians, 6 of them ( 8 percent) developed COVID-19 disease with laboratory confirmation. All of the involved physicians were working in the private section as well as a state-run system. From about 200 patients with different rheumatic diseases came to the clinic in eight weeks, 11 of them (5.5\%) were in the relapse of their disease which in comparison to the same time in the last year, 26 from 760 patients (3\%), a remarkable increase (almost double) is seen for which different causes can be considered. Most of the patients who came to the clinic in the COVID-19 outbreak were those who had to come due to the severity of their disease. In the patients who came before the epidemic, the most causes were musculoskeletal pains such as hypermobility and skeletal benign reasons and rheumatic diseases were in the next rank but in the epidemic outbreak, the prior group came in a lower number. Follow up on the patients after the epidemic will reveal this matter better.

Conclusion: Employing personal and patient protective equipments, patients' triage, postponement of face-to-face appointments, social distancing, telemedicine, and using the virtual social networks may be effective policies in outpatient clinics in the COVID-19 outbreak.

\section{Disclosure of Interest}

None declared

\author{
AB011 \\ Severe cardiac involvement with myocardial dysfunction and \\ arrhythmias in SARS-COV-2-related Kawasaki-like disease \\ M. E. Pinto', G. Di Salvo', E. Reffo', G. Cendon', A. Meneghel', F. Zulian', \\ G. Martini \\ ${ }^{1}$ Pediatric Rheumatology; ${ }^{2}$ Pediatric Cardiology, University-Hospital of \\ Padua, Padua, Italy \\ Correspondence: M. E. Pinto \\ Pediatric Rheumatology 2020, 18(Suppl 2):AB011
}

Introduction: During SARS-COV-2 pandemic, different reports have been published regarding children who developed hyperinflammatory syndrome with certain or probable relationship with SARS-COV2. These patients presented incomplete or atypical manifestations of Kawasaki disease (KD), particularly abdominal pain, myocarditis and macrophage activation syndrome features.

Objectives: To report a case of SARS-COV-2-related Kawasaki-like disease with severe cardiac involvement.

Methods: case report description.

Results: A 10-year-old previously healthy girl presented progressively worsening abdominal pain, high grade fever for 3 days and vomiting. Lab tests showed WBC $11680 / \mathrm{mmc}, \mathrm{N} 9370 / \mathrm{mmc}, \mathrm{C}$ reactive protein (CRP) $329 \mathrm{mg} / \mathrm{L}$, procalcitonin (PCT) 0,74 ug/L, PT-INR 1,35 and elevated D-dimer and fibrinogen levels (817 ug/L and $9,45 \mathrm{~g} / \mathrm{L}$ respectively). Abdomen ultrasound revealed lymphadenopathies and hyperechogenic mesentery in the right lower quadrant, although the appendix was not visualized. She underwent laparoscopy showing moderate quantity of free fluid and appendectomy was performed. Thereafter she continued to complain of high-grade fever, abdominal pain and diarrhoea, despite broad-spectrum antibiotics. Blood, urine and stool cultures were negative. Bilateral non-exudative conjunctivitis was present. Moreover, the lab tests showed persistent marked elevation of CRP (370 mg/L), WBC 15590/mmc, N 14070/mmc, hypoalbuminemia $(23 \mathrm{~g} / \mathrm{L})$, elevated ferritin and triglycerides ( $458 \mathrm{ug} / \mathrm{L}$ and $221 \mathrm{mg} / \mathrm{dl})$. By taking into consideration the concomitant SARS-COV-2 pandemic, nasopharyngeal and rectal swabs were taken with negative results. Conversely, serological test showed anti-SARS-COV-2 IgG antibodies and absence of IgM. The family medical history showed that the mother had presented fever, cough, ageusia and anosmia one month before, preceded by a contact with a SARS-COV-2 positive case, while the patient was asymptomatic at that time.

Suspecting a KD-like disease she was referred to our Paediatric Rheumatology Unit: cardiological assessment revealed negative Twaves in V4-V5-V6 on EKG while standard and advanced echocardiography showed mild mitral and tricuspid insufficiencies, mild dilatation of the left main coronary artery (LMCA, z score +2$)$, normal global function (FEVS 2D 58\%) but reduced longitudinal strain (GLS -16\%). Lab tests confirmed myocardial injury with troponin (Tnl) 100,1 ng/l and brain natriuretic peptide (P-BNP) $593 \mathrm{ng} / \mathrm{L}$.

A single infusion of intravenous immunoglobulin $2 \mathrm{~g} / \mathrm{kg}$ associated with methylprednisolone (1 mg/kg/day) led to a rapid clinical improvement with apyrexia and resolution of abdominal pain and conjunctivitis. Blood test confirmed gradual normalization of inflammatory markers, ferritin, troponin and BNP and EKG showed positive T-waves. Shortly after the discharge, while she was on prednisone $0.5 \mathrm{mg} / \mathrm{kg} /$ day and acetylsalicylic acid $100 \mathrm{mg} /$ day, she referred some episodes of heart pounding, lasting about ten minutes with spontaneous resolution. Three weeks after onset cardiac MRI was normal, however, speckle tracking echocardiography showed persistent dilatation of LMCA and reduction of global longitudinal strain (GLS -14\%). 24-hour EKG-Holter detected episodes of supraventricular tachycardia and several ventricular and supraventricular extrasystoles. Thus, oral atenolol therapy was started.

Conclusion: In our patient SARS-COV-2 induced a possible postinfectious antibody or immune-complex mediated reaction that led to KD-like disease with acute surgical abdomen presentation and persistent myocardial damage and arrythmias. Speckle tracking echocardiography appears more reliable than MRI in early detection of myocardial damage in patients with preserved left ventricular ejection fraction.

Disclosure of Interest

None declared

\section{AB012}

Spectrum of systemic inflammatory syndrome in children during COVID 19 pandemic in India

D. B. Pandya, on behalf of Dr Haresh Dobariya, Dr Vinod Bhadukiya, Dr Rakesh Patel, Dr Hitesh Bhambhani and Dr Sameer Dhami.

Paediatric Rheumatology \& Immunology, Dev Children's Hospital, Rajkot, India

Correspondence: D. B. Pandya

Pediatric Rheumatology 2020, 18(Suppl 2):AB012

\section{Introduction}

Recently reports from Europe and North America have described clusters of children and adolescents with a multisystem inflammatory condition with some features similar to those of incomplete Kawasaki disease. ${ }^{1-3}$

\section{Objectives}

To unveil characteristics of systemic inflammatory syndrome (SIS) in children during COVID-19 pandemic in India

Methods

This is a case series which included three such pediatric patients who were evaluated between April 2020 and May 2020 at five different hospitals from an Indian state of Gujarat. Our electronic data included demographics, clinical presentation, laboratory results and follow up. 
Results

Table 1 (abstract AB012). showed characteristics of SIS in children during COVID-19 pandemic in India

\begin{tabular}{|c|c|c|c|}
\hline Age (years) / Sex (M/F) & $3 / \mathrm{M}$ & $7.7 / \mathrm{M}$ & $7 / F$ \\
\hline Fever (duration) & 2 weeks & 11 days & 11 days \\
\hline Containment Zone & Yes & No & Yes \\
\hline Rash & + & + & + (evanescent) \\
\hline Red eyes & + & + & - \\
\hline Red Tongue & - & + & - \\
\hline Pharyngeal congestion & + & - & - \\
\hline Distal edema & + & - & - \\
\hline Skin exfoliation & - & + & - \\
\hline Swollen lips & + & - & - \\
\hline Joint involvement & + & + & - \\
\hline Serositis & + & - & - \\
\hline Organomegaly & + & - & - \\
\hline Gl symptoms & + & + & - \\
\hline Respiratory symptoms & + & - & - \\
\hline Chest X-Ray findings & + & - & - \\
\hline Shock or Hypotension & - & - & - \\
\hline $2 \mathrm{~d}$ Echo findings & - & - & - \\
\hline HB $(11.5-14.5 \mathrm{gm} / \mathrm{dl})$ & 7.2 & 9.1 & 9.6 \\
\hline TLC (4000-12,000/cumm) & 7800 & 19,700 & 23,230 \\
\hline Platelets $(1,50,000-4,50,000 / \mathrm{ul})$ & 46,000 & $5,20,000$ & $5,57,000$ \\
\hline Blood C/S & Negative & Negative & Negative \\
\hline ESR $(0-20 \mathrm{~mm} / \mathrm{hr})$ & 68 & 110 & 120 \\
\hline CRP $(0-6 \mathrm{mg} / \mathrm{L})$ & 234 & 44.7 & 226 \\
\hline Ferritin $(7-140 \mathrm{ng} / \mathrm{ml})$ & 125.4 & 122 & 6235 \\
\hline Procalcitonin $(<0.15 \mathrm{ng} / \mathrm{ml})$ & 3.84 & Not done & 7.14 \\
\hline Triglycerides (35-138mg/dl) & 333 & Not done & Not done \\
\hline Fibrinogen $(150-400 \mathrm{mg} / \mathrm{dl})$ & 209.5 & Not done & Not done \\
\hline LDH (24-59 IU/L) & 663 & Not done & Not done \\
\hline PT, aPTT & Mild derangements & Not done & Not done \\
\hline COVID-19 RT PCR & Negative & Negative & Not done \\
\hline \multirow[t]{3}{*}{ Treatment } & IV antibiotics & IV $\lg$ & Oral naproxen \\
\hline & Oral Azithromycin & Oral aspirin & Oral steroids \\
\hline & IV albumin & & \\
\hline Last follow up & better & better & better \\
\hline
\end{tabular}

( $\$=$ IV albumin infusion was given in view of progressive generalized edema and hypoalbuminemia)

\section{Conclusion}

Above three cases reemphasize the need for a high index of suspicion for COVID 19 (coronavirus) as a possible culprit in a child with unexplained multisystem inflammatory syndrome.

Trial registration identifying number

1. Riphagen S, Gomez X, Gonzalez-Martinez C, Wilkinson N, Theocharis P. Hyperinflammatory shock in children during COVID-19 pandemic. Lancet. 2020. Epub 2020/05/11.

2. DeBiasi RL, Song X, Delaney M, Bell M, Smith K, Pershad J, et al. Severe COVID-19 in Children and Young Adults in the Washington, DC Metropolitan Region. J Pediatr. 2020

3. Jones VG, Mills M, Suarez D, Hogan CA, Yeh D, Bradley Segal J, et al. COVID-19 and Kawasaki Disease: Novel Virus and Novel Case. Hosp Pediatr. 2020. Epub 2020/04/09
Disclosure of Interest

None declared

\section{AB013}

A child with a severe multi-system inflammatory syndrome following COVID-19 infection

A. Mauro', L. Zenzeri' , N. Improda', T. Gagliardo' ', C. Granato' ${ }^{1}$, C. D'Anna', I. Parente', I. R. Schiavone', M. Oreste', R. Sottile², V. Tipo'

${ }^{1}$ Emergency department; ${ }^{2}$ pediatrics, AORN Santobono-Pausilipon, Naples, Italy

Correspondence: A. Mauro

Pediatric Rheumatology 2020, 18(Suppl 2):AB013

Introduction: Despite the mild clinical course during the acute phase of COVID-19 infection in children, latest ongoing researches are pointing the attention towards a hyperinflammatory shock in pediatric patients as a possible consequence to COVID19 exposure.

Objectives: We report the case of a child with a severe systemic inflammatory syndrome following an asymptomatic COVID-19 infection.

Methods: A 9-year-old male was admitted to the Pediatric Emergency Unit due to fever and abdominal pain. Symptoms started 7 days before admission, with fever, vomiting and non-bloodydiarrhea. Family history revealed that the father had been admitted to a COVID-19 Sub-Intensive Unit with bilateral interstitial pneumonia until 7 days before the onset of symptoms in the child. On the basis of familial history and because of the presence of fever, patient entered the COVID-19 pathway and was isolated. He had no chronic underlying disease nor history of previous hospitalization. At admission, he appeared stable. Body temperature was $38.1^{\circ} \mathrm{C}, \mathrm{O} 2$ saturation was $98 \%$ in ambient area, blood pressure was $106 / 60 \mathrm{~mm} \mathrm{Hg}$, heart rate was $140 \mathrm{bpm}$, respiratory rate was 21 breaths per minute. On examination he was alert, there were no cough, runny nose or other respiratory symptoms. No conjunctivitis, rash or peripheral edema was detected. He had mild hepatomegaly.

Results: The patient underwent blood and microbiological exams including blood specimens for cultures and nasopharyngeal swabs for SARS-COV2 nucleic acid (by RT-PCR- assay). At baseline, leukocytosis with neutrophilia and relative lymphopenia were found. Hemoglobin was below the normal range, while platelets count was normal. Inflammatory markers were strongly elevated, particularly CRP(420.8 mg/L), ferritin(4488 ng/mL), D-dimer(5106 ng/ $\mathrm{mL}$ ). Several significantly altered parameters suggested liver function abnormality, with hypertransaminasemia, acute renal injury, with elevated blood urea nitrogen and serum creatinine, and myocardial injury, with elevated high sensitivity cardiac troponin (434 ng/L) and brain natriuretic peptide (825 pg/mL). Lymphocyte subsets were within the normal range, while NK cells were slightly reduced. Patient was also tested for respiratory syncytial virus (RSV) and for influenza viruses $A$ and $B$, resulted all negative. Bacteria and fungi blood cultures were sterile, as well as urine and stool cultures. He was tested for COVID-19 antibodies which showed positivity of both IgG and IgM (qualitative test), confirmed by a quantitative analysis which showed a high level of $\operatorname{lgG}(5066 \mathrm{AU} / \mathrm{ml})$ and a weak positivity of $\operatorname{lgM}(0.532 \mathrm{AU} / \mathrm{mL})$. Echocardiography showed no ventricular dysfunction, no dilatated coronaries or pericoronary iperechogenicity. Chest CT on the $2^{\text {nd }}$ day showed two small bilateral areas of atelectasis associated to minimal pleural effusion more evident on the right side.

The diagnosis of Hyperinflammatory syndrome COVID-19 related was made.

Conclusion: Because of the high levels of BNP and troponin, IV methylprednisolone $(5 \mathrm{mg} / \mathrm{kg} /$ day) and subcutaneous heparin (100 $\mathrm{U} / \mathrm{kg} /$ day) were started after 24 hours since admission.

Search for COVID-19 on nasopharyngeal swabs collected for 3 consecutive days resulted negative, The patient gradually recovered and fever disappeared after 48 hours. He presented no vomiting or diarrhea during the hospital stay, nor respiratory symptoms. Laboratory exams dramatically improved. According to his clinical and 
laboratoristic improvement, methylprednisolone was tapered to $3 \mathrm{mg} / \mathrm{kg} /$ day and he started oral prednisone $1.25 \mathrm{mg} / \mathrm{Kg} /$ day four days after. He was discharged with steroid and heparin therapy and a close follow-up was planned.

\section{Disclosure of Interest}

None declared

\author{
AB014 \\ Campatodactly-Arthropathy -Coxavara-Pericarditis (CACP) \\ syndrome in a libyan female child \\ A. B. Malek' , A. M. Abushhaiwia ${ }^{2}$, M. A. Zletni ${ }^{2}$, A. J. Ateeq², F. \\ Bencheikh ${ }^{2}$ \\ ${ }^{1}$ Radiology, Emitiga Hospital; ${ }^{2}$ Rheumatology, Tripoli children Hospital, \\ Tripoli, Libya \\ Correspondence: A. B. Malek \\ Pediatric Rheumatology 2020, 18(Suppl 2):AB014
}

Introduction: Campatodactly-arthropathy -coxavara-pericarditis (CACP) syndrome is a rare autosomal recessive disorder caused by a mutation in the gene proteoglycan 4(PRG4) affecting lubricin production, which is an essential protein for joint function. We present this case of a female child to describe the clinical, laboratory and radiological findings of CACP syndrome that should help in differentiation of CACP syndrome from other childhood inflammatory arthritis.

Objectives: To increase the awareness of this familial condition to prevent confusion with other inflammatory conditions

Methods: A case report

Results: an 8-year-old female patient was referred to the Rheumatology clinic with complaints of joint deformities of her fingers namely campatodactly was noticed through the first year of life that treated surgically, while other joint involvement at age 3 was starting to have multiple joints swelling, she was the products of $3^{\text {rd }}$ degree consanguineous marriage and other 2 siblings are healthy, she was referred as a case of poly juvenile idiopathic arthritis. She has normal intelligence with normal school performance, she's able to perform all daily activities except that she has difficulty in the traditional cross sitting.At the time of our assessment, articular findings revealed bilateral flexion deformity of proximal interphalangeal joints and limited flexion and extension of both wrist joints, additionally there was a thickened rubbery synovium, significant knee joints swelling with moderate to large effusion associated with it, significant limitation of hip joint movements mainly in the internal and external rotation as well as abduction, and she was unable to sit crosslegged, although she didn't have joint tenderness or hotness, she had sometimes a non-specific diffuse joint pain. Moreover, she had normal cardiac examination and echocardiography study, her ophthalmological assessment was normal, however ; her height was below $3^{\text {rd }}$ percentile, there were no other associated systemic involvement. Her laboratory tests revealed that she had normal inflammatory marks (ESR, CRP) and the result of rheumatoid factor test was negative, she had low vitamin D and slightly elevated alkaline phosphatase level, her PTH, serum calcium and phosphate were within normal range. Regarding her radiological findings, her $\mathrm{x}$ ray showed that there were pictures of bilateral coxa vara with a short femoral neck and flat, irregular femoral head and intra-osseous cysts, increased joint space and abnormal modeling acetabulum with small iliac wings. Other joints (knees, ankle, elbow and wrist) showed soft tissue swelling consistent with thick cartilage. Her hand $x$ ray showed a soft tissue swelling around the interphalangeal and wrist joints, periarticular osteopenia and flexion deformities of the interphalangeal joints, affecting the 5 th joints bilaterally .A bilateral hip MRI showed effusion. Patient was treated at the referral hospital with a (NSIDs) and antirheumatic drugs, however ; they were ineffective.We treated her in the rheumatology clinic with vitamin D and calcium supplements as a treatment for osteopenia. We believe that synovial biopsy is not indicated in the correct clinical and radiological setting for atypical presentation, thus it is recommended if genetic testing is not affordable.
Conclusion: CACP syndrome should be considered in juvenile patients presenting with non-inflammatory arthropathy. We hope that this report will increase the awareness of this familial arthropathy condition and the clinical characteristics and the radiological findings well facilitate the differentiation from the common childhood rheumatic diseases.

\section{Disclosure of Interest}

None declared

\author{
AB015 \\ Relationship of Raynaud's phenomenon on qualitative and \\ quantitative videocapillaroscopy evaluation of pediatric patients \\ with rheumatic diseases \\ S. Jiménez H., K. Jiron, A. V. Villarreal T., F. Garcia R., M. De la O C., N. \\ Rubio P. \\ Reumatología Pediátrica, Hospital Universitario "Dr. José Eleuterio \\ González", Monterrey, Mexico \\ Correspondence: $\mathrm{S}$. Jiménez H \\ Pediatric Rheumatology 2020, 18(Suppl 2):AB015
}

Introduction: Capillaroscopy is a tool that helps to assess microcirculation (MC) by observing the shape, density, structure, and alterations in the capillaries of the nailfold. $M C$ has an important association with rheumatic diseases (RD), which is why it has become an important tool in rheumatology, and is considered a low-cost, non-invasive method. Raynaud's phenomenon (RP), a clinical condition commonly found in patients with $\mathrm{RD}$, requires capillaroscopic evaluation to differentiate primary RP from the secondary, and it also helps to monitor the activity of the disease, assess response to treatment and prognosis. To date, there are no standardized capillaroscopic patterns in pediatric populations.

Objectives: Describe qualitative and quantitative capillaroscopic differences between pediatric RD patients with RP and those without RP. Methods: Patients from the pediatric rheumatology department of a tertiary center in Monterey, Nuevo León, Mexico from July 2017 to March 2020 were included. Eight fingers, excluding the thumb, taking for analysis the most representative $1 \mathrm{~mm}$ image of each finger with x200 videocapillaroscope (Optilia), all measurements were taken by the same observer. Demographic data captured to describe the diagnosis, age, and RP presence. Capillaroscopy data, qualitative data: pattern, density, abnormal shapes, microbleedings and edema; for quantitative characteristics length, width, inter capillary distance, afferent diameter and efferent diameter were obtained.

Results: Sixteen patients were included, of which $13(81.2 \%)$ were female, the mean age was 12 years, RP was present in 12 (75\%) patients. Regarding the diagnoses in the RP group, we found Systemic Lupus Erythematosus (5/12), Juvenile Idiopathic Arthritis (3/12), undifferentiated connective tissue disease (1/12), systemic sclerosis $(1 / 12)$, IgA vasculitis $(1 / 12)$ and primary RP (1/12); in the group without RP the diagnosis where psoriatic arthritis (1/4), psoriasis (1/4), localized scleroderma (1/4) and polymyositis (1/4). Among the quantitative characteristics, we found a greater length in R2, R4, R5, L4 and L5 of the patients with RP. Similarly, we observed a difference in the afferent diameter of R3, R4, R5, L2, L3, L4 and $L 5$, being bigger in patients with RP. In L4 we found a difference in length, afferent and efferent diameter, being greater in those patients with $\mathrm{RP}$, on the contrary, the width is usually smaller.

Conclusion: We found both qualitative and quantitative differences in patients with RP versus those without RP, mainly in afferent diameter for all fingers, and in $L 4$ we found a difference in most of the measurements. This study shows the quantitative data of the videocapilaroscopic analysis, and therefore it is proposed as a possible tool for the diagnosis and monitoring of $\mathrm{RD}$ in pediatric patients. Despite finding capillaroscopic alterations in both, the sample size in this cohort is small, so studies with a greater sample must be done, as well as studies with controls on healthy children, to make more conclusions.

Disclosure of Interest

None declared 
Table 1 (abstract AB015). See text for description

\begin{tabular}{|c|c|c|c|c|c|c|c|c|c|}
\hline & $\begin{array}{l}\text { Raynaud's } \\
\text { phenomenon }\end{array}$ & R2 & R3 & R4 & R5 & L2 & L3 & L4 & L5 \\
\hline \multirow{2}{*}{$\begin{array}{l}\text { Length } \\
(\mu \mathrm{m})\end{array}$} & Yes & 230.878 & 234.351 & 239.687 & 217.900 & 217.930 & 215.636 & 223.755 & 203.186 \\
\hline & No & 253.036 & 281.273 & 180.926 & 200.546 & 275.238 & 268.120 & 192.640 & 194.445 \\
\hline \multirow{2}{*}{$\begin{array}{l}\text { Width } \\
(\mu \mathrm{m})\end{array}$} & Yes & 54.442 & 69.112 & 51.100 & 51.091 & 53.070 & 46.854 & 51.963 & 45.713 \\
\hline & No & 57.696 & 54.518 & 45.658 & 48.696 & 52.281 & 60.571 & 53.029 & 42.710 \\
\hline \multirow{2}{*}{$\begin{array}{l}\text { Afferent } \\
\text { diameter } \\
(\mu \mathrm{m})\end{array}$} & Yes & 18.511 & 21.858 & 20.718 & 18.033 & 18.106 & 17.241 & 18.143 & 17.149 \\
\hline & No & 18.075 & 19.573 & 15.568 & 16.319 & 15.302 & 15.815 & 15.239 & 15.307 \\
\hline \multirow{2}{*}{$\begin{array}{l}\text { Efferent } \\
\text { diameter } \\
(\mu \mathrm{m})\end{array}$} & Yes & 25.403 & 26.675 & 24.918 & 24.037 & 23.804 & 22.895 & 25.008 & 22.229 \\
\hline & No & 26.493 & 27.900 & 22.088 & 22.763 & 30.990 & 22.396 & 20.148 & 18.834 \\
\hline
\end{tabular}

\section{AB016}

Is subclinical synovitis assessed by ultrasound able to predict flares in children with Juvenile Idiopathic Arthritis in clinical remission? A systematic review of the literature

T. Giani ${ }^{1,2}$, O. De Lucia ${ }^{3}$, R. Caporali ${ }^{4}$, R. Cimaz ${ }^{5}$

${ }^{1}$ Rheumatology Unit, AOU Meyer, Florence; ${ }^{2}$ Department of Medical

Biotechnology, University of Siena, Siena; ${ }^{3}$ Rheumatology and

Orthopedic Department, ASST-Gaetano Pini/CTO Orthopedic and

Traumatology Specialist Center; ${ }^{4}$ Division of Clinical Rheumatology, ASST

Gaetano Pini-CTO Institute; ${ }^{5}$ Department of Clinical Sciences and

Community Health, Research Center for Adult and Pediatric Rheumatic

Diseases, University of Milan, Milan, Italy

Correspondence: $T$. Giani

Pediatric Rheumatology 2020, 18(Suppl 2):AB016

Introduction: Clinical examination may not detect mild degrees of synovitis that can be present in subjects deemed to be in clinical remission and with negative inflammatory markers. In adults, ultrasound (US) Power Doppler positivity in patients with RA in clinical remission has been suggested by some authors to have a predictive value for flare. In pediatrics there are less definite data, in relation to difficulties in practical application of US and physiological changes in growing joints. In asymptomatic JIA children with a normal joint examination the long term significance of subclinical arthritis detected by US is not clear.

Objectives: Aim of the present study is to review published articles related to the predictive value for flare of subclinical synovitis assessed by ultrasound in juvenile idiopathic arthritis (JIA) in clinical remission. This could have implications in the practical management both for adjustments in drug treatments and for the timing of clinical surveillance during follow-up.

Methods: Medline, Embase and Cochrane databases were searched from 1990 to 2020 by two authors, using PICO methodology to build the following strategy. Population: JIA patients, aged $\leq 16$ years, in clinical remission. Interventions: Clinical assessment plus ultrasound. Comparison: Clinical assessment only. Outcome: Clinical flare. Reviews and case series $<10$ patients were excluded. The study is built and reported according to PRISMA guidelines.

Results: The search found a total of 208 records. After removing duplicates, 168 articles were selected by title and abstract, and of those 163 were excluded since they did not meet the PICO inclusion criteria. Five articles were identified suitable for analysis. One of these, after reading the full text, lacked detailed data related to US findings at baseline and was therefore excluded. We considered four articles comprising a total of 202 patients with JIA in clinical remission from at least 3 months. Because of the small number of the articles and data heterogeneity meaningful statistical analysis could not be performed. Two of the articles found US subclinical signs of synovitis to be predictive for flare, with a five times higher risk (with Power Doppler signal as an important feature), while in the other two articles baseline US abnormalities could did not predict a clinical flare. The articles differed for protocols, definitions, and length of follow-up.

Conclusion: US has an expanding role in pediatric rheumatology. Although its usefulness in the early diagnostic phase may be questionable, a more interesting use could be applied during followup, for the possibility of identifying subclinical inflammatory signs predictive of a future flare. The small number of studies available, data heterogeneity, and conflicting results however do not allow yet any definite conclusions in this regard.

Disclosure of Interest

None declared

\section{AB018}

Etanercept and Juvenile Idiopathic Arthritis, experience of the pediatric center of Sétif Hospital

K. Okka, Z. Benarab, S. Bouabdallah, A. Dehimi, M. Fellahi, M. Belghazi, B. Bioud

Pediatrics, Ward, Sétif, Algeria

Correspondence: $\mathrm{K}$. Okka

Pediatric Rheumatology 2020, 18(Suppl 2):AB018

Introduction: Etanercept (ETN) was the first anti-TNFa agent to obtain Marketing Authorization in 2000 for juvenile idiopathic arthritis (JIA) of polyarticular progression resistant to Methotrexate.

etanercept (ETN), a human $\operatorname{lgG} 1$ fusion molecule - soluble p75 receptor for human TNF alpha.

The indications of the ETN currently cover the JIA of polyarticular evolution, that is to say the polyarticular, extended oligoarticular forms and the systemic forms become polyarticular, the form associated with enthesopathies and psoriatic arthritis

Objectives: To analyze the effectiveness of Etanercept, as well as its tolerance in juvenile idiopathic arthritis (JIA).

Methods: We carried out a retrospective study of children diagnosed with JIA according to the ILAR classification criteria and treated with Etanercept at the pediatric center of Setif University Hospital since 2015.

15 children were included and judged at 3 months, 6 months, 1 year, 2 years, 3 years, 4 years on epidemiological criteria, efficacy criteria (Joint scores, uveitis, SV, CHAQ) and the occurrence of possible Side effects .

We defined the improvement of 30\% (ACR 30), 50\% (ACR 50), 70\% (ACR 70), 90\% (ACR 90), and 100\% (ACR 100) as the improvement of minus 3 out of 6 criteria of $30 \%, 50 \%, 70 \%, 90 \%, 100 \%$; patients must not have an aggravation of more than $30 \%$ from one of the 6 criteria.

Results: The epidemiological characteristics were as follows: 9 girls and 6 boys, 7 have a polyarticular form, 5 have an oligoarticular form, 2 cases with psoriatic arthritis, and only one case of arthritisassociated enthesitis (ARF).

ACR 30 is obtained in $75 \%, 84 \%, 88 \%$ of cases respectively at 3 months, 6 months, 1 year.

The most marked responses were obtained in the polyarticular, oligoarticular and and arthritis-related enthesitis. for psoriatic arthritis $=72 \%$.

Complete remission was maintained in the majority of the patients for varying durations depending on the outcomes.

Furthermore, no clinical or biological undesirable effects have been noted.

Conclusion: Etanercept has a spectacular efficacy in children suffering from juvenile idiopathic arthritis, particularly in polyarticular, oligoarticular forms and IBAs.

His overall tolerance is very good.

Disclosure of Interest

None declared 


\section{AB019}

Fibroblast growth factor and hepatocyte growth factor in adolescents with Juvenile Idiopathic Arthritis treated with methotrexate

L. Parkhomenko, L. Strashok, O. Pavlova

Department of Adolescent Medicine, Kharkiv medical academy of postgraduate education, Kharkiv, Ukraine

Correspondence: $\mathrm{O}$. Pavlova

Pediatric Rheumatology 2020, 18(Suppl 2):AB019

Introduction: Risk of liver fibrosis development during juvenile idiopathic arthritis (JIA) treatment with methotrexate (MTX) in adolescents remains relevant.

Objectives: To study levels of basic fibroblast growth factor (BFGF) and hepatocyte growth factor (HGF) in adolescents with JIA treated with MTX

Methods: 68 children were observed, 25 boys (36.8\%) and 43 girls (63.2\%).The average age of the patients was $13.3 \pm 0.3$ years. Children were divided into four groups: those with JIA who didn't receive MTX yet (group 1); those who received MTX less than one gram during whole treatment (group 2); those who received MTX from 1 to 3 grams (group 3); children, received more than 3 grams of MTX (group 4).

Results: The autoimmune inflammatory process in JIA can cause formation of pathological changes in the liver, even before the start of treatment. It is confirmed by a statistically significant correlation of BFGF level in 1st group with liver steatosis according to ultrasound examination $(r=0.8)$ and the level of $C$-reactive protein $(r=0.7)$. This indicates a close relationship between the intensity of the inflammatory process and collagen synthesis activation, which can further provoke liver fibrosis. Alterative processes in the liver associated with autoimmune inflammation, as evidenced by the presence of a positive correlation between the level of ALT and BFGF $(r=0.5)$. Upon reaching MTX dose $1 \mathrm{gram}$ and 3 grams, it is possible that compensatory processes in the liver are triggered, as evidenced by the negative correlation between the content of BFGF and HGF $(r=-0.6)$.

Conclusion: The use of modern markers with routine laboratory and instrumental studies is appropriate for the timely determination of the risks of developing irreversible pathological changes in the liver during JIA treatment with MTX.

\section{Disclosure of Interest}

None declared

\section{AB020}

Predictors of the effectiveness of intra-articular glucocorticoid injections in juvenile hip involvement

L. Nacef, H. Ferjani, L. Ben Ammar, K. Maatallah, D. Kaffel, W. Hamdi

Rheumathology, Mohamed Kassab Hospital, Tunisia, Tunisia

Correspondence: $L$. Nacef

Pediatric Rheumatology 2020, 18(Suppl 2):AB020

Introduction: Juvenile idiopathic arthritis (JIA) is the most common chronic inflammatory arthritis in children. Intra-articular glucocorticoids (IAG) are recommended as the first-line therapy for oligoarticular JIA. The safety and the benefits of use of IAG in other subtypes should be more studied.

Objectives: The aim of our study is to evaluate the efficacy of (IAG) injections in hip in children with (JIA) and to assess the factors predicting the improvement of this management.

Methods: This is a retrospective study, between 2006 and 2009, including patients with JIA diagnosed according to the ILAR criteria. The socio-demographic data were collected as well as the parameters of the disease. The activity was evaluated by JADAS. The functional impact was assessed by the Lequesne score. The treatments taken have been specified as well as the infiltrations received. The improvement after infiltration was assessed by JADAS and Lequesne score.

Results: Fourteen patients were included, with mean age 17.21 +6.8 [6-33]. The mean age at the onset of symptoms was $11+0.5$ [3-15]. Subtypes of JIA according to The ILAR were: enthesitis- related arthritis in 7 cases, seropositive polyarticular JIA in 2 cases, seronegative polyarticular JIA in 2 cases, oligoarticular JIA in 2 cases and juvenile psoriatic arthritis in one case. All the patients had hip arthritis, inaugural in $90 \%$ of the cases. Of these, $92.8 \%$ had a flexion deformity and lower limb inequality. The average Lequesne index was $8.5+4.6$. The treatments taken were Methotrexate in $57.14 \%$ of the cases, Sulfasalazine in $14.28 \%$ of the cases, and the combination of the two in $21.4 \%$ of the cases. Eleven patients underwent hip infiltration, and three of them required more than one. Eighty one percent improved thereafter. The number of infiltrations was not statistically associated with the Lequesne index $(p=0.069)$. Improvement after infiltration was negatively associated with the prior existence of an inequality of the lower limbs $(p=0.04)$. The existence of a flexion deformity was not associated with good results after infiltration ( $p$ $=0.476, r=-0,624)$. Ten patients $(90 \%)$ among those who had an infiltration did not have to resort to surgery.

Conclusion: IAG injection is an adjunct therapy in AJl with hip involvement offering a good results and delay surgery in the majority of cases. The presence of lower limb inequality is associated with less improvement of IAG.

\section{Disclosure of Interest}

None declared

\section{AB021}

Rice bodies at juvenile idiopathic arthritis onset

P. Mesa-Del-Castillo B. ${ }^{1}$, M. J. Lorente Sanchez', L. Barona Garcia², P. Alcañiz Rodriguez ${ }^{1}$, P. Castillo Dayer ${ }^{3}$

'Pediatric Rheumatology, Hospital Clínico Universitario Virgen de la Arrixaca; ${ }^{2}$ Pathology, Hospital Universitario Virgen de la Arrixaca;

${ }^{3}$ Rheumatology, Hospital Clínico Universitario Virgen de la Arrixaca, Murcia, Spain

Correspondence: P. Mesa-Del-Castillo B

Pediatric Rheumatology 2020, 18(Suppl 2):AB021

Introduction: Rice bodies are detached synovial fragments surrounded by fibrine concentric layers, giving them the aspect of a white oval rice grain. The pathologic mechanism is related to synovial hypertrofic growth and has been frecuently reported in rheumatoid ant tuberculous arthritis. This formations have been scarcely reported in juvenile idiopathic arthritis individual patients.

Objectives: To describe the difficulties and particularities of three cases Juvenile Idiopathic Arthritis in which rice bodies were present at onset.

Methods: Retrospective chart review, collection of clinical, radiological and microscopic images.

Results: CASE 1: A 3 years old girl presenting with five week history of right knee swelling. Two joint punctures with a $21 \mathrm{G}$ needle were unable to completely drain the joint; with a $16 \mathrm{G}$ needle the joint was finally drained and injected with triamcinolone acetonide. Synovial fluid had plenty of white oval shaped bodies. Culture of synovial fluid and mantoux were negative. Pathology showed to be compatible with synovial rice bodies. MRI and ultrasound images show an intense synovial hypertrophy.

CASE 2: A 3 years old girl presenting with an undetermined duration right knee effusion, recieved an steroid joint injection with good response. One month later she presents a left knee swelling; joint punture was performed with a $21 \mathrm{G}$ needle obtaining synovial fluid with tiny oval white formations compatible with rice bodies. Culture of synovial fluid and mantoux were negative. Ultrasound images show intense synovial hypertrophy and positive synovial Doppler signal.

CASO 3: A 3 years old girl presenting with a nine week history of right knee swelling. Joint puncture was performed with a $21 \mathrm{G}$ needle obtaining $10 \mathrm{ml}$ of inflammatory fluid ank steroids injected in the joint. After two weeks a second procedure was performed with a 16G needle: giant white oval bodies were obtained but complete drenaige of the joint was not achieved. Finaly she underwent arthroscopic surgery for complete clearance of intraarticular loose 
bodies. Pathology showed synovial hypertrophy fragments surrounded by fibrine, compatible with rice bodies. Culture of synovial fluid and mantoux were both negative. MRI and ultrasound images show an intense sinovial hypertrophy with suprapatelar recess occupation and positive synovial Doppler signal.

\begin{tabular}{lllllll}
\hline CASE & SEX & AGE & $\begin{array}{l}\text { JOINT } \\
\text { AFFECT } \\
\text { ED }\end{array}$ & $\begin{array}{l}\text { DURATION } \\
\text { OF ARTHRI } \\
\text { TIS }\end{array}$ & $\begin{array}{l}\text { NUMBER OF } \\
\text { PROCEDURES }\end{array}$ & $\begin{array}{l}\text { DIAMETER NEED } \\
\text { ED FOR } \\
\text { EVACUATION }\end{array}$ \\
\hline $\mathbf{1}$ & $\begin{array}{l}\text { FEMA } \\
\text { LE }\end{array}$ & Knee & 5 weeks & 2 & $16 \mathrm{G}$ \\
$\mathbf{2}$ & $\begin{array}{l}\text { LE } \\
\text { FEMA }\end{array}$ & $3 Y$ & Knee & Acute & 1 & $21 \mathrm{G}$ \\
$\mathbf{3}$ & $\begin{array}{l}\text { LE } \\
\text { FEMA }\end{array}$ & $3 Y$ & Knee & 9 weeks & 3 & Arthroscopy \\
& LE & & & & & \\
\hline
\end{tabular}

Conclusion: Synovial rice bodies are rarely described in juvenile idiopathic arthritis, even less at disease onset. Their presence has not been associated to a worse disease prognosis or joint outcome but awareness of the existence of this particular form of intraarticular loose bodies may encourage the clinician to use lower gauge needle during arthrocentesis procedure; this can prevent arthroscopy, as occurred in our case 1. Arthroscopy may be necessary in some cases to achieve full drainage of the joint. In our series the duration of arthritis correlated with the size of rice bodies and the number and agressiveness of procedures needed to evacuate them.

\section{Disclosure of Interest}

None declared

\section{AB022}

Spectrum of Juvenile Idiopathic Arthritis in $\mathbf{2 7}$ patients from a single center in an Indian state of Gujarat

D. B. Pandya, on behalf of Dr Haresh Dobariya, Dr Hitesh Bhambhani,Dr Harshad Takvani , Dr Yogesh Soni , Dr Jay Virani, Dr Rohan Parwani and Dr Akash Mankadia.

Pediatric Rheumatology \& Immunology, Dev Children's Hospital, Rajkot, India

Correspondence: D. B. Pandya

Pediatric Rheumatology 2020, 18(Suppl 2):AB022

\section{Introduction}

There is very limited information and awareness about juvenile idiopathic arthritis (JIA) amongst primary physicians in Gujarat and to make this matter even worse, we are not having a single exclusive pediatric rheumatology center for a population of around 60 million. ${ }^{1-3}$

Objectives

To study characteristics of twenty seven JIA children managed at Dev Children's Hospital.

Methods

I gathered a data of 27 pediatric patients with confirmed diagnosis of JIA who were seen at Dev Children's Hospital between January 2019 and January 2020. It included demographics, clinical presentation, laboratory results and treatment. All cases were reclassified according to preliminary PRINTO JIA classification. ${ }^{4}$

Results

Conclusion

Most common JIA sub-type in our cohort is RF positive JIA. Knee is the most common affected joint irrespective of JIA sub-type. Only one patient showed eye involvement. Most of the patients have been given steroids at onset. One patient with RF positive JIA and two patients with systemic JIA have been given intravenous tocilizumab infusion. Anti-inflammatory diet and yoga were advised for almost all patients of more than 5 years of age.

Trial registration identifying number

1. Review.Indian J Pediatr2010 Sep;77(9):993-6.doi: 10.1007/s12098010-0134-x. Epub 2010 Sep 3.

The Place of Pediatric Rheumatology in India

Sujata Sawhney 1, Prudence Manners
2. Journal of Natural science,Biology \& Medicine-2018

Clinico-epidemiological profile of pediatric rheumatology disorders in

Eastern India

PratapKumarPatra, ManishKumar

Department of Pediatrics, All Institute of Medical Sciences, Patna, Bihar, India

3. International Journal of Advanced Medical Health \& Research (JIPMER)

Pediatric rheumatology: An under-recognized subspecialty in India

Year : 2017 | Volume : 4 | Issue : 2 | Page : 47-53

AkhilaKavirayani1, SumaBalan2

1 Department of Paediatric Rheumatology, Oxford University Hospitals NHS Foundation Trust, Oxford, United Kingdom

2 Department of Paediatric Rheumatology, Amrita Institute of Medical Sciences, Kochi, Kerala, India

4.Research Article: Pediatric Rheumatology: Toward New Classification Criteria for Juvenile Idiopathic Arthritis: First Steps, Pediatric Rheumatology International Trials Organization International Consensus

Alberto Martini, Angelo Ravelli, Tadej Avcin, Michael W. Beresford, Ruben Burgos-Vargas, Ruben Cuttica, Norman T. llowite, Raju Khubchandani, Ronald M. Laxer, Daniel J. Lovell, Ross E. Petty, Carol A. Wallace, Nico M. Wulffraat, Angela Pistorio, Nicolino Ruperto and for the Pediatric Rheumatology International Trials Organization (PRIN TO)

The Journal of Rheumatology February 2019, 46 (2) 190-197; DOI: https://doi.org/10.3899/jrheum.180168

5. Ann Rheum Dis, volume 79, supplement 1, year 2020, page 1777

The impact of yoga, anti inflammatory diet and self monitoring in children with rheumatic diseases

D. B. Pandya1

1Dev Children's Hospital, Pediatric Department, Rajkot, India

\section{Disclosure of Interest}

None declared

Table 1 (abstract AB022). showed characteristics of $27 \mathrm{JIA}$ patients managed at Dev Children's Hospital.

\begin{tabular}{|c|c|c|c|c|c|}
\hline $\begin{array}{l}\text { Sub-types of } \\
\text { JIA } \\
\text { Total no of } \\
\text { Cases } \\
27\end{array}$ & $\begin{array}{l}\text { Early-onset } \\
\text { ANA positive } \\
\text { JIA } \\
4(15 \%)\end{array}$ & $\begin{array}{l}\text { RF } \\
\text { positive } \\
\text { JIA } \\
9(33 \%)\end{array}$ & $\begin{array}{l}\text { sJA } \\
7 \\
(26 \%)\end{array}$ & $\begin{array}{l}\text { Enthesitis- } \\
\text { spondylitis related } \\
\text { arthritis (ESRA) } \\
3(11 \%)\end{array}$ & $\begin{array}{l}\text { Other and } \\
\text { unclassified } \\
4(15 \%)\end{array}$ \\
\hline $\begin{array}{l}\text { Average age of } \\
\text { onset } \\
\text { Sex } \\
\text { predilection }\end{array}$ & $\begin{array}{l}3.7 \text { years } \\
\mathrm{F}>\mathrm{M}\end{array}$ & $\begin{array}{l}9.4 \text { yrs } \\
\text { All } \\
\text { females }\end{array}$ & $\begin{array}{l}5.1 \\
\text { yrs } \\
F> \\
M\end{array}$ & $\begin{array}{l}12.9 \\
\text { All males }\end{array}$ & $\begin{array}{l}6.6 \text { years } \\
M=F\end{array}$ \\
\hline $\begin{array}{l}\text { Average } \\
\text { number of } \\
\text { joints affected }\end{array}$ & 4.5 & 7 & 3 & 1.6 & 4 \\
\hline $\begin{array}{l}\text { Most common } \\
\text { affected joint }\end{array}$ & Knee & Wrist & Knee & Ankle & Knee \\
\hline $\begin{array}{l}\text { Eye } \\
\text { involvement }\end{array}$ & None & None & None & One patient & None \\
\hline \multicolumn{6}{|l|}{ Drugs used } \\
\hline Naproxen & All & All & All & All & All \\
\hline Methotrexate & All & All & Five & All & All \\
\hline Steroids & One & All & All & None & Two \\
\hline Tocilizumab & None & One & Two & None & None \\
\hline \multicolumn{6}{|c|}{ Alternative therapy } \\
\hline Anti- & & & & $\begin{array}{l}\text { inflammatory } \\
\text { diet }\end{array}$ & All \\
\hline All & All & All & All & & \\
\hline Yoga 5 & None & All & One & All & Two \\
\hline
\end{tabular}




\section{AB023}

Ruxolitinib therapeutic trial in refractory cutaneous Juvenile Dermatomyositis(case report)

H. M. Abd El-Lateef

Pediatric Rheumatology Immunology department, Ain Shams University Hospitals, Cairo, Egypt

Pediatric Rheumatology 2020, 18(Suppl 2):AB023

Introduction: Juvenile dermatomyositis (JDM) is the most recognizable paediatric-onset systemic autoimmune inflammatory myopathy .

Objectives: To confirm the significance of Type I interferon signature as a proven fascinating contributor in the pathogenesis of the disease that strongly correlates with disease measures in JDM.

Methods: An 8 years old female patient, $2^{\text {nd }}$ sibling of non consanginous marriage, presented at the age of seven with a two month history of high grade persistent fever, progressive proximal muscle weakness with evolving dysphonia, nasal tone, velopharyngeal dysfunction, dysphagia and severe dyspnea.

Results: On physical examination,patient was average built for age,conscious and alert . Systemic examination revealed evidence of heart failure, generalized muscle weakness more evident in the proximal groups( 2 on Manual Muscle Testing Grading System), marked hypotonia and hyporeflexia with no affection of sensation, dermatologic manifestations showed only Gottron's papules on metacarpophalangeal joints of both hands and no organomegaly.Routine Laboratory evaluation showed a within normal CBC picture, ESR $11 \mathrm{~mm} / 1 \mathrm{st} \mathrm{hr}$,CRP $96 \mathrm{mg} / \mathrm{dl}$, elevated ALT, AST, LDH and highly elevated CK (6000 U/L),non significant 24 hrs proteinuria level and negative viral markers.Immunological profile showed a within normal levels serum immunoglobulins assay, CD markers, negative immune markers (ANA , Anti-DNA , ANCA. Echo showed evidence of carditis and EF $38 \%$, pelviabdomial US was free. Patient was diagnosed as a case of juvenile dermatomyositis(JDM), started on pulse steroid therapy (Methylprednisolone; 30mg/kg x 5 days)and IV cyclophosphamide $(600$ $\mathrm{mg} / \mathrm{m}^{2}$ planned monthly $x 6$ doses),patient showed relative improvement and was maintained further on oral full dose steroids(FDS) and cardiac anti-failures.One month later, erythematous rash started on both elbows,regained weakness and dyspagia, CK (12000 U/L),24 hr proteinuria $(500 \mathrm{mg})$ so patient was given another pulse steroid therapy combined with IVIG $(1 \mathrm{gm} / \mathrm{kg})$ and an every 2 weeks course of Rituximab $\left(375 \mathrm{mg} / \mathrm{m}^{2} x\right.$ 4 doses) together with monthy cyclophosphamide.Patient went into clinical remission with a CK level 570 U/L. Four months later, patient developed full cutaneous manifestations (heliotrope rash, facial rash, nodular violaceous erythema and annular plaques on both elbows with periungual telangiectasia) and a CK rise to $790 \mathrm{U} / \mathrm{L}$ with fair general condition so a push on course of pulse steroids and IVIG were given and Mycophenolate Mofetil(MMF) was added to her FDS together as maintenance with Etanercept $(0.8 \mathrm{mg} /$ $\mathrm{kg} /$ week SC ) for three months where the patient entered into a complete clinical and laboratory remission(CK $56 \mathrm{U} / \mathrm{L}$ ) except for a progressive non responsive cutaneous manifestations ( V-sign ,Shawl sign, photosensitivity ,vasculopathic ear rash and starting upper eye lid calcinosis) adding to the previous ones.Considering the severity and progression of the cutaneous maifestations the dependence on FDS, partial failure of the previous therapies, low CD19 \& CD20 follow up levels and assuming involvement of the type I IFN pathway led to initiating treatment with the JAK $1 / 2$ selective inhibitor ruxolitinib (5 mg twice a day, i.e. $0.5 \mathrm{mg} / \mathrm{kg} /$ day) with consecutive gradual tapering of oral steroids and maintenance on $\mathrm{MMF}\left(600 \mathrm{mg} / \mathrm{m}^{2} /\right.$ $12 \mathrm{hr}$ ) . Patient's cutaneous manifestation went into a significant remission with good tolerance to ruxolitinib and no recorded disease activity or relapses for six months till now. Close follow up is done.

Conclusion: Refractory cutaneous dermatomyositis is a challenging situation.Steroid dependence, limited success of anti-TNF and difficult implementation of rituximab drove to applying JAK inhibitors. Ruxolitinib observed great efficacy emphasizes the importance of interferon in the cutaneous disease arm and provides a new therapeutic avenue.

Disclosure of Interest

None declared
AB024

An unique case of multiple autoimmune syndrome combined with gout in a 16-years boy

M. I. Kaleda, I. Nikishina, T. Pachkoria, M. Eliseev

Pediatrics, V.A. Nasonova Research Institute of Rheumatology, Moscow,

Russian Federation

Correspondence: I. Nikishina

Pediatric Rheumatology 2020, 18(Suppl 2):AB024

Introduction: Multiple autoimmune syndrome is a condition characterized by three or more autoimmune disorders in a same individual. About 25 percent of people with autoimmune diseases have a tendency to develop additional other autoimmune disorders. Objectives: To describe a rare case of a boy with combination of juvenile idiopathic arthritis (JIA), RF and ACCP positive, Sjogren's syndrome, autoimmune thyroiditis and gout.

Methods: Case report.

Results: A 14 yo male patient with normal physical, psychosocial and cognitive development presented with polyarthralgia in October $2016 \mathrm{y}$, two months later he had acute onset arthritis in the right shoulder and right knee (intensive pain, swelling, hyperemia and local hyperthermia), which developed without potential triggering factors. The patient received short course of diclofenac i.m. with temporal positive effect. This episode was followed by stable swelling of metacarpophalangeal and proximal interphalangeal joints of 2-3 fingers on both hands with morning stiffness lasting up to 1 hour and multiple short episodes of acute arthritis in 1st metatarsophalangeal joint of the left foot, metacarpophalangeal joints of 2-5 fingers on both hands independently. The patient was hospitalized to local hospital in February 2019, lab findings were as follows: ESR $44 \mathrm{~mm} / \mathrm{h}$, CRP $36 \mathrm{mg} / \mathrm{l}, \mathrm{RF} 24 \mathrm{U} / \mathrm{ml}$, ANA screen neg, the serum level of uric acid was $653,0 \mu \mathrm{mol} / \mathrm{L}$. Diagnosis $\mathrm{JIA}, \mathrm{RF}+$ polyarthritis was established, but NSAIDs (i/m and per os) and sulfasalazine therapy was ineffective followed by increasing number of flares and the persistence of high laboratory activity. He was first admitted to our hospital in April 2019 (at 16 yo) with active arthritis of metacarpophalangeal and proximal interphalangeal joints of 2-3 fingers on both hands, of right knee, $1^{\text {st }}$ metatarsophalangeal joint of the left foot. Lab findings included ESR $36 \mathrm{~mm} / \mathrm{h}$, CRP $6.9 \mathrm{mg} / \mathrm{l}, \mathrm{RF}$ $484 \mathrm{U} / \mathrm{ml}$, ACCPhs $1000 \mathrm{U} / \mathrm{ml}$, ANF Hep-2 1/640h+sp, a-TPO 1585.5 $\mathrm{U} / \mathrm{ml}$, a-TG $4028.9 \mathrm{U} / \mathrm{ml}$, serum creatinine $122 \mu \mathrm{mol} / \mathrm{L}$, urea 7.8 $\mathrm{mMol} / \mathrm{L}$, uric acid $577 \mu \mathrm{mol} / \mathrm{L}$. Schwartz estimate glomerular filtration rate (GFR) was $110.9 \mathrm{ml} / \mathrm{min}$, diurnal proteinuria $0.04 \mathrm{~g} /$ day. Chronic erosive arthritis of the hands and distal parts of the feet by ultrasound and X-ray imaging was confirmed. Crystals of sodium monurate were found in the synovial fluid from the right knee and 1st metatarsophalangeal joint of the left foot. Ultrasound finding of the thyroid gland revealed signs of autoimmune thyroiditis. Imaging modalities (US, sialography) identified visible changes of salivary glands characteristic for Sjogren's syndrome. Sialometry confirmed the presence of the sicca syndrome. Thus, JIA RF+ and ACCP+, secondary Sjogren's syndrome, autoimmune thyroiditis and gout were verified. Molecular-genetic testing didn't confirm the any genetic mutation. The patient was treated by methotrexate ( $15 \mathrm{mg}$ per week), folic acid ( $1 \mathrm{mg} / 6$ days a week), allopurinol with a titration of up to $300 \mathrm{mg}$ per day, L-thyroxine $100 \mathrm{mg}$ per day with gradual positive dynamics and good tolerance.

Conclusion: The presence of one autoimmune disease should be an indicator for the possibility of another one. Occurrence of multiple autoimmune phenomenon indicates the need for continued surveillance for the development of new autoimmune disease in predisposed patients. The simultaneous presence of several autoimmune conditions and gout, which is of autoinflammatory origin, seems to be unique, especially in pediatric patients.

Disclosure of Interest

None declared 


\section{AB025}

Rituximab for treatment of resistant Paediatric MCTD

V. Paisal, S. Compeyrot-Lacassagne

Paediatric Rheumatology, Great Ormond Street Hospital, London, United Kingdom

Correspondence: $\vee$. Paisal

Pediatric Rheumatology 2020, 18(Suppl 2):AB025

Introduction: First described in 1972 by Sharp et al, Mixed Connective Tissue Disease (MCTD) is a rare entity in children. Diagnosis is made by clinical-immunological overlapping features of different Connective tissue diseases. Typical clinical features are Raynaud's phenomenon, myositis, arthritis, sclerodactyly along with positive anti RNP Antibody. There is a higher risk of interstitial lung disease and pulmonary hypertension. Treatment is tailored to the patient based on features present ${ }^{(1)}$.

Objectives: We present a challenging case of Paediatric Mixed Connective Tissue Disease (MCTD) with Sjogren overlap resistant to standard treatment and requiring the addition of Rituximab to control recurrent debilitating parotitis.

Methods: We conducted a retrospective case review using electronic patient record.

Results: A 15 year old female, first presented at the age of 7 with Raynaud's phenomenon and a high ANA 1:5120 was referred from local hospital. Double stranded DNA was negative, ENA typing revealed anti U1-RNP positive. She then developed symptoms of Parotitis and arthritis when oral steroids were commenced. A lower lip biopsy revealed periductal lymphocytic infiltrate admixed with scattered plasma cells. USS of parotid glands showed bilateral chronic sialadenitis in submandibular and parotid glands. Increased vascularity in submandibular glands bilaterally may represent acute on chronic inflammation. She did not have dry mouth, pain with swallowing or dry eyes. Her capillary folds were abnormal. She was commenced on Hydroxychloroquine whilst awaiting Biopsy, which was consistent with Sjogren's syndrome. Methotrexate was added, which soon led to transaminitis and she also continued to have flares and Methotrexate was switched to Azathioprine. This helped with her symptoms but led to Neutropenia. Eventually decision was made to switch to Mycophenolate Mofetil (MMF). Initially MMF helped her with arthritis and energy levels improved but after a year of being on MMF her disease started to flare, requiring oral steroids, and leading to cushingoid facies. Her Lipase was elevated and Amylase was borderline elevated.

Due to ongoing disease activity with recurrent and painful parotitis, decision was made to use Rituximab. She gradually recovered from this. Since Rituximab infusion in January 2019 her disease has been continuously in remission and she has recently started to wean MMF down.

Conclusion: We report the successful use of rituximab in a difficult to treat patient with Sjogren-MCTD overlap ${ }^{(2)}$. Rituximab was chosen due to the well-established role of B cell hyperactivity in the immunopathogenesis of primary Sjogren disease ${ }^{(3)}$. Rituximab has mixed results in the management of primary Sjogren syndrome in adults. There is no evidence of the use of rituximab in paediatric Sjogren but it was successful at controlling paediatric Sjogren syndrome in our patient and allowed weaning of long-term DMARD.

\section{References}

1. Tani C,Carli L, Vagnani S, Talarico R, Baldini C, Mosca M, Bombardieri S. The diagnosis and classification of of mixed connective tissue disease. J Autoimmun. 2014 Feb-Mar;48-49:46-9

2. Deepak S, Warrier KC. Mixed connective tissue disease in children - case series. Rheumatol Orthop Med. 2017;2(2):1-7

3. Verstappen GM, van NimwegenJF, Vissink A, Kroese FGM, Bootsma H. The value of Rituximab treatment in primary Sjögren's syndrome. Clin Immunol. 2017 Sep;182:62-71

Disclosure of Interest

None declared

\section{AB026}

Juvenile Idiopathic Arthritis a multifaceted approach is essential for robust rehabilitation

M. Nice', M. E. Thomsen', M. S. Jensen², L. A. Bistrup ${ }^{1}$, A.-H. Bohr ${ }^{1}$

${ }^{1}$ Paediatrics; ${ }^{2}$ Physiotherapy, Slagelse Hospital, Slagelse, Denmark

Correspondence: M. E. Thomsen

Pediatric Rheumatology 2020, 18(Suppl 2):AB026

Introduction: The prognosis for JIA regarding joint destruction and disability has changed significantly during the last two decades due to the emergence of effective anti-inflammatory treatment regimens. However, approximately $50 \%$ of the patients will need ongoing medication far into adult life. The prospect of having a long lasting chronic disease requiring continuing medication, with possible side effects, frequent clinical controls and periods of pain can, by itself, for some patients lead to a passive and inactive lifestyle overshadowing the primary positive effect of the anti-inflammatory medication. In these cases, it can be difficult for the patient (child or adolescent) to resume a normal physically and mentally active lifestyle so important in childhood and youth and of great importance for general health in adulthood.

Recently a Danish version of the Juvenile Arthritis Multidimensional Assessment Report (JAMAR) was published, and we now have an internationally validated tool for assessment of our patients' life circumstances and their personal view of the burden of their disease. Using this tool, in combination with structured clarifying talks between nurse and/or physiotherapist and patient (and parents according to age of the patient) we aim to uncover unmet special needs that we may help to fulfil in the municipal system, in schools, in sports clubs or in other institutions. This structured approach may also by itself help the patient and the family to gain constructive insight in their life situation, with a chronically ill member, and find possible hitherto unknown resources.

Objectives: The purpose of this project is to investigate the effect of a structured multifaceted treatment of juvenile idiopathic arthritis, focusing on lifestyle and physical activity in parallel with antiinflammatory medication.

We hypothesize that this strategy will help patients to obtain an early and robust rehabilitation and thus get the full benefits of the effective medicine currently available.

Methods: This is a prospective, non-randomized intervention project. A pilot design is chosen with the purpose of early evaluation of practical circumstances.

Inclusion criteria:

- Danish speaking patients with JIA, followed in the outpatient clinic at the Pediatric Department at Slagelse Hospital

- having a low objective clinical disease activity and a high subjective burden of disease according to JAMAR

We aim to include 20 patients.

Intervention:

- structured clarifying talks between patient (and parents according to age of the patient) and the caregiving nurse or physiotherapist with the purpose of uncovering unmet needs

- special needs are sought fulfilled by individual agreements between patients/families and the municipal system, with schools, with sports clubs or with other institutions. The staff in the rheumatology clinic shall be drivers in this process.

Endpoints:

- Change in JAMAR score at 3 and 6 months after inclusion; the patient will serve as his/her own control.

Results: Pending

Delayed by the Corona Pandemic 
Conclusion: Pending

Disclosure of Interest

None declared

\section{AB027}

Autoimmune Hepatitis-SLE overlap syndrome (Lupoid Hepatitis)

A. A. Abushhaiwia', A. Ravelli ${ }^{2}$, M. A Zletni ${ }^{3}$, A. MALEK ${ }^{4}$

${ }^{1}$ Pediatric Rheumatology, Tripoli Children's Hospital,Tripoli Faculty of Medicine, Tripoli , Libya; ${ }^{2}$ head division of Rheumatology, Giannina Gaslini Institute, Giannina Gaslini Institute, Genova, Italy; ${ }^{3}$ Pediatric Rheumatology, Tripoli Children's Hospital ,Tripoli Faculty of Medicine; ${ }^{4}$ Radiology department, Tripoli faculty of Medicine, Tripoli, Libya Correspondence: A. A. Abushhaiwia

Pediatric Rheumatology 2020, 18(Suppl 2):AB027

Introduction: Systemic lupus erythematosus (SLE) is a systemic autoimmune disease classically involving the skin, kidneys, and central nervous system. Several previous reports showed the manifestation of AlH in adult lupus patients; however, there are no reports of such an association between AlH and SLE in children. Both AlH and SLE have an autoimmune basis and hence can occur simultaneously or masquerading as presentations of each other. In this case report, we report AlH as the first manifestation of SLE in an 8-year-old girl.

Objectives: We described a case of liver involvement in SLE presenting with emphasis on the differential diagnosis with autoimmune hepatitis.

Methods: case report study

Results: : An 8-year-old female patient was referred to the Rheumatology clinic with complaints icteric sclera for 10 months anorexia, malaise, pain in the both knees, ankles joints and both wrists accompanied by swelling, and remarkable motion limitations. Laboratory revealed T bilirubin 4.9 mainly direct 3.9 with elevated liver enzymes GOT 401, GPT 189, ALKP 520, high Glutamyl transpeptidase 56UL her WBC 7.4 HGB 10, PTL 317, except very high ESR $105 \mathrm{ml} / \mathrm{hr}$, CRP was positive $190 \mathrm{mg} / \mathrm{dl}$, viral screen (HCV, HBSAg, HIV) was normal, serology tests ANA was positive with high titer 1280 , anti ds- DNA AB was positive 320 , anti-Sm was negative, ANTI LKM1 antibodies negative, anti smooth muscle $A B$ negative soluble liver antigen were negative, antimitohondrial $A B(M 1, M 2, M 3)$.

ultrasound abdomen revealed mild enlarged spleen, abnormal diffused increased liver echogenicity with early stage of liver cirrhosis treated her by fresh frozen plasma 5 times, ViT k $10 \mathrm{mg}$ oncel day then was referred to rheumatology clinic regarding her serology tests \& developed arthritis of her joints suspected PSLE! She was performed liver biopsy showed lesions necrotic inflammatory portal and lobular severe in eosinophilic polynuclear with cirrhosis evoking a syndrome of overlap associating a primary biliary cirrhosis and an autoimmune hepatitis. Laboratory data revealed liver dysfunction and liver biopsy Suggesting autoimmune hepatitis, and she underwent treatment for hepatitis (prednisolone with azathioprine), Urosdoxycholic acid with fat-soluble vitamins K, D\&A, E. However, with the elimination of jaundice and decreased hepatic enzyme levels, the prednisolone dose was tapered within 2 months and stopped before they were referred to Rheumatology clinic. On her review of systems, she has malar rash, generalized fatigability. On physical examination, we found malar rash, levidoreticularis of her skin, swelling and limitation of movement in the knees, ankles, wrists joints. There was hepatosplenomegaly. Laboratory data revealed liver treatment for hepatitis, ANA still high titer 1:1280, Antids DAN positive with titer $307 \mathrm{IU} \backslash \mathrm{ml}$, antiSMA was negative .WBC 4.5, HGB 11.8, PLT 268, ESR 68ml/hr, Her ultrasound abdomen: revealed slightly heterogeneous liver with coarse echotexture without focal lesion with liver span $14 \mathrm{~cm}$. These paraclinical results together with the clinical findings strongly suggested systemic lupus erythematosus (SLE) as the definitive diagnosis. Indeed, in this case, AlH was associated with SLE, prednisolone orally for 2 months, after that dose was tapered and continued, rapid clinical improvement in arthritis, malaise, and general condition. Azathioprine was continued. In addition, daily hydroxycholoquine sulfate
Conclusion: Overlapping of SLE and AlH should be suspected when AlH patients present with a malar or other skin rash. The prompt diagnosis and adjustment of further treatment plans can improve disease outcomes and prevent liver disease progression.

Disclosure of Interest

None declared

\author{
AB028 \\ Hematological presentation as the first manifestation of a positive \\ triple marker antiphospholipid syndrome case presentation and \\ literature review \\ M. C. TERCERO', M. G. E. PLAZA², F. I. E. ROQUE ${ }^{2}$, Y. R. LOYOLA ${ }^{3}$ \\ ${ }^{1}$ PEDIATRIC; ${ }^{2}$ HOSPITAL DE ESPECIALIDADES DEL NINNO Y LA MUJER, \\ QUERETARO; ${ }^{3}$ HOSPITAL DE ESPECIALIDADES DEL NIÑO Y LA MUJER, \\ QUERATRAO, Mexico \\ Correspondence: M. C. TERCERO \\ Pediatric Rheumatology 2020, 18(Suppl 2):AB028
}

Introduction: Pediatric Antiphospholipid syndrome (p-AFS) is a rare autoimmune multisystemic syndrome, with a reported incidence around $3 \%$ in patients younger tan 15 years with a higher prevalence in female : male ratio of 1.2:1. Secondary p-AFS is associated with Systemic Erythematosus Lupus in $50 \%$ of the cases. The Sapporo criteria require al least 1 clinical criteria, one or more thrombosis events or comorbidities during pregnancy, associated with the finding of antiphospholipid antibodies, positive lupus anticoagulant, $\lg \mathrm{M}$ or $\lg$ anticardiolipin antibodies, IgM or IgG anti-b2glycoproteins in two or more determinations separated by 12 weeks. In pediatric patients we take for consideration antiphospholipid antibodies and clinical manifestations, such as hematological (thrombocytopenia, anemia) and neurological (Chorea).

Objectives: To describe the clinical presentation of a 13 yo patient with positive triple marker for antiphospholipid syndrome (APS) secondary to Systemic Erythematosus Lupus (SLE), with hematologic manifestations.

Methods: Thirteen-year-old female patient under hematology department follow up due to pancytopenia, discarding an hematological malignancy. Presenting to our department complaining of anomalous uterine bleeding, clinically with a malar erythema. Routine labs were performed, reporting pancytopenia with Anemia (3.2), Thrombocytopenia $(23,000)$ and lymphopenia (850). Immunology test with hypocomplementemia (C3 56mg/dl and C4 $2.9 \mathrm{mg} / \mathrm{dl}$ ), Positive Anti-DNA antibodies $(410 \mathrm{Ul} / \mathrm{ml})$, Positive Antinuclear antibodies with a speckled pattern (1:1280), positive lupus anticoagulant (1:8), IgG anticardiolipin $) 11.8 \mathrm{U} / \mathrm{ml})$, IgM anticardiolipin $(37.7 \mathrm{U} / \mathrm{ml})$, IgM anti.b2, glycoprotein $(32 \mathrm{U} / \mathrm{ml})$. With triple positive marker for APS and SLE, Initiating treatment with methylprednisolone bolus, hydroxychloroquine, acetyl salicylic acid and immunomodulation with Azathioprine. We performed a second determination 12 weeks after with positive IgG Anticardiolipin $(10.9 \mathrm{U} / \mathrm{ml}) \mathrm{lgM}(56.8 \mathrm{U} / \mathrm{l})$, Positive lupus anticoagulant (1.9), positive anti b2-glycoprotein $(40.7 \mathrm{U} / \mathrm{ml})$. During follow up as an outpatient without thrombotic events.

Results: Our patient meets the 1997 ACR criteria for SLE and Sapporo criteria for APS. With APS positive triple marker. Clinically with anomalous uterine bleeding and hematological manifestations, persistent thrombocytopenia and lymphopenia, having discarded hematological malignancies.

Conclusion: Pediatric antiphospholipid syndrome is a rare condition, in most cases associated with Systemic Erythematosus Lupus. Triple positive marker increases the risk of thrombotic events significantly. Our patient, at presentation and during follow up, only hematological manifestations were presented as the only clinical symptom associated with pancytopenia, nevertheless the risk of thrombotic events is high during the natural course of APS.

Disclosure of Interest

None declared 


\section{AB029}

Juvenile SLE and Autism: a case of rare combinations and diagnostic dilemma

A. Sridhar, C. Devanahalli

Leicester Children's Hospital , University Hospitals of Leicester NHS Trust, Leicester, United Kingdom

Correspondence: A. Sridhar

Pediatric Rheumatology 2020, 18(Suppl 2):AB029

\section{Objectives: Case}

We present a case of a $7 \mathrm{yr}$ old with severe Autism presenting with 2 week history of facial and bilateral neck swelling with bilateral cervical lymphadenopathy initially thought to be lymphoma but later diagnosed as systemic lupus with nephritis and neuro-lupus. Her initial course was stormy needing admission to intensive care unit and referral to oncology centre for work up of lymphoma.

This child had no significant hospitalisations in the past.

Methods: Her initial blood results and CT (Computerised tomography) chest suggestive of lymphoma with Tumor lysis. She was transferred to a local paediatric oncology centre for lymph node and bone marrow testing which showed no evidence of Leukemia or Lymphoma. She developed focal seizure secondary to hypertension and PRES (Posterior reversible encephalopathy), needing intubation and transferred to intensive care unit for 48 hours.

Magnetic resonance (MRI) imaging brain showed signs consisted with cerebral lupus. Autoimmune screen strongly positive for antibodies ANA (Antinuclear), dsDNA antibodies, Anti-Ro, Anti-Smith, RNP, RNP70, low C3 (complement) and C4 in keeping with Juvenile SLE. Renal biopsy was not performed as the child remained hypertensive despite being on antihypertensive medications and later due to parental refusal for Renal Biopsy. Echo showed evidence of mild Pericardial effusion, which was managed conservatively. She also had ascites and pleural effusion

Results: Course

She was managed with IV Pulse Methylprednisolone for 3 days, followed by oral prednisolone, and IV cyclophosphamide and later switched to Mycophenolate (MMF) therapy with which she made good recovery. She stayed in the hospital for 2 months for Nuro rehab with good neurological recovery back to her previous status.

At 3 months - She remains on immunosuppressive medication i.e. Mycophenolate therapy and small tapering dose of Oral Prednisolone and Hydroxychloroquine

Follow up MRI head shows significant improvement in comparison to her previous images, Echo showed complete resolution of the pericardial effusion and urine examination no evidence of proteinuria or haematuria.

At $1 \mathrm{yr}$ follow up, she remains clinically stable with complete neurological recovery with no ongoing neurological, cardiac or Renal flare ups. She has been maintained on Oral Mycophenolate (MMF), and Hydroxychloroquine. Her inflammatory markers, complement profile, double stranded DNA levels remain normal and Urine shows no evidence of Haematuria or Proteinuria

Conclusion: Conclusion

Juvenile SLE with multi-system involvement in an Autistic child can be difficult to diagnose and manage. The case highlights that JSLE is a disease of thousand faces with varied clinical presentations and the need for high index of suspicion. This case also highlights the role of multidisciplinary teams in the management of such complex cases.

Trial registration identifying number: Autism and Juvenile SLE diagnostic

\section{dilemma- A case of rare combinations}

Case

We present a case of a $7 \mathrm{yr}$ old with severe Autism presenting with 2 week history of facial and bilateral neck swelling with bilateral cervical lymphadenopathy initially thought to be lymphoma but later diagnosed as systemic lupus with nephritis and neuro-lupus. Her initial course was stormy needing admission to intensive care unit and referral to oncology centre for work up of lymphoma.
This child had no significant hospitalisations in the past. Her initial blood results and CT (Computerised tomography) chest suggestive of lymphoma with tumor lysis. She was transferred to a local paediatric oncology centre for lymph node and bone marrow testing which showed no evidence of Leukemia or Lymphoma. She developed focal seizure secondary to hypertension and PRES (Posterior reversible encephalopathy), needing intubation and transferred to intensive care unit for 48 hours.

Magnetic resonance (MRI)imaging brain showed signs consisted with cerebral lupus. Autoimmune screen strongly positive for antibodies ANA (Antinuclear), dsDNA antibodies, Anti-Ro, Anti-Smith, RNP, RNP70, low C3(complement) and C4 in keeping with Juvenile SLE. Renal biopsy was not performed as the child remained hypertensive despite being on antihypertensive medications and later due to parental refusal for Renal Biopsy. Echo showed evidence of mild Pericardial effusion, which was managed conservatively. She also had ascites and pleural effusion

Course

She was managed with IV Pulse Methylprednisolone for 3 days, followed by oral prednisolone, and IV cyclophosphamide and later switched to Mycophenolate (MMF) therapy with which she made good recovery. She stayed in the hospital for 2 months for Nuro rehab with good neurological recovery back to her previous status.

At 3 months - She remains on immunosuppressive medication i.e. Mycophenolate therapy and small tapering dose of Oral Prednisolone and Hydroxychloroquine

Follow up MRI head shows significant improvement in comparison to her previous images, Echo showed complete resolution of the pericardial effusion and urine examination no evidence of proteinuria or haematuria.

At $1 \mathrm{yr}$ follow up, she remains clinically stable with complete neurological recovery with no ongoing neurological, cardiac or Renal flare ups. She has been maintained on Oral Mycophenolate (MMF), and Hydroxychloroquine. Her inflammatory markers, complement profile, double stranded DNA levels remain normal and Urine shows no evidence of Haematuria or Proteinuria

Conclusion

Juvenile SLE with multi-system involvement in an Autistic child can be difficult to diagnose and manage. The case highlights that JSLE is a disease of thousand faces with varied clinical presentations and the need for high index of suspicion. This case also highlights the role of multidisciplinary teams in the management of such complex cases.

Disclosure of Interest

None declared

АВ030

Juvenile Systemic Lupus Erythematosus, experience of the

pediatric center

K. Okka, B. Bioud

Pediatrics, word, SETIF, Algeria

Correspondence: $\mathrm{K}$. Okka

Pediatric Rheumatology 2020, 18(Suppl 2):AB030

Introduction: Juvenile systemic lupus erythematosus (SLE) is a chronic autoimmune disease characterized by multi-visceral involvement with an unpredictable prognosis. The diagnosis is usually made in young women aged between 20 to 40 years, however, it can affect people at any age and it is classified as a juvenile illness when it starts before the age of 16 .

Objectives: We are reporting the epidemical, clinical, therapeutical and evolutional characteristics of a series done in the pediatric Pole in setif with 13 girls and 1 boy.

Methods: The average age of onset is 13 years. The average time limits of the diagnosis is 7 months. The clinical features is done with cutaneous, articular manifestations and fever respectively in 100\% $71 \%$ and $57 \%$ of the cases ,followed by kidney damage in $42 \%$ of the cases, 
the cardiac, pulmonary and ophthalmological participations are reported with low percentage.

Haematological involvement was detected in $85 \%$ of the patients and the inflammatory syndrome was almost constant. A positive titer of anti-nuclear antibodies and anti-DNA is objectified, as well as a reduction in the complement rate. Antibodies anti GP 2 and anti cardiolopine are positive in $57 \%$ of cases. Kidney damage was diagnosed in $42 \%$ of the cases, and only one case of overlap syndrome with dermatomyosits was reported. Concerning the neurological form it was present in only one addolecent girl, and only one case of familial lupus.

Results: The diagnosis is based on the classification of the American College of rheumatology (ACR) 1982 revised on 1997 and the new criteria SLICC"Systemic Lupus International Collaborating Clinics" .

The clinical characteristics of our series relies on global data of literature with the Predominance of Cutaneous and articular involvement. with however some specific characteristics which are individualized by a more advanced age of onset, 13 years on average in our study versus 10 years and 12 years, the rarity of familial forms (1 case), a lower percentage of kidney damage ( $42 \%$ versus $63 \%$ and $80 \%$ ).

The therapeutic management was based on corticosteroid therapy and Hydroxychloroquine in the majority of cases, the use of immunosuppressants has been reserved for severe forms.

Conclusion: Lupus is an autoimmune disease with protean clinical manifestations, the prognosis of which is dominated by renal, neurological and thrombotic disorders. Cortisonic treatments and immunosuppressants have significantly improved the prognosis for life .

Trial registration identifying number: Lupus is an autoimmune disease with protean clinical manifestations, the prognosis of which is dominated by renal, neurological and thrombotic disorders. Cortisonic treatments and immunosuppressants have significantly improved the prognosis for life .

\section{Disclosure of Interest}

None declared

\section{AB031}

Systemic lupus erythematosus with unusual presentation: a single centre experience from North India

Y. Sharma, A. Sharma

Pediatrics, Dr Rajendra Prasad Government Medical College, kangra, India

\section{Correspondence: $Y$. Sharma}

Pediatric Rheumatology 2020, 18(Suppl 2):AB031

Introduction: Systemic lupus erythematosus (SLE) is a multisystem autoimmune disease. SLE mimics many clinical entities and diagnosis may become difficult at times,especially if presentation is atypical.

Objectives: To highlight unusual presentation of Pediatric SLE

Methods: Retrospective case review of all children diagnosed as SLE from July 2017-November 2018 at a single tertiary care hospital in North India was done. We here describe 4 children who presented with unusual manifestations.

CASE 1: 8 years,girl, presented with fever for 1 month and bilateral pleural effusion. She had fever, epistaxis, hepatosplenomegaly and bicytopenia and was managed as tropical infections initially.She was later on diagnosed to have SLE and managed for the same.

CASE 2: 11 years,boy, presented with arthritis in multiple small and large joints of body. On evaluation ANA and anti dsDNA was found to be positive. He was started on subcutaneous, weekly methotrexate and responded to treatment. However, he developed lupus nephritis during disease course after 6 months of diagnosis. Renal biopsy revealed ClassIII+V lupus nephritis. Intravenous Cyclophosphamide pulses were started for induction along with tapering doses of corticosteroids.

CASE 3: 8 years, girl, presented with early morning periorbital puffiness and abdominal distension with history of photosensitivity and Raynaud phenomenon. Urine routine examination revealed nephrotic range proteinuria with no hematuria. Clinical possibility of nephrotic syndrome secondary to SLE was kept.Renal biopsy revealed Class V Lupus nephritis.
CASE 4: 17 years old,girl,presented with high grade fever and pain in bilateral knee joints and malar rash. Investigation were suggestive of SLE with MAS(macrophage activation syndrome) .Child was started on iv antibiotics and iv immunoglobulins but died during the disease process.

ANA positivity, hypocomplimentimia and Anti ds DNA positivity were seen in all the 4 cases.

Results: out of 11 children diagnosed to have SLE, 4 presented with unusual manifestations as described above.

Conclusion: In this report we present unusual presentations as the predominant manifestation of SLE and emphasize the fact that early recognition and awareness of unusual presentation of SLE help institute timely initiation of appropriate treatment

\section{Disclosure of Interest \\ None declared}

\section{AB032}

Retrospective analysis of intraarticular effectiveness of triamcinolone acetonide in treatment of oligoarticular juvenile arthritis in children

A. Kozhevnikov ${ }^{1,2}$, N. Pozdeeva ${ }^{2}$, M. Nikitin ${ }^{2}$, G. Novik $^{1}$

${ }^{1}$ Saint-Petersburg State Pediatric Medical University; ${ }^{2} H$.Turner National

Medical Research Center for Children's Orthopedics and Trauma Surgery, Saint-Petersburg, Russian Federation

Correspondence: A. Kozhevnikov

Pediatric Rheumatology 2020, 18(Suppl 2):AB032

Introduction: Despite significant advances in treatment and increased understanding of pediatric chronic disease the choice of first-line antirheumatic drugs of oligoarticular onset juvenile arthritis still remains relevant. Pediatric rheumatologists and to this day there are no consensus on the best modality for treatment.

Objectives: The aim of this study was to assessment of feasibility and efficacy of local steroid therapy oligoarticular onset JIA in children in the Russian Federation.

Methods: In a retrospective study 92 children (89\% girls) aged median (IQR) 4,2 (1,6 - 7,6) years with oligoarticular onset JIA without extra-articular manifestations (oligo-JA) who did not received DMARDs were monitored. All children were met ILAR criteria. Ttriamcinolone acetonide (TA) was administered intra-articular at a dose of 20-40 mg with an injection interval of 3-6-12 months which was depended on the activity of the disease. The maximum allowable number of consecutive isolated intra-articular injections (is-IAl) was 34. A total of 218 active joints were injected with TA: knees - 156 injections, ankles - 62 injections. All children were divided into two groups: active / inactive arthritis based on the effectiveness of local corticosteroid treatment. The average follow-up was 48 [38; 62] months.

Results: 32 children (35\%; all girls) were achieved remission oligo-JA after is-IAI of TA with mean of $2[1,75 ; 2]$ injection per joint (inactive arthritis $>24$ months). The mean interval between two consecutive is-IAI was $7[5,25 ; 10]$ months. Other children did not achieve inactive oligo-JA after is-IAI of TA with mean of $3[2 ; 4]$ injection per joint. The mean interval between first two consecutive injection was 5,5 $[4,25 ; 7]$ months and other injections - $2[2 ; 3]$ months. All children who did not achieve remission oligo-JA for is-IAI were treated by DMARDs. Statistical analyses were performed to determine the relationships between clinical, instrumental, laboratory signs and efficacy is-IAI of TA. Measures included the number of swollen or tender joints [active joint counts]; biological inflammatory markers [erythrocyte sedimentation rate (ESR), C-reactive protein (CRP), serum and synovial fluid level of interleukin 6 (IL6) and tumor necrosis factor alfa (TNF-a)]; autoimmunity [titer of antinuclear factor (ANF)] and physicians' assessment of JIA disease activity [clinical Juvenile Arthritis Disease Activity Score including maximal 10 joints (cJADAS10)]. Efficacy is-IAI of TA was no associated significantly with number of active joint of onset oligo-JA, CJADAS10, serum level of CRP $\mathrm{mg} / \mathrm{ml}$, ESR $\mathrm{mm} / \mathrm{h}, \mathrm{IL} 6 \mathrm{pg} / \mathrm{ml}$ and TNF- $\mathrm{a} \mathrm{pg} / \mathrm{ml}$, titer of ANF. The mean inflamed synovial fluid of IL6 levels 2208 [710; 4564] / 3234 [1265; 16902] $\mathrm{pg} / \mathrm{ml}$ and TNF-a levels $3,3[2,5 ; 3,8] / 1,1[0,6 ; 3,7] \mathrm{pg} / \mathrm{ml}$ at 
onset of inactive and active oligo-JA were not significantly differ. The analysis revealed a correlation between a short phase of beneficial effect after is-IAI of TA and risk of activity disease (with an inactive phase of arthritis less than 3 months, the risk activity was $\mathrm{OR}=2.09$, $\mathrm{p}<0.001$; with an inactive phase less than 2 months - $O R=8.9, p<$ $0.001)$.

Conclusion: Trimacinolone acetonide is an effective and safe treatment in children with oligoarticular juvenile arthritis. Research was revealed that about a third of children with oligo-JA achieved inactive arthritis of average after two intra-articular injections of triamcinolone acetonide. Despite the widespread use of biological treatment should not be neglected topic corticosteroid drugs as the first-line treatment in children with oligo-JIA.

\section{Disclosure of Interest}

None declared

\section{AB033}

The use of off-label biologics in children with systemic connective tissue diseases

O. A. Oshlianska', L. I. Omelchenko², O. M. Okhotnikova ${ }^{3}$, A. G. Artsymovych

11.Department of Pediatrics № 1, 2. Department of connective tissue disorders in children, 1.Shupyk National Medical Academy of

postgraduate education, 2.State Institute of Pediatrics, Obstetrics and Gynecology, Academy of Medical Sciences of Ukraine; ${ }^{2} 2$. Department of connective tissue disorders in children, 2.State Institute of Pediatrics, Obstetrics and Gynecology, Academy of Medical Sciences of Ukraine; ${ }^{3}$ 1.Department of Pediatrics № 1, 1.Shupyk National Medical Academy of postgraduate education, Kyiv, Ukraine

Correspondence: $\mathrm{O}$. A. Oshlianska

Pediatric Rheumatology 2020, 18(Suppl 2):AB033

Introduction: Off-label prescribing often occurs in pediatric rheumatology. This is due to insufficient evidence base, the severity and unpredictability of the course of the disease.

Objectives: To analyze the cases of off-label use of biologicals among patients with rheumatic diseases.

Methods: Analysis of patient treatment was carried out using data from the registry of children's rheumatological patients: 38 systemic scleroderma (SSD), 47 systemic lupus erythematosus (SLE), 42 juvenile dermatomyositis (JDM), 19 systemic vasculitis (SV), 11 unspecified systemic involvement of connective tissue, 8 autoinflammatory syndromes.

Results: The possibility of providing patients with systemic connective tissue diseases with expensive therapy in Ukraine is absent. Biologicals are prescribed only in cases of extremely severe courses of the diseases with the previous ineffectiveness of all other available methods of therapy CS and DMARDs. Biologicals were prescribed off-label 10 times in 8 patients. In case with SLE (SELENASLEDAl=60, ESR $30 \mathrm{~mm} / \mathrm{h}$ ) rituximab (RTX) weas used after tocilizumab (TCZ). RTX was prescribed in another 4 patients (polyarteritis nodosa $\mathrm{VDI}=11$, ESR $64 \mathrm{~mm} / \mathrm{h}$; ANCA+SV VDI=12, ESR $56 \mathrm{~mm} / \mathrm{h}, \mathrm{CRP}=$ $18 \mathrm{mg} / \mathrm{l}$; JDM DAS 31, CMAS 4, CK 8000 IO//; SLE SELENA-SLEDAl=59, ESR $45 \mathrm{~mm} / \mathrm{h}$ ). RTX was administered to patients who had received high-dose CS with 2-3 DMARDS; in all cases combined pulse therapy CS № 2-10 was preliminarily used. RTX 500 mg № 2 was applied after 6 mo-2y from the debut of the disease. In all 5 cases, its use led to clinical improvement after 1-5 mo with normalization of laboratory activity indicators, in 4 cases a decrease in the level of B cells to 00.56 in $\mu \mathrm{l}$ was noted ( 3 with agammaglobulinemia). After 2 months 3 patients had severe infectious complications, 2 of them ended fatally. 2 another patients had a second stroke. The 1st patient survived, had a kidney allotransplantation, there is no disease activity. The 2nd patient, in connection with the development of the demyelinating process of CNS, attempted to continue therapy using golimumab with IVIG. It led to an increase in the infectious syndrome, therefore, we decided to refrain from continuing with iTNF as well. The patient died after 2 years from the administration of RTX due to the progression of neurological disorders. 2 cases with auto-inflammatory syndromes were: chronic infantile neurologic cutaneous and articular syndrome received TCZ; it was unsuccessful (hyperthermia and rash persisted, eye lesions progressed, there were no increase in height), later switched to anakinra. Family mediterranean fever, received adalimumab (ADA). The 1-year-course of ADA leaded to the disappearance of articular and abdominal syndrome while maintaining persistent increased levels of ESR and CRP and periodic fever. The use of TCZ in 2 patients with SSD was more successful. The first patient received it subcutaneously for 1 year, CS\&DMARDs (3 were used) had already been canceled, lung and kidney lesions were contained, blood pressure normalized, EScSG-Al decreased from 7 to 1 , MRSS decreased from 18 to 14 . In the second case, the patient received TCZ for 6 months i/v, decrease of EScSG-Al 6.5 to 1, MRSS 33 to 21 were noted, the dose of CS was halved, he also continued treatment with cyclophosphamide.

Conclusion: The presented experience cannot be called completely positive; maybe this can be improved by earlier prescribing of medicines. An attempt to off-label use of biological cannot be rejected in pediatric rheumatology since sometimes this is the only chance for our patients to survive.

\section{Disclosure of Interest}

None declared

\author{
AB034 \\ latrogenic cushing after local infiltration with triamcinolone \\ acetate. About two cases \\ N. Palmou ${ }^{1}$, M. Bertholt ${ }^{2}$, C. Naranjo ${ }^{2}$, H. Ortega ${ }^{3}$, A. López ${ }^{4}$ \\ ${ }^{1}$ Rheumatology; ${ }^{2}$ Paediactric Endocrinology; ${ }^{3}$ Endocrinology; \\ ${ }^{4}$ Dermatology, Hospital Universitario Marquez De Valdecilla, Santander \\ (Cantabria), Spain \\ Correspondence: N. Palmou \\ Pediatric Rheumatology 2020, 18(Suppl 2):AB034
}

Objectives: To describe two cases of Cushing's Syndrome secondary to local infiltration with triamcinolone acetate, presented at our Hospital during 2019

Methods: CASE 1: 6-year-old girl referred, for asthenia, polyphagia, weight gain ( $4 \mathrm{~kg}$ in 30 days) and progressive face and abdomen oedema, one month in evolution. It associates high TA (129/ $92 \mathrm{mmHg}$ ) and frontal headache (without alarm data). The only antecedent: triamcinolone acetonide depot infiltration in ankle's ganglion (equipotent $x 4: 16 \mathrm{mg} / \mathrm{m} 2$ ) one week before starting symptoms .No family history.CASE 2: 7-year-old gir ,father with psoriasis without joint involvement. Consultation in July 2019 to traumatology for right knee pain with functional impotence after trauma .In ultrasound and MRI, sinovial hypertrophy is visualized, with minimal joint effusion. Referred to Rheumatology, she was diagnosed by Polyarticular Juvenile Idiopathic Arthritis. In the analysis, hypochromic microcytic anaemia and PCR $3.6 \mathrm{mg} / \mathrm{dl}$ stand out. Haematology rules out haematological process and starts treatment with oral iron. She received corticosteroid treatment:Oral prednisolone(equipotential $\mathrm{x} 5$ :supposes $31.5 \mathrm{mg} / \mathrm{m} 2$ for 20 days and descending regimen) and intra-articular infiltration (knee and ankles) with Triamcinolone acetonide depot (equipotent $x 4$ : supposes $432.4 \mathrm{mg} / \mathrm{m} 2$ ). Also, she receives treatment with methotrexate, folic acid, tocilizumab and abatacept. From the start of the corticosteroid treatment, she presented a Cushing phenotype and weight gain ( $7 \mathrm{~kg}$ in 4.5 months). She did not have fatigue or any other symptom. It refers to paedriatic endocrine Results: CASE 1 Complementary ambulatory tests: leukocytosis (neutrophilia) and mild reticulocytosis, hypercholesterolemia $232 \mathrm{mg} /$ dl (HDL: 104mg/dl), Exploration: Age 6.59 years, height $122.3 \mathrm{~cm}$ (0.5SDS), weight 27.2kg (0.76SDS), P/T 107.97\%, BMI 18.19 (0.46SDS). TAS $121 \mathrm{mmHg} \quad(\mathrm{p} 97) / T A D \quad 81 \mathrm{mmHg}$ (p89).Cushing phenotype, prepubertal. Complementary tests: Ultrasound without pathological findings. ACTH and cortisol suppressed (ACTH $<5 \mathrm{pg} / \mathrm{ml}$, cortisol $<1 \mu \mathrm{g} /$ dl). Suspecting exogenous Cushing síndrome secondary to local infiltration with corticosteroid, The study was expanded with normal imaging tests (brain MRI, normal bonescan, total-body PET with normal F18-FDG PET/CT) and ACTH test with a suppressed response. In monthly controls, ACTH and suppressed cortisol persist up to five months after the infiltration that initiates analytical recovery of the 
adrenal axis. Clinically, phenotype persists. Continue to follow up. CASE 2 Exploration: Age 8.12 years, TA $99 / 55 \mathrm{mmHg}$, height $125.1 \mathrm{~cm}$ (-0.73SDS), Weight 37.4kg (1.28SDS), P/T 138.9\%, BMI 23.9 (2.14SDS). Good general condition, Cushing phenotype (full moonface, globular abdomen, few stretch marks in the inner thighs). Prepubertal. Complementary tests: ACTH plasma $17 \mathrm{pg} / \mathrm{ml}$, Cortisol serum $10.9 \mu \mathrm{g} / \mathrm{dl}$, Suppression test with normal dexamethasone. 24-hour cortisoluria decreased.

Conclusion: DISCUSSION: The use of intra-muscular or intra-synovial triamcinolone acetate is approved in children from 6 years. There are few cases described that develop a secondary Cushing syndrome. We found a systematic review describing 18 cases of Cushing after infiltrations and injuries in scars. Although there are no clear recommendations regarding its use in paediatrics, we must be cautious, use the lowest effective dose and adequately monitor these patients.

\section{Disclosure of Interest}

None declared

\section{AB035 \\ Successful haplo-identical allogenic bone marrow transplantation in a child with refractory systemic Juvenile Idiopathic Arthritis \\ G. Morelle, D. Moshous, P. Quartier \\ Paediatric Hematology-Immunology and Rheumatology Department, Hôpital Necker-Enfants Malades, Paris, France \\ Correspondence: $\mathrm{G}$. Morelle \\ Pediatric Rheumatology 2020, 18(Suppl 2):AB035}

Introduction: Some patients with systemic juvenile idiopathic arthritis (sJIA) and severe, refractory disease achieved remission through intensive immunosuppressive treatment followed by autologous hematopoietic stem cell transplantation (HSCT). However, disease relapsed in most cases. More recently selected SJIA patients received allogenic HSCT from a HLA-identical sibling or a HLA matched unrelated donor. While most transplanted patients achieved sustained sJIA remission off-treatment, the procedure-related morbidity was high.

Objectives: We try to demonstrate that haplo-identical allogenic HSCT in refractory SJIA can be an alternative treatment for refractory SJIA

Methods: We report the case of a child who was successfully treated with haplo-identical allogenic HSCT.

Results: A girl presented SJIA since the age of 15 months with a severe disease course. She was refractory to the combination of methotrexate and steroids to anti-interleukin(IL)-1, then anti-IL-6, tumor necrosis factor alpha inhibitors and thalidomide. Therefore allogenic HSCT was considered. In the absence of any possible HLA matched donor, a multidisciplinary team assessed carefully risks and benefits of an alternative graft. Given the high disease burden and treatment related toxicity the indication for a haploidentical HSCT from her mother was validated. The patient was. treated with intensive immunosuppression and received a T replete bone marrow graft at the age of 3.7 years. Conditioning contained Rituximab, Alemtuzumab, Busulfan, and Fludarabine, as well as Cyclophosphamide at D+3 and +4 post HSCT for GHVD prophylaxis, followed by Cyclosporine A and Mycophenolate Mofetil. Post HSCT complications included severe infections, grade 3 intestinal graft versus host disease, autoimmune thyroiditis, and immune thrombocytopenia. Three years after HSCT, the child was alive and well, notwithstanding persistent hypothyroidy requiring substitution. Immune thrombocytopenia had resolved. Most importantly, sJIA was in complete remission, off immunosuppressive drugs.

Conclusion: Allogenic HSCT may be a therapeutic option, even with a HLA haplo-identical donor, in patients with inflammatory diseases such as SJIA. Despite increased experience with this treatment, the risk of life-threatening complications restrains its indication to selected patients with severe, refractory diseases.

\section{AB036}

Analysis of published randomized controlled trials in pediatric patients with rheumatic inflammatory diseases treated with biologics

T. Welzel ${ }^{1,2}$, C. Winskill ${ }^{3}$, N. Zhang ${ }^{3}$, A. Woerner ${ }^{2}$, M. Pfister ${ }^{1,3}$

${ }^{1}$ Pediatric Pharmacology and Pharmacometrics; ${ }^{2}$ Pediatric

Rheumatology, University Children's Hopital Basel (UKBB), University of

Basel, Basel, Switzerland; ${ }^{3}$ Certara, Princeton, New Jersey, United States

Correspondence: $T$. Welzel

Pediatric Rheumatology 2020, 18(Suppl 2):AB036

Introduction: The same biologic disease modifying antirheumatic drugs (bDMARDs) are prescribed in adult and pediatric rheumatology. Due to age-dependent changes, disease course, and pharmacokinetic (PK) processes pediatric patients with inflammatory rheumatic diseases (PiRD) differ from adult rheumatology patients.

Objectives: A systematic literature review was performed to assess current knowledge about bDMARDs use in PiRD patients by analyzing published randomized controlled trials (RCTs) in this pediatric population.

Methods: A systematic literature search for RCTs in PiRD with bDMARDs intervention such as rituximab, abatacept, anakinra, canakinumab, rilonacept, tocilizumab, ustekinumab, secukinumab, adalimumab, etanercept, infliximab, certolizumab pegol, golimumab, belimumab, baricitinib, tofacitinib, upadacitinib was conducted on Medline, PubMed, EMBASE, the Cochrane Library, the US National Institutes of Health Ongoing Trials Register ClinicalTrials.gov and the EU Clinical Trials Register in March 2020. Abstracts from conferences and relevant studies were added. RCTs were included if (i) patients were aged $\leq 20$ years, (ii) patients had a previous defined pediatric rheumatic diagnosis and (iii) RCT met predefined outcomes. Studies were excluded in case of (i) observational or single arm study or (ii) sample size $\leq 5$ patients. Study design, location, duration, treatment, population, sample size, age criteria, gender, concomitant treatments and primary outcome was extracted.

Results: Out of 550 screened references, 62 references reporting 35 unique RCTs in PiRD. All 35 RCTs reported efficacy while 34/35 RCTs provided safety outcomes and 15/35 RCTs provided PK data. Ten of 17 reviewed bDMARDs are approved for PiRDs by the Food and Drug Administration (FDA). Of these, seven had $\leq 2$ RCTs. The most common intervention was TNF inhibitors (63\%), IL-1 inhibitors (17\%) and IL-6 inhibitors (8\%). No RCTs with published results were identified for rituximab, secukinumab, certolizumab pegol, baricitinib, or upadacitinib. In patients with juvenile idiopathic arthritis (JIA) 26/35 RCTs were conducted. The remaining 9 RCTs were performed in nonJIA patients including plaque psoriasis, Kawasaki Disease (KD), systemic lupus erythematosus and idiopathic uveitis. In JIA-RCTs, the control arm was mainly placebo and the concomitant treatments were either methotrexate, NSAIDS or corticosteroids. Non-JIA patients received mostly NSAIDS. A majority of JIA-RCTs were global studies or otherwise conducted in Europe, the US, or Japan. The non-JIA RCTs took place in North America, Europe or globally and for KD mainly in Asia or the US. Currently three Phase III RCTs are investigating baricitinib and one Phase III global RCT investigating tofacitinib. Further, there are recruiting studies for secukinumab and certolizumab pegol in psoriasis, for adalimumab in JIA-associated uveitis, for abatacept and etanercept in JIA.

Conclusion: Despite the FDA Modernization Act and support of major pediatric rheumatology networks, such as the Pediatric Rheumatology Collaborative Study Group (PRCSG) and the Pediatric Rheumatology International Trials Organization (PRINTO), which resulted in bDMARDs approval for PiRD indications, there are limited RCTs in pediatric rheumatology. As bDMARDs therapy response is influenced by age-dependent changes, PK processes and disease course it is important to consider developmental changes in bDMARDs use in PiRD patients. As such it is critical to collaborate and conduct randomized clinical trials in PiRD patients with the goal to appropriately investigate PK, efficacy and safety in bDMARDs used in pediatric rheumatology. 
Disclosure of Interest

None declared

\section{AB037}

Efficacy and safety on the use of bisphosphonates for secondary osteoporosis in paediatrics

S. Odorico ${ }^{1}$, E. Jeziorski ${ }^{2}$, F. Rivier ${ }^{3}$, T.-A. Tran ${ }^{1}$

${ }^{1}$ Pediatrics, Nîmes University Hospital, Nîmes; ${ }^{2}$ Pediatrics; ${ }^{3}$ Pediatric

Neurology, Montpellier University Hospital, Montpellier, France

Correspondence: T.-A. Tran

Pediatric Rheumatology 2020, 18(Suppl 2):AB037

Introduction: The use of bisphosphonates has increased in paediatrics in the last twenty years.

Objectives: The study describes efficacy and safety of bisphosphonate therapy for secondary osteoporosis in children in Montpellier and Nimes University Hospitals, France.

Methods: In our retrospective study, all patients treated with bisphosphonates for secondary osteoporosis, between January 2012 and August 2018, were included, using medical files. The main endpoint was efficacy using fracture rate, bone mineral density (BMD) change, and bone pain frequency before and after treatment. Adverse effects were also analysed.

Results: 77 children, median age [IQR 25\%>75\%] of 14 years [11-15 years], with secondary osteoporosis: immobility $(n=63)$, nutritional diseases $(n=5)$, corticosteroids $(n=7)$, sickle cell anaemia $(n=1)$, growth hormone deficiency $(n=1)$, were included. The median duration of treatment [IQR $25 \%>75 \%]$ was 9 months [1-25 months]: 25 using zoledronate, 52 using pamidronate. Fracture rate significantly decreased from $41.6 \%$ to $5.2 \%$ and bone pain frequency significantly decreased from $57 \%$ to $26 \%(p<0.01)$. Lumbar spine BMD Z-score significantly improved by $0.74(p<0.01)$. Adverse events were reported for $79.2 \%$ of patients: flu-like symptoms (65\%), hypocalcaemia $(44.2 \%)$ and hypophosphatemia (27.3\%). Only one serious hyponatremia occurred corresponding to a patient with renal failure before treatment.

Conclusion: Our results were similar to those previously published: bisphosphonates are effective and safe for secondary osteoporosis in children. The use of bisphosphonates beforehand requires dietary measures (vitamin D and calcium supplementation). Growth periods amplify bisphosphonates effects as we have shown with a maximum mean increase in the early years of life: this suggests a better time to start treatment in young people. Furthers systematic collection on efficacy and safety parameters for each bisphosphonates drug should confirm these data.

\section{Disclosure of Interest}

None declared

\section{AB038}

Cutaneous or systemic polyarteriitis nodosa?

M. Nashawi', F. Blankenburg ', A. Heinkele', F. Glowatzki ', C. Strinkorb' ${ }^{1}$, F. Noll', D. Hübner ${ }^{1}$, A. Enninger ${ }^{2}$, S. Dammann², T. Heigele², P. Ostendorf ${ }^{2}$, P. Driesch ${ }^{3}$, C. Eisenbeis ${ }^{3}$, T. Hospach

${ }^{1}$ Pediatric rheumatology department; ${ }^{2}$ Pediatric gastroenterology department; ${ }^{3}$ Dermatology, Klinikum Stuttgart, Stuttgart, Germany Correspondence: M. Nashawi

Pediatric Rheumatology 2020, 18(Suppl 2):AB038

Introduction: Polyarteriitis nodosa (PAN) is a rare vasculitis of medium and small vessels. There is ongoing controversy about if systemic and cutaneous forms are different diseases or only a spectrum of symptoms

Objectives: Presenting a case of cutaneous polyarteritis nodosa and discussing the possibility evolving to a systemic disease

Methods: We introduce a 13-year-old girl patient who has been admitted to our clinic with suspicion of an erythema nodosum. She had painful subcutaneous nodules for 4 weeks, especially on the lower extremities and her face. Macroscopically, central necrotizing skin rashes could be seen. She had frank arthritis of both knee and ankle joints. The comprehensive serological diagnosis (including hepatitis serology and anti-streptolysin titer) were normal except for a slight increase in CRP $0,9 \mathrm{mg} / \mathrm{dl}$ and ESR $36 \mathrm{~mm} / \mathrm{h}$. The patient also complained of abdominal pain and bloody stools. Calprotectin was $3613 \mu \mathrm{g} / \mathrm{g}$. A Gastro-coloscopy revealed a small mariske and a minimal inflammation of the lleocecal valve, without signs of vasculitis or chronic bowel disease. A skin biopsy revealed leukocytoclastic vasculitis of the small arteries. Angiography of the intestinal arteries was rejected by the family. Initially we started a treatment with methylprednisolone pulses followed by oral prednisolone. The patient showed a very good response with quick resolution of the skin symptoms and abdominal pain. The medication could be quickly tapered and discontinued at full remission after one month

Results: PAN is classified as a cutaneous PAN (CPAN) when there are exclusive skin manifestations, besides arthralgia or arthritis. A systemic PAN must be diagnosed with the involvement of internal organs. However, cutaneous PAN may evolve into systemic PAN. In our patient, the skin and joints were primarily affected. If the existing gastrointestinal complaints are part of a systemic PAN or chronic bowel disease could not be cleared yet, due to refusal of further investigations.

Conclusion: CPAN must be considered as a suspected diagnosis in patients with necrotizing skin nodules. As transition of the cutaneous into the systemic form cannot be predicted regular monitoring is mandatory.

\section{Disclosure of Interest \\ None declared}

\section{AB039}

Efficacy of anakinra in reducing giant coronary aneurysms in refractory Kawasaki disease: a case report

M. E. Pinto, A. Meneghel, G. Martini, M. Patti, F. Zulian

Pediatric Rheumatology, University-Hospital of Padua, Padua, Italy

Correspondence: $M$. E. Pinto

Pediatric Rheumatology 2020, 18(Suppl 2):AB039

Introduction: Coronary artery aneurysms (CAAs) are the most serious complication in Kawasaki Disease (KD). Treatment with intravenous immunoglobulin (IVIG) significantly reduces the risk of CAAs. However, up to $20 \%$ of cases are IVIG resistant with a higher risk of cardiovascular complications. Currently several second-line treatments are available for refractory KD. Nonetheless, the existing literature is still unable to identify which treatment is the most effective. Recent studies suggest that a IL-1 receptor antagonist (anakinra) may be an effective therapy in refractory KD.

Objectives: We report the case of a 3 year-old boy diagnosed with $\mathrm{KD}$ refractory to conventional treatment, who developed giant CAAs successfully treated with subcutaneous (sc) anakinra.

Methods: Case report.

Results: A 3 year-old boy was referred to our Pediatric Rheumatology Unit 18 days after the onset of a Typical refractory KD. He had been previously treated at a local hospital with two doses of IVIG $(2 \mathrm{~g} / \mathrm{kg})$, infused respectively 8 and 11 days after the onset of the fever. Afterwards, given the persisting fever, doses of pulse intravenous (IV) methylprednisolone (MPDN $30 \mathrm{mg} / \mathrm{kg} /$ day) have been used for 3 days followed by oral prednisone $(2 \mathrm{mg} / \mathrm{kg} /$ day). Treatment with Acetylsalicylic Acid (60 mg/Kg/day q8h) was also started. Following a transient defervescence the day after the first IV pulse MPDN, fever relapsed and the echocardiography showed CAAs of Left Main Coronary Artery (LMCA), Left Anterior Descending (LAD) and Right Coronary Artery (RCA) with z score 7.4, 11.7, 4.1 respectively. Laboratory tests showed a persistent elevation of inflammatory markers (WB 20810/mm3, PMNc 12860/mm3, CRP $50 \mathrm{mg} / \mathrm{L}$ ). The patient was then transferred to our Unit. Therapy with sc anakinra ( $2 \mathrm{mg} / \mathrm{kg} /$ day) was started with rapid defervescence and reduction of CRP. Echocardiography was performed 5 days after and it showed a worsening of all 
coronary aneurisms, with evidence of giant CAAs (LMCA, LAD, RCA z score: 12.7, 15.1, 10.4). The anakinra dosage was increased to $4 \mathrm{mg} /$ $\mathrm{kg} /$ day and anticoagulant therapy with warfarin was started. Echocardiography showed a significant reduction of CAAs (LMCA, LAD, RCA z score: $10.1,12.29,7.9) 6$ days after, with complete resolution of giant CAA (LMCA, LAD, RCA z score 7.4, 9.4, 7.17) 13 days after. Therapy with sc Anakinra (4 mg/kg/day) was continued for a month, then it was gradually tapered down and completely stopped after 4 months. Follow up is still ongoing but serial echocardiographies confirmed a persistent positive remodelling of all CAAs.

Conclusion: It has been reported that therapy with Anakinra in refractory KD leads to a rapid and sustained improvement in clinical and biological inflammation. However, its effects on the size of CAAs are still unclear. In our patient with refractory $K D$, subcutaneous Anakinra was quickly effective in controlling the systemic and laboratory inflammatory aspects of the disease and, at a higher dose, led to a rapid and significant reduction of CAAs with complete resolution of giant aneurisms. No side effects were reported. Therefore Anakinra can represent a valuable and safe treatment approach in refractory KD patients, reducing cardiovascular damage. Further studies are needed to reach a standardized treatment protocol to better define the optimal dosage, duration and tapering of Anakinra therapy, facilitating improvement and uniformity of care.

\section{References}

1) Tremoulet $\mathrm{AH}$ et al. Rationale and study design for a phase I/lla trial of anakinra in children with Kawasaki disease and early coronary artery abnormalities (The ANAKID Trial). Contemp Clin Trials. 2016 May; 48: 7075.

2) Kone-Paut I et al. The use of interleukin 1 receptor antagonist (anakinra) in Kawasaki disease: A retrospective cases series. Autoimmun Rev 2018 Aug;17(8):768-774.

Disclosure of Interest

None declared

\section{AB040}

Kawasaki disease complicated by acute kidney injury: a case report

A. Mauro, F. Orlando, M. Tardi, R. Borrelli, L. Martemucci, R. Sottile

Pediatrics, AORN SANTOBONO-PAUSILIPON, Naples, Italy

Correspondence: A. Mauro

Pediatric Rheumatology 2020, 18(Suppl 2):AB040

Introduction: Kawasaki disease (KD) is a febrile vasculitis that affect young children $<5$ years of age. Diagnosis of KD is a clinical challenge, given the wide variety of clinical presentations. There are few reports of acute kidney injury (AKI) in KD, defined as serum creatinine level elevation to more than 1.5 times of baseline level. Objectives: To describe the case of Kawasaki Disease complicated by AKI

Methods: A 5-year-old female was admitted to our Rheumatology Unit with persistent fever (6 days), widespread polymorphous exanthema, change in lips and in oral mucosa. Family history was unremarkable. She had no chronic underlying disease nor history of previous hospitalization. At admission, she appeared stable. Body temperature was $38.9^{\circ} \mathrm{C}, \mathrm{O} 2$ saturation was $96 \%$ in ambient area blood pressure was 118/75 mm Hg, heart rate was $90 \mathrm{bpm}$, respiratory rate was 21 breaths per minute. On examination she presented widespread polymorphous exanthema, changes in lips and in oral mucosa, cervical lymphadenopathy and bilateral conjunctival injection.

Results: Exams revealed: white blood cells 11980/ul, Hb $10.4 \mathrm{~g} / \mathrm{dL}$, platelets $389.000 / \mu \mathrm{l}$, albumin $2.5 \mathrm{~g} / \mathrm{dL}$, serum sodium $126 \mathrm{mEq} / \mathrm{L}$, serum chloride $90 \mathrm{mEq} / \mathrm{L}$. Transaminases were in normal range. creatinine was $1.5 \mathrm{mg} / \mathrm{dL}$ (nv 0.20-0.45). Inflammatory markers were strongly elevated (CRP $108 \mathrm{mg} / \mathrm{L}$, ESR $45 \mathrm{~mm} / 1 \mathrm{~h}$, ferritin
$1421 \mathrm{ng} / \mathrm{mL}$ ). Fluid balance evidenced oliguria. She underwent echocardiography that showed no dilatated coronaries or pericoronary hyperechogenicity. Abdomen ultrasound and ChestRX were negative. Intravenous Immunoglobulin (IGIV) at the dose of $2 \mathrm{~g} / \mathrm{Kg}$ and antinflammatory dosage of ASA were started at 7 days of fever. After the first dose of IVIG, fever, conjunctivitis and polymorphous rash disappeared. Blood tests revealed a reduction of inflammatory markers (CRP $89.9 \mathrm{mg} / \mathrm{L}$, ESR $28 \mathrm{~mm} / 1 \mathrm{~h}$ ) and creatinine $(0.69 \mathrm{mg} / \mathrm{dL})$. She presented also normalization of fluid balance.

Conclusion: Acute kidney injury (AKI) has rarely been reported in patients with Kawasaki disease (KD). The pathogenic mechanism underlying renal involvement in patients with $\mathrm{KD}$ and $\mathrm{AKI}$ is unknown. A recent review concluded that patients with $K D$ and $A K I$ had good outcomes. The reported patient presented rapid improvement of clinical and laboratory parameters after only one dose of IGIV.

- Kidney Disease: Improving Global Outcomes (KDIGO) Acute Kidney Injury Work Group. KDIGO Clinical Practice Guideline for Acute Kidney Injury. Kidney Int 2012;2:1-138.

- Watanabe T. Clinical features of acute kidney injury in patients with Kawasaki disease. World J Clin Pediatr. 2018;7(3):83-88. Published 2018 Aug 30.

\section{Disclosure of Interest}

None declared

\section{AB041}

Paediatric extra-pulmonary large vessel arteritis, a forme fruste of pediatric Behcet's disease?

S. A. Ganu', R. K. Kumar', S. Balan'

${ }^{1}$ Department of Clinical Immunology and Rheumatology; ${ }^{2}$ Dept of Pediatric Cardiology, Amrita Institute of Medical Sciences, Kochin, India

Correspondence: S. A. Ganu

Pediatric Rheumatology 2020, 18(Suppl 2):AB041

Introduction: Prevalence of Behcet's disease in children is not known, but is probably very low. Extra-pulmonary large vessel arteritis in these cases is even rarer as a presenting manifestation.

Objectives: To report two cases of paediatric extrapulmonary large vessel arteritis with a 'Behcet like disease'.

Methods: We present case reports of two cases who presented to paediatric rheumatology OPD to our department. Ms. F, a 16 year old girl was referred to us with history of short duration of fever, generalized lymphadenopathy, neutrophilic leucocytosis, thrombocytosis, hyperglobulinemia and high inflammatory markers. On detailed history and examination she was found to have a healed palatal ulcer and her maternal aunt was found to have a history of recurrent oral ulcer, genital ulcer and enthesitis. Patient's Montoux test was positive but the gene expert for MTB was negative. MD-CT showed a circumferential thickening of aorta, subclavian and bilateral renal artery with stenosis at origin of both renal arteries indicating a vasculitis. Few necrotic nodes were also noted in lungs. Lymph node biopsy suggested a reactive hyperplasia. Tissue typing showed presence of HLA B 44, B 51. She improved clinically with oral Prednisolone and Mycophenolate Mofetil and had no recurrence till her recent follow up visit. Second case, master FKN an 11 year old child was referred to us with a background of 2 week history of fever, non migratory arthritis, raised inflammatory markers and a symptomatic severe aortic regurgitation with pandiastolic flow reversal on $2 \mathrm{D}$ echo. His evaluation showed negative Montoux, normal IGG4 levels and HLA B35 B 51 on tissue typing. His aortic wall thickness resolved with $1 \mathrm{mg} / \mathrm{kg}$ oral Prednisolone and Mycofenolate mofetil.

Results: Both these cases have features similar to Behcet's disease. These cases do not fulfil ISG, ICBD 2014 or ICBD criteria for pediatric Behcet's disease. However, the aortitis and other clinical features responded well to the treatment in both cases. 
Conclusion: Paediatric case with extra-pulmonary Large vessel arteritis that do not meet criteria for Behcet's disease but have specific clinical or laboratory features do respond well to immunosuppression. Therefore, after ruling out other causes of the large vessel vasculitis, a possibility of form fruste of Behcet's disease should be under consideration.

Disclosure of Interest: None declared

\section{Publisher's Note}

Springer Nature remains neutral with regard to jurisdictional claims in published maps and institutional affiliations.

\section{About this supplement}

These abstracts have been published as part of Pediatric Rheumatology, Volume 18, Supplement 2, 2020: Proceedings of the 26th European Paediatric Rheumatology Congress (PReS 2020). The full contents of the supplement are available at https://ped-rheum. biomedcentral.com/articles/supplements/volume-18-supplement-2.

Please note that this is part 2 of 2 .
Ready to submit your research? Choose BMC and benefit from:

- fast, convenient online submission

- thorough peer review by experienced researchers in your field

- rapid publication on acceptance

- support for research data, including large and complex data types

- gold Open Access which fosters wider collaboration and increased citations

- maximum visibility for your research: over $100 \mathrm{M}$ website views per year

At $\mathrm{BMC}$, research is always in progress.

Learn more biomedcentral.com/submissions 